Alternative testing strategy to quantify inter-species, inter-ethnic, and interindividual variation in bioactivation and toxicity of food-borne alkenylbenzenes and pyrrolizidine alkaloids

Jia Ning 


\section{Thesis committee:}

\section{Promotor}

Prof. Dr I.M.C.M. Rietjens

Professor of Toxicology

Wageningen University \& Research

\section{Co-promotor}

Dr M. Strikwold

Associate professor of Safety in the Food Chain

Van Hall Larenstein University of Applied Sciences, Leeuwarden, the Netherlands

\section{Other members}

-Prof. Dr E.J.M. Feskens, Wageningen University \& Research

-Prof. Dr J. Kleinjans, Maastricht University

-Dr N.I. Kramer, Utrecht University

-Dr A.A.C.M. Peijnenburg, RIKILT - Wageningen University \& Research

This research was conducted under the auspices of the Graduate School VLAG (Advanced studies in Food Technology, Agrobiotechnology, Nutrition and Health Sciences). 


\section{Alternative testing strategy to quantify inter-species, inter-ethnic, and inter- individual variation in bioactivation and toxicity of food-borne alkenylbenzenes and pyrrolizidine alkaloids}

\section{Jia Ning}

Thesis

submitted in fulfilment of the requirements for the degree of doctor

at Wageningen University

by the authority of the Rector Magnificus,

Prof. Dr A.P.J.Mol,

in the presence of the

Thesis Committee appointed by the Academic Board

to be defended in public

on Monday 16 September 2019

at 13:30 p.m. in the Aula. 
Jia Ning

Alternative testing strategy to quantify inter-species, inter-ethnic, and inter-individual variation in bioactivation and toxicity of food-borne alkenylbenzenes and pyrrolizidine alkaloids

273 pages

PhD thesis, Wageningen University, Wageningen, the Netherlands (2019)

With references, with summary in English

ISBN: 978-94-6343-999-2

DOI: https://doi.org/10.18174/478360 


\section{Table of Contents}

\section{Chapter 1}

General introduction

\section{Chapter 2}

Risk assessment of genotoxic and carcinogenic alkenylbenzenes in botanical containing products present on the Chinese market

\section{Chapter 3}

Study on inter-ethnic human differences in bioactivation and detoxification of estragole using physiologically based kinetic modelling

\section{Chapter 4}

Use of physiologically based kinetic modelling-facilitated reverse dosimetry to convert in vitro cytotoxicity data to predicted in vivo liver toxicity of lasiocarpine and riddelliine in rat

\section{Chapter 5}

Use of an in vitro-in silico testing strategy to predict inter-species and interethnic human differences in liver toxicity of the pyrrolizidine alkaloids lasiocarpine and riddelliine

\section{Chapter 6}

Integrating physiologically based kinetic (PBK) and Monte Carlo modelling to predict inter-individual and inter-ethnic variation in bioactivation and liver toxicity of lasiocarpine

\section{Chapter 7}

General discussion

Summary

Acknowledgements

About the author

List of publications

Overview of completed training activities 
<smiles>CCC(C)C(CC)C(C)C</smiles> 

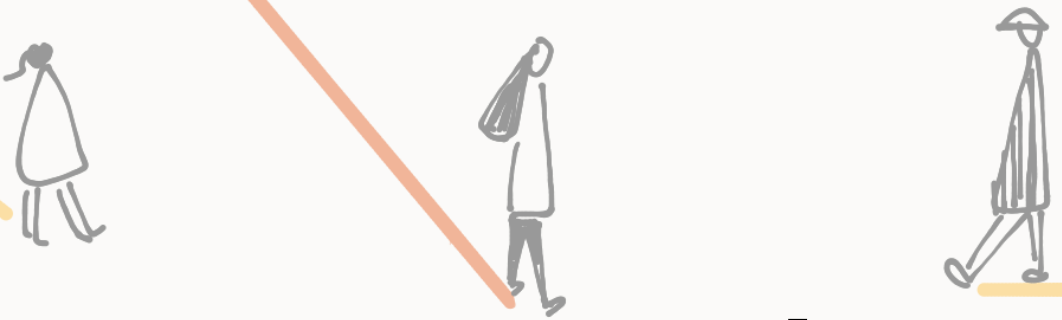

Chapter 1

General Introduction
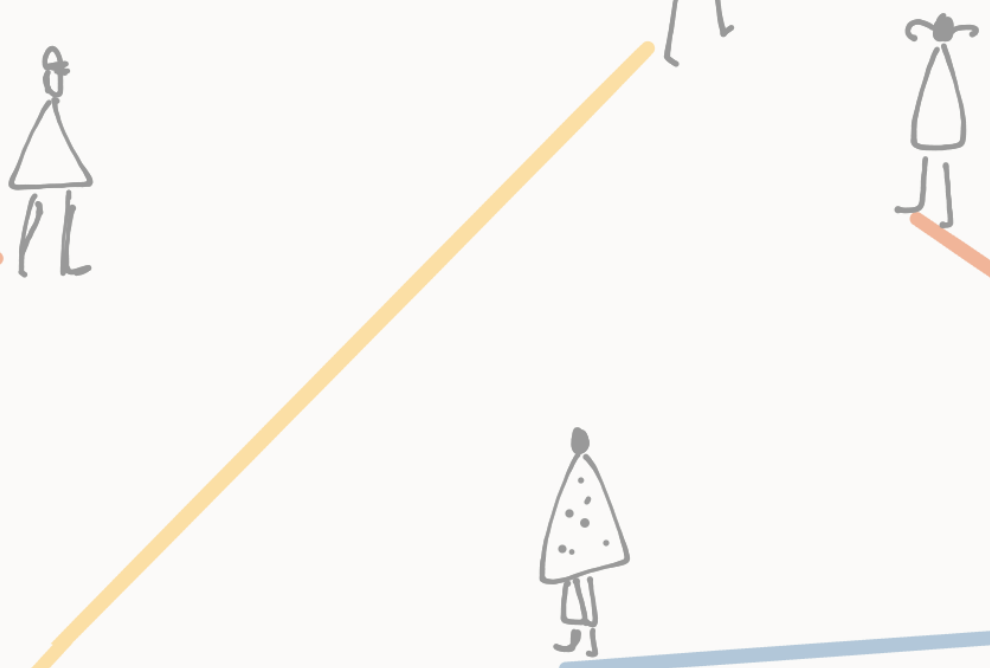


\section{Background}

Use of botanical preparations in China has a long history, which dates back to 2,000 years ago (Liu et al. 2015). From ancient times to the present, a health concept which is a combination of diet, the use of herbal containing products including plant food supplement (PFS), traditional Chinese medicine (TCM) and herbal teas, acupuncture and physical exercise has been integrated as part of Chinese culture (Liu et al. 2015). Thus, a wide range of botanical preparations has been integrated into the daily diet for the Chinese population to maintain general wellness or prevent illness. In China, botanical preparations are easily accessible with relatively low prices. Meanwhile, many consumers consider that "natural" equals "safe" (Rietjens et al. 2008). However, botanical preparations may contain compounds that are of concern for health. Some natural toxins have even been shown to be genotoxic and carcinogenic (EFSA 2012a; Van den Berg et al. 2011a). Examples are the alkenylbenzenes and pyrrolizidine alkaloids (PAs), studied in the present thesis. The safety and risk assessment of such compounds is complicated and methods for evaluation of these type of compounds are not yet internationally harmonised (EFSA 2005). Several risk assessments of the presence of alkenylbenzenes and PAs in PFS and herbal teas have been performed for the Caucasian population (Al-Malahmeh et al. 2017a; Alajlouni et al. 2016a; Alajlouni et al. 2017; Chen et al. 2017; Dusemund et al. 2018; EFSA 2017; Van Den Berg et al. 2014). Given that possible inter-ethnic differences in toxicokinetics and toxicodynamics for these compounds may exist and that a risk assessment for the Chinese population is currently lacking, the aim of the present thesis was to perform a mode-of-action (MOA) based risk and safety assessment for two groups of natural toxins, namely alkenylbenzenes and PAs for the Chinese population as compared to the overall Caucasian population using the Margin of Exposure (MOE) approach, physiologically based kinetic (PBK) modelling-based reverse dosimetry and Monte Carlo simulation. The US Environmental Protection Agency (EPA) provides the definition of the term MOA for a carcinogenic compound as "a sequence of key events and processes, starting with interaction of an agent with a cell, proceeding through operational and anatomical changes, and resulting in cancer formation" (EPA 2005). The ultimate goals for MOA based risk assessment are determination of key events and deriving dose-response and time-course data of the measurable parameters which are associated with those key events (Edwards and Preston 2008). Based on insight in the key events in the MOA, dose-response and time-course data for the inter-ethnic and inter-individual differences can be determined focussing on these key events. Thus, this work should finally provide insights in the existence of inter-ethnic and inter-individual differences for the possible adverse health effects of these carcinogenic and genotoxic botanical ingredients and when risk management action is needed.

\section{The model compounds tested in the present thesis}

In the present thesis two groups of natural toxins were selected, i.e. alkenylbenzenes and PAs. These two groups of compounds are of possible concern in the botanical preparations because of their genotoxic and carcinogenic properties (Van den Berg et al. 2011a). 


\subsection{Alkenylbenzenes}

\subsubsection{Background}

Figure 1 shows the six alkenylbenzenes of interest studied in the present thesis. These alkenylbenzenes are all allylalkoxybenzenes. The common structural features of allylalkoxybenzene compounds are an allylbenzene structure with one or more alkoxy ring substituents. Three compounds of this group contain a 3,4-methylenedioxy substituent and may contain additional methoxy substituents, i.e. safrole (3,4-methylenedioxyallylbenzene), myristicin (5-methoxy-3,4-methylenedioxyallylbenzene) and apiol (2,5-dimethoxy-3,4methylenedioxyallylbenzene). Three other compounds only contain methoxy substituents, i.e. estragole (4-methoxyallylbenzene), methyleugenol (3,4-dimethoxyallylbenzene) and elemicin (3,4,5-trimethoxyallylbenzene) (JEFCA 2009). These alkenylbenzenes are naturally present in herbs and spices such as anise, nutmeg, fennel, mace and basil (Rietjens et al. 2014). Human exposure to these alkenylbenzenes occurs through the consumption of products containing these herbs or their extracts (Smith et al. 2002). In the alkenylbenzene group, several compounds are proven to be genotoxic and carcinogenic and to cause liver tumors in experimental animals when given at high doses (Miller et al. 1983; Phillips et al. 1984; Randerath et al. 1984). A number of studies reported that the carcinogenicity of these alkenylbenzenes is related to their bioactivation by cytochromes P450 and sulfotransferases, leading to the formation of 1'-sulfooxy metabolites, which are able to bind to DNA, forming DNA adducts (Al-Malahmeh et al. 2017c; Al-Subeihi et al. 2012; Alajlouni et al. 2016b; Martati et al. 2012; Punt et al. 2009; Wiseman et al. 1987). The alkenylbenzenes listed in Figure 1 are considered as carcinogenic and genotoxic compounds. Figure 2 shows the metabolic pathways of estragole which are also relevant for the other alkenylbenzenes presented in Figure 1.

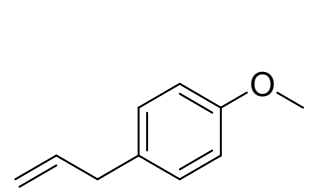

estragole<smiles>C=CCc1ccc2c(c1)OCO2</smiles>

safrole<smiles>C=CCc1ccc(OC)c(OC)c1</smiles>

methyleugenol<smiles>C=CCc1ccc2c(c1OC)OCO2</smiles>

myristicin<smiles>C=CCc1cc(OC)c(OC)c(OC)c1</smiles>

elemicin<smiles>C=CCc1cc(OC)c2c(c1OC)OCO2</smiles>

apiol

Fig. 1 Chemical structures of the six alkenylbenzenes of interest 


\subsubsection{Human exposure to alkenylbenzenes}

Human exposure to alkenylbenzenes may occur via the consumption of herbs and spices containing alkenylbenzenes and of botanical preparations such as PFS, traditional medicines and herbal teas (Al-Malahmeh et al. 2017a; Alajlouni et al. 2016a; Alajlouni et al. 2017; Smith et al. 2002; Van Den Berg et al. 2014; Van Den Berg et al. 2011b). The amount of alkenylbenzenes in herbs can vary substantially depending on the geographical region, plant maturity at harvest, harvesting technique, methods of processing and storage conditions (Smith et al. 2002). Thus, the daily intake of products containing alkenylbenzenes may differ for similar type of products from different origins. Data for the estimated daily intake for the Caucasian population are available but information for the average daily intake of alkenylbenzenes by the Chinese population are lacking. For example, for the Caucasian population, the Flavor and Extract Manufacturers Association (FEMA) reported an estimated daily intake (EDI) of estragole amounting to $10 \mu \mathrm{g} / \mathrm{kg}$ bw/day based on the annual production volume data of herbs, essential oils and flavourings containing estragole in the US (Smith et al. 2002). The Scientific Committee on Food (SCF) of the European Union estimated that the EDI of methyleugenol and estragole from all sources was $217 \mu \mathrm{g} / \mathrm{kg}$ bw/day and $72 \mu \mathrm{g} / \mathrm{kg}$ bw/day, respectively for a $60 \mathrm{~kg}$ person (SCF 2001a; SCF 2001b). The SCF also indicated that estragole and methyleugenol have been shown to be genotoxic and carcinogenic and that the presence of a threshold cannot be assumed for both substances, and that therefore a safe exposure limit could not be established. Consequently, the SCF indicated that reductions in exposure and restrictions in use levels of both compounds were indicated. As a consequence, the European Commission (EC) prohibited the use of estragole, methyleugenol and safrole as pure compound in foods in Regulation No 1334/2008 of the European Parliament and of the Council (EC 2008). However, alkenylbenzenes can still be naturally found in a variety of products such as PFS, herbal teas, pesto, tomato sauce and meat-based products (AlMalahmeh et al. 2017a; Al-Malahmeh et al. 2017b; Alajlouni et al. 2016a; Alajlouni et al. 2017; Siano et al. 2003; Van Den Berg et al. 2014; Van Den Berg et al. 2011b). A recent study, for example, reported EDIs of alkenylbenzenes resulting from consumption of pesto sauces containing basil purchased from the Dutch market to amount to $14.4-43.5 \mu \mathrm{g} / \mathrm{kg}$ bw/day (AlMalahmeh et al. 2017b). Thus, despite the prohibition of using estragole, methyleugenol and safrole as pure compounds in foods according to Regulation No 1334/2008 of the European Parliament and of the Council (EC 2008), daily consumption of food products naturally containing alkenylbenzenes still occurs.

\subsubsection{Absorption, Distribution, Metabolism and Excretion (ADME) of alkenylbenzenes}

A study in humans revealed that upon oral intake, alkenylbenzenes such as estragole are rapidly absorbed, metabolized, distributed and eliminated (Sangster et al. 1987). The metabolic fate of estragole was investigated in two volunteers at an oral dose of $1 \mu \mathrm{g} / \mathrm{kg}$ bw of methoxy- ${ }^{14} \mathrm{C}$ labelled estragole. It was shown that after 8 hours about $58 \%$ of estragole was excreted via urine and more than $11 \%$ of the radioactivity was found in the exhaled air. After 48 hours, approximately $70 \%$ radioactivity was recovered (Sangster et al. 1987). Figure 2 shows the metabolic pathways of estragole, which are similar to those for other structurally related alkenylbenzenes (Al-Malahmeh et al. 2017c; Al-Subeihi et al. 2012; Alajlouni et al. 
2016b; Martati et al. 2012; Punt et al. 2009). Following absorption in the gastrointestinal tract, alkenylbenzenes are distributed to the liver where the main metabolism occurs. $O$ demethylation, epoxidation and 3'-hydroxylation are recognized as detoxification pathways, leading to the metabolites 4-allylphenol, estragole-2',3'-oxide and 3'-hydroxyanethole, respectively. In vivo the estragole-2',3'-oxide is rapidly detoxified by epoxide hydrolases and/or GST and does not form DNA adducts (Guenthner and Luo 2001; Luo et al. 1992). Based on human and rat microsomal tissue incubations, it was found that $O$-demethylation of estragole also occurs to some extent in the lung and kidney of rat, whereas for humans no significant microsomal metabolism of alkenylbenzenes has been found for these organs. Also no conversion of estragole has been found in incubation with rat and human intestinal microsomes (Punt et al. 2008; Punt et al. 2009). The 1'-hydroxylation reaction, initiates a bioactivation pathway resulting in the formation of the proximate carcinogenic 1'hydroxymetabolite. For estragole this 1'-hydroxylation was shown to be mainly mediated by CYP 1A2 and 2A6, and to a small extent by CYP 2D6, 2C19 and 2E1, while for the related alkenylbenzenes, CYP 2A6 was shown to be the most important enzyme for safrole 1'hydroxylation, and for methyleugenol CYP 1A2 was reported to be the main enzyme for 1'hydroxylation (Jeurissen et al. 2004; Jeurissen et al. 2006; Jeurissen et al. 2007). The 1'hydroxymetabolite can be further conjugated with glucuronic acid or sulfate, or further oxidized forming an 1'-oxometablite. Of these pathways, glucuronidation and oxidation are considered to be detoxification pathways, whereas sulfation of the 1'-hydroxymetabolite results in the formation of an unstable and reactive electrophilic 1'-sulfooxymetabolite, which is able to bind with nucleophilic agents such as DNA and proteins, forming adducts (AlMalahmeh et al. 2017c; Al-Subeihi et al. 2012; Alajlouni et al. 2016b; Martati et al. 2012; Punt et al. 2009). The involvement of the 1'-sulfooxymetabolite metabolite in the carcinogenicity of the alkenylbenzenes was corroborated by the observation that co-exposure to a sulfotransferase inhibitor significantly inhibited induction of hepatocellular carcinomas in mice given an intraperitoneal injection of estragole (Wiseman et al. 1987).

Dose-dependent effects in metabolism of alkenylbenzenes have been shown in rodents. Anthony et al. (1987) reported that in rodents the relative extent of the $O$-demethylation pathway decreased, while the extent of glucuronidation increased with an increasing dose of estragole. This observation can be explained by the PBK model for estragole in male rat showing that $O$-demethylation is the major pathway, whereas saturation of this metabolic pathway at increasing dose level results in the relatively increased formation of the 1'hydroxymetabolite (Punt et al. 2008). Besides dose-dependent effects, species differences in metabolism have also been described. It has been shown that formation of the 1 'oxometabolite is a major conversion in humans whereas in rat this pathway is recognized as a minor metabolic route and the 1'-hydroxymetabolite is mainly detoxified by glucuronidation (Punt et al. 2009). 


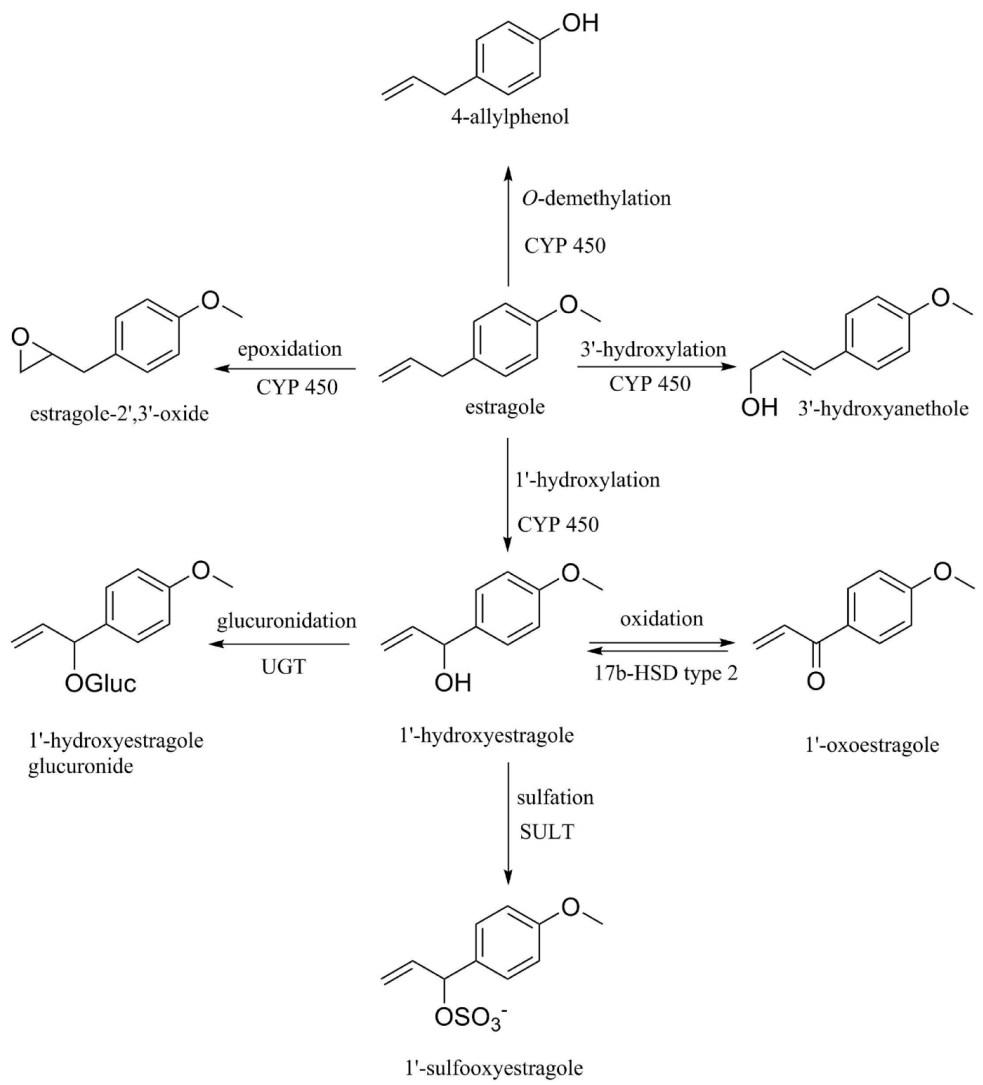

Fig. 2 Metabolic pathway of estragole in humans. CYP 450=cytochromes P450, UGT=glucuronosyltransferase, SULT=sulfotransferase, $17 \beta$-HSD type $2=17 \beta$-hydroxysteroid dehydrogenase type 2

\subsubsection{Genotoxicity and carcinogenicity of alkenylbenzenes}

The bioactivation pathway as described above has been confirmed by studies showing that DNA adduct formation is observed upon dosing rodents with alkenylbenzenes (Phillips et al. 1984; Randerath et al. 1984). Randerath et al. (1984) reported that the alkenylbenzenes are able to induce DNA adduct formation in female CD-1 mice liver $24 \mathrm{~h}$ after administration. Of the tested alkenylbenzenes, estragole, methyleugenol and safrole exhibited the strongest DNA binding activities. These results were comparable to the findings obtained in neonatal mice showing that DNA adducts formed in livers treated with the alkenylbenzenes, safrole, estragole and methyleugenol induced relatively high levels of adducts (Phillips et al. 1984). The formation of DNA adducts has also been reported for the 1'-hydroxymetabolites of estragole and safrole in vivo (Wiseman et al. 1987). Randerath et al. (1984) further showed the prevention of safrole-DNA adduct formation by pre-treatment of female CD-1 mice with the SULT inhibitor pentachlorophenol, indicating that DNA adduct formation strongly 
depends on SULT mediated catalysis. Besides DNA adduct formation, several in vitro and in vivo studies have shown that estragole, methyleugenol and safrole are able to induce unscheduled DNA synthesis (UDS) (Burkey et al. 2000; Chan and Caldwell 1992; Hasheminejad and Caldwell 1994; Howes et al. 1990). Moreover, Chan and Caldwell (1992) reported that the induction of UDS was higher in rat hepatocytes exposed to the 1'hydroxymetabolites of estragole, safrole and methyleugenol than to their parent compounds, indicating that the 1 '-hydroxymetabolites of alkenylbenzenes are the proximate carcinogenic metabolites. These observations also indicate that in rat hepatocytes the P450 mediated conversion of the parent alkenylbenzenes to their 1 '-hydroxymetabolites represents the rate limiting step in the bioactivation pathway. Overall, the results from the DNA adduct formation measurements and the UDS test suggested that the alkenylbenzenes studied in the present thesis are genotoxic compounds.

With respect to the carcinogenicity of the alkenylbenzenes, long-term carcinogenicity studies are available for estagole, methyleugenol and safrole. Estragole and its 1'-hydroxy metabolite induced liver tumor formation in mice upon intra peritoneal (i.p.) injection (Miller et al. 1983; Wiseman et al. 1987), subcuteneous (s.c.) injection (Drinkwater et al. 1976; Miller et al. 1983) or when administered via the diet (Miller et al. 1983). In these studies 1'hydroxyestragole was shown to be more potent than estragole (Miller et al. 1983). In addition to liver tumors, angiosarcomas and lung adenomas were also observed (Miller et al. 1983). Within the same study, methyleugenol was shown to induce hepatomas in male mice upon i.p. treatment for 12 weeks (Miller et al. 1983). In a 2-year NTP study, a dose-dependent increase in tumor incidence in the liver was observed in both male and female rats upon dosing methyleugenol by gavage at doses of 37,75 , and $150 \mathrm{mg} / \mathrm{kg}$ bw (NTP 2000). Additionally, male rats also developed renal tubule adenoma, malignant mesothelioma, fibroadenoma in the mammary gland and fibroma or fibrosarcoma (NTP 2000). Safrole was found to be carcinogenic in mice (Borchert et al. 1973; Miller et al. 1983) and rats (Miller et al. 1983; Wislocki et al. 1977). In these studies, mice and rats exposed to safrole by injections or diet developed hepatomas and lung adenomas (Borchert et al. 1973; Miller et al. 1983; Wislocki et al. 1977). Overall, estragole, methyleugenol and safrole were shown to be multi-site carcinogens with liver being the most sensitive target organ.

\subsection{Pyrrolizidine alkaloids (PAs)}

\subsubsection{Background}

Pyrrolizidine alkaloids (PAs) are exclusively biosynthesized by approximately 6000 plant species worldwide (Smith and Culvenor 1981). PAs generally contain an amino alcohol, referred to as necine or necine base and an acid part, referred to as necic acid (EFSA 2011). The necine bases of the PAs relevant in food and feed are platynecine, retronecine, heliotridine, and otonecine as shown in Figure 3A, among which the 1,2-unsaturated PAs containing necine bases (retronecine, heliotridine, and otonecine) can be metabolized in the liver into reactive intermediates, so-called dehydro-PA metabolites (also referred to as reactive pyrroles) and these metabolites are able to bind covalently to DNA and proteins forming adducts (EFSA 2011; Fu et al. 2004). Among the 1,2-unsaturated necine bases, 
retronecine and heliotridine are enantiomers at the C-7 position, and PAs containing these type of necine bases have received the most attention because of their abundance in food and feed and their toxicities, including hepatotoxicity and carcinogenicity (Fu et al. 2002). In the present thesis, lasiocarpine (heliotridine-type PA) and riddelliine (retronecine-type PA), shown in Figure 3B, are the compounds of interest. Based on the available data in animal experiments and in vitro systems, 1,2-unsaturated PAs are considered as genotoxic and carcinogenic substances (EFSA 2011; EFSA 2017; Fu et al. 2004). To date, lasiocarpine, monocrotaline and riddelliine are classified as category $2 \mathrm{~B}$ carcinogens being possibly carcinogenic to humans (IARC 2017).

A

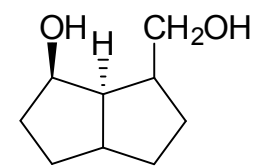

Platynecine

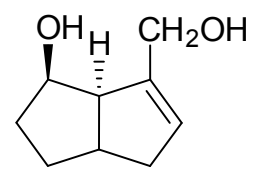

Retronecine

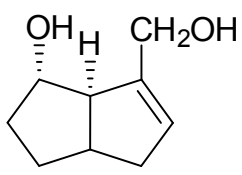

Heliotridine<smiles>CN1CC=C(CO)C(=O)[C@H](O)CC1</smiles>

Oconecine

B

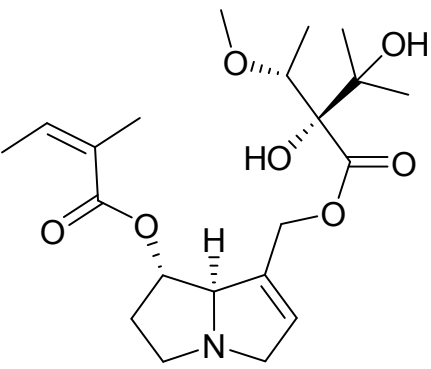

Lasiocarpine<smiles>C=C(C/C(=C/C)C(=O)O[C@H]1CCN2CC=C(COC(=O)[C@](O)(CO)C(=O)O)[C@H]12)C1CC1</smiles>

Riddelliine

Fig. 3 Common necine bases of PAs (A) and chemical structures of the two PAs of interest in the present thesis $(\mathrm{B})$.

\subsubsection{Human exposure to PAs}

In general, humans are exposed to PAs through contaminated staple foods such as wheat, honey and milk, herbal teas, and via herbal medicines and PFS (EFSA 2011; EFSA 2017), and these exposure routes are suspected to cause worldwide problems ( $\mathrm{Fu}$ et al. 2004). Human poisoning with 1,2-unsaturated PAs is characterized by acute hepatic veno-occlusive disease (HVOD) (EFSA 2011; EFSA 2017). Several outbreaks of HVOD and other liver disorders in humans have been reported in Central Asia, India and Afghanistan with the last documented outbreak in 2008, where flour contaminated with Heliotropium seeds and goat milk seemed to be the main source for PA related adverse health effects (EFSA 2017; Kakar et al. 2010). According to the risk assessment reported by the European Food Safety Authority (EFSA), the acute exposure estimated considering the presence of high contamination levels in all different food products, ranged from 1 to $300 \mathrm{ng} / \mathrm{kg} \mathrm{bw} / \mathrm{day}$ for 
mean consumers in the younger age class and from 6 to $170 \mathrm{ng} / \mathrm{kg}$ bw/day for mean adult consumers (EFSA 2017). However, short-term exposure to PAs via botanical preparations was estimated to vary significantly depending on the type of preparations. For example, consumption of herbal infusion containing PA producing plants may lead to exposure levels amounting to $890 \mathrm{ng} / \mathrm{kg}$ bw/day (EFSA 2017). Consumption of PFS tablets or capsules containing PA producing plants may result in exposure levels amounting to levels as high as $1800 \mu \mathrm{g} / \mathrm{kg}$ bw/day (EFSA 2017).

For the Chinese population, besides the exposure sources described above, exposure to PAs through TCM represents an important exposure route. According to the review described by Roeder, there are about 90 PAs found in 38 TCM herbs (Roeder 2000). The study of Griffin et al. (2014) analyzed 54 TCM samples and found that $78 \%$ of the TCM samples contained PAs and/or PA-N-oxides, at levels ranging from 10 to $3668 \mu \mathrm{g} / \mathrm{kg}$ sample. Besides TCMs, exposure levels to PAs via honey for the Chinese population are also available. A recent study reported that of among 120 honey samples collected from the Chinese market, more than half of the samples contained PAs in a concentration range from 0.04 to $288.07 \mu \mathrm{g} / \mathrm{kg}$, estimated to result in exposure levels amounting to 0.013 to $90 \mathrm{ng} / \mathrm{kg}$ bw/day assuming ingestion of a spoon of honey $(20 \mathrm{~g})$ per day by a $60 \mathrm{~kg}$ person (Zhu et al. 2018). A recent risk assessment performed by the Centre Food Safety of the Food and Environmental Hygiene Department (FEHD) of the Government of the Hong Kong Special Administrative Region estimated that for the local adult population the average dietary exposure to PAs via food such as herbal teas, honey, staple foods was $0.33-1.5 \mathrm{ng} / \mathrm{kg}$ bw/day (CFS 2017).

\subsubsection{Absorption, Distribution, Metabolism and Excretion of PAs}

In vivo toxicokinetic studies in rat and mice revealed that a rapid uptake of PAs such as riddelliine and senecionine was observed upon oral administration with the peak concentration in plasma achieved within $30 \mathrm{~min}$ (Wang et al. 2011; Williams et al. 2002). Brauchli et al. (1982) exposed rats to a PA mixture by gavage and via the skin and estimated that the oral absorption was 20-50 times higher than dermal absorption by comparing the excretion through these two exposure routes. Following absorption in the gastrointestinal tract, PAs are distributed to the liver where the main metabolism occurs (EFSA 2011; Fu et al. 2004). Generally, three metabolic pathways have been identified for lasiocarpine (heliotridine-type PA) and riddelliine (retronecine-type PA) (Figure 4). The esterase-mediated hydrolysis resulting in the formation of necines and necic acids, and the N-oxygenation pathway mediated by cytochromes $\mathrm{P} 450$ and flavin-containing monooxygenase (FMO) resulting in the formation of PA N-oxides are considered detoxification pathways, because these metabolites have relatively higher water solubility and are rapidly excreted via the urine compared to their parent compounds (EFSA 2011). The bioactivation pathway is mainly mediated by cytochromes $\mathrm{P} 450$ to form a pyrrolic ester via hydroxylation at the C-3 or C-8 position of the necine base followed by dehydration (EFSA 2011; Fu et al. 2004). This metabolite can be further metabolized via hydrolysis to the formation of a racemic compound called $( \pm$ ) 6,7-dihydro-7-hydroxy-1-hydroxymethyl-5H-pyrrolizine (DHP). Due to the electrophilic nature of the pyrrolic esters and DHP, these metabolites are chemically and 
biologically reactive and form adducts with cellular components such as protein and DNA (EFSA 2011; Fu et al. 2004). The pyrrolic esters and DHP were generally considered to be detoxified by reacting with glutathione (GSH) to form 7-GS-DHP or 7,9-diGSH-DHP and 9GS-DHP (Chen et al. 2016; EFSA 2011; Fu et al. 2004). However, recently, Xia et al. (2018) found that 7-GS-DHP also produced DHP-DNA adducts in incubations of rat and human liver microsomes in the presence of calf thymus DNA and upon incubation with HepG2 cells, indicating that 7-GS-DHP adducts can be reactive metabolites of PAs leading to DNA adduct formation. In human, CYP 3A4 is identified as the main enzyme for the bioactivation pathway of PAs (Fu et al. 2004; Li et al. 2011; Miranda et al. 1991). Miranda et al. (1991) studied the metabolism of senecionine in human liver microsomes showing that DHP and Noxide PAs were the main metabolites, also reporting large individual variability in the rate of this metabolic conversion. With respect to inter-species differences, DHP and the N-oxide PA metabolites of riddelliine were identified as the main metabolites formed in incubations with human and rat liver microsomes while the same set of DNA adducts was identified in both species (Xia et al. 2003). However, the quantities of DNA adducts formed in incubations with human liver microsomes and calf thymus DNA showed either similar or 2-3 fold higher levels than the mean quantities of adducts formed in incubations with rat liver microsomes and calf thymus DNA (Xia et al. 2003), indicating that humans might be more sensitive to 1,2unsaturated PA toxicity than rats. 


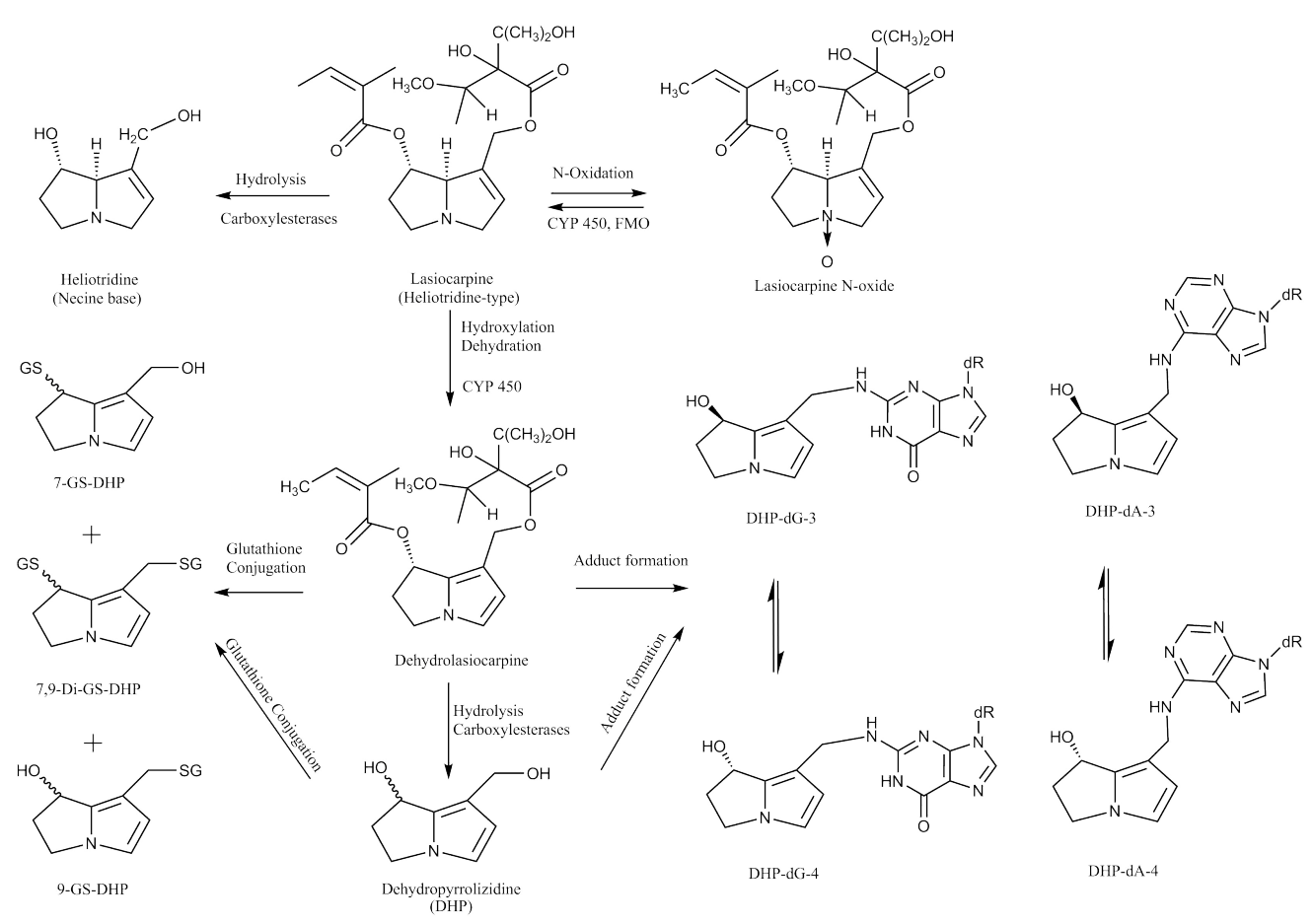

Fig. 4 Major metabolic pathways of lasiocarpine and adduct formation by lasiocarpine metabolites relevant for rat and human (EFSA 2011; Forsch et al. 2018; Fu et al. 2004; Xia et al. 2013). FMO=flavin-containing monooxygenase, CYP 450=cytochromes $\mathrm{P} 450$

\subsubsection{Hepatotoxicity, genotoxicity and carcinogenicity of PAs}

The hepatotoxic effects of acute exposure to PAs in livestock and humans include hemorrhagic necrosis, hepatic megalocytosis, hepatic veno-occlusive, liver cirrhosis, nodular hyperplasia and hepatic carcinomas (Edgar et al. 2015; Lin et al. 2011). In particular, human poisoning with 1,2-unsaturated PAs is known to cause acute HVOD (also called hepatic sinusoidal obstruction syndrome), which may lead to liver necrosis, fibrosis, nodular hyperplasia and eventually to cirrhosis and liver failure (Edgar et al. 2015; Lin et al. 2011). Based on the limited information available for the human poisoning by PAs, the EFSA Panel on Contaminants in the Food Chain (CONTAM Panel) could not set an acute reference dose (ARfD) (EFSA 2017). The lowest known dose associated with acute toxicity in humans is approximately $2 \mathrm{mg}$ PA/kg bw/day (EFSA 2017). This estimation was based on acute PA poisoning cases in 2 infants, a 6-month-old girl and a 2 month-old boy exposed to an extract of Senecio longilobus, in which retrorsine and riddelliine were the main constituents. The 6month-old girl who received around $0.7-1.5 \mathrm{mg} \mathrm{PA} / \mathrm{kg}$ bw for 2 weeks, developed liver cirrhosis after 6 months and was diagnosed for HVOD. The 2-month-old boy was exposed to a dose of 1.4-3 mg PA/kg bw for less than 2 weeks, resulting in a fatal outcome with liver necrosis (Culvenor 1983; EFSA 2017). Hepatic metabolic activation of PAs resulting in dehydro-PAs formation and their subsequent reaction with cellular proteins forming 2,3- 
dihydro-1H-pyrrolizine-protein (pyrrole-protein) adducts are generally considered as the initial steps of PA poisoning (Fu et al. 2004; Lin et al. 2011). Generally, two hypothesized mechanisms for protein adduct-induced cytotoxicity have been proposed: 1) the protein adducts impair the physiological function of the targeted proteins, 2) the protein adducts may trigger immune responses (Ma et al. 2018; Pumford and Halmes 1997; Zhou et al. 2005). Additionally, cellular GSH depletion caused by reactive metabolites resulting in oxidative stress in the target organs is another hypothesized mechanism for hepatotoxicity of PAs (Liang et al. 2011; Ma et al. 2018). For PA induced liver injury (PA-ILI), Lin et al. (2011) first identified serum pyrrole-protein adducts in a PA-induced HVOD patient who was confirmed to intake of a PA-containing herb, Gynura segetum (Tusanqi as Chinese name), in which senecionine and seneciphylline were detected. Subsequently, Lin and co-workers detected pyrrole-protein adducts at levels ranging from 0.14 to $74.4 \mathrm{nM}$ in the blood of 15 PA-ILI patients who consumed the PA-containing herb, Gynura segetum during periods varying from 5 to 200 days (Gao et al. 2015; Ruan et al. 2015). Thus, Lin and co-workers suggested that blood pyrrole-protein can serve as a specific biomarker of PA-ILI (Gao et al. 2015; Ruan et al. 2015). Different PAs may induce similar effects but the degree of hepatotoxicity may vary markedly. Recently, Yang et al. (2017) performed a comparative study of hepatotoxicity of retrorsine and monocrotaline. By treating male Kunming mice with retrorsine or monocrotaline at a dose of $0.2 \mathrm{mmol} / \mathrm{kg}$, compared to monocrotaline, the mice treated with retrorsine showed a 10.3-fold higher serum alanine transaminase (ALT) level, a 3.6-fold higher serum aspartate transaminase (AST) level, a 2.9-fold higher level of plasma pyrroleGSH adducts, a 2.7-fold higher level of liver pyrrole-GSH adducts and a 3.8-fold higher level of liver pyrrole-protein adducts. Yang et al. (2017) concluded that protein covalent binding by reactive metabolites is an important mechanism of hepatotoxicity of PAs.

Extensive studies have been conducted on the genotoxicity of PAs. For the DNA adduct formation in vivo, the levels of DNA adduct formation in rats administered with different doses of riddelliine were analyzed by ${ }^{32} \mathrm{P}$-postlabeling/HPLC analysis, and the levels of adducts correlated closely with the tumorigenic potency of riddelliine, indicating that DNA adduct formation presumably induces the genotoxic and tumorigenic effects of PAs (Chou et al. 2003; Yang et al. 2001). In addition, a more sensitive LC-ESI-MS/MS method was developed to identify and quantify the DHP-derived adducts in vivo and in vitro formed from a series of different PAs (Fu et al. 2010). With this method, different DNA adducts could be quantified at levels varying in the range of 0.1-1.5 adducts in $10^{8}$ nucleotides, based on 100 $\mu \mathrm{g}$ sample. The authors indicated that this sensitivity is potentially adequate for measuring adduct level in humans (Fu et al. 2010). Xia et al. (2013) recently reported that 12 tumorigenic PAs and PA N-oxides undergo an identical bioactivation mechanism by the formation of four major DNA adducts which are two predominant adducts, a pair of epimers of 7-hydroxy-9-(deoxyguanosin- $N^{2}$-yl)-dehydrosupinidine adducts (DHP-dG-3 and DHP$\mathrm{dG}-4)$ and two minor adducts, a pair of epimers of 7-hydroxy-9-(deoxyadenosin- $N 6$-yl) dehydrosupinidine adducts (DHP-dA-3 and DHP-dA-4) in rats as shown in Figure 4. Additionally, this study also reported that there was a correlation between the potency of liver tumor formation and levels of DNA adduct formation in vivo (retrorsine $>$ lasiocarpine $>$ 
riddelliine $\sim$ monocrotaline $>$ riddelliine N-oxide $>$ senkirkine $>$ heliotrine $\geq$ clivorine $>$ lycopsamine $\sim$ retronecine $\sim$ platyphylliine) (Xia et al. 2013). Besides DNA adduct formation, the genotoxicity of PAs and extracted plants were also found in other in vitro and in vivo assays such as assays for DNA strand breakage, the micronucleus assay, assays for mutations in bacteria and the sister chromatid exchange assay, the UDS assay, and assays for chromosomal damage (Chen et al. 2010). Studies of Mei et al. (2004a; 2004b), reported that riddelliine induced a dose-dependent increase in mutant frequency of the cII gene in liver endothelial cells in big blue transgenic rats, and DHP adducts were also found in the endothelial cells of the liver (Mei et al. 2004a; Mei et al. 2004b). These studies strongly suggest that mutation by riddelliine is a key event in the carcinogenesis pathway (EFSA 2011). Dietary comfrey containing 9 PAs of which the major unsaturated PAs are lycopsamine and intermedine, and symphytine (Vollmer et al. 1987), also induced mutations such as $\mathrm{G}: \mathrm{C}$ to T:A transversions and tandem base substitutions in big blue transgenic rats (Mei et al. 2005; Mei and Chen 2007; Mei et al. 2010). This study further supports that mutations by PAs in comfrey are responsible for its carcinogenicity (EFSA 2011).

Studies on carcinogenicity of PAs are limited. There are no human epidemiological data on PA carcinogenesis. Long-term rodent oral carcinogenicity studies for PAs are only available for lasiocarpine and riddelliine, conducted by NTP (Chan et al. 2003; NTP 1978; NTP 2003). The carcinogenicity of lasiocaprine was studied for 2 years in rats. Compared to the controls an increased incidence of liver angiosarcomas was observed in 5 of 24 males and 8 of 22 females at $0.35 \mathrm{mg} / \mathrm{kg}$ bw/day, in 11 of 23 males and 7 out of 24 females at $0.75 \mathrm{mg} / \mathrm{kg} \mathrm{bw} /$ day, and in 13 of 23 male and 2 of 23 female rats following dietary administration of $1.5 \mathrm{mg} /$ $\mathrm{kg}$ bw/day (NTP 1978). For riddelliine, a 2-year carcinogenicity study was conducted in mice and rats. In this 2-year NTP study of riddelliine, liver tumors mainly developed from endothelial cells in the liver (NTP 2003). Liver haemangiosarcomas were seen in 43 of 50 male rats and 38 of 50 female rats at $0.7 \mathrm{mg} / \mathrm{kg}$ bw/day (Chan et al. 2003; NTP 2003). For mice, liver haemangiosarcomas were observed in 31 of 50 male mice at a dose of $3 \mathrm{mg} / \mathrm{kg}$ bw/day, whereas female mice showed alveolar and bronchiolar neoplasms in 13 of 50 animals (Chan et al. 2003; NTP 2003). Other limited carcinogenicity studies of PAs following oral, s.c. or i.p. administration are available, such as for clivorine (oral), senkirkine (i.p.), symphytine (i.p.), monocrotaline (s.c.), isatidine (oral) and retrorsine (oral) showing different potencies of liver tumor formation for the different PAs (Hirono et al. 1979; Kuhara et al. 1980; Schoental and Bensted 1963; Schoental et al. 1954; Shumaker et al. 1976). Overall, the carcinogenicity studies of PAs showed there was clear evidence of carcinogenic effects of PAs, especially for lasiocarpine and riddelliine. The International Agency Research on Cancer (IARC) classified these two compounds as group 2B "possibly carcinogenic to humans" (IARC 2017). 


\section{Approaches used in the present thesis to perform a mode of action based risk assessment}

\subsection{Margin of Exposure (MOE) approach}

Many different risk assessment approaches for the risk and safety assessment of compounds that are both genotoxic and carcinogenic are available including the history of use, the Threshold of Toxicology concern (TTC), the ALARA (as low as reasonably achievable) approach, the virtual safe dose (VSD) approach and the MOE approach (Van den Berg et al. 2011a). The TTC approach could be used for chemicals for which the chemical structure is known but for which toxicological data are not available. This approach compares the estimated oral intake of the compound of interest to the TTC values for structurally related compounds. In general, for genotoxic compounds, a TTC value was defined at $0.15 \mu \mathrm{g}$ per person per day, equal to $0.0025 \mu \mathrm{g} / \mathrm{kg}$ bw/day for a $60 \mathrm{~kg}$ person (Kroes et al. 2004; Munro et al. 1999). However, for high potency carcinogens like aflatoxin-like, azoxy or N-nitroso compounds and benzidines, the TTC approach is not applicable because these type of chemicals were not included in the data set used to define the TTC (EFSA 2016; Kroes et al. 2004). Overall, the TTC approach can be used for an initial safety evaluation of relatively low dose chemical exposures and to determine whether further assessment is needed (EFSA 2016).

The ALARA approach, indicating that the intake of a genotoxic carcinogen should be as low as reasonably achievable, is a risk management tool and has been applied by advisory bodies and regulatory agencies (Barlow et al. 2006; Dybing et al. 2008). The approach can be based on only hazard identification data that confirms that the substance is genotoxic in vivo. For avoidable compounds that test positive for genotoxicity in vivo zero tolerance policy is applied, but for unavoidable compounds that raise a concern for genotoxicity one may apply ALARA. For risk assessors, the ALARA approach has the advantage that there is no need to evaluate exposure data and the carcinogenicity data in a quantitative manner (Barlow et al. 2006). Thus, the ALARA approach is applicable for genotoxic and carcinogenic substances which are technically unavoidable contaminants in foods such as aflatoxin and acrylamide. However, this approach only identifies the hazard and does not provide a quantitative estimate of the cancer risk under the relevant intake for human exposure. Therefore, this approach does not provide the risk manager with information for setting priorities for action (Dybing et al. 2008). In modern risk assessment, ALARA may be applied when the risk assessment by other methods reveals that the unavoidable contaminant raises a concern that is difficult to mediate (like for acrylamide and aflatoxin). In such cases ALARA may be the final advice (EFSA 2005).

An approach to quantitatively assess the risk for carcinogenic and genotoxic compounds is deriving a virtual safe dose (VSD) by linear extrapolation from high dose to low dose to define a dose level at which the additional lifetime cancer risk should be one in a million, the VSD. However, the VSD value is highly affected by the shape of the dose-response curves and the selected models to perform the extrapolation outside the range of experimentally available data as shown in Figure 5 (EFSA 2005). Furthermore, the tumor data in 
experimental animals are directly translated to a VSD for human cancer risks without taking inter-species and inter-individual variation into account (EFSA 2005). Therefore, an alternative approach for risk assessment of carcinogenic and genotoxic compounds was developed.

For the risk and safety assessment of compounds for which there is a threshold below which no adverse effects will be observed different health-based guidance values can be defined. These include a tolerable daily intake (TDI) for unavoidable compounds, or an acceptable daily intake (ADI) in case of intentionally added avoidable substances. These values reflect the amount of a compound that can be consumed every day over a lifetime without presenting an appreciable risk to health. For acute exposure, acute reference Dose (ARfD) is defined which is an estimate of the amount that can be ingested within a period of 24 hours, without appreciable health risks. To derive these health-based guidance values, a PoD, such as the benchmark dose lower confidence limit (BMDL) or a no observed adverse effect level (NOAEL) obtained from the appropriate chronic or acute toxicity study, is divided by relevant uncertainty factors. A default uncertainty factor of 100 , consisting of a factor 10 for inter-species differences and another factor 10 for intra-species differences, is usually applied, although additional factors may be used for example in case of deficiencies in the dataset (EFSA 2012b). As an alternative for applying these health-based guidance values, also the TTC and the MOE (also called margin of safety (MOS)) approach can be applied in the risk and safety assessment of compounds for which a threshold exists.

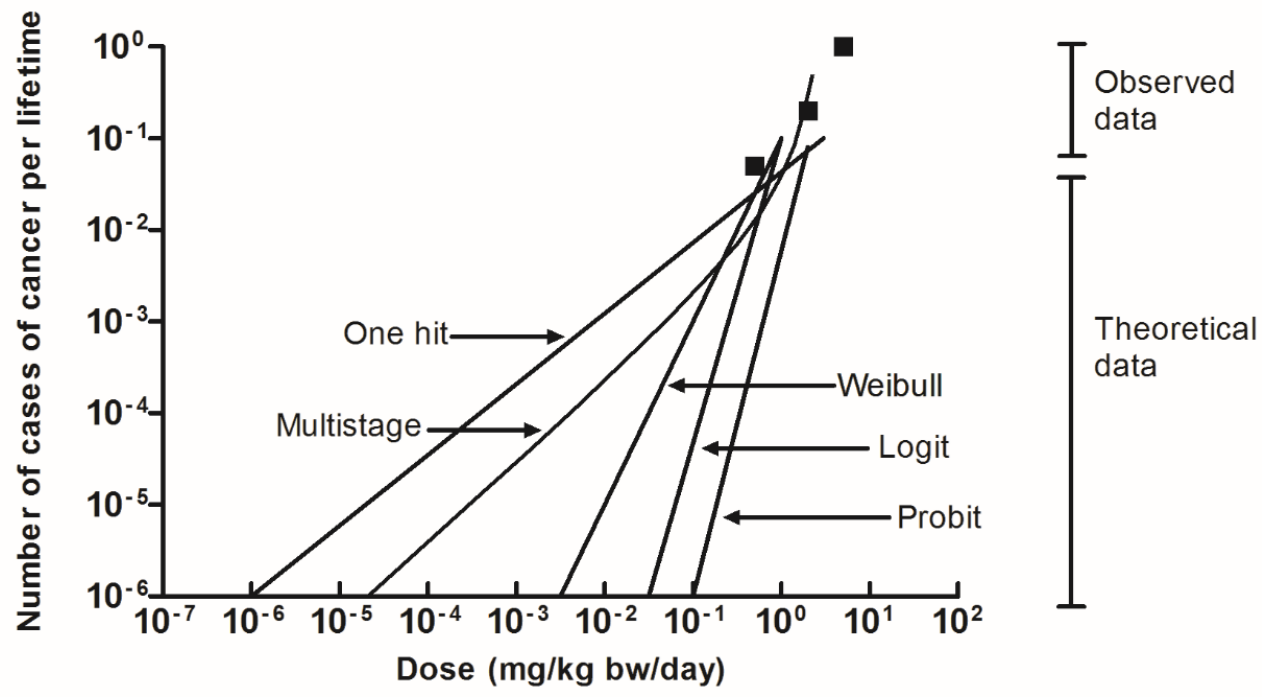

Fig. 5 Low dose extrapolation from animal carcinogenicity data using various models (EFSA 2005)

The so-called MOE approach is recommended by expert groups of the EFSA, the Joint FAO/ WHO expert committee on Food Additives (JECFA) and the International Life Sciences 
Institute (ILSI) (EFSA 2005). The MOE value for genotoxic compounds is a ratio of a reference point usually obtained from epidemiological or animal experimental data on tumor incidence, and the EDI in humans. Also for non-genotoxic chemicals and adverse effects that have a threshold a MOE or MOS can be used in risk assessment, calculating the ratio between a lower bound of the BMD confidence interval (BMDL) or the NOAEL for the relevant adverse effects and the EDI. In general, the preferred reference point used as PoD for calculating the MOE is derived using the BMD approach which analyses the available (tumor) data with mathematical models, using for example the recently released BMDS 3.0 software from the EPA (EPA 2018) or PROAST software 65.6 from the RIVM (RIVM 2018). Commonly, the BMDL, that is the lower $95 \%$ confidence bound of the BMD (Figure 6) is used as the PoD. To define a PoD first the Benchmark Dose Response (BMR) should be selected, which is a dose that causes a low but measurable change in response compared to the background response (EFSA 2005). BMR values of $1 \%, 5 \%$ or $10 \%$ extra risk have been proposed to be used to analyse data from animal studies (EPA 1995). In general, $10 \%$ extra risk is more appropriate for quantal data, such as tumor incidences, because the $\mathrm{BMDL}_{1}$ or $\mathrm{BMDL}_{5}$ may be outside what can be experimentally determined and thus become more dependent on the choice of the mathematical models (Sand et al. 2002). For continuous data, a BMR of $5 \%$ is recommended as a default for defining a PoD (EFSA 2009).

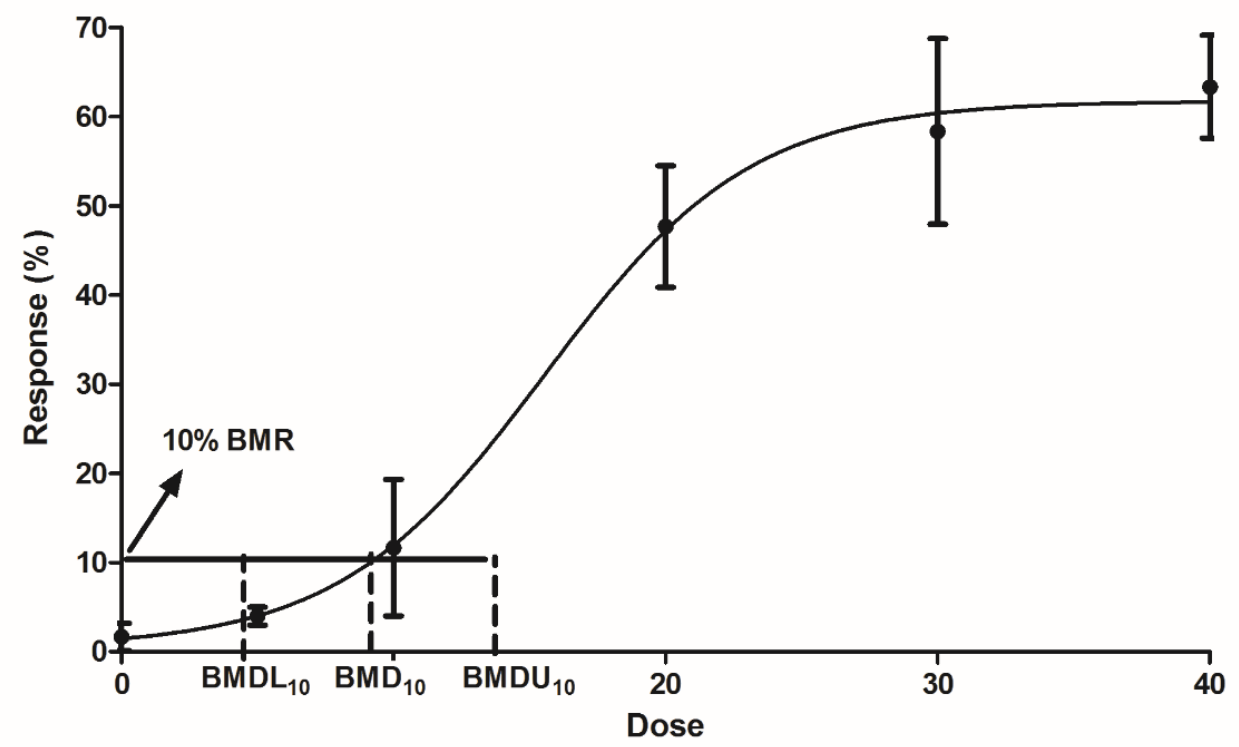

Fig. 6 Dose-response curve illustrating the key concepts for the BMD approach. $B M R=10 \%$ change in response relative to the background effect, $\mathrm{BMD}_{10}=$ benchmark dose resulting in 10 $\%$ extra effect, $\mathrm{BMDL} 10 / \mathrm{U}_{10}=95 \%$ Lower/Upper confidence limit of the benchmark dose resulting in $10 \%$ extra effect 
EFSA advised that, when using a BMDL 10 derived from animal tumor data, a MOE value lower than 10000 for genotoxic and carcinogenic compounds indicates a high priority for risk management actions and would be a concern for human health (EFSA 2005). The value of 10000 takes uncertainty factors into account including (1) a factor 100 for inter-species differences and human variability in kinetics and dynamics (intra-species); (2) a factor 10 for inter-individual differences in cell control and DNA repair; and (3) a factor 10 for the fact that the $\mathrm{BMDL}_{10}$ or the reference point is not identical to a NOAEL (EFSA 2005). A MOE is calculated based on lifetime daily exposure. For the carcinogenic and genotoxic compounds that have other adverse effects such as hepatotoxicity in addition to the genotoxic carcinogenicity, EFSA advised that a MOE or MOS value higher than 100 is considered as no or low concerns for these thresholded additional adverse effects. The value of 100 is based on (1) a factor of 10 for inter-species differences including both kinetic and dynamic differences between experimental animals and humans and (2) a factor of 10 for inter-individual differences in kinetics and dynamics within the human population (EFSA 2012b; EFSA 2015).

\subsection{Physiologically based kinetic (PBK) modelling}

A refinement of the MOE approach, both for genotoxic or non-genotoxic endpoints, may be implemented when information on the mode of action provides a basis to modify the uncertainty factors used to define the value of 10000 (for compounds that are both genotoxic and carcinogenic) or of 100 (for threshold adverse effects). To this end it is relevant to take into account that the uncertainty factor of 100 for inter-species differences and human variability in kinetics and dynamics is subdivided into a factor 10 for inter-species differences and a factor 10 for intra-species differences. Also these factors can be further subdivided into a factor 4.0 to account for potential inter-species differences in toxicokinetics and a factor 2.5 for interspecies differences in toxicodynamic responses (IPCS 2005). For inter-individual differences, the 10-fold uncertainty factor can be subdivided into a factor 3.16 for toxicokinetic and toxicodynamic differences each (IPCS 2005).

EFSA previously stated that these factors of 10 could be adjusted when appropriate chemical specific data are available (EFSA 2005). However, a clear guidance on how to make this adjustment is currently not available. Mode of action based arguments can support a change in either one of these uncertainty factors defining what is called a compound specific assessment factor (CSAF) (IPCS 2005). Thus, the mode of action approach may be used to provide insight in inter-and intra-species differences and define CSAF values.

For the mode of action approach, physiologically based kinetic (PBK) modelling can be used. PBK modelling assumes that the whole body can be described as a set of compartments with each compartment representing a single region of the body as shown in Figure 7. Within each compartment, a set of differential equations is defined that describes the absorption, distribution, metabolism and/or excretion (ADME) of the compounds in time. The mathematical equations are established based on three types of parameters including (1) physiological parameters (e.g. tissue volumes, tissue blood flows and cardiac output) of the species for which the model is defined, (2) physico-chemical parameters (e.g. tissue /blood 
partition coefficients), and (3) metabolic parameters (e.g. kinetic constants for metabolic conversions) (Rietjens et al. 2011). Once the models have been defined and evaluated they can be used to make predictions. The PBK model predictions can consist of the internal concentration of the compound or its metabolites in the tissues and blood over time at any dose level. Furthermore, by applying specific parameters for certain species, ethnic groups or individuals, the PBK models can be used to facilitate inter-species extrapolation or define inter-ethnic and inter-individual differences or CSAF values.

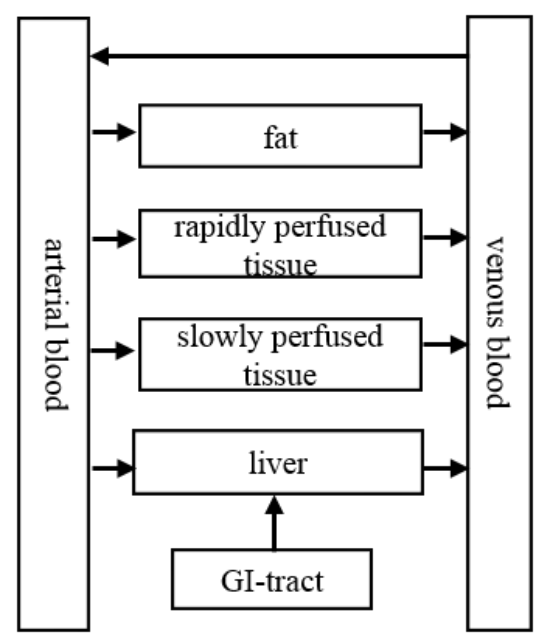

Fig. 7 Schematic overview of a physiologically based kinetic (PBK) model

The development of a PBK model as performed in the present thesis proceeds by several steps including (1) definition of the conceptual model, (2) translation into a mathematical model, (3) defining parameter values, (4) solving the model equations, (5) evaluation of the model performance, (6) making predictions (Rietjens et al. 2011). In the first step, several compartments should be defined to represent the most relevant target organs for describing the ADME characteristics of the test compound. The remaining organs can be pooled together in so-called slowly perfused tissue or richly perfused tissue. The defined compartments are mutually connected via the systemic circulation. In the second and third step, a set of mathematical equations needs to be defined for each compartment including physiological parameters, physico-chemical parameters and metabolic parameters. The physiological parameters can usually be obtained from available literature. The physico-chemical parameters and metabolic parameters are mainly derived from in vitro incubations and in silico approaches. In the fourth step, the model equations need to be solved to be able to predict the concentration of the compound or its metabolites in the defined compartments over time at selected dose levels. This can be performed using software such as Berkeley Madonna (Macey and Oster, UC Berkeley, CA, USA), acslXtreme (Aegis Technologies Group), or MATLAB (the MathWorks). In the fifth step, the developed model needs to be evaluated against available (in vivo) data to support the validity of the model. Apart from that, a sensitivity analysis can be done to study which parameters have the largest influence on the 
model predictions. Once the performance of the model has been adequately evaluated, in the last step, the model can be used to make predictions for the purpose of interest. PBK modelling can be used to define CSAF values, but also to define PoDs using so-called reverse dosimetry converting data from in vitro toxicity studies to in vivo dose-response curves, as explained in the next section.

\subsection{Physiologically based kinetic (PBK) modelling-based reverse dosimetry}

To obtain a PoD for performing a risk assessment using the MOE approach, animal data to establish the dose-response curve and a BMDL for a specified response are required. However, use of experimental animals for safety testing of compounds is considered unethical and also unacceptable from an economic point of view, because it is time-consuming and requires extensive laboratory equipment and skilled people (Rovida and Hartung 2009). Also it is questioned to what extent animal data adequately match the human situation. Because of these limitations, applying alternatives to animal testing to obtain PoDs for risk assessment is of interest. A novel strategy called PBK modelling-based reverse dosimetry can be used to translate the in vitro concentration-response curves to in vivo dose-response curves, from which PoDs can be derived as schematically shown in Figure 8. If both the in vitro model as well as the PBK model are human based models, in vivo dose-response curves and PoDs for the human situation can be predicted. Traditionally, PBK models defined as described in section 3.2 allow estimation of tissue or blood concentrations of the compound of interest and its metabolites at specified external dose levels, and this type of prediction is called forward dosimetry. Reverse dosimetry refers to the use of the PBK models to translate internal concentrations (for example plasma concentrations set equal to the in vitro concentrations) into external administered dose levels, using the model to calculate these corresponding external dose level. Previously, it was shown that the reverse dosimetry approach was able to predict in vivo neurotoxicity (Forsby and Blaauboer 2007), developmental toxicity (Louisse et al. 2010; Strikwold et al. 2017; Strikwold et al. 2013) and kidney toxicity (Abdullah et al. 2016). In the current thesis, the same approach was applied to predict liver toxicity for the PAs and also to investigate inter-species and inter-ethnic human differences for this toxicity.

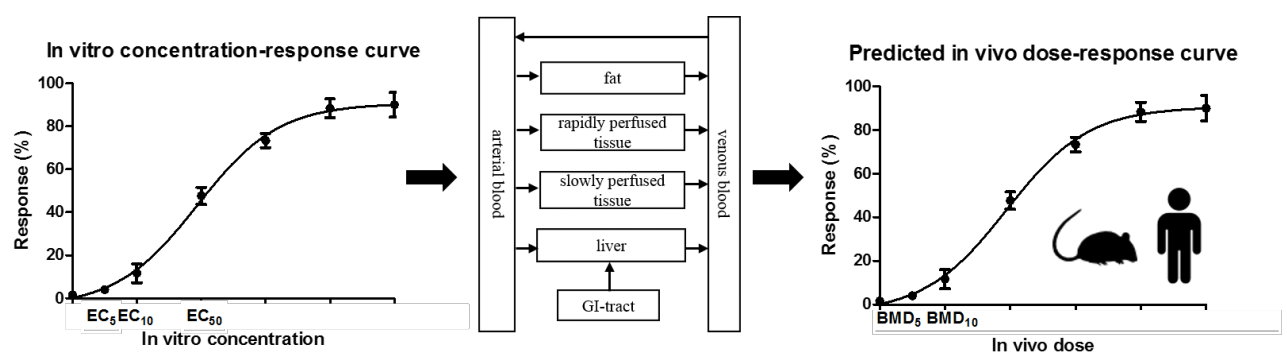

Fig. 8 Principle of the physiologically based kinetic (PBK) modelling-based reverse dosimetry 
The development of PBK modelling-based reverse dosimetry in the current thesis contains six steps including (1) establishment of a concentration-response curve for hepatotoxicity using relevant in vitro models, (2) development of PBK models to describe the ADME of the compound, (3) evaluation of the model performance against available literature data, (4) translation of the in vitro concentration-response curves obtained in step 1 to in vivo doseresponse curves using PBK modelling-based reverse dosimetry, (5) BMD analysis of the predicted dose-response curves obtained in step 4 to derive a PoD, (6) evaluation of the doseresponse curve and the related $\mathrm{PoD}$ against available literature data.

In the present thesis this approach was applied for liver toxicity induced by PAs. The first step was therefore establishing the concentration-response curves of the test compounds in selected in vitro models to study liver toxicity. To study liver biology and function, druginduced hepatotoxicity and liver disease, primary hepatocytes are currently considered as the gold standard for in vitro model systems (Bell et al. 2016; Gomez-Lechon et al. 2014). In the present thesis, primary rat and human hepatocytes were selected to study liver toxicity of the test compounds. When the in vitro concentrations are translated into in vivo external dose level, the concentrations used in vitro are set equal to the predicted concentration in plasma or the relevant organ in the PBK model, after correction for the difference in protein binding of the test compound in the in vitro culture medium and the rat or human plasma. Reverse dosimetry can be based on the maximum concentration $\left(\mathrm{C}_{\max }\right)$ or the area under the concentration-time curves (AUC). The choice of the dose metric to be used for the reverse dosimetry depends on the mode of action of the test compound and the endpoint of interest. When predicting toxicity for which a threshold exists and thus a concentration below which there will be no effect, $\mathrm{C}_{\max }$ may be the selected parameter for the reverse dosimetry, while for an endpoint like tumor formation the total dose over time and thus an AUC may be a better choice (Groothuis et al. 2015).

\subsection{Combining physiologically based kinetic (PBK) modelling-based reverse dosimetry and Monte Carlo simulation}

The present thesis also used PBK modelling in combination with Monte Carlo simulation to investigate inter-individual differences in sensitivity toward a chemical and to define CSAF values for risk assessment as schematically shown in Figure 9. This inter-individual variation was studied within both the Chinese population and the Caucasian population providing further insight in inter-ethnic differences and the applicability of the default uncertainty factor for intra-species variability in human risk assessment. To this end PBK modelling was combined with Monte Carlo simulation. PBK models themselves do not take uncertainty or variability of parameters into account and thus the outcomes of the model predictions describe the behaviour of a chemical for the average person in a population (when using input parameters for the average individual). In order to estimate the inter-individual variability within a human population, Monte Carlo simulation can be used to incorporate the uncertainty or variability of parameters into the PBK model based predictions, such as the variability in the kinetic parameters for formation of a toxic metabolite in the liver. Monte Carlo simulation is a computer-based method of analysis that uses statistical sampling 
techniques to evaluate the impact of variability of input parameters on the model output (Krishnan et al. 2013). In a Monte Carlo simulation, a probability distribution for one or more parameters is randomly sampled for a large number of simulations creating a frequency distribution for the model output of interest. In general, 1000 or more simulations are performed to derive the distribution for the model output, from which subsequently for example the geometric mean, $90^{\text {th }}$ and $99^{\text {th }}$ percentiles can be derived.

Input parameter distributions PBK modelling and Monte Carlo simulation Output parameter distribution
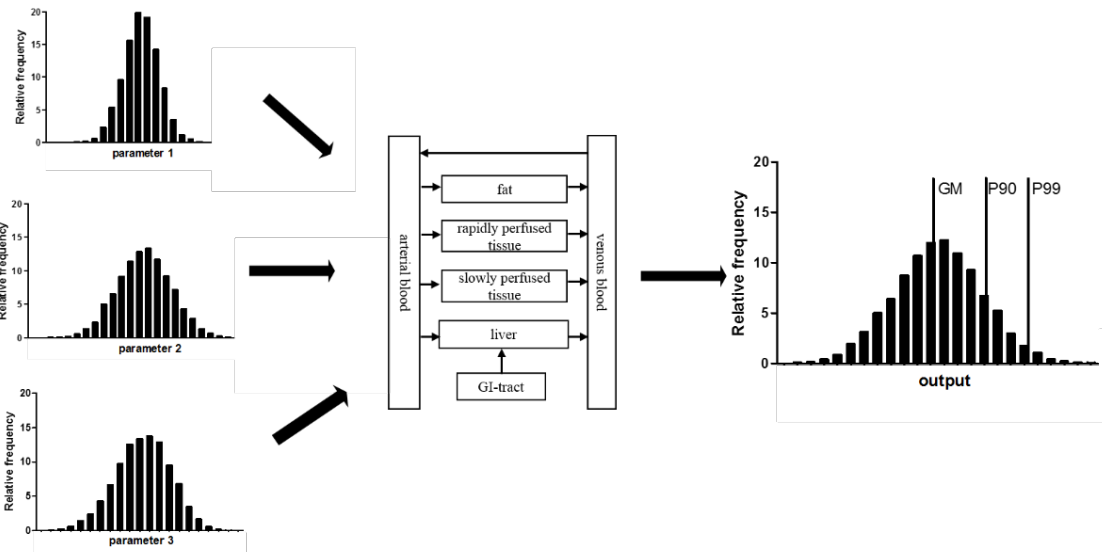

Fig. 9 Illustration of the combination of Monte Carlo simulations with PBK modelling. $\mathrm{GM}=$ geometric mean, $\mathrm{P} 90=90^{\text {th }}$ percentile of the population, $\mathrm{P} 99=99^{\text {th }}$ percentile of the population

Combining the PBK modelling-based reverse dosimetry approach with Monte Carlo simulations in the present thesis consisted of the following steps (1) selecting the input parameters of the PBK model of highest interest for the predictions and their frequency distribution, (2) random sampling of the input parameters from their specified distributions, (3) solving the PBK model equations, (4) computing the distribution of the output of interest at a selected dose level based on a large number of simulations, (5) deriving GM, 90 th and $99^{\text {th }}$ percentiles from the generated distribution of output, (6) calculation of a CSAF for the inter-individual variability by comparison of the $90^{\text {th }}$ or $99^{\text {th }}$ percentile value of the output of interest to the GM value (Krishnan et al. 2013). In the present thesis, the input parameters for the Monte Carlo simulations were selected based on the results of the sensitivity analysis of the PBK model parameters, which is used to specify which parameters have the largest influence on the PBK model output. The probability distribution for the selected input parameters is used as input for the PBK modelling. To define these distributions, the physicochemical and biochemical parameters of interest are considered to display a log-normal distribution (Zhang et al. 2007). The log-normal distributions parameters can be integrated into the Berkeley Madonna model codes so that after performing a large number of Monte Carlo simulations, a distribution is generated that represents the variability of the output of interest within in human population. From this predicted distribution, the GM, $90^{\text {th }}$ or $99^{\text {th }}$ 
percentile values of the output of interest can be obtained to define the CSAF for interindividual kinetic differences. The CSAF can replace default UFs when deriving a safe guidance value for risk assessment of chemicals (IPCS 2005).

\section{Regulatory status of botanical preparations in China and the EU}

The present thesis focussed on selected ingredients of concern present in herbal preparations. For herbal preparations, different countries and regulatory bodies have different regulations. In China, botanical food supplements and dietary food supplements are all defined as "health food", which is required to obtain a Health Food Approval certificate from the China Food and Drug Administration (CFDA) before marketing (Low et al. 2017). In 2016, CFDA revised the regulations requiring manufacturers to provide a package of toxicological data if the product ingredients are not listed in the Catalogue of Health Food Ingredients (CFDA 2016; Low et al. 2017). The Chinese health authorities have developed regulations in 2002 to define dietary herbs (allowed as herbal drug or food) and herbs that can be used in health food products (CFDA 2013a; CFDA 2013b). In the lists of dietary herbs, botanicals potentially containing alkenylbenzenes such as Illicium verum Hook.f, Foeniculum vulgare Mill. and Myristica fragrans Houtt. can be found (CFDA 2013a). Additionally, in the list of herbs allowed for use in health food products in China, botanicals potentially containing PAs can be found such as Eupatorium fortunei Turcz. (CFDA 2013b). Herbal teas are considered as food in China and are under regulation by CFDA (CFDA 2006; Fu et al. 2018). This authority is responsible to control the quality of herbal teas throughout the supply chain from collection until storage. All herbal teas are required to comply with the national regulations on agricultural products and in this regulation, several chemical contaminant levels have been regulated. For example, the arsenic level in Dendranthema morifolium must not exceed 0.5 $\mathrm{mg} / \mathrm{kg}$ (Sun et al. 2013). Herbal medicines, which are classified as medicines, account for 30 $\%-50 \%$ of the total medicinal consumption and are under regulation by CFDA (Liu and Salmon 2010). The qualification and standardization of TCM is based on the Chinese Pharmacopoeia formulated by the State Pharmacopeia Commission. In this standard system, the standards of purity, dosage, description, precaution, and storage of each medicine are regulated (Liu and Salmon 2010).

In the EU, food supplements are regulated under Directive 2002/46/EC for a specific category of foodstuffs (EC 2002). However, this directive is applicable only for the use of vitamins and minerals in food supplements, while PFS are not included in this directive. PFS are required to comply with EU General Food Law (regulation [EC] No 178/2002) and requirements for novel foods (Regulation [EC] No 258/97 and Regulation [EU] 2015/2283) (Low et al. 2017). Herbal medicines are considered as medicinal products and are regulated under Directive 2004/24/EC (EC 2004). The European Medicines Agency (EMA) is responsible for assessing the safety and efficacy of these products (Low et al. 2017). For alkenylbenzenes, the European Commission (EC) has prohibited the use of estragole, methyleugenol and safrole as pure compound in foods based on Regulation No 1334/2008 of the European Parliament and of the Council (EC 2008). For PAs, different Member States have their specific limits for these substances. For example, for herbal medicines, Germany regulated the total amount of 
1,2-unsaturated PAs (including N-oxides) in botanical preparations, which must not result in an intake that exceeds $0.1 \mu \mathrm{g}$ per day when the products containing PAs for oral use are consumed for periods more than 6 months. When the consumption period is less than 6 months, the limit is $1 \mu \mathrm{g}$ per day (Merz and Schrenk 2016). Bundesinstitut für Risikobewertung (BfR) recommended to keep the intake of the total amount of PAs from different foods as low as possible (ALARA) and not to exceed an intake of $0.007 \mu \mathrm{g} \mathrm{1,2-}$ unsaturated PAs and their N-oxides/kg bw/day. In the Netherlands, the total content of PAs in herbal preparations and herbal extracts may not exceed $1 \mu \mathrm{g} / \mathrm{kg}$ preparation (Merz and Schrenk 2016).

\section{Consumer behaviour towards botanical preparations}

Due to more than two thousand years dietary herbal therapy and recent economic growth, disease prevention and non-medical approaches of health maintenance are becoming increasingly popular. A survey on usage of PFS in 2011 in China revealed that $70 \%$ of 300 respondents have used PFS, from which around $35 \%$ would continue consuming PFS because of their anticipated beneficial effects. Regarding the reasons for PFS consumption, $63.5 \%$ of respondents believed that PFS consumption is helpful for maintenance or preservation of wellness and health or prevention from disease, and $46.9 \%$ of the respondents were of the opinion that PFS have little adverse side effects ( $\mathrm{Xu}$ and Li 2011). A recent survey on usage of PFS conducted in 2017 in NanNing, China, included 462 respondents and revealed that the prevalence of PFS consumption in elder respondents was relatively higher amounting to $48.5 \%$ compared to the age group of 35-55 years amounting to $26.8 \%$. In this study $65.4 \%$ of the respondents were of the opinion that PFS have little adverse side effect (Liu and Zeng 2017). For herbal teas, these products are considered by the Chinese people as food with preventive and therapeutic effects. Drinking herbal tea can be a daily habit without restrictions regarding dose, frequency and time of consumption (Fu et al. 2018). Overall, it can be concluded that in China, elderly people are the major consumer group for botanical preparations and cogitation of "natural" and "safe" provides a main reason for consuming botanical preparations.

In the EU, from May 2011 to September 2012, a cross-sectional, 12-month retrospective survey on usage of PFS was conducted in six European countries (Finland, Germany, Italy, Romania, Spain and the United Kingdom) consisting of 2400 respondents (Garcia-Alvarez et al. 2014). The results showed that an estimated $18.8 \%$ of screened survey respondents used at least one PFS. For the survey of PFS product usage patterns, $37.3 \%$ of the consumers indicated to use PFS "periodically", $22.2 \%$ of the consumers used PFS when experiencing a "flare up or worsening of a condition", $19.8 \%$ of the consumers used PFS on a more "sporadic basis" and $17.8 \%$ of consumers took PFS on "other non-specified occasions". For the characteristics of the PFS consumers, the proportion of PFS consumers aged more than 60 years was relatively higher amounting to $25.2 \%$ compared to other age groups i.e.,18-29 years $(17.7 \%), 30-39$ years $(18.9 \%), 40-49$ years $(19.5 \%)$ and $50-59$ years $(18.7 \%)$. The majority of participating consumers were medium educated, amounting to $65.7 \%$ (GarciaAlvarez et al. 2014). A similar survey recently conducted in the Netherlands showed that the 
prevalence of PFS users in the Dutch population was about $10 \%$ for men, $17 \%$ for women and $13 \%$ for children. For the sociodemographic characteristics, PFS users tended to have a low educational level but a high social class (Jeurissen et al. 2018). Compared to the survey results conducted in the EU, consumption of botanical preparations seems to be more prevalent in China.

\section{Outline of the present thesis}

The aim of the present thesis was to perform a mode of action based risk and safety assessment for two groups of natural botanical toxins, namely alkenylbenzenes and PAs for the Chinese population as compared to the overall Caucasian population using the Margin of Exposure (MOE) approach, physiologically based kinetic (PBK) modelling-based reverse dosimetry and Monte Carlo simulation. Thus, this work should provide insights in the existence of inter-ethnic and inter-individual differences for these carcinogenic and genotoxic botanical ingredients and whether risk management actions are indicated.

Chapter 1 of this thesis, the present chapter presents the aim of the thesis, the model compounds used, namely alkenylbenzenes and 1,2-unsaturated PAs, which both are botanical ingredients that are hepatotoxic, genotoxic and carcinogenic, and the strategies applied to perform a mode of action based safety and risk assessment. It outlines the regulations and consumer behaviour towards botanical preparations in China and in several EU countries and introduces the outline of the thesis.

Chapter 2 describes the analysis of alkenylbenzenes in botanical preparations including PFS, herbal teas and TCM purchased on the Chinese market. A risk assessment for combined exposure to different alkenylbenzenes for the Chinese population based on the MOE approach was performed.

Chapter 3 defines a PBK model for metabolism of the alkenylbenzene estragole in the average Chinese population. The development of this PBK model allowed describing the bioactivation and detoxification of estragole in the average Chinese population. The outcomes predicted by the PBK model for the average Chinese population were compared to those predicted by the PBK model for Caucasians to evaluate the inter-ethnic differences in metabolic activation and detoxification of estragole and to demonstrate the potential of PBK modelling to study such inter-ethnic variability.

Chapter 4 presents the development of a PBK model for the PAs lasiocarpine and riddelliine in rat and mouse. Using PBK modelling-based reverse dosimetry, concentration-response curves obtained for toxicity of lasiocarpine and riddelliine in primary rat hepatocytes were translated to in vivo dose-response curves for acute liver toxicity. The model performance and predictions for in vivo liver toxicity were evaluated against in vivo data available in the literature.

Chapter 5 describes the development of PBK models for lasiocarpine and riddelliine in humans for two different ethnic populations (Chinese and Caucasian). These models were used to convert in vitro toxicity data obtained in pooled primary human hepatocytes to predict 
in vivo dose-response data for liver toxicity in humans. From these curves, PoD (BMDL5$\mathrm{BMDU}_{5}$ ) values were derived to predict the acute liver toxicity of lasiocarpine and riddelliine in humans. Subsequently, these PoDs were compared to PoDs derived previously for rats (chapter 4) to evaluate the species differences in the metabolism and toxicity of lasiocarpine and riddelliine, while the PoDs obtained for the average Chinese and average Caucasians provided insight in inter-ethnic differences in sensitivity to lasiocarpine and riddelliine.

Chapter 6 describes the combination of PBK modelling and Monte Carlo simulations to evaluate the consequences of inter-individual and inter-ethnic differences in kinetics for the sensitivity for the development of acute liver toxicity upon exposure to lasiocaprine, and to derive chemical specific adjustment factors (CSAFs). PBK modelling-based reverse dosimetry converting in vitro liver toxicity data provided in vivo dose-response curves for acute liver toxicity for the average as well as the sensitive (90 th and $99^{\text {th }}$ percentile) population.

Finally, chapter 7 presents a discussion of the results obtained in this thesis and provides future perspectives that can be addressed in the near future for further improvement of the risk assessment of botanicals containing ingredients that are both genotoxic and carcinogenic for different ethnic populations. 


\section{References}

Abdullah R, Alhusainy W, Woutersen J, Rietjens IMCM, Punt A (2016) Predicting points of departure for risk assessment based on in vitro cytotoxicity data and physiologically based kinetic (PBK) modeling: The case of kidney toxicity induced by aristolochic acid I. Food Chem Toxicol 92:104-116.

Al-Malahmeh AJ, Alajlouni AM, Ning J, Wesseling S, Vervoort J, Rietjens IMCM (2017a) Determination and risk assessment of naturally occurring genotoxic and carcinogenic alkenylbenzenes in nutmeg-based plant food supplements. J Appl Toxicol 37:1254-1264

Al-Malahmeh AJ, Al-Ajlouni AM, Wesseling S, Vervoort J, Rietjens IMCM (2017b) Determination and risk assessment of naturally occurring genotoxic and carcinogenic alkenylbenzenes in basil-containing sauce of pesto. Toxicol Rep 4:1-8

Al-Malahmeh AJ, Al-Ajlouni A, Wesseling S, Soffers AEMF, Al-Subeihi A, Kiwamoto R, Vervoort J, Rietjens IMCM (2017c) Physiologically based kinetic modeling of the bioactivation of myristicin. Arch Toxicol 91:713-734

Al-Subeihi AA, Spenkelink B, Punt A, Boersma MG, Van Bladeren PJ, Rietjens IMCM (2012) Physiologically based kinetic modeling of bioactivation and detoxification of the alkenylbenzene methyleugenol in human as compared with rat. Toxicol Appl Pharmacol 260:271-284

Alajlouni AM, Al-Malahmeh AJ, Isnaeni FN, Wesseling S, Vervoort J, Rietjens IMCM (2016a) Level of alkenylbenzenes in parsley and dill based teas and associated risk assessment using the Margin of Exposure approach. J Agr Food Chem 64:8640-8646

Alajlouni AM, Al Malahmeh AJ, Kiwamoto R, Wesseling S, Soffers AEMF, Al-Subeihi AAA, Vervoort J, Rietjens IMCM (2016b) Mode of action based risk assessment of the botanical food-borne alkenylbenzene apiol from parsley using physiologically based kinetic (PBK) modelling and read-across from safrole. Food Chem Toxicol 89:138-150

Alajlouni AM, Al-Malahmeh, AJ, Wesseling S, Kalli M, Vervoort J, Rietjens IMCM (2017) Risk assessment of combined exposure to alkenylbenzenes through consumption of plant food supplements containing parsley and dill. Food Addit Contam Part A 34:2201-2211

Anthony A, Caldwell J, Hutt AJ, Smith RL (1987) Metabolism of estragole in rat and mouse and influence of dose size on excretion of the proximate carcinogen 1'-hydroxyestragole. Food Chem Toxicol 25:799-806

Barlow S, Renwick AG, Kleiner J, Bridges JW, Busk L, Dybing E, Edler L, Eisenbrand G, FinkGremmels J, Knaap A, Kroes R, Liem D, Müller DJG, Page S, Rolland V, Schlatter J, Tritscher A, Tueting W, Würtzen G, (2006) Risk assessment of substances that are both genotoxic and carcinogenic: Report of an International Conference organized by EFSA and WHO with support of ILSI Europe. Food Chem Toxicol 44:1636-1650

Bell CC, Hendriks DFG, Moro SML, Ellis E, Walsh J, Renblom A, Fredriksson Puigvert L, Dankers ACA, Jacobs F, Snoeys J, Sison-Young RL, Jenkins RE, Nordling A, Mkrtchian S, Park BK, Kitteringham NR, Goldring CEP, Lauschke VM, Ingelman-Sundberg M (2016) Characterization of primary human hepatocyte spheroids as a model system for drug-induced liver injury, liver function and disease. Sci Rep 6:25187 
Borchert P, Wislocki PG, Miller JA, Miller EC (1973) The metabolism of the naturally occurring hepatocarcinogen safrole to 1'-hydroxysafrole and the electrophilic reactivity of 1'-acetoxysafrole. Cancer Res 33:575-589

Brauchli J, Luthy J, Zweifel U, Schlatter C (1982) Pyrrolizidine alkaloids from Symphytum officinale L. and their percutaneous absorption in rats. Experientia 38:1085-1087

Burkey JL, Sauer JM, McQueen CA, Sipes IG (2000) Cytotoxicity and genotoxicity of methyleugenol and related congeners-a mechanism of activation for methyleugenol. Mutat Res 453:25-33.

Centre for Food Safety (CFS) (2017) Pyrrolizidine Alkaloids in Food.

Chan PC, Haseman JK, Prejean JD, Nyska A (2003) Toxicity and carcinogenicity of riddelliine in rats and mice. Toxicol Lett 144:295-311

Chan VS, Caldwell J (1992) Comparative induction of unscheduled DNA synthesis in cultured rat hepatocytes by allylbenzenes and their 1'-hydroxy metabolites. Food Chem Toxicol 30:831-836

Chen L, Mulder PPJ, Louisse J, Peijnenburg A, Wesseling S, Rietjens IMCM (2017) Risk assessment for pyrrolizidine alkaloids detected in (herbal) teas and plant food supplements. Regul Toxicol Pharmacol 86:292-302

Chen M, Li L, Zhong D, Shen S, Zheng J, Chen X (2016) 9-Glutathionyl-6,7-dihydro-1hydroxymethyl-5H-pyrrolizine Is the Major Pyrrolic Glutathione Conjugate of Retronecine-Type Pyrrolizidine Alkaloids in Liver Microsomes and in Rats. Chem Res Toxicol 29:180-189

Chen T, Mei N, Fu PP (2010) Genotoxicity of pyrrolizidine alkaloids. J Appl Toxicol v 30:183-196

China Food and Drug Administration (CFDA) (2006) Regulation on production of herbal tea products

China Food and Drug Administration (CFDA) (2013a) List of herbs allowed for health food products (in Chinese)

China Food and Drug Administration (CFDA) (2013b) List of dietary herbs (in Chinese)

China Food and Drug Administration (CFDA) (2016) Detailed rules for the examination and approval of health food registration (in Chinese)

Chou MW, Yan J, Nichols J, Xia Q, Beland FA, Chan PC, Fu PP (2003) Correlation of DNA adduct formation and riddelliine-induced liver tumorigenesis in $\mathrm{F} 344$ rats and $\mathrm{B} 6 \mathrm{C} 3 \mathrm{~F} 1$ mice. Cancer letters 193:119-125

Culvenor CC (1983) Estimated intakes of pyrrolizidine alkaloids by humans A comparison with dose rates causing tumors in rats. J Toxicol Environ Health 11:625-635

Drinkwater NR, Miller EC, Miller JA, Pitot HC (1976) Hepatocarcinogenicity of Estragole (1-Allyl-4methoxybenzene) and 1'-Hydroxyestragole in the Mouse and Mutagenicity of 1'-Acetoxyestragole in Bacteria. J Natl Cancer Inst 57:1323-1331

Dusemund B, Nowak N, Sommerfeld C, Lindtner O, Schafer B, Lampen A (2018) Risk assessment of pyrrolizidine alkaloids in food of plant and animal origin. Food Chem Toxicol 115:63-72 
Dybing E, O'Brien J, Renwick AG, Sanner T (2008) Risk assessment of dietary exposures to compounds that are genotoxic and carcinogenic - An overview. Toxicol Lett 180:110-117

Edgar JA, Molyneux RJ, Colegate SM (2015) Pyrrolizidine Alkaloids: Potential Role in the Etiology of Cancers Pulmonary Hypertension Congenital Anomalies and Liver Disease. Chem Res Toxicol 28:4-20

Edwards SW, Preston RJ (2008) Systems biology and mode of action based risk assessment. Toxicol Sci 106:312-318

European Commission (EC) (2002) Directive 2002/46/EC of the European Parliament and of the Council of 10 June 2002 on the approximation of the laws of the Member States relating to food supplements. Official Journal of the European Communities 183:51-57

European Commission (EC) (2004) Directive 2004/24/EC of the European Parliament and of the Council of 31 March 2004 amending as regards traditional herbal medicinal products Directive 2001/83/ EC on the Community code relating to medicinal products for human use. Official Journal of the European Communities 136:85-90

European Commission (EC) (2008) No 1334/2008 of the European Parliament and of the Council of 16 December 2008 on flavourings and certain food ingredients with flavouring properties for use in and on foods and amending Council Regulation (EEC) No 1601/91 Regulations (EC) No 2232/96 and (EC) No 110/2008 and Directive 2000/13/EC. Official Journal of the European Communities 354:34-50

European Food Safety Authority (EFSA) (2005) Opinion of the Scientific Committee on a request from EFSA related to A Harmonised Approach for Risk Assessment of Substances Which are both Genotoxic and Carcinogenic. EFSA J 3:282

European Food Safety Authority (EFSA) (2009) Guidance of the Scientific Committee on Use of the benchmark dose approach in risk assessment. EFSA J 7:1150

European Food Safety Authority (EFSA) (2011) Scientific opinion on pyrrolizidine alkaloids in food and feed. EFSA J 9:2406

European Food Safety Authority (EFSA) (2012a) Compendium of botanicals reported to contain naturally occuring substances of possible concern for human health when used in food and food supplements. EFSA J 10: 2663

European Food Safety Authority (EFSA) (2012b) Guidance on selected default values to be used by the EFSA Scientific Committee Scientific Panels and Units in the absence of actual measured data. EFSA J 10:2579

European Food Safety Authority (EFSA) (2015) Scientific Opinion on acrylamide in food. EFSA J 13:4104

European Food Safety Authority (EFSA) (2016) Review of the Threshold of Toxicological Concern (TTC) approach and development of new TTC decision tree. EFSA J 13:1006E

European Food Safety Authority (EFSA) (2017) Risks for human health related to the presence of pyrrolizidine alkaloids in honey tea herbal infusions and food supplements. EFSA J 15:4908

Environmental Protection Agency (EPA) (1995) The use of the benchmark dose approach in health risk assessment. 
Environmental Protection Agency (EPA) (2005) Guidelines for Carcinogen Risk Assessment.

Environmental Protection Agency (EPA) (2018) BMDS Version 3.0 User Guide.

Forsby A, Blaauboer B (2007) Integration of in vitro neurotoxicity data with biokinetic modelling for the estimation of in vivo neurotoxicity. Hum Exp Toxicol 26:333-338

Forsch K, Schoning V, Disch L, Siewert B, Unger M, Drewe J (2018) Development of an in vitro screening method of acute cytotoxicity of the pyrrolizidine alkaloid lasiocarpine in human and rodent hepatic cell lines by increasing susceptibility. J Ethnopharmacol 217:134-139

Fu PP, Xia Q, Lin G, Chou MW (2002) Genotoxic Pyrrolizidine Alkaloids - Mechanisms Leading to DNA Adduct Formation and Tumorigenicity. Int J Mol Sci 3:948-964

Fu PP, Xia Q, Lin G, Chou MW (2004) Pyrrolizidine Alkaloids-Genotoxicity Metabolism Enzymes Metabolic Activation and Mechanisms. Drug Metab Rev 36:1-55

Fu PP, Chou MW, Churchwell M, Wang Y, Zhao Y, Xia Q, Gamboa da Costa G, Marques MM, Beland FA, Doerge DR (2010) High-performance liquid chromatography electrospray ionization tandem mass spectrometry for the detection and quantitation of pyrrolizidine alkaloid-derived DNA adducts in vitro and in vivo. Chem Res Toxicol 23:637-652

Fu Y, Yang JC, Cunningham AB, Towns AM, Zhang Y, Yang HY, Li JW, Yang XF (2018) A billion cups: The diversity traditional uses safety issues and potential of Chinese herbal teas. J Ethnopharmacol 222:217-228

Gao H, Ruan JQ, Chen J, Li N, Ke CQ, Ye Y, Lin G, Wang JY (2015) Blood pyrrole-protein adducts as a diagnostic and prognostic index in pyrrolizidine alkaloid-hepatic sinusoidal obstruction syndrome. Drug Des Devel 9:4861-4868

Garcia-Alvarez A, Egan B, de Klein S, Dima L, Maggi FM, Isoniemi M, Ribas-Barba L, Raats MM, Meissner EM, Badea M, Bruno F, Salmenhaara M, Mila-Villarroel R, Knaze V, Hodgkins C, Marculescu A, Uusitalo L, Restani P, Serra-Majem L (2014) Usage of plant food supplements across six European countries: findings from the PlantLIBRA consumer survey. PLoS One 9:e92265

Gomez-Lechon MJ, Tolosa L, Conde I, Donato MT (2014) Competency of different cell models to predict human hepatotoxic drugs. Expert Opin Drug Metab Toxicol 10:1553-1568

Griffin C, Gosetto F, Danaher M, Sabatini S, Furey A (2014) Investigation of targeted pyrrolizidine alkaloids in traditional Chinese medicines and selected herbal teas sourced in Ireland using LC-ESI-MS/ MS. Food Addit Contam Part A Chem Anal Control Expo Risk Assess 31(5):940-61

Groothuis FA, Heringa MB, Nicol B, Hermens JL, Blaauboer BJ, Kramer NI (2015) Dose metric considerations in in vitro assays to improve quantitative in vitro-in vivo dose extrapolations. Toxicology 332:30-40

Guenthner TM, Luo G (2001) Investigation of the role of the 2'3'-epoxidation pathway in the bioactivation and genotoxicity of dietary allylbenzene analogs. Toxicology 160:47-58

Hasheminejad G, Caldwell J (1994) Genotoxicity of the alkenylbenzenes alpha- and beta-asarone myristicin and elimicin as determined by the UDS assay in cultured rat hepatocytes. Food Chem Toxicol 32:223-231 
Hirono I, Haga M, Fujii M, Matsuura S, Matsubara N, Nakayama M, Furuya T, Hikichi M, Takanashi H, Uchida E, Hosaka S, Ueno I (1979) Induction of hepatic tumors in rats by senkirkine and symphytine. J Natl Cancer Inst 63:469-472

Howes AJ, Chan VS, Caldwell J (1990) Structure-specificity of the genotoxicity of some naturally occurring alkenylbenzenes determined by the unscheduled DNA synthesis assay in rat hepatocytes. Food Chem Toxicol 28: 537-542

International Agency for Research on Cancer (IARC) (2017) Agents Classified by the IARC Monographs International Agency for Research on Cancer. Lyon France

International Programme on Chemical Safety (IPCS) (2005) Chemical-specific adjustment factors for interspecies differences and human variability : guidance document for use of data in dose/ concentration-response assessment. World Health Organization.

Jeurissen SM, Bogaards JJ, Awad HM, Boersma MG, Brand W, Fiamegos YC, van Beek TA, Alink GM, Sudholter EJ, Cnubben NH, Rietjens IMCM (2004) Human cytochrome p450 enzyme specificity for bioactivation of safrole to the proximate carcinogen 1'-hydroxysafrole. Chem Res Toxicol 17:1245-1250

Jeurissen SM, Bogaards JJ, Boersma MG, ter Horst JP, Awad HM, Fiamegos YC, van Beek TA, Alink GM, Sudholter EJ, Cnubben NH, Rietjens IMCM (2006) Human cytochrome p450 enzymes of importance for the bioactivation of methyleugenol to the proximate carcinogen 1'hydroxymethyleugenol. Chem Res Toxicol 19:111-116

Jeurissen SM, Punt A, Boersma MG, Bogaards JJ, Fiamegos YC, Schilter B, van Bladeren PJ, Cnubben NH, Rietjens IMCM (2007) Human cytochrome p450 enzyme specificity for the bioactivation of estragole and related alkenylbenzenes. Chem Res Toxicol 20:798-806

Jeurissen SM, Buurma-Rethans EJM, Beukers MH, Jansen-van der Vliet M ,van Rossum CTM, Sprong RC (2018) Consumption of plant food supplements in the Netherlands. Food Funct 9:179-190

Joint FAO/WHO Expert Committee on Food Additives (JEFCA) (2009) Safety evaluation of certain food additives / prepared by the sixty-ninth meeting of the Joint FAO/WHO Expert Committee on Food Additives (JEFCA). World Health Organization.

Kakar F, Akbarian Z, Leslie T, Mustafa ML, Watson J, van Egmond HP, Omar MF, Mofleh J (2010) An outbreak of hepatic veno-occlusive disease in Western afghanistan associated with exposure to wheat flour contaminated with pyrrolizidine alkaloids. J Toxicol:313280

Krishnan K, McPhail B, Chiu W, White P, Fowler BA (2013) Computational Toxicology: Chapter 4 Modeling of Sensitive Subpopulations and Interindividual Variability in Pharmacokinetics for Health Risk Assessments Computational Toxicology. Academic Press San Diego pp 45-66

Kroes R, Renwick AG, Cheeseman M, Kleiner J, Mangelsdorf I, Piersma A, Schilter B, Schlatter J, van Schothorst F, Vos JG, Wurtzen G (2004) Structure-based thresholds of toxicological concern (TTC): guidance for application to substances present at low levels in the diet. Food Chem Toxicol 42:65-83

Kuhara K, Takanashi H, Hirono I, Furuya T, Asada Y (1980) Carcinogenic activity of clivorine a pyrrolizidine alkaloid isolated from Ligularia dentata. Cancer Lett 10:117-122

Li N, Xia QS, Ruan JQ, Fu PP, Lin G (2011) Hepatotoxicity and Tumorigenicity Induced by Metabolic Activation of Pyrrolizidine Alkaloids in Herbs. Curr Drug Metab 12:823-834 
Liang Q, Sheng Y, Jiang P, Ji L, Xia Y, Min Y, Wang Z (2011) The gender-dependent difference of liver GSH antioxidant system in mice and its influence on isoline-induced liver injury. Toxicology 280:61-69

Lin G, Wang JY, Li N, Li M, Gao H, Ji Y, Zhang F, Wang H, Zhou Y, Ye Y, Xu HX, Zheng J (2011) Hepatic sinusoidal obstruction syndrome associated with consumption of Gynura segetum. J Hepatol 54:666-673

Liu FX, Salmon JW (2010) Herbal medicine regulation in China Germany and the United States. Integr Med 9: 54-61

Liu SM, Zeng XW (2017) 基于多元定序Logit模型的中药保健品消费影响因素研究一一南宁市为 例 中国市场. China Market 27:91-95 (in Chinese)

Liu YZ, Wang ZM, Zhang JZ (2015) Dietary chinese herbs: Chemistry pharmacology and clinical evidence. Springer-Verlag Wien

Louisse J, de Jong E, van de Sandt JJ, Blaauboer BJ, Woutersen RA, Piersma AH, Rietjens IMCM, Verwei M (2010) The use of in vitro toxicity data and physiologically based kinetic modeling to predict dose-response curves for in vivo developmental toxicity of glycol ethers in rat and man. Toxicol Sci $118: 470-484$

Low TY, Wong KO, Yap ALL, Haan LHJ, Rietjens IMCM (2017) The regulatory framework across international jurisdictions for risks associated with consumption of botanical food supplements. Compr Rev Food Sci F 16:821-834

Luo G, Qato MK, Guenthner TM (1992) Hydrolysis of the 2'3'-allylic epoxides of allylbenzene estragole eugenol and safrole by both microsomal and cytosolic epoxide hydrolases. Drug Metab Dispos 20:440-445

Ma J, Xia Q, Fu PP, Lin G (2018) Pyrrole-protein adducts - A biomarker of pyrrolizidine alkaloidinduced hepatotoxicity. J Food Drug Anal 26:965-972

Martati E, Boersma MG, Spenkelink A, Khadka DB, van Bladeren PJ, Rietjens IMCM, Punt A (2012) Physiologically based biokinetic (PBBK) modeling of safrole bioactivation and detoxification in humans as compared with rats. Toxicol Sci 128:301-316

Mei N, Heflich RH, Chou MW, Chen T (2004a) Mutations induced by the carcinogenic pyrrolizidine alkaloid riddelliine in the liver cII gene of transgenic big blue rats. Chem Res Toxicol 17:814-818

Mei N, Chou MW, Fu PP, Heflich RH, Chen T (2004b) Differential mutagenicity of riddelliine in liver endothelial and parenchymal cells of transgenic big blue rats. Cancer Lett 215:151-158

Mei N, Guo L, Fu PP, Heflich RH, Chen T (2005) Mutagenicity of comfrey (Symphytum officinale) in rat liver. Br J Cancer 92:873-875

Mei X, Chen T (2007) The mutant frequency and types of mutations induced by comfrey in the lungs of transgenic Big Blue rats. J Food Drug Anal 15:458-465

Mei N, Guo L, Fu PP, Fuscoe JC, Luan Y, Chen T (2010) Metabolism genotoxicity and carcinogenicity of comfrey. J Toxicol Environ Health B Crit Rev 13:509-526 
Merz KH, Schrenk D (2016) Interim relative potency factors for the toxicological risk assessment of pyrrolizidine alkaloids in food and herbal medicines. Toxicol Lett 263:44-57

Miller EC, Swanson AB, Phillips DH, Fletcher L, Liem A, Miller JA (1983) Structure-activity studies of the carcinogenicities in the mouse and rat of some naturally occurring and synthetic alkenylbenzene derivatives related to safrole and estragole. Cancer Res 43:1124-1134

Miranda C,L Reed RL, Guengerich FP, Buhler DR (1991) Role of cytochrome P450IIIA4 in the metabolism of the pyrrolizidine alkaloid senecionine in human liver. Carcinogenesis 12:515-519

Munro IC, Kennepohl E, Kroes R (1999) A procedure for the safety evaluation of flavouring substances Joint FAO/WHO Expert Committee on Food Additives. Food Chem Toxicol 37:207-232

National Toxicology Program (NTP) (1978) Bioassay of lasiocarpine for possible carcinogenicity. Natl Cancer Inst Carcinog Tech Rep Ser 39:1-66

National Toxicology Program (NTP) (2000) Toxicology and Carcinogenesis Studies of Methyleugenol (CAS NO 93-15-2) in F344/N Rats and B6C3F1 Mice (Gavage Studies). Natl Toxicol Program Tech Rep Ser 491:1-412

National Toxicology Program (NTP) (2003) Toxicology and carcinogenesis studies of riddelliine (CAS No 23246-96-0) in F344/N rats and B6C3F1 mice (gavage studies). Natl Toxicol Program Tech Rep Ser 508:1-280

Phillips DH, Reddy MV, Randerath K (1984) 32P-post-labelling analysis of DNA adducts formed in the livers of animals treated with safrole estragole and other naturally-occurring alkenylbenzenes II Newborn male B6C3F1 mice. Carcinogenesis 5:1623-1628

Pumford NR, Halmes NC (1997) Protein targets of xenobiotic reactive intermediates. Annu Rev Pharmacol Toxicol 37:91-117

Punt A, Freidig AP, Delatour T, Scholz G, Boersma MG, Schilter B, van Bladeren PJ, Rietjens IMCM (2008) A physiologically based biokinetic (PBBK) model for estragole bioactivation and detoxification in rat. Toxicol Appl Pharmacol 231:248-259

Punt A, Paini A, Boersma MG, Freidig AP, Delatour T, Scholz G, Schilter B, Bladeren PJV, Rietjens IMCM (2009) Use of physiologically based biokinetic (PBBK) modeling to study estragole bioactivation and detoxification in humans as compared with male rats. Toxicol Sci 110:255-269

Randerath K, Haglund RE, Phillips DH, Reddy MV (1984) ${ }^{32}$ P-post-labelling analysis of DNA adducts formed in the livers of animals treated with safrole estragole and other naturally-occurring alkenylbenzenes I Adult female CD-1 mice. Carcinogenesis 5:1613-1622

Rietjens IMCM, Louisse J, Punt A (2011) Tutorial on physiologically based kinetic modeling in molecular nutrition and food research. Mol Nutr Food Res 55:941-956

Rietjens IMCM, Slob W, Galli C, Silano V (2008) Risk assessment of botanicals and botanical preparations intended for use in food and food supplements: emerging issues. Toxicol Lett 180:131-136

Rietjens IMCM, Cohen SM, Fukushima S, Gooderham NJ, Hecht S, Marnett LJ, Smith RL, Adams TB, Bastaki M, Harman CG, Taylor SV (2014) Impact of Structural and Metabolic Variations on the Toxicity 
and Carcinogenicity of Hydroxy- and Alkoxy-Substituted Allyl- and Propenylbenzenes. Chem Res Toxicol 27:1092-1103

Rijksinstituut voor Volksgezondheid en Milieu (RIVM) (2018) PROAST

Roeder E (2000) Medicinal plants in China containing pyrrolizidine alkaloids. Die Pharmazie 55:711-726

Rovida C, Hartung T (2009) Re-evaluation of animal numbers and costs for in vivo tests to accomplish REACH legislation requirements for chemicals - a report by the transatlantic think tank for toxicology $(\mathrm{t}(4))$. Altex 26:187-208

Ruan J, Gao H, Li N, Xue J, Chen J, Ke C, Ye Y, Fu PP, Zheng J, Wang J, Lin G (2015) Blood PyrroleProtein Adducts-A Biomarker of Pyrrolizidine Alkaloid-Induced Liver Injury in Humans. J Environ Sci Health C Environ Carcinog Ecotoxicol Rev 33:404-421

Sand S, Filipsson AF, Victorin K (2002) Evaluation of the Benchmark Dose Method for Dichotomous Data: Model Dependence and Model Selection. Regul Toxicol Pharmacol 36:184-197

Sangster SA, Caldwell J, Hutt AJ, Anthony A, Smith RL (1987) The metabolic disposition of [methoxy-14C]-labelled trans-anethole estragole and p-propylanisole in human volunteers. Xenobiotica $17: 1223-1232$

Scientific Committee on Food (SCF) (2001a) Opinion of the Scientific Committee on Food on Methyleugenol (4-Allyl-12-dimethoxybenzene).

Scientific Committee on Food (SCF) (2001b) Opinion of the scientific committee on food on estragole (1-Allyl-4-methoxybenzene).

Schoental R, Head MA, Peacock PR (1954) Senecio alkaloids: primary liver tumours in rats as a result of treatment with (1) a mixture of alkaloids from $\mathrm{S}$ jacobaea Lin; (2) retrorsine; (3) isatidine. Br J Cancer 8:458-465

Schoental R, Bensted JP (1963) Effects of Whole Body Irradiation and of Partial Hepatectomy on the Liver Lesions Induced in Rats by a Single Dose of Retrorsine a Pyrrolizidine (Senecio) Alkaloid. Br J Cancer 17:242-251

Shumaker RC, Robertson KA, Hsu IC, Allen JR (1976) Neoplastic transformation in tissues of rats exposed to monocrotaline or dehydroretronecine. J Natl Cancer Inst 56:787-790

Siano F, Ghizzoni C, Gionfriddo F, Colombo E, Servillo L, Castaldo D (2003) Determination of estragole safrole and eugenol methyl ether in food products. Food Chem 81:469-475

Smith LW, Culvenor CCJ (1981) Plant sources of hepatotoxic pyrrolizidine alkaloids. J Nat Prod $44: 129-152$

Smith RL, Adams TB, Doull J, Feron VJ, Goodman JI, Marnett LJ, Portoghese PS, Waddell WJ, Wagner BM, Rogers AE, Caldwell J, Sipes IG (2002) Safety assessment of allylalkoxybenzene derivatives used as flavouring substances-methyleugenol and estragole. Food Chem Toxicol 40:851-870 
Strikwold M, Spenkelink B, Woutersen RA, Rietjens IMCM, Punt A (2013) Combining in vitro embryotoxicity data with physiologically based kinetic (PBK) modelling to define in vivo dose-response curves for developmental toxicity of phenol in rat and human. Arch Toxicol 87:1709-1723

Strikwold M, Spenkelink B, de Haan LHJ, Woutersen RA, Punt A, Rietjens IMCM (2017) Integrating in vitro data and physiologically based kinetic (PBK) modelling to assess the in vivo potential developmental toxicity of a series of phenols. Arch Toxicol 91:2119-2133

Sun HX, Wang W, Liu XR, Yang SS, Wang ZX, Liu WJ (2013) Arsenic concentrations in chrysanthemum tea and assessment of arsenic risk to human. Asian J Ecotoxicol 8:230-237

Van den Berg SJ, Serra-Majem L, Coppens P, Rietjens IMCM (2011a) Safety assessment of plant food supplements (PFS). Food Funct 2:760-768

Van Den Berg SJ, Restani P, Boersma MG, Delmulle L, Rietjens IMCM (2011b) Levels of genotoxic and carcinogenic compounds in plant food supplements and associated risk assessment. Food Sci Nutr 2:989-1010

Van Den Berg SJ, Alhusainy W, Restani P, Rietjens IMCM (2014) Chemical analysis of estragole in fennel based teas and associated safety assessment using the Margin of Exposure (MOE) approach. Food Chem Toxicol 65:147-154

Vollmer JJ, Steiner NC, Larsen GY, Muirhead KM, Molyneux RJ (1987) Pyrrolizidine alkaloids: Testing for toxic constituents of comfrey. J Chem Educ 64:1027

Wang C, Li Y, Gao J, He Y, Xiong A, Yang L, Cheng X, Ma Y, Wang Z (2011) The comparative pharmacokinetics of two pyrrolizidine alkaloids senecionine and adonifoline and their main metabolites in rats after intravenous and oral administration by UPLC/ESIMS. Anal Bioanal Chem 401:275-287

Williams L, Chou MW, Yan J, Young JF, Chan PC, Doerge DR (2002) Toxicokinetics of Riddelliine a Carcinogenic Pyrrolizidine Alkaloid and Metabolites in Rats and Mice. Toxicol Appl Pharm 182:98-104

Wiseman RW, Miller EC, Miller JA, Liem A (1987) Structure-activity studies of the hepatocarcinogenicities of alkenylbenzene derivatives related to estragole and safrole on administration to preweanling male C57BL/6J x C3H/HeJ F1 mice. Cancer Res 47:2275-2283

Wislocki PG, Miller EC, Miller JA, McCoy EC, Rosenkranz HS (1977) Carcinogenic and mutagenic activities of safrole 1'-hydroxysafrole and some known or possible metabolites. Cancer Res 37:1883-1891

Xia Q, Chou MW, Kadlubar FF, Chan PC, Fu PP (2003) Human liver microsomal metabolism and DNA adduct formation of the tumorigenic pyrrolizidine alkaloid riddelliine. Chem Res Toxicol 16:66-73

Xia Q, Zhao Y, Von Tungeln LS, Doerge DR, Lin G, Cai L, Fu PP (2013) Pyrrolizidine alkaloid-derived DNA adducts as a common biological biomarker of pyrrolizidine alkaloid-induced tumorigenicity. Chem Res Toxicol 26:1384-1396

Xia Q, He X, Ma L, Chen S, Fu PP (2018) Pyrrolizidine Alkaloid Secondary Pyrrolic Metabolites Construct Multiple Activation Pathways Leading to DNA Adduct Formation and Potential Liver Tumor Initiation. Chem Res Toxicol 31:619-628 
Xu MY, Li XR (2011) Market Analysis of Development and Application of Chinese medicine Healthcare Products. J Tradit Complement Med 7:1-3

Yang XJ, Li WW, Sun Y, Guo XC, Huang WL, Peng Y, Zheng J (2017) Comparative Study of Hepatotoxicity of Pyrrolizidine Alkaloids Retrorsine and Monocrotaline. Chem Res Toxicol 30:532-539

Yang YC, Yan J, Doerge DR, Chan PC, Fu PP, Chou MW (2001) Metabolic activation of the tumorigenic pyrrolizidine alkaloid riddelliine leading to DNA adduct formation in vivo. Chem Res Toxicol 14:101-109

Zhang X, Tsang AM, Okino MS, Power FW, Knaak JB, Harrison LS, Dary CC (2007) A physiologically based pharmacokinetic/pharmacodynamic model for carbofuran in Sprague-Dawley rats using the exposure-related dose estimating model. Toxicol Sci 100:345-359

Zhou S, Chan E, Duan W, Huang M, Chen YZ (2005) Drug bioactivation covalent binding to target proteins and toxicity relevance. Drug Metab Rev 37:41-213

Zhu L, Wang Z, Wong L, He Y, Zhao Z, Ye Y, Fu PP, Lin G (2018) Contamination of hepatotoxic pyrrolizidine alkaloids in retail honey in China. Food Control 85:484-494 
<smiles>CCC(C)C(CC)C(C)C</smiles> 


\section{Chapter 2}

Risk assessment of genotoxic and carcinogenic alkenylbenzenes in botanical containing products present on the Chinese market
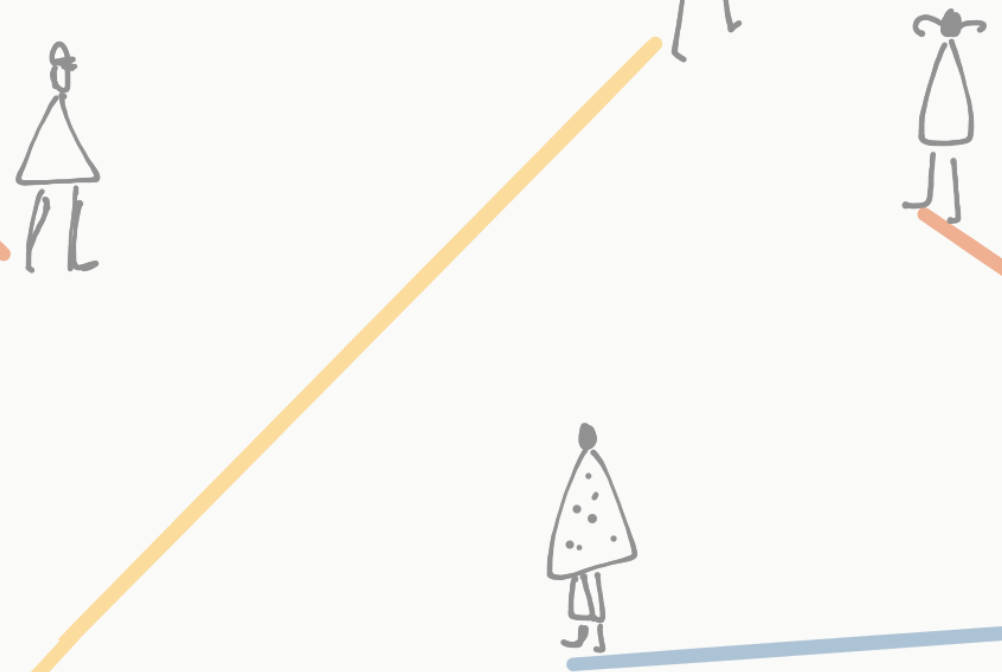

Jia Ning, XinYue Cui, XiangNan Kong, YiFei Tang, Riana Wulandari, Lu Chen, Sebas Wesseling, Ivonne M.C.M. Rietjens

Published in: Food and Chemical Toxicology (2018) 115, 344-357 


\begin{abstract}
In the present study, a risk assessment of plant food supplements (PFS), traditional Chinese medicines (TCM) and herbal teas containing alkenylbenzenes was performed using the Margin of Exposure (MOE) approach. The levels of alkenylbenzenes in botanical preparations collected on the Chinese market were quantified and the combined estimated daily intake (EDI) was determined using dose additivity. The combined EDI values obtained assuming equal potency of all alkenylbenzenes detected in the PFS, TCM and herbal teas were $0.3-14.3,0.05-539.4$ and $0.04-42.5 \mu \mathrm{g} / \mathrm{kg}$ bw/day, respectively. Calculating combined EDI values taking into account the toxic equivalency (TEQ) approach, the values for PFS, TCM and herbal teas were 0.3-7.7, 0.05-278.0 and 0.02-16.5 $\mu \mathrm{g}$ estragole equivalents $/ \mathrm{kg} \mathrm{bw} /$ day, respectively. The MOE values resulting from consumption of these PFS, TCM and one cup of herbal tea per day during life-time were generally lower than 10000 , suggesting a potential priority for risk management. For short-term exposure such as two weeks consumption, applying Haber's rule, only one TCM 6 (四神丸) still had an MOE value below 10 000. It is concluded that consumption of Chinese botanical preparations raise a concern because of exposure to alkenylbenzenes, especially when exposure is for longer periods of time.
\end{abstract}




\section{Introduction}

In China, there is a long history of using botanical containing products which can date back to 221-220 B.C. (Liu et al. 2015). After more than two thousand years of learning and summarizing, more than 6000 Chinese botanicals have been identified and more than 600 botanicals have been used to produce medical raw materials (Liu and Salmon 2010). From ancient times to the present, a health concept consisting of a combination of diet, herbal containing products including plant food supplement (PFS), traditional Chinese medicine (TCM), herbal teas, acupuncture and physical exercise has been integrated as part of Chinese culture. According to the literature, TCM are traditionally used as drugs to prevent and treat diseases and comprised of $30 \%-50 \%$ of the total medical consumption in China (Liu and Salmon 2010). Botanical preparations in China are easily accessible in most supermarkets, health-food shops or pharmacies where they are sold for relatively low prices. In the meantime, many consumers equate "natural" with "safe" when considering botanical preparations (Rietjens et al. 2008). However, several botanicals are known to contain toxic or even genotoxic and carcinogenic compounds (Rietjens et al. 2008).

In such herbal containing products, one of the main group of compounds of possible concern is the group of alkenylbenzenes, including compounds like estragole, methyleugenol, safrole and analogues (Figure 1) which are generally present in herbs like basil, nutmeg, fennel, dill and parsley (EFSA 2012; Van Den Berg et al. 2011). In the alkenylbenzene group, several compounds are proven to be genotoxic and carcinogenic and to cause liver tumors in experimental animals when given at high doses (Miller et al. 1983; Phillips et al. 1984; Randerath et al. 1984). The bioactivation pathway of these compounds proceeds by formation of a reactive 1'-sulfoxy metabolite in the liver, where this metabolite can bind with DNA and proteins to form adducts that will contribute to the formation of liver tumors (Al-Malahmeh et al. 2017a; Al-Subeihi et al. 2012; Alajlouni et al. 2016b; Martati et al. 2012; Punt et al. 2009; Van Den Berg et al. 2012).

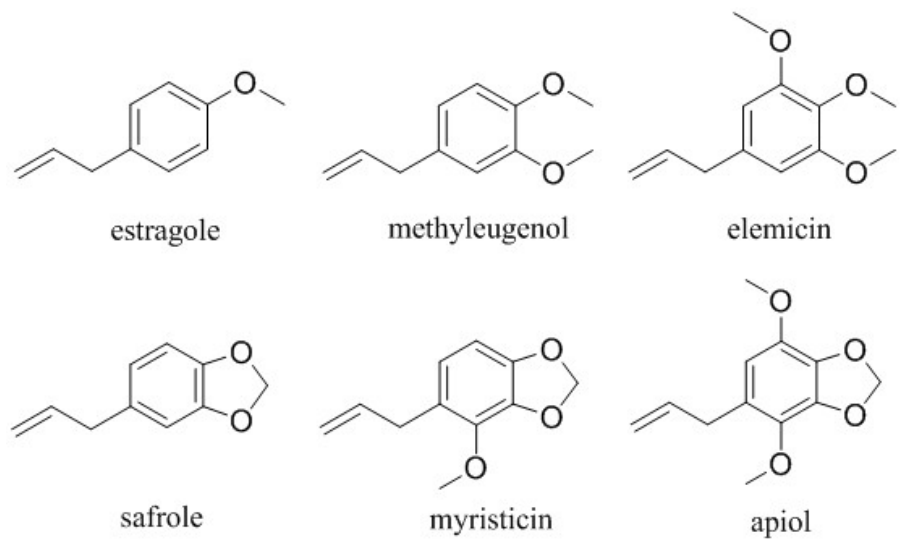

Fig. 1 Structural formulas of the six alkenylbenzenes of interest 
Due to their genotoxic and carcinogenic properties, the European Commission (EC) has prohibited the use of estragole, methyleugenol and safrole as pure compound in foods based on Regulation No 1334/2008 of the European Parliament and of the Council (EC 2008). However, there is no regulation specifically made regarding to the use of supplements containing alkenylbenzenes other than the general food law regulation [EC] No 178/2002 and the regulation of novel food (Regulation [EC] No 258/97 and Regulation [EU] 2015/2283) (EFSA 2009a). In China, PFS are regulated as "health foods" and there is a national standard for health food which includes the criteria of heavy metals and microbiologicals (Low et al. 2017; NHFPC 2014). However, there is no specific requirement for the relevant criteria for botanicals. Botanicals containing alkenylbenzenes have been used in China for a long time and the China Food and Drug Administration (CFDA) has developed a list of dietary botanicals and botanicals allowed for health food products (CFDA 2013a; CFDA 2013b). In this list, botanicals potentially containing alkenylbenzenes such as Illicium verum Hook.f(八 角茴香), Foeniculum vulgare Mill. (小茴香) and Myristica fragrans Houtt. (肉豆冦) can be found. Therefore, the risks associated with the levels of alkenylbenzenes present in a variety of botanical preparations on the Chinese market remain to be evaluated. Many different qualitative and quantitative risk assessment approaches for the risk and safety assessment of compounds that are both genotoxic and carcinogenic are available, and the Margin of Exposure (MOE) approach is recommended by expert groups of European Food Safety Authority (EFSA), the Joint FAO/WHO expert committee on Food Additives (JECFA) and the International Life Sciences Institute (ILSI) (EFSA 2005). The MOE is the ratio between a reference point usually obtained from epidemiological or experimental data on tumor incidence (i.e. the BMDL 10 , the lower confidence bound of the benchmark dose that gives $10 \%$ extra cancer incidence), and the estimated daily intake (EDI) in humans (EFSA 2005). An MOE value lower than 10000 is considered to indicate a priority for risk management (EFSA 2005).

Several risk assessment studies of products containing alkenylbenzenes present in western countries have been done using the MOE approach (Al-Malahmeh et al. 2017b; Alajlouni et al. 2016a; Alajlouni et al. 2017; Van Den Berg et al. 2014; Van Den Berg et al. 2011). The study of Van Den Berg et al. (2011) revealed that use of plant food supplements (PFS) containing methyleugenol, safrole and estragole might raise a potential concern for human health upon life-time consumption (Van Den Berg et al. 2011). The risk assessment of combined exposure to PFS containing parsley and dill or nutmeg showed that consumption of these products is only of concern for long periods of time (Al-Malahmeh et al. 2017b; Alajlouni et al. 2017). In the case of herbal teas containing alkenylbenzenes, Van Den Berg et al. (2014) reported that consumption of one cup of tea containing fennel on a daily basis during life-time for adults was a low priority for risk management (Van Den Berg et al. 2014). However, a combined risk assessment of alkenylbenzenes in parsley and dill based tea showed that there was a concern if the teas would be consumed on a daily basis over longer periods of time (Alajlouni et al. 2016a). Since China has a long history of using botanical preparations especially TCM, use of dietary herb applications has been integrated into people's daily life. Therefore, the aim of this study was to analyse the presence of different 
alkenylbenzenes in botanical products including TCM, PFS and herbal teas on the Chinese market and perform a combined risk assessment based on the MOE approach.

\section{Materials and Methods}

\subsection{Chemicals and materials:}

Estragole (purity $98 \% \mathrm{w} / \mathrm{w}$ ), methyleugenol (purity $99 \% \mathrm{w} / \mathrm{w}$ ), safrole (purity $>97 \% \mathrm{w} / \mathrm{w}$ ) and myristicin (purity $>97 \% \mathrm{w} / \mathrm{w}$ ) were obtained from Sigma Aldrich (Zwijdrecht, Netherland). Trifluoroacetic acid (TFA) was obtained from VWR International (Darmstadt, Germany). Acetonitrile (ULC/MS gradient) was obtained from Biosolve BV (Valkenswaard, Netherlands). Apiol (purity > $99 \%$ ) was obtained from Extrasynthese (Genay Cedex, France). Elemicin was obtained from Synchem OHG (Felsberg, Germany). Dimethyl sulfoxide (DMSO) and methanol were acquired from Acros Organics (Geel, Belgium). $0.45 \mu \mathrm{m}$ and $0.25 \mu \mathrm{m}$ Cellulose acetate membrane filter were obtained from Chromafil (Duren, Germany). Nanopure water was obtained from a pro UF/VF water purification system (Sartorius Weighting Technology GmbH, Goettingen, Germany).

\subsection{Sampling}

The alkenylbenzenes are naturally present in the plant families Acoraceae, Umbelliferae, Myristicaceae, Labiatae, Lauraceae and Compositeae (EFSA 2012; JEFCA 2009). Table 1 shows a list of plants generally containing alkenylbenzenes. According to the book of named Flora of China, the corresponding Chinese common names of these plants were derived and were given in Pin Yin, which is a phonetic representation of Chinese characters. And the alternative names for the corresponding botanicals in Pin Yin were also presented, since different regions of China might use alternative names for the same botanicals. For example, Luo Le (罗勒) is often called Jiu Ceng Ta (九层塔) and some regions of China name fennel as Xiao Hui Xiang (小茴香) rather than as Hui Xiang (茴香). For sampling, products were selected by screening the ingredient list of the samples for the suspected herbs through the common or alternative name in Chinese.

In total, 71 botanical preparations including plant food supplement (10 samples), traditional Chinese medicine (23 samples) and herbal tea products ( 38 samples) were purchased from the Chinese market via the internet or health shops. For PFS and TCM, the products were provided in the form of capsules, tablets or powder. For tea samples, the products were provided as tea bags, powder, whole fruit or leaves. Some herbal teas were provided as the coarse herbal materials without labelling. The product information is summarized in Table S1 in the supplementary materials. This Table S1 also presents the recommended use of the product as listed on the label by the manufacturer, which was used in calculating the estimated daily intake (EDI) (see section 2.6). 
Table 1 Examples of botanicals generally containing alkenylbenzenes.

\begin{tabular}{|c|c|c|c|c|}
\hline Pin Yin name & $\begin{array}{l}\text { Alternative } \\
\text { name in Pin Yin }\end{array}$ & $\begin{array}{l}\text { Botanical } \\
\text { name }\end{array}$ & Latin name & References \\
\hline Hui Qin & N.A. ${ }^{a}$ & Anise & Pimpinella anisum L. & $\begin{array}{l}\text { (She et al. } \\
\text { 2005) }\end{array}$ \\
\hline Ba Jiao & $\begin{array}{l}\text { Ba Jiao Hui } \\
\text { Xiang, Da Hui } \\
\text { Xiang }\end{array}$ & Star anise & Illicium verum Hook.f. & $\begin{array}{l}\text { (Xia and } \\
\text { Saunders } \\
\text { 2008) }\end{array}$ \\
\hline Hui Xiang & Xiao Hui Xiang & Fennel & $\begin{array}{l}\text { Foeniculum vulgare } \\
\text { Mill. }\end{array}$ & $\begin{array}{l}\text { (She et al. } \\
\text { 2005) }\end{array}$ \\
\hline Ou Qin & $\begin{array}{l}\text { Ba Xi Li, Xiang } \\
\text { Qin }\end{array}$ & Parsley & $\begin{array}{l}\text { Petroselinum crispum } \\
\text { (Mill.) Hill }\end{array}$ & $\begin{array}{l}\text { (She et al. } \\
\text { 2005) }\end{array}$ \\
\hline Rou Dou Kou & $\begin{array}{l}\text { Rou Guo, Yu } \\
\text { Guo }\end{array}$ & Nutmeg & $\begin{array}{l}\text { Myristica fragrans } \\
\text { Houtt. }\end{array}$ & $\begin{array}{l}\text { (Li and } \\
\text { Wilson 2008) }\end{array}$ \\
\hline Luo Le & $\begin{array}{l}\text { Jiu Ceng Ta, Lan } \\
\text { Xiang }\end{array}$ & Basil & Ocimum basilicum L. & $\begin{array}{l}\text { (Li and } \\
\text { Hedge 1994) }\end{array}$ \\
\hline Long Hao & $\begin{array}{l}\text { She Hao, Qing } \\
\text { Hao }\end{array}$ & Tarragon & $\begin{array}{l}\text { Artemisia dracunculus } \\
\text { L. }\end{array}$ & $\begin{array}{l}\text { (Lin et al. } \\
\text { 2011) }\end{array}$ \\
\hline Rou Gui & Yu Gui, Gui Pi & Cinnamon & $\begin{array}{l}\text { Cinnamomum cassia } \\
\text { Presl }\end{array}$ & $\begin{array}{l}\text { (Li et al. } \\
2008)\end{array}$ \\
\hline Chang Pu & $\begin{array}{l}\text { Shi Chang Pu, } \\
\text { Bai Chang Pu }\end{array}$ & $\begin{array}{l}\text { Acorus } \\
\text { calamus }\end{array}$ & Acorus calamus L. & $\begin{array}{l}\text { (Li et al. } \\
2010)\end{array}$ \\
\hline $\begin{array}{l}\text { Ning Meng } \\
\text { Cao }\end{array}$ & Xiang Mao & Lemongrass & $\begin{array}{l}\text { Cymbopogon citratus } \\
\text { (DC.) Stapf }\end{array}$ & $\begin{array}{l}\text { (Chen and } \\
\text { Sylvia 2006) }\end{array}$ \\
\hline
\end{tabular}

a N.A. not available

\subsection{Methanolic extraction}

Methanolic extraction was applied to completely extract and quantify the amount of different alkenylbenzenes present in the samples purchased from the Chinese market. The extracts were prepared based on the method previously described (Alajlouni et al. 2016a; Gursale et al. 2010). Briefly, $0.5 \mathrm{~g}$ of sample was suspended in $50 \mathrm{ml}$ methanol followed by $15 \mathrm{~min}$ of ultrasonication at room temperature. Subsequently, the sample was filtered through a $0.45 \mu \mathrm{m}$ cellulose acetate membrane filter and the filtrate was stored at $-20{ }^{\circ} \mathrm{C}$ until Ultra Performance Liquid Chromatography (UPLC) analysis. Each of the samples was extracted in triplicate. A recovery test was performed by spiking pure alkenylbenzene compounds on three samples to correct for the possible loses during the extraction process. The spiked samples were 
cellulose acetate membrane filter and the filtrate was stored at $-20{ }^{\circ} \mathrm{C}$ until Ultra Performance Liquid Chromatography (UPLC) analysis. Each of the samples was extracted in triplicate. A recovery test was performed by spiking pure alkenylbenzene compounds on three samples to correct for the possible loses during the extraction process. The spiked samples were extracted following the same extraction method as described above. The average recovery percentages were found to be $100 \pm 8.3 \%$ for estragole, $105.3 \pm 5.9 \%$ for elemicin, $105.8 \pm$ $9.6 \%$ for methyleugenol, $82.4 \pm 8.9 \%$ for safrole, $109.7 \pm 8.1 \%$ for myristicin and $80.3 \pm 12$ $\%$ for apiol.

\subsection{Hot water extraction}

Hot water extraction was performed to mimic the way of preparation of the homemade tea and performed as described previously (Alajlouni et al. 2016a; Van Den Berg et al. 2014). According to the safety assessment of fennel teas reported by the EFSA Scientific Cooperation (ESCO) Working Group on Botanicals and Botanical Preparations, 1.5-2.5 g of tea would be used to prepare a cup of tea within $250 \mathrm{ml}$ boiling water for an adult (EFSA 2009a). To mimic this preparation, $0.5 \mathrm{~g}$ of tea sample was suspended in $50 \mathrm{ml}$ boiling water, followed by incubation for $7 \mathrm{~min}$ in a covered beaker. During this incubation period, the samples were mixed three times over $10 \mathrm{~s}$ with a teaspoon at $0 \mathrm{~min}, 3 \mathrm{~min}$ and $6 \mathrm{~min}$, respectively. After $7 \mathrm{~min}$ of incubation, the water extract was separated from tea samples and was left for cooling to room temperature. Subsequently, the extract was centrifuged at 15000 $\mathrm{g}$ for $5 \mathrm{~min}$, and the supernatants were collected and stored at $-20{ }^{\circ} \mathrm{C}$ until UPLC analysis. Each of the herbal teas was extracted in triplicate.

\subsection{UPLC analysis}

For quantification of alkenylbenzene compounds, $3.5 \mu \mathrm{l}$ of each sample was subjected to UPLC analysis. The UPLC system consisted of an ACQUITY UPLC photodiode array detector, a quaternary solvent manager and an ACQUITY UPLC BEH C18 $1.7 \mu \mathrm{m}$ column, $2.1 \times 50 \mathrm{~mm}$. The column was set at $35^{\circ} \mathrm{C}$ and the sample manager was set at $7{ }^{\circ} \mathrm{C}$. The gradient was made with $0.1 \%$ TFA (v/v) in nanopure water and $100 \%$ acetonitrile. The flow rate was set at $0.6 \mathrm{ml} / \mathrm{min}$. The mobile phase started with $32 \%$ acetonitrile for $1 \mathrm{~min}$, after which the percentage of acetonitrile was linearly increased to $33.5 \%$ in $2.5 \mathrm{~min}$, then further increased to $60 \%$ in $2.5 \mathrm{~min}$, followed by continued increasing to $80 \%$ in $0.5 \mathrm{~min}$ and finally kept at this level for $0.5 \mathrm{~min}$, after which the starting condition was reset. Under these conditions, alkenylbenzenes were identified by comparing their retention time and UV spectrum to those of reference compounds. Quantification of alkenylbenzenes was achieved by comparison of the peak area obtained at $206 \mathrm{~nm}$ for elemicin, $202 \mathrm{~nm}$ for myristicin and safrole, $209 \mathrm{~nm}$ for apiol and methyleugenol, and $225 \mathrm{~nm}$ for estragole to the calibration curves of the reference compounds. 


\subsection{Estimation of daily intake}

The estimated daily intakes (EDIs) of the individual alkenylbenzenes were obtained based on the content of alkenylbenzenes in the samples, the recommended daily intake provided by the manufacturer (Table S1) and a body weight of $60 \mathrm{~kg}$ for a Chinese adult (NHFPC 2007).

Since some of the samples seemed to contain more than one alkenylbenzene, there is a need to perform a combined exposure and risk assessment. The combined EDI was determined based on the dose additivity concept, because alkenylbenzenes show similarities in structure, adverse effect, target organ and mode of action proceeding by bioactivation to form a 1'sulfooxymetabolite that contributes to the formation of liver tumors (Al-Malahmeh et al. 2017a; Al-Subeihi et al. 2012; Alajlouni et al. 2016b; Martati et al. 2012; Punt et al. 2009; Van Den Berg et al. 2012). According to these similarities, the combined EDI was calculated by two approaches: summing up the individual EDI of the individual alkenylbenzenes as such assuming the equal potency, or using a so-called toxic equivalency (TEQ) approach.

For the TEQ approach, the interim relative potency factors (REPs) of each alkenylbenzene were defined using three endpoints and estragole was selected as the index compound (REP $=1$ ) resulting in the combined EDI expressed as estragole equivalents. The first approach used to obtain interim REP values was based on the data of DNA adduct formation of different alkenylbenzenes in CD-1 mice as reported by Randerath et al. (1984), from which the slopes of dose-response curves for DNA adduct formation of the different alkenylbenzenes in the liver were derived. The second endpoint was based on the data obtained from human physiologically based kinetic (PBK) models which were used to predict the ultimate carcinogenic 1'-sulfoxy metabolite formation of the alkenylbenzenes of interest representing the relative importance of their bioactivation route (Al-Malahmeh et al. 2017a; Al-Subeihi et al. 2012; Alajlouni et al. 2016b; Martati et al. 2012; Punt et al. 2009; Van Den Berg et al. 2012). The third approach was based on the benchmark dose lower confidence limit for $10 \%$ extra tumor incidence above background levels $\left(\mathrm{BMDL}_{10}\right)$ of the different alkenylbenzenes of interest derived from carcinogenicity data reported by Miller et al. (1983) and NTP (2000) or obtained by read-across (Al-Malahmeh et al. 2017a; Alajlouni et al. 2016b; Van Den Berg et al. 2012). Specifically, the $\mathrm{BMDL}_{10}$ of elemicin was determined by read-across from estragole and methyleugenol (Van Den Berg et al. 2012) and the BMDL 10 of safrole was used for derivation of the BMDL 10 of myristicin (Al-Malahmeh et al. 2017a) and apiol (Alajlouni et al. 2016b). An overview of these $B_{M D L} 10$ values from which interim REP values were derived are presented in Table 2. Finally, the interim REP values were taken as the average of the results from the three endpoints. These interim REP values were used to calculate EDIs of alkenylbenzenes detected in the samples expressed in $\mu \mathrm{g}$ estragole equivalents $/ \mathrm{kg} \mathrm{bw} / \mathrm{day}$. An overview of the interim REP values acquired from the three different endpoints and the average values derived from them is presented in Table 3. 
Table 2 BMDL $_{10}$ values for the different alkenylbenzenes derived from the literature.

\begin{tabular}{ccc}
\hline Compound & $\begin{array}{c}\text { BMDL } 10 \\
(\mathrm{mg} / \mathrm{kg} \text { bw/day) }\end{array}$ & Reference \\
\hline Estragole & 3.3 & (Van Den Berg et al. 2011) \\
Myristicin & 1.9 & (Al-Malahmeh et al. 2017a) \\
Apiol & 5.7 & (Alajlouni et al. 2016b) \\
Safrole & 1.9 & (Van Den Berg et al. 2011) \\
Methyleugenol & 15.3 & (Smith et al. 2010; Van Den Berg et al. 2011) \\
Elemicin & 30.6 & (Van Den Berg et al. 2012) \\
\hline
\end{tabular}

a Details of the BMD analysis can be found in the study of Van Den Berg et al. (2011)

Table 3 An overview of the interim REF values obtained using three endpointsa,b,c.

\begin{tabular}{ccccc}
\hline Compound & $\begin{array}{c}\text { REP derived } \\
\text { from in vivo } \\
\text { DNA adduct } \\
\text { formation }^{\mathrm{a}}\end{array}$ & $\begin{array}{c}\text { REP derived from } \\
\text { human PBK model } \\
\text { based prediction of } \\
1^{\text {'-sulfoxy formation }}{ }^{\mathrm{b}}\end{array}$ & $\begin{array}{c}\text { REP derived from } \\
\text { the BMDL } 10 \text { for } \\
\text { liver tumor } \\
\text { formation }^{\mathrm{c}}\end{array}$ & $\begin{array}{c}\text { Average } \\
\text { REP value }\end{array}$ \\
\hline Estragole & 1.00 & 1.00 & 1.00 & 1.00 \\
Myristicin & 0.16 & 0.17 & 1.74 & $0.69 \pm 0.91$ \\
Apiol & 0.04 & 0.11 & 0.58 & $0.24 \pm 0.29$ \\
Safrole & 0.68 & 0.23 & 1.74 & $0.88 \pm 0.78$ \\
$\begin{array}{c}\text { Methyl- } \\
\text { eugenol }\end{array}$ & 0.65 & 0.22 & 0.22 & $0.36 \pm 0.25$ \\
Elemicin & 0.06 & 0.09 & 0.11 & $0.09 \pm 0.03$ \\
\hline
\end{tabular}

a Randerath et al. 1984

b Al-Malahmeh et al. 2017a; Al-Subeihi et al. 2012; Alajlouni et al. 2016b; Martati et al. 2012; Punt et al. 2009; Van Den Berg et al. 2012

c Al-Malahmeh et al. 2017a; Alajlouni et al. 2016b; Miller et al. 1983; Van Den Berg et al. 2012

\subsection{Calculation of Margin of Exposure}

The MOE values were calculated in three ways: 1) the MOE values for individual alkenylbenzenes present in the samples were calculated via dividing the respective BMDL 10 of the relevant alkenylbenzene as shown in Table 2 by the individual EDI; 2) MOE values were calculated via dividing the $\mathrm{BMDL}_{10}$ of the major alkenylbenzene present in the sample 
were calculated via dividing the $\mathrm{BMDL}_{10}$ of the major alkenylbenzene present in the sample by the combined EDI assuming the equal potency of all alkenylbenzenes; 3) the MOE values were calculated based on the TEQ approach, where the BMDL 10 of estragole was divided by the combined EDI expressed in estragole equivalents.

Affected by advertisements of PFS and limited knowledge of side effect of TCMs, some Chinese consumers might take the botanical preparations for long periods of time. However, most Chinese consumers take botanical preparations, especially TCMs, for shorter time intervals. Therefore, it is of interest to evaluate what exposure frequency and duration would result in MOE value higher than 10 000, indicating a low priority of risk management. This was estimated using Haber's rule to convert EDI values for 1, 2 and 4 weeks exposure to lifetime exposure and comparison of these corrected life-time EDI values to the BMDL 10 values of estragole. This approach using Haber's rule assumes that the short-term exposure can be converted to a life-time exposure assuming the dose-response relationship to be linear and the product of time and exposure level to be constant (Felter et al. 2011).

\section{Results}

\subsection{Chemical analysis of samples}

Table 4 displays the levels of different alkenylbenzenes as detected in PFS and TCM obtained from the Chinese market. In the case of PFS, it appeared that 7 out of 10 samples contained alkenylbenzenes of interest and 4 out of 7 positively tested samples contained more than one alkenylbenzene. The levels of alkenylbenzenes of interest in PFS ranged from 7.2-341.1 $\mu \mathrm{g} / \mathrm{g}$. Among the six alkenylbenzenes, estragole was the most frequently detected compound in the PFS samples ( 5 samples), followed by myristicin ( 2 samples), apiol (2 samples), elemicin (2 samples), and methyleugenol (1 sample). Safrole was non-detectable in the PFS samples.

For TCMs, 12 out of 23 samples contained the alkenylbenzenes of interest, among which 2 out of 12 positively tested samples comprised more than one alkenylbenzene compounds. The levels of alkenylbenzenes of interest in TCM ranged from 1.4-1160.3 $\mu \mathrm{g} / \mathrm{g}$. Both estragole and myristicin were detected the most frequent ( 5 samples), followed by methyleugenol (4 samples), elemicin (2 samples) and safrole (1 sample). It is important to point out that the amount of the different alkenylbenzenes varies greatly among the TCM samples analysed, and it is quite interesting to notice that TCM 6 contained the highest amount of myristicin, elemicin, methyleugenol and safrole, with a level of myristicin that was about 5 times higher than the level in other myristicin containing samples. 
Table 4 The levels of different alkenylbenzenes presented in PFS and TCM as determined by methanol extraction.

\begin{tabular}{|c|c|c|}
\hline $\begin{array}{l}\text { Botanical } \\
\text { preparation }^{\mathrm{a}}\end{array}$ & $\begin{array}{c}\text { Alkenylbenzenes } \\
\text { detected }\end{array}$ & $\begin{array}{c}\text { Alkenylbenzene levels } \\
\text { (average } \pm \mathrm{SD} \mu \mathrm{g} / \mathrm{g})\end{array}$ \\
\hline \multicolumn{3}{|l|}{ PFS } \\
\hline \multirow[t]{2}{*}{ PFS 1} & elemicin & $26.4 \pm 5.9$ \\
\hline & myristicin & $28.2 \pm 0.7$ \\
\hline PFS 2 & estragole & $10.0 \pm 1.5$ \\
\hline \multirow[t]{2}{*}{ PFS 3} & apiol & $12.0 \pm 0.7$ \\
\hline & estragole & $252.1 \pm 9.9$ \\
\hline \multirow[t]{2}{*}{ PFS 4} & methyleugenol & $17.8 \pm 0.8$ \\
\hline & estragole & $9.6 \pm 1.0$ \\
\hline PFS 5 & estragole & $7.2 \pm 1.4$ \\
\hline PFS 6 & estragole & $83.2 \pm 3.6$ \\
\hline \multirow[t]{3}{*}{ PFS 7} & elemicin & $37.1 \pm 7.6$ \\
\hline & myristicin & $341.1 \pm 48.3$ \\
\hline & apiol & $270.8 \pm 39.2$ \\
\hline PFS 8 & N.D.b & - \\
\hline PFS 9 & N.D. & - \\
\hline PFS 10 & N.D. & - \\
\hline \multicolumn{3}{|l|}{ TCMs } \\
\hline TCM 1 & N.D. & - \\
\hline TCM 2 & methyleugenol & $12.4 \pm 4.2$ \\
\hline TCM 3 & N.D. & - \\
\hline TCM 4 & myristicin & $216.9 \pm 5.3$ \\
\hline TCM 5 & myristicin & $176.2 \pm 6.4$ \\
\hline \multirow[t]{4}{*}{ TCM 6} & safrole & $18.8 \pm 3.0$ \\
\hline & methyleugenol & $214.2 \pm 36.0$ \\
\hline & elemicin & $404.8 \pm 49.2$ \\
\hline & myristicin & $1160.3 \pm 155.7$ \\
\hline
\end{tabular}


Table 4 (continued) The levels of different alkenylbenzenes presented in PFS and TCM as determined by methanol extraction.

\begin{tabular}{|c|c|c|}
\hline $\begin{array}{c}\text { Botanical } \\
\text { preparationa }\end{array}$ & $\begin{array}{c}\text { Alkenylbenzenes } \\
\text { detected }\end{array}$ & $\begin{array}{l}\text { Alkenylbenzene levels } \\
\text { (average } \pm \mathrm{SD} \mu \mathrm{g} / \mathrm{g})\end{array}$ \\
\hline TCM 7 & estragole & $32.0 \pm 2.4$ \\
\hline TCM 8 & estragole & $17.7 \pm 7.2$ \\
\hline TCM 9 & estragole & $126.3 \pm 1.6$ \\
\hline \multirow[t]{3}{*}{ TCM 10} & methyleugenol & $33.2 \pm 8.4$ \\
\hline & elemicin & $65.7 \pm 13.5$ \\
\hline & myristicin & $147.6 \pm 11.3$ \\
\hline TCM 11 & myristicin & $41.1 \pm 2.9$ \\
\hline TCM 12 & N.D. & - \\
\hline TCM 13 & estragole & $1.4 \pm 0.7$ \\
\hline TCM 14 & N.D. & - \\
\hline TCM 15 & N.D. & - \\
\hline TCM 16 & N.D. & - \\
\hline TCM 17 & N.D. & - \\
\hline TCM 18 & N.D. & - \\
\hline TCM 19 & N.D. & - \\
\hline TCM 20 & estragole & $8.4 \pm 4.4$ \\
\hline TCM 21 & methyleugenol & $60.5 \pm 1.5$ \\
\hline TCM 22 & N.D. & - \\
\hline TCM 23 & N.D. & - \\
\hline
\end{tabular}

a The detailed information of botanical preparations are summarized in Table S1.

b N.D. not detected.

Table 5 shows the level of alkenylbenzenes detected in herbal tea samples after both methanol and hot water extraction as well as the corresponding extraction efficiencies. The extraction efficiency was calculated by comparing the amount of alkenylbenzenes in the hot water extracts to those in the methanolic extracts taking the amounts of tea extracted in the two extraction procedures into account. The extraction efficiency varied from 1.6 to $97.6 \%$ and this wide variation in the hot water extraction efficiency might be related to the form of the herbal teas. For example, the herbal teas provided as whole fruit resulted in the average extraction efficiency of $19.4 \%$, while the average extraction efficiency for the grinded leaf or 
fine cut was $37.3 \%$. In line with these findings, Alajlouni et al. (2016a) also reported that the extraction efficiency of fine cut materials is higher compared to that of the whole fruit materials (Alajlouni et al. 2016a). Table 5 also reveals that 28 out of 38 herbal teas contained alkenylbenzenes and 7 out of these 28 samples appeared to contain more than one alkenylbenzene. In line with the nature of the botanical present in the herbal teas (often including fennel), estragole was the most frequently detected alkenylbenzene.

\subsection{EDI of alkenylbenzenes resulting from consumption of the botanical preparations}

The EDI values of the alkenylbenzenes resulting from consumption of the PFS, TCMs and herbal teas were determined based on the amount of the different alkenylbenzenes detected in the samples, the recommended daily intake and use of the sample provided by the suppliers (Table S1) and assuming a body weight of $60 \mathrm{~kg}$. For those herbal teas, where the information of a recommended daily intake was not available, it was assumed that $2 \mathrm{~g}$ of dry tea materials is used to prepare a cup of tea of $200 \mathrm{ml}$ based on the assumption of the ESCO working group (EFSA 2009a).

Table 6 shows the EDI values of the individual alkenylbenzenes and also the combined EDI assuming equal potency or based on the TEQ approach expressed in estragole equivalents for PFS, TCM and herbal teas assuming use of one cup of tea per day. Combined exposure was expressed in estragole equivalents because estragole appeared to be the major alkenylbenzene detected in the botanical samples analysed. For individual alkenylbenzenes, the EDI values amounted to $0.3-7.6 \mu \mathrm{g} / \mathrm{kg}$ bw/day for PFS, $0.05-348.1 \mu \mathrm{g} / \mathrm{kg} \mathrm{bw} /$ day for TCMs, and 0.03-16.7 $\mu \mathrm{g} / \mathrm{kg}$ bw/day for herbal teas. When assuming equal potency of the different alkenylbenzenes, the EDI values for PFS, TCM and herbal teas amounted to 0.3-14.3, $0.05-539.4$ and $0.04-42.5 \mu \mathrm{g} / \mathrm{kg}$ bw/day, respectively. For combined EDI values based on TEQ approach, the values amounted to 0.3-7.7 $\mu \mathrm{g}$ estragole equivalents $/ \mathrm{kg} \mathrm{bw} /$ day for PFS, 0.05-278.0 $\mu \mathrm{g}$ estragole equivalents/kg bw/day for TCM and 0.02-16.5 $\mu \mathrm{g}$ estragole equivalents/kg bw/day for herbal tea. The EDIs obtained using the TEQ approach with estragole as the reference compound showed relatively lower values compared with the combined EDI values assuming equal potency of the alkenylbenzenes, because the interim REP value of the other alkenylbenzenes that were present in the samples are lower than the value of 1.0 used for estragole as the reference compound.

\subsection{Risk assessment of consumption of the botanical preparations based on the MOE approach}

Figure 2 shows the MOE values for the combined risk assessment based on assuming equal potency of the alkenylbenzenes. Figure S2.1-S2.3 in the supplementary materials 2 present the MOE values for consumption of botanical preparations based on consideration of the levels of the individual alkenylbenzenes (A) and calculated using their respective individual EDIs shown in Table 6 and their respective BMDL 10 values taken from literature (AlMalahmeh et al. 2017a; Alajlouni et al. 2016b; Van Den Berg et al. 2012; Van Den Berg et al. 2011) as shown in Table 2. Figure S2.1-S2.3 in the supplementary materials 2 present the MOE values obtained for the combined risk assessment based on the TEQ approach (B). All 
MOE values are based on life-time exposure in line with the MOE approach as defined (EFSA 2005).

For PFS, 6 out of 10 samples give rise to MOE values for consumption of the individual alkenylbenzenes lower than 10000 and almost all PFS samples give MOE values lower than 10000 when taking combined exposure into account in both approaches except for sample PFS 5. For TCM samples, 10 out of 23 samples had individual MOE values lower than 10 000, and TCM 6 had an individual MOE value even lower than 10, which means that the daily intake of the alkenylbenzene of interest, myristicin, would be within the range of dose levels causing liver tumors in experimental animals (Van Den Berg et al. 2011). For the MOE values derived taking combined exposure into account, 10 out of 23 TCM samples had MOE values lower than 10000 in both methods. The MOE of TCM 6 was even lower than 10 when assuming equal potency of the alkenylbenzenes. For consumption of one cup of herbal tea per day, 19 out of 38 tea samples would result in MOE values for exposure to individual alkenylbenzenes lower than 10000 and 19 out of these 38 tea samples had a MOE value lower than 10000 in both combined exposure approaches. Overall, the results show that the MOE values for PFS, TCM and herbal teas using the two combined exposure methods do not vary substantially and both methods for combined exposure and risk assessment indicate there is a potential priority for risk management for several botanical preparations especially when considering life-long exposure. 


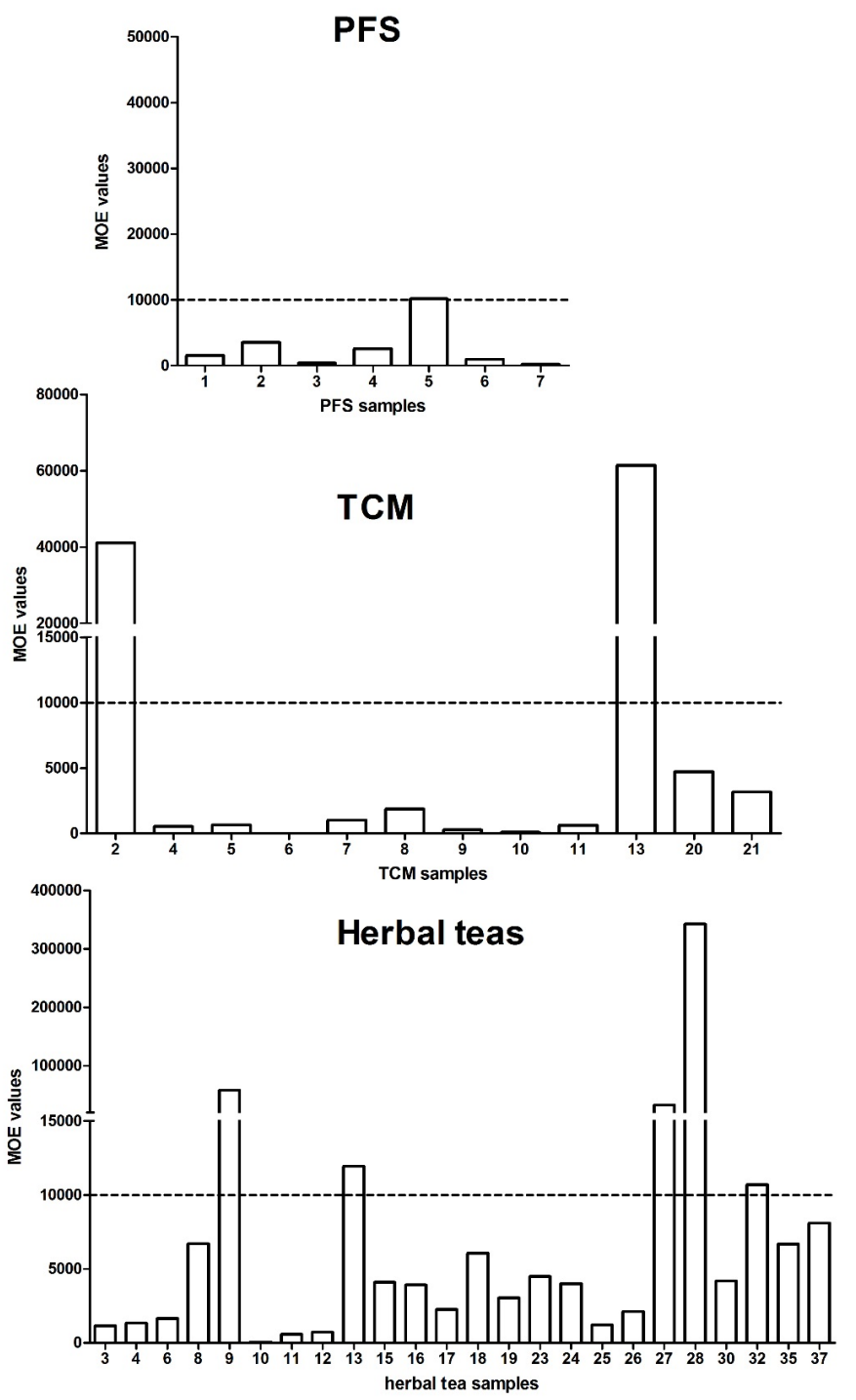

Fig. 2 MOE values for combined exposure to alkenylbenzenes based on assuming equal potency evaluating life-time use of PFS, TCM and herbal teas. The black dash line represents the limit of MOE of 10000 . MOE values lower than 10000 suggest a potential priority for risk management. 


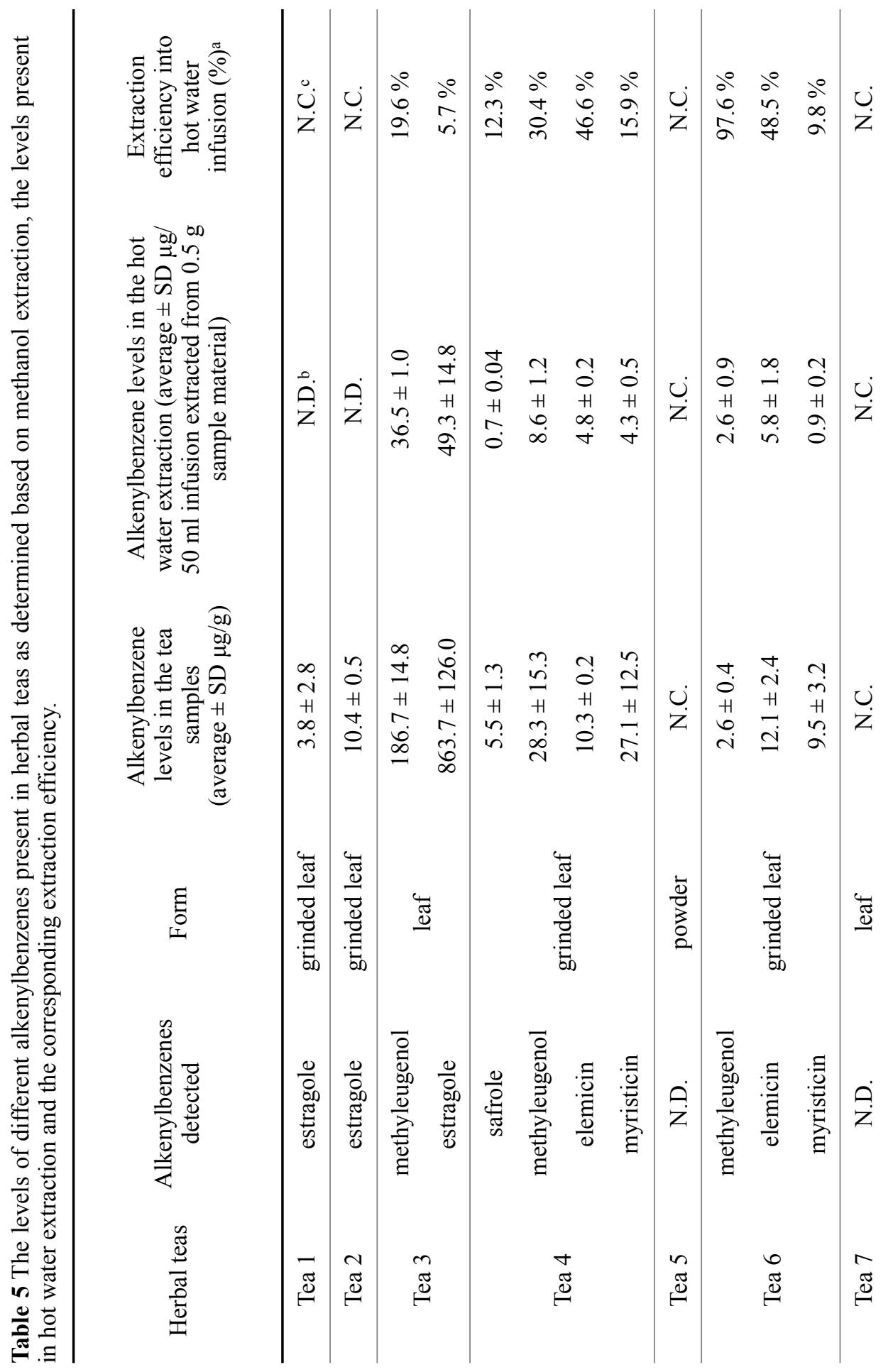




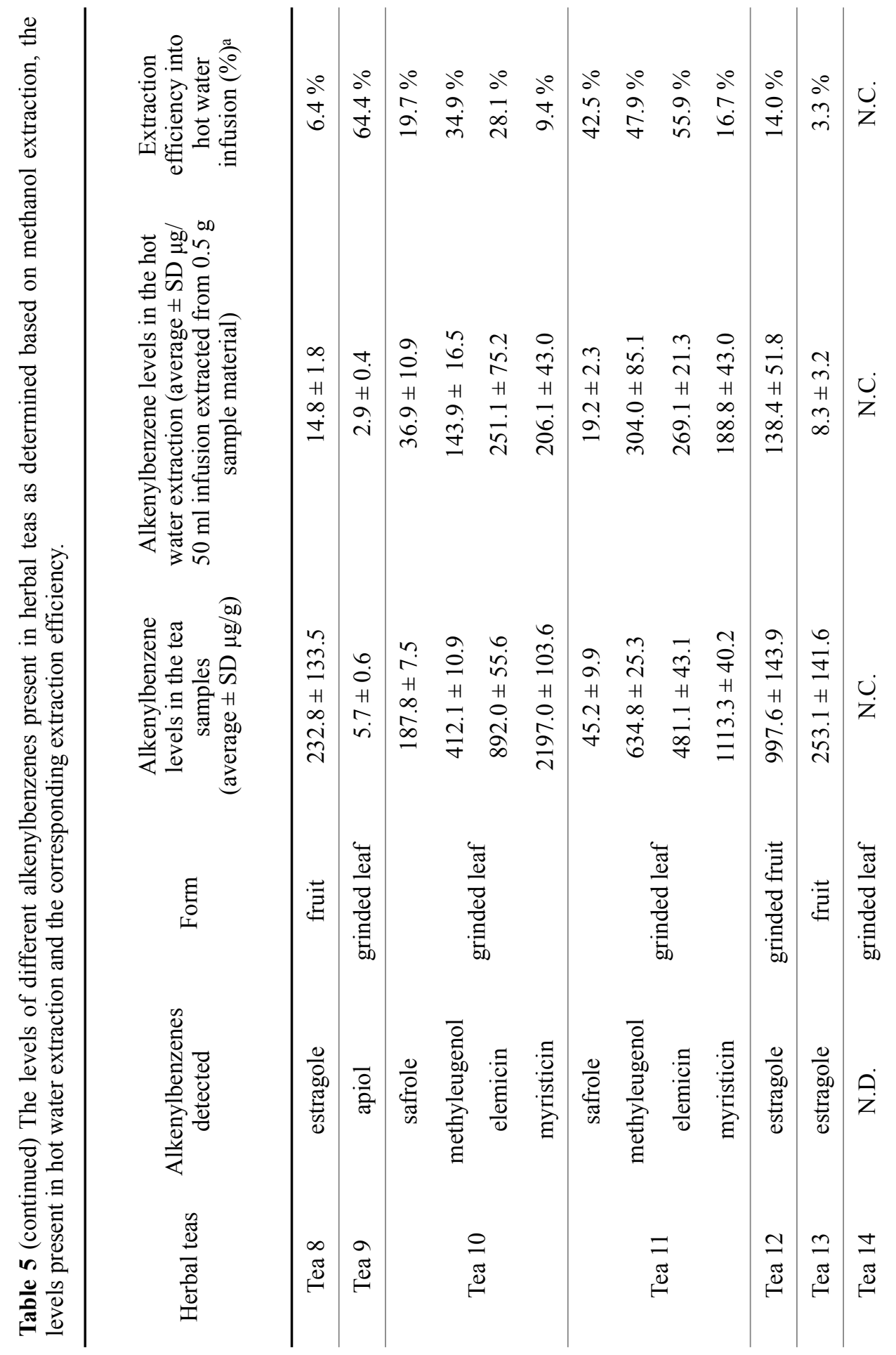




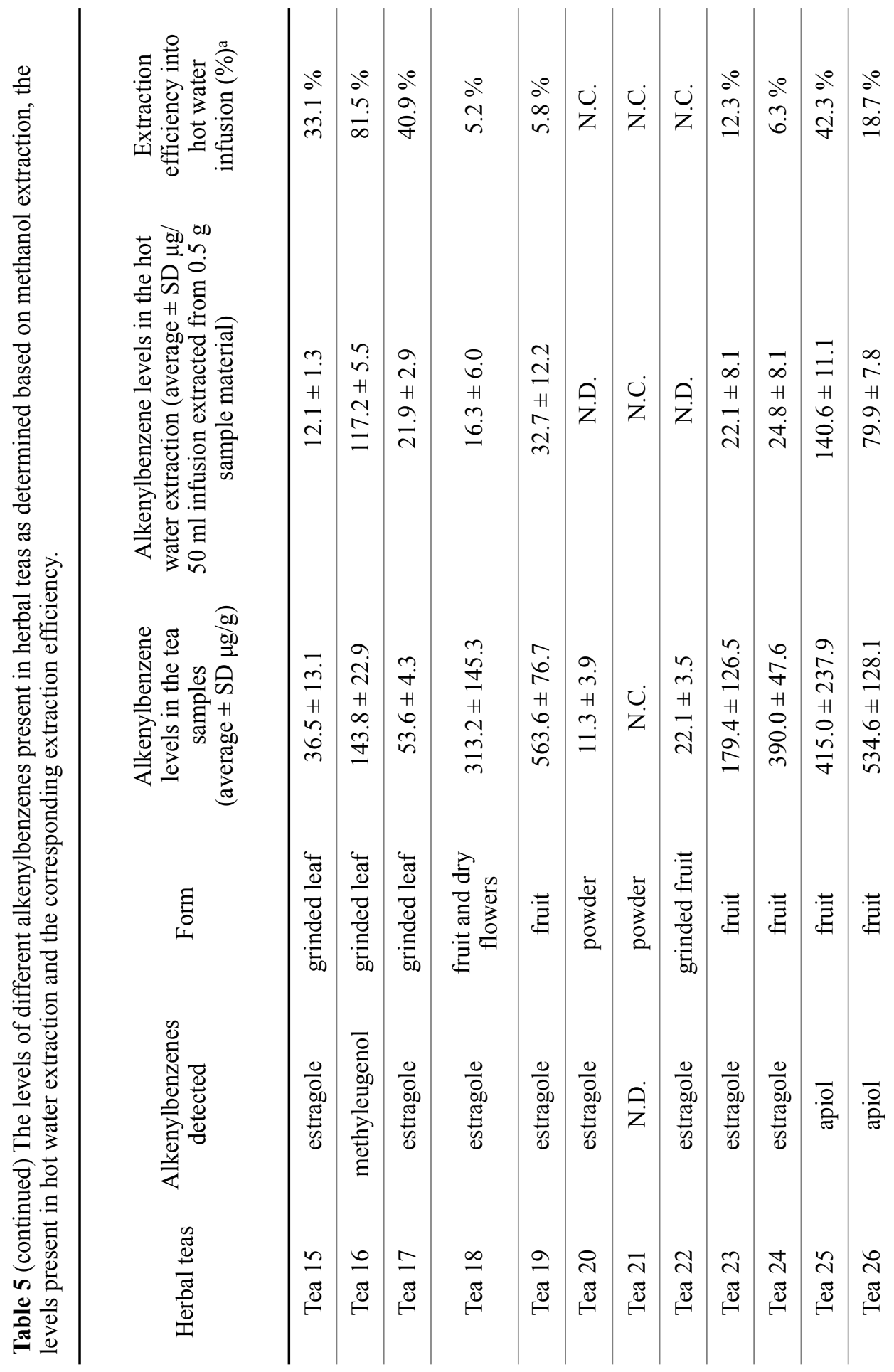




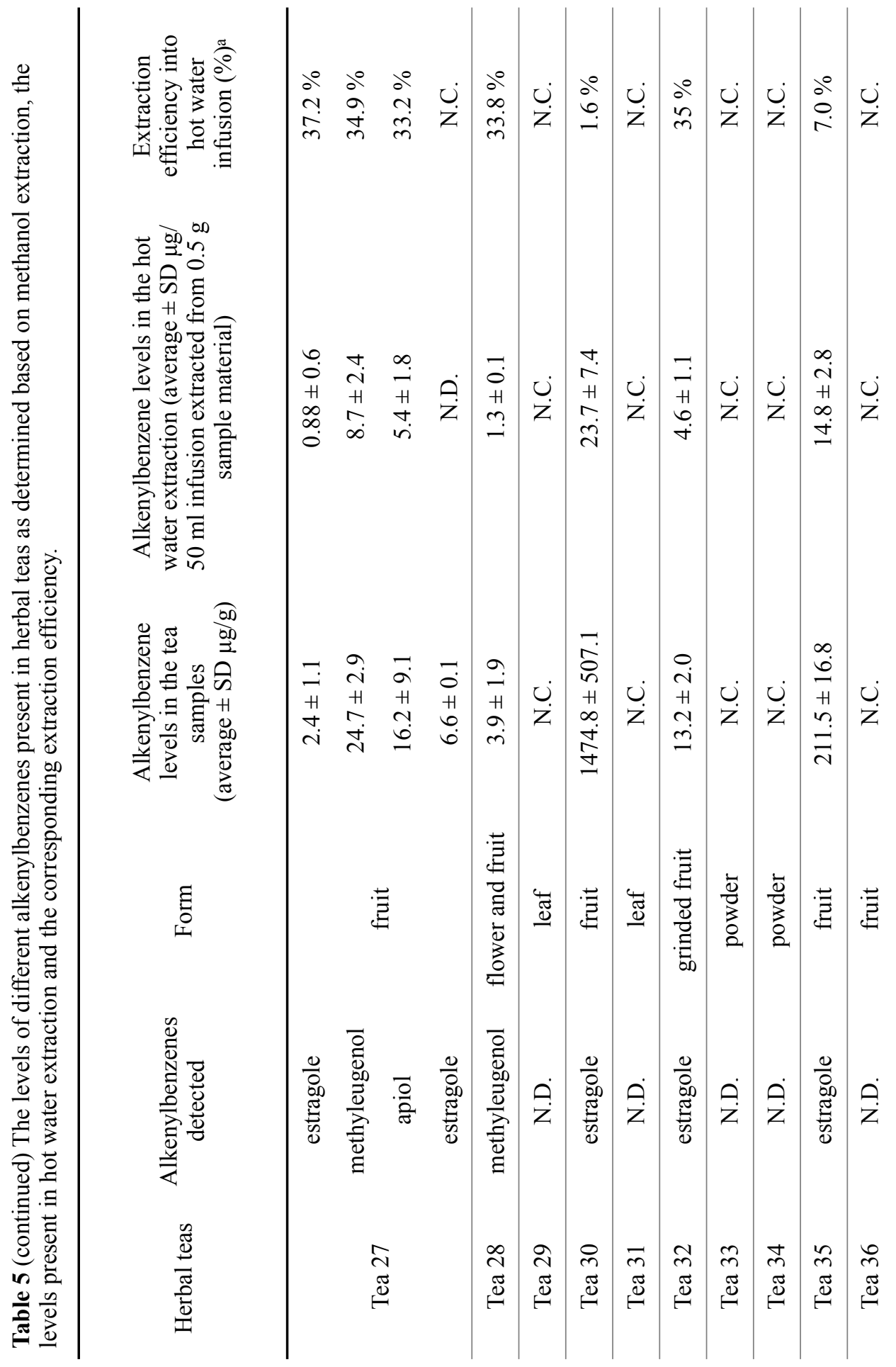




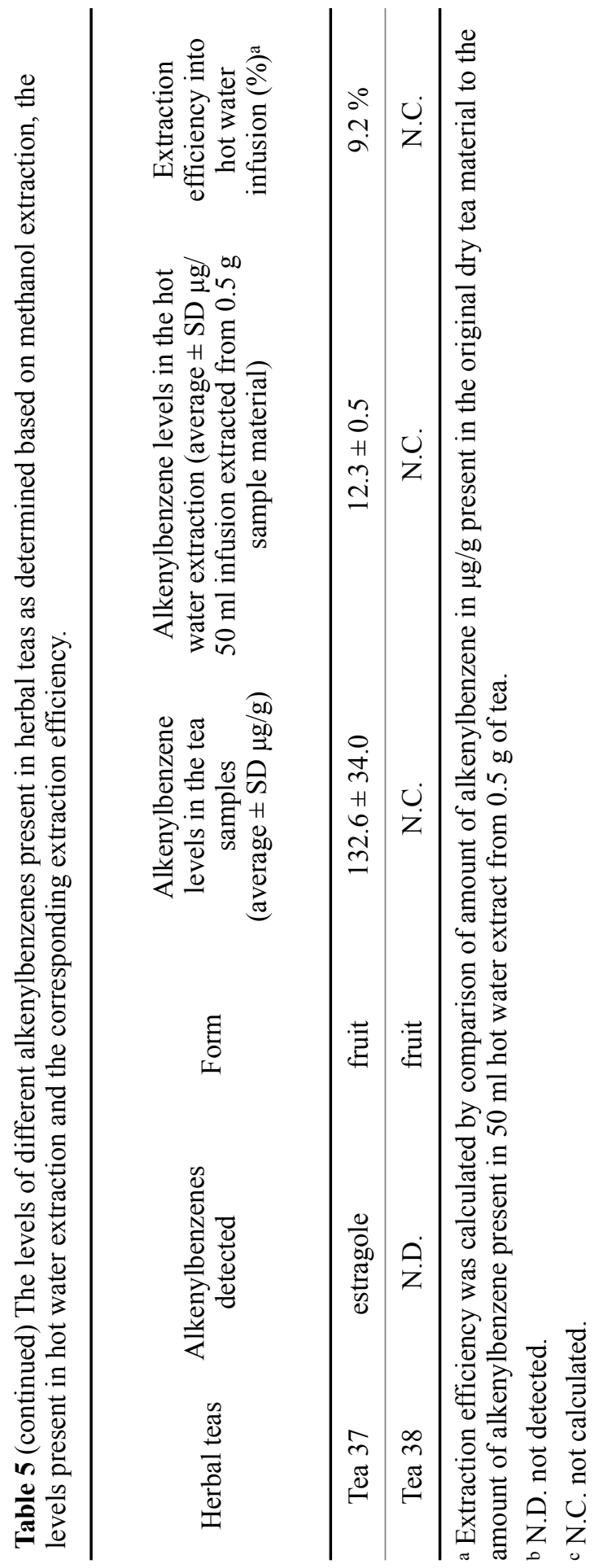




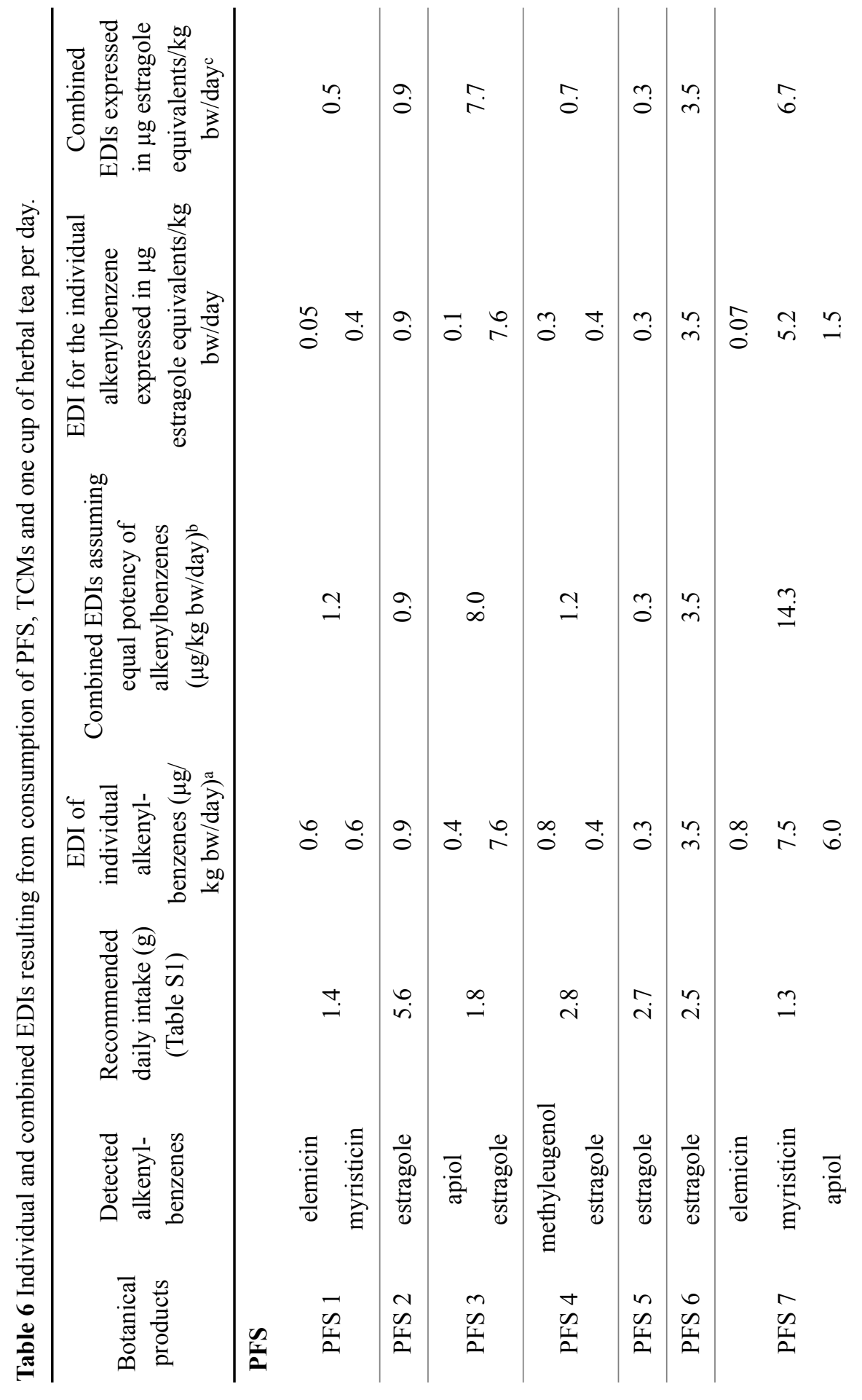




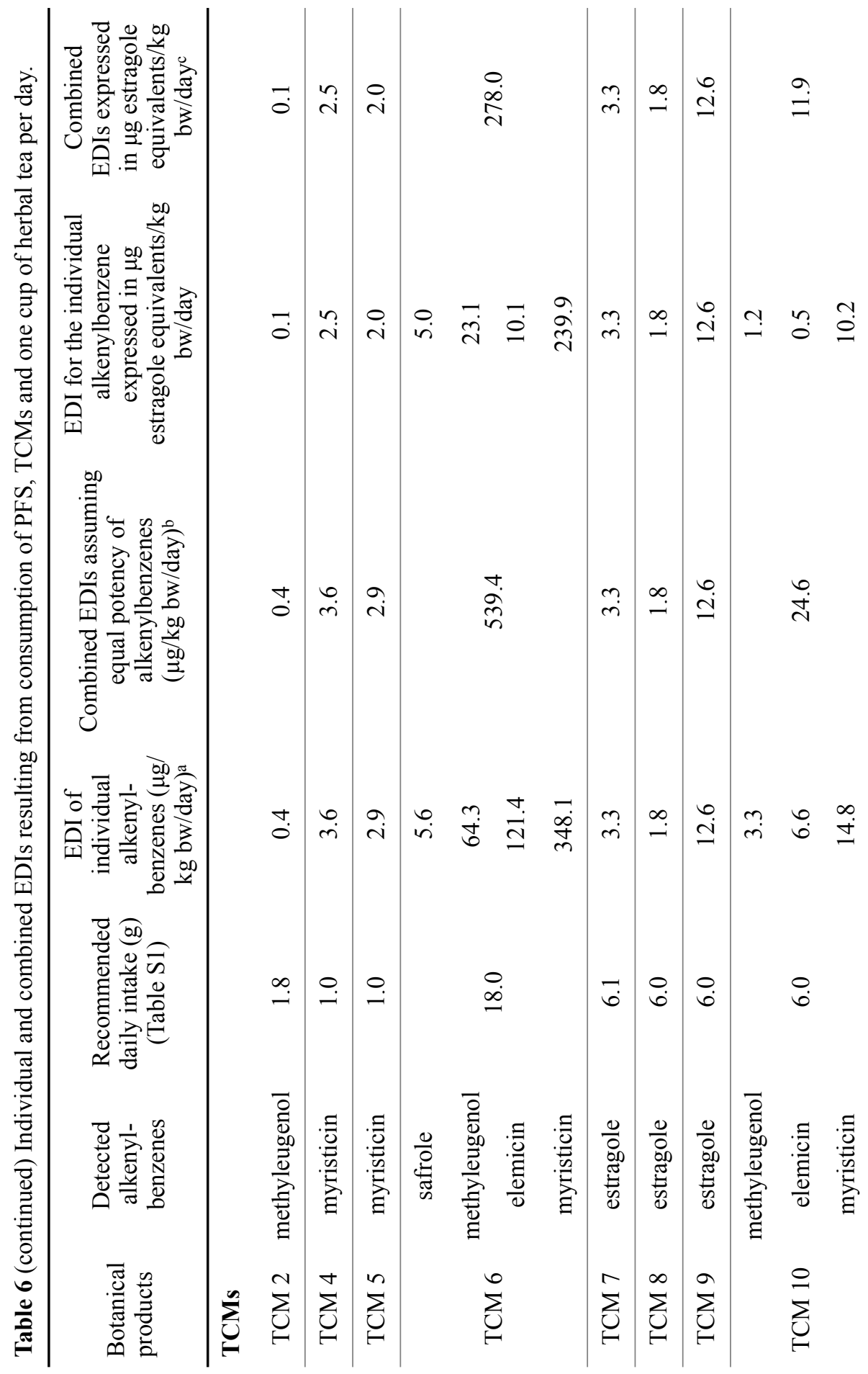




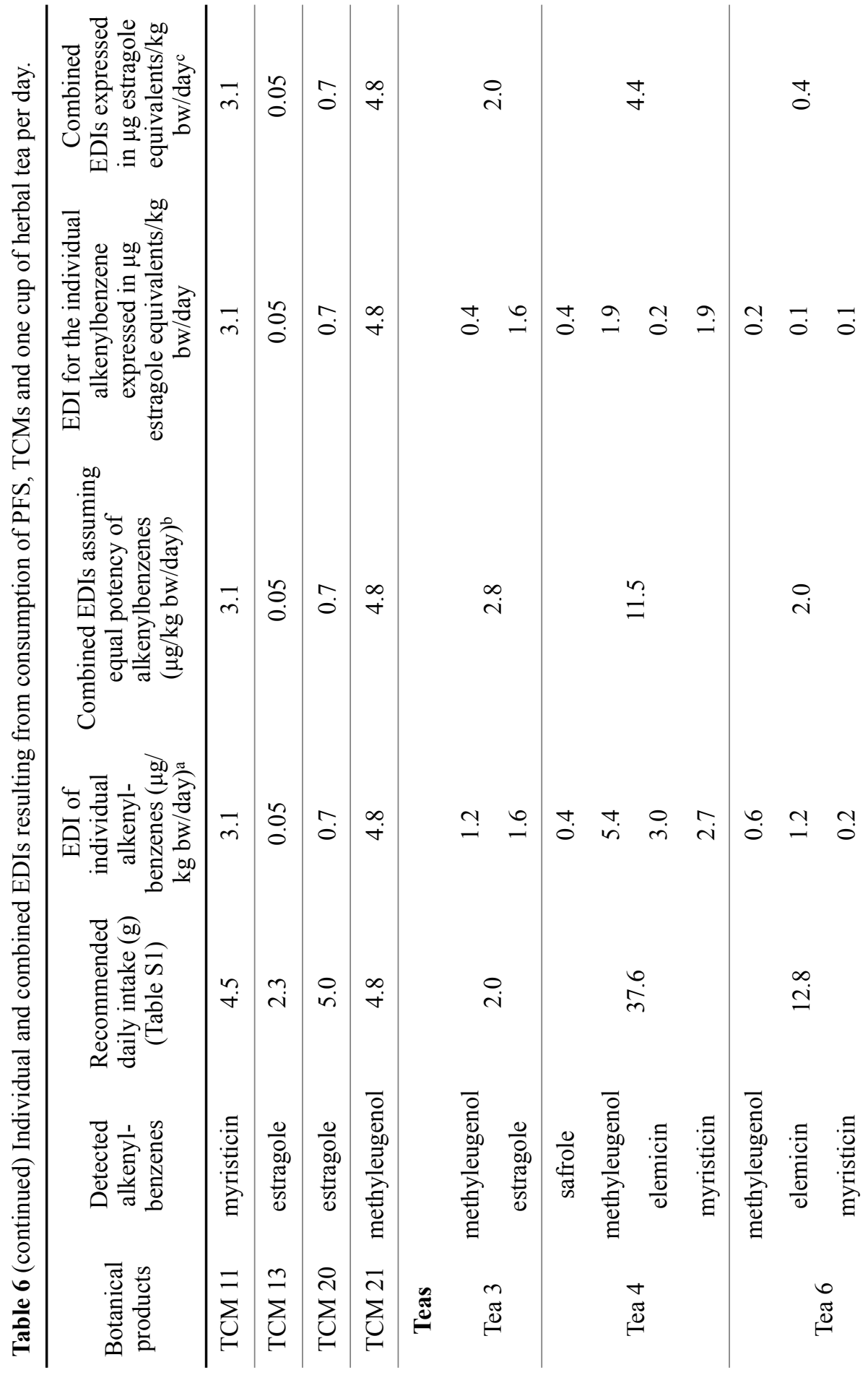




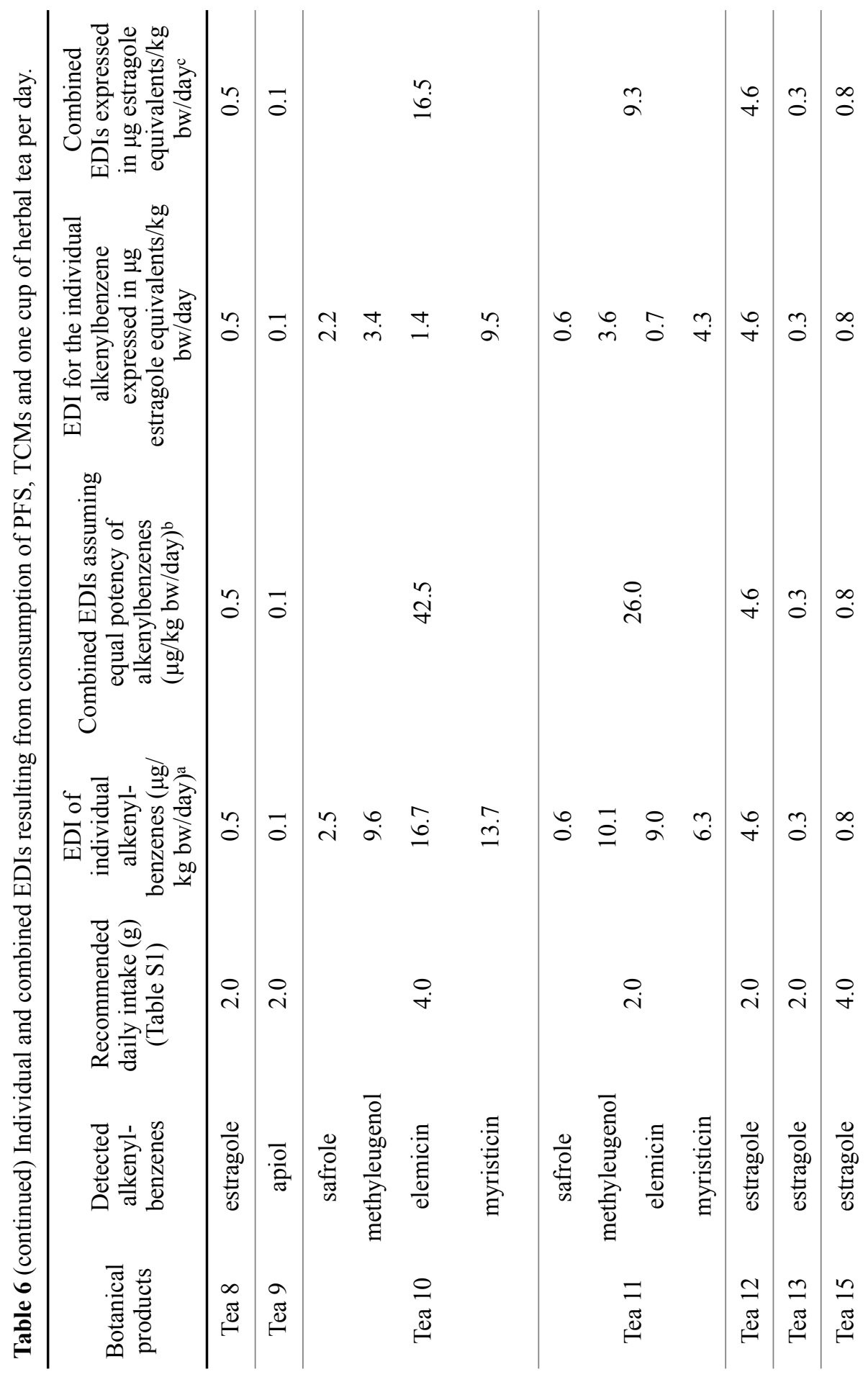




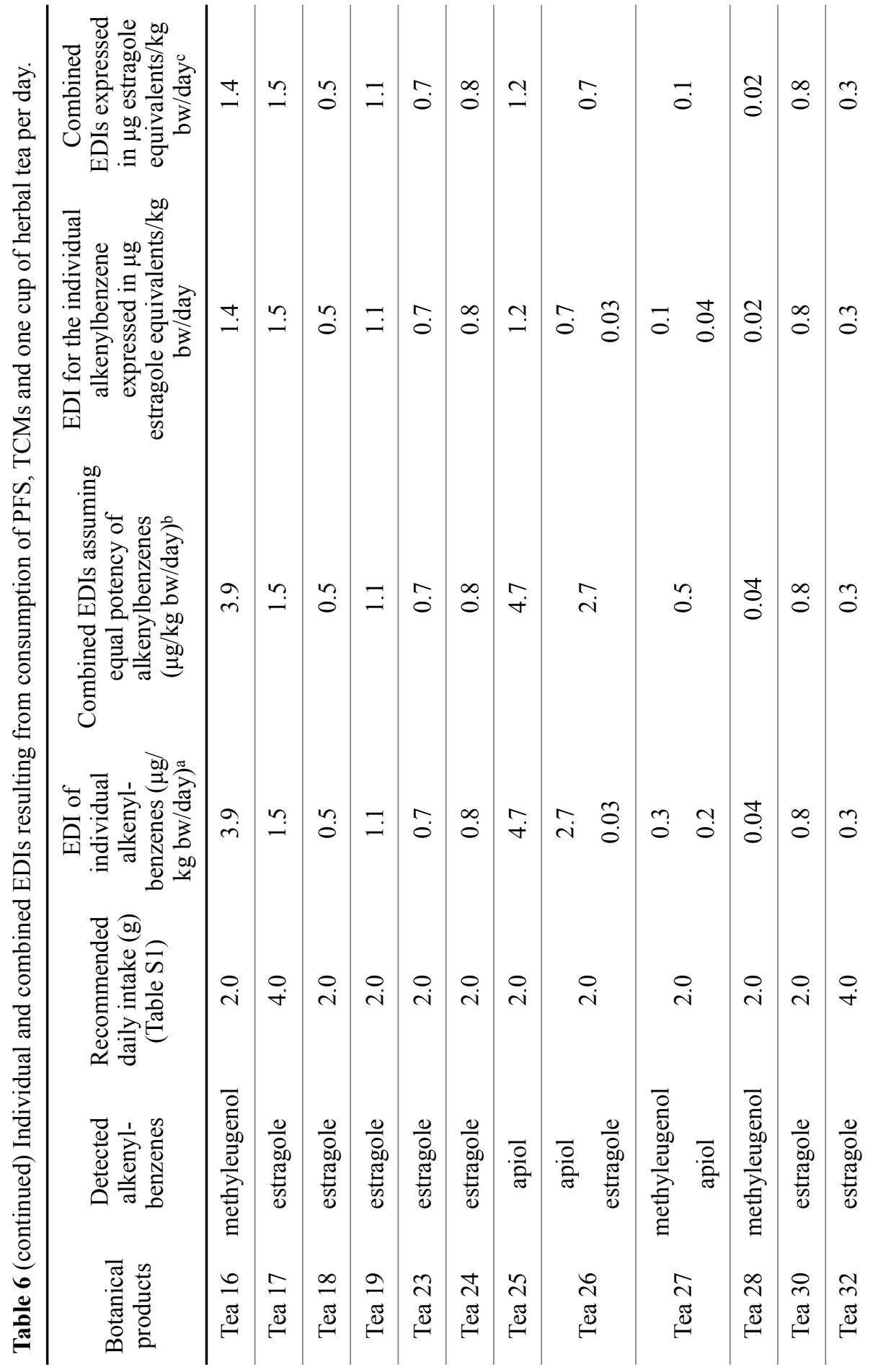




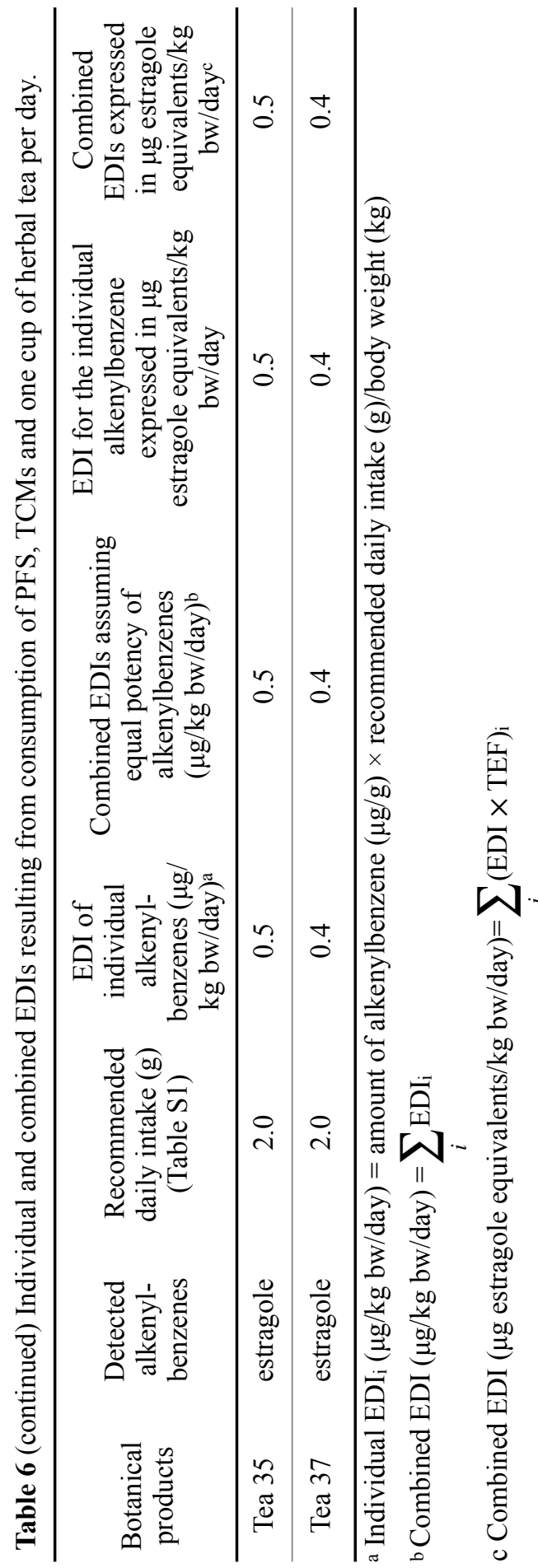


In the default MOE method, and the data presented in Figure 2, risk assessment is based on life-time (75 years) exposure. However for consumption of PFS, TCM and herbal teas real life exposure may be for shorter duration. A formal method to correct the MOE approach for short-term exposure has not been defined. Previously, Alajlouni et al. (2017) and AlMalahmeh et al. (2017b) presented a risk assessment for short-term use of PFS applying Haber's rule $(\mathrm{k}=\mathrm{C} \times \mathrm{T}$, where $\mathrm{C}$ is the concentration or dose of the toxic chemical, $\mathrm{T}$ is the period of exposure and $\mathrm{k}$ is the toxic outcome, which is considered to be constant) (Felter et al. 2011).

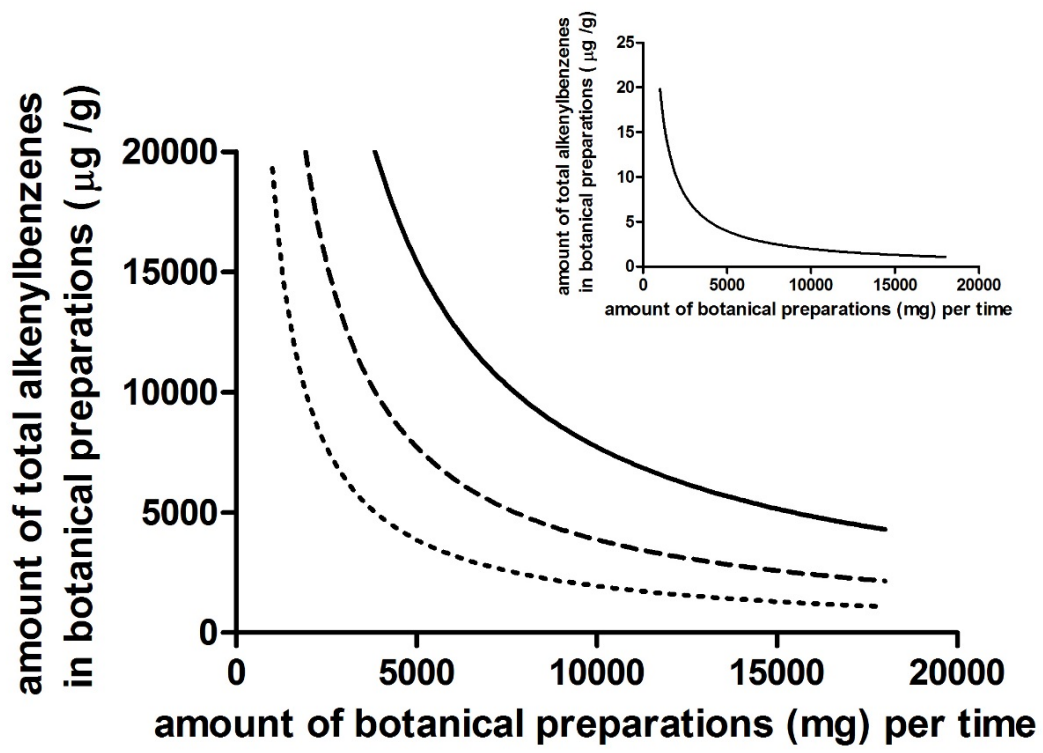

Fig. 3 The relation between the amount of total alkenylbenzenes in botanical preparations based on equal potency, and recommended daily intake that would result in MOE of 10000 by life-time (insert), 1 week (- ), 2 weeks (--) and 4 weeks $(\cdots)$ consumption.

Based on this method, Figure 3 displays the relationship between the amount of total alkenylbenzenes detected in the botanical preparations based on equal potency, and the amount of botanical preparations consumed that would result in an MOE of 10000 upon 1, 2 and 4 weeks consumption calculated using Haber's rule. For these calculations the BMDL 10 of estragole was used, since estragole is the major alkenylbenzene detected in the samples. Figure 3 indicates that daily consumption of $1000-18000 \mathrm{mg}$ botanical preparations would be of low priority for risk management at a total alkenylbenzene level lower than 19.8-1.1 $\mu \mathrm{g} /$ $\mathrm{g}$ for life-time consumption, $1.9 \cdot 10^{4}-1.1 \cdot 10^{3} \mu \mathrm{g} / \mathrm{g}$ for 4 weeks consumption, $3.9 \cdot 10^{4}-2.1 \cdot 10^{3}$ $\mu \mathrm{g} / \mathrm{g}$ for 2 weeks consumption and $7.7 \cdot 10^{4}-4.3 \cdot 10^{3} \mu \mathrm{g} / \mathrm{g}$ for 1 week consumption. From figure 3 it can also be derived that the total level of alkenylbenzenes determined in the present study amounting to 7.2-649.0 $\mu \mathrm{g} / \mathrm{g}$ for PFS, 1.4-1798.1 $\mu \mathrm{g} / \mathrm{g}$ for TCM and 1.3-781.3 $\mu \mathrm{g} / \mathrm{g}$ for herbal 
teas, would allow consumption of $2.8 \cdot 10^{3}-31 \mathrm{mg}$ for PFS, $1.4 \cdot 10^{4}-11 \mathrm{mg}$ for TCM and $1.5 \cdot 10^{4}-25 \mathrm{mg}$ for consuming one cup of herbal teas to be of low concern for life-long consumption. For 4 weeks consumption, the daily intake that would not raise a concern for PFS, TCM and herbal teas would be lower than $2.6 \cdot 10^{6}-3.0 \cdot 10^{4} \mathrm{mg}, 1.4 \cdot 10^{7}-1.1 \cdot 10^{4} \mathrm{mg}$ and $1.5 \cdot 10^{7}-2.4 \cdot 10^{4} \mathrm{mg}$, respectively to achieve MOE values higher than 10000 . For 1 and 2 week consumption, the daily intake of the botanical preparations that would be of no concern would be 4 - and 2-fold higher than the values for 4 weeks consumption. Figure 4 shows the MOE values for the botanical preparations assuming 2 weeks consumption based on assuming equal potency (MOE values for consumption of individual alkenylbenzene and MOE for combined exposure to alkenylbenzenes based on the TEQ approach can be found in the supplementary materials 3 ) and the results thus obtained reveal that almost all of the botanical preparations were of low concern except TCM 6, since the recommended daily intake of this sample was $18000 \mathrm{mg}$ and the amount of total alkenylbenzenes levels was 1798 $\mu \mathrm{g} / \mathrm{g}$, resulting in a corrected EDI of $0.28 \mu \mathrm{g} / \mathrm{kg}$ bw/day and an MOE of 6868 assuming equal potency approach, that was thus lower than 10000 even when correcting for the short term 2 week exposure.

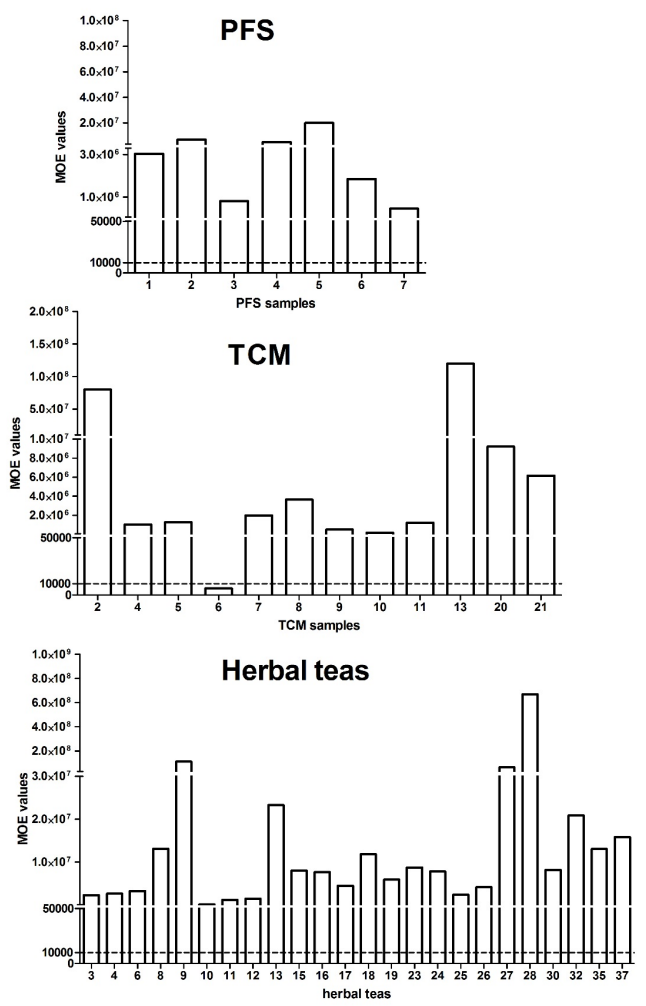

Fig. $4 \mathrm{MOE}$ values for combined exposure to alkenylbenzenes based on assuming equal potency evaluating two weeks use of PFS, TCM and herbal teas. The black dash line represents the limit of MOE of 10000 . MOE values lower than 10000 suggest a potential priority for risk management. 


\section{Discussion}

In the present study, a risk assessment for alkenylbenzenes in 71 botanical preparations including PFS, TCMs and herbal teas obtained from the Chinese market was made using the MOE approach taking into account combined exposure based on the dose additivity concept. The results indicate that the consumption of botanical preparations containing alkenylbenzenes suggest a potential priority for risk management actiomns when these preparations would be used on a daily basis for life-time. For short-term exposure such as 2 weeks consumption, almost all of the samples analysed in the present study showed low priority for risk management, except TCM 6 having an MOE value for myristicin below 10 000 .

It is important to note that the botanical preparations were selected by a targeted approach, that is by screening for suspicious botanicals which might contain alkenylbenzenes of interest in the ingredient list of the samples. Table 1 presents the list of botanicals potentially containing alkenylbenzenes used for this targeted selection. Since the interpretation of the names of botanicals differs between geographical regions in China, there is confusion over nomenclature. Several plant species share a Chinese common name with different prefix. For example, the common name "Dou Kou" refers to at least three plant species but in combination with "Rou" it refers to nutmeg. And botanicals such as basil and calamus have even more than 30 different alternative names originating from different regions in China, which can lead to confusion as well. Therefore, there is a need to develop an effective protocol for better nomenclature to facilitate more extended and dedicated sampling in the future and it is evident that complexities on nomenclature systems increase the risk of inadvertent exposure to the toxic compounds. This is further illustrated by the unintentional exposure to aristolochic acids that occurred in the 1990s in Belgium due to name confusion, where there was a replacement of the Stephania tetrandra (Fang Ji) with Aristolochia fangchi (Guang Fang Ji), which contained aristolochic acids (AAs). More than 100 young women suffered from renal failure, some of them developing renal and bladder cancer (Vanherweghem et al. 1993). Therefore, use of the Latin naming system for the botanicals might be useful to avoid confusion caused by the use of common names of botanicals and add to safe use of botanicals (Wu et al. 2007).

Based on the chemical analysis of 71 botanical preparations, estragole was the most frequently detected compound, being detected in 30 out of 71 samples, followed by methyleugenol, myristicin, elemicin, apiol and safrole. This observation is in line with the fact that 37 out of the 71 samples were claimed to contain fennel (Table S1) and estragole is known to be the major alkenylbenzene in fennel (Van Den Berg et al. 2014; Van Den Berg et al. 2011). Interestingly, PFS samples containing cinnamon did not contain alkenylbenzenes at detectable levels. These results are consistent with the study of Van Den Berg et al. (2011), showing negative results for alkenylbenzenes of interest in PFS samples containing cinnamon. In contrast to this, EFSA previously reported that $0.01 \%$ of methyleugenol was identified in the essential oil of leaves of cinnamomum verum J. Presl (EFSA 2009b). The possible reasons of negative results for cinnamon containing PFS could be related to the 
relatively low levels of methyleugenol in cinnamomum species, in line with the report of EFSA indicating a level of only $0.01 \%$ of methyleugenol in the essential oil of cinnamon.

The levels of estragole, methyleugenol, myristicin, elemicin, apiol and safrole in botanical preparations in the present study varied from 1.4-1474.8, 2.6-634.8, 9.5-2197.0, 10.3-892.0, 5.7-534.6 and 5.5-187.8 $\mu \mathrm{g} / \mathrm{g}$, respectively. This high variation between the different samples may in part be caused by the botanical composition as such, but may also be due to the influence of variation induced by genetic factors, geographical influences in cultivation, harvesting time, processing and manufacturing conditions, and the method of drying (Smith et al. 2002). A previous study reported that drying, irradiation and microwave treatment could cause degradation of safrole in certain spices like black pepper (Farag and Abo-Zeid 1997). And the various processing methods for TCM such as roasting, frying and stewing, claimed to reduce toxicity and potentiate the effects, have a long history in China (Zhu 1998). Due to all factors mentioned above, alkenylbenzenes of interest may be present at variable levels as observed in the botanical preparations analyzed.

Previously, other studies have evaluated the risk of alkenylbenzenes in cinnamon, calamus, nutmeg, fennel, parsley and basil-based PFS and teas in western countries (Al-Malahmeh et al. 2017b; Alajlouni et al. 2016a; Alajlouni et al. 2017; Van Den Berg et al. 2014; Van Den Berg et al. 2011). In addition to those botanical based preparations, the present study also analysed lemongrass and cloves based products. According to the literature, lemongrass might contain methyleugenol and estragole, and methyleugenol can be present in cloves (BfR 2002; Mcdonald 1999). Herbal tea 1, 2, and 9 were claimed to containing lemongrass, and tea 27 , 28 and 29 contained cloves (Table S1). In these samples, estragole, methyleugenol and apiol were identified in the methanol extractions (Table 5). The presence of apiol in those samples was unexpected, and might be due to (unintentional) contamination with apiol containing botanicals, such as parsley and dill, during harvesting, processing or blending of the teas. The MOE values of teas containing lemongrass and cloves determined in the current study were generally of low concern even upon life-time exposure.

The assessment of combined exposure to alkenylbenzenes was made based on the assumption of dose additivity. This assumption was made because of the similarity of chemical structures, metabolic pathways, route for bioactivation and mode of action resulting in the adverse effects by a similar mechanism, from which it can be assumed that alkenylbenzenes display similar shaped dose-response curves. However, empirical data supporting this dose additive assumption are not available and future experimental work may further support this assumption. Nevertheless, the combined exposure to alkenylbenzenes in the present study was assessed using the concept of dose addition as a conservative default approach for assessing cumulative risk based on equal potencies and interim REP values for the different alkenylbenzenes.

Ideally, REP values should be defined based on the tumor formation as the endpoint. This requires availability of data from long term carcinogenicity studies. Such data on in vivo tumor formation are available for estragole, safrole and methyleugenol (Miller et al. 1983; 
NTP 2000), but data on in vivo tumor formation for elemicin, myristicin and apiol are lacking. Because the $\mathrm{BMDL}_{10}$ values for elemicin, myristicin and apiol were estimated by read-across from structurally related alkenylbenzenes by comparing the predicted relative formation of the proximate carcinogenic 1'-sulfooxy metabolites using physiologically based kinetic modelling (Al-Malahmeh et al. 2017a; Alajlouni et al. 2016b; Van Den Berg et al. 2012), the REP values could be defined using only these BMDL 10 values for tumor formation. However, given the uncertainty in these tumor data and corresponding BMDL 10 values and the fact that additional data on relative bioactivation and DNA adduct formation of the alkenylbenzenes were available, we decided to include data from these surrogate endpoints in estimation of the REP values. Use of such surrogate endpoints is in line with recent published literature on definition of REP values. For example interim REP values for pyrrolizidine alkaloids were recently defined based on data from in vitro cytotoxicity, genotoxicity in Drosophila, and LD 50 values (Merz and Schrenk 2016). Therefore, the REP values for the different alkenylbenzenes defined in the present study can be considered as interim values and further studies will be required to further refine these REP values. It is important to note, however, that the MOE values obtained in the present study for combined exposure to the alkenylbenzenes based on the TEQ approach using the REP values and assuming equal potency do not vary substantially. It can even be concluded that performing a combined risk assessment of the alkenylbenzenes assuming dose additivity without taking the relative potencies into account is adequate.

Based on the chemical analysis of botanical preparations and the recommended daily intake of the samples provided by the manufacturers, the EDI for consumption of PFS, TCMs and one cup of herbal tea for the Chinese population were determined. The combined EDI values based on assuming equal potency of the different alkenylbenzene of PFS ranged from $0.3-14.3 \mu \mathrm{g} / \mathrm{kg}$ bw/day with a median value of $1.2 \mu \mathrm{g} / \mathrm{kg}$ bw/day, which was 3-60 times lower than the combined EDI values of alkenylbenzenes resulting from consuming nutmeg or parsley based PFS obtained on the western market amounting to $76.6 \mu \mathrm{g} / \mathrm{kg}$ bw/day or $3.8 \mu \mathrm{g} /$ $\mathrm{kg}$ bw/day, respectively (Al-Malahmeh et al. 2017b; Alajlouni et al. 2017). For herbal teas, the combined EDI assuming equal potency for consuming one cup of tea were $0.04-42.5 \mu \mathrm{g} /$ $\mathrm{kg} \mathrm{bw} /$ day with a median value of $0.8 \mu \mathrm{g} / \mathrm{kg}$ bw/day. Compared to the previous results showing a range of $0.6-13.1 \mu \mathrm{g} / \mathrm{kg}$ bw/day with a median value of $4.5 \mu \mathrm{g} / \mathrm{kg}$ bw/day (Alajlouni et al. 2016a) for consuming one cup of parsley based teas, the EDI value of alkenylbenzenes resulting from herbal tea consumption for the Chinese population was at the median value 6-fold lower although maximum exposure as estimated for the Chinese population may be 3.2-fold higher. The relatively lower median EDI values obtained for consumption of PFS and herbal teas in the current study probably results from the fact that most of the PFS and herbal tea samples purchased from the Chinese market consist of a mixture of botanicals, in which the percentage of the herbal ingredient containing alkenylbenzenes might be relatively low, resulting in lower concentrations of alkenylbenzenes in those samples compared to the samples analysed by Alajlouni et al. (2016a; 2017) and AlMalahmeh et al. (2017b). 
Although the combined median EDI values of the botanical preparations included in the current study were lower than those of previous studies, 6 out of 10 PFS, 10 out of 23 TCM and 19 out of 38 herbal teas had MOE values in both combined exposure methods for lifetime exposure below 10 000, suggesting a potential priority for risk management. Use of the TEQ approach to express the EDI in estragole equivalents resulted in the combined EDI values ranging from $0.02-278.0 \mu \mathrm{g}$ estragole equivalents $/ \mathrm{kg}$ bw/day resulting from consumption of the PFS, TCM and one cup of herbal teas. These values were generally 39 $\%-54 \%$ of the combined EDI values assuming equal potency for each alkenylbenzene, which is mainly due to the fact that the interim REP values of the other alkenylbenzenes that were detected are lower than the value of 1.0 used for estragole as the reference compound. In line with these findings, Alajlouni et al. (2017) and Al-Malahmeh et al. (2017b) reported that combined EDI values obtained using a TEQ approach with safrole as the reference compound were also lower than those obtained from assuming equal potency (Al-Malahmeh et al. 2017b; Alajlouni et al. 2017). In spite of the relatively lower combined EDI values obtained when using the TEQ approach, the MOE obtained using the EDI values expressed in estragole equivalents and the $\mathrm{BMDL}_{10}$ of estragole was found to be lower than 10000 for 35 out of 71 botanical preparations. Thus, the overall conclusion did not change with the method used for combined exposure to estimate MOE values.

It is important to note that the MOE approach assumes life-time consumption, which for the botanical samples of the present study might be an overestimation of realistic human consumption, especially for TCM considered as medicines in China. Risk assessment for short-term consumption of these botanicals preparations might better reflect the actual situation. Currently, there is no generally established method or guidance on how to take a shorter than life-time exposure into account in risk assessment of genotoxic carcinogens, but one could consider Haber's rule to estimate the impact of less-than-lifetime exposure to genotoxic carcinogens (Abdullah et al. 2017; Al-Malahmeh et al. 2017b; Alajlouni et al. 2017; Van Den Berg et al. 2014). By applying this rule, MOE values will be three orders of magnitude higher than those obtained for life-time exposure (Van Den Berg et al. 2014). In this study, the MOE values for short-term exposure such as two weeks consumption were obtained by using Haber's rule and were shown in Figure 4 and Figure S3.1-3.3 in supplementary materials 3. The MOE values for two weeks consumption of most botanicals preparations determined in the current study were above 10000 , indicating a low priority for risk management. This does not hold for TCM 6, named Si Shen Wan (四神丸) for which the MOE after correction for 2 weeks exposure was still below 10000 based on assuming equal potency of the alkenylbenzenes. Although this sample is considered as drug in China, it is used for the treatment of diarrhea and bad appetite and there is no information on duration for consumption on the label, which make people easily consume it for relatively longer periods. For life-time exposure, the MOE of this sample when assuming equal potency of the alkenylbenzenes was even lower than 10 , which means that the daily intake of the compounds of interest are within the range of dose levels causing tumors in experimental animals (Van Den Berg et al. 2011). It is important to note that the MOE values determined in the current study were calculated for Chinese adults assuming a body weight of $60 \mathrm{~kg}$ (NHFPC 2007). 
The MOE values for infants and children, who have a lower bodyweight, will be even lower because at the same use levels per person the intake per $\mathrm{kg}$ body weight will be higher. As a result, for infants and children who use TCM 6 for two weeks to cure bad appetite, the MOE values in both combined exposure approaches would be lower than 10000 .

However, in real life people may consume the botanical preparations, especially TCM, for short periods but on a regular basis for example two weeks every year for a whole life time. To take this assumption into account, we estimated the MOE values showing that 1 out of 10 PFS, 3 out of 23 TCM and 1 out of 38 herbal teas would then result in MOE values $<10000$, suggesting a potential priority for risk management (data not shown).

Applying Haber's rule for correcting the MOE approach for short-term exposure in risk assessment gives a first indication but is not yet current practice, and future developments in risk assessment of botanicals and botanical preparations containing genotoxic carcinogens should consider how to further advance this issue (Felter et al. 2011; Geraets et al. 2016).

The current study focused on safety and risk evaluation of alkenylbenzenes present in botanical preparations. Depending on the botanicals present in the preparation also other botanical genotoxic and carcinogens, such as aristolochic acids, or pyrrolizidine alkaloids (PAs) might raise a concern. For example, TCM 5 (二十五味松石丸) is claimed to contain Aristolochia species, and the BMDL 10 of a mixture of AAs (71\% of AAI and $21 \%$ of AAII) was $0.01 \mathrm{mg} / \mathrm{kg}$ bw (Abdullah et al. 2017), which is 2 orders of magnitude lower than the $\mathrm{BMDL}_{10}$ values of alkenylbenzenes. According to the published data, the averaged levels of AAs detected in herbal medicines containing AA producing plants may vary between 133-900 $\mu \mathrm{g} / \mathrm{g}$ (Cheung et al. 2006; Huang et al. 2005; Martena et al. 2007; Wang and Chan 2014). If the level of AAs in TCM 5 would be comparable to the published data, then the EDI would be 2.2-15 $\mu \mathrm{g} / \mathrm{kg} \mathrm{bw} /$ day resulting in MOE value for AAs in the range of 4.5-0.7, which is substantially lower than the MOE for the alkenylbenzene in TCM 5 amounting to 646. For risk assessment of this combined presence of different classes of genotoxic carcinogens in one preparation also no established method is available, and using dose additivity may not be appropriate because the modes of action of alkenylbenzenes (Al-Malahmeh et al. 2017a; AlSubeihi et al. 2012; Alajlouni et al. 2016b; Martati et al. 2012; Punt et al. 2009; Van Den Berg et al. 2012), AAs (Abdullah et al. 2016) and other groups of botanical compounds that are genotoxic and carcinogenic is different. Furthermore, a single herb might contain hundreds of compounds, and mixed herbal products like TCMs might contain several times that number. This may result in synergism or antagonism occurring in the matrix of herbal products, causing for example modification of the bioactivation and/or detoxification pathways of the genotoxic carcinogens. A previous study showed for example that the flavonoid nevadensin in basil extract can inhibit the bioactivation pathway of estragole by inhibiting sulfotransferase (SULT) activity and DNA adduct formation both in vitro (Jeurissen et al. 2008) and in vivo (Alhusainy et al. 2013). However, PBK modelling revealed that this matrix-derived combination effect might be limited at dose levels of realistic human intake, because at these levels the plasma and liver concentrations of the inhibitor nevadensin are unlikely to reach the $\mathrm{K}_{\mathrm{i}}$ of nevadensin for the SULTs (Rietjens et al. 2015). This result illustrates that risk 
assessment taking into account combination effects that may occur upon exposure to botanical preparations should be done on a case-by-case basis taking into account the dosedependency of effects. However, due to the complex constituents in most botanicals, it may be possible that combination effects may affect the overall outcome predicted based on a risk assessment focusing on single mixture ingredients. The MOE based risk assessment presented may set priorities for not only risk management actions but also for further studies on such combination effects enhancing or decreasing the respective concerns.

In conclusion, genotoxic and carcinogenic alkenylbenzenes can be found in botanicals preparations present on the Chinese market. The MOE values for alkenylbenzene exposure resulting from consumption of these botanical preparations suggest a potential priority for risk management especially upon long-term exposure. For short-time exposure such as two weeks consumption, only one of the TCM samples still showed a potential priority for risk management actions. It is concluded that consumption of selected botanical preparations purchased from the Chinese market for especially longer periods of time raise a concern because of potential exposure to alkenylbenzenes.

\section{Acknowledgements}

This work was funded by a grant from the China Scholarship Council (No. 201507720019 to NING JIA)

\section{Note}

The authors declare that they have no conflict of interest

\section{References}

Abdullah R, Alhusainy W, Woutersen J, Rietjens IMCM, Punt A (2016) Predicting points of departure for risk assessment based on in vitro cytotoxicity data and physiologically based kinetic (PBK) modeling: The case of kidney toxicity induced by aristolochic acid I. Food Chem Toxicol 92:104-116

Abdullah R, Diaz LN, Wesseling S, Rietjens IMCM (2017) Risk assessment of plant food supplements and other herbal products containing aristolochic acids using the margin of exposure (MOE) approach. Food Addit Contam Part A 34:135-144

Al-Malahmeh AJ, Al-Ajlouni A, Wesseling S, Soffers AEMF, Al-Subeihi A, Kiwamoto R, Vervoort J, Rietjens IMCM (2017a) Physiologically based kinetic modeling of the bioactivation of myristicin. Arch Toxicol 91:713-734

Al-Malahmeh AJ, Alajlouni AM, Ning J, Wesseling S, Vervoort J, Rietjens IMCM (2017b) Determination and risk assessment of naturally occurring genotoxic and carcinogenic alkenylbenzenes in nutmeg-based plant food supplements. J Appl Toxicol 37:1254-1264

Al-Subeihi AA, Spenkelink B, Punt A, Boersma MG, Van Bladeren PJ, Rietjens IMCM (2012) Physiologically based kinetic modeling of bioactivation and detoxification of the alkenylbenzene methyleugenol in human as compared with rat. Toxicol Appl Pharmacol 260:271-84 
Alajlouni AM, Al-Malahmeh AJ, Isnaeni FN, Wesseling S, Vervoort J, Rietjens IMCM (2016a) Level of alkenylbenzenes in parsley and dill based teas and associated risk assessment using the Margin of Exposure approach. J Agr Food Chem 64:8640-8646

Alajlouni AM, Al-Malahmeh AJ, Wesseling S, Kalli M, Vervoort J, Rietjens IMCM (2017) Risk assessment of combined exposure to alkenylbenzenes through consumption of plant food supplements containing parsley and dill. Food Addit Contam Part A 34:2201-2211

Alajlouni AM, Al Malahmeh AJ, Kiwamoto R, Wesseling S, Soffers AEMF, Al-Subeihi AAA, Vervoort J, Rietjens IMCM (2016b) Mode of action based risk assessment of the botanical food-borne alkenylbenzene apiol from parsley using physiologically based kinetic (PBK) modelling and read-across from safrole. Food Chem Toxicol 89:138-150

Alhusainy W, Paini A, Van Den Berg JH, Punt A, Scholz G, Schilter B, Van Bladeren PJ, Taylor S, Adams TB, Rietjens IMCM (2013) In vivo validation and physiologically based biokinetic modeling of the inhibition of SULT-mediated estragole DNA adduct formation in the liver of male Sprague-Dawley rats by the basil flavonoid nevadensin. Mol Nutr Food Res 57:1969-78

Bundesinstitut für Risikobewertung (BfR) (2002) Reduce estragole and methyleugenol contents in foods China Food and Drug Administration (CFDA) (2013a) List of dietary herbs (in Chinese).

China Food and Drug Administration (CFDA) (2013b) List of herbs allowed for health food product s(in Chinese).

Chen S, Sylvia MP (2006) Poaceae-Flora of China, vol 22. Science Press (Beijing) \& Missouri Botanical Garden (St.Louis)

Cheung TP, Xue C, Leung K, Chan K, Li CG (2006) Aristolochic acids detected in some raw chinese medicinal herbs and manufactured herbal products - A consequence of inappropriate nomenclature and imprecise labelling? Clin Toxicol 44:371-378

European Commission (EC) (2008) No. 1334/2008 of the European Parliament and of the Council of 16 December 2008 on flavourings and certain food ingredients with flavouring properties for use in and on foods and amending Council Regulation (EEC) No. 1601/91, Regulations (EC) No. 2232/96 and (EC) No. 110/2008 and Directive 2000/13/EC. Official Journal of the European Communities 354:34-50

European Food Safety Authority (EFSA) (2005) Opinion of the scientific committee on a request from EFSA related to a harmonised approach for risk assessment of substances which are both genotoxic and carcinogenic. EFSA J 282:1-31

European Food Safety Authority (EFSA) (2009a) Advice on the EFSA guidance document for the safety assessment of botanicals and botanical preparations intended for use as food supplements, based on real case studies. EFSA J 7:280

European Food Safety Authority (EFSA) (2009b) EFSA Compendium of botanicals that have been reported to contain toxic, addictive, psychotropic or other substances of concern. EFSA J 7:281

European Food Safety Authority (EFSA) (2012) Compendium of botanicals reported to contain naturally occuring substances of possible concern for human health when used in food and food supplements. EFSA J 10:2663 
Farag SE, Abo-Zeid M (1997) Degradation of the natural mutagenic compound safrole in spices by cooking and irradiation. Mol Nutr Food Res 41:359-61

Felter SP, Conolly RB, Bercu JP, Bolger PM, Boobis AR, Bos PMJ, Carthew P, Doerrer NG, Goodman JI, Harrouk WA, Kirkland DJ, Lau SS, Llewellyn GC, Preston RJ, Schoeny R, Schnatter AR, Tritscher A, van Velsen F, Williams GM (2011) A proposed framework for assessing risk from less-than-lifetime exposures to carcinogens. Crit Rev Toxicol 41:507-544

Geraets L, Nijkamp MM, Ter Burg W (2016) Critical elements for human health risk assessment of less than lifetime exposures. Regul Toxicol Pharmacol 81:362-371

Gursale A, Dighe V, Parekh G (2010) Simultaneous quantitative determination of cinnamaldehyde and methyleugenol from stem bark of cinnamomum zeylanicum blume using RP-HPLC. J Chromatogr Sci 48:59-62

Huang CY, Tseng MC, Lin JH (2005) Analyzing aristolochic acids in Chinese herbal preparations using LC/MS/MS. J Food Drug Anal 13:125-131

Jeurissen SM, Punt A, Delatour T, Rietjens IMCM (2008) Basil extract inhibits the sulfotransferase mediated formation of DNA adducts of the procarcinogen 1'-hydroxyestragole by rat and human liver S9 homogenates and in HepG2 human hepatoma cells. Food Chem Toxicol 46:2296-2302

Joint FAO/WHO Expert Committee on Food Additives (JEFCA) (2009) Safety evaluation of certain food additives / prepared by the sixty-ninth meeting of the Joint FAO/WHO Expert Committee on Food Additives (JEFCA). World Health Organization.

Li BT, Wilson TK (2008) Myristicaceae-Flora of China. Science Press (Beijing) \& Missouri Botanical Garden (St.Louis)

Li H, Zhu G, Josef B (2010) Acoracea-Flora of China, vol 23. Science Press (Beijing) \& Missouri Botanical Garden (St.Louis)

Li XW, Hedge IC (1994) Lamiaceae-Flora of China, vol 17. Science Press (Beijing) \& Missouri Botanical Garden (St.Louis)

Li XW, Li J, Huang P, Wei FN, Cui HB, Van Der Werff H (2008) Lauraceae-Flora of China, vol 7. Science Press (Beijing) \& Missouri Botanical Garden (St. Louis)

Lin Y, Humphries CJ, Gilbert MG (2011) Asteraceae-Flora of China, vol 20. Science of Press (Beijing) \& Missouri Botanical Garden Press (St. Louis)

Liu FX, Salmon JW (2010) Herbal medicine regulation in China, Germany, and the United States. Integr Med 9:54-61

Liu Y, Wang Z, Zhang J (2015) Dietary chinese herbs: Chemistry, pharmacology and clinical evidence. Springer-Verlag Wien

Low TY, Wong KO, Yap ALL, Haan LHJ, Rietjens IMCM (2017) The regulatory framework across international jurisdictions for risks associated with consumption of botanical food supplements. Compr Rev Food Sci F 16:821-834 
Martati E, Boersma MG, Spenkelink A, Khadka DB, van Bladeren PJ, Rietjens IMCM, Punt A (2012) Physiologically based biokinetic (PBBK) modeling of safrole bioactivation and detoxification in humans as compared with rats. Toxicol Sci 128:301-316

Martena MJ, van der Wielen JCA, van de Laak LFJ, Konings EJM, de Groot HN, Rietjens IMCM (2007) Enforcement of the ban on aristolochic acids in Chinese traditional herbal preparations on the Dutch market. Anal Bioanal Chem 389:263-275

Mcdonald TA (1999) Evidence on the carcinogenicity of estragole. Reproductive and Cancer Assessment Section, Office of Environmental Health Hazard Assessment, California Environmental Protection Agency

Merz KH, Schrenk D (2016) Interim relative potency factors for the toxicological risk assessment of pyrrolizidine alkaloids in food and herbal medicines. Toxicol Lett 263:44-57

Miller EC, Swanson AB, Phillips DH, Fletcher L, Liem A, Miller JA (1983) Structure-activity studies of the carcinogenicities in the mouse and rat of some naturally occurring and synthetic alkenylbenzene derivatives related to safrole and estragole. Cancer Res 43:1124-1134

National Health and Family Planning Commission (NHFPC) (2007) Reference individuals for use in radiation protection-Part2: Masses of main organs and tissues. National Health and Family Planning Commission of the People's Republic of China (in Chinese)

National Health and Family Planning Commission (NHFPC) (2014) Notice on the Issuance of 37 National Standards for Food Safety, such as GB2760-2014, the National Standard for the Use of Food Additives in Food Safety (in Chinese).

National Toxicology Program (NTP) (2000) NTP Toxicology and Carcinogenesis Studies of Methyleugenol (CAS NO. 93-15-2) in F344/N Rats and B6C3F1 Mice (Gavage Studies). Natl Toxicol Program Tech Rep Ser 491:1-412

Phillips DH, Reddy MV, Randerath K (1984) 32P-post-labelling analysis of DNA adducts formed in the livers of animals treated with safrole, estragole and other naturally-occurring alkenylbenzenes. II. Newborn male B6C3F1 mice. Carcinogenesis 5:1623-1628

Punt A, Paini A, Boersma MG, Freidig AP, Delatour T, Scholz G, Schilter B, Bladeren PJV, Rietjens IMCM (2009) Use of physiologically based biokinetic (PBBK) modeling to study estragole bioactivation and detoxification in humans as compared with male rats. Toxicol Sci 110:255-269

Randerath K, Haglund RE, Phillips DH, Reddy MV (1984) ${ }^{32}$ P-post-labelling analysis of DNA adducts formed in the livers of animals treated with safrole, estragole and other naturally-occurring alkenylbenzenes. I. Adult female CD-1 mice. Carcinogenesis 5:1613-1622

Rietjens IMCM, Slob W, Galli C, Silano V (2008) Risk assessment of botanicals and botanical preparations intended for use in food and food supplements: emerging issues. Toxicol Lett 180:131-136

Rietjens IMCM, Tyrakowska B, Van Den Berg SJ, Soffers AEMF, Punt A (2015) Matrix-derived combination effects influencing absorption, distribution, metabolism and excretion (ADME) of foodborne toxic compounds: implications for risk assessment. Toxicol Res 4:23-35 
She M, Pu F, Pan Z, Watson MF, Cannon JFM, Smith IH, Kljuykov EV, Phillippe LR, Pimenov MG (2005) Apiaceae-Flora of China, vol 14. Science Press (Beijing) \& Missouri Botanical Garden (St.Louis)

Smith B, Cadby P, Leblanc JC, Setzer RW (2010) Application of the margin of exposure (MoE) approach to substances in food that are genotoxic and carcinogenic Example: Methyleugenol, CASRN: 93-15-2. Food Chem Toxicol 48:S89-S97

Smith RL, Adams TB, Doull J, Feron VJ, Goodman JI, Marnett LJ, Portoghese PS, Waddell WJ, Wagner BM, Rogers AE, Caldwell J, Sipes IG (2002) Safety assessment of allylalkoxybenzene derivatives used as flavouring substances-methyleugenol and estragole. Food Chem Toxicol 40:851-870

Van Den Berg SJ, Alhusainy W, Restani P, Rietjens IMCM (2014) Chemical analysis of estragole in fennel based teas and associated safety assessment using the Margin of Exposure (MOE) approach. Food Chem Toxicol 65:147-154

Van Den Berg SJ, Punt A, Soffers AE, Vervoort J, Ngeleja S, Spenkelink B, Rietjens IMCM (2012) Physiologically based kinetic models for the alkenylbenzene elemicin in rat and human and possible implications for risk assessment. Chem Res Toxicol 25:2352-2367

Van Den Berg SJ, Restani P, Boersma MG, Delmulle L, Rietjens IMCM (2011) Levels of genotoxic and carcinogenic compounds in plant food supplements and associated risk assessment. Food Sci Nutr 2:989-1010

Vanherweghem JL, Depierreux M, Tielemans C, Abramowicz D, Dratwa M, Jadoul M, Richard C, Vandervelde D, Verbeelen D, Vanhaelen-Fastre RMJ (1993) Rapidly progressive interstitial renal fibrosis in young women: association with slimming regimen including Chinese herbs. Lancet 341:387-391

Wang YA, Chan W (2014) Determination of Aristolochic Acids by High-Performance Liquid Chromatography with Fluorescence Detection. J Agr Food Chem 62:5859-5864

Wu KM, Farrelly JG, Upton R, Chen J (2007) Complexities of the herbal nomenclature system in traditional Chinese medicine (TCM): lessons learned from the misuse of Aristolochia-related species and the importance of the pharmaceutical name during botanical drug product development. Phytomedicine 14:273-279

Xia NH, Saunders RMK (2008) Illiciaceae-Flora of China, vol 7. Science Press (Beijing) \& Missouri Botanical Garden (St.Louis)

Zhu YP (1998) Chinese materia medica: chemistry, pharmacology, and applications. Harwood Academic, Amsterdam, The Netherlands

\section{Supplementary materials}

For convenience, supplementary materials 1 and 2 can be found in http://edepot.wur.nl/496948 



$$
3
$$



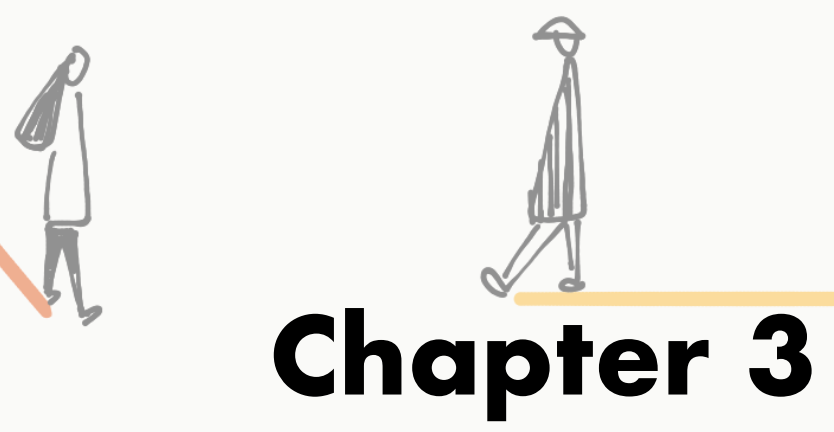

Study on inter-ethnic human differences in bioactivation and detoxification of estragole using physiologically based Kinetic modelling
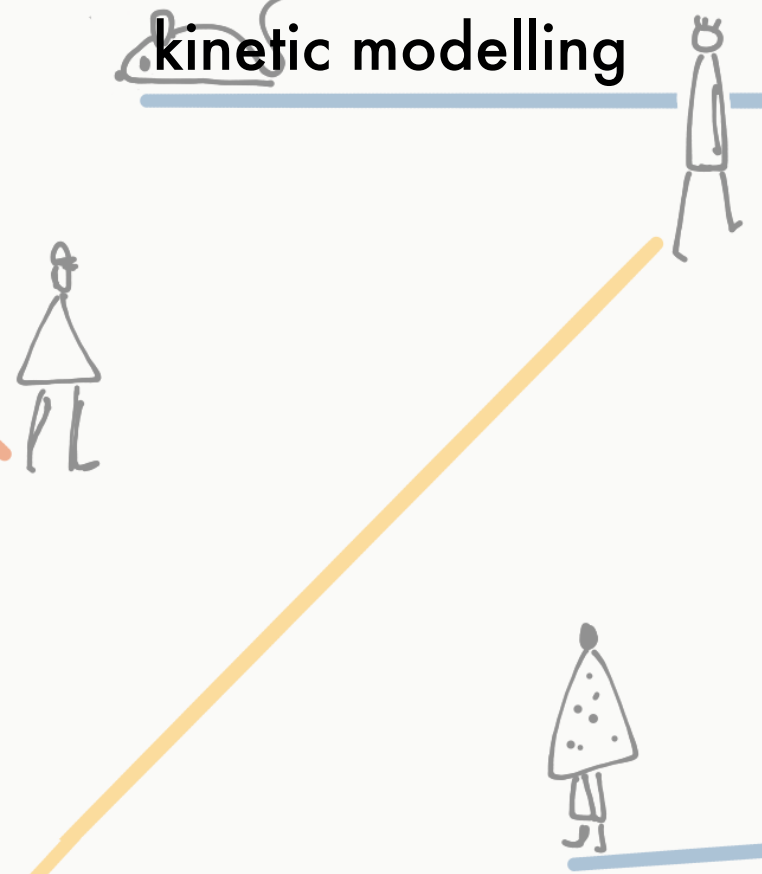

Jia Ning, Jochem Louisse, Bert Spenkelink, Sebas Wesseling, Ivonne M.C.M. Rietjens

Published in: Archives of Toxicology (2017) 91(9), 3093-3108 


\begin{abstract}
Considering the rapid developments in food safety in the past decade in China, it is of importance to obtain insight into what extent safety and risk assessments of chemicals performed for the Caucasian population apply to the Chinese population. The aim of present study was to determine physiologically based kinetic (PBK) modelling based predictions for differences between Chinese and Caucasians in terms of metabolic bioactivation and detoxification of the food-borne genotoxic carcinogen estragole. The PBK models were defined based on kinetic constants for hepatic metabolism derived from in vitro incubations using liver fractions of the two ethnic groups, and used to evaluate the inter-ethnic differences in metabolic activation and detoxification of estragole. The models predicted that at realistic dietary intake levels only $0.02 \%$ of the dose was converted to the ultimate carcinogenic metabolite 1'-sulfooxyestragole in Chinese subjects, whereas this amounted to $0.09 \%$ of the dose in Caucasian subjects. Detoxification of 1'-hydroxyestragole, mainly via conversion to 1'oxoestragole was similar within the two ethnic groups. The 4.5 -fold variation in formation of the ultimate carcinogenic metabolite of estragole accompanied by similar rates of detoxification may indicate a lower risk of estragole for the Chinese population at similar levels of exposure. The study provides a proof-of-principle for how PBK modelling can identify differences in ethnic sensitivity and provide a more refined risk assessment for a specific ethnic group for a compound of concern.
\end{abstract}




\section{Introduction}

Recently the dose dependent bioactivation and detoxification of estragole in different species including human has been studied by physiologically based kinetic (PBK) and dynamic (PBD) modelling (Punt et al. 2008; Punt et al. 2009; Punt et al. 2016). The models defined for the human population were specific for Caucasians, since the parameters used to describe the kinetics were derived using samples from relevant tissues from Caucasian origin. Considering the rapid developments in food safety in the past decade in China, it is of importance to obtain insight into to what extent safety and risk assessments of chemicals performed for the Caucasian population would also apply to the Chinese population. Given the fact that race diversity might result in the variability of dose-response relationships affecting the safety and efficacy of chemical exposures (Malinowski et al. 2008), the absence of knowledge in this field implies that harmonization in legislation on chemicals between regulatory bodies of Europe, USA and Asia is hampered. Several studies have shown ethnic differences in cytochrome P450 enzymes. Significant differences in reactions catalyzed by CYP 1A2, CYP 2C9, CYP 2C19 and CYP 2E1 in liver microsomes have been observed between Chinese and Caucasian samples (Yang et al. 2012). Barter et al. (2013) developed PBK models and used them for in vitro to in vivo extrapolation to predict the P450 mediated pharmacokinetics of selected drugs in the Chinese population. The results showed that the predicted clearances for phenacetin, tolbutamide, desipramine, omeprazole, alprazolam (intravenous), alprazolam (oral), midazolam (intravenous) and midazolam (oral) in Chinese subjects were predicted to be $36,25,43,51,21,22,24$ and $17 \%$ lower, respectively, than in Caucasian subjects and the experimentally observed clearances in treated Chinese individuals were 28, 2, 42, 75, 20, 21, 19 and $62 \%$ lower, respectively, than in Caucasian volunteers (Barter et al. 2013).

Thus, inter-ethnic differences in metabolism and metabolic bioactivation and detoxification may occur. However, systematic attempts to predict the kinetic processes and related toxicity in different populations by using physiologically based kinetic (PBK) modelling are lacking. The aim of the present study was to determine PBK modelling based predictions for differences between Chinese and Caucasians in terms of metabolic bioactivation and detoxification of the food-borne genotoxic carcinogen estragole (Figure 1). Estragole (1-allyl4-methoxybenzene) is an alkenylbenzene that is naturally present in a variety of herbs and spices such as fennel, basil and tarragon (Smith et al. 2002). Consumption of herbs, spices and their essential oils and food products containing these is an important route of exposure to estragole. The Flavor and Extract Manufacturers Association (FEMA) estimated the daily intake of estragole to be less than $0.01 \mathrm{mg} / \mathrm{kg}$ bw/day based on the annual production volume data of estragole for use in flavorings (Smith et al. 2002). Estragole is known to be genotoxic and carcinogenic in rodents at high dose levels (Drinkwater et al. 1976; Miller et al. 1983). Bioactivation of estragole to a DNA reactive ultimate carcinogen proceeds by cytochrome P450 mediated conversion to 1'-hydroxyestragole and subsequent conversion of 1'hydroxyestragole to the ultimate carcinogen 1'-sulfooxyestragole by sulfotransferases (SULTs) (Figure 1). Detoxification of 1'-hydroxyestragole proceeds by glucuronidation to 1'hydroxyestragole glucuronide and oxidation to 1'-oxoestragole (Figure 1). Given the variety 
of biotransformation enzymes involved in estragole bioactivation and detoxification, estragole was selected as an adequate model compound to study ethnic differences in bioactivation and detoxification. To define the PBK models for the Chinese and Caucasian populations, kinetic constants for the various biotransformation reactions of estragole were quantified by using in vitro incubations with relevant tissue fractions of the two ethnic groups. The outcomes predicted by the PBK model for the Chinese population were compared to those predicted by the PBK model for Caucasians to evaluate the inter-ethnic differences in metabolic activation and detoxification of estragole and to demonstrate the potential of PBK modelling to study such inter-ethnic variability.

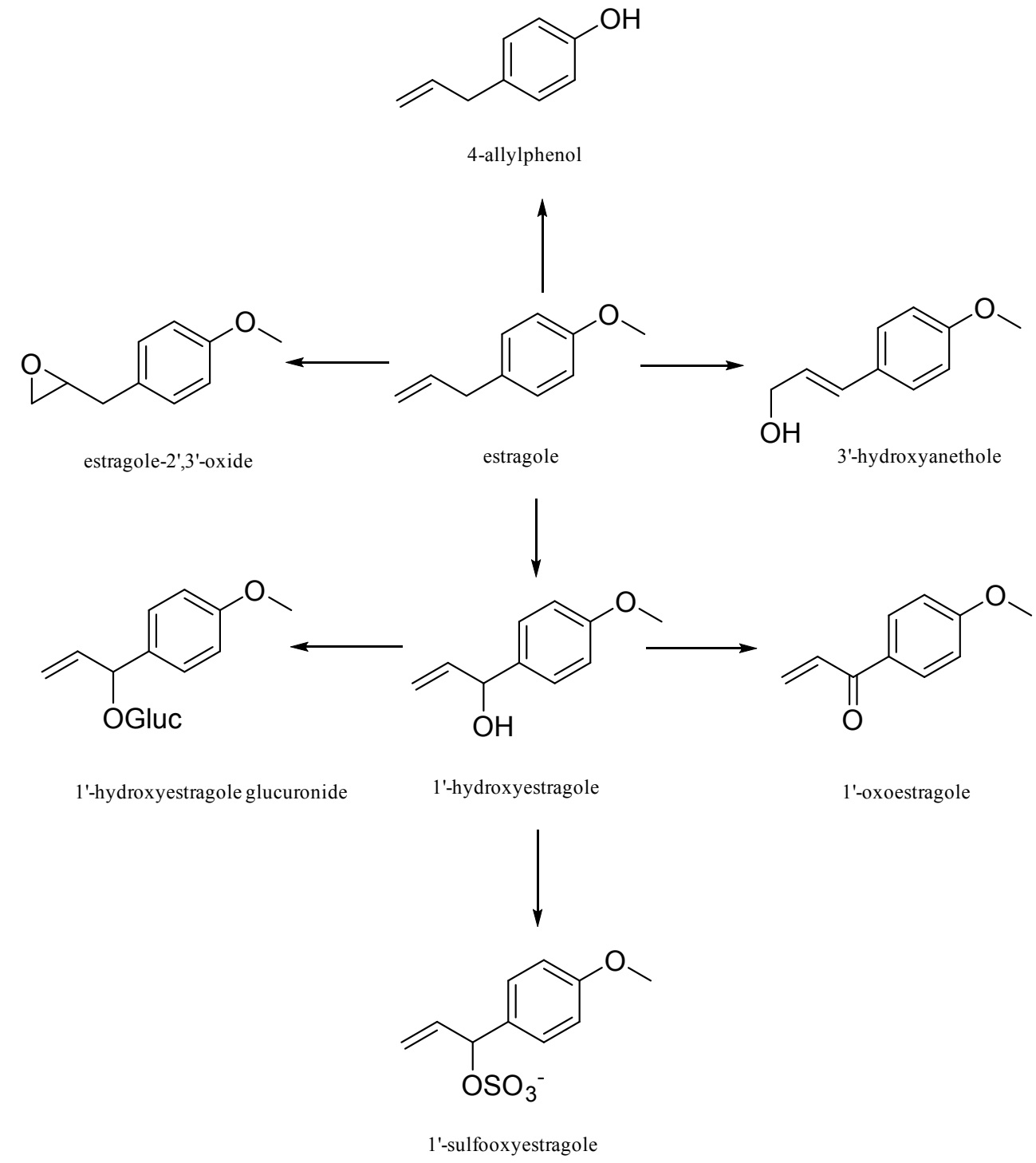

Fig. 1 Metabolic pathways of estragole 


\section{Material and methods}

\subsection{Chemicals and biological materials}

Estragole (1-allyl-4-methoxybenzene), dimethylsulfoxide (DMSO), alamethicin, uridine 5'diphosphoglucuronic acid (UDPGA), 3'-phosphoadenosine-5'-phosphosulfate (PAPS), phenacetin, acetaminophen, coumarin, 7-hydroxycoumarin, 7-hydroxycoumarin sulfate, glucose-6-phosphate dehydrogenase and reduced L-glutathione (GSH) were purchased from Sigma-Aldrich (Steinheim, Germany). Potassium dihydrogen phosphate, dipotassium hydrogen phosphate trihydrate, hydrochloric acid (37\%), trifluoroacetic acid (TFA) and magnesium chloride were purchased from VWR International (Darmstadt, Germany). Reduced nicotinamide adenine dinucleotide phosphate (NADPH), nicotinamide adenine dinucleotide phosphate nicotinamide $\left(\mathrm{NADP}+\right.$ ), adenine dinucleotide $\left(\mathrm{NAD}^{+}\right)$and glucose-6phosphate were obtained from Roche Diagnostics (Mannheim, Germany). Acetonitrile (UPLC/MS grade) was obtained from Biosolve BV (Valkenswaard, Netherlands). 1'Hydroxyestragole, 4-allylphenol, estragole-2',3'-oxide, 3'-hydroxyanethole and 1'oxoestragole were synthesized as previously described by Punt et al. (Punt et al. 2007; Punt et al. 2008). Chinese liver microsomes and Chinese S9, made from 40 donors, were purchased from PrimeTox (Wuhan, China). Caucasian liver microsomes were purchased from BD Gentest (Woburn, MA, USA) and Caucasian liver S9 was purchased from Corning (Amsterdam, Netherlands). All microsomes and S9 were of pooled mixed gender.

\subsection{In vitro incubations}

\section{Assessment of metabolic capabilities of CYP and SULT enzymes in Chinese and Caucasian liver samples}

The quality of the Chinese and Caucasian liver microsomes was checked by measuring the activity of CYP 1A2 and CYP 2A6 by the method of Yang et al. (2012). The quality of Chinese and Caucasian liver S9 samples was checked by measuring the SULT activity based on the method provided by Wang et al. (2006). The details of the methods for the microsomal and S9 in vitro incubations and UPLC analysis for assessing the metabolic capabilities of CYP and SULTs enzymes can be found in the supplementary materials 1 .

\section{Microsomal metabolism of estragole}

The kinetic constants for the microsomal conversion of estragole were determined as previously described by Punt et al. (2009). Briefly, mixed gender Chinese or Caucasian liver microsomes were incubated with estragole in the presence of NADPH. The incubation mixtures contained (final concentrations) $3 \mathrm{mM} \mathrm{NADPH}$ and $1 \mathrm{mg} / \mathrm{ml} \mathrm{microsomal} \mathrm{protein} \mathrm{in}$ $0.2 \mathrm{M}$ Tris- $\mathrm{HCl}$ ( $\mathrm{pH} 7.4$ ). Incubations were performed for $10 \mathrm{~min}$ at substrate concentrations ranging from 25 to $1000 \mu \mathrm{M}$, after which the reaction was terminated by adding $25 \mu \mathrm{l}$ icecold acetonitrile. Blank incubations were performed in the absence of the cofactor NADPH. All incubations were performed in triplicate. In the supplementary materials 2, detailed information can be found (Table S1 can be used to keep track of detailed information). 


\section{Glucuronidation of 1'-hydroxyestragole}

Pooled mixed gender Chinese or Caucasian liver microsomes were incubated with 1'hydroxyestragole in the presence of UDPGA. As previously described by Punt et al. (2009), the incubation mixtures contained (final concentrations) $10 \mathrm{mM}$ UDPGA, and $1 \mathrm{mg} / \mathrm{ml}$ microsomal protein in $0.2 \mathrm{M}$ Tris- $\mathrm{HCl}(\mathrm{pH} 7.4)$ with $10 \mathrm{mM} \mathrm{MgCl}_{2}$. Incubations were carried out for 6 hours and the reaction was terminated by adding $25 \mu$ ice-cold acetonitrile. Blank incubations were performed in the absence of the cofactor UDPGA. All incubations were performed in triplicate. In the supplementary materials 2 , detailed information can be found (Table S1 can be used to keep track of detailed information).

\section{Oxidation of 1'-hydroxyestragole}

Mixed gender Chinese or Caucasian liver S9 was incubated with 1'-hydroxyestragole in the presence of $\mathrm{NAD}^{+}$and $\mathrm{GSH}$, the latter added to trap the transient 1'-oxoestragole. Formation of the 1'-oxoestragole adducts with GSH forming GS-1'-oxoestragole reflects the formation of 1'-oxoestragole (Punt et al. 2009). The incubations had a final volume of $100 \mu$ l, containing (final concentrations) $3 \mathrm{mM} \mathrm{NAD}{ }^{+}, 2 \mathrm{mM} \mathrm{GSH}$ and $1 \mathrm{mg} / \mathrm{ml}$ liver S9 in $0.2 \mathrm{M}$ Tris- $\mathrm{HCl}$ (pH 7.4), as described previously by Punt et al. (2016). The reactions were terminated after $10 \mathrm{~min}$ by the addition of $25 \mu \mathrm{l}$ ice-cold acetonitrile. Blank incubations were performed without cofactor $\mathrm{NAD}^{+}$. All incubations were performed in triplicate. In the supplementary materials 2, detailed information can be found (Table S1 can be used to keep track of detailed information).

\section{Sulfation of 1'-hydroxyestragole}

The formation of 1 '-sulfooxyestragole was determined by incubating $0.2 \mathrm{mg} / \mathrm{ml}$ pooled mixed gender Chinese or Caucasian liver S9 in the presence of $0.2 \mathrm{mM}$ PAPS as cofactor and $10 \mathrm{mM} \mathrm{GSH}$ as trapping agent for the reactive 1'-sulfooxyestragole in $0.1 \mathrm{M}$ potassium phosphate $(\mathrm{pH} 8.0)$. The incubations were carried out for 2 hours and the reactions were terminated by adding $25 \mu \mathrm{l}$ ice-cold acetonitrile. The blank samples were performed without cofactor. All incubations were performed in triplicate. In the supplementary materials 2, detailed information can be found (Table S1 can be used to keep track of detailed information).

\subsection{UPLC analysis}

\section{UPLC analysis of estragole metabolites}

Before UPLC analysis, all samples were centrifuged for $5 \mathrm{~min}$ at $16000 \mathrm{~g}$ to precipitate microsomal proteins. Supernatant of each sample was analyzed on UPLC using a BEH C18 $(1.7 \mu \mathrm{m} 2.1 \times 50 \mathrm{~mm})$ column with a guard column and a diode array detector (Acquity, Waters). The gradient for analysis of metabolites of estragole can be found in supplementary materials 2. Table S1 can be used to keep track of detailed information. Identification of microsomal metabolites of estragole including 4-allylphenol, estragole-2',3'-oxide, 1'hydroxyestragole, 3'-hydroxyanethole and M5 was achieved by comparison of the UV spectra and retention times of formed metabolites to those of synthesized reference compounds 
identified previously (Punt et al. 2007; Punt et al. 2008). Formation of 4-allylphenol, estragole-2',3'-oxide and 1'-hydroxyestragole was quantified by comparing the peak areas to those of the corresponding reference standard curves at wavelength $225 \mathrm{~nm}$ (Agharahimi M.R 1995; Drinkwater et al. 1976; Iyer et al. 2003; Luo et al. 1992). Because the UV spectrum of M5 is similar to that of estragole-2',3'-oxide, quantification of M5 could be achieved by comparison of the peak area to the calibration curve of estragole-2',3'-oxide at $225 \mathrm{~nm}$. 3'Hydroxyanethole was quantified by comparison of the peak areas of the metabolite in the chromatograms obtained at a wavelength of $206 \mathrm{~nm}$ to the calibration curve of the synthesized reference compound (Agharahimi M.R 1995; Drinkwater et al. 1976; Iyer et al. 2003; Luo et al. 1992). The amounts of formed microsomal estragole metabolites were corrected for the amounts detected in the blank incubations performed without the respective cofactor NADPH.

\section{UPLC analysis of 1'-hydroxyestragole metabolites}

Before UPLC analysis, all samples were centrifuged for $5 \mathrm{~min}$ at $16000 \mathrm{~g}$ to precipitate microsomal or cytosolic proteins. Supernatant of each sample was analyzed on UPLC using a BEH C18 $(1.7 \mu \mathrm{m} 2.1 \times 50 \mathrm{~mm})$ column with a guard column and a diode array detector (Acquity, Waters). Secondary metabolism of 1'-hydroxyestragole includes glucuronidation, oxidation and sulfation. The gradient of analysis the metabolites of 1'-hydroxyestragole can be found in supplementary materials 2. Table S1 can be used to track more detailed information.

Identification of 1'-hydroxyestragole glucuronide was achieved by the fact that it was the only metabolite formed and by LC-MS analysis as reported previously (Punt et al. 2008). Both 1'hydroxyestragole glucuronide and 1'-hydroxyestragole have the same UV spectrum and the same extinction coefficient at a wavelength of $225 \mathrm{~nm}$. Thus the quantification of 1'hydroxyestragole glucuronide could be achieved by comparing the peak area to the calibration curve of 1'-hydroxyestragole at wavelength $225 \mathrm{~nm}$ (Punt et al. 2009). The amounts of 1'-hydroxyestragole glucuronide formed were corrected for the amounts detected in the blank incubations performed without the respective cofactor UDPGA.

Identification of the GS-1'-oxoestragole which reflects the formation of 1'-oxoestragole was done by comparing the UV spectra and retention time of the formed GSH adduct to those of GS-1'-oxoestragole identified as described previously (Punt et al. 2009). Quantification of the GSH conjugate of 1'-oxoestragole was achieved by comparing the peak area to the calibration curve of GS-1'-oxoestragole at a wavelength of $280 \mathrm{~nm}$ prepared as described previously by Punt et al. (2009; 2016). Briefly, the calibration curve of GS-1'-oxoestragole was prepared by incubating $40 \mu \mathrm{M} 1$ 1'-oxoestragole with a range of GSH concentrations. The reactions were incubated for 4 hours after which maximal formation of GS-1'-oxoestragole was previously shown to be reached (Punt et al. 2009). The amounts of 1'-oxoestragole formed were corrected for the amounts detected in the blank incubations performed without the respective cofactor $\mathrm{NAD}^{+}$. 
Since the UV spectrum of 3'-hydroxyanethole is similar to that of the GSH adduct of 1'sulfooxyestragole, quantification of 1'-sulfooxyestragole could be achieved by comparing the peak area of the GSH adduct of 1'-sulfooxyestragole to the calibration curve of 3'hydroxyanethole at a wavelength $260 \mathrm{~nm}$ as described for the quantification of 1'sulfooxysafrole and 1'-sulfooxyelemicin (Martati et al. 2012; Van den Berg et al. 2012). The amount of 1'-sulfooxyestragole GSH adduct formed was corrected for the amount detected in the blank samples performed without the respective cofactor PAPS.

\subsection{Kinetic analysis}

The data for the formation of estragole and 1'-hydroxyestragole metabolites with increasing substrate concentration [S] were fitted to the standard Michaelis-Menten equation:

$\mathrm{v}=\mathrm{V}_{\max } /\left(1+\left(\mathrm{K}_{\mathrm{m}} /[\mathrm{S}]\right)\right.$

The apparent maximum velocity $\left(\mathrm{V}_{\max }\right)$ and the apparent Michaelis-Menten constant $\left(\mathrm{K}_{\mathrm{m}}\right)$ were determined by fitting the data to this equation using GraphPad Prism version 5.04 (GraphPad software, San Diego California U.S.A.).

\subsection{PBK model structure}

A schematic diagram of the PBK models for estragole kinetics is presented in Figure 2 and the code of the models can be found in the supplementary materials 6.The PBK models developed in this study were based on the PBK models previously defined by Punt et al. (2008). The models have six compartments including blood, fat, rapidly perfused tissue, slowly perfused tissue, liver and GI-tract that are mutually connected through the systemic circulation. Table 1 summarizes the physiological parameters for Caucasian and Chinese subjects, respectively, which were derived from the literature (Brown et al. 1997; NHFPC 2007; NHFPC 2014b). Based on the method described by DeJongh et al. (DeJongh et al. 1997; Punt et al. 2016), partition coefficients were estimated based on the $\log \mathrm{K}_{\mathrm{ow}}$. The $\log$ $\mathrm{K}_{\mathrm{ow}}$ values for estragole and 1'-hydroxyestragole were estimated by ChemBio 3D 2010 (CambrigeSoft, USA). Model equations were coded and numerically integrated in Berkely Madonna (Macey and Oster, UC Berkeley, CA, USA) using the Rosenbrock's algorithm for stiff systems. 


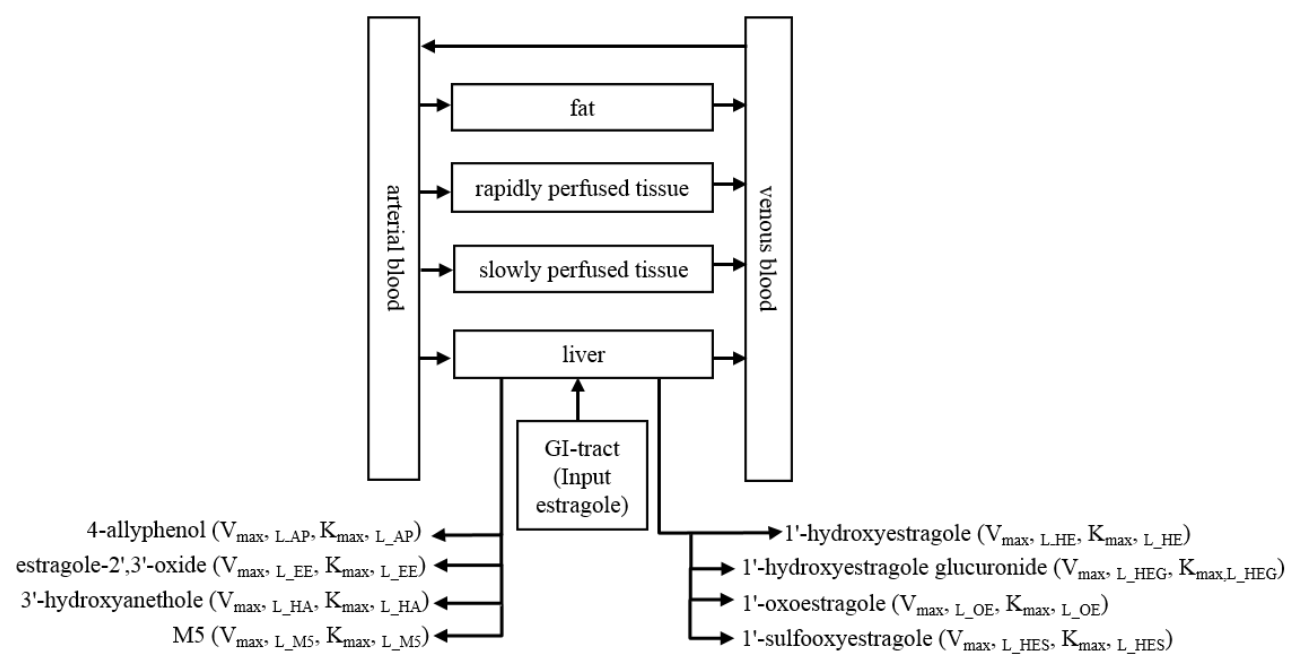

Fig. 2 Schematic diagram of the PBK model for estragole in human, including the name of kinetic parameters for metabolites of estragole and 1'-hydroxyestragole as used in the mass balance equations of the PBK model.

Estragole was assumed to directly enter from the gastrointestinal tract via the portal vein into the liver with an absorption rate constant $\left(\mathrm{K}_{\mathrm{a}}\right)$ of $1.0 \mathrm{~h}^{-1}$ as a first order process. This value was used based on the fact that complete and fast absorption of estragole from the GI tract has been observed (Anthony et al. 1987). As reported by Punt et al. (2009), conversion of estragole mainly occurs in the liver and not in other organs. Also, since the liver is known to be the major target organ for estragole-induced tumor formation in rat and mice, the PBK model focused on metabolism of estragole and 1'-hydroxyestragole in the liver. 1'Hydroxyestragole, 2',3'-estragole oxide, 3'-hydroxyanethole, 4-allyphenol and an unidentified minor metabolite referred to as M5 were formed in incubations with liver microsomes from both populations (see Results section), and conversions of estragole into these metabolites were included in the liver compartment of the models. In both models, descriptions of the conversions of 1'-hydroxyestragole into its further metabolites were included, but the conversions of 2',3'-estragole oxide, 3'-hydroxyanethole, 4-allyphenol and M5 to their further metabolites were not included, since these are assumed to not influence the formation of the ultimate carcinogenic metabolite 1'-sulfooxyestragole. The mass balance equations for microsomal conversion of estragole when using Chinese or Caucasian liver microsomes were described as follows: 


$$
\begin{aligned}
\mathrm{dAL}_{\mathrm{E}} / \mathrm{dt}= & \mathrm{dUptake}_{\mathrm{E}} / \mathrm{dt} \\
& +\mathrm{QL} \times\left(\mathrm{CA}_{\mathrm{E}}-\mathrm{CL}_{\mathrm{E}} / \mathrm{PL}_{\mathrm{E}}\right) \\
& -\mathrm{V}_{\max , \mathrm{L}_{-} \mathrm{AP}} \times\left(\mathrm{CL}_{\mathrm{E}} / \mathrm{PL}_{\mathrm{E}}\right) /\left(\mathrm{K}_{\mathrm{m}, \mathrm{L}_{-} \mathrm{AP}}+\mathrm{CL}_{\mathrm{E}} / \mathrm{PL}_{\mathrm{E}}\right) \\
& -\mathrm{V}_{\max , \mathrm{L}_{-} \mathrm{HE}} \times\left(\mathrm{CL}_{\mathrm{E}} / \mathrm{PL}_{\mathrm{E}}\right) /\left(\mathrm{K}_{\mathrm{m}, \mathrm{L}_{-} \mathrm{HE}}+\mathrm{CL}_{\mathrm{E}} / \mathrm{PL}_{\mathrm{E}}\right) \\
& -\mathrm{V}_{\max , \mathrm{L}_{-} \mathrm{EE}} \times\left(\mathrm{CL}_{\mathrm{E}} / \mathrm{PL}_{\mathrm{E}}\right) /\left(\mathrm{K}_{\mathrm{m}, \mathrm{L}_{-} \mathrm{EE}}+\mathrm{CL}_{\mathrm{E}} / \mathrm{PL}_{\mathrm{E}}\right) \\
& -\mathrm{V}_{\max , \mathrm{L}_{-} \mathrm{HA}} \times\left(\mathrm{CL}_{\mathrm{E}} / \mathrm{PL}_{\mathrm{E}}\right) /\left(\mathrm{K}_{\mathrm{m}, \mathrm{L}_{-} \mathrm{HA}}+\mathrm{CL}_{\mathrm{E}} / \mathrm{PL}_{\mathrm{E}}\right) \\
& -\mathrm{V}_{\max , \mathrm{L}_{-} \mathrm{M} 5} \times\left(\mathrm{CL}_{\mathrm{E}} / \mathrm{PL}_{\mathrm{E}}\right) /\left(\mathrm{K}_{\mathrm{m}, \mathrm{L}_{-} \mathrm{M} 5}+\mathrm{CL}_{\mathrm{E}} / \mathrm{PL}_{\mathrm{E}}\right)
\end{aligned}
$$

$\mathrm{dUptake}_{\mathrm{E}} / \mathrm{dt}=-\mathrm{dAGI}_{\mathrm{E}} / \mathrm{dt}=\mathrm{Ka} \times \mathrm{AGI}_{\mathrm{E}}, \mathrm{AGI}_{\mathrm{E}}(0)=$ oral dose $\mathrm{CL}_{\mathrm{E}}=\mathrm{AL}_{\mathrm{E}} / \mathrm{VL}$

Where $\mathrm{AL}_{\mathrm{E}}$ is the amount of estragole in the liver tissue $(\mu \mathrm{mol})$. Uptake $\mathrm{E}$ is the amount of estragole taken up from the GI tract $(\mu \mathrm{mol})$, QL is the blood flow rate to and from the liver tissue $(\mathrm{L} / \mathrm{hr}), \mathrm{CA}_{\mathrm{E}}$ is the estragole concentration in the arterial blood ( $\left.\mu \mathrm{mol} / \mathrm{L}\right), \mathrm{CL}_{\mathrm{E}}$ is the estragole concentration in the liver tissue. $\mathrm{PL}_{\mathrm{E}}$ is the liver/blood partition coefficient, $\mathrm{V}_{\max , \mathrm{L}_{-} \mathrm{M}}$

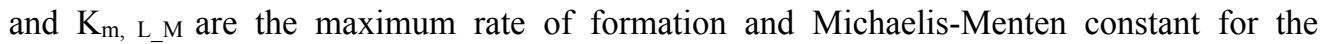
metabolites 4-allylphenol (AP), 1'-hydroxyestragole (HE), estragole-2',3'-oxide (EE), 3'hydroxyanethole (HA) and metabolite 5 (M5) in the liver tissue, AGI $(\mu \mathrm{mol})$ is the amount of estragole in the GI tract, and VL is the volume of the liver. The mass balance equation for the metabolism of 1'-hydroxyestragole in the liver was as follows:

$$
\begin{aligned}
& \mathrm{dAL} L_{\mathrm{HE}} / \mathrm{dt}=\mathrm{V}_{\max , \mathrm{L}_{-} \mathrm{HE}} \times\left(\mathrm{CL}_{\mathrm{E}} / \mathrm{PL}_{\mathrm{E}}\right) /\left(\mathrm{K}_{\mathrm{m}, \mathrm{L}_{-} \mathrm{HE}}+\mathrm{CL}_{\mathrm{E}} / \mathrm{PL}_{\mathrm{E}}\right) \\
& -\mathrm{V}_{\text {max, } \mathrm{L}_{-} \mathrm{HEG}} \times\left(\mathrm{CL}_{\mathrm{HE}} / \mathrm{PL}_{\mathrm{HE}}\right) /\left(\mathrm{K}_{\mathrm{m}, \mathrm{L}_{-} \mathrm{HEG}}+\mathrm{CL}_{\mathrm{HE}} / \mathrm{PL}_{\mathrm{HE}}\right) \\
& -\mathrm{V}_{\max , \mathrm{L}_{-} \mathrm{OE}} \times\left(\mathrm{CL}_{\mathrm{HE}} / \mathrm{PL}_{\mathrm{HE}}\right) /\left(\mathrm{K}_{\mathrm{m}, \mathrm{L}_{-} \mathrm{OE}}+\mathrm{CL}_{\mathrm{HE}} / \mathrm{PL}_{\mathrm{HE}}\right) \\
& -\mathrm{V}_{\max , \mathrm{L}_{-} \mathrm{HES}} \times\left(\mathrm{CL}_{\mathrm{HE}} / \mathrm{PL}_{\mathrm{HE}}\right) /\left(\mathrm{K}_{\mathrm{m}, \mathrm{L}_{-} \mathrm{HES}}+\mathrm{CL}_{\mathrm{HE}} / \mathrm{PL}_{\mathrm{HE}}\right) \\
& \mathrm{CL}_{\mathrm{HE}}=\mathrm{AL}_{\mathrm{HE}} / \mathrm{VL}
\end{aligned}
$$

Where $A L_{H E}$ is the amount of 1'-hydroxyestragole in the liver tissue ( $\left.\mu \mathrm{mol}\right), \mathrm{CL}_{\mathrm{HE}}$ is the 1'hydroxyestragole concentration in the liver tissue $(\mu \mathrm{mol} / \mathrm{L}), \mathrm{PL}_{\mathrm{HE}}$ is the liver/blood partition coefficient of 1'-hydroxyestragole, $\mathrm{V}_{\max }, \mathrm{L}_{-} \mathrm{M}$ and $\mathrm{K}_{\mathrm{m}, \mathrm{L}_{-} \mathrm{M}}$ are the maximum rate and the Michaelis-Menten constant for the formation of 1'-hydroxyestragole glucuronide (HEG), 1'oxoestragole (OE) and 1'-sulfooxyestragole (HES) in the liver tissue.

The kinetic constants for metabolites formed were determined in vitro in the present study. $\mathrm{V}_{\max }$ values expressed as $\mathrm{nmol} / \mathrm{min} /\left(\mathrm{mg}\right.$ microsomal or $\mathrm{S} 9$ protein) were scaled to the $\mathrm{V}_{\max }$ per $\mu \mathrm{mol} / \mathrm{h} /(\mathrm{g}$ liver) using mirosomal and S9 protein yields of 35 and $143 \mathrm{mg} / \mathrm{g}$ liver, respectively as previously described by Punt et al. (2009), Al-Subeihi et al. (2012), Martati et al. (2012) and Van den Berg et al. (2012). Currently, no data are available specifically for the liver microsomal and S9 protein relevant for the Chinese tissue samples. Therefore, the value of liver microsomal protein and liver S9 protein available for the Caucasians were used for the Chinese population. 
Table 1 Parameters used in the physiologically based kinetic model for estragole in Chinese and Caucasian populations as obtained from literature.

\begin{tabular}{|c|c|c|}
\hline Model parameters & Chinese ${ }^{a, c}$ & Caucasian $^{b, c}$ \\
\hline \multicolumn{3}{|l|}{ Physiological parameters } \\
\hline Body weight (kg) & 60 & 70 \\
\hline \multicolumn{3}{|l|}{ Percentage of body weight } \\
\hline Liver & 2.3 & 2.6 \\
\hline Fat & 18.7 & 21.4 \\
\hline Rapidly perfused & 5.3 & 5 \\
\hline Slowly perfused & 54.3 & 51.7 \\
\hline Blood & 7.9 & 7.9 \\
\hline Cardiac output $\left(\mathrm{L} / \mathrm{hr} / \mathrm{kg} \mathrm{bw}{ }^{0.74}\right)$ & 15 & 15 \\
\hline \multicolumn{3}{|l|}{ Percentage of cardiac output } \\
\hline Liver & 26.3 & 22.7 \\
\hline Fat & 6.75 & 5.2 \\
\hline Rapidly perfused & 43.8 & 47.3 \\
\hline Slowly perfused & 23.3 & 24.8 \\
\hline \multicolumn{3}{|l|}{ Tissue: blood partition coefficients } \\
\hline \multicolumn{3}{|l|}{ Estragole } \\
\hline Liver & 6.5 & 6.5 \\
\hline Fat & 105 & 105 \\
\hline Rapidly perfused & 6.5 & 6.5 \\
\hline Slowly perfused & 4.1 & 4.1 \\
\hline \multicolumn{3}{|l|}{ 1'-Hydroxyestragole } \\
\hline Liver & 1.6 & 1.6 \\
\hline
\end{tabular}

a NHFPC (2007); NHFPC (2014b)

b Brown et al. (1997)

c DeJongh et al. (1997) 


\subsection{Sensitivity analysis}

To identify which parameters have the greatest impact on the model predictions on the formation of 1'-hydroxyestragole and 1'-sulfooxyestragole, a sensitivity analysis was performed. Normalized sensitivity coefficients (SC) were determined using the following equation:

$\mathrm{SC}=\left(\mathrm{C}^{\prime}-\mathrm{C}\right) /\left(\mathrm{P}^{\prime}-\mathrm{P}\right) \times(\mathrm{P} / \mathrm{C})$

Where $\mathrm{C}$ is the initial value of the model output; $\mathrm{C}^{\prime}$ is the modified model output resulting from a $5 \%$ increase of the parameter value; $\mathrm{P}$ is the initial parameter value; $\mathrm{P}^{\prime}$ is the modified parameter value (Evans and Andersen 2000). A $5 \%$ increase in parameter values was chosen to analyze the effect of a change in parameter values on formation of 1'-hydroxyestragole and 1 '-sulfooxyestragole at a dose $0.01,5$ and $150 \mathrm{mg} / \mathrm{kg}$ bw/day for 24 hours exposure, representing respectively a realistic daily intake (Smith et al. 2002), an intake that may result from supplement use (Van Den Berg et al. 2011) and a dose level known to cause liver tumors in rodent bioassays (Drinkwater et al. 1976; Miller et al. 1983). Each parameter was analyzed individually while other parameters were kept as their initial value.

\subsection{Comparison of the PBK model based predictions for bioactivation and detoxification of estragole in the Chinese and Caucasian population}

The PBK model based predictions for the formation of metabolites of estragole and 1'hydroxyestragole in the Chinese population were compared with the predicted formation of these metabolites in the Caucasian population. Model predictions were made for a period of 24 hours after exposure.

\section{Results}

\subsection{Metabolic capabilities of CYP and SULTs enzymes}

The results of metabolic capabilities of CYP and SULTs enzymes can be found in supplementary materials 3 . The quality of the Chinese tissue samples used to determine the various kinetic parameters appeared to be in line with what has been reported before (Yang et al. 2012).

\subsection{Microsomal conversion of estragole}

The microsomal conversion of estragole was determined by incubating estragole with Chinese or Caucasian liver microsomes in the presence of NADPH. Figure S1 in supplementary materials 4 presents a representative chromatogram of an incubation with Chinese liver microsomes showing the formation of estragole-2',3'-diol ( Rt $=2.9 \mathrm{~min}), 1^{\prime}$ hydroxyestragole $(\mathrm{Rt}=4.7 \mathrm{~min}), 3^{\prime}$-hydroxyanethole $(\mathrm{Rt}=4.9 \mathrm{~min})$, M5 $(\mathrm{Rt}=5 \mathrm{~min})$ and 4allylphenol $(\mathrm{Rt}=6.8 \mathrm{~min})$. Since the metabolite referred to as M5 was only formed in a relatively small amount in incubations with liver microsomes from both ethnic groups, its identification was not deemed essential. Due to the presence of epoxide hydrolase in the 
human liver microsomes, the formation of estragole-2',3'-diol reflects formation of estragole2',3'-oxide (Guenthner and Luo 2001; Luo and Guenthner 1996; Luo et al. 1992).

The estragole concentration-dependent rates of formation of estragole-2',3'-oxide, 1'hydroxyestragole, 3'-hydroxyanethole, M5 and 4-allylphenol by Chinese or Caucasian liver microsomes is shown in Figure 3. The kinetic constants for these metabolic conversions of estragole and the catalytic efficiency, calculated as $\mathrm{V}_{\max } / \mathrm{K}_{\mathrm{m}}$, obtained from these data are summarized in Table 2.

a

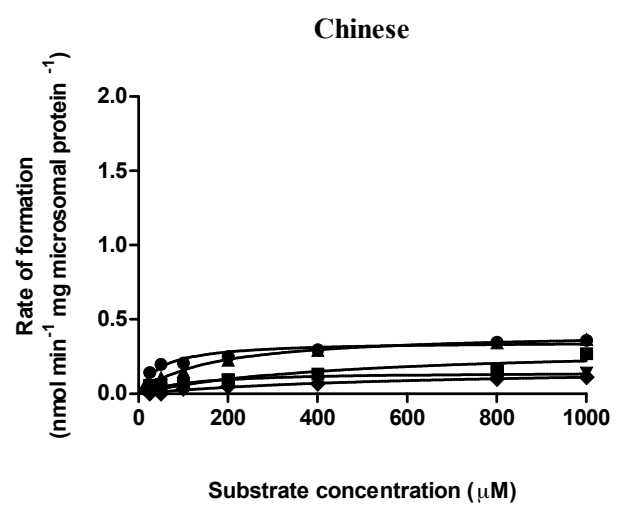

b

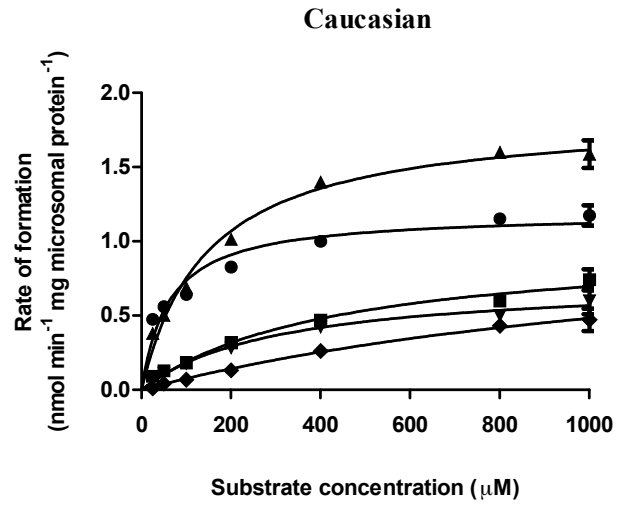

Fig. 3 Concentration-dependent rate of metabolic conversion of estragole in incubations with Chinese (a) or Caucasian (b) liver microsomes. Data points represent mean values \pm SEM of three individual experiments for each metabolite including estragole-2',3'-oxide $(\boldsymbol{\Delta}), 1^{\prime}$ -

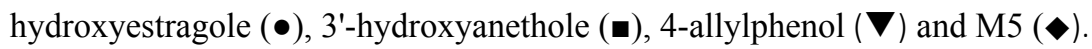

The results showed that estragole-2',3'-oxide was the most abundant metabolite formed in incubations with liver microsomes from both populations, followed by 1'-hydroxyestragole. Analysis of catalytic efficiencies for the formation of the microsomal estragole metabolites, revealed that the catalytic efficiency for formation of 1'-hydroxyestragole was the highest, followed by the formation of estragole-2',3'-oxide, 4-allylphenol, 3'-hydroxyanethole and M5 in both populations. Thus, formation of 1'-hydroxyestragole was the major microsomal metabolic pathway of estragole, whereas formation of M5 was the least important route of estragole metabolism. The catalytic efficiency for the formation of 1'-hydroxyestragole was higher than that for the other microsomal metabolites because of a relatively low $\mathrm{K}_{\mathrm{m}}$. Comparison of the catalytic efficiencies obtained for the two ethnic groups revealed that the catalytic efficiencies for the formation of 4-allylphenol, estragole-2',3'-oxide, 1'hydroxyestragole, 3'-hydroxyanethole and M5 in incubations with the Chinese samples was 1.8-, 4.9-, 2.4-, 3.2-, and 2.9-fold lower than those in incubations with Caucasian samples. The relatively low catalytic efficiencies for the Chinese samples were mainly due to relatively low $\mathrm{V}_{\max }$ values, which were 5.0-, 4.4-, 3.4-, 3.1- and 6.5-fold lower than the $\mathrm{V}_{\max }$ for $O$ demethylation, epoxidation, 1'-hydroxylation, 3'-hydroxylation and the reaction for the 
1.8-, 4.9-, 2.4-, 3.2-, and 2.9-fold lower than those in incubations with Caucasian samples. The relatively low catalytic efficiencies for the Chinese samples were mainly due to relatively low $\mathrm{V}_{\max }$ values, which were 5.0-, 4.4-, 3.4-, 3.1- and 6.5-fold lower than the $\mathrm{V}_{\max }$ for $O$ demethylation, epoxidation, 1'-hydroxylation, 3'-hydroxylation and the reaction for the formation of M5 in Caucasian samples. The apparent $K_{m}$ values for these reactions by Chinese and Caucasian samples were similar.

\subsection{Glucuronidation of 1'-hydroxyestragole}

Kinetic constants for the formation of 1'-hydroxyestragole glucuronide were determined by incubations with Chinese or Caucasian liver microsomes in the presence of UDPGA and 1'hydroxyestragole. 1'-Hydroxyestragole glucuronide eluted at $2.6 \mathrm{~min}$ and was identified by LC-MS as previously described (Punt et al. 2008). The metabolite was quantified using the calibration curve of 1'-hydroxyestragole on the basis of the similarity in their UV spectra assuming a similar molar extinction coefficient.

Figure $4 \mathrm{a}$ shows the 1'-hydroxyestragole concentration-dependent rate of formation of 1'hydroxyestragole glucuronide by liver microsomes and kinetic constants derived from these plots are displayed in Table 2. The apparent $\mathrm{K}_{\mathrm{m}}$ and $\mathrm{V}_{\max }$ for formation of 1'-hydroxyestragole glucuronide by Chinese samples were $4656 \mu \mathrm{M}$ and $1.63 \mathrm{nmol} / \mathrm{min} /(\mathrm{mg}$ microsomal protein), respectively, whereas $\mathrm{K}_{\mathrm{m}}$ and $\mathrm{V}_{\max }$ for formation of 1'-hydroxyestragole glucuronide by Caucasian samples were $4607 \mu \mathrm{M}$ and $4.29 \mathrm{nmol} / \mathrm{min} /(\mathrm{mg}$ microsomal protein), respectively. These values result in a catalytic efficiency that is 2.7-fold lower for Chinese than for Caucasian subjects.

\subsection{Oxidation of 1'-hydroxyestragole}

1'-Oxoestragole was formed in incubations with 1'-hydroxyestragole and Chinese or Caucasian liver S9 using $\mathrm{NAD}^{+}$as cofactor and GSH to trap 1'-oxoestragole forming GS-1'oxoestragole. Chromatographic analysis revealed that GS-1'-oxoestragole eluted at $2.6 \mathrm{~min}$ based on its previous identification (Punt et al. 2009).

The rate of oxidation of 1'-hydroxyestragole with increasing concentration of 1'hydroxyestragole is shown in Figure $4 \mathrm{~b}$ and Table 2 displays the kinetic constants for formation of 1'-oxoestragole derived from these data. The apparent $\mathrm{K}_{\mathrm{m}}$ for formation of 1'oxoestragole by Chinese samples was $403 \mu \mathrm{M}$ and the $\mathrm{V}_{\max }$ was $1.82 \mathrm{nmol} / \mathrm{min} /(\mathrm{mg} \mathrm{S} 9$ protein). For Caucasian samples, the apparent $\mathrm{K}_{\mathrm{m}}$ and $\mathrm{V}_{\max }$ were $521 \mu \mathrm{M}$ and $2.8 \mathrm{nmol} / \mathrm{min} /$ (mg S9 protein), respectively. The catalytic efficiencies for formation of 1'-oxoestragole by both ethnic groups were similar.

\subsection{Sulfation of 1'-hydroxyestragole}

Formation of the ultimate carcinogenic metabolite 1'-sulfooxyestragole upon sulfation of 1'hydroxyestragole was observed in incubations with Chinese as well as Caucasian liver S9 in the presence of PAPS and GSH. GSH was used to trap the transient 1'-sulfooxyestragole forming the GSH adduct of 1'-sulfooxyestragole. Chromatographic analysis revealed a peak at $1.4 \mathrm{~min}$, identified as the GSH adduct of 1'-sulfooxyestragole. This identification was based on the fact that the peak was absent in the incubations with PAPS and S9 in the absence 
of GSH (chromatogram not shown), and the previous identification of this (Punt et al. 2007) and similar alkenylbenzene 1'-sulfooxy metabolites by LC-MS (Al-Malahmeh et al. 2016; AlSubeihi et al. 2012; Alajlouni et al. 2016; Martati et al. 2012).

Figure 4c presents the 1'-hydroxyestragole concentration dependent rate of formation of 1'sulfooxyestragole in incubations with Chinese or Caucasian liver S9 as determined by quantification of the GSH adduct of 1'-sulfooxyestragole. Table 2 presents a summary of the kinetic constants for formation of 1'-sulfooxyestragole in the two ethnic groups derived from these data. The catalytic efficiencies for formation of 1'-sulfooxyestragole in incubation with Chinese samples was 5-fold lower than those in incubations with Caucasian samples.

Chinese

a

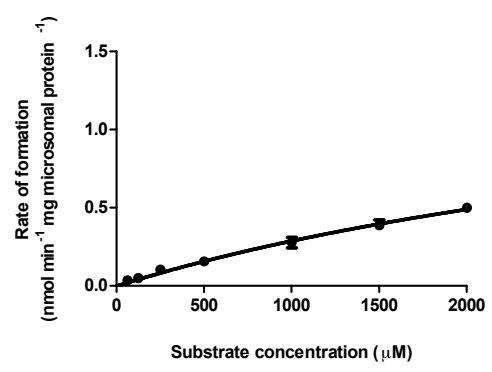

b

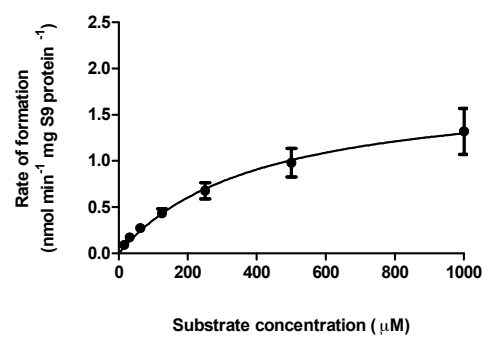

c

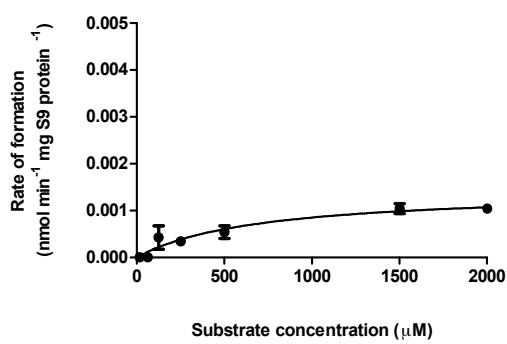

Caucasian

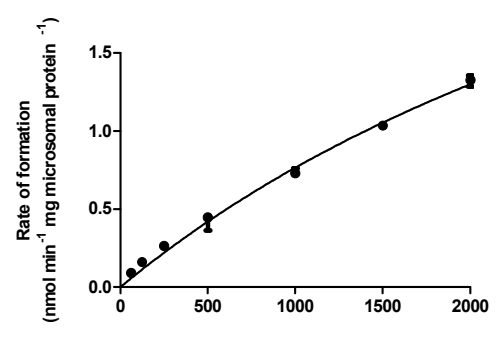

Substrate concentration $(\mu \mathrm{M})$
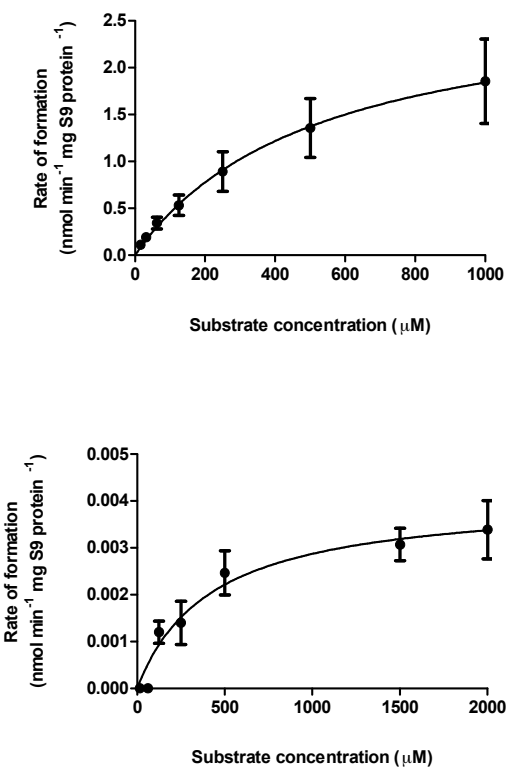

Fig. 4 Concentration-dependent rate of metabolic conversion of 1'-hydroxyestragole to (a) 1'hydroxyestragole glucuronide, (b) 1'-oxoestragole and (c) 1'-sulfooxyestragole in incubations with Chinese or Caucasian liver tissue fractions. Data points represent mean values \pm SEM of three individual experiments for each metabolite. 
3.6 Comparison of the kinetic constants for conversion of estragole and 1'hydroxyestragole by Chinese and Caucasian liver tissue fractions

Table 3 displays the scaled kinetic parameters for conversion of estragole and 1'hydroxyestragole by Chinese or Caucasian liver tissue fractions. In this table, $\mathrm{V}_{\max }$ values that were obtained in vitro expressed as $\mathrm{nmol} / \mathrm{min} /(\mathrm{mg}$ microsomal protein or S9) (Table 2) were scaled to whole liver and expressed in $\mu \mathrm{mol} / \mathrm{h} /\left(\mathrm{g}\right.$ tissue). A scaled catalytic efficiency $\left(\mathrm{V}_{\max }\right.$ in vivo $/ K_{m} \times 1000$ ) for formation of the different metabolites of estragole and 1'hydroxyestragole could be calculated. The catalytic efficiencies thus obtained reveal that formation of the proximate carcinogenic metabolite of estragole, 1'-hydroxyestragole, was 2.5fold lower for Chinese liver microsomes as compared to Caucasian liver micorosomes. This difference in catalytic efficiency was mainly due to the low $V_{\max }$ in Chinese liver microsomes, since the value of $\mathrm{V}_{\max }$ in Chinese liver incubations was 3.5-fold lower than in Caucasian liver incubations. Epoxidation of estragole turned out to be the main route of microsomal metabolism of estragole in both ethnic groups, although the catalytic efficiency for formation of estragole-2',3'-oxide was 5-fold lower for the Chinese population. This lower catalytic efficiency was mainly due to a 4-fold lower $\mathrm{V}_{\max }$ for Chinese liver samples.

Regarding the metabolic reactions of 1'-hydroxyestragole, formation of 1'-oxoestragole was the main detoxification pathway for this proximate carcinogenic metabolite in both populations. The catalytic efficiencies for formation of 1'-oxoestragole in both populations were similar. However, catalytic efficiency for formation 1'-hydroxyestragole glucuronide, another detoxification route of 1'-hydroxyestragole was found to be 3-fold lower in incubations with Chinese than with Caucasian liver microsomes, resulting from a 3-fold lower $\mathrm{V}_{\max }$. Sulfation of 1'-hydroxyestragole in both populations, reflecting bioactivation to a reactive ultimate carcinogen, was found to be the least efficient pathway for 1'hydroxyestragole metabolism for both ethnic groups. For Chinese samples, the scaled catalytic efficiency for this bioactivation reaction was 4.5 -fold lower than for the Caucasian samples. 


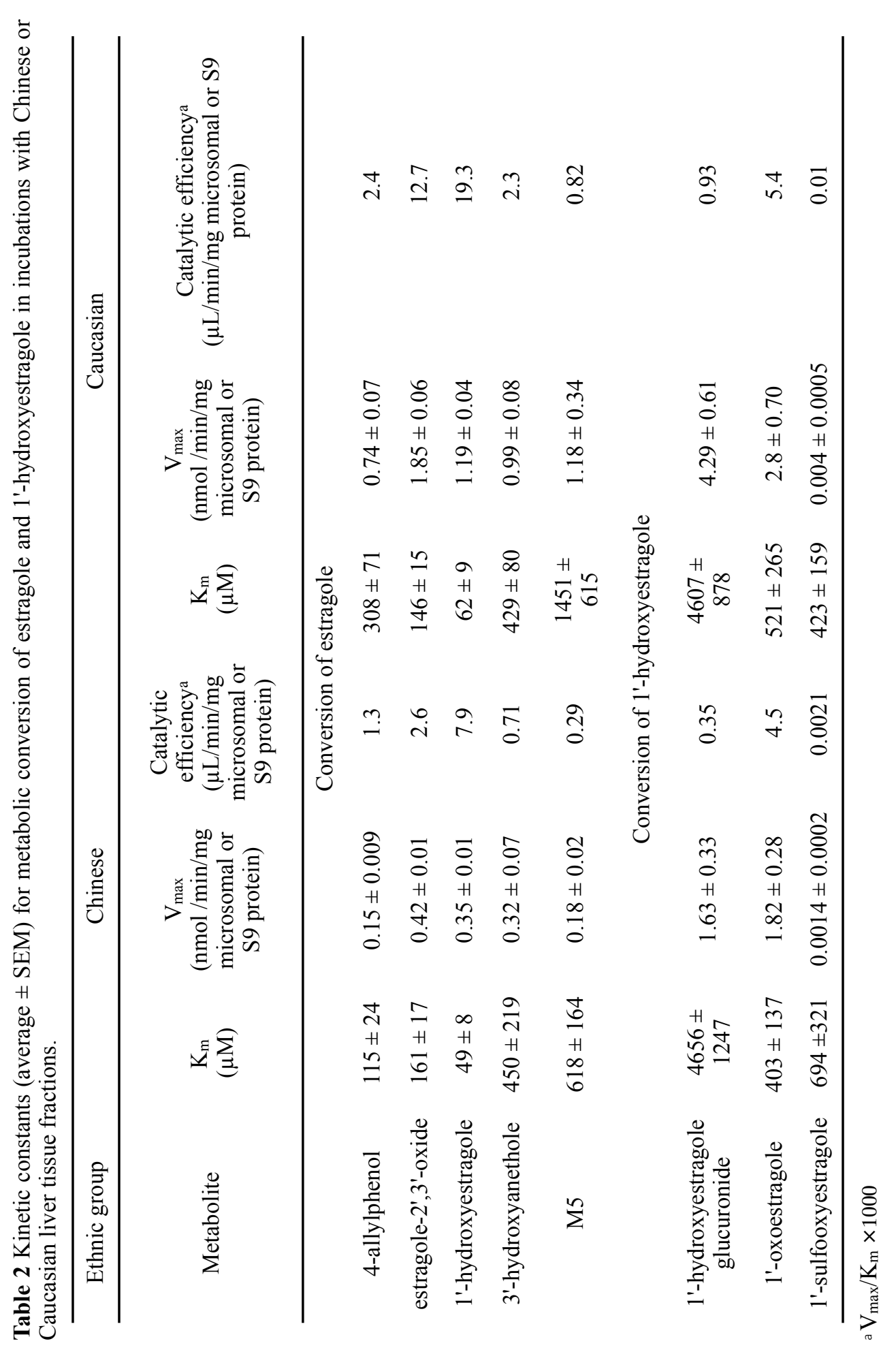




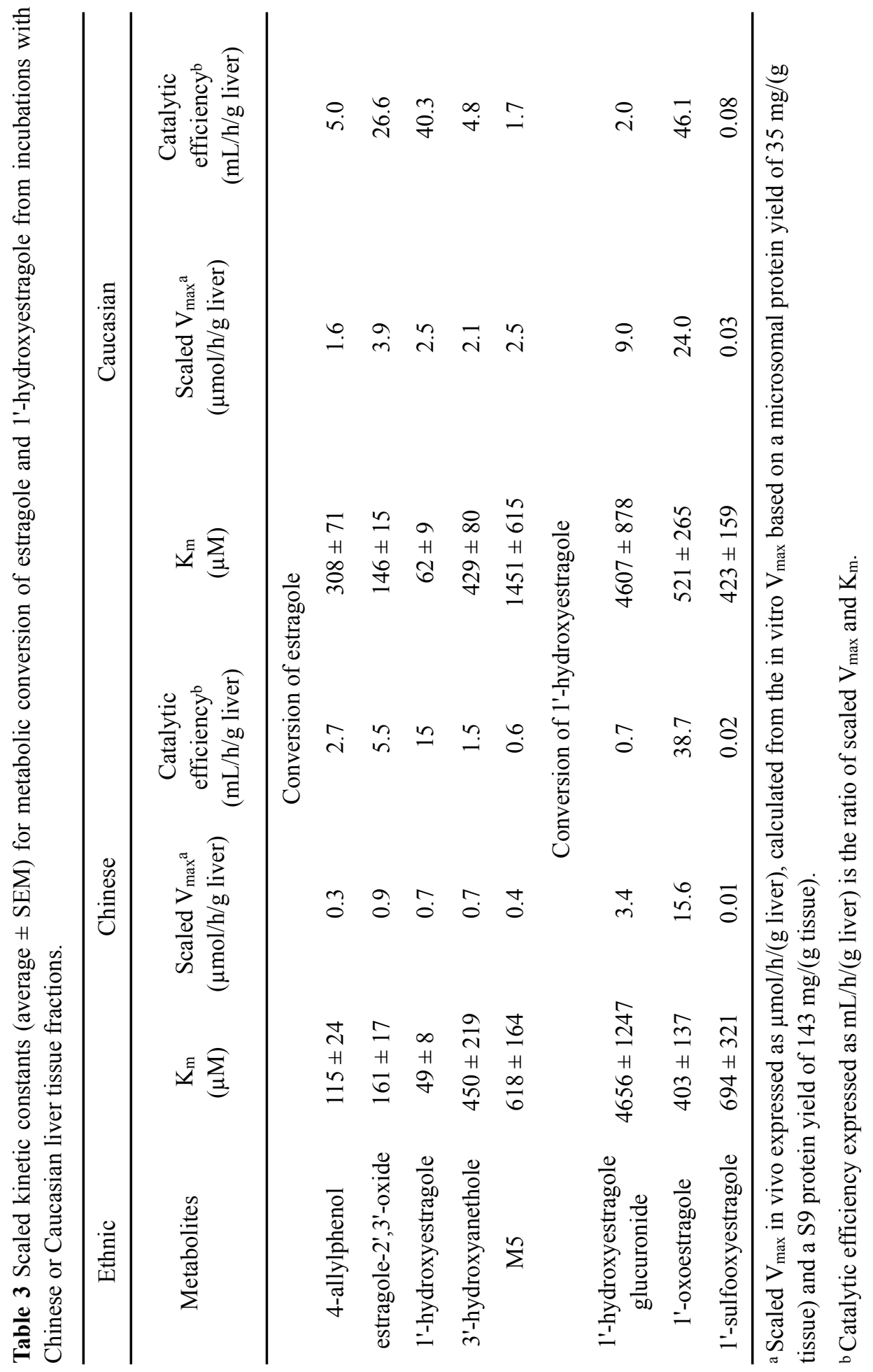




\subsection{Comparison of the PBK model based predictions for bioactivation and detoxification of estragole in Chinese and Caucasians}

Comparison of the overall consequences of the observed differences in kinetic constants for all the individual bioactivation and detoxification reactions of estragole between the two ethnic groups requires integration of all data in a PBK model. In the present study these PBK models were defined based on the PBK model for estragole in human previously developed (Punt et al. 2009), using the population specific physiological and kinetic parameters defined in the present study. The PBK model for estragole for Caucasians was evaluated previously by comparing the predicted formation of 4-allyphenol and 1'-hydroxyestragole glucuronide to the observed dose levels of these metabolites in vivo (Punt et al. 2009). The performance of the PBK model for estragole for the Chinese population could not be evaluated against Chinese in vivo data because quantitative data on the excretion of different metabolites of estragole in Chinese subjects after oral exposure to estragole are not available. Evaluation of the model could only be made by comparing the predicted inter-ethnic differences in formation of 1'-hydroxyestragole to reported ethnic differences in hepatic drug-metabolizing enzymes that mainly catalyze the formation of metabolites of estragole to 1'hydroxyestragole. CYP 1A2 and CYP 2A6 predominantly catalyze formation of 1'hydroxyestragole with CYP 2C19, CYP 2D6 and CYP 2E1 contributing to some extent at high concentrations of estragole (Jeurissen et al. 2007). The PBK model based predicted formation of 1'-hydroxyestragole at an oral dose of $0.01 \mathrm{mg} / \mathrm{kg}$ bw, a realistic dietary intake level (Smith et al. 2002), in Chinese liver, amounted to $43 \%$ of the dose, which was similar to the relative amount of 1'-hydroxyestragole predicted to be formed in the Caucasian liver, which was $47 \%$ of the dose as shown in Figure 6 . Although the catalytic efficiency in formation of 1'-hydroxyestragole in Chinese subjects is 2-3 fold lower than that of those in Caucasian incubations, the predicted overall formation of 1'-hydroxyestragole over 24 hours in both ethnic groups is similar. So although the catalytic efficiency for estragole 1'hydroxylation in the Chinese subjects is lower, when considering a time for conversion of 24 hours the overall $\%$ of the dose converted to 1'-hydroxestragole still appears to be similar. The prediction of similar overall formation of 1'-hydroxyestragole in both ethnic groups is in line with the experimental observation that peak plasma concentrations achieved at $0.5-2 \mathrm{~h}$ and apparent oral clearance values within 7 hours of administration for phenacetin, a marker for CYP1A2 activity, did also not differ between Chinese and Caucasian subjects (Bartoli et al. 1996). At a high dose level of $300 \mathrm{mg} / \mathrm{kg}$ bw/day estragole, the predicted formation of 1'hydroxyestragole in the liver of Chinese subjects was 2 -fold lower $(13 \%$ versus $25 \%$ of the dose) than the predicted formation in the liver of Caucasian subjects. This would be in line with the fact that at higher estragole concentrations CYP 2C19 might become involved in estragole 1'-hydroxylation and the fact that the frequency of poor metabolizers (PM) for CYP 2C19 in the Chinese populations was $14.6 \%$ compared to $3.3 \%$ in a Swedish population (Bertilsson et al. 1992). Altogether, the PBK models obtained were considered adequate for further predictions of inter-ethnic differences in estragole metabolism, also because of the previous evaluation of the model for human as reported in literature (Punt et al. 2009). 
a

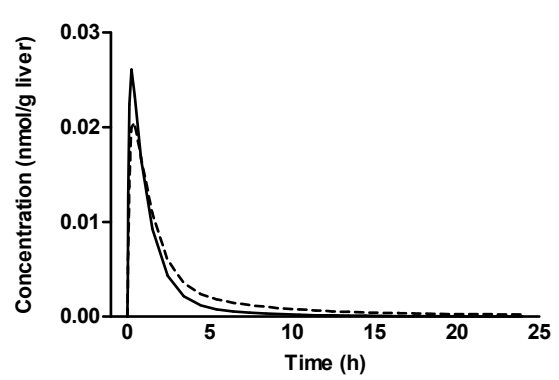

b

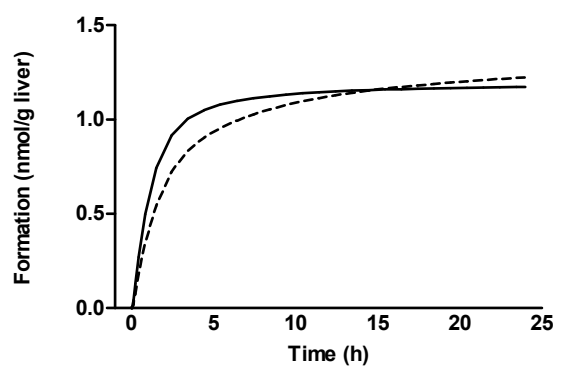

C

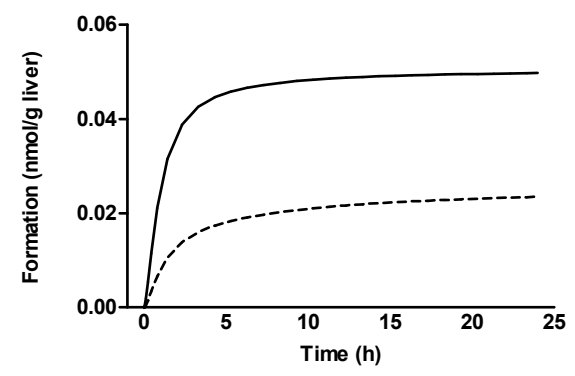

d

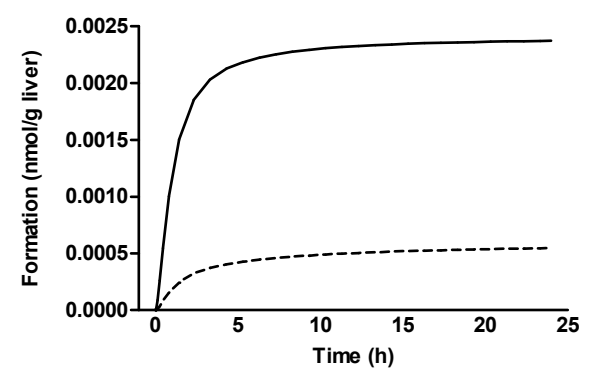

Fig. 5 PBK model based predictions for the time-dependent concentration of 1'hydroxyestragole in liver (a), and overall formation of 1'-oxoestragole (b), 1'hydroxyestragole glucuronide (c) and 1'-sulfooxyestragole (d) in Chinese (---) and Caucasian (一) liver following exposure to $0.01 \mathrm{mg} / \mathrm{kg}$ bw estragole.

Figure 5 shows the PBK model-based predictions for the time-dependent formation of 1' hydroxyestragole and 1'-hydroxyestragole metabolites in both ethnic groups at a dose level of $0.01 \mathrm{mg} / \mathrm{kg} \mathrm{bw} /$ day of estragole. Although the catalytic efficiency in formation of 1'hydroxyestragole in incubations with liver asmaples from Chinese subjects is 2-3 fold lower than that in incubations with Caucasian samples, the predicted $\mathrm{C}_{\max }$ and $24 \mathrm{~h}$ AUC of 1'hydroxyestragole in both the groups are similar. The predicted formation of the different metabolites amounted to $0.02 \mathrm{nmol} /(\mathrm{g}$ liver) for 1 '-hydroxyestragole glucuronide, $1.2 \mathrm{nmol} /(\mathrm{g}$ liver) for 1'-oxoestragole and $0.0005 \mathrm{nmol} /(\mathrm{g}$ liver) for 1'-sulfooxyestragole in Chinese subjects, corresponding to $0.8 \%, 42 \%$ and $0.02 \%$ of the dose, respectively (Figure 5 ). For the Caucasians, these values amounted to $0.05 \mathrm{nmol} /(\mathrm{g}$ liver) for 1'-hydroxyestragole glucuronide, $1.2 \mathrm{nmol} /(\mathrm{g}$ liver) for 1'-oxoestragole and $0.002 \mathrm{nmol} /(\mathrm{g}$ liver) for 1'sulfooxyestragole, corresponding to $2 \%, 45 \%$ and $0.09 \%$ of the dose, respectively (Figure $5)$. 
Figure 6 presents the PBK model based predictions for the dose-dependent formation of 1'hydroxyestragole and metabolites of 1'-hydroxyestragole in both ethnic groups. Differences on predicted formation of the various estragole metabolites in both ethnic groups can be considered following estragole exposure at $0.01,5$ and $150 \mathrm{mg} / \mathrm{kg}$ bw/day, representing respectively a realistic daily intake (Smith et al. 2002), an intake that may result from supplement use (Van Den Berg et al. 2011) and a dose level known to cause liver tumors in rodent bioassays (Drinkwater et al. 1976; Miller et al. 1983). Figure 6 shows that the ethnic differences in predicted formation of estragole metabolites in both ethnic groups at a dose of $0.01 \mathrm{mg} / \mathrm{kg} \mathrm{bw} /$ day are similar to the situation for these metabolites formed in both ethnic groups at a dose of $5 \mathrm{mg} / \mathrm{kg}$ bw/day estragole. When the dose level increases up to $150 \mathrm{mg} / \mathrm{kg}$ bw/day, the ethnic difference in predicted metabolites of estragole increases. Figure $6 \mathrm{a}$ displays the predicted formation of 1'-hydroxyestragole in both ethnic groups. At dose level of $0.01 \mathrm{mg} / \mathrm{kg} \mathrm{bw} /$ day of estragole, no ethnic difference in the formation of 1'hydroxyestragole is observed. When the dose level increases up to $150 \mathrm{mg} / \mathrm{kg} \mathrm{bw} / \mathrm{day}$, the relative formation of 1'-hydroxyestragole in both ethnic subjects decreased due to the saturation of this metabolic route. However, the formation of 1'-hydroxyestraogle predicted for Chinese liver was 1.5-fold lower than for Caucasian liver. At relatively highs concentration of estragole, CYP 2C19 might start to be involved in 1'-hydroxyestragole formation and a genetic polymorphism between Chinese and Caucasians has been reported with higher frequency of slow metabolizers in the Chinese population (Bertilsson et al. 1992) which may provide an explanation for the observed effect. Figure $6 \mathrm{~b}$ presents the predicted formation of 1'-oxoestragole in both ethnic groups. Oxidation of 1'-hydroxyestragole represents the main metabolic route for 1'-hydroxyestragole in both ethnic groups at all three dose levels of estragole. The predicted formation of 1'-oxoestragole was similar at $0.01 \mathrm{mg} / \mathrm{kg}$ bw/day of estragole, $41.2 \%$ of the dose for Chinese and $45 \%$ of the dose for Caucasians. At $5 \mathrm{mg} / \mathrm{kg}$ bw/day of estragole, $39 \%$ of dose was converted to 1'-oxoestragole in Chinese and $44 \%$ of the dose in Caucasians. When a dose of $150 \mathrm{mg} / \mathrm{kg}$ bw/day estragole was applied, only $19 \%$ of the dose was converted to 1'-oxoestragole for Chinese and $28 \%$ for Caucasians. The ethnic difference in formation of this metabolite was increased from 1 -fold at $0.01 \mathrm{mg} / \mathrm{kg}$ bw/day to 1.5 -fold at $150 \mathrm{mg} / \mathrm{kg}$ bw/day estragole. Figure 6c shows that the PBK model based predicted formation of 1'-hydroxyestragole glucuronide in the Chinese liver was 2.4fold lower than the levels predicted to be formed in Caucasians at a dose level of $0.01 \mathrm{mg} / \mathrm{kg}$ bw/day of estragole. At a dose level of $5 \mathrm{mg} / \mathrm{kg}$ bw/day of estragole, there was a 2.6-fold difference in formation of 1'-hydroxyestragole glucuronide between the ethnic subjects, and at a dose level of $150 \mathrm{mg} / \mathrm{kg}$ bw/day of estragole, the difference increased to 3.4-fold. Figure $6 \mathrm{~d}$ reveals that the PBK model based predicted formation of 1'-sulfooxestragole was 4.5 -fold lower for Chinese than Caucasian liver at $0.01 \mathrm{mg} / \mathrm{kg}$ bw/day of estragole. The ethnic difference in formation of 1'-sulfooxyestragole was 5.1 -fold at $5 \mathrm{mg} / \mathrm{kg}$ bw/day and 6.6 -fold at $150 \mathrm{mg} / \mathrm{kg}$ bw/day of estragole pointing at lower levels of bioactivation to the ultimate carcinogenic metabolite for the Chinese population. 

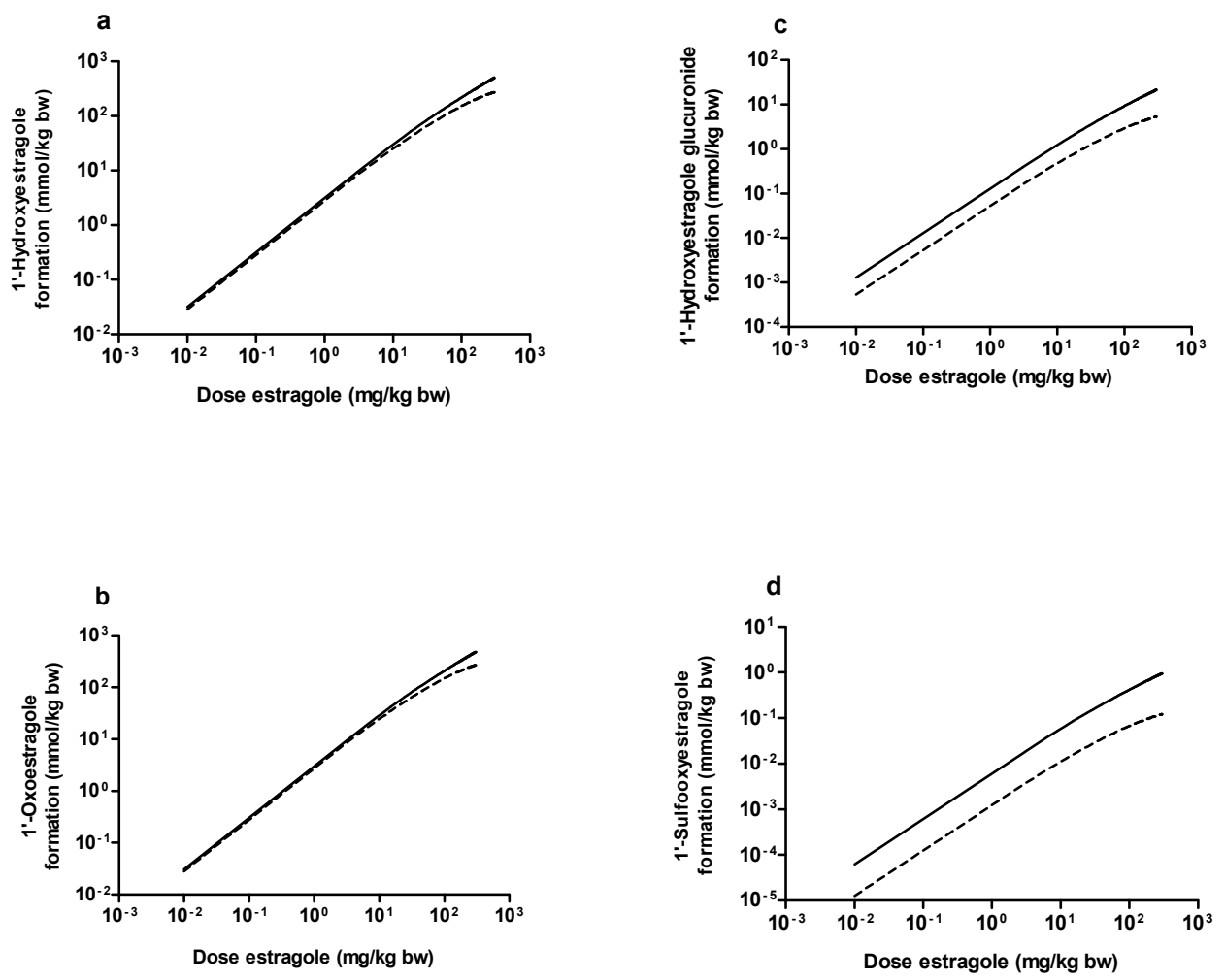

Fig. 6 PBK model based predictions for the dose-dependent formation of (a) 1'hydroxyestragole, (b) 1'-oxoestragole, (c) 1'-hydroxyestragole glucuronide and (d) 1'sulfooxyestragole in Chinese (---) and Caucasian (-) liver.

\subsection{Sensitivity analysis}

A sensitivity analysis was performed to determine model parameters that are able to influence the formation of 1'-hydroxyestragole and 1'-sulfooxyestragole in both ethnic groups to the largest extent. Sensitivity coefficients were calculated for all model parameters at a dose of $0.01,5$ and $150 \mathrm{mg} / \mathrm{kg}$ bw/day estragle, but only the parameters that appeared to have a sensitivity coefficient higher than $|0.1|$ are presented in Figure S2 in supplementary materials 5 .

In Chinese and Caucasian liver at $0.01 \mathrm{mg} / \mathrm{kg}$ bw of estragole (Figure S2a), formation of 1'hydroxyestragole is predominantly influenced by the kinetic constant for 1'-hydroxylation, and to a minor extent by the kinetic constants for formation of estragole-2',3'-oxide. Focusing on inter-ethnic differences it can be concluded that parameters including liver volume, blood flow to fat and microsome protein yield of liver have higher effect on the formation of 1'hydroxyestragole in Chinese liver compared to Caucasian liver. Formation of 1'sulfooxyestragole mainly depends on the kinetic constants for its formation from 1'hydroxyestragole, the formation of 1'-hydroxyestragole itself and the kinetic constants for 
formation of 1'-oxoestragole, which is an important competing metabolic pathway to sulfation in both ethnic groups. At $5 \mathrm{mg} / \mathrm{kg}$ bw/day of estragole (Figure S2b), the results of the sensitivity analysis of both ethnic groups are similar to the situation at a dose of $0.01 \mathrm{mg} / \mathrm{kg}$ bw/day. When the dose increases to $150 \mathrm{mg} / \mathrm{kg}$ bw/day (Figure S2c), besides the influential parameters at a dose of $0.01 \mathrm{mg} / \mathrm{kg} \mathrm{bw} /$ day and $5 \mathrm{mg} / \mathrm{kg}$ bw/day of estragole, the parameters related to fat tissue like volume of fats, fat/blood partition coefficient, also have influence on the formation of 1'-hydroxyestragole and 1'-sulfooxyestragole in Chinese liver. For the Caucasian group, the results of the sensitivity analysis at $150 \mathrm{mg} / \mathrm{kg}$ bw/day are similar to those at 0.01 or $5 \mathrm{mg} / \mathrm{kg}$ bw/day of estragole.

\section{Discussion}

In the present study, bioactivation and detoxification of estragole in Chinese and Caucasian populations upon oral exposure to estragole were examined using PBK modeling. The structure of the PBK models was based on the model developed and validated previously for estragole in Causcasians (Punt et al. 2009). The parameter values for the Chinese PBK model were derived from literature or from incubation experiments using human liver fractions. To guarantee adequate experimental comparison the parameters for hepatic metabolism in the Caucasian model were redefined in the present study as well. The kinetic constants of estragole metabolism obtained from incubation with Caucasian liver fractions and PBK model predictions of estragole in Caucasians in this study were similar to results reported in the study by Punt et al. (2009).

Since the actual kinetic data on plasma or urinary estragole metabolite levels were not available for the Chinese population, the performance of the model was evaluated by comparing the predicted inter-ethnic differences in formation of 1'-hydroxyestragole to reported ethnic differences in hepatic drug-metabolizing enzymes CYP 1A2 that mainly catalyze the formation of metabolites of estragole to 1'-hydroxyestragole. Furthermore, the PBK model for the Chinese population was based on the model for estragole defined and validated previously (Punt et al. 2009). Also the quality of the Chinese tissue samples used to determine the various kinetic parameters appeared to be in line with what has been reported before (Yang et al. 2012; Wang et al. 2006). Considering these facts, it can be concluded that the developed PBK model for the Chinese population are expected to adequately describe the in vivo levels of metabolites upon oral exposure.

The ethnic difference in bioactivation of estragole was evaluated by predicting the formation of 1'-sulfooxyestragole at dose level of 0.01 and $5 \mathrm{mg} / \mathrm{kg}$ bw/day of estragole. The PBK models predicted that following an exposure of $0.01 \mathrm{mg} / \mathrm{kg} \mathrm{bw} /$ day estragole, only $0.02 \%$ of the estragole dose was predicted to be converted to 1'-sulfooxyestragole in the Chinese population, whereas $0.09 \%$ of the dose was converted to 1 '-sulfooxyestragole in Caucasians, which was 4.5 -fold higher. At $5 \mathrm{mg} / \mathrm{kg}$ bw/day of estragole, a worst case scenario intake for people using plant food supplements containing estragole (Van Den Berg et al. 2011), the difference in the predicted formation of 1'-sulfooxyestragole between the two ethnic groups was increased to 5-fold. This 4 to 5 -fold difference in the level of bioactivation of estragole between the two ethnic groups mainly originates from the difference in SULT mediated 
conversion of 1'-hydroxyestragole. The human SULTs involved in this sulfation reaction have not been identified to date, but sulfation of the 1'-hydroxy metabolite of the related alkenylbenzene methyleugenol in the liver has been shown to be catalyzed mainly by SULT 1A1 and to a small extent by SULT 1E1 (Herrmann et al. 2012). It has also been shown that exon 7 of the SULT1A1 gene may contain a G to A transition at codon 213 (rs9282861), which induces an Arg to His amino acid substitution (Raftogianis et al. 1997). This SULT 1A1 Arg213His (rs9282861) polymorphism is reported to be associated with increased risk of various cancer types (Xiao et al. 2014). In the ethnic subgroup analysis of Xiao et al. (2014), they found that the genotype distributions of the SNP site are different for different ethnic groups. Occurrence of the His allele in Asians (9.58 \%) was reported to be significantly lower than in Caucasians $(35.2 \%)$. This difference of the His allele frequency could contribute to differential drug responses between these two ethnic groups. It is tempting to speculate that the SNP difference in SULT 1A1 between the ethnic groups may explain the differences in formation of 1'-sulfooxyestragole as observed in the present study, although a definite conclusion should await measurement of the respective SULT reaction using samples from individuals from both genotypes.

It is of interest to consider the possible consequences of the ethnic variation in bioactivation of estragole for the related health risks. Among the different available qualitative and quantitative approaches for making such a risk assessment for a genotoxic and carcinogenic compounds, the use of the Margin of exposure (MOE) approach was advised by EFSA (EFSA 2005). The MOE is a dimensionless value that is the ratio of a reference point derived from experimental data on tumor incidence and the estimated daily intake of human. Generally, the BMDL $_{10}$ which is the lower confidence bound of the Benchmark Dose that gives $10 \%$ extra cancer incidence above background levels can be used as the point of departure (Barlow et al. 2006). Using an estimated daily intake of $0.01 \mathrm{mg} / \mathrm{kg}$ bw/day and the BMDL 10 of 3.3-6.5 mg/ $\mathrm{kg}$ bw/day for estragole (Van Den Berg et al. 2011), the MOE value would amount to 330 up to 650 , which is lower than 10000 , suggesting a priority for risk management. In Europe since 2008 the use of estragole as pure flavoring substance in foodstuffs has been prohibited, and for a few food categories maximum levels of naturally occurring estragole in foodstuffs have been defined (EC 2008). When estimated daily intakes would amount to $5 \mathrm{mg} / \mathrm{kg} / \mathrm{day}$ for example as a result of daily use of estragole containing plant food supplements (Van Den Berg et al. 2011), then the MOE values would amount to 0.66 up to 1.3 , indicating that the daily intake of estragole by using plant food supplements are within the range of the dose levels causing tumors in the experimental animals. Considering the consequences of 5-fold reduction in bioactivation of estragole at similar dose levels in the Chinese as compared to the Caucasian population, as predicted by the results of the present study, one could argue that, assuming a linear relationship between the formation of the ultimate carcinogenic metabolite and the hepatoma incidence, intake levels in the Chinese population could be 5-fold higher before reaching the same risk as expected for the Caucasian population. This is confirmed by the PBK model predictions that reveal that the amount of 1'-sulfooxyestragole formed within 24 hours upon an intake of respectively 0.01 or $5 \mathrm{mg} / \mathrm{kg}$ bw for a Caucasian would be reached upon an estragole intake of respectively 0.05 or $25 \mathrm{mg} / \mathrm{kg}$ bw for a Chinese individual. 
Converting this 5-fold difference in estragole bioactivation to the MOE values presented above would still result in MOE values below 10000 pointing at a priority for risk management also for the Chinese population. According to the national food safety standard for uses of food additives in China, estragole is allowed to be used in foods as synthetic flavoring substance and fennel oil is permitted to be used in foods as natural flavoring substance (NHFPC 2014a). The maximum levels of estragole present in foods are not wellregulated in China and thus may lead to a potential risk for the Chinese population when consuming estragole-containing food products.

It is important to note that the PBK model used in the present study was developed to predict the formation of the ultimate carcinogen metabolite, 1'-sulfooxyestragole. Further metabolic reactions of 1'-sulfooxyestragole such as conjugation with GSH, RNA, DNA, and other cellular macromolecules, were not included in the PBK model. Possible ethnic differences in the capacity of DNA repair and reversibility of DNA-adduct formation may also be present. For example, genetic polymorphisms in DNA repair genes ERCC1 and ERCC2/XPD, which are involved in the nucleotide excision repair pathway, have been identified (Yin et al. 2005). Previous studies reported that the A-allele frequency of ERCC1 G19007A in the northeastern Chinese population was much lower compared with Caucasian, indicating another genetic contribution to differences in sensitivity to genotoxic carcinogens between ethnic groups (Yin et al. 2005). The current PBK model could be extended to a PBD model by including DNA adduct formation in hepatocytes from Chinese donors, which allows taking into account possible ethnic difference in DNA repair.

In summary, the present study elucidates a possible approach to determine the inter-ethnic human difference in bioactivation and detoxification of compounds of concern taking estragole as the model compound. The outcomes obtained clearly illustrate that PBK modelling is essential to integrate information on ethnic differences in the various metabolic conversions to enable their combined consequence for the bioactivation to the ultimate carcinogenic metabolite. The 4.5 -fold difference in formation of the ultimate carcinogenic metabolite of estragole accompanied by similar rates of detoxification may indicate a lower risk of estragole for the Chinese population at similar levels of exposure. The study provides a proof-of-principle for how PBK modelling can identify ethnic sensitivity and provide a more refined risk assessment for a specific ethnic group for a compound of concern.

\section{Note}

The authors declare that they have no conflict of interest

\section{Acknowledgements}

This work was funded by a grant from the China Scholarship Council (No. 201507720019 to NING JIA) 


\section{References}

Agharahimi MR, LeBel NA (1995) Synthesis of (-)-Monoterpene-magnolol and Magnolol. J Org Chem 60:1856-1863

Al-Malahmeh AJ, Al-Ajlouni A, Wesseling S, Soffers AEMF, Al-Subeihi A, Kiwamoto R, Vervoort J, Rietjens IMCM (2016) Physiologically based kinetic modeling of the bioactivation of myristicin. Arch Toxicol 91:713-734

Al-Subeihi AA, Spenkelink B, Punt A, Boersma MG, van Bladeren PJ, Rietjens IMCM (2012) Physiologically based kinetic modeling of bioactivation and detoxification of the alkenylbenzene methyleugenol in human as compared with rat. Toxicol Appl Pharmacol 260:271-284

Al-Subeihi AA, Spenkelink B, Rachmawati N, Boersma MG, Punt A, Vervoort J, van Bladeren PJ, Rietjens IMCM (2011) Physiologically based biokinetic model of bioactivation and detoxification of the alkenylbenzene methyleugenol in rat. Toxicol in Vitro 25:267-85

Alajlouni AM, Al-Malahmeh AJ, Kiwamoto R, Wesseling S, Soffers AE, Al-Subeihi AA, Vervoort J, Rietjens IMCM (2016) Mode of action based risk assessment of the botanical food-borne alkenylbenzene apiol from parsley using physiologically based kinetic (PBK) modelling and read-across from safrole. Food Chem Toxicol 89:138-150

Anthony A, Caldwell J, Hutt AJ, Smith RL (1987) Metabolism of estragole in rat and mouse and influence of dose size on excretion of the proximate carcinogen 1'-hydroxyestragole. Food Chem Toxicol 25:799-806

Barlow S, Renwick A, Kleiner J, Bridges J, Busk L, Dybing E, Edler L, Eisenbrand G, Fink-Gremmels J, Knaap A (2006) Risk assessment of substances that are both genotoxic and carcinogenic: Report of an International Conference organized by EFSA and WHO with support of ILSI Europe. Food Chem Toxicol 44:1636-1650

Barter ZE, Tucker GT, Rowland-Yeo K (2013) Differences in cytochrome p450-mediated pharmacokinetics between chinese and caucasian populations predicted by mechanistic physiologically based pharmacokinetic modelling. Clin Pharmacokinet 52:1085-1100

Bartoli A, Xiaodong S, Gatti G, Cipolla G, Marchiselli R, Perucca E (1996) The influence of ethnic factors and gender on CYP1A2-mediated drug disposition: A comparative study in Caucasian and Chinese subjects using phenacetin as a marker substrate. Ther Drug Monit 18:586-591

Bertilsson L, Lou YQ, Du YL, Liu Y, Kuang TY, Liao XM, Wang KY, Reviriego J, Iselius L, Sjoqvist F (1992) Pronounced Differences between Native Chinese and Swedish Populations in the Polymorphic Hydroxylations of Debrisoquin and S-Mephenytoin. Clin Pharmacol Ther 51:388-397

Brown RP, Delp MD, Lindstedt SL, Rhomberg LR, Beliles RP (1997) Physiological parameter values for physiologically based pharmacokinetic models. Toxicol Ind Health 13:407-484

DeJongh J, Verhaar HJ, Hermens JL (1997) A quantitative property-property relationship (QPPR) approach to estimate in vitro tissue-blood partition coefficients of organic chemicals in rats and humans. Arch Toxicol 72:17-25 
Drinkwater NR, Miller EC, Miller JA, Pitot HC (1976) Hepatocarcinogenicity of estragole (1-allyl-4methoxybenzene) and 1'-hydroxyestragole in the mouse and mutagenicity of 1'-acetoxyestragole in bacteria. J Natl Cancer I 57:1323-1331

European Commission (EC) (2008) No 1334/2008 of the European Parliament and of the Council of 16 December 2008 on flavourings and certain food ingredients with flavouring properties for use in and on foods and amending Council Regulation (EEC) No 1601/91 Regulations (EC) No 2232/96 and (EC) No 110/2008 and Directive 2000/13/EC. Official Journal of the European Communities 354:34-50

European Food Safety Authority (EFSA) (2005) Opinion of the Scientific Committee on a request from EFSA related to A Harmonised Approach for Risk Assessment of Substances Which are both Genotoxic and Carcinogenic. EFSA J 3:282

Evans MV, Andersen ME (2000) Sensitivity analysis of a physiological model for 2,3,7,8tetrachlorodibenzo-p-dioxin (TCDD): assessing the impact of specific model parameters on sequestration in liver and fat in the rat. Toxicol Sci 54:71-80

Fisher MB, Campanale K, Ackermann BL, VandenBranden M, Wrighton SA (2000) In vitro glucuronidation using human liver microsomes and the pore-forming peptide alamethicin. Drug Metab Dispos 28:560-566

Guenthner TM, Luo G (2001) Investigation of the role of the 2', 3'-epoxidation pathway in the bioactivation and genotoxicity of dietary allylbenzene analogs. Toxicology 160:47-58

Herrmann K, Engst W, Appel KE, Monien BH, Glatt H (2012) Identification of human and murine sulfotransferases able to activate hydroxylated metabolites of methyleugenol to mutagens in Salmonella typhimurium and detection of associated DNA adducts using UPLC-MS/MS methods. Mutagenesis $27: 453-462$

Iyer LV, Ho MN, Shinn WM, Bradford WW, Tanga MJ, Nath SS, Green CE (2003) Glucuronidation of 1'-hydroxyestragole (1'-HE) by human UDP-glucuronosyltransferases UGT2B7 and UGT1A9. Toxicol Sci 73:36-43

Jeurissen SM, Punt A, Boersma MG, Bogaards JJ, Fiamegos YC, Schilter B, van Bladeren PJ, Cnubben NH, Rietjens IMCM (2007) Human cytochrome P450 enzyme specificity for the bioactivation of estragole and related alkenylbenzenes. Chem Res Toxicol 20:798-806

Lin JH, Wong BK (2002) Complexities of glucuronidation affecting in vitro-in vivo extrapolation. Curr Drug Metab 3:623-646

Luo G, Guenthner TM (1996) Covalent binding to DNA in vitro of 2', 3'-oxides derived from allylbenzene analogs. Drug Metab Dispos 24:1020-1027

Luo G, Qato MK, Guenthner TM (1992) Hydrolysis of the 2', 3'-allylic epoxides of allylbenzene, estragole, eugenol, and safrole by both microsomal and cytosolic epoxide hydrolases. Drug Metab Dispos 20:440-445

Malinowski HJ, Westelinck A, Sato J, Ong T (2008) Same drug, different dosing: differences in dosing for drugs approved in the United States, Europe, and Japan. J Clin Pharmacol 48:900-908 
Martati E, Boersma M, Spenkelink A, Khadka DB, van Bladeren P, Rietjens IMCM, Punt A (2012) Physiologically based biokinetic (PBBK) modeling of safrole bioactivation and detoxification in humans as compared with rats. Toxicol Sci 128:301-306

Martati E, Boersma MG, Spenkelink A, Khadka DB, Punt A, Vervoort J, van Bladeren PJ, Rietjens IMCM (2011) Physiologically based biokinetic (PBBK) model for safrole bioactivation and detoxification in rats. Chem Res Toxicol 24:818-834

Miller EC, Swanson AB, Phillips DH, Fletcher L, Liem A, Miller JA (1983) Structure-activity studies of the carcinogenicities in the mouse and rat of some naturally occurring and synthetic alkenylbenzene derivatives related to safrole and estragole. Cancer Res 43:1124-1134

National Health and Family Planning Commission (NHFPC) (2007) Reference individuals for use in radiation protection-Part2: Masses of main organs and tissues. vol GBZ/T 200.2. National Health and Family Planning Commission of the People's Republic of China (in Chinese)

National Health and Family Planning Commission (NHFPC) (2014a) National Food Safety Standard for Uses of Food Additives. vol GB2760 - 2014. National Health and Family Planning Commission of the People's Republic of China (in Chinese)

National Health and Family Planning Commission (NHFPC) (2014b) Reference individiuals for use in radiation protection-Part3: main physiological parameters. vol GBZ/T 200.3. National Health and Family Planning Commission of the People's Republic of China (in Chinese)

Punt A, Delatour T, Scholz G, Schilter B, van Bladeren PJ, Rietjens IMCM (2007) Tandem Mass Spectrometry Analysis of N 2-(trans-Isoestragol-3'-yl)-2'-deoxyguanosine as a Strategy to Study Species Differences in Sulfotransferase Conversion of the Proximate Carcinogen 1'-Hydroxyestragole. Chem Res Toxicol 20:991-998

Punt A, Freidig AP, Delatour T, Scholz G, Boersma MG, Schilter B, van Bladeren PJ, Rietjens IMCM (2008) A physiologically based biokinetic (PBBK) model for estragole bioactivation and detoxification in rat. Toxicol Appl Pharmacol 231:248-259

Punt A, Paini A, Boersma MG, Freidig AP, Delatour T, Scholz G, Schilter B, van Bladeren PJ, Rietjens IMCM (2009) Use of Physiologically Based Biokinetic (PBBK) Modeling to Study Estragole Bioactivation and Detoxification in Humans as Compared with Male Rats. Toxicol Sci 110:255-269

Punt A, Paini A, Spenkelink A, Scholz G, Schilter B, van Bladeren P, Rietjens IMCM (2016) Evaluation of interindividual human variation in bioactivation and DNA adduct formation of estragole in liver predicted by physiologically based kinetic/dynamic (PBK/D) and Monte Carlo modeling. Chem Res Toxicol 29:659-668

Raftogianis RB, Wood TC, Otterness DM, Van Loon JA, Weinshilboum RM (1997) Phenol sulfotransferase pharmacogenetics in humans: association of common SULT1A1 alleles with TS PST phenotype. Biochem Biophys Res Commun 239:298-304

Smith R, Adams T, Doull J, Feron V, Goodman J, Marnett L, Portoghese P, Waddell W, Wagner B, Rogers A (2002) Safety assessment of allylalkoxybenzene derivatives used as flavouring substancesmethyleugenol and estragole. Food Chem Toxicol 40:851-870 
Van den Berg SJ, Punt A, Soffers AE, Vervoort J, Ngeleja S, Spenkelink B, Rietjens IMCM (2012) Physiologically based kinetic models for the alkenylbenzene elemicin in rat and human and possible implications for risk assessment. Chem Res Toxicol 25:2352-2367

Van Den Berg SJ, Restani P, Boersma MG, Delmulle L, Rietjens IMCM (2011) Levels of genotoxic and carcinogenic compounds in plant food supplements and associated risk assessment. FNS 2:989

Wang Q, Ye C, Jia R, Owen AJ, Hidalgo IJ, Li J (2006) Inter-species comparison of 7-hydroxycoumarin glucuronidation and sulfation in liver S9 fractions. In Vitro Cell Dev Biol Anim 42:8-12

Xiao J, Zheng Y, Zhou Y, Zhang P, Wang J, Shen F, Fan L, Kolluri VK, Wang W, Yan X, Wang M (2014) Sulfotransferase SULT1A1 Arg213His polymorphism with cancer risk: a meta-analysis of 53 case-control studies. PloS one 9:e106774

Yang J, He MM, Niu W, Wrighton SA, Li L, Liu Y, Li C (2012) Metabolic capabilities of cytochrome $\mathrm{P} 450$ enzymes in Chinese liver microsomes compared with those in Caucasian liver microsomes. Brit $\mathrm{J}$ Clin Pharmaco 73:268-284

Yin J, Li J, Vogel U, Wang H (2005) Polymorphisms of DNA repair genes: ERCC1 G19007A and ERCC2/XPD C22541A in a northeastern Chinese population. Biochem Genet 43:543-548

\section{Supplementary materials}

For convenience, supplementary materials $4-6$ can be found in http://edepot.wur.nl/496947

\section{Supplementart materials 1}

\section{Material and methods}

\section{Assessment of metabolic capabilities of CYP and SULT enzymes in Chinese and Caucasian liver samples}

\section{In vitro incubations}

The quality of the Chinese and Caucasian liver microsomes was checked by measuring the activity of CYP 1A2 and CYP 2A6 by the method of Yang et al. (2012). CYP 1A2 and CYP 2A6 are predominant enzymes for formation of 1'-hydroxyestragole (Jeurissen et al. 2007). The activity of CYP 1A2 was measured with the substrate phenacetin, and the activity of CYP 2A6 was checked using the substrate coumarin. Briefly, the incubations were performed in triplicate containing $0.1 \mathrm{mg} / \mathrm{ml}$ microsomal protein in $0.1 \mathrm{M}$ potassium phosphate $\mathrm{pH} 7.4$ (for CYP 1A2) / 0.1 M Tris buffer pH 7.4 (for CYP 2A6) with (final concentrations) 10 to 2000 $\mu \mathrm{M}$ phenacetin or 1.6 to $50 \mu \mathrm{M}$ coumarin added from 100 times concentrated stock solutions in DMSO in the presence of an NADPH-generating system consisting of (final concentrations) $3.3 \mathrm{mM}$ of glucose-6-phosphate, $0.5 \mathrm{U} / \mathrm{ml}$ glucose-6-phosphate dehydrogenase and $1.3 \mathrm{mM} \mathrm{NADP}{ }^{+}$. Before adding the NADPH-generating system, the mixture was incubated for $3 \mathrm{~min}$. The optimal incubation time was $20 \mathrm{~min}$ for phenacetin $O$ deethylation and coumarin 7-hydroxylation (Yang et al. 2012). The reactions were terminated by adding $100 \mu \mathrm{l}$ of ice-cold methanol. The samples were centrifuged for $5 \mathrm{~min}$ at $16000 \mathrm{~g}$ to precipitate microsomal proteins and analyzed by UPLC. 
The quality of Chinese and Caucasian liver S9 samples was checked by measuring the SULT activity based on the method provided by Wang et al. (2006). Incubations with Chinese liver S9 were performed in $100 \mu \mathrm{l}$ incubation mixture containing (final concentrations) $1 \mathrm{mM}$ PAPS, $0.4 \mathrm{mg} / \mathrm{ml} \mathrm{S} 9$ and the substrate 7-hydroxycoumarin at concentrations ranging from 1.5 to $25 \mu \mathrm{M}$ in $0.1 \mathrm{M}$ Tris buffer ( $\mathrm{pH} 7.4)$. Incubations were carried out for $10 \mathrm{~min}$ and the reactions were terminated by adding $25 \mu \mathrm{l}$ ice-cold acetonitrile. The samples were centrifuged for $5 \mathrm{~min}$ at $16000 \mathrm{~g}$ to precipitate proteins and were analyzed by UPLC.

\section{UPLC analysis of conversion of phenacetin, coumarin and 7-hydroxycoumarin in incubations with Chinese and Caucasian liver samples}

Before UPLC analysis, all samples were centrifuged for $5 \mathrm{~min}$ at $16000 \mathrm{~g}$ to precipitate microsomal or cytosolic proteins. Supernatant of each sample was analyzed on UPLC using a BEH C18 $(1.7 \mu \mathrm{m} 2.1 \times 50 \mathrm{~mm})$ column with a guard column and a diode array detector (Acquity, Waters). The flow rate was $0.6 \mathrm{ml} / \mathrm{min}$ and the mobile phase was made with ultrapure water with $0.1 \%(\mathrm{v} / \mathrm{v})$ TFA and acetonitrile. Identification of metabolites of phenacetin, coumarin and 7-hydroxycoumarin was achieved by comparison of the UV spectra and retention times of formed metabolites to those of commercially available reference compounds. Quantification of metabolites was done by comparing the peak areas of formed metabolites to the calibration curve of the corresponding standard compounds. For the analysis of acetaminophen formed by O-deethylation of phenacetin to check the activity of CYP 1A2, a gradient was applied from 5 to $30 \%$ acetonitrile in ultrapure water in 5 min, after which the percentage of acetonitrile was increased to $70 \%$ in $1 \mathrm{~min}$ and kept at this level for 1 min longer. Under these conditions, acetaminophen had a retention time of $0.7 \mathrm{~min}$. For detection of 7-hydroxycoumarin by hydroxylation of coumarin to check the activity of CYP 2A6, the gradient started with $20 \%$ acetonitrile in ultrapure water for $0.3 \mathrm{~min}$, followed by a linear gradient from 20 to $30 \%$ during $0.7 \mathrm{~min}$, and a subsequent increase to $80 \%$ acetonitrile in $1.5 \mathrm{~min}$. This percentage was kept for $0.5 \mathrm{~min}$, after which the column was set back to the starting conditions. Under these conditions 7-hydroxycoumarin eluted at 0.6 min. For measuring the sulfate metabolite of 7-hydroxycoumarin to check SULT activity in Chinese and Caucasian liver S9, the gradient was started from 20 to $30 \%$ acetonitrile in 4.5 min, after which the percentage of acetonitrile was increased to $100 \%$ in $0.3 \mathrm{~min}$ and kept at $100 \%$ for another $0.2 \mathrm{~min}$. Under these conditions, 7-hydroxycoumarin sulfate had a retention time of $1 \mathrm{~min}$. 


\section{Supplementary materials 2}

\subsection{Material and methods}

Table S1 tracks the detailed methods for in vitro incubations with estragole and 1'hydroxyestragole and for UPLC analysis of metabolite formation for obtaining the kinetic parameters.

Table S1 The kinetic parameters of metabolites of estragole and 1'-hydroxyestragole were derived from corresponding methods of in vitro incubations and UPLC analysis.

\begin{tabular}{|c|c|c|c|}
\hline Metabolites & Kinetic parameters & $\begin{array}{c}\text { In vitro } \\
\text { incubations }\end{array}$ & UPLC analysis \\
\hline 4-allylphenol & $\mathrm{V}_{\max }, \mathrm{L}_{-} \mathrm{AP}, \mathrm{K}_{\max }, \mathrm{L}_{-} \mathrm{AP}$ & \multirow{5}{*}{$\begin{array}{l}\text { Microsomal } \\
\text { metabolism of } \\
\text { estragole }\end{array}$} & \multirow{5}{*}{$\begin{array}{l}\text { UPLC analysis of } \\
\text { estragole metabolites }\end{array}$} \\
\hline 1'-hydroxyestragole & $\mathrm{V}_{\max }, \mathrm{L}_{-} \mathrm{HE}, \mathrm{K}_{\max }, \mathrm{L}_{-} \mathrm{HE}$ & & \\
\hline estragole-2',3'-oxide & $\mathrm{V}_{\max }, \mathrm{L}_{-} \mathrm{EE}, \mathrm{K}_{\max }, \mathrm{L}_{-} \mathrm{EE}$ & & \\
\hline 3'-hydroxyanethole & $\mathrm{V}_{\max }, \mathrm{L}_{-} \mathrm{HA}, \mathrm{K}_{\max }, \mathrm{L}_{-} \mathrm{HA}$ & & \\
\hline M5 & $\mathrm{V}_{\max }, \mathrm{L}_{-} \mathrm{M} 5, \mathrm{~K}_{\max }, \mathrm{L} \_\mathrm{M} 5$ & & \\
\hline 1'-hydroxyestragole & $\mathrm{V}_{\max }, \mathrm{L}_{-} \mathrm{HEG}, \mathrm{K}_{\max }$, & $\begin{array}{c}\text { Glucuronidation } \\
\text { of 1'- }\end{array}$ & $\begin{array}{c}\text { UPLC analysis of 1'- } \\
\text { hydroxyestragole }\end{array}$ \\
\hline glucuronide & L_HEG & hydroxyestragole & metabolites \\
\hline 1'-oxoestragole & $\mathrm{V}_{\max }, \mathrm{L}_{-} \mathrm{OE}, \mathrm{K}_{\max }, \mathrm{L}_{-} \mathrm{OE}$ & $\begin{array}{l}\text { Oxidation of 1'- } \\
\text { hydroxyestragole }\end{array}$ & $\begin{array}{l}\text { UPLC analysis of 1'- } \\
\text { hydroxyestragole } \\
\text { metabolites }\end{array}$ \\
\hline 1'-sulfooxyestragole & $\begin{array}{c}\mathrm{V}_{\max }, \mathrm{L}_{-} \mathrm{HES}, \mathrm{K}_{\max }, \\
\text { L_HES }_{-}\end{array}$ & $\begin{array}{l}\text { Sulfation of 1'- } \\
\text { hydroxyestragole }\end{array}$ & $\begin{array}{c}\text { UPLC analysis of 1'- } \\
\text { hydroxyestragole } \\
\text { metabolites }\end{array}$ \\
\hline
\end{tabular}

\subsection{In vitro incubations}

\section{Microsomal metabolism of estragole}

The kinetic constants for the microsomal conversion of estragole were determined as previously described by Punt et al. (2009). Briefly, mixed gender Chinese or Caucasian liver microsomes were incubated with estragole in the presence of NADPH. The incubation mixtures contained (final concentrations) $3 \mathrm{mM} \mathrm{NADPH}$ and $1 \mathrm{mg} / \mathrm{ml}$ microsomal protein in $0.2 \mathrm{M}$ Tris- $\mathrm{HCl}\left(\mathrm{pH}\right.$ 7.4). After $1 \mathrm{~min}$ pre-incubation at $37^{\circ} \mathrm{C}$, the reaction was started by addition of the substrate estragole from 100 times concentrated stock solutions in DMSO. Incubations were performed for $10 \mathrm{~min}$ at substrate concentrations ranging from 25 to 1000 
$\mu \mathrm{M}$, after which the reaction was terminated by adding $25 \mu \mathrm{l}$ ice-cold acetonitrile. Under these conditions, the formation of the different estragole metabolites was linear with time and microsomal protein concentration (Punt et al. 2009). Blank incubations were performed in the absence of the cofactor NADPH. All incubations were performed in triplicate.

\section{Glucuronidation of 1'-hydroxyestragole}

Pooled mixed gender Chinese or Caucasian liver microsomes were incubated with 1'hydroxyestragole in the presence of UDPGA. As previously described by Punt et al. (2009), the incubation mixtures contained (final concentrations) $10 \mathrm{mM} \mathrm{UDPGA}$, and $1 \mathrm{mg} / \mathrm{ml}$ microsomal protein in $0.2 \mathrm{M}$ Tris- $\mathrm{HCl}(\mathrm{pH} 7.4)$ with $10 \mathrm{mM} \mathrm{MgCl}$. To overcome enzyme latency and obtain maximal glucuronidation activity (Fisher et al. 2000; Lin and Wong 2002), the mixtures were pre-treated on ice for $15 \mathrm{~min}$ with $0.025 \mathrm{mg} / \mathrm{ml}$ of the pore forming peptide alamethicin added from a 200 times concentrated stock in methanol. After 1 min preincubation at $37{ }^{\circ} \mathrm{C}$, the reactions were started by adding the substrate 1'-hydroxyestragole from 100 times concentrated stock solutions in DMSO at final concentrations ranging from $60-2000 \mu \mathrm{M}$. Incubations were carried out for 6 hours and the reaction was terminated by adding $25 \mu \mathrm{l}$ ice-cold acetonitrile. Under these conditions, the formation of the glucuronide of 1'-hydroxyestragole was linear with time and microsomal protein concentration (Punt et al. 2009). Blank incubations were performed in the absence of the cofactor UDPGA. All incubations were performed in triplicate.

\section{Oxidation of 1'-hydroxyestragole}

Mixed gender Chinese or Caucasian liver S9 was incubated with 1'-hydroxyestragole in the presence of $\mathrm{NAD}^{+}$and $\mathrm{GSH}$, the latter added to trap the transient 1'-oxoestragole. Formation of the 1'-oxoestragole adducts with GSH forming GS-1'-oxoestragole reflects the formation of 1'-oxoestragole (Punt et al. 2009). The incubations had a final volume of $100 \mu$, containing (final concentrations) $3 \mathrm{mM} \mathrm{NAD}^{+}, 2 \mathrm{mM} \mathrm{GSH}$ and $1 \mathrm{mg} / \mathrm{ml}$ liver $\mathrm{S} 9$ in $0.2 \mathrm{M}$ Tris- $\mathrm{HCl}(\mathrm{pH}$ 7.4), as described previously by Punt et al. (2016). The mixtures were pre-incubated for $1 \mathrm{~min}$ at $37^{\circ} \mathrm{C}$, after which the reactions were started by adding the substrate 1'-hydroxyestragole at concentrations ranging from 15 to $1000 \mu \mathrm{M}$ added from 100 times concentrated stock solutions in DMSO. The reactions were terminated after $10 \mathrm{~min}$ by the addition of $25 \mu \mathrm{l}$ icecold acetonitrile. Under these conditions, the formation of the GSH adduct of 1'-oxoestragole was linear with time and cytosolic protein concentration (Punt et al. 2009). Blank incubations were performed without cofactor $\mathrm{NAD}^{+}$. All incubations were performed in triplicate.

\section{Sulfation of 1'-hydroxyestragole}

The formation of 1'-sulfooxyestragole was determined by incubating $0.2 \mathrm{mg} / \mathrm{ml}$ pooled mixed gender Chinese or Caucasian liver S9 in the presence of $0.2 \mathrm{mM}$ PAPS as cofactor and $10 \mathrm{mM} \mathrm{GSH}$ as trapping agent for the reactive 1'-sulfooxyestragole in $0.1 \mathrm{M}$ potassium phosphate $(\mathrm{pH} \mathrm{8.0)}$. The final volume of the incubations was $100 \mu \mathrm{l}$ with the substrate 1'hydroxyestragole ranging from 15 to $2000 \mu \mathrm{M}$ added from 100 times concentrated stock solutions in DMSO. As previously described by Martati et al. (2011) and Al-Subeihi et al. 
(2011), the reactions were started by adding substrate after a pre-incubation at $37{ }^{\circ} \mathrm{C}$ for 1 min. The incubations were carried out for 2 hours and the reactions were terminated by adding $25 \mu \mathrm{l}$ ice-cold acetonitrile. Under these conditions, the formation of the GSH adduct of 1 '-sulfooxyestragole was linear with time and microsomal protein concentration (Martati et al. 2011). The blank samples were performed without cofactor. All incubations were performed in triplicate.

\subsection{UPLC analysis}

\section{UPLC analysis of estragole metabolites}

For detection of microsomal estragole metabolites by UPLC, the gradient was made with 100 $\%$ ultrapure water and $100 \%$ acetonitrile. The flow rate was $0.6 \mathrm{ml} / \mathrm{min}$ and the gradient started with $100 \%$ ultrapure water. After $1 \mathrm{~min}$, the amount of acetonitrile was increased to $10 \%$ and kept at that level for $1 \mathrm{~min}$, after which the percentage of acetonitrile was increased to $35 \%$ in $2.5 \mathrm{~min}$ and then increased to $100 \%$ over 0.5 minute and kept at $100 \%$ acetonitrile for another $1 \mathrm{~min}$, and finally back to the starting conditions.

\section{UPLC analysis of 1'-hydroxyestragole metabolites}

The gradient for analysis of 1'-hydroxyestragole glucuronide was performed with ultrapure water containing $0.1 \%(\mathrm{v} / \mathrm{v})$ TFA and $100 \%$ acetonitrile with a $0.6 \mathrm{ml} / \mathrm{min}$ flow rate. A gradient was applied from 10 to $25 \%$ acetonitrile over $3.5 \mathrm{~min}$, after which the acetonitrile was increased to $100 \%$ in $0.5 \mathrm{~min}$, kept at $100 \%$ for $0.5 \mathrm{~min}$ and finally set back to the starting condition.

The gradient for analysis of the metabolites contains a mixture of acetonitrile and $0.1 \%$ TFA in ultrapure water with $0.6 \mathrm{ml} / \mathrm{min}$ flow rate. A gradient from 0 to $25 \%$ acetonitrile was applied over $1.2 \mathrm{~min}$ and kept at $25 \%$ acetonitrile for $0.2 \mathrm{~min}$, after which the percentage of acetonitrile was increased to $100 \%$ in $0.5 \mathrm{~min}$ and then kept at $100 \%$ for another $0.6 \mathrm{~min}$.

For detection of the GSH conjugate of 1'-sulfooxyestragole, the flow rate was $0.6 \mathrm{ml} / \mathrm{min}$ and the mobile phase was made with acetonitrile and ultrapure water with $0.1 \%(\mathrm{v} / \mathrm{v}) \mathrm{TFA}$. A linear gradient started from 20 to $30 \%$ acetonitrile in $4.5 \mathrm{~min}$, after which the percentage of acetonitrile was increased to $100 \%$ in $0.3 \mathrm{~min}$ and kept at $100 \%$ for another $0.2 \mathrm{~min}$ and finally set back to the starting condition.

\section{Supplementary materials 3}

\section{Results}

\section{Metabolic capabilities of CYP and SULTs enzymes}

The quality of the Chinese and Caucasian liver microsomes was checked by measuring CYP 1 A2 mediated $O$-deethylation of phenacetin, and CYP 2A6 mediated 7-hydroxylation of coumarin. The catalytic efficiency $\left(\mathrm{V}_{\max } / \mathrm{K}_{\mathrm{m}}\right)$ for CYP $1 \mathrm{~A} 2$ mediated $O$-deethylation of phenacetin, and CYP 2A6 mediated 7-hydroxylation of coumarin by Chinese liver microsomes were 3.1 and $525.8 \mu \mathrm{L} / \mathrm{min} /(\mathrm{mg}$ microsomal protein $)$, respectively, which were in 
the range of results reported before in the literature showing a catalytic efficiency of 0.6-51.4 $\mu \mathrm{L} / \mathrm{min} /(\mathrm{mg}$ microsomal protein) for CYP $1 \mathrm{~A} 2$ and of $150-1776 \mu \mathrm{L} / \mathrm{min} /(\mathrm{mg}$ microsomal protein) for CYP 2D6 in Chinese liver microsomes (Yang et al. 2012). These catalytic efficiencies for the CYP 1A2 and CYP 2D6 mediated conversions by Chinese liver microsomes were 3- and 1.6-fold lower than the corresponding catalytic efficiency values by Caucasian liver microsomes, which was consistent with the data reported by Yang et al. (2012) indicating a 2-fold lower value for catalytic efficiency of CYP 1A2 for Chinese liver microsomes and no difference between Chinese and Caucasian liver microsomes in the case of CYP 2 A6 catalyzed activity.

The quality of the Chinese and Caucasian liver S9 preparations was checked by measuring the kinetics for SULT mediated conversion of 7-hydroxycoumarin to 7-hydroxycoumarin sulfate. The $\mathrm{K}_{\mathrm{m}}$ for SULT mediated conversion of 7-hydroxycoumarin in incubations with Chinese liver S9 was $1.7 \mu \mathrm{M}$ and thus similar to reported data by Wang et al. (2006) and the $\mathrm{V}_{\max }$ for both ethic groups appeared to be similar which was also in line with previously reported data (Wang et al. 2006). 

<smiles>CCCC(C)C(C)CC</smiles> 

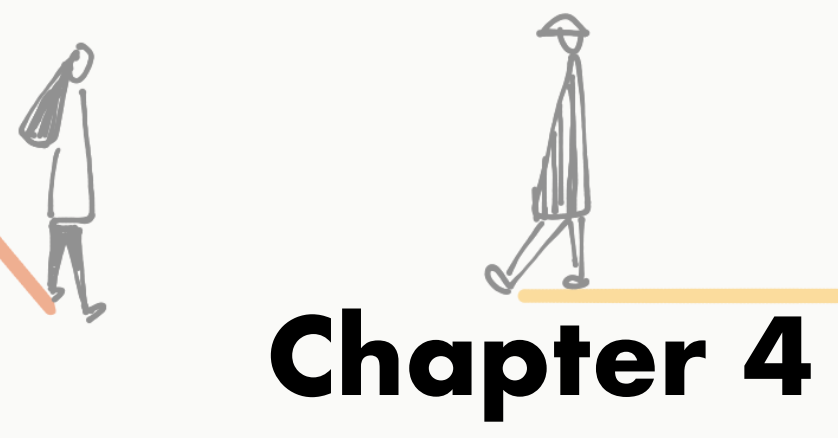

Use of physiologically based kinetic modellingfacilitated reverse dosimetry to convert in vitro cytotoxicity data to predicted in vivo liver foxicity of lasiocarpine and riddelliine in rat
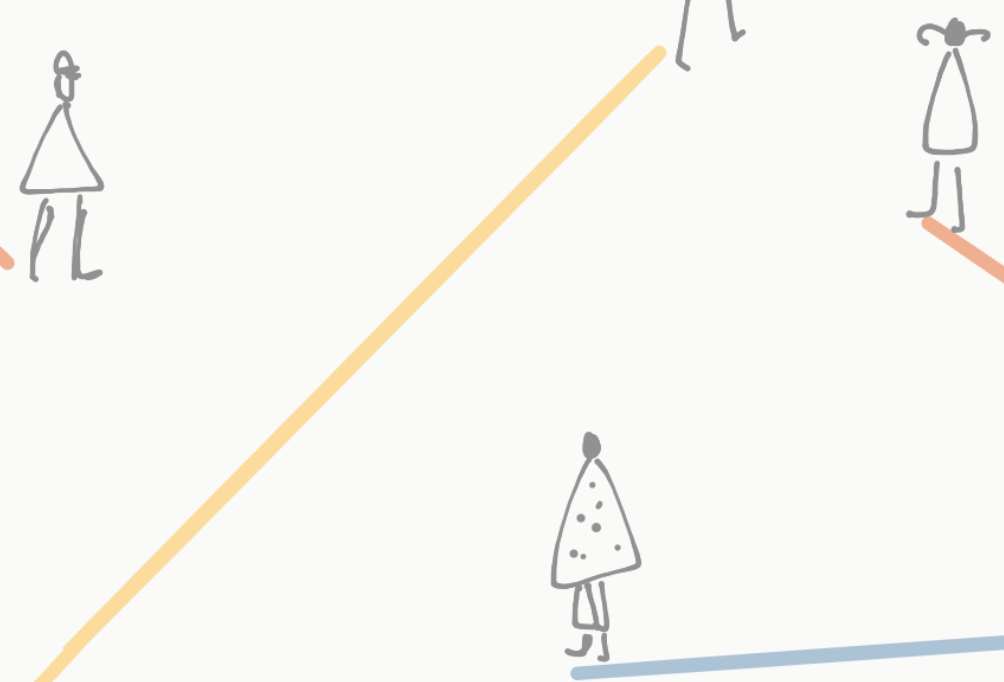

Lu Chen, Jia Ning, Jochem Louisse,

Sebas Wesseling, Ivonne M.C.M. Rietjens

Published in: Food and Chemical Toxicology (2018) 116 (Pt B), 216-226 


\begin{abstract}
Lasiocarpine and riddelliine are pyrrolizidine alkaloids (PAs) present in food and able to cause liver toxicity. The aim of this study was to investigate whether physiologically based kinetic (PBK) modelling-facilitated reverse dosimetry can adequately translate in vitro concentration-response curves for toxicity of lasiocarpine and riddelliine to in vivo liver toxicity data for the rat. To this purpose, PBK models were developed for lasiocarpine and riddelliine, and predicted blood concentrations were compared to available literature data to evaluate the models. Concentration-response curves obtained from in vitro cytotoxicity assays in primary rat hepatocytes were converted to in vivo dose-response curves from which points of departure (PoDs) were derived and that were compared to available literature data on in vivo liver toxicity. The results showed that the predicted PoDs fall well within the range of PoDs derived from available in vivo toxicity data. To conclude, this study shows the proof-ofprinciple for a method to predict in vivo liver toxicity for PAs by an alternative testing strategy integrating in vitro cytotoxicity assays with in silico PBK modelling-facilitated reverse dosimetry. The approach may facilitate prediction of acute liver toxicity for the large number of PAs for which in vivo toxicity data are lacking.
\end{abstract}




\section{Introduction}

Pyrrolizidine alkaloids (PAs) are plant secondary metabolites (Fu et al. 2004; Mattocks 1986) naturally occurring in several food items (EFSA 2011; Wiedenfeld 2011). To date, more than 660 PAs and PA $N$-oxides have been identified from more than 6000 plants (Wiedenfeld 2013; Wiedenfeld and Edgar 2011). PAs are a class of heterocyclic chemicals, and most PAs are derived from esters of basic alcohols, known as the necine bases. Especially the PAs with an 1,2-unsaturated necine base tend to be toxic (Mori et al. 1985). Of the four common necine bases encountered in PAs, including platynecine, retronecine, heliotridine and otonecine, especially the later three give rise to 1,2-unsaturated PAs that have been shown to be hepatotoxic, genotoxic and carcinogenic in rats and other experimental rodents (Chan 1993; Chan et al. 2003; Hirono et al. 1979; Hirono et al. 1976; Hirono et al. 1977; Kuhara et al. 1980; NTP 1978; Schoental 1970; Shumaker et al. 1976). Human poisonings caused by intake of PA-containing plants have been reported in several countries (Kumana et al. 1985; Mohabbat et al. 1976; Prakash et al. 1999; Roulet et al. 1988; Sperl et al. 1995; Tandon et al. 1976). Due to the carcinogenicity of PAs, the International Agency for Research on Cancer (IARC) classified lasiocarpine and riddelliine in Group 2B, possibly carcinogenic to humans (IARC 1976, 2002). In addition, the European Food Safety Authority (EFSA) recently stated that 1,2-unsaturated PAs may act as carcinogens in humans based on the actual knowledge of metabolism, activation, DNA adduct-formation, genotoxicity and carcinogenicity (EFSA 2011).

PAs need metabolic activation to form pyrrolic metabolites to exhibit their hepatotoxicity and genotoxicity (Chan et al. 2003; Chou et al. 2003; Fu et al. 2002; Fu et al. 2004; Lin et al. 2000; Mattocks 1986; Miranda et al. 1991; Stegelmeier et al. 1999; Yan et al. 2002). In general, there are three principal metabolic pathways for the metabolism of 1,2-unsaturated PAs such as lasiocarpine (Figure 1). In the first pathway, the necine base is formed by hydrolysis of the ester group. For the second pathway, the necine base is N-oxidised to generate pyrrolizidine alkaloid N-oxide. In addition, the necine base can also be oxidised to form the corresponding reactive pyrrolic ester metabolite ( $\mathrm{Fu} \mathrm{2017}$; $\mathrm{Li}$ et al. 2011). The generated pyrrolic ester can immediately bind to DNA and proteins in the liver resulting in DNA cross-links, DNA-protein cross-links, protein adducts and DNA adducts, that may induce hepatotoxicity and liver cancer (Bovee et al. 2015; Lin et al. 1998, 2000; Lin et al. 2011; White 1976; Yan and Huxtable 1995a, b).

In spite of the large number of PAs known at present, toxicity data on only a limited number of PAs are available. Among the 1,2-unsaturated PAs, lasiocarpine and riddelliine have been tested in two year rodent carcinogenicity bioassays by the National Toxicology Program (NTP), and were shown to cause liver carcinogenicity (NTP 1978, 2003). In addition to lasiocarpine and riddelliine, limited tumour data are available for some other PAs, including monocrotaline, clivorine, isatidine, retrosine, senkirkine and symphytine (Hirono et al. 1979; Kuhara et al. 1980; Schoental and Bensted 1963; Schoental et al. 1954; Shumaker et al. 1976). However, of all these PAs, data on liver toxicity are only available for lasiocarpine 
(Dalefield et al. 2016; Jago 1970; Nolan et al. 1966). Given the large number of PAs and the limited number of PAs for which actual in vivo data on liver toxicity and carcinogenicity are available, it is of importance to investigate whether information on relative potency for hepatotoxicity of the different PAs can be obtained from alternative testing strategies reducing the need for a large number of animal experiments. In our previous work, we have shown that data on in vivo toxicity can be adequately predicted by translation of in vitro concentrationresponse curves for toxicity to in vivo dose-response curves for toxicity using so-called physiologically based kinetic (PBK) modelling-facilitated reverse dosimetry (Abdullah et al. 2016; Li et al. 2017; Louisse et al. 2015; Louisse et al. 2010; Strikwold et al. 2017). In this way in vivo dose-response curves for developmental toxicity and kidney toxicity were defined (Abdullah et al. 2016; Li et al. 2017; Louisse et al. 2015; Louisse et al. 2010; Strikwold et al. 2017). The aim of the present study was to investigate whether PBK modelling-facilitated reverse dosimetry can adequately translate in vitro concentrationresponse curves for toxicity of PAs, to in vivo dose-response curves for liver toxicity for the rat. Riddelliine and lasiocarpine were used as model PAs because for these two PAs in vivo data on kinetics (Williams et al. 2002) and liver toxicity (Dalefield et al. 2016; Jago 1970; Nolan et al. 1966), respectively, are available enabling evaluation of the PBK models and the toxicity predictions made.

To achieve the aim of this study, rat PBK models were developed for lasiocarpine and riddelliine, and concentration-response curves obtained from in vitro cytotoxicity studies with rat hepatocytes were translated into in vivo dose-response curves by using PBK modellingfacilitated reverse dosimetry from which PoDs for evaluation liver toxicity induced by lasiocarpine and riddelliine could be derived. For evaluating the performance of the PBK models, PBK model-predicted blood concentrations were compared with in vivo kinetic data. Because of the lack of any in vivo kinetic data of lasiocarpine, we also developed a PBK model for riddelliine in rat and mouse to enable examination of model performance based on available blood concentration values in the literature for this related PA. To evaluate the prediction of liver toxicity, PoDs derived from predicted liver toxicity induced by lasiocarpine were compared with available in vivo liver toxicity data of this PA. 


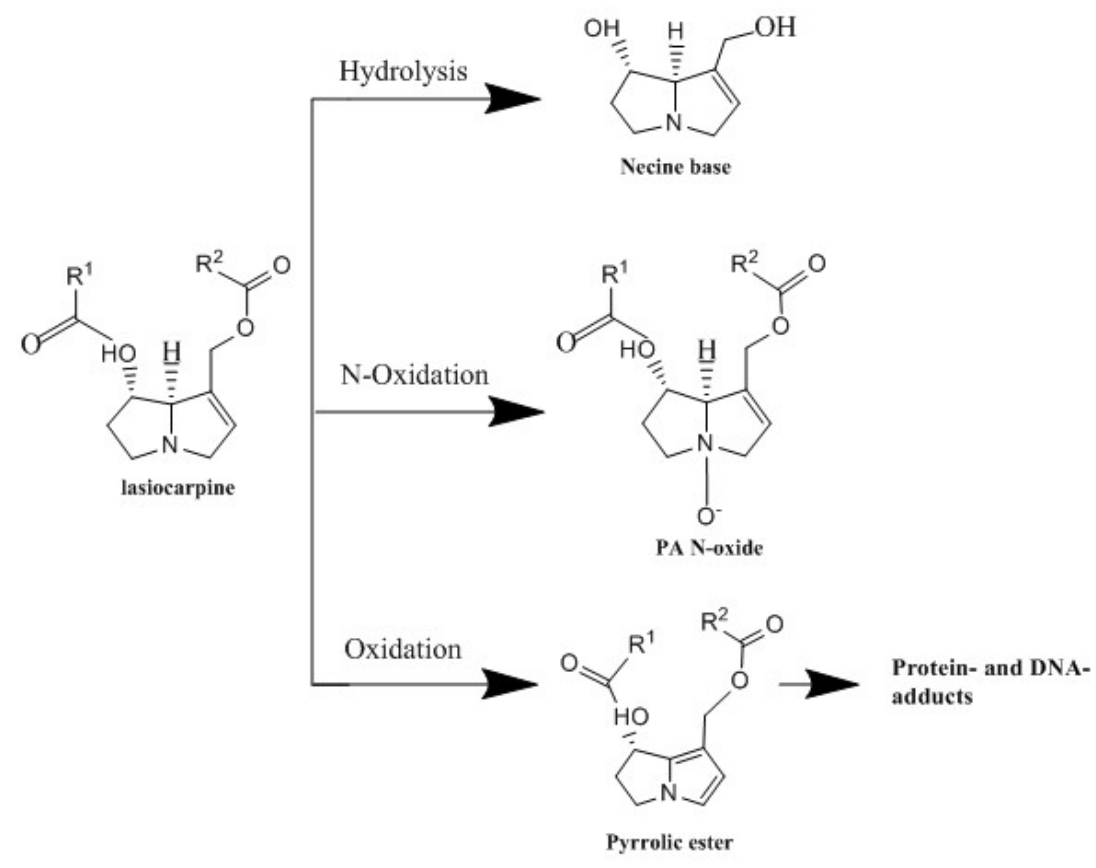

Fig. 1 The proposed pathways for metabolism of lasiocarpine, also relevant for riddelliine (Bovee et al. 2015; Fu 2017; Li et al. 2011; Lin et al. 2011).

\section{Materials and Methods}

\subsection{Chemicals}

Lasiocarpine (> 97 \%) was purchased from PhytoLab (PhytoLab GmbH \& Co. KG, Germany), and riddelliine (90\%) was kindly provided by RIKILT Wageningen University \& Research (Wageningen, The Netherlands). The cryopreserved rat (Sprague-Dawley) hepatocytes, the thawing/plating supplement pack, the cell maintenance supplement pack, fetal bovine serum and Williams E Medium (WEM, A1217601) were purchased from ThermoFisher (Naarden, The Netherlands). Trypsin-EDTA was purchased from Gibco (Paisley, Scotland, UK). Dimethyl sulfoxide (DMSO) was obtained from Acros Organics (Geel, Belgium). Acetonitrile (UPLC/MS grade) was obtained from Biosolve (Valkenswaard, The Netherlands). Potassium hydrogen phosphate $\left(\mathrm{K}_{2} \mathrm{HPO} 4\right)$ and trifluoroacetic acid (TFA) was purchased from Merck (Darmstadt, Germany). The reduced form of nicotinamide adenine dinucleotide phosphate (NADPH) was obtained from Sigma-Aldrich (Zwijndrecht, The Netherlands). Pooled liver microsomes from male Sprague-Dawley rats were obtained from Corning (Amsterdam, The Netherlands) and pooled liver microsomes from male CD-1 mice were purchased from Sigma Aldrich (Zwijndrecht, The Netherlands). Pooled intestinal microsomes from male Sprague-Dawley rats and male CD-1 mice were purchased from Xenotech (Lenexa, USA). 


\subsection{General outline for PBK modelling-facilitated reverse dosimetry approach}

The combined in vitro PBK modelling approach to predict in vivo dose-response curves and a PoD for risk assessment using in vitro cytotoxicity data consisted of the following steps: (1) establishment of in vitro concentration-response curves for the toxicity of lasiocarpine and riddelliine in rat hepatocytes, (2) development of PBK models describing in vivo kinetics of lasiocarpine and riddelliine, using kinetic parameters defined based on in vitro assays using tissue fractions of rat and mouse, (3) evaluation of the PBK models against available literature data on blood levels and liver toxicity upon oral dosing of the PAs, (4) translation of the in vitro concentration-response curves for acute liver toxicity into in vivo dose-response curves for acute liver toxicity in rat using the PBK models, (5) BMD analysis on the predicted in vivo dose-response data to obtain a PoD for risk assessment, and (6) evaluation of the predicted PoD against available literature data.

\subsection{In vitro liver toxicity}

Rat hepatocytes were used to define the in vitro concentration-response curves for cytotoxicity. To this end rat hepatocytes were seeded at concentrations of $5 \times 10^{5}$ cells $/ \mathrm{ml}$ into 96-well plates according to the supplier's protocol, and cultured in plating medium for 4-6 h. After incubation, medium was replaced by exposure medium (serum free), containing different concentrations of lasiocarpine or riddelliine ranging from 0 to $300 \mu \mathrm{M}$ (final concentrations), added from 200 times concentrated stock solutions in DMSO. After $23 \mathrm{~h}$ treatment, $10 \mu \mathrm{l}$ MTT $(5 \mathrm{mg} / \mathrm{mL})$ was added and cells were incubated for an additional $1 \mathrm{~h}$. Cells were lysed and the MTT formazan crystals formed were dissolved by addition of $100 \mu 1$ DMSO, and the absorbance was measured at $562 \mathrm{~nm}$ and $620 \mathrm{~nm}$ using a microplate reader (SpectraMax M2, USA). Relative cell viability (\%) was calculated as (mean absorbance of sample/mean absorbance of vehicle control) $\times 100 \%$. Each concentration was tested in three replicates, and three independent experiments with 3 different batches of hepatocytes were carried out.

\subsection{Determination of fraction unbound (fub) of lasiocarpine and riddelliine in rat serum}

Since it is assumed that the toxicity is caused by the fraction unbound ( $f_{u b}$ ) of the chemical and since the $f_{u b}$ in vitro and in vivo differ, a correction for the differences in $f_{u b}$ needs to be made. The $f_{u b}$ of lasiocarpine and riddelliine in rat serum were determined by using rapid equilibrium dialysis (RED) (Waters et al. 2008). The RED device insert has two chambers, a blood and a buffer chamber. Briefly, a sample of $300 \mu \mathrm{l}$ of spiked rat serum containing $5 \mu \mathrm{M}$ of lasiocarpine or $50 \mu \mathrm{M}$ of riddelliine (final concentration, $0.5 \% \mathrm{DMSO}$ ) were added to the blood chamber, while $500 \mu \mathrm{l}$ PBS (containing $100 \mathrm{mM}$ sodium phosphate and $150 \mathrm{mM}$ sodium chloride) were added to the buffer chamber. The device was sealed with tape and incubated at $37{ }^{\circ} \mathrm{C}$ on a shaker at $250 \mathrm{rpm}$. After incubation for 5 hours the system reaches equilibrium (van Liempd et al. 2011) and $25 \mu \mathrm{L}$ of post-dialysis samples were collected from the blood and buffer chambers in separate tubes. Simultaneously, $25 \mu \mathrm{L}$ of corresponding buffer was added to the sample taken from the blood chamber and the same volume of corresponding rat serum was added to the sample taken from the buffer chamber. Then, 300 $\mu \mathrm{L}$ cold precipitation buffer (90/10 acetonitrile/water) was added to both samples to 
precipitate the proteins and release the compound. The samples were put on ice for $30 \mathrm{~min}$ and subsequently centrifuged for $15 \mathrm{~min}$ at $15000 \mathrm{~g}$. Then, the supernatants were collected for UPLC analysis. By determining the compound concentration in each chamber, the $f_{u b}$ can be calculated with the following equation: $f_{u b}=$ (concentration in buffer chamber) / (concentration in blood chamber) (van Liempd et al. 2011; Waters et al. 2008). Then the effect concentration of lasiocarpine (LC) or riddelliine $(\mathrm{RD})$ in rat serum $\left(\mathrm{C}_{\mathrm{LC} / \mathrm{RD}}\right.$, rat serum) used for reverse dosimetry was corrected by the following equation: $\mathrm{C}_{\mathrm{LC}} / \mathrm{RD}$, rat serum $=\mathrm{C}_{\mathrm{ub}}$, in vitro / $\mathrm{f}_{\mathrm{ub}}$, rat serum, where $\mathrm{C}_{\mathrm{ub}}$, in vitro is the unbound concentration of lasiocarpine or riddelliine in the in vitro culture medium. Since the exposure medium for the rat hepatocytes was serum free, the concentration of lasiocarpine or riddelliine in the in vitro cytotoxicity assay was considered to be equal to the unbound concentration in rat serum.

\subsection{In vitro incubations of lasiocarpine or riddelliine to derive the kinetic parameters for the PBK model}

Microsomal incubations were performed with rat tissue fractions to determine kinetic parameter values for lasiocarpine and riddelliine clearance, using a substrate depletion approach. The incubation mixtures for lasiocarpine contained, in a final volume of $100 \mu \mathrm{l}$ (final concentrations): $0.1 \mathrm{M} \mathrm{K}_{2} \mathrm{HPO}_{4} \quad(\mathrm{pH} 7.4)$, pooled rat liver or intestinal microsomes (0.04 $\mathrm{mg}$ protein $/ \mathrm{ml}$ ), and lasiocarpine ranging from 0 to $200 \mu \mathrm{M}$ (added from 100 times concentrated stock solutions in DMSO). Under these conditions PA conversion was linear with time and the amount of protein added (data not shown). For riddelliine, the incubation mixtures contained in a final volume of $100 \mu \mathrm{l}$ (final concentrations): $0.1 \mathrm{M} \mathrm{K}_{2} \mathrm{HPO}_{4} \quad(\mathrm{pH}$ 7.4), pooled rat liver or intestinal microsomes $(0.5 \mathrm{mg}$ protein $/ \mathrm{ml})$, and riddelliine ranging from 0 to $400 \mu \mathrm{M}$ (added from 100 times concentrated stock solutions in DMSO). Under these conditions PA conversion was linear with time and the amount of protein added (data not shown). After $5 \mathrm{~min}$ of pre-incubation in a shaking water bath at $37^{\circ} \mathrm{C}$, the reactions were started by the addition of $2 \mathrm{mM}$ NADPH. The reactions were carried out for $30 \mathrm{~min}$ with liver microsomes and $2 \mathrm{~h}$ with intestinal microsomes. Control incubations were performed without NADPH. The incubations were terminated by the addition of $25 \mu$ l ice-cold acetonitrile followed by centrifugation at $16000 \mathrm{~g}$ for $5 \mathrm{~min}$ before analyse of the supernatant by UPLC (Waters Acquity). The incubation conditions for metabolism of riddelliine by pooled mouse liver and intestinal microsomes were performed in a similar way. The clearance of lasiocarpine and riddelline in lung or kidney was determined using either lung or kidney microsomes of rat or mouse, by following the same incubation procedure for intestinal microsomes using final microsomal concentrations of $0.04 \mathrm{mg} / \mathrm{mL}$ for rat and $0.5 \mathrm{mg} / \mathrm{mL}$ for mouse.

From each incubation, $3.5 \mu \mathrm{l}$ of supernatant were injected into the UPLC (Waters Acquity) equipped with a Waters BEH C18 $1.7 \mathrm{~mm}$ column, 2.1×50 mm (Waters, Ireland). A gradient was made with ultra-pure water containing $0.1 \%(\mathrm{v} / \mathrm{v})$ TFA as solvent $\mathrm{A}$ and $100 \%$ acetonitrile as solvent $\mathrm{B}$. The flow rate was set to $0.6 \mathrm{ml} / \mathrm{min}$. The starting condition was 100:0 (A:B), changing to 50:50 from 0 to $2 \mathrm{~min}$, then to $0: 100$ from 2 to $5 \mathrm{~min}$, then to 100:0 from 5 to $8 \mathrm{~min}$. Detection was carried out with a photodiode array detector (Waters, Milford, 
MA, USA) at a wavelength of $220 \mathrm{~nm}$. The amount of substrate depletion of lasiocarpine or riddelliine was quantified as the amount detected in the control incubations performed without the respective cofactor NADPH minus the amount detected in the incubations with NADPH using a calibration curve made with available reference compounds. The data for the rate of depletion of lasiocarpine and riddelliine with increasing lasiocarpine or riddelliine concentrations were fitted to the standard Michaelis-Menten equation:

$V=\operatorname{Vmax} *[S] /(K m+[S])$

With [S] being the substrate concentration, $\mathrm{V}_{\max }$ being the apparent maximum velocity, and $\mathrm{K}_{\mathrm{m}}$ being the apparent Michaelis-Menten constant. This was done with GraphPad Prism, 5.0 software (San Diego, CA, USA).

\subsection{PBK models for rat and mouse}

A schematic diagram of the structure of the PBK models developed for rat and mouse is shown in Figure 2. The models include separate compartments for liver and intestine (as metabolizing compartments), fat, arterial blood, venous blood, rapidly perfused and slowly perfused tissue. Lasiocarpine and riddelliine were assumed to be taken up from the gastrointestinal tract following first order kinetics and absorbed directly by the intestine. No absorption rate constants $(\mathrm{Ka})$ are available for lasiocarpine and riddelliine, but for the PA adonifoline, a Ka of $0.6 / \mathrm{h}$ has been reported (Wang et al. 2011). The Ka of lasiocarpine and riddelliine was estimated based on two approaches. In the first approach, the Ka was estimated by relating the Ka values of the PAs to the permeability coefficients ( $P$ app values) of the PAs as predicted by Qikprop (Schrödinger, trial version, Germany). The $P_{\text {app }}$ values of adonifoline, lasciocarpine and riddelliine as derived from Qikprop amount to 241, 542 and $107 \mathrm{~nm} / \mathrm{s}$ for adonifoline, lasiocarpine and riddelliine, respectively. The Ka values for lasioicarpine and riddelliine were calculated relative to adonifoline as the standard compound $\left(P_{\text {app LC/RD }} / \mathrm{Ka}\right.$ LC/RD $\left.=P_{\text {app adonifoline }} / 0.6\right)$. Based on the $P_{\text {app }}$ for lasicoarpine and riddelliine of $542 \mathrm{~nm} / \mathrm{s}$ and $107 \mathrm{~nm} / \mathrm{s}, \mathrm{Ka}$ values for lasiocarpine and riddelliine were calculated to amount to $1.35 / \mathrm{h}$ and $0.27 / \mathrm{h}$, respectively. Ka values for lasiocarpjne and riddelliine were also estimated based on $P_{\text {app }}$ values obtained from the correlation between Caco- 2 permeation and molecular properties described by Hou et al. (2004) reflected by the equation $\log P_{\text {app }}=$ $-5.469+0.236 \log \mathrm{P}$. The $\log \mathrm{P}$ values for lasiocarpine, riddelliine and adonifoline were obtained using the ChemBio 3D 2010 software (CambrigeSoft, USA) and amounted to 0.48, -0.26 and -1.49 , respectively. Based on these $\log \mathrm{P}$ values, the corresponding $\log P_{\text {app }}$ values were calculated to be -5.35 for lasiocarpine, -5.53 for riddelliine and -5.82 for adonifoline. Then, the relative Ka values for lasiocarpine or riddelliine were calculated relative to adonifoline as the standard compound as described above $\left(\log P_{\text {app }} \quad\right.$ LC/RD $/ \mathrm{Ka}$ LC/RD $=\log$ $P_{\text {app }}$ adonifoline / 0.6). The Ka values thus obtained for lasiocarpine and riddelliine were 1.75/ $\mathrm{h}$ and $1.17 / \mathrm{h}$, respectively.

In this study, we calculated the average Ka values for lasiocarpine and riddelliine from those two methods, which amounted to $1.55 / \mathrm{h}$ and $0.72 / \mathrm{h}$, respectively. Physiological parameter values for the model, such as for tissue volumes and blood flows, were obtained from literature (Brown et al. 1997). The tissue/blood partition coefficients applied in the rat model 
were calculated based on the method reported by DeJongh et al. (1997). All physiological parameters and partition coefficients used in the rat PBK models are presented in Table 1. For evaluation of the generic PBK models developed in the present study, we also developed a mouse PBK model for riddelliine, because for riddelliine also kinetic data in mice were available for model evaluation (Williams et al. 2002). The physiological parameters for the mouse PBK model were taken from literature (Brown et al. 1997) and the tissue/blood partition coefficients were assumed to be the same as in rat. These values used in this mouse PBK model are also presented in Table 1. On the basis of data obtained in the in vitro incubations with liver and intestinal fractions with lasiocarpine and riddelline, the conversion of lasiocarpine and riddelliine were described in the liver and intestine. The $\mathrm{V}_{\max }$ values for conversion of lasiocarpine or riddelliine by rat or mouse liver and intestinal microsomal fractions were scaled accordingly using an estimated microsomal protein yield of $35 \mathrm{mg} / \mathrm{g}$ liver (Medinsky et al. 1994) and $20.6 \mathrm{mg} / \mathrm{g}$ small intestine (Cubitt et al. 2009), respectively as scaling factors. The in vivo $\mathrm{K}_{\mathrm{m}}$ values were assumed to equal the in vitro $\mathrm{K}_{\mathrm{m}}$ values.

In the present study it was assumed that clearance of the PAs fully depends on their metabolic conversion and that excretion of the parent compound in its unmodified form does not add significantly to the systemic clearance. This assumption is supported by data reported by Bull et al. (1968) who delivered lasiocarpine to rats through ip injection, after which only 1-1.5\% of total lasiocarpine was found in unmodified form in the urine (Bull et al. 1968), so excretion of the parent compound was not included in the current PBK model. The PBK model equations were coded and numerically integrated in Berkeley Madonna (version 8.3.18, UC Berkeley, CA, USA) using Rosenbrock's algorithms for solving stiff systems.

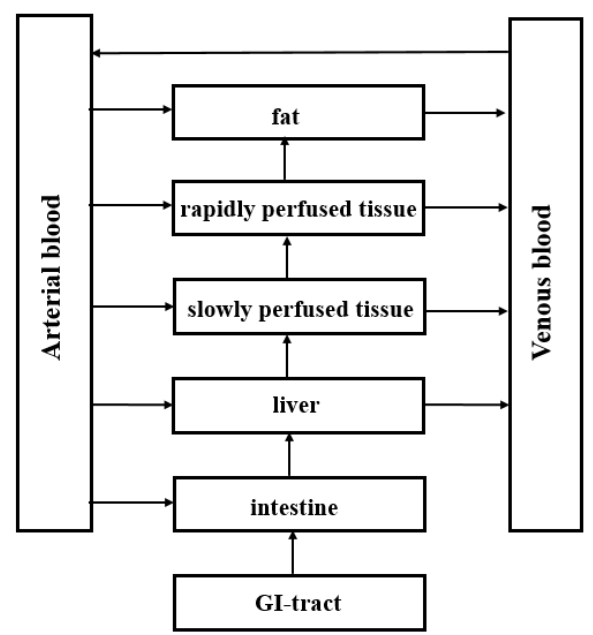

Fig. 2 Schematic diagram of the generic PBK model for lasiocarpine and riddelliine in rat and mouse. 
Table 1 Parameters used in the rat or mouse PBK models for lasiocarpine and riddelliine.

\begin{tabular}{|c|c|c|}
\hline Model parameters & Rat & Mouse \\
\hline \multicolumn{3}{|l|}{ Physiological parameters ${ }^{a}$} \\
\hline Body weight (kg) & 0.25 & 0.0025 \\
\hline \multicolumn{3}{|l|}{ Tissue volumes ( $\%$ body weight) } \\
\hline Liver & 3.4 & 5.5 \\
\hline Fat & 7.0 & 10 \\
\hline Small intestine & 1.4 & 2.5 \\
\hline Richly perfused tissue & 4.2 & 1 \\
\hline Slowly perfused tissue & 75 & 72 \\
\hline Blood & 7.4 & 4.9 \\
\hline Cardiac output $\left(\mathrm{L} \cdot \mathrm{h}^{-1} \cdot \mathrm{kg} \mathrm{bw}^{-0.74}\right)$ & 15.0 & 15.0 \\
\hline \multicolumn{3}{|l|}{ Blood flow to tissue ( $\%$ cardiac output) } \\
\hline Liver & 13.2 & 6.7 \\
\hline Fat & 7 & 9 \\
\hline Small intestine & 11.8 & 18.3 \\
\hline Richly perfused tissue & 51 & 51 \\
\hline Slowly perfused tissue & 17 & 15 \\
\hline \multicolumn{3}{|l|}{ Physicochemical parameters ${ }^{b}$} \\
\hline \multicolumn{3}{|l|}{ Tissue/blood partition coefficients } \\
\hline \multicolumn{3}{|l|}{ Lasiocarpine } \\
\hline Liver & 0.88 & 0.88 \\
\hline Fat & 2.44 & 2.44 \\
\hline Small intestine & 0.88 & 0.88 \\
\hline Richly perfused tissue & 0.88 & 0.88 \\
\hline Slowly perfused tissue & 0.48 & 0.48 \\
\hline \multicolumn{3}{|l|}{ Riddelliine } \\
\hline Liver & 0.77 & 0.77 \\
\hline Fat & 0.44 & 0.44 \\
\hline Small intestine & 0.77 & 0.77 \\
\hline Richly perfused tissue & 0.77 & 0.77 \\
\hline Slowly perfused tissue & 0.43 & 0.43 \\
\hline
\end{tabular}

a Brown et al. (1997)

b Derived using the approach described by DeJongh et al. (1997) 


\subsection{PBK model evaluation}

Normalized sensitivity coefficients (SC) were determined to identify parameters that largely influence the prediction of the maximal blood concentrations $\left(\mathrm{C}_{\max }\right)$ in liver blood by the PBK model, using the equation:

$$
\mathrm{SC}=\left(\left(\mathrm{C}^{\prime}-\mathrm{C}\right) /\left(P^{\prime}-P\right)\right) *(P / C)
$$

where $\mathrm{P}$ and $\mathrm{P}^{\prime}$ are the initial and modified parameter values respectively, and $\mathrm{C}$ and $\mathrm{C}^{\prime}$ are the initial and modified values of the model output resulting from an increase in parameter value, respectively (Evans and Andersen 2000). A 5\% increase in parameter value was chosen to analyse the effect of a change in a parameter. The sensitivity analysis was conducted at an oral dose of 0.008 and $0.144 \mu \mathrm{g} / \mathrm{kg}$ bw/day PAs representing the lowest and the highest daily intake previously estimated to originate from use of herbal teas (BfR 2013). Each parameter was analysed individually, keeping the other parameters to their initial values.

Because of the lack of available in vivo kinetic data for lasiocarpine, to evaluate the performance of the PBK model developed in the present study, the predicted riddelliine blood concentrations obtained with the rat and mouse models were compared with blood concentrations from in vivo kinetic studies in rat and mouse reported in the literature (Williams et al. 2002).

\subsection{Translation of the in vitro concentration-response curves to in vivo dose-response curves}

Based on the reverse dosimetry approach, the in vitro effect concentrations of lasiocarpine and riddelliine inducing cytotoxicity were considered as surrogate in vivo (free) concentrations in blood that could result in adverse effect on the liver. In the present study, each concentration used in the cytotoxicity assay, corrected by $\mathrm{fub}_{\mathrm{ub}}$ (determined as described above), was set equal to the maximum concentration $\left(\mathrm{C}_{\max }\right)$ of lasiocarpine or riddelliine in the liver blood and the developed PBK models were used to determine the oral dose required that results in this concentration.

\subsection{BMD analysis of in vitro concentration-response data and of predicted in vivo dose- response data}

The continue model in PROAST programme (version 38.9, The Dutch National Institute for Public Health and the Environment, The Netherlands) was used to analyse the predicted in vivo dose-response data and to obtain the BMDL5-BMDU5 (lower/upper limit of the $90 \%$ confidence interval of the benchmark dose that gives a $5 \%$ response) values for liver toxicity. The aim of the use of continue model was to determine BMDL5-BMDU 5 values. The goodness of fit application of the models was used to judge if the model can be accepted, using the $\mathrm{P}$ value with $\mathrm{P}>0.05$. Only the models which met the requirements for acceptance of the model fit were considered for the determination of BMDL5-BMDU 5 values. The same analysis was performed to predict in vivo dose-response curves derived upon extrapolation of the concentration-response curves for in vitro cytotoxicity derived from the literature (Hayes et al. 1984). 


\subsection{Evaluation of the combined in vitro-PBK modelling approach}

To support the validity and use of the in vitro-in silico approach to obtain a PoD for risk assessment, the predicted BMDL5-BMDU5 values of lasiocarpine for rat were compared with PoDs derived from in vivo rat acute liver toxicity data from the literature (Dalefield et al. 2016; Jago 1970; Nolan et al. 1966). For comparison of the predicted in vivo toxicity data to the available in vivo toxicity data form the literature it was assumed that the BMDL $5-\mathrm{BMDU}_{5}$ from such work, could be a reasonable approximation of an in vivo NOAEL-LOAEL (No/ Lowest observed adverse effect level) value.

\section{Results}

\subsection{In vitro cytotoxicity}

Figure 3 shows the concentration-response curves for the cytotoxicity of lasiocarpine or riddelliine in incubations with rat hepatocytes. The $\mathrm{IC}_{50}$ of lasiocarpine and riddelliine derived from these curves amounted to $10.9 \mu \mathrm{M}$ and $6.3 \mu \mathrm{M}$, respectively as shown in Table 2. The in vitro concentration-response curve of lasiocarpine in the present study was comparable with in vitro concentration-response curves previously reported by Hayes et al. (1984) (Figure 3). Table 2 shows the $\mathrm{IC}_{50}$ values obtained from the concentration-response curves in Figure 3.

- Present study, Lasiocarpine, Rat hepatocytes

- Present study, Riddelliine, Rat hepatocytes

A Hayes et al., 1984, Lasiocarpine, Rat hepatocytes

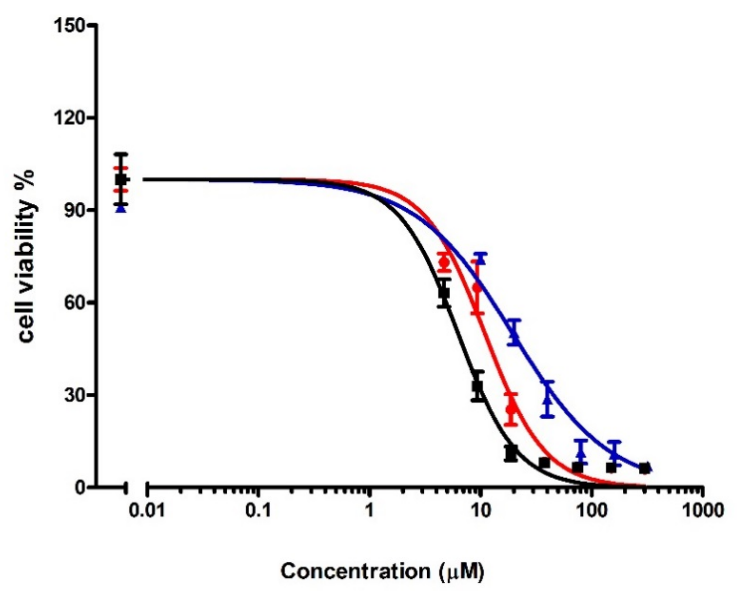

Fig. 3 Concentration-response curves for primary rat hepatocytes treated with increasing concentrations of lasiocarpine (circles) and riddelliine (squares) for 24 hours (mean $\pm \mathrm{SD}$ ) and as reported by Hayes et al. (1984) (triangles) for lasiocarpine. 
Table $2 \mathrm{IC}_{50}$ values derived from the concentration-response curves presented in Figure 3.

\begin{tabular}{ccc}
\hline Compound & $\mathrm{IC}_{50} \pm \mathrm{SD}(\mu \mathrm{M})$ & Studies \\
\hline Lasiocarpine & $10.9 \pm 1.6$ & Present study \\
& $19.5 \pm 4.6$ & (Hayes et al., 1984) \\
\hline Riddelliine & $6.3 \pm 0.9$ & Present study \\
\hline
\end{tabular}

\subsection{In vitro kinetic data}

Figure $4 \mathrm{a}$ and $\mathrm{b}$ shows the rate of depletion of the PAs in incubations with rat liver and intestinal microsomes with increasing concentrations of lasiocarpine or riddelliine. The results reveal that lasiocarpine and riddelliine are converted in a concentration-dependent manner. For each reaction, metabolism followed Michaelis-Menten kinetics. The apparent $\mathrm{V}_{\max }$ and $\mathrm{K}_{\mathrm{m}}$ values and the (scaled) catalytic efficiency $\left(\mathrm{V}_{\max } / \mathrm{K}_{\mathrm{m}}\right)$ for the depletion of lasiocarpine and riddelliine derived from these curves are presented in Table 3. The scaled in vivo catalytic efficiency (calculated as $\mathrm{V}_{\max } / \mathrm{K}_{\mathrm{m}}$ ) of liver for conversion of lasiocarpine and riddelliine by rat was 6.3 times and 107.8 times higher than the catalytic efficiency for conversion by intestine, respectively. The total scaled in vivo catalytic efficiency (sum of liver and intestine) for depletion of lasiocarpine was 11 times higher than that for riddelliine. The results reveal that in rat, lasiocarpine and riddelliine are especially converted in the liver, and that intestinal conversion contributes only to a minor extent.

The rate of depletion of riddelliine in incubations with mouse tissue samples and increasing concentrations of riddelliine is shown in Figure 4c. The (scaled) catalytic efficiencies $\left(\mathrm{V}_{\max } /\right.$ $\mathrm{K}_{\mathrm{m}}$ ) for the depletion of riddelliine in mouse tissue are also shown in Table 3. For mouse tissues, the scaled catalytic efficiency of the liver for conversion of riddelliine was 9.6 times higher than that of the intestine. The total catalytic efficiency for metabolic conversion of riddelliine by rat and mouse liver and intestine was comparable, being only 1.8 times higher in rat than in mouse. Incubations with lung and kidney rat and mouse tissue samples did not result in detectable conversion (data not shown). 

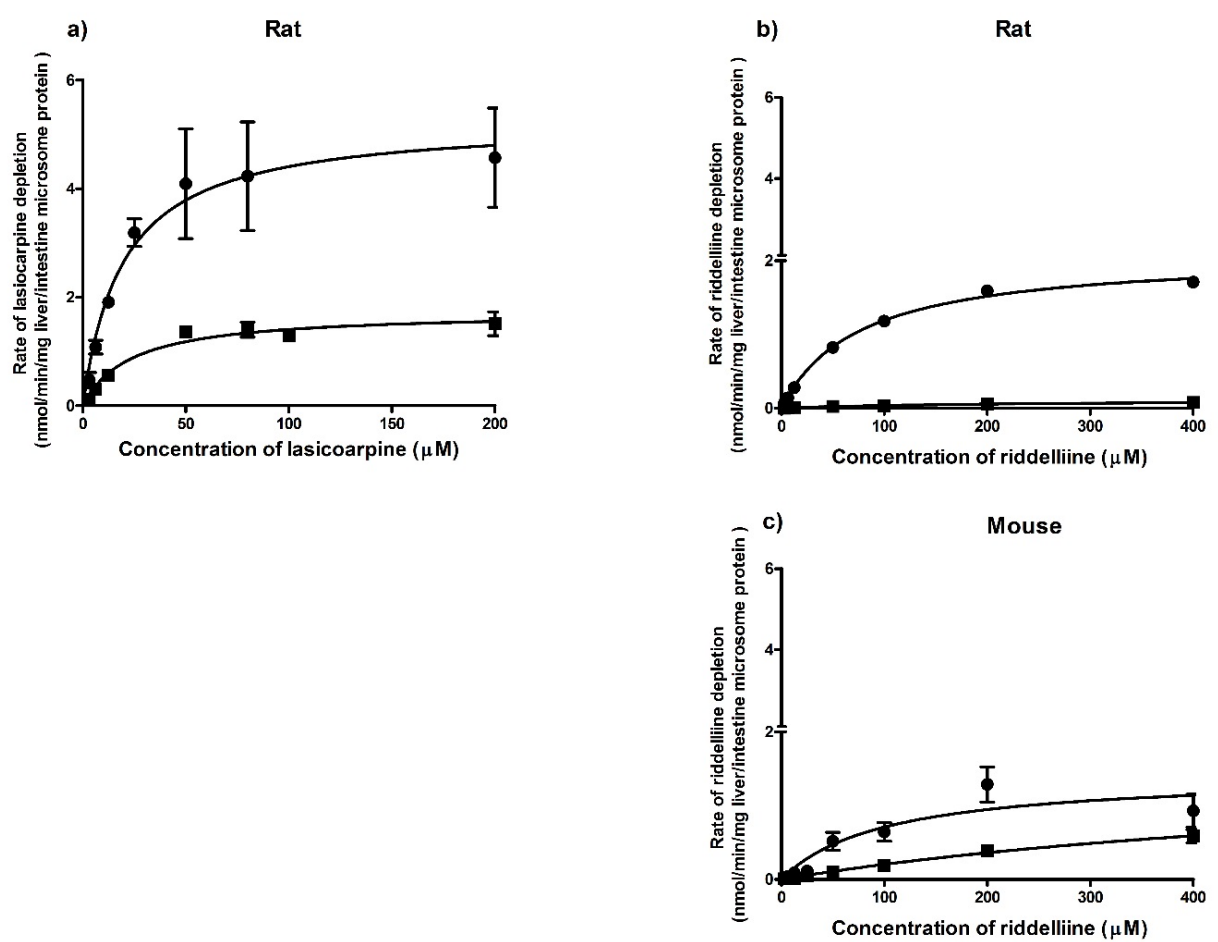

Fig. 4 Concentration-dependent rate of lasiocarpine (a) and riddelliine (b and c) depletion in incubations with rat liver (filled circle) and intestinal (filled square) microsomes, and mouse liver (filled circle) and intestinal (filled square) microsomes (c). Results represent data of three independent experiments. 
Table 3 Kinetic parameters for lasiocarpine and riddelliine depletion by pooled rat tissue fractions, and for riddelliine depletion by pooled mouse tissue fractions.

\begin{tabular}{|c|c|c|c|c|c|}
\hline $\begin{array}{l}\text { Species } \\
\text { Organ }\end{array}$ & $\begin{array}{c}\mathrm{V}_{\max } \\
(\mathrm{nmol} / \mathrm{min} / \mathrm{mg} \\
\text { microsomal } \\
\text { protein) }\end{array}$ & $\mathrm{K}_{\mathrm{m}}(\mu \mathrm{M})$ & $\begin{array}{c}\text { Catalytic } \\
\text { efficiencyb } \\
\text { (mL/min } / \mathrm{mg} \\
\text { microsomal } \\
\text { protein) }\end{array}$ & $\begin{array}{l}\text { Scaled } V_{\max ^{a}} \\
\text { (nmol/min } / \mathrm{g} \\
\text { tissue) }\end{array}$ & $\begin{array}{c}\text { Scaled } \\
\text { catalytic } \\
\text { efficiency } \\
\text { (mL/min } / g \\
\text { tissue) }\end{array}$ \\
\hline
\end{tabular}

\section{Rat}

\section{Lasiocarpine}

$\begin{array}{cccccc}\text { Liver } & 5.3 \pm 0.6 & 19.5 \pm 7.7 & 0.27 & 185.5 & 9.5 \\ \text { Intestine } & 1.7 \pm 0.1 & 23.4 \pm 6.3 & 0.07 & 35 & 1.5\end{array}$

\section{Riddelliine}

$\begin{array}{cccccc}\text { Liver } & 2.1 \pm 0.07 & 75.7 \pm 7.4 & 0.03 & 73.5 & 0.97 \\ \text { Intestine } & 0.1 \pm 0.02 & 221.0 \pm 71.2 & 0.0005 & 2.06 & 0.009\end{array}$

\section{Mouse}

\section{Riddelliine}

$\begin{array}{llllll}\text { Liver } & 1.4 \pm 0.3 & 101.3 \pm 49.9 & 0.01 & 49 & 0.48\end{array}$

$\begin{array}{llllll}\text { Intestine } & 1.6 \pm 0.5 & 695.8 \pm 342.7 & 0.002 & 33 & 0.05\end{array}$

a Scaled $\mathrm{V}_{\max }$ calculated from the in vitro $\mathrm{V}_{\max }$ based on a microsome protein yield of $35 \mathrm{mg} /$ (g liver) or $20.6 \mathrm{mg} /(\mathrm{g}$ small intestine)

b Catalytic efficiency expressed as $\mathrm{mL} / \mathrm{min} /(\mathrm{g}$ liver $)$ or $\mathrm{mL} / \mathrm{min} /(\mathrm{g}$ intestine $)$ is the ratio of scaled $\mathrm{V}_{\max }$ and $\mathrm{K}_{\mathrm{m}}$

\subsection{PBK model evaluation}

Due to unavailability of in vivo kinetic data for lasiocarpine, to evaluate the performance of the PBK models, the blood concentration-time curves of riddelliine as predicted by the developed PBK models were compared with the in vivo kinetic data from the literature on the blood concentration of riddelliine in rat and mouse exposed to riddelliine. Williams et al. (2002) reported riddelliine blood concentrations in rat and mouse that were orally exposed to $10 \mathrm{mg} / \mathrm{kg}$ bw of riddelliine, and from which blood samples were collected from 0.5-24 hours (Williams et al. 2002). Figure 5a shows that for the mouse model there is only 2- to 6.5- 
$10 \mathrm{mg} / \mathrm{kg}$ bw of riddelliine, and from which blood samples were collected from $0.5-24$ hours (Williams et al. 2002). Figure 5a shows that for the mouse model there is only 2- to 6.5(average $4.6 \pm 2.2$ ) fold difference at time points of $0.5,2,3,4,8,24$ hours between the predicted blood concentrations using the developed PBK model and the observed blood concentration of riddelliine upon a dose of $10 \mathrm{mg} / \mathrm{kg}$ bw in mouse (Williams et al. 2002). When comparing the predicted blood concentrations with in vivo blood concentrations of riddelliine upon a dose of $10 \mathrm{mg} / \mathrm{kg}$ bw in rat, the results showed that the predicted values are 0.9- to 23.7- (average $14.5 \pm 9.1$ ) fold higher at time points of $0.5,1,2,3,4,8,24$ hours than the observed blood concentrations. Further, the differences between the predicted and observed $\mathrm{C}_{\max }$ of riddelliine at a dose of $10 \mathrm{mg} / \mathrm{kg}$ bw, were 2-fold for mouse and 9.7-fold for rat (Figure 5). The predicted blood concentration-time curves of riddelliine in mouse using the two individual Ka values are shown in the Supplementary data (Figure S1). As shown in Figure $\mathrm{S} 1$, the two Ka values for riddelliine result in different predicted blood concentrationtime curves and in $\mathrm{C}_{\max }$ values that are 3.2- fold different.

In addition, the predicted AUC values were also calculated. The observed AUC were $516 \mathrm{ng}$ $\mathrm{h} / \mathrm{mL}$ for rat and $1307 \mathrm{ng} \mathrm{h} / \mathrm{mL}$ for mouse (Williams et al. 2002), while the predicted AUC values were $5323 \mathrm{ng} \mathrm{h} / \mathrm{mL}$ for rat and $6113 \mathrm{ng} \mathrm{h} / \mathrm{mL}$ for mouse, respectively. Thus, the predicted AUC for rat was 10.3-fold higher than the observed AUC, while the predicted AUC for mouse was 4.7-fold higher than the observed AUC.
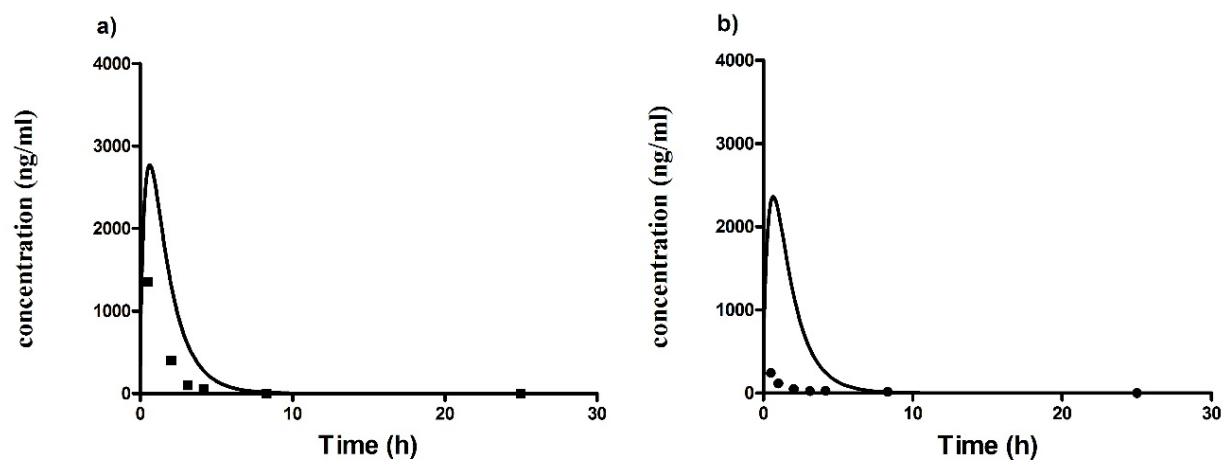

Fig. 5 PBK modelling-predicted time-dependent blood concentrations (black line) and reported time-dependent blood concentrations (filled squares) for riddelliine in mouse (a) or (filled circles) in rat (b) upon a dose of $10 \mathrm{mg} / \mathrm{kg}$ bw.

\subsection{Sensitivity analysis}

To further evaluate the performance of the developed models, a sensitivity analysis was performed to assess the parameters that affect the prediction of the $\mathrm{C}_{\max }$ of lasiocarpine and riddelliine in liver blood. The sensitivity analysis was performed at an oral dose of 0.008 and $0.144 \mu \mathrm{g} / \mathrm{kg}$ bw/day. These PA dose levels represent the reported lowest and the highest daily intake originating from use of herbal teas (BfR 2013). Only the parameters that result in a normalized sensitivity coefficient higher than 0.1 (in absolute value) are shown in Figure 6. The results obtained reveal that the fraction of liver volume, partition coefficient of liver, the 
uptake rate constant, the liver microsomal protein yield, the kinetic parameters $\left(\mathrm{V}_{\max }\right.$ and $\left.\mathrm{K}_{\mathrm{m}}\right)$ for depletion of lasiocarpine and riddelliine in the liver were the most sensitive parameters in the rat PBK models for lasiocarpine and riddelliine. Compared to the rat PBK for riddelliine, the parameters related to the intestine are more influential in the rat PBK model for lasiocarpine (Figure 6A). Comparison of the sensitivity analysis performed at 0.008 and 0.144 $\mu \mathrm{g} / \mathrm{kg} \mathrm{bw} /$ day reveals that the influence of all parameters was not dose-dependent in the rat PBK model for lasiocarpine and riddelliine (Figure 6).

\subsection{Translation of the in vitro concentration-response curves to in vivo dose-response curves, and application of PROAST modelling on predicted dose-response data to derive PoDs}

Figure 7 shows the predicted in vivo dose-response curves for liver toxicity of lasiocarpine and riddelliine in rat using PBK modelling-facilitated reverse dosimetry. The two predicted dose-response curves were derived from the in vitro cytotoxicity concentration-response curves obtained with rat hepatocytes (Figure 3) based on the $\mathrm{C}_{\max }$ assuming the in vitro concentration to be equal to the $\mathrm{C}_{\max }$ of unbound $\mathrm{PA}$ in liver blood. The $\mathrm{fub}_{\mathrm{ub}}$ of lasiocarpine and riddelliine in rat serum was estimated to be 0.64 and 0.66 , respectively. Table 4 displays the range of $\mathrm{BMDL}_{5}-\mathrm{BMDU}_{5}$ values derived from these predicted dose-response curves. The predicted $\mathrm{BMDL}_{5}-\mathrm{BMDU}_{5}$ values for lasiocarpine and riddelliine in rats were 23-34.4 and 4.9-8.4 mg/kg bw/day, respectively. When converting the concentration-response curves from Hayes et al. (1984) for rat hepatocytes depicted in Figure 3 and using the same PBK model, the predicted $\mathrm{BMDL}_{5}-\mathrm{BMDU}_{5}$ values for lasiocarpine were $34.5-72.1 \mathrm{mg} / \mathrm{kg}$ bw/day (Table 4). When using the individual Ka for prediction of the $\mathrm{BMDL}_{5}-\mathrm{BMDU}_{5}$ values, the predicted $\mathrm{BMDL}_{5}-\mathrm{BMDU}_{5}$ values for riddelliine in rat result in the ranges of 3.3-14.6 and 1.0-2.2 mg/ $\mathrm{kg}$ bw/day, respectively. 

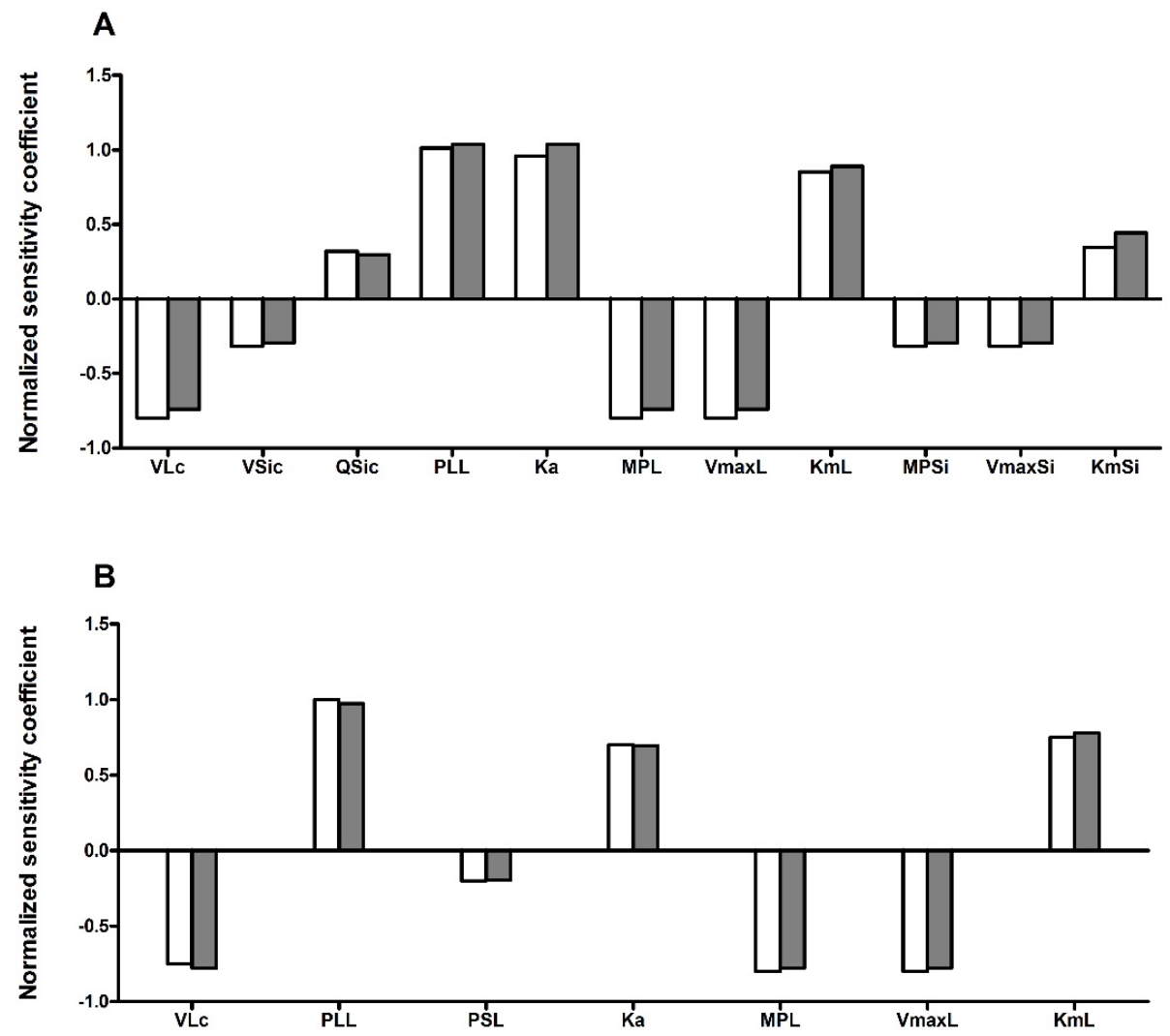

Fig. 6 Normalized sensitivity coefficients for the parameters of the rat PBK model for lasiocarpine (A) and riddelliine (B) on predicted $\mathrm{C}_{\max }$ in liver blood values at a single oral dose of $0.008 \mu \mathrm{g} / \mathrm{kg}$ bw/day (white bars) and $0.144 \mu \mathrm{g} / \mathrm{kg}$ bw/day (grey bars) PAs. VLc $=$ fraction of liver volume, $\mathrm{VSi}=$ fraction of small intestine volume, $\mathrm{QSi}=$ blood flow to small intestine, PLL = liver/blood partition coefficient, PSL = slowly perfused tissue/blood partition coefficient, $\mathrm{Ka}=$ uptake rate constant, $\mathrm{MPL}$ and $\mathrm{MPSi}=$ liver and small intestinal microsomal protein yield, $\mathrm{V}_{\max } \mathrm{L}$ and $\mathrm{K}_{\mathrm{m}} \mathrm{L}=$ the maximum rate of depletion and the Michaelis-Menten constant for depletion of lasiocarpine or riddelliine in liver, $\mathrm{V}_{\max } \mathrm{Si}$ and $\mathrm{K}_{\mathrm{m}} \mathrm{Si}$ = the maximum rate of depletion and the Michaelis-Menten constant for depletion of lasiocarpine or riddelliine in small intestine. 


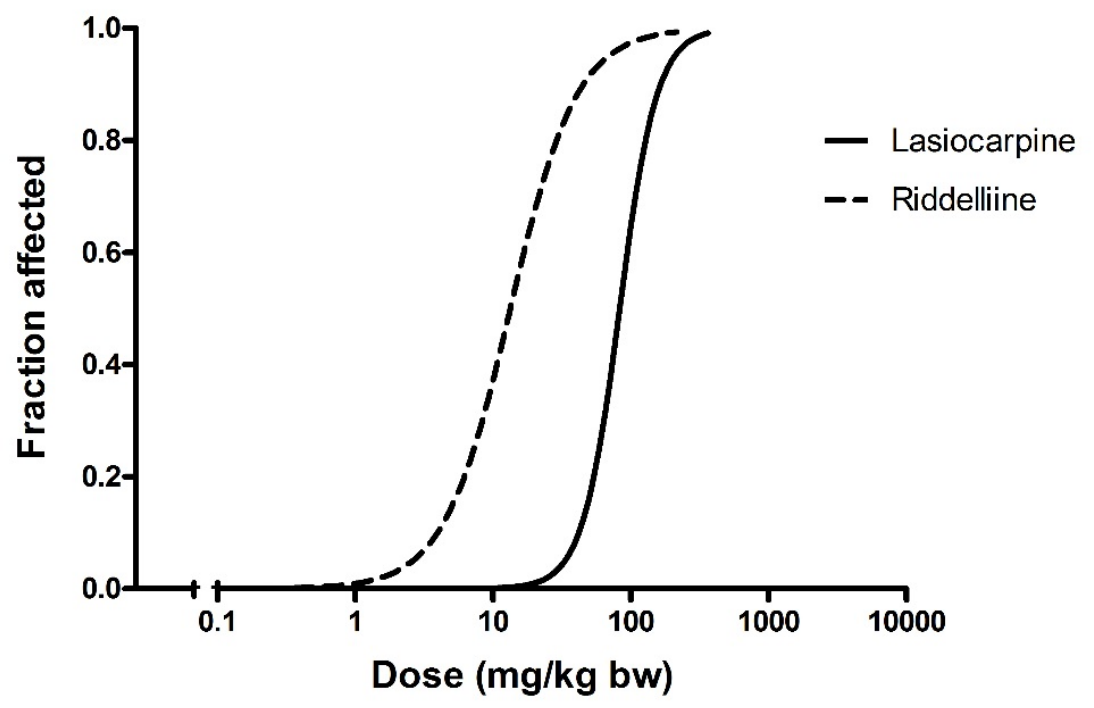

Fig. 7 Predicted in vivo dose-response curves for acute liver toxicity of lasiocarpine and riddelliine in rat. The solid line represents the predicted in vivo dose-response curves for lasiocarpine and the dashed line represents the predicted dose-response curve for riddelliine.

Table 4 Predicted BMDL5-BMDU 5 values derived from the dose-response curves predicted using PBK modelling-facilitated reverse dosimetry to convert the in vitro concentrationresponse curves as obtained in the present study and obtained from the literature (Figure 3) to in vivo dose-response curves.

\begin{tabular}{cccc}
\hline Compound & Cell model & $\begin{array}{c}\text { Predicted BMDL5-BMDU5 } \\
(\mathrm{mg} / \mathrm{kg} \text { bw/day })\end{array}$ & $\begin{array}{c}\text { Source in vitro } \\
\text { concentration-response } \\
\text { curve }\end{array}$ \\
\hline Lasiocarpine & Rat hepatocytes & $23.0-34.4$ & Present study \\
Riddelliine & Rat hepatocytes & $4.9-8.4$ & Present study \\
Lasiocarpine & Rat hepatocytes & $34.5-72.1$ & (Hayes et al. 1984) \\
\hline
\end{tabular}




\subsection{Evaluation of the predicted PoDS for in vivo liver toxicity}

Due to the absence of in vivo acute liver toxicity data reported in the literature for riddelliine, the predicted PoDs for in vivo liver toxicity could only be evaluated for lasiocarpine. To this end, the $\mathrm{BMDL}_{5}-\mathrm{BMDU}_{5}$ values presented in Table 4 were compared with in vivo reported data for acute liver toxicity of lasiocarpine as available in the literature (Dalefield et al. 2016; Jago 1970; Nolan et al. 1966). Table 5 presents an overview of literature data on acute liver toxicity of lasiocarpine in rat, measuring endpoints including megalocytosis, the mitotic index value, or the alanine amino transferase concentration in serum as a measure of acute liver toxicity. The data from these studies were not suitable for BMD analysis due to the limited number of data points and insufficient distribution of the data points over the dose-response curves. Therefore, the NOAEL values and/or the range of estimated NOAEL to LOAEL values that could be derived from these studies were taken as the PoDs (Table 5). When only the LOAEL was available, the NOAEL was calculated using the LOAEL divided by a factor of 10 (Barnes et al. 1988).

Figure 8 shows a comparison of the predicted range of BMDL5-BMDU5 values for in vivo liver toxicity of lasiocarpine and the PoDs derived from in vivo literature rat data for liver toxicity of lasiocarpine. The literature values for single oral exposure are the ones that can best be compared to the values predicted in the present study. Values for IP or oral repeated exposure appear to be somewhat lower probably because of these different dose regimes. The predicted $\mathrm{BMDL}_{5}-\mathrm{BMDU}_{5}$ values derived from the present study appear to fall well within the range of PoDs obtained from the available oral single dose in vivo studies. The BMDL5, a possible PoD for evaluation of acute toxicity of PAs, determined from predicted concentration-response curves was 1.9- to 2.9-fold higher than the NOAELs derived as corresponding PoD from the experimental data. The BMDU 5 predicted in the present study was 2.3- to 3.5-fold lower compared to the LOAELs derived as corresponding PoD from the in vivo studies. The results indicate that the data and range of $\mathrm{PoD}$ values derived using our combined in vitro-PBK modelling approach are a good approximation to the in vivo acute liver toxicity of lasiocarpine. 


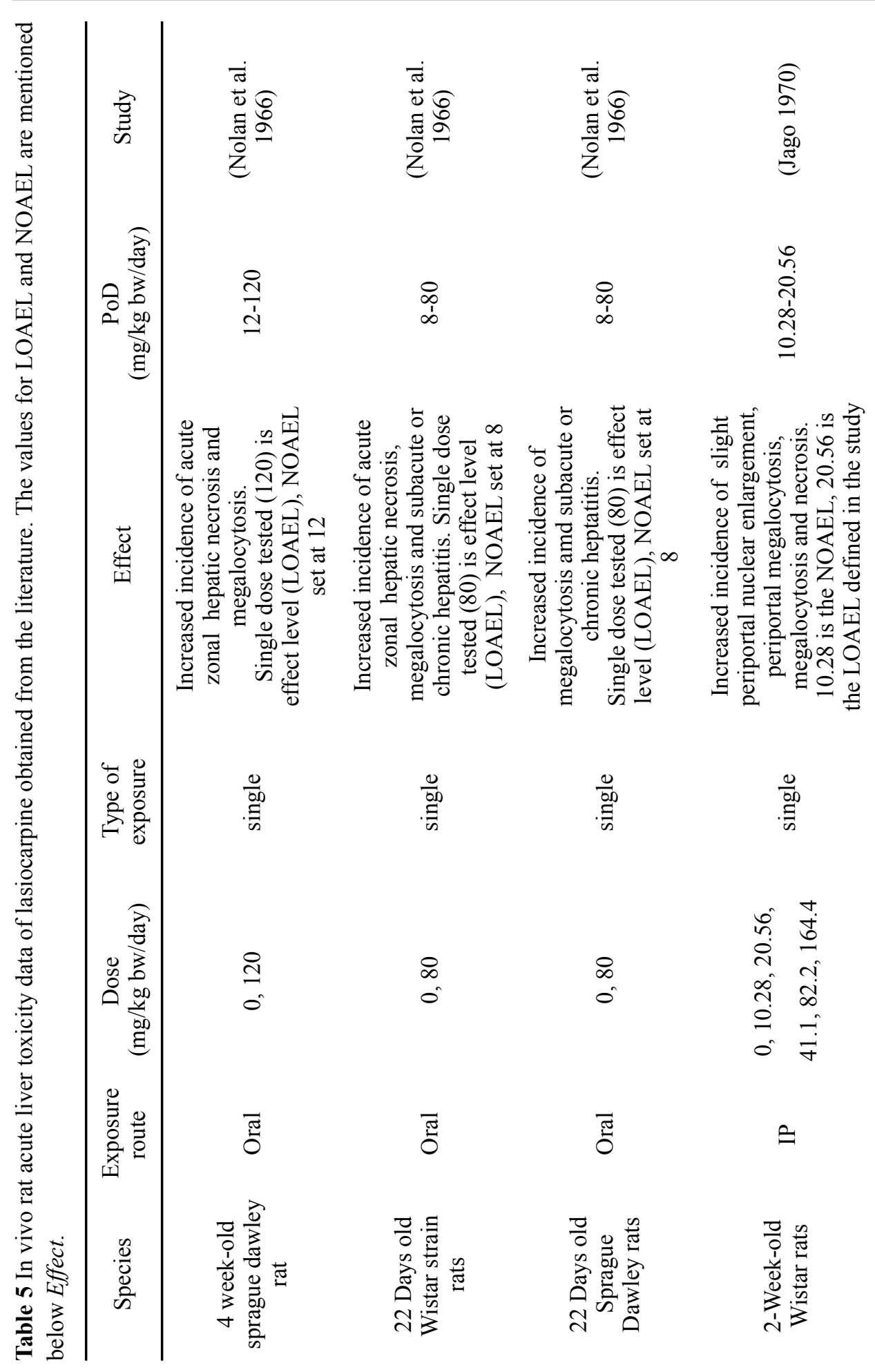




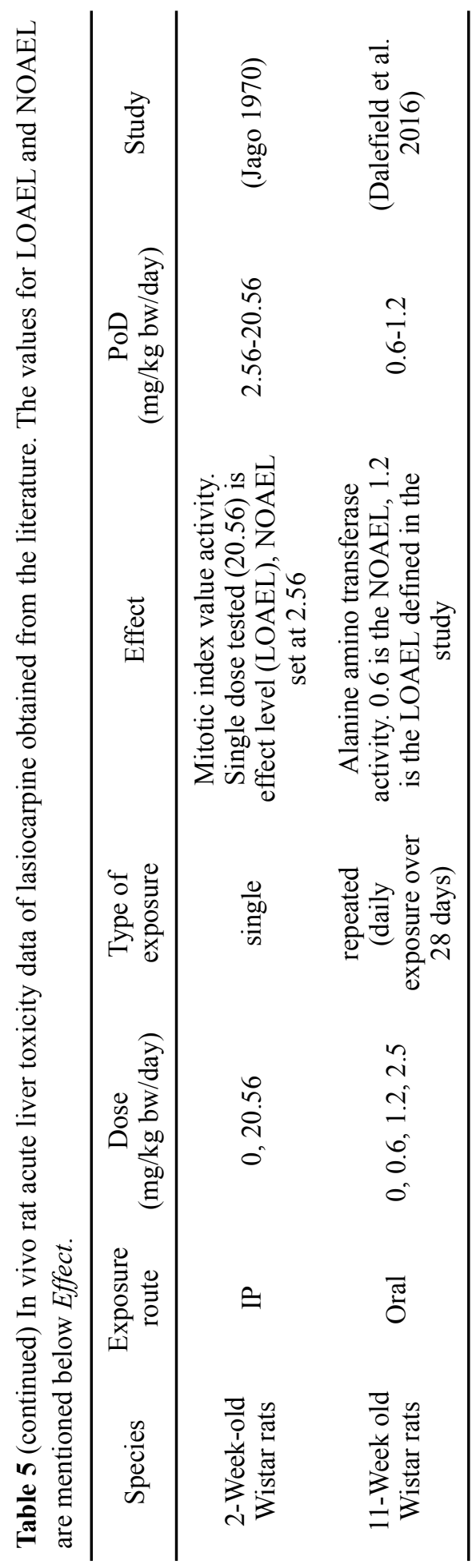




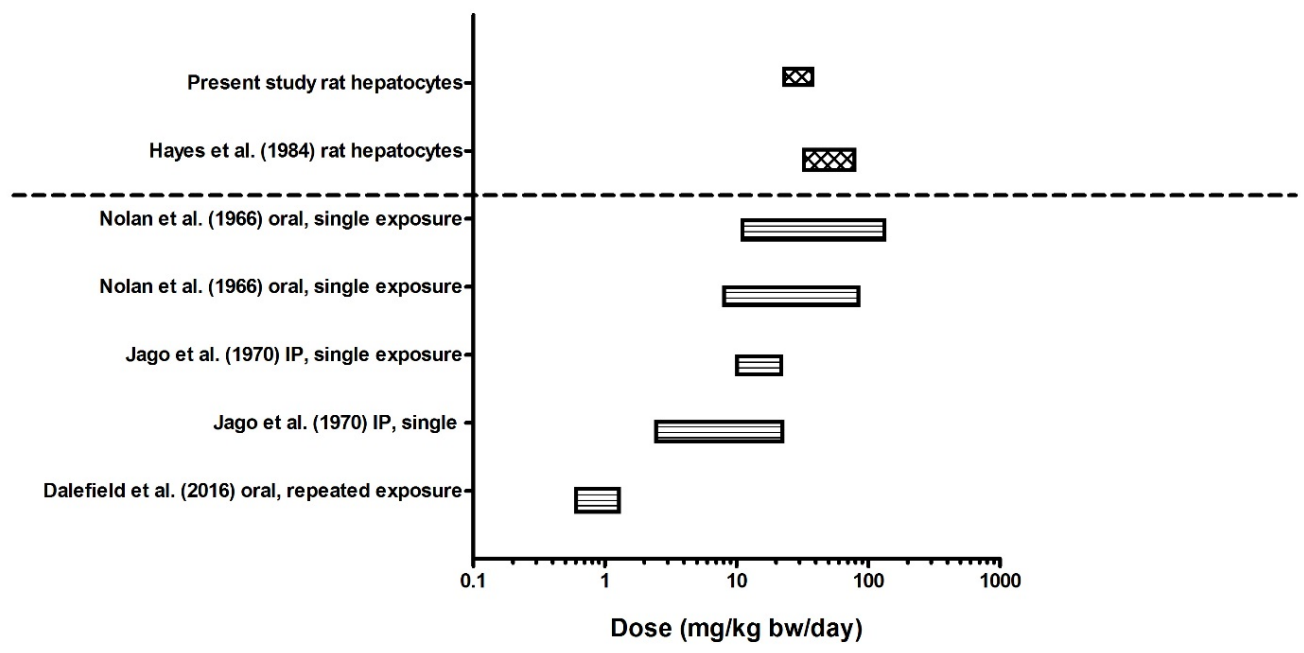

Fig. $8 \mathrm{BMDL}_{5}-\mathrm{BMDU}_{5}$ values for acute liver toxicity of lasiocarpine in rat predicted by the PBK modelling-facilitated reverse dosimetry approach using data for rat hepatocytes from the present study and literature data for rat hepatocytes (rectangle bars filled with grid) as compared to comparable PoDs (rectangle bars filled with horizontal lines) derived from in vivo liver toxicity studies as shown in Tabls 5 upon oral or IP exposure. Range of estimated PoD from in vivo data $=$ estimated NOAEL to LOAEL.

\section{Discussion}

The aim of present study was to assess whether a physiologically based kinetic (PBK) modelling-facilitated reverse dosimetry approach could adequately predict in vivo doseresponse curves for hepatotoxicity of PAs in rats. Especially 1,2-unsaturated PAs are of concern because of their hepatotoxicity and carcinogenicity. However, of the large group of 1,2-unsaturated PAs in vivo data for liver toxicity are only available for lasiocarpine (Dalefield et al. 2016; Jago 1970; Nolan et al. 1966). A method to estimate liver toxicity for related PAs using read-across from lasiocarpine based on quantitative insight into the relative level of their bioactivation and detoxification would enable priority setting in the further evaluation of the toxicity of PAs for which no toxicity data are availabe. To facilitate this approach the aim of the present study was to develop a PBK model for PAs and use this model to generate a proof-of-principle that liver toxicty of the model PA lasiocarpine can be adequately predicted by this comined in silico-in vitro alternative tetsing strategy.

In the present study, the in vitro concentration-response curves of lasiocarpine and riddelliine in primary rat hepatocytes were used as the in vitro toxicity data. Riddelliine and lasiocarpine were selected as the model PAs, because for riddelliine data on dose- and time-dependent blood levels in mouse and rats were available enabling evaluation of the PBK models, while for lasiocarpine in vivo data for liver toxicity were available, enabling valuation of the final prediction of the in vivo liver toxicity. Primary rat liver hepatocytes were selected as the in vitro model because they are expected to best reflect the intrinsic sensitivity and metabolism 
of liver cells in vivo. Furthermore, hepatocytes were used before to characterise in vitro liver toxicity of lasiocarpine (Hayes et al. 1984; Moore et al. 1989). The results for the in vitro cytotoxicity of lasiocarpine in rat primary hepatocytes obtained in the present study were comparable to the literature data (Hayes et al. 1984).

The PBK models developed could be evaluated based on in vivo kinetic data available for riddelliine (Williams et al. 2002). The predicted riddelliine blood concentrations by the PBK model for mouse were 2- to 6.5 - (average $4.6 \pm 2.2$ ) fold different from those reported by Williams et al. (2002) and for the rat PBK model the predicted blood concentrations were 0.9to 23.7- (average 14.5 \pm 9.1) fold different from those reported by Williams et al. (2002). In a subsequent step, the PBK models were used to convert the in vitro cytotoxicity data for lasiocarpine and riddelliine to in vivo dose-response curves for liver toxicity, enabling to assess the validity and use of the PBK model for reverse dosimetry, by comparison of the predicted toxicity data to available in vivo toxicity data for lasiocarpine. The predicted $\mathrm{BMDL}_{5}-\mathrm{BMDU}_{5}$ values derived from the present study appeared to fall well within the range of PoDs obtained from the available oral single dose in vivo studies. The results indicate that the data and range of PoD values derived using our combined in vitro-in silico PBK modelling approach are a good approximation of the in vivo acute liver toxicity of lasiocarpine.

In addition, the $\mathrm{BMDL}_{5}-\mathrm{BMDU}_{5}$ values predicted for riddelliine were 2.7 to 7.0 times lower than those values predicted for lasiocarpine. This indicates that in vivo riddelliine is predcited to induce liver toxicity at lower dose levels than lasiocarpine. In line with this, the in vitro cytotoxicity of riddelliine was also higher than that of lasiocarpine. The higher in vivo toxicity of ridelliine may in part be due to the fact that there are differences in kinetics which revealed a slower clearance of riddelliine compared to lasiocarpine. Given all the results above, it is concluded that the developed PBK modeling-facilitated reverse dosimetry approach provides a promising method to predict acute liver toxicity in vivo for lasiocarpine, and related PAs. Based on the results of the sensitivity analysis (Figure 6), the model preditions are highly influenced by the kinetic constant Ka for uptake of the PAs in the small intestine. In current study, the predicted $\mathrm{BMDL}_{5}-\mathrm{BMDU}_{5}$ values derived when using the average $\mathrm{Ka}$ value fall within the ranges of predicted $\mathrm{BMDL}_{5}-\mathrm{BMDU}_{5}$ values using the individual Ka values. Nevertheless the method used to determine the $P_{\text {app }}$ value and resulting Ka for intestinal uptake of PAs may need further refinement.

In addition it is also of interest to compare the PoDs predicted for acute oral toxicity in rats, to what can be derived from reported cases on actue toxicity in humans. This includes for example cases in Afghanistan in 1976 (Mohabbat et al. 1976), in Jamaica in 1954 (Bras et al. 1954), and in India in 1975 (Tandon et al. 1976). In fact, these reports reveal that during the past century, a lot of acute PAs poisoning cases took place in central Asia, while other cases have been reported for other countries with poorly developed agricultural systems (Dharmananda 2001). Culvenor estimated the dose and duration of exposure to the toxic PAs that may lead to liver toxicity in humans (Culvenor 1983) and concluded that an intake of 0.7$1.5 \mathrm{mg} / \mathrm{kg} \mathrm{bw} /$ day for riddelliine for 2 weeks can induce hepatotoxicity. The predicted 
$\mathrm{BMDL}_{5}-\mathrm{BMDU}_{5}$ value for acute liver toxicity of riddelliine in rats reported in the present study amounted to $4.9-8.4 \mathrm{mg} / \mathrm{kg}$ bw/day. Comparison of these values suggests that humans might be more sensitive than rats, although it should be kept in mind that the human study was based on more than a single exposure and that estimation of dose levels in human case studies is generally not acurate. In a next step, the PBK modelling based approach developed in the present study will be modified to predict human liver toxicity using human hepatocytes and the PBK model modified to describe human kinetics.

In conclusion, we showed that PBK modelling-facilitated reverse dosimetry can translate in vitro concentration-response curves to in vivo dose-response curves for acute liver toxicity of lasiocarpine and riddelliine. BMDL5-BMDU 5 values could be derived that were in line with available in vivo data. This study provides another proof- of-principle for integrating in vitro toxicity data and PBK modelling-facilitated reverse dosimetry to obtain a promising alternative testing strategy in risk and safety evaluation of chemicals.

\section{Acknowledgements}

This work was funded by a grant from the China Scholarship Council to Lu Chen (grant No. 201508650023).

The riddelliine was kindly provided by Patrick P.J. Mulder from RIKILT Wageningen University \& Research, Wageningen, The Netherlands. 


\section{References}

Abdullah R, Alhusainy W, Woutersen J, Rietjens IMCM, Punt A (2016) Predicting points of departure for risk assessment based on in vitro cytotoxicity data and physiologically based kinetic (PBK) modeling: The case of kidney toxicity induced by aristolochic acid I. Food Chemical Toxicol 92:104-116

Barnes DG, Dourson M, Dourson M, Preuss P, Barnes DG, Bellin J, Derosa C, Engler R, Erdreich L, Farber T, Fenner-Crisp P, Francis E, Ghali G, Hill R, Irene S, Marcus W, Patrick D, Perlin S, Revesz A, Rubenstein R, Stara J, Wiltse J, Zaragosa L (1988) Reference dose (RfD): Description and use in health risk assessments. Regul Toxicol Pharmacol 8:471-486

Bundesinstitut für Risikobewertung (BfR) (2013) pyrrolizidine alkaloids in herbal teas and teas.

Bovee TF, Helsdingen RJ, Hoogenboom RL, de Nijs MW, Liu X, Vrieling K, Klinkhamer PG, Peijnenburg AA, Mulder PP (2015) Are effects of common ragwort in the Ames test caused by pyrrolizidine alkaloids? Mutat Res 778:1-10

Bras G, Jelliffe DB, Stuart KL (1954) Veno-occlusive disease of liver with nonportal type of cirrhosis occurring in Jamaica. Arch Pathol 57:285-300

Brown RP, Delp MD, Lindstedt SL, Rhomberg LR, Beliles RP (1997) Physiological Parameter Values for Physiologically Based Pharmacokinetic Models. Toxicol Ind Health 13:407-484

Bull LB, Culvenor Ct, Dick A (1968) The pyrrolizidine alkaloids: their chemistry pathogenicity and other biological properties. The Quarterly Review of Biology 45 no 1 (Mar 1970):67

Cubitt HE, Houston JB, Galetin A (2009) Relative importance of intestinal and hepatic glucuronidation -impact on the prediction of drug clearance. Pharm Res 26:1073-1083

Chan P (1993) NTP technical report on the toxicity studies of Riddelliine (CAS No 23246-96-0) Administered by Gavage to F344 Rats and B6C3F1 Mice. Toxic Rep Ser 27:1-D9

Chan PC, Haseman JK, Prejean J, Nyska A (2003) Toxicity and carcinogenicity of riddelliine in rats and mice. Toxicol Lett 144:295-311

Chou MW, Jian Y, Williams LD, Xia Q, Churchwell M, Doerge DR, Fu PP (2003) Identification of DNA adducts derived from riddelliine a carcinogenic pyrrolizidine alkaloid. Chem Res Toxicol 16:1130-1137

Culvenor C (1983) Estimated intakes of pyrrolizidine alkaloids by humans A comparison with dose rates causing tumors in rats. J Toxicol Environ Health Part A Current Issues 11:625-635

Dalefield RR, Gosse MA, Mueller U (2016) A 28-day oral toxicity study of echimidine and lasiocarpine in Wistar rats. Regul Toxicol Pharmacol 81:146-154

DeJongh J, Verhaar HJ, Hermens JL (1997) A quantitative property-property relationship (QPPR) approach to estimate in vitro tissue-blood partition coefficients of organic chemicals in rats and humans. Arch Toxicol 72:17-25

Dharmananda S (2001) Safety issues affecting herbs: pyrrolizidine alkaloids 
European Food Safety Authority (EFSA) (2011) Scientific opinion on pyrrolizidine alkaloids in food and feed. EFSA J 9:1-134

Evans MV, Andersen ME (2000) Sensitivity analysis of a physiological model for 2, 3, 7, 8tetrachlorodibenzo-p-dioxin (TCDD): assessing the impact of specific model parameters on sequestration in liver and fat in the rat. Toxicol Sci 54:71-80

Fu PP (2017) Pyrrolizidine Alkaloids: Metabolic activation pathways leading to liver tumor initiation. Chem Res Toxicol 30:81-93

Fu PP, Xia Q, Lin G, Chou MW (2002) Genotoxic pyrrolizidine alkaloids-mechanisms leading to DNA adduct formation and tumorigenicity. Int J Mol Sci 3:948-964

Fu PP, Xia Q, Lin G, Chou MW (2004) Pyrrolizidine alkaloids-genotoxicity metabolism enzymes metabolic activation and mechanisms. Drug Metab Rev 36:1-55

Hayes MA, Roberts E, Jago MV, Safe SH, Farber E, Cameron RC (1984) Influences of various xenobiotic inducers on cytocidal toxicity of lasiocarpine and senecionine in primary cultures of rat hepatocytes. J Toxicol Environ Health 14:683-694

Hirono I, Haga M, Fujii M, Matsuura S, Matsubara N, Nakayama M, Furuya T, Hikichi M, Takanashi H, Uchida E (1979) Induction of hepatic tumors in rats by senkirkine and symphytine. J Natl Cancer Inst 63:469-472

Hirono I, Mori H, Culvenor CC (1976) Carcinogenic activity of coltsfoot Tussilago farfara L. Jpn J Cancer Res 67:125-129

Hirono I, Mori H, Yamada K, Hirata Y, Haga M, Tatematsu H, Kanie S (1977) Carcinogenic activity of petasitenine a new pyrrolizidine alkaloid isolated from Petasites japonicus Maxim. J Natl Cancer Inst 58:1155-1157

Hirono I, Haga M, Fujii M, Matsuura S, Matsubara N, Nakayama M, Furuya T, Hikichi M, Takanashi H, Uchida E, Hosaka S, Ueno I (1979) Induction of Hepatic Tumors in Rats by Senkirkine and Symphytine. JNCI 63:469-472

Hou TJ, Zhang W, Xia K, Qiao XB, Xu XJ (2004) ADME evaluation in drug discovery 5 Correlation of Caco-2 permeation with simple molecular properties. J Chem Inf Comput Sci 44:1585-1600

International Agency for Research on Cancer (IARC) (1976) IARC Monographs on Evaluation of Carcinogenic Risk of chemicals to Man: Some Naturally Occuring Substances 10

International Agency for Research on Cancer (IARC) (2002) Some traditional herbal medicines some mycotoxins naphthalene and styrene IARC monographs on the evaluation of carcinogenic risks to humans 82

Jago MV (1970) A method for the assessment of the chronic hepatoxicity of pyrrolizidine alkaloids. Immunol Cell Biol 48:93-103

Kuhara K, Takanashi H, Hirono I, Furuya T, Asada Y (1980) Carcinogenic activity of clivorine a pyrrolizidine alkaloid isolated from Ligularia dentata. Cancer Lett 10:117-122 
Kumana CR, Ng M, Lin HJ, Ko W, Wu PC, Todd D (1985) Herbal tea induced hepatic veno-occlusive disease: quantification of toxic alkaloid exposure in adults. Gut 26:101-104

Li H, Zhang M, Vervoort J, Rietjens IMCM, van Ravenzwaay B, Louisse J (2017) Use of physiologically based kinetic modeling-facilitated reverse dosimetry of in vitro toxicity data for prediction of in vivo developmental toxicity of tebuconazole in rats. Toxicol Lett 266:85-93

Li N, Xia Q, Ruan J, Fu PP, Lin G (2011) Hepatotoxicity and tumorigenicity induced by metabolic activation of pyrrolizidine alkaloids in herbs. Curr Drug Metab 12:823-834

Lin G, Cui YY, Hawes EM (1998) Microsomal formation of a pyrrolic alcohol glutathione conjugate of clivorine. Drug Metab Dispos 26:181-184

Lin G, Cui YY, Hawes EM (2000) Characterization of rat liver microsomal metabolites of clivorine an hepatotoxic otonecine-type pyrrolizidine alkaloid. Drug Metab Dispos 28:1475-1483

Lin G, Wang JY, Li N, Li M, Gao H, Ji Y, Zhang F, Wang H, Zhou Y, Ye Y (2011) Hepatic sinusoidal obstruction syndrome associated with consumption of Gynura segetum. J Hepatol 54:666-673

Louisse J, Bosgra S, Blaauboer BJ, Rietjens IMCM, Verwei M (2015) Prediction of in vivo developmental toxicity of all-trans-retinoic acid based on in vitro toxicity data and in silico physiologically based kinetic modeling. Arch Toxicol 89:1135-1148

Louisse J, de Jong E, van de Sandt JJ, Blaauboer BJ, Woutersen RA, Piersma AH, Rietjens IMCM, Verwei M (2010) The use of in vitro toxicity data and physiologically based kinetic modeling to predict dose-response curves for in vivo developmental toxicity of glycol ethers in rat and man. Toxicol Sci $118: 470-484$

Mattocks A (1986) Chemistry and toxicology of pyrrolizidine alkaloids. London: Academic Press

Medinsky MA, Leavens TL, Csanády GA, Gargas ML, Bond JA (1994) In vivo metabolism of butadiene by mice and rats: a comparison of physiological model predictions and experimental data. Carcinogenesis 15:1329-1340

Miranda CL, Reed RL, Guengerich FP, Buhler DR (1991) Role of cytochrome P450IIIA4 in the metabolism of the pyrrolizidine alkaloid senecionine in human liver. Carcinogenesis 12:515-519

Mohabbat O, Younos MS, Merzad A, Srivastava R, Sediq GG, Aram G (1976) An outbreak of hepatic veno-occlusive disease in north-western Afghanistan. The Lancet 308:269-271

Moore DJ, Batts KP, Zalkow LL, Fortune GT Jr, Powis G (1989) Model systems for detecting the hepatic toxicity of pyrrolizidine alkaloids and pyrrolizidine alkaloid N-oxides. Toxicol Appl Pharmacol 101:271-284

Mori H, Sugie S, Yoshimi N, Asada Y, Furuya T, Williams GM (1985) Genotoxicity of a variety of pyrrolizidine alkaloids in the hepatocyte primary culture-DNA repair test using rat mouse and hamster hepatocytes. Cancer Res 45:3125-3129

Nolan JP, Scheig RL, Klatskin G (1966) Delayed hepatitis and cirrhosis in weanling rats following a single small dose of the senecio alkaloid lasiocarpine. Am J Pathol 49:129-151 
National Toxicology Program (NTP) (1978) Bioassay of lasiocarpine for possible carcinogenicity. Natl Cancer Inst Carcinog Tech Rep Ser 39:1-66

National Toxicology Program (NTP) (2003) Toxicology and carcinogenesis studies of riddelliine (CAS No 23246-96-0) in F344/N rats and B6C3F1 mice (gavage studies). Natl Toxicol Program Tech Rep Ser 508:1-280

Prakash AS, Pereira TN, Reilly PE, Seawright AA (1999) Pyrrolizidine alkaloids in human diet. Mutat Res Genet Toxicol Environ Mutagen 443:53-67

Roulet M, Laurini R, Rivier L, Calame A (1988) Hepatic veno-occlusive disease in newborn infant of a woman drinking herbal tea. J Pediatr 112:433-436

Schoental R (1970) Hepatotoxic activity of retrorsine senkirkine and hydroxysenkirkine in newborn rats and the role of epoxides in carcinogenesis by pyrrolizidine alkaloids and aflatoxins. Nature 227:401-402

Schoental R, Bensted J (1963) Effects of whole body irradiation and of partial hepatectomy on the liver lesions induced in rats by a single dose of retrorsine a pyrrolizidine (Senecio) alkaloid. Br J Cancer $17: 242$

Schoental R, Head MA, Peacock P (1954) Senecio alkaloids: primary liver tumours in rats as a result of treatment with (1) a mixture of alkaloids from S jacobaea Lin;(2) retrorsine;(3) isatidine. Br J Cancer $8: 458$

Shumaker R, Robertson K, Hsu I, Allen J (1976) Neoplastic transformation in tissues of rats exposed to monocrotaline or dehydroretronecine. J Natl Cancer Inst 56:787-790

Sperl W, Stuppner H, Gassner I, Judmaier W, Dietze O, Vogel W (1995) Reversible hepatic venoocclusive disease in an infant after consumption of pyrrolizidine-containing herbal tea. Eur J Pediatr 154:112-116

Stegelmeier B, Edgar J, Colegate S, Gardner D, Schoch T, Coulombe R, Molyneux R (1999) Pyrrolizidine alkaloid plants metabolism and toxicity. J Nat Toxins 8:95-116

Strikwold M, Spenkelink B, de Haan LH, Woutersen RA, Punt A, Rietjens IMCM (2017) Integrating in vitro data and physiologically based kinetic (PBK) modelling to assess the in vivo potential developmental toxicity of a series of phenols. Arch Toxicol 91:2119-2133

Tandon BN, Tandon H, Tandon R, Narndranathan M, Joshi Y (1976) An epidemic of veno-occlusive disease of liver in central India. The Lancet 308:271-272

van Liempd S, Morrison D, Sysmans L, Nelis P, Mortishire-Smith R (2011) Development and validation of a higher-throughput equilibrium dialysis assay for plasma protein binding. J Lab Autom 16:56-67

Waters NJ, Jones R, Williams G, Sohal B (2008) Validation of a rapid equilibrium dialysis approach for the measurement of plasma protein binding. J Pharm Pharm Sci 97:4586-4595

White IN (1976) The role of liver glutathione in the acute toxicity of retrorsine to rats. Chem Biol Interact 13:333-342

Wiedenfeld H (2011) Plants containing pyrrolizidine alkaloids: toxicity and problems. Food Addit Contam: Part A 28:282-292 
Wiedenfeld H (2013) Alkaloids derived from ornithine: pyrrolizidine alkaloids. Natural Products 359-379

Wiedenfeld H, Edgar J (2011) Toxicity of pyrrolizidine alkaloids to humans and ruminants. Phytochem Rev 10:137-151

Williams L, Chou MW, Yan J, Young JF, Chan PC, Doerge DR (2002) Toxicokinetics of riddelliine a carcinogenic pyrrolizidine alkaloid and metabolites in rats and mice. Toxicol Appl Pharmacol 182:98-104

Yan CC, Huxtable RJ (1995a) The effect of the pyrrolizidine alkaloids monocrotaline and trichodesmine on tissue pyrrole binding and glutathione metabolism in the rat. Toxicon 33:627-634

Yan CC, Huxtable RJ (1995b) Relationship between glutathione concentration and metabolism of the pyrrolizidine alkaloid monocrotaline in the isolated perfused liver. Toxicol Appl Pharmacol 130:132-139

Yan J, Nichols J, Yang YC, Fu PP, Chou MW (2002) Detection of riddelliine-derived DNA adducts in blood of rats fed riddelliine. Int J Mol Sci 3:1019-1026 


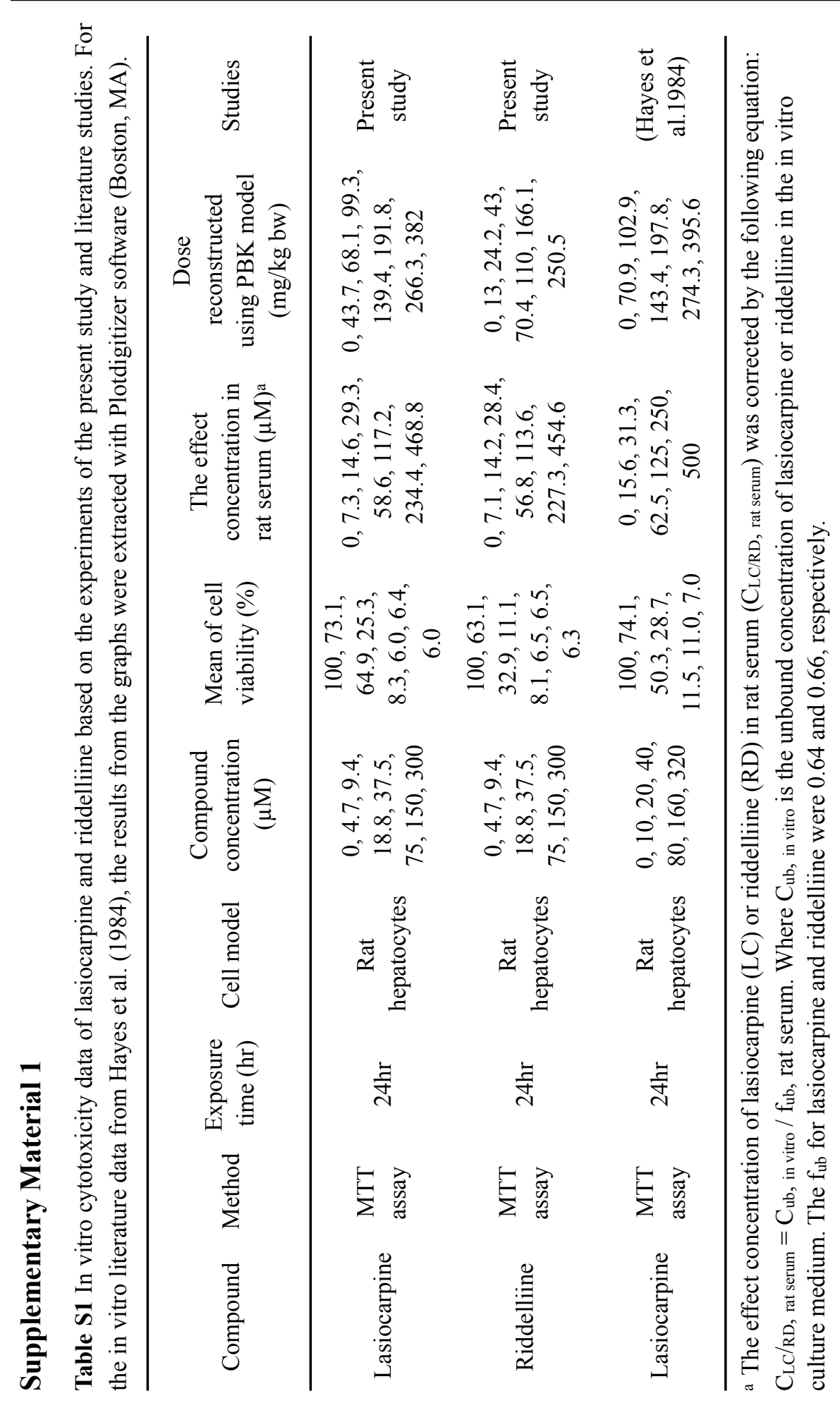



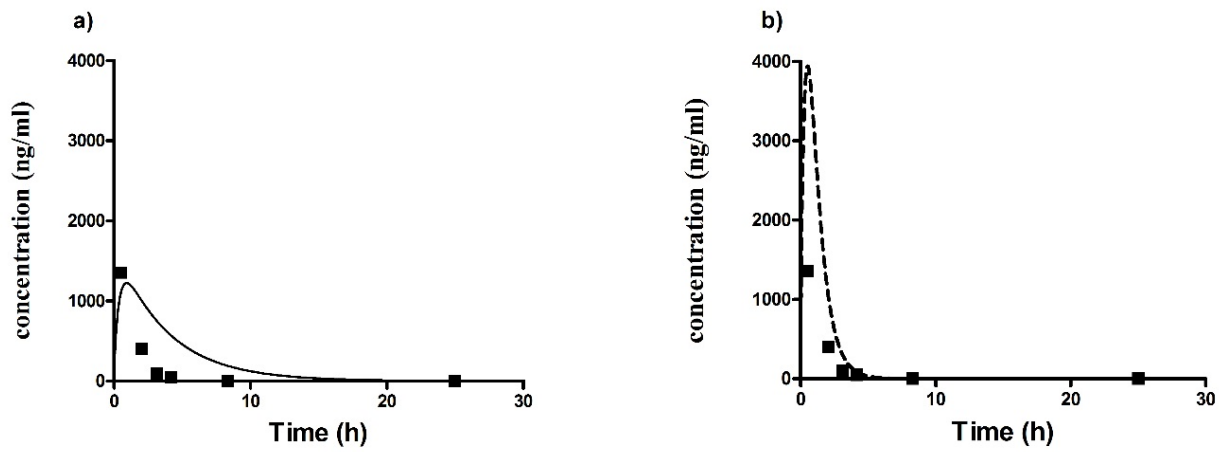

Fig. S1 Reported time-dependent blood concentrations (filled square) for riddelliine in mouse and PBK modelling-predicted time-dependent blood concentrations when using Ka value of $0.27 / \mathrm{h}$ (black line) (a) or using Ka value of $1.17 / \mathrm{h}$ (dotted line) upon a dose of $10 \mathrm{mg} / \mathrm{kg}$ bw. 



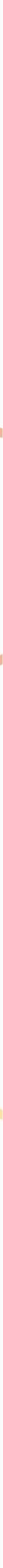



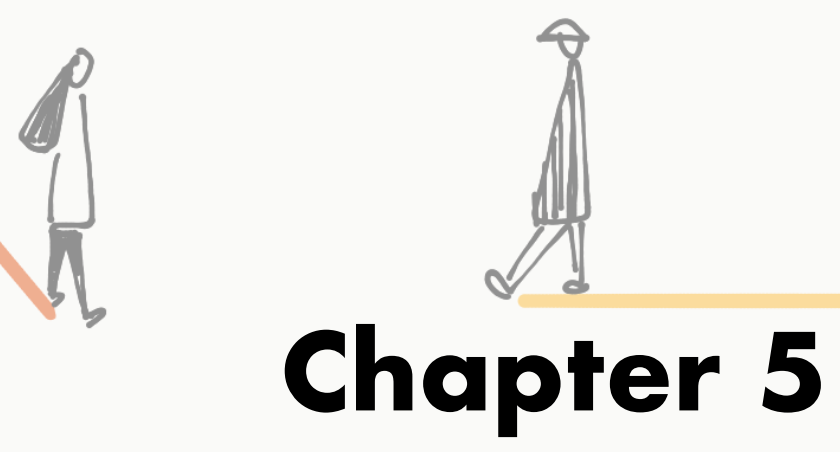

Use of an in vitro-in silico testing strategy to predict inter-species and inter-ethnic human differences in liver toxicity of the pyrrolizidine Calkaloids lasiocarpine and riddelliine
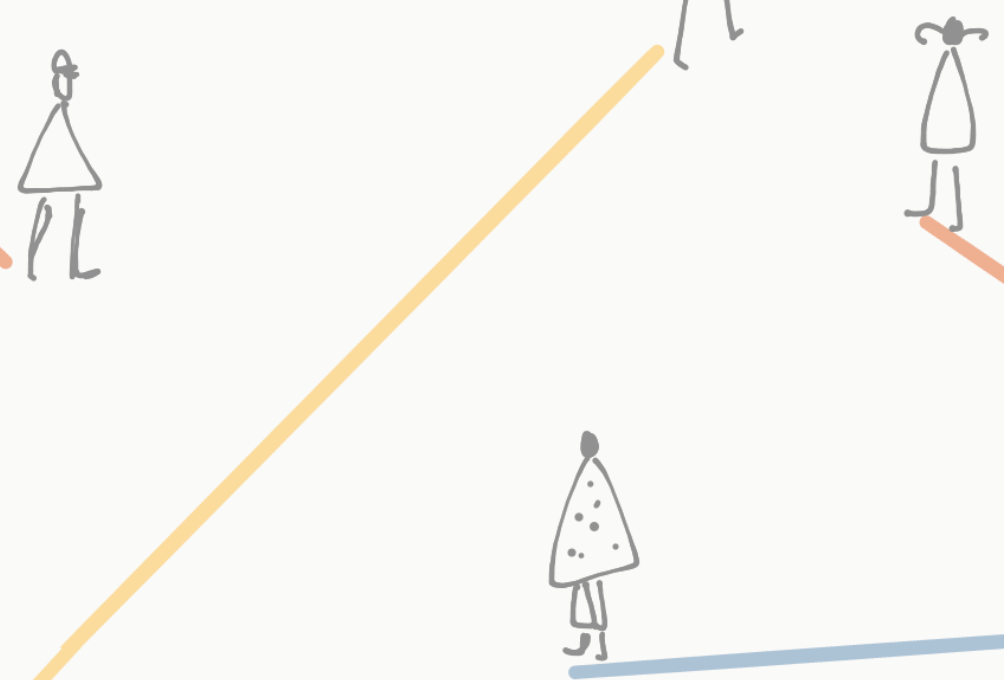

Jia Ning, Lu Chen, Marije Strikwold, Jochem Louisse, Sebas Wesseling, Ivonne M.C.M. Rietjens

Published in: Archives of Toxicology (2019) 93 (3), 801-818 


\begin{abstract}
Lasiocarpine and riddelliine are pyrrolizidine alkaloids (PAs) known to cause liver toxicity. The aim of this study was to predict the inter-species and inter-ethnic human differences in acute liver toxicity of lasiocarpine and riddelliine using physiologically based kinetic (PBK) modelling-based reverse dosimetry of in vitro toxicity data. The concentration-response curves of in vitro cytotoxicity of lasiocarpine and riddelliine defined in pooled human hepatocytes were translated to in vivo dose-response curves by PBK models developed using kinetic data obtained from incubations with pooled tissue fractions from Chinese and Caucasian individuals, providing PBK models for the average Chinese and average Caucasian, respectively. From the predicted in vivo dose-response curves, the benchmark dose lower and upper confidence limits for $5 \%$ effect (BMDL5 and BMDU 5 ) were derived and subsequently compared to those previously obtained in rat to evaluate inter-species differences. The inter-species differences amounted to 2.0-fold for lasiocarpine and 8.2-fold for riddelliine with humans being more sensitive than rats. The inter-ethnic human differences varied 2.0-fold for lasiocarpine and 5.0-fold for riddelliine with the average Caucasian being more sensitive than the average Chinese. In conclusion, the present study provides the proofof-principle to predict inter-species and inter-ethnic differences in in vivo liver toxicity for PAs by an alternative testing strategy integrating in vitro cytotoxicity data with PBK modelling-based reverse dosimetry.
\end{abstract}




\section{Introduction}

Lasiocarpine and riddelliine (Figure 1) are chemicals belonging to the group of pyrrolizidine alkaloids (PAs) which are important secondary metabolites of approximately 6000 plant species distributed around the world (Smith and Culvenor 1981). Lasiocarpine is widely distributed in the Heliotropium and Symphytum genus (Boraginaceae species), and riddelliine is present in the genus Senecio (Asteraceae species) (Adams et al. 1942; Smith and Culvenor 1981). PA-containing plants can be toxic to livestock, wildlife and humans (Stegelmeier et al. 1999). Human exposure to lasiocarpine and riddelliine may result from the consumption of contaminated food staples, plant food supplements, herbal medicines, herbal teas, honey and milk (EFSA 2011; EFSA 2017). Exposure to lasiocarpine, as well as riddelliine has been shown to cause fatal liver veno-occlusive disease in animals and humans (Bane et al. 2012; Mohabbat et al. 1976; Tandon et al. 1978). A 2-year National Toxicology Program (NTP) study testing the genotoxicity and carcinogenicity of lasiocarpine and riddelliine showed that these PAs are also carcinogenic and genotoxic compounds (Chan et al. 2003; NTP 1978; NTP 2003). Liver angiosarcomas were observed in 11 of 23 male rats and 7 of 24 female rats following dietary administration of $0.75 \mathrm{mg} / \mathrm{kg}$ bw/day of lasiocarpine. Riddelliine induced a high incidence of liver hemangiosarcoma in both male (43 out of 50 rats) and female rats (38 out of 50 rats) at $0.7 \mathrm{mg} / \mathrm{kg}$ bw/day (Chan et al. 2003; NTP 1978; NTP 2003). The International Agency for Research on Cancer (IARC) classified these alkaloids in Group 2B, potentially carcinogenic to humans (IARC 2017).

Following absorption in the gastrointestinal tract, PAs are distributed to the liver where the main metabolism occurs ( $\mathrm{Fu}$ et al. 2004). In general, three metabolic pathways have been identified for lasiocarpine (heliotridine-type PA) and riddelliine (retronecine-type PA) (Figure 1) (EFSA 2011; Fu et al. 2004). The bioactivation pathway is the conversion of lasiocarpine or riddelliine into a toxic intermediate, known as a dehydropyrrolizidine alkaloid, in a reaction mainly catalysed by cytochrome P450 3A4 (Fu et al. 2004). Dehydropyrrolizidine alkaloids react with cellular proteins and DNA leading to liver toxicity, genotoxicity and carcinogenicity (Fu et al. 2004). Other metabolic pathways include hydrolysis and Noxidation, leading to the formation of necine bases and PA N-oxides, respectively (Figure 1). These latter two metabolic pathways are considered to lead to detoxification (EFSA 2011).

Significant variations between species regarding the toxicity of PAs have been observed. Recently, Fashe et al. (2015) conducted a comparative study on the metabolism of lasiocarpine by human and rat liver microsomes and found that humans might be more susceptible to lasiocarpine-induced toxicity, because the overall metabolic fingerprint detected upon incubation with human liver microsomes differed from that obtained with rat liver microsomes in the following two major aspects: 1) the amount of detoxified metabolites was lower in incubations with human liver microsomes than with rat liver microsomes, and 2) higher levels of GSH-reactive metabolites were formed in incubations with the human liver microsomes than with the rat liver microsomes. These species differences may be mediated by interspecies differences in biotransformation capacities including CYP 3A4 catalysed bioactivation, carboxylesterase-mediated hydrolysis and the level of cellular GSH (Fashe et 
al. 2015). In addition to these possible species differences in biotransformation capacities, other studies reported ethnic differences in P450 dependent metabolism and these may potentially result in inter-ethnic differences in PA bioactivation and detoxification. Yang et al. (2012) reported significant differences in phenacetin $O$-deethylation, diclofenac $4^{\prime}-$ hydroxylation, $(S)$-mephenytoin 4'-hydroxylation and chlorzoxazone 6-hydroxylation reactions, mediated by CYP 1A2, CYP 2C9, CYP 2C19 and CYP 2E1, respectively between Chinese and Caucasian liver microsomal preparations. Previously, we also reported differences between Chinese and Caucasians regarding the metabolic bioactivation and detoxification of the food-borne genotoxic carcinogen estragole, resulting in a predicted 4.5fold lower formation of the ultimate carcinogenic metabolite of estragole with similar rates of detoxification in Chinese as compared to the Caucasians, and thus a possible lower risk of estragole exposure for the average Chinese at similar levels of exposure (Ning et al. 2017).

Thus, inter-species and inter-ethnic human differences in metabolism might occur and may in theory also affect the sensitivity towards PA toxicity. The aim of this study was to predict the inter-species and inter-ethnic human differences in acute liver toxicity of lasiocarpine and riddelliine using physiologically based kinetic (PBK) modelling-based reverse dosimetry of in vitro toxicity data. Previously, we developed a PBK modelling-based approach to predict acute liver toxicity of lasiocarpine and riddelliine in rats (Chen et al. 2018). The predicted $\mathrm{BMDL}_{5}-\mathrm{BMDU}_{5}$ values for in vivo liver toxicity of lasiocarpine and riddelliine in rats amounted to 23.0-34.4 and 4.9-8.4 mg/kg bw/day, respectively (Chen et al. 2018). That study already showed that the PBK modelling-based approach was able to define inter-compound differences in acute liver toxicity of PAs. The study of Culvenor (1983) estimated that a dose of $0.7-3 \mathrm{mg} / \mathrm{kg}$ bw/day of riddelliine may lead to acute liver toxicity in humans, indicating that humans might be more sensitive than rats towards liver toxicity of PAs. In the present study we developed PBK models for lasiocarpine and riddelliine in different ethnic populations (Chinese and Caucasian) and used these models to convert in vitro toxicity data obtained in pooled human hepatocytes to predict in vivo dose-response data and BMDL5$\mathrm{BMDU}_{5}$ values for acute liver toxicity of lasiocarpine and riddelliine in humans. Subsequently, these points of departure (PoDs) were compared to PoDs derived previously for rats by the same methodology (Chen et al. 2018) to evaluate the occurrence of species differences in the metabolism and toxicity of lasiocarpine and riddelliine while the PoDs obtained for the average Chinese and average Caucasian provided insight in inter-ethnic differences. 


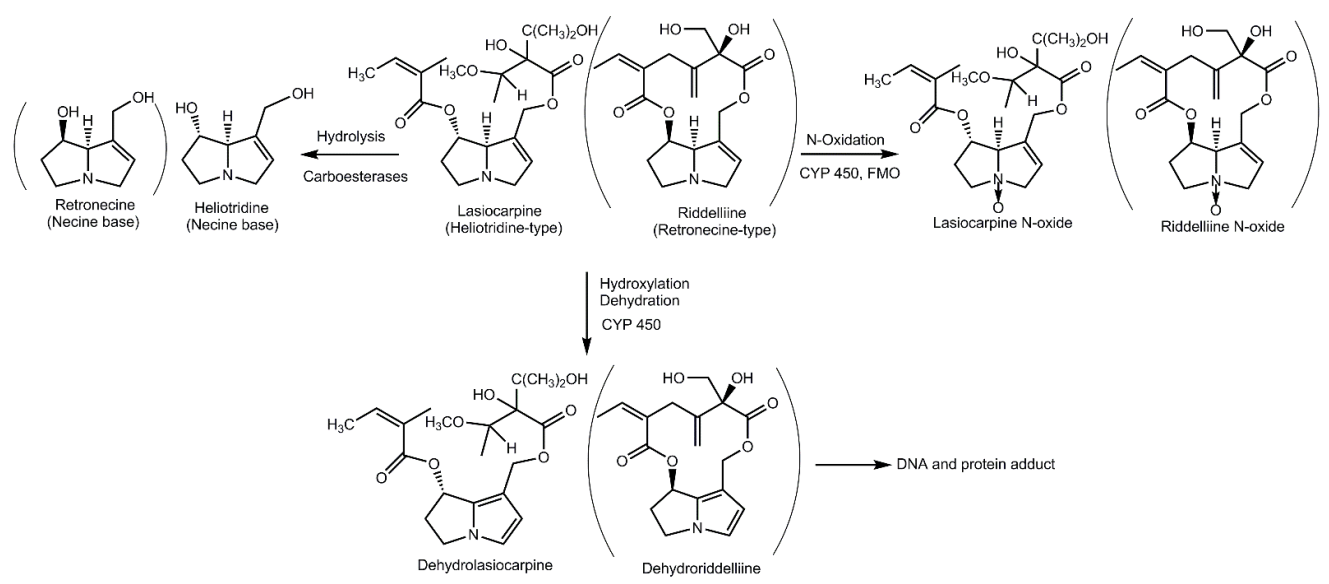

Fig. 1 Schematic presentation of the major metabolic pathways of lasiocarpine (heliotridinetype PA) and riddelliine (retronecine-type PA), the latter shown in brackets. FMO = flavincontaining monooxygenase, CYP $450=$ cytochromes $\mathrm{P} 450$.

\section{Materials and Methods}

\subsection{Chemicals and biological materials}

Lasiocarpine (purity $>97 \%$ ) was purchased from Phytolab (Vestenbergsgreuth, Germany). Riddelliine (purity $>90 \%$ ) was a gift from RIKILT (Wageningen, The Netherlands) and from the National Institute for Environmental Health Sciences (NIEHS) (Durham, USA). The HepaRG cell line was obtained from Biopredic International (Saint-Grégoire, France). Cryopreserved pooled human hepatocytes ( 5 mixed gender donors), cryopreserved hepatocyte plating medium (CHPM) (CM 9000) and cryopreserved hepatocyte recovery medium (CHRM) (CM 7000) were purchased from Invitrogen (Cheshire, UK). Willliams medium E (without glutamine), penicillin/streptomycine-solution, L-glutamine, phosphate buffered saline (PBS), and trypsin-EDTA were purchased from Gibco (Paisley, Scotland, UK). Fetal bovine serum was purchased from the American Type Culture Collection (ATCC) (Manassas, USA). Dimethylsulfoxide (DMSO), human insulin and hydrocortisone-21-hemisuccinate were purchased from Sigma-Aldrich (Steinheim, Germany). Potassium dihydrogen phosphate, dipotassium hydrogen phosphate trihydrate, sodium phosphate, sodium chloride and trifluoroacetic acid (TFA) were purchased from VWR International (Darmstadt, Germany). Reduced nicotinamide adenine dinucleotide phosphate (NADPH) was obtained from Roche Diagnostics (Mannheim, Germany). Acetonitrile (UPLC/MS grade) was obtained from Biosolve BV (Valkenswaard, Netherlands). Human plasma and rapid equilibrium dialysis (RED) materials, including RED inserts, RED base plate and sealing tape were obtained from Thermo Fisher Scientific (Bleiswijk, the Netherlands). Pooled Caucasian liver microsomes (33 donors) were purchased from Corning (Woburn, MA, USA). Pooled Caucasian intestinal microsomes (15 donors) were obtained from Xenotech (Lenexa, USA). Pooled Caucasian human lung and kidney microsomes were purchased from Biopredic 
International (Rennes, France). Pooled Chinese liver microsomes (40 donors) were purchased from PrimeTox (Wuhan, China).

\subsection{Outline of the PBK modelling-based reverse dosimetry approach in human}

The PBK modelling-based reverse dosimetry approach to predict the in vivo dose-response curves for risk assessment from in vitro cytotoxicity concentration-response data included the following steps: 1) establishment of in vitro cytotoxicity concentration-response curves for lasiocarpine and riddelliine in HepaRG cells and in cryopreserved pooled human hepatocytes from Caucasian donors and correcting for toxicity expected in hepatocytes from the Chinese population as described below (section 2.6), 2) development of PBK models for lasiocarpine and riddelliine in human using the kinetic parameters defined in the present study using both Chinese and Caucasian human liver subcellular factions, 3) translation of in vitro concentration-response curves to the in vivo dose-response curves using the PBK models, 4) BMD analysis based on the predicted in vivo dose-response curves to obtain the BMDL5 and $\mathrm{BMDU}_{5}$ values as points of departure (PoDs), 5) comparison of the predicted PoDs in human (Chinese and Caucasian) to one another and to the previously predicted PoDs in rats (Chen et al. 2018) to assess for inter-ethnic and inter-species differences in acute liver toxicity.

\subsection{In vitro liver toxicity data}

Two types of cells were used to determine the in vitro concentration-response curves for acute cytotoxicity in human liver including cells from the HepaRG cell line and human hepatocytes. The HepaRG cell line is a human hepatoma cell line, which can differentiate into hepatocyte-like morphology by treatment with DMSO (Kanebratt and Andersson 2008). HepaRG cells were seeded at a density of 9000 cells/well in 96-well plates in growth medium consisting of Williams' medium E, supplemented with $10 \%$ fetal bovine serum (FCS), 100 $\mathrm{IU} / \mathrm{ml}$ penicillin, $100 \mu \mathrm{g} / \mathrm{ml}$ streptomycin, $1 \%$ (v/v) L-glutamine, $5 \mu \mathrm{g} / \mathrm{ml}$ human insulin and $50 \mu \mathrm{M}$ hydrocortisone-21-hemisuccinate. The medium was renewed every two to three days. After two weeks, the cells were nearly confluent and were cultured in the differentiation medium supplemented with $1.7 \%(\mathrm{v} / \mathrm{v})$ DMSO. The medium was refreshed every two to three days. After two weeks differentiation treatment, HepaRG cells were fully differentiated and were used for experiments within four weeks with refreshing the medium every two to three days according to the protocol provided by Biopredic International.

Cryopreserved mixed gender primary human hepatocytes from five Caucasian donors were also used to perform the cytotoxicity experiments and define in vitro concentration-response curves. Cryopreserved human hepatocytes were thawed according to the protocol provided by Invitrogen. Briefly, human hepatocytes were thawed in a $37{ }^{\circ} \mathrm{C}$ water bath and transferred to $50 \mathrm{ml}$ thawing medium (CM 7000). The cells were centrifuged at room temperature at 100 $\mathrm{g}$ for $10 \mathrm{~min}$ and re-suspended in the plating medium (CM 9000). After determination of the cell viability using trypan blue exclusion, the cells were seeded in 96-well plates at a density of 50000 cells/well and incubated in a $5 \% \mathrm{CO}_{2}: 95 \%$ air-humidified incubator for $6 \mathrm{~h}$, after which the cells were ready to be exposed to the compounds. Only batches with a cell viability $>90 \%$ were used for experiments. 
Cytotoxicity was determined with the MTT assay by assessing the activity of mitochondrial dehydrogenase enzymes according to the method of Field et al. (2015). In brief, the fully differentiated HepaRG cells and human hepatocytes were exposed to serum free medium containing different concentrations of lasiocarpine and riddelliine ranging from 4 to $300 \mu \mathrm{M}$ (final concentrations), added from 200 times concentrated stock solutions in DMSO. Each concentration was measured in triplicate and three independent experiments were performed. A solvent control was included in each independent experiment. After 24 hours exposure, MTT $(0.45 \mathrm{mg} / \mathrm{ml}$ final concentration) was added and cells were incubated for another 2 hours in the incubator. The formed MTT formazan crystals were dissolved in DMSO and the absorbance was measured at a microplate reader at $562 \mathrm{~nm}$ (SpectraMax M2, USA). All the data are presented as mean values $\pm \mathrm{SD}$. The half maximal inhibitory concentration ( $\left.\mathrm{IC}_{50}\right)$ values used to assess the in vitro acute liver toxicity of lasiocarpine and riddelliine were obtained using GraphPad Prism 5.04 (GraphPad software, San Diego California U.S.A.) using a non-linear regression model for curve fitting $\mathrm{Y}=$ Bottom $+($ Top-Bottom $) /\left(1+10^{\wedge}((\mathrm{X}\right.$ $\log (\mathrm{C} 50)))$. The in vitro concentration-response curve for liver toxicity for Chinese hepatocytes was obtained as described in section 2.6.

\subsection{In vitro microsomal incubations}

The in vitro incubation for the conversion of lasiocarpine with Chinese and Caucasian liver microsomes were performed as described by Fashe et al. (2015). Briefly, mixed gender human liver microsomes were incubated with lasiocarpine in the presence of NADPH. The incubation mixtures contained (final concentrations) $2 \mathrm{mM} \mathrm{NADPH}$ and $0.04 \mathrm{mg} / \mathrm{ml}$ microsomal protein in $0.1 \mathrm{M}$ potassium phosphate ( $\mathrm{pH} 7.4$ ). After pre-incubation for $5 \mathrm{~min}$, the reaction was started by adding NADPH. Incubations were performed for $30 \mathrm{~min}$ at substrate concentrations ranging from 3 to $200 \mu \mathrm{M}$ (final concentrations) (added from 100 times concentrated stock solutions in DMSO), after which the reaction was terminated by adding ice-cold acetonitrile $(20 \% \mathrm{v} / \mathrm{v})$. Under these conditions conversion of lasiocarpine was linear in time (1-30 min) and with the amount of microsomal protein $(0.01-0.04 \mathrm{mg} / \mathrm{ml})$ (data not shown). Blank incubations were performed in the absence of the cofactor NADPH.

The incubation conditions for metabolism of riddelliine with human liver microsomes contained (final concentrations) $2 \mathrm{mM} \mathrm{NADPH}, 0.5 \mathrm{mg} / \mathrm{ml}$ microsomal protein for Chinese and Caucasian samples in $0.1 \mathrm{M}$ potassium phosphate $(\mathrm{pH} 7.4$ ) and 3 to $400 \mu \mathrm{M}$ (final concentrations) riddelliine added from 100 times concentrated stock solutions in DMSO. After $5 \mathrm{~min}$ pre-incubation at $37{ }^{\circ} \mathrm{C}$, the reactions were started by adding NADPH. Incubations were carried out for $30 \mathrm{~min}$ and the reaction was terminated by adding ice-cold acetonitrile $(20 \% \mathrm{v} / \mathrm{v})$. Under these conditions conversion of riddelliine was linear in time (1$30 \mathrm{~min}$ ) and with the amount of microsomal protein $(0.04-0.5 \mathrm{mg} / \mathrm{ml})$ (data not shown). Blank incubations were performed in the absence of the cofactor NADPH.

To compare the inter-ethnic differences in metabolic activation of lasiocarpine and riddelliine, incubations with Chinese and Caucasian liver microsomes were performed as described above in the presence of $4 \mathrm{mM}$ glutathione (GSH) to scavenge dehydropyrrolizidine alkaloids 
metabolites. The level of GSH in the incubations was optimized to obtain the maximum scavenging of dehydropyrrolizidine alkaloids metabolites as major glutathione conjugate, namely 7-GS-DHP (Tamta et al. 2012), which was analyzed by LC-MS/MS analysis as described in section 2.6.

The kinetic constants for conversion of lasiocarpine and riddelliine by Caucasian intestinal, lung and kidney microsomal preparations were determined as described above for liver microsomes but increasing the incubation time to $2 \mathrm{~h}$. Under these conditions conversion of lasiocarpine and riddelliine was linear with time (1-120 minutes) and with the amount of microsomal protein added $(0.01-0.04 \mathrm{mg} / \mathrm{ml}$ for lasiocarpine, $0.04-0.5 \mathrm{mg} / \mathrm{ml}$ for riddelliine) for the intestine, whereas negligible conversion was observed for lung and kidney (data not shown). Before Ultra Performance Liquid Chromatography photodiode array analysis (UPLCPDA) or LC-MS/MS analysis, all samples were centrifuged for $5 \mathrm{~min}$ at $15000 \mathrm{~g}$ to precipitate microsomal proteins. Intestine, lung and kidney microsomes from the Chinese population are not available. However, the kinetic parameters obtained using pooled intestinal microsomal preparations from Caucasian individuals were used for the Chinese PBK model, because the results of the sensitivity analysis (see section 3.6) revealed that the parameters related to the intestinal metabolism of lasiocarpine and riddelliine do not have a large influence on the predicted maximum concentration in the liver blood $\left(\mathrm{C}_{\max }\right)$. Because conversion by kidney and lung microsomes was negligible (see result section), no further kinetic data for these organs were required.

The substrate depletion approach was used to obtain the kinetic parameters. The amount of substrate depletion of lasiocarpine and riddelliine was determined as the amount detected in the blank incubations in the absence of the cofactor NADPH minus the amount detected in the incubations with NADPH. The amounts of 7-GS-DHP formed were corrected for the amounts detected in the blank incubations performed without cofactor NADPH. The data for the rate of conversion of lasiocarpine and riddelliine with increasing substrate concentration $[\mathrm{S}]$ were fitted to the standard Michaelis-Menten equation (1):

$$
\mathrm{v}=\mathrm{V}_{\max } /\left(1+\mathrm{K}_{\mathrm{m}} /[\mathrm{S}]\right)
$$

The apparent maximum velocity $\left(\mathrm{V}_{\max }\right)$ and the apparent Michaelis-Menten constant $\left(\mathrm{K}_{\mathrm{m}}\right)$ were determined by fitting the data to this equation using GraphPad Prism version 5.04 (GraphPad software, San Diego California U.S.A.). $V_{\max }$ values expressed as $\mathrm{nmol} / \mathrm{min} /(\mathrm{mg}$ microsomal protein) were scaled to the $\mathrm{V}_{\max }$ expressed as $\mu \mathrm{mol} / \mathrm{h} / \mathrm{kg}$ bw using microsomal protein yields of $35 \mathrm{mg} / \mathrm{g}$ liver (Medinsky et al. 1994) and $20.6 \mathrm{mg} / \mathrm{g}$ small intestine (Cubitt et al. 2009). The $K_{m}$ values determined in vitro were assumed to be equal to in vivo $K_{m}$ values.

\subsection{Protein binding correction}

To correct for protein binding in the in vivo situation in human liver blood, while the in vitro toxicity was determined in serum free medium, the fraction of unbound lasiocarpine and riddelliine under the in vivo conditions $\left(f_{u b}\right)$ was determined. To this end the $f_{u b}$ for both compounds in human blood was determined using the RED (rapid equilibrium dialysis) device. According to the protocol described by Waters et al. (2008), $300 \mu \mathrm{l}$ of spiked human 
plasma containing $5 \mu \mathrm{M}$ lasiocarpine and 5 or $50 \mu \mathrm{M}$ riddelliine (final concentration, $1 \% \mathrm{v} / \mathrm{v}$ DMSO) were added to the plasma chambers of the RED device insert, while $500 \mu$ dialysis buffer (PBS containing $100 \mathrm{mM}$ sodium phosphate and $150 \mathrm{mM}$ sodium chloride) were added to the buffer chamber. The device was sealed with tape and incubated at $37^{\circ} \mathrm{C}$ on a shaker at $250 \mathrm{rpm}$ for $5 \mathrm{~h}$. After the system reached equilibrium, $50 \mu \mathrm{l}$ of post-dialysis samples were collected from the plasma and buffer chambers into separate tubes. Subsequently, $50 \mu$ of human plasma was added to the buffer samples and the same volume of dialysis buffer was added to the plasma samples, after which protein was precipitated by addition of ice-cold acetonitrile $(75 \% \mathrm{v} / \mathrm{v})$. After incubation on ice for $30 \mathrm{~min}$, the mixtures were centrifuged at $15000 \mathrm{~g}$ for $15 \mathrm{~min}$ at $4{ }^{\circ} \mathrm{C}$, and the supernatants were analyzed by UPLC-PDA. The measurements were performed in three independent experiments.

The fraction of unbound lasiocarpine and riddelliine in human blood ( $\left.f_{u b}\right)$ was assumed to be the same as in human plasma in the present study and was defined as the ratio of the concentration of the test compound in the buffer chamber and the concentration in the plasma chamber (van Liempd et al. 2011; Waters et al. 2008). The $f_{u b}$ thus obtained was used to correct for the difference in protein binding in the in vitro assay and the in vivo situation when performing the PBK modelling-based reverse dosimetry. To this end the effect concentration of lasiocarpine and riddelliine in human liver blood $\left(\mathrm{C}_{\mathrm{Lc} / \mathrm{Rd}}\right.$, human blood $)$ used for reverse dosimetry was corrected by the following equation (2):

(2) $\mathrm{C}_{\mathrm{Lc} / \mathrm{Rd} \text {, human blood }}=\mathrm{C}_{\mathrm{ub}}$, in vitro $/ \mathrm{f}_{\mathrm{ub}}$

Where $f_{u b}$ is the fraction of the unbound lasiocarpine and riddelliine in human blood and $\mathrm{C}_{\mathrm{ub}}$, in vitro is the unbound concentration of lasiocarpine and riddelliine in the in vitro culture medium. Since the exposure medium for the HepaRG and human hepatocyte cell lines was serum free, the concentrations of lasiocarpine and riddelliine in the in vitro cytotoxicity assay were considered to be the unbound concentration. This correction implies that the unbound fraction in vitro is set equal to the unbound fraction in vivo.

\subsection{UPLC and LC-MS/MS analysis}

The supernatant of each sample was analyzed on UPLC using a BEH C18 $(1.7 \mu \mathrm{m} 2.1 \times 50$ $\mathrm{mm}$ ) column with a guard column and a photodiode array detector (PDA) (Acquity, Waters). The flow rate was $0.6 \mathrm{ml} / \mathrm{min}$ and the mobile phase consisted of ultrapure water with $0.1 \%$ (v/ v) TFA and acetonitrile. For the analysis of lasiocarpine, a gradient was applied from 0 to 50 $\%$ acetonitrile in ultrapure water in $2 \mathrm{~min}$, after which the percentage of acetonitrile was increased to $100 \%$ in 3 min and kept at this level for 2 min longer, after which the column was set back to the starting conditions. Under these conditions, lasiocarpine had a retention time of $1.9 \mathrm{~min}$. For detection of riddelliine, a linear gradient from 0 to $50 \%$ acetonitrile in ultrapure water during 4 min was applied, followed by an increase to $100 \%$ acetonitrile in 1 min. This percentage was kept for 1 min to wash the column, after which the column was set back to the starting conditions. Under these conditions, riddelliine eluted at $1.8 \mathrm{~min}$. Identification of lasiocarpine and riddelliine was achieved by comparison of the UV spectra and retention times of these compounds to those of reference compounds. Quantification of 
lasiocarpine and riddelliine was done by comparing the peak areas of these compounds at a wavelength of $220 \mathrm{~nm}$ to the calibration curve of the corresponding standard compounds.

The major glutathione conjugate of lasiocarpine and riddelliine, 7-GS-DHP was analyzed and quantified by LC-MS/MS. LC-MS/MS analysis was performed on a Shimadzu Nexera XR LC-20AD SR UPLC system coupled with a Shimadzu LCMS-8040 mass spectrometer (Kyoto, Japan). The samples $(1 \mu \mathrm{l})$ were loaded onto a reverse phase $\mathrm{C} 18$ column $(1.7 \mu \mathrm{m}$ $2.1 \times 50 \mathrm{~mm}$ ) with a flow rate of $0.3 \mathrm{ml} / \mathrm{min}$. The column temperature was set to $40{ }^{\circ} \mathrm{C}$. The mobile phase consisted of ultrapure water with $0.1 \%(\mathrm{v} / \mathrm{v})$ formic acid and acetonitrile containing $0.1 \%(\mathrm{v} / \mathrm{v})$ formic acid. The gradient began with $0 \%$ acetonitrile for one minute to wash away unwanted salts, followed by a linear gradient from 0 to $5 \%$ in 8 min and a further increase to $100 \%$ in $6 \mathrm{~min}$. This percentage was kept for $0.5 \mathrm{~min}$, after which the column was set back to the starting conditions. A Shimadzu LCMS-8040 triple quadrupole with electrospray ionization (ESI) interface was used to perform the MS-MS analysis. The instrument was operated in positive mode in the multiple reaction monitoring (MRM) mode with a spray voltage of $4.5 \mathrm{KV}$. The 7-GS-DHP was monitored at the $[\mathrm{M}+\mathrm{H}]^{+}$of precursor to product $443.2 \rightarrow 425.15(\mathrm{CE}=-7 \mathrm{kV}), 443.2 \rightarrow 118.1(\mathrm{CE}=-24 \mathrm{kV})$ and $443.2 \rightarrow 247.2(\mathrm{CE}=-$ $15 \mathrm{kV}) \mathrm{m} / \mathrm{z}$. Some of these MRMs were also used in study of Tamta et al. (2012). Under these conditions, 7-GS-DHP eluted at $8.97 \mathrm{~min}$ and the chromatogram of 7-GS-DHP is presented in the supplementary materials 1 . The peak area of total ion chromatogram (TIC) of 7-GS-DHP was acquired using Postrun analysis in the software LabSolution (Shimadzu). The ratio of the peak areas of 7-GS-DHP formed in incubations with Chinese and Caucasian liver microsomes at each substrate concentration, was assumed to reflect the inter-ethnic differences in metabolic activation of lasiocarpine and riddelliine. Thus these ratios were used to convert the concentration-response curve for in vitro toxicity obtained with human hepatocytes derived from Caucasian donors to a curve for Chinese donors. To this end the percentage of cytotoxicity at each concentration was multiplied by the amount of 7-GS-DHP formed in incubations with Caucasian liver samples divided by the amount formed in similar incubations with Chinese liver microsomes. This provided an estimated concentrationresponse curve for cytotoxicity towards Chinese liver hepatocytes.

\subsection{Development of a PBK model for lasiocarpine and riddelliine in human}

PBK models for lasiocapine and riddelliine for the average Chinese and average Caucasian were developed based on the PBK models previously defined and evaluated by Chen et al. (2018) for lasiocarpine and riddelliine in rats. The structure of the model for lasiocarpine and riddelliine is presented in Figure 2. The model has 6 compartments including blood, fat, rapidly perfused tissue, slowly perfused tissue, liver and intestine that are mutually connected through the systemic circulation which is represented by a blood compartment. The intestine and liver compartments are included in the model, since the conversion of lasiocarpine and riddelliine occurs in these organs. The physiological parameters for the average Chinese and average Caucasian were derived from the literature (Brown et al. 1997; NHFPC 2007; NHFPC 2014) and are presented in Table S2 in the supplementary materials 2. Several reports from the literature indicate that Chinese have significantly smaller livers than Caucasians 
(Chan et al. 2006; Shi et al. 2009; Yuan et al. 2008). The physiological parameters provided by National Health Commission of the People's Republic of China (NHFPC) also show that the Chinese liver volume is 1.3-fold lower than the Caucasian liver volume. The tissue:blood partition coefficients were estimated from log $\mathrm{K}_{\mathrm{ow}}$ according to the method described by DeJongh et al. (1997). The log $\mathrm{K}_{\mathrm{ow}}$ values for lasiocarpine and riddelliine were estimated by ChemBio 3D 2010 software (CambrigeSoft, USA).

Lasiocarpine and riddelliine were assumed to enter the body from the gastrointestinal tract lumen following first order uptake. The in vivo oral absorption rate constant (Ka) of lasiocarpine and riddelliine in human were assumed to be the same as previously determined (Chen et al. 2018). The Ka obtained for lasiocarpine was $1.55 / \mathrm{h}$ and for riddelliine the value amounted to $0.72 / \mathrm{h}$. Model equations were coded (supplementary materials 3) and numerically integrated in Berkeley Madonna 8.0.1 (Macey and Oster, UC Berkeley, CA, USA) using the Rosenbrock's algorithm for stiff systems.

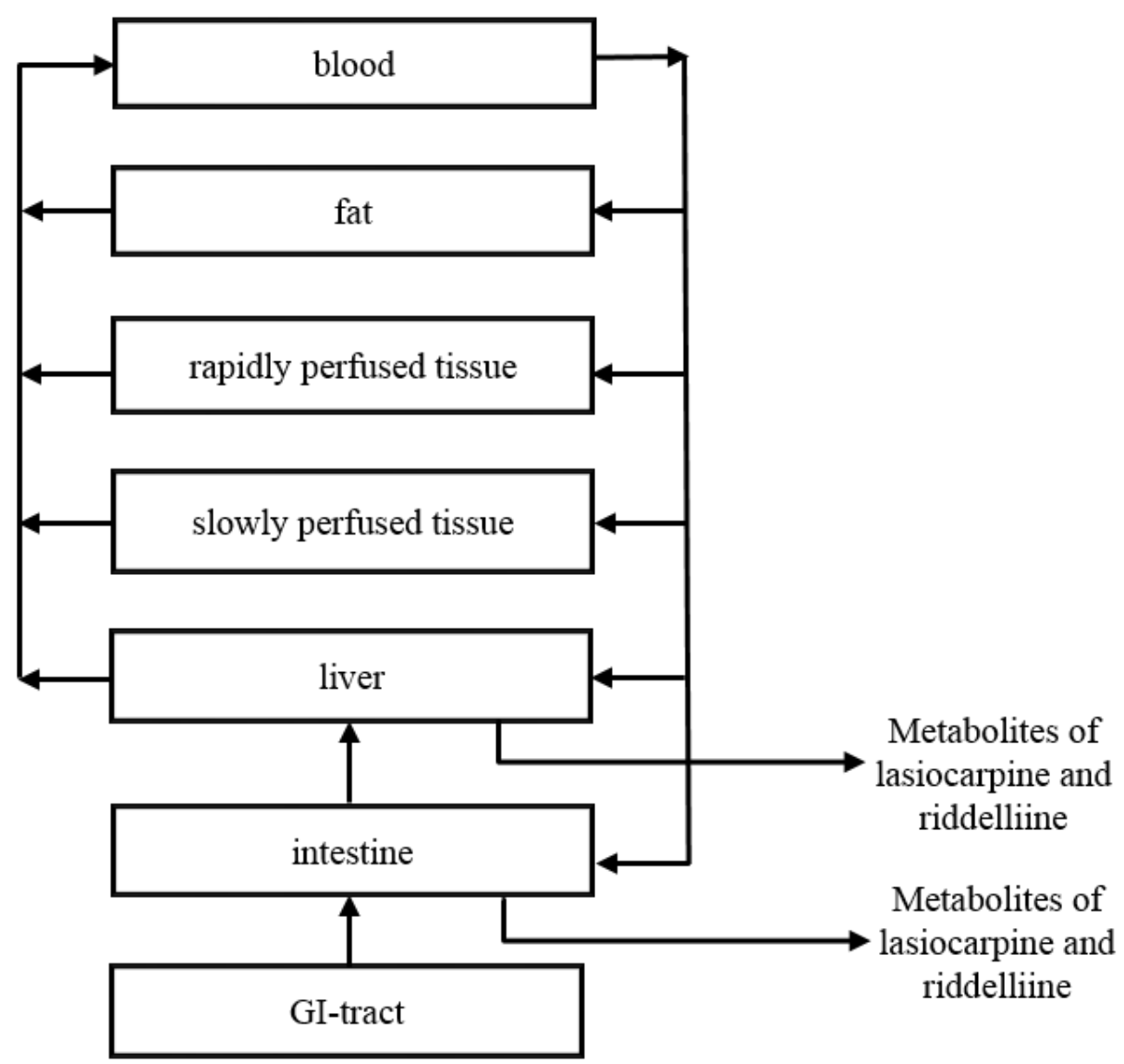

Fig. 2 Schematic diagram of the PBK models for lasiocarpine and riddelliine in human 


\subsection{Sensitivity analysis}

A sensitivity analysis was performed to identify which parameters have the largest influence on the model predictions. Normalized sensitivity coefficients (SC) were determined using the following equation (3) for the prediction of maximum liver blood concentration $\left(\mathrm{C}_{\max }\right)$ of lasiocarpine and riddelliine:

(3) $\mathrm{SC}=\left(\mathrm{C}^{\prime}-\mathrm{C}\right) /\left(\mathrm{P}^{\prime}-\mathrm{P}\right) \times(\mathrm{P} / \mathrm{C})$

Where $\mathrm{C}$ is the initial value of the model output $\left(\mathrm{C}_{\max }\right.$ in liver blood); $\mathrm{C}^{\prime}$ is the modified model output resulting from a $5 \%$ increase of the parameter value; $\mathrm{P}$ is the initial parameter value; $\mathrm{P}^{\prime}$ is the modified parameter value (Evans and Andersen 2000). A $5 \%$ increase in parameter values was chosen to analyze the effect of a change in parameter values on $\mathrm{C}_{\max }$ of lasiocarpine and riddelliine at a dose of $8 \mathrm{ng} / \mathrm{kg}$ bw/day and $3 \mathrm{mg} / \mathrm{kg}$ bw/day for 24 hours exposure, representing respectively an average daily intake of PAs via consumption of herbal tea (Bfr 2013) and a dose level known to cause liver toxicity in humans (Culvenor 1983). Each parameter was analyzed individually while other parameters were kept at their initial value.

\subsection{Translation of the in vitro concentration-response curves to in vivo dose-response curves}

The in vitro concentration-response curves on acute cytotoxicity were translated to in vivo dose-response curves on hepatotoxicity of lasiocarpine and riddelliine by PBK modellingbased reverse dosimetry. For this translation, each concentration applied in the cytotoxicity experiments was converted to a corresponding $\mathrm{C}_{\mathrm{Lc} / \mathrm{Rd}}$, human blood which was then assumed to be the $\mathrm{C}_{\max }$ (the maximum concentration) of lasiocarpine and riddelliine in the liver blood and used as input in the PBK model to determine the corresponding oral dose which would result in acute hepatotoxicity. By performing this exercise for each concentration used in the in vitro cytotoxicity assay, the concentration-response curve was translated to an in vivo doseresponse curve.

The in vitro concentration-response curve on cytotoxicity of lasiocarpine and riddelliine for the average Chinese was obtained based on in vitro concentration-response curves on cytotoxicity derived from mixed gender human (Caucasian) liver hepatocytes, correcting the curve for the differences in bioactivation between Chinese and Caucasians as explained above (section 2.6). This was done because mixed gender Chinese liver hepatocytes were not available. To this end each concentration in the cytotoxicity experiment was first multiplied by the fold difference in the peak area of the major glutathione conjugate, 7-GS-DHP formed in liver microsomal incubations in the presence of GSH for Chinese and Caucasian samples. Subsequently, the corrected in vitro concentration-response curves for Chinese was translated to the in vivo dose-response curves based on the same approach as described above for Caucasians using the PBK model for the average Chinese. 


\subsection{BMD analysis of predicted in vivo dose-response data}

BMD modelling was applied on the predicted in vivo dose-response curves to derive the BMDL5-BMDU $_{5}$ (lower-upper $90 \%$ confidence interval of the benchmark dose that gives a 5 $\%$ response) for hepatotoxicity by the PROAST software version 38.9 developed by RIVM (the Dutch National Institute for Public Health and the Environment) (Slob 2002). This BMD modelling was performed using Exponential and Hill models for continuous data. Models were selected if they passed the goodness-of-fit test at $\mathrm{P}=0.05$ and the ratio of the $\mathrm{BMD}_{5}$ and $\mathrm{BMDL}_{5}$ was not more than 3-fold (EPA 2017).

\section{Results}

\subsection{In vitro cytotoxicity}

Figure 3 shows the concentration-response curves for the cytotoxicity of lasiocarpine and riddelliine in HepaRG cells and human pooled hepatocytes from Caucasian donors. Table 1 shows the $\mathrm{IC}_{50}$ values derived from these concentration-response curves. The $\mathrm{IC}_{50}$ for the cytotoxicity of lasiocarpine and riddelliine towards rat hepatocytes reported in the previous study of Chen et al. (2018) are also shown in Table 1 for comparison (Chen et al. 2018). The results show that rat hepatocytes are more sensitive to lasiocarpine and riddelliine than the HepaRG and human hepatocytes, with the $\mathrm{IC}_{50}$ of lasiocarpine in rat hepatocytes being 20and 2-fold lower than the $\mathrm{IC}_{50}$ in HepaRG and human hepatocytes, respectively and the $\mathrm{IC}_{50}$ of riddelliine in rat hepatocytes being 22- and 7-fold lower than the $\mathrm{IC}_{50}$ for riddelliine in HepaRG and human hepatocytes, respectively. Therefore, the sensitivity towards the toxicity of lasiocarpine and riddelliine decreases in the order: rat hepatocytes $>$ human hepatocytes $>$ HepaRG cells. In the different cell models the cytotoxicity of lasiocarpine and riddelliine is comparable. For HepaRG cells the $\mathrm{IC}_{50}$ of lasiocarpine is 1.7-fold higher than that of riddelliine while for human hepatocytes, the $\mathrm{IC}_{50}$ of lasiocarpine is 2.1-fold lower than that of riddelliine. Chen et al. (2018) already reported that the $\mathrm{IC}_{50}$ of lasiocarpine was 1.8-fold higher than that of riddelliine in rat hepatocytes (Chen et al. 2018).

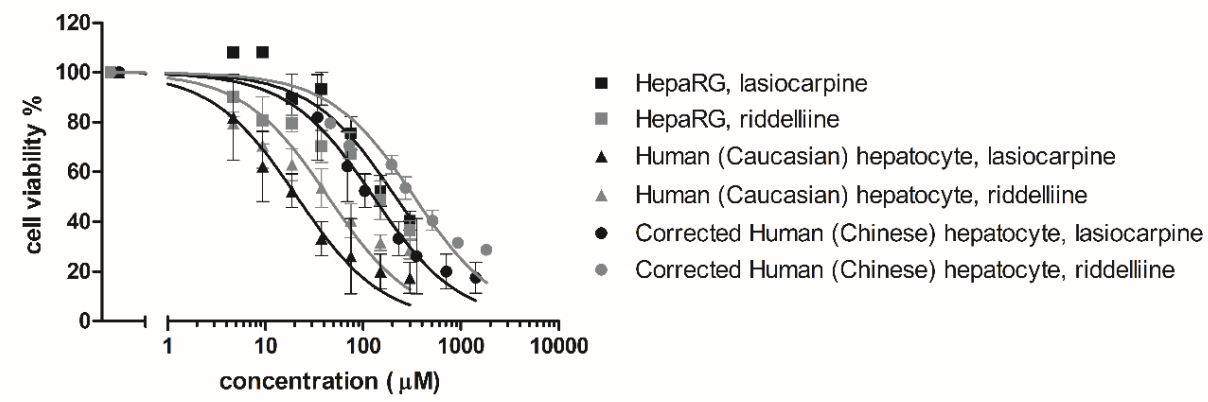

Fig. 3 Concentration-response curves for the cytotoxicity of lasiocarpine (black line) and riddelliine (grey line) in HepaRG cells (squares), human (Caucasian) hepatocytes (triangles) and corrected human (Chinese) hepatocytes (circles) upon 24 hours exposure (mean values \pm $\mathrm{SD})$. 
Given that Chinese human liver hepatocytes are not available, the cytotoxicity of lasiocarpine and riddelliine towards Chinese liver hepatocytes was modelled based on the concentrationresponse curves obtained with mixed gender Caucasian liver hepatocytes, making a correction for the relative difference in bioactivation as determined in in vitro incubations. Figure 3 also shows the in vitro concentration-response curves of lasiocarpine and riddelliine for the Chinese population obtained in this way. From Figure 3, the $\mathrm{IC}_{50}$ of lasiocarpine and riddelliine were derived for the average Chinese amounting to $126 \pm 39 \mu \mathrm{M}$ and $380 \pm 67 \mu$ $\mathrm{M}$, respectively. Comparing these $\mathrm{IC}_{50}$ values to the values obtained for the average Caucasian (Table 1), reveals that the $\mathrm{IC}_{50}$ values of lasiocarpine and riddelliine for the Chinese is estimated to be 6.0-fold and 8.6-fold higher, respectively. The cytotoxicity data are presented in the supplementary materials 4 .

Table $1 \mathrm{IC}_{50}$ values for the cytotoxicity of lasiocarpine and riddelliine in different cell models

\begin{tabular}{lll}
\hline Cell line & $\mathrm{IC}_{50}(\mu \mathrm{M}) \pm \mathrm{SD}$ & Studies \\
\hline Lasiocarpine & & \\
HepaRG cells & $215 \pm 47$ & Present study \\
Human (Caucasian) hepatocytes & $21 \pm 7$ & Present study \\
Corrected human (Chinese) hepatocytes & $126 \pm 39$ & Present study \\
Rat hepatocytes & $11 \pm 2$ & Chen et al. (2018) \\
Riddelliine & & \\
HepaRG cells & $130 \pm 30$ & Present study \\
Human (Caucasian) hepatocytes & $44 \pm 9$ & Present study \\
Corrected human (Chinese) hepatocytes & $380 \pm 67$ & Present study \\
Rat hepatocytes & $6 \pm 1$ & Chen et al. (2018) \\
\hline
\end{tabular}

\subsection{Microsomal conversion of lasiocarpine and riddelliine by Chinese and Caucasian tissue fractions}

Upon incubation of increasing concentrations of lasiocarpine and riddelliine with Chinese and Caucasian tissue fractions and $\mathrm{NADPH}$, the extent of substrate depletion was quantified by UPLC-PDA analysis. Table 2 displays the apparent $\mathrm{V}_{\max }$ and $\mathrm{K}_{\mathrm{m}}$ values obtained from the plots as shown in Figure 4 and the catalytic efficiency, calculated as $V_{\max } / K_{m}$. Conversion of lasiocarpine and riddelliine was detected in incubations with liver and intestinal microsomes. Incubations of lasiocarpine and riddelliine with lung and kidney microsomes of Caucasians did not result in detectable metabolic conversion. Lung and kidney microsomes from Chinese individuals were not available, but it was assumed that they would also not cause metabolic conversion of lasiocarpine and riddelliine. 
Liver was the major organ for lasiocarpine and riddelliine metabolism, as reflected by the fact that the catalytic efficiency for the depletion of lasiocarpine or riddelliine in incubations with pooled liver microsomes from Caucasian individuals is around 15-fold higher than the catalytic efficiency derived from incubations with intestinal microsomes. Comparison of the kinetic constants for lasiocarpine and riddelliine reveals that for depletion of lasiocarpine by Chinese liver fractions, the $\mathrm{V}_{\max }$ is 15.3 -fold higher and the $\mathrm{K}_{\mathrm{m}}$ is 3.7-fold lower than for riddelliine, resulting in a 54.8-fold higher catalytic efficiency for lasiocarpine metabolism. For Caucasian liver samples, the $\mathrm{V}_{\max }$ and $\mathrm{K}_{\mathrm{m}}$ for depletion of lasiocarpine are 2.6-fold higher and 10.5-fold lower, respectively, than those of riddelliine, resulting in a 27.1-fold higher catalytic efficiency for depletion of lasiocarpine. In the case of the intestine, the apparent $\mathrm{V}_{\max }$ and $\mathrm{K}_{\mathrm{m}}$ for the depletion of lasiocarpine in incubations with small intestinal microsomes from Caucasians are 5.0-fold higher and 5.3-fold lower, respectively, than those of riddelliine. The catalytic efficiency for depletion of lasiocarpine in these intestinal incubations is 25 -fold higher than for depletion of riddelliine.

\section{Lasiocarpine}
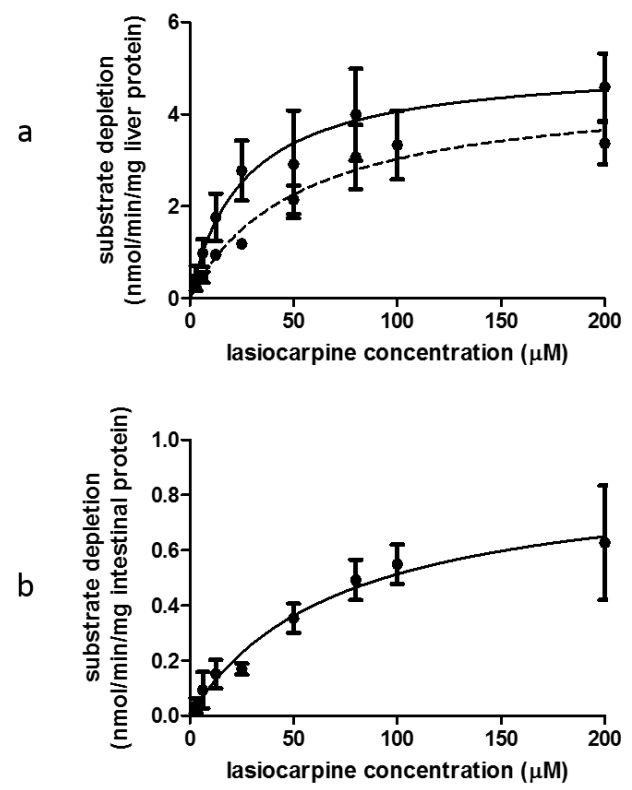

Riddelliine
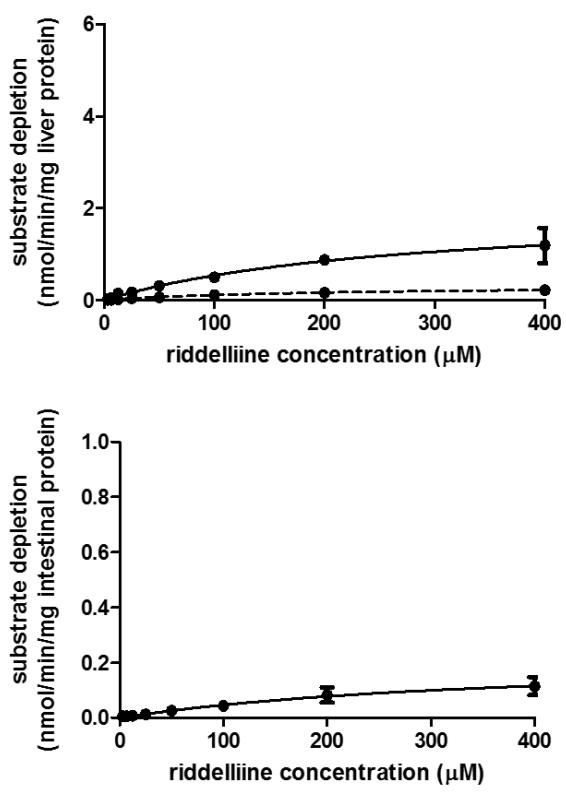

Fig. 4 Concentration-dependent rate of depletion of lasiocarpine and riddelliine in incubations with Chinese (dotted line) and Caucasian (solid line) liver microsomes (a) and Caucasian intestinal microsomes (b) in the presence of the cofactor NADPH. Data points represent mean values $\pm \mathrm{SD}$ of three individual experiments.

Comparison of the kinetic data for the depletion of lasiocarpine and riddelliine by liver fractions from the Chinese and Caucasians reveals that the catalytic efficiency for both compounds by the Chinese liver samples is 2.3 to 4 .6-fold lower, which is mainly due to a 2.0fold higher $\mathrm{K}_{\mathrm{m}}$ for depletion of lasiocarpine and 6.7-fold lower $\mathrm{V}_{\max }$ for depletion of 
riddelliine in Chinese liver. Comparison of the kinetic data for formation of 7-GS-DHP of both compounds by Chinese and Caucasian liver fractions, reveals that over the substrate concentration range used in incubations with Chinese liver microsomes bioactivation of lasiocarpine and riddelliine is 7.5-fold and 8.1-fold lower, respectively. $\mathrm{V}_{\max }$ values in these incubations were not quantified because for correction of the in vitro curves only the relative ratio in bioactivation were required. The curves of the concentration-dependent rate of formation of 7-GS-DHP of both compounds, expressed in peak area $/ \mathrm{min} / \mathrm{mg}$ liver protein and the ratio of the peak areas of 7-GS-DHP formed in incubations with Chinese and Caucasian liver microsomes at each substrate concentrationare presented in the supplementary materials 5. Since the 7-GS-DHP metabolite formed from lasiocarpine and riddelliine is the same, also bioactivation of the two compounds can be compared based on peak areas.

\subsection{Comparison of the scaled kinetic constants for lasiocarpine and riddelliine by human and rat tissue fractions}

In a subsequent step the kinetic constants presented in table 2 were scaled to human tissues. To this end, the in vitro $\mathrm{V}_{\max }$ values expressed as nmol/min/mg microsomal protein (Table 2) were scaled to $\mu \mathrm{mol} / \mathrm{h} / \mathrm{kg}$ bw using the microsomal protein yields of $35 \mathrm{mg} /(\mathrm{g}$ liver) or 20.6 $\mathrm{mg} /(\mathrm{g}$ small intestine) as scaling factors as described previously (Cubitt et al. 2009; Medinsky et al. 1994), and bodyweight and tissue fractions of human (Chinese and Caucasian) and rat (Chen et al. 2018). This scaling allows the comparison of the kinetic constants obtained from tissue fractions of different species and from different organs. Table 3 presents a summary of the kinetic parameters for lasiocarpine and riddelliine by human (Chinese and Caucasian) tissue fractions thus obtained and the comparable kinetic parameter values previously reported for rat tissue fractions (Chen et al. 2018).

Comparison of the kinetic constants between human (Chinese and Caucasians) and rat shows that liver is the major organ for lasiocarpine and riddelliine metabolism. Compared to the human (Caucasian), the contribution of intestinal metabolism for both compounds is higher in the rat. The catalytic efficiency for the depletion of lasiocarpine is generally 3.2-fold lower in human liver (Chinese and Caucasian) and 6-fold lower in human intestine (Caucasian) than in rat. The overall catalytic efficiency for depletion of lasiocarpine in human (Caucasian) is 1.9fold lower than in rat. The catalytic efficiency for the depletion of riddelliine is generally 16.8fold lower in human liver (Chinese and Caucasian) and 1.2-fold lower in small intestine (Caucasian) compared to those values in rat tissues. The overall catalytic efficiency for depletion of riddelliine in human (Caucasian) is 5.0-fold lower. Those generally lower values of catalytic efficiency for depletion of lasiocarpine and riddelliine in human liver and small intestine compared to the rat were mainly due to relatively higher $\mathrm{K}_{\mathrm{m}}$ values for depletion of both compounds in human. 


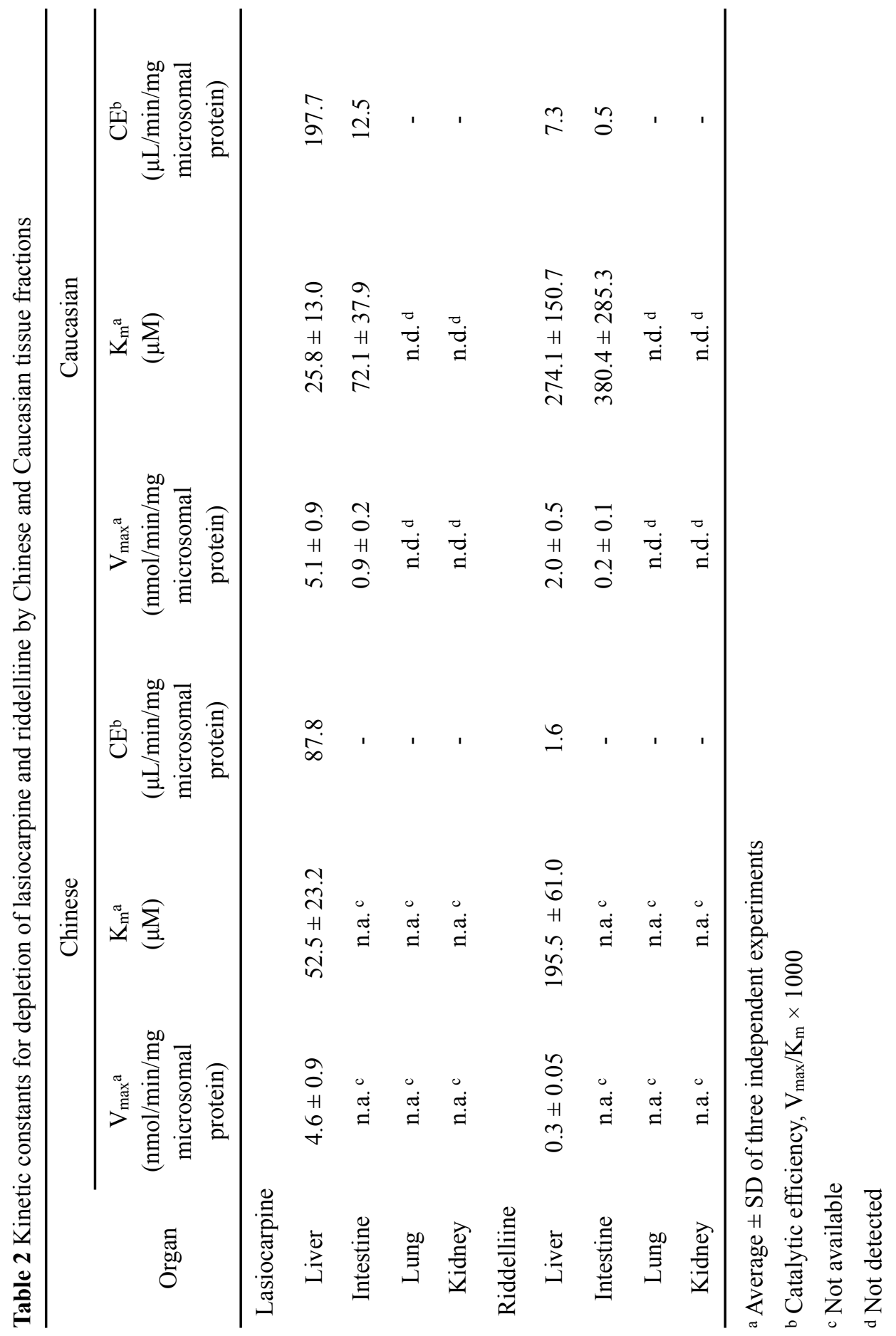


Table 3 Scaled kinetic constants for depletion of lasiocarpine and riddelliine by human (Chinese and Caucasian) and rat tissue fractions

\begin{tabular}{|c|c|c|c|c|c|c|}
\hline \multirow[b]{2}{*}{ Organ } & \multicolumn{3}{|c|}{ Human (Chinese/Caucasian) } & \multicolumn{3}{|c|}{ Rat } \\
\hline & $\begin{array}{c}\text { Scaled } V_{\max ^{\mathrm{a}}} \\
(\mu \mathrm{mol} / \mathrm{h} / \mathrm{kg} \\
\text { bw })\end{array}$ & $\begin{array}{c}\mathrm{K}_{\mathrm{m}} \\
(\mu \mathrm{M})\end{array}$ & $\begin{array}{c}\text { Scaled } \\
\text { catalytic } \\
\text { efficiency } \\
(\mathrm{L} / \mathrm{h} / \mathrm{kg} \mathrm{bw})\end{array}$ & $\begin{array}{l}\text { Scaled } \\
\mathrm{V}_{\max ^{\mathrm{a}, \mathrm{c}}} \\
(\mu \mathrm{mol} / \mathrm{h} / \\
\mathrm{kg} \mathrm{bw})\end{array}$ & $\begin{array}{l}\mathrm{K}_{\mathrm{m}^{\mathrm{a}, \mathrm{c}}} \\
(\mu \mathrm{M})\end{array}$ & $\begin{array}{c}\text { Scaled } \\
\text { catalytic } \\
\text { efficiency }{ }^{\mathrm{b}, \mathrm{c}} \\
(\mathrm{L} / \mathrm{h} / \mathrm{kg} \mathrm{bw})\end{array}$ \\
\hline \multicolumn{7}{|c|}{ Lasiocarpine } \\
\hline Liver & $222.2^{\mathrm{e} / 278.2^{\mathrm{f}}}$ & $52.5^{\mathrm{e} / 25.8^{\mathrm{f}}}$ & $4.2^{\mathrm{e} / 10.8^{\mathrm{f}}}$ & 377.4 & 19.5 & 19.4 \\
\hline Intestine & n. $a^{\mathrm{d}, \mathrm{e} / 9} \cdot 9^{\mathrm{f}}$ & $\mathrm{n} \cdot \mathrm{a}^{\mathrm{d}, \mathrm{e} / 72.1^{\mathrm{f}}}$ & $\mathrm{n} . \mathrm{a}^{\mathrm{d}, \mathrm{e} / 0.2^{\mathrm{f}}}$ & 29.4 & 23.4 & 1.3 \\
\hline \multicolumn{7}{|c|}{ Riddelliine } \\
\hline Liver & $14.5^{\mathrm{e}} / 109.2^{\mathrm{f}}$ & $195.5^{\mathrm{e} / 274.1^{\mathrm{f}}}$ & $0.07 \mathrm{e} / 0.4^{\mathrm{f}}$ & 149.6 & 75.7 & 2.0 \\
\hline Intestine & $\mathrm{n} . \mathrm{a}^{\mathrm{d}, \mathrm{e}} / 1.8^{\mathrm{f}}$ & $\mathrm{n} \cdot \mathrm{a}^{\mathrm{d}, \mathrm{e} / 380.4^{\mathrm{f}}}$ & $\mathrm{n} . \mathrm{a}^{\mathrm{d}, \mathrm{e} / 0.005^{\mathrm{f}}}$ & 1.4 & 221.0 & 0.006 \\
\hline
\end{tabular}

a Scaled $\mathrm{V}_{\max }$ calculated from the in vitro $\mathrm{V}_{\max }$ using the scaling factor of microsome protein yields of $35 \mathrm{mg} /(\mathrm{g}$ liver) or $20.6 \mathrm{mg} /$ (g small intestine), bodyweight and tissue fractions of human (Chinese and Caucasian) and rat

b Scaled catalytic efficiency (scaled $\mathrm{V}_{\max } / \mathrm{K}_{\mathrm{m}}$ )

c Chen et al. 2018

d Not available

e Scaled kinetic constants by Chinese tissue fractions

f Scaled kinetic constants by Caucasian tissue fractions

\subsection{Unbound fraction in human blood as determined in the RED assay}

The unbound fraction for lasiocarpine in human plasma was determined to be 0.43 . For as yet unknown reasons, and in contrast to lasiocarpine, the unbound faction $\left(f_{u b}\right)$ of riddelliine could not be detected after incubation in the RED device, even not when the concentration was increased from 5 to $50 \mu \mathrm{M}$. Previously, Chen et al. (2018) defined $\mathrm{f}_{\mathrm{ub}}$ values for lasiocarpine and riddelliine by rat serum using the same method as provided in section 2.5 , and reported that the $f_{u b}$ values of lasiocarpine and riddelliine in rat plasma were comparable, amounting to 0.64 for lasiocarpine and to 0.66 for riddelliine. Therefore, in the present study the $f_{u b}$ of riddelliine in human plasma was assumed to be the same as the $f_{u b}$ value of lasiocarpine in human plasma amounting to 0.43 .

\subsection{PBK model prediction and evaluation}

To evaluate the performance of the human PBK models, ideally the model outcomes should be compared to available human data. Since such human in vivo data on the kinetics of lasiocarpine and riddelliine are not available, model evaluation is mainly based on evaluation 
of the same model in our previous study where we developed and evaluated the model for riddelliiine and lasiocarpine in rat and mouse (Chen et al. 2018). Chen et al. (2018) described the performance of the rat and mouse PBK model of riddelliine by comparing the predicted $\mathrm{C}_{\max }$ of riddelliine in rat and mouse blood to the observed in vivo data reported in the literature (Chen et al. 2018). Data revealed that the predicted levels of $\mathrm{C}_{\max }$ of riddelliine in rat and mouse blood and the $\mathrm{C}_{\max }$ values observed in these species in the in vivo studies matched adequately (Chen et al. 2018). An additional evaluation of the model emerged from comparison of the PBK modelling-based predicted BMDL5-BMDU 5 values derived from the predicted in vivo dose-response curve for liver toxicity of lasiocarpine in rats. The results fell well within the range of PoDs obtained from actual in vivo studies on acute liver toxicity of lasiocarpine in rats, indicating that the PoDs derived from the combined in vitro and in silico PBK modelling approach provided a good approximation of in vivo acute liver toxicity of lasiocarpine (Chen et al. 2018). Based on these results for the rat model, the developed PBK model for lasiocarpine or riddelliine are considered an adequate first approximation to describe the in vivo situation for human. An additional approach to describe the performance of the human PBK model for lasiocarpine and riddelliine for the human populations, especially for the difference between the Chinese and the Caucasian populations, was done based on the approach described previously (Ning et al. 2017). In this approach a comparison is made between the predicted inter-ethnic differences for the dose-dependent concentration of lasiocarpine and riddelliine in the liver blood and the observed inter-ethnic differences in hepatic metabolising enzymes that mainly catalyse the depletion of lasiocarpine or riddelliine. CYP 3A4 predominately catalyses the depletion of lasiocarpine and riddelliine in human liver (Fu et al. 2004; Miranda et al. 1991; Wiedenfeld and Edgar 2011). Figure 5 shows that at similar dose levels the predicted $\mathrm{C}_{\max }$ of lasiocarpine and riddelliine in the liver blood from average Chinese subjects is generally 2- to 3-fold higher than in the liver from average Caucasian subjects. This is mainly due to the fact that the catalytic efficiency for depletion of lasiocarpine and riddelliine by Chinese liver microsomes is 2- to 4-fold lower compared to Caucasian liver mirosomes. The prediction of higher $\mathrm{C}_{\max }$ values for lasiocarpine and riddelliine for Chinese subjects is in line with the reported data for the $\mathrm{C}_{\max }$ and AUC of alprazolam, the substrate for CYP 3A4/5, for which the $\mathrm{C}_{\max }$ and AUC for Asian subjects (ten Chinese, three Filipino, and one Japanese) were 1.2- and 1.3-fold, respectively, higher than for Caucasian subjects $(n=14)$ (Lin et al. 1988). Furthermore, in a meta-analysis Barter et al. (2013) showed that the plasma clearance of the CYP 3A4/5 substrate midazolam following an oral dosing was 2.4-fold higher in Caucasian than in Chinese subjects. 

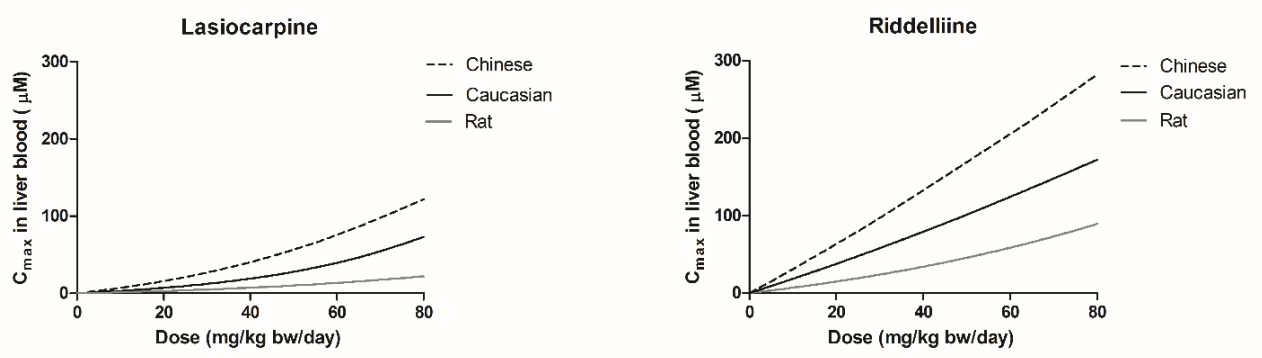

Fig. 5 PBK modelling-based predictions for the dose-dependent $\mathrm{C}_{\max }$ of lasiocarpine and riddelliine in liver blood in Chinese (black dotted line), Caucasian (black solid line) and rat (grey line).

For the inter-species comparison, the predicted $\mathrm{C}_{\max }$ of lasiocarpine and riddelliine in the human (Chinese and Caucasian) liver blood is generally 2- to 5-fold higher than those in the rat liver blood as shown in Figure 5. This is because the catalytic efficiency for depletion of both compounds in human (Caucasian) is 2- to 5-fold lower compared to rat. Altogether, the PBK models obtained in the present study were considered adequate for further prediction of the inter-species and inter-ethnic differences in hepatotoxicity of lasiocarpine and riddelliine.

\subsection{Sensitivity analysis}

The normalized sensitivity coefficients of the model parameters for prediction of the $\mathrm{C}_{\max }$ of lasiocarpine and riddelliine in liver blood of Chinese and Caucasian was analyzed. The sensitivity coefficients were calculated at a dose of $8 \mathrm{ng} / \mathrm{kg}$ bw and $3 \mathrm{mg} / \mathrm{kg}$ bw lasiocarpine and riddelliine and all model parameters with sensitivity coefficients higher than $|0.1|$ are shown in the supplementary materials 6 . The results obtained reveal that for both Chinese and Caucasian subjects at a dose of $8 \mathrm{ng} / \mathrm{kg}$ bw lasiocarpine the predicted $\mathrm{C}_{\max }$ in liver blood in the PBK model is most sensitive to liver related parameters and the absorption rate constant (Ka) for uptake from the GI tract. At $3 \mathrm{mg} / \mathrm{kg}$ bw lasiocarpine, the results of the sensitivity analysis of both ethnic groups are similar to the situation at $8 \mathrm{ng} / \mathrm{kg}$ bw lasiocarpine. The results for the sensitivity analysis of the PBK models for riddelliine shown in Figure S6 reveal similar results showing that $\mathrm{Ka}$ and liver related parameters have the highest influence on the predicted $\mathrm{C}_{\max }$ of riddelliine in the liver blood in both ethnic groups. At $8 \mathrm{ng} / \mathrm{kg}$ bw riddelliine the results of the sensitivity analysis of both ethnic groups are similar to the analysis at $3 \mathrm{mg} / \mathrm{kg}$ bw. For both compounds, $\mathrm{Ka}$ and liver related parameters have less influence on the predicted $\mathrm{C}_{\max }$ in the liver blood in average Chinese compared to average Caucasian.

\subsection{Predicted inter-ethnic differences in hepatotoxicity of lasiocarpine and riddelliine}

Figure 6 shows the predicted in vivo dose-response curves for liver toxicity of lasiocarpine and riddelliine in Chinese and Caucasian subjects obtained by translation of the in vitro concentration-response curves obtained from human hepatocytes (Figure 3) by reverse dosimetry using the respective PBK models. The response \% indicated at the y-axis of the in 
vivo dose-response curve was calculated based on the equation (4): response $\%=100 \%$ (solvent control) - cell viability $\%$ in the in vitro concentration response curve.
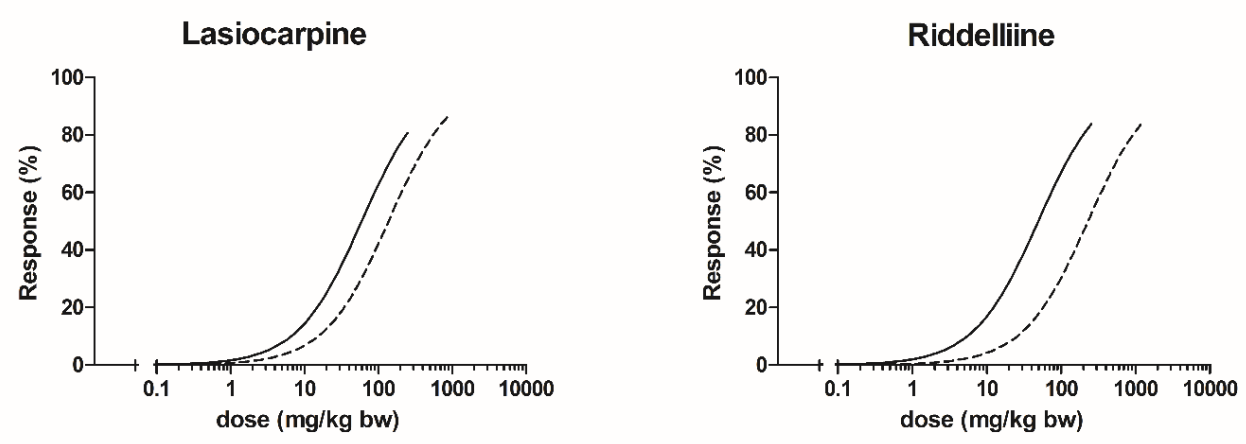

Fig. 6 Predicted dose-response curves for acute liver toxicity of lasiocarpine and riddelliine in average Chinese (black dotted line) and average Caucasians (black solid line) as obtained by translation of cytotoxicity concentration-response data obtained with human hepatocytes using PBK modelling-based reverse dosimetry.

Table 4 presents the $\mathrm{BMDL}_{5}-\mathrm{BMDU}_{5}$ values derived from the predicted dose-response curves as shown in Figure 6 for Chinese and Caucasian subjects. The values of the $\mathrm{BMD}_{5}$ (benchmark dose corresponding to $5 \%$ extra risk) were also presented to enable calculation of the ratio between the $\mathrm{BMD}_{5}$ and $\mathrm{BMDL}_{5} / \mathrm{BMDU}_{5}$ showing they are generally lower than 3 , which was one of the criteria for acceptance of the model fit.

Table 4 Predicted $\mathrm{BMD}_{5}$ and $\mathrm{BMDL}_{5}-\mathrm{BMDU}_{5}$ values derived from dose-response curves predicted using PBK modelling-based reverse dosimetry of in vitro cytotoxicity data with human hepatocytes

\begin{tabular}{ccc}
\hline \multirow{2}{*}{ Compounds } & Predicted BMDL5-BMDU5 $\left(\mathrm{BMD}_{5}\right)(\mathrm{mg} / \mathrm{kg}$ bw/day $)$ \\
\cline { 2 - 3 } & Chinese & Caucasian \\
\hline Lasiocarpine & $14.7-41.2(26.0)$ & $7.4-23.7(14.1)$ \\
Riddelliine & $1.0-5.9(2.6)$ & $0.2-1.2(0.5)$ \\
\hline
\end{tabular}

Interestingly, the PBK modelling-based predictions for in vivo human toxicity of riddelliine could be evaluated using available human data, supporting further evaluation of the PBK models and the PBK modelling-based reverse dosimetry approach. The study of Culvenor (1983) estimated that in human subjects a dose of $1.4-3 \mathrm{mg} / \mathrm{kg}$ bw/day and $0.7-1.5 \mathrm{mg} / \mathrm{kg} \mathrm{bw} /$ day of riddelliine may lead to liver necrosis (exposure of a boy for shorter than 2 weeks) and liver cirrhosis (exposure of a girl for 2 weeks), respectively (Culvenor 1983). The BMD5 values for Caucasian and Chinese subjects predicted in the present study are comparable since they amount to 0.5 and $2.6 \mathrm{mg} / \mathrm{kg}$ bw/day, respectively. For the inter-ethnic comparison, the 
predicted BMDL $_{5}$ of lasiocarpine was observed to be 2.0-fold higher for Chinese than for Caucasian subjects and for riddelliine the predicted BMDL 5 value was 5.0-fold higher for Chinese than for Caucasian subjects, indicating Chinese may be less sensitive to the liver toxicity of these PAs than the Caucasian population. This difference can be mainly ascribed to the lower relative level of bioactivation resulting in lower toxicity at similar dose levels in spite of the slower metabolic clearance.

\subsection{Predicted species differences in hepatotoxicity of lasiocarpine and riddelliine}

To obtain insight in possible species differences in sensitivity toward liver toxicity of the PAs, the $\mathrm{BMDL}_{5}-\mathrm{BMDU}_{5}$ values of lasiocarpine and riddelliine for acute liver toxicity for humans (Chinese and Caucasians) (Table 4) defined in the present study were compared to the same PoDs defined previously for rats using in vitro toxicity data in rat hepatocytes and PBK models for rats (Chen et al. 2018) (see Figure 7). The BMDL $-\mathrm{BMDU}_{5}$ values derived from the predicted dose-response curves for lasiocarpine for humans amounting to $11.1-32.3 \mathrm{mg} /$ $\mathrm{kg}$ bw (average PoDs of Chinese and Caucasians) are up to 2.0-fold lower than the predicted $\mathrm{BMDL}_{5}-\mathrm{BMDU}_{5}$ values of Chen et al. (2018) for lasiocarpine for rat which amounted to 23.0$34.4 \mathrm{mg} / \mathrm{kg}$ bw/day. For riddelliine, the predicted BMDL5-BMDU5 values for humans amounting to $0.6-3.6 \mathrm{mg} / \mathrm{kg}$ bw (average PoDs of Chinese and Caucasians) are up to 8.2-fold lower than the BMDL5-BMDU5 for rat amounting to $4.9-8.4 \mathrm{mg} / \mathrm{kg}$ bw/day. Thus humans seem to be somewhat more sensitive to liver toxicity of these PAs than rats.

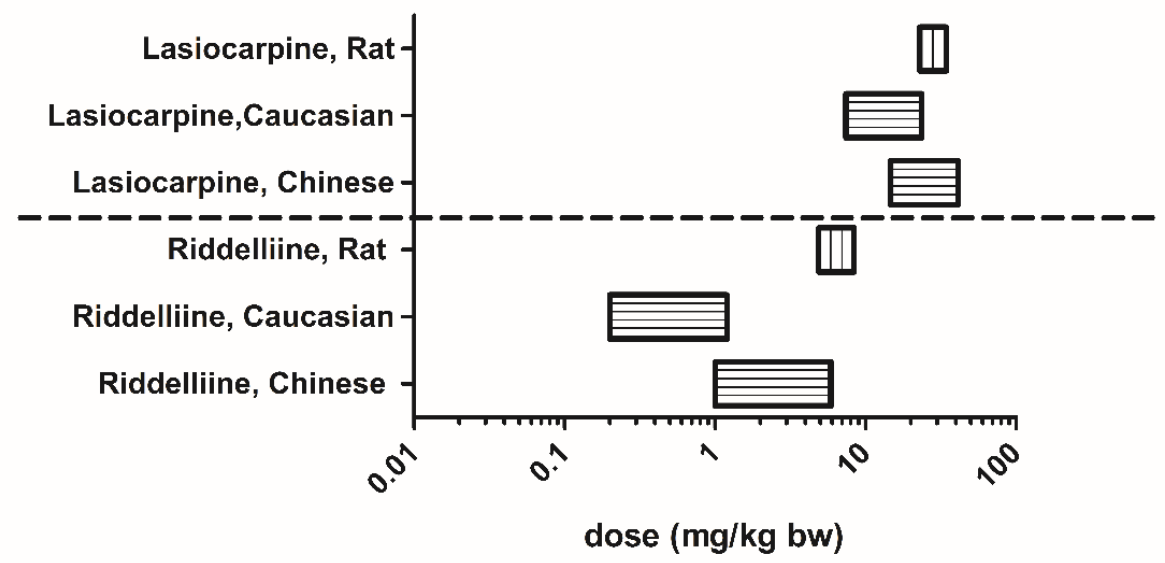

Fig. 7 Predicted BMDL5-BMDU 5 values for acute liver toxicity of lasiocarpine (above the dashed line) and riddelliine (below the dashed line) in humans (Caucasians and Chinese) (bars containing horizontal lines) predicted by the PBK modelling-based reversed dosimetry approach using data for human hepatocytes from the present study as compared to the predicted BMDL5-BMDU 5 values for acute liver toxicity of lasiocarpine (above the dashed line) and riddelliine (below the dashed line) in rat (bars containing vertical lines) derived from our previous study using data for rat hepatocytes and a rat PBK model (Chen et al. 2018). 


\section{Discussion}

The aim of the present study was to use PBK modelling-based reverse dosimetry to translate human cytotoxicity data of lasiocarpine and riddelliine to in vivo dose-response data in humans (Chinese and Caucasians) and study ethnic human differences. Comparison of the results to a similar study previously performed for rats (Chen et al. 2018) would provide insight in species differences in sensitivity to these two PAs.

The PBK model developed in the present study describes the kinetics of lasiocarpine and riddelliine and not of their metabolites, since we assume that the acute liver toxicity of lasiocarpine and riddelliine can be modelled based on cytotoxicity data of the parent compound provided that the in vitro model used contains the adequate enzymes required for their bioactivation to hepatotoxic metabolites. Especially the dehydropyrrolizidine alkaloids metabolites of PAs are reactive with proteins and DNA, thereby causing toxic effects (Fu et al. 2004). The in vitro cytotoxicity experiment using HepaRG cells and human hepatocytes can be expected to take this bioactivation into account. The HepaRG cell line is a human hepatoma cellular model consisting of a mixture of both hepatocyte-like and biliary-like cells (Lambert et al. 2009). After the treatment with DMSO, HepaRG cells differentiate into hepatocyte-like morphology. Kanebratt and Andersson (2008) reported that the expression of P450 in HepaRG cells was generally lower compared with human hepatocytes, except for a higher expression of CYP 3A4 and CYP 7A1. However, the CYP 3A4 activity was still about 1.5-fold higher in human hepatocytes in 2 out of 3 individuals compared to DMSO treated HepaRG cells as determined by measuring the clearance of the CYP 3A4 probe substrate midazolam (Kanebratt and Andersson 2008). Other studies also showed that P450 activities such as CYP 1A2, CYP 2B6, CYP 2C8, CYP 2C9, CYP 2C19 and CYP 2D6 were generally lower in HepaRG cells than in human hepatocytes, expect for CYP 3A4 showing a generally 1.5-fold higher activity in HepaRG cells (Kvist et al. 2018; Lubberstedt et al. 2011). However, Gerets et al. (2012) reported that CYP 3A4 activity was about 17.0-fold lower in the HepaRG cells than in freshly isolated human hepatocytes from three different donors. This conflicting result may result from inter-individual variability in CYP 3A4 activity and/ or from differences in the level of enzyme induction the DMSO treated HepaRG cells. However based on the limited difference in the cytotoxicity of riddelliine and lasiocarpine in primary hepatocytes and the HepaRG cells observed in the present study (Figure 3), we conclude that induction of the CYPs involved in bioactivation of these PAs in the HepRG cells was adequate. Gerets et al. (2012) also reported that cryopreserved primary human hepatocytes showed a 3 -fold higher sensitivity for the detection of hepatotoxic compounds compared with HepaRG cells. In the present study, the $\mathrm{IC}_{50}$ value for lasiocarpine and riddelliine derived from HepaRG cells was 10- and 3-fold higher, respectively, than the $\mathrm{IC}_{50}$ derived from studies with human hepatocytes, probably reflecting the lower metabolic capacity of the HepaRG cells for bioactivation of the PAs. Given these differences, prediction of the acute liver toxicity of both PAs was based on the in vitro concentration-response curves obtained with human primary hepatocytes. 
Based on the sensitivity analysis, the predicted $\mathrm{C}_{\max }$ values of lasiocarpine and riddelliine in average Chinese and average Caucasians are highly affected by the absorption rate constant (Ka) for uptake from the GI tract and the kinetic constants for metabolic clearance of the parent compounds in the liver. Variability in the kinetic constants for metabolic clearance can result from the individual differences in the expression of CYP 3A4 which is the primary enzyme for metabolizing lasiocarpine and riddelliine in humans (Miranda et al. 1991). The literature reports that the abundance of CYP 3A4 in the liver varies significantly in individuals ( $\mathrm{Fu}$ et al. 2004), and the inter-individual variation in the level of CYP 3A4 expression can vary up to 100-fold (Westlind-Johnsson et al. 2003). In addition to the kinetic constants, the Ka value also has a large influence on the model predictions. In the present study, the Ka values of lasiocarpine and riddelliine were $1.55 / \mathrm{h}$ and $0.72 / \mathrm{h}$, respectively as reported by Chen et al. (2018). In the study of Chen et al. (2018), Ka values of both compounds were defined based on $\mathrm{P}_{\mathrm{app}}$ values derived by two methods: Qikprop software (SchrÖdinger, trial version, Germany) and a QSAR method developed by Hou et al. (2004). The $\mathrm{P}_{\text {app }}$ values obtained from these two methods differ only 1.3-fold for lasiocarpine, but 4.5fold for riddelliine (Chen et al. 2018). The large difference in $P_{\text {app }}$ values for riddelliine may therefore largely affect PBK model-predicted internal concentrations and related predicted dose levels that cause acute toxicity. Previously, Chen et al. (2018) reported the predicted $\mathrm{BMDL}_{5}-\mathrm{BMDU}_{5}$ for riddelliine in rat amounting to $1.0-2.2 \mathrm{mg} / \mathrm{kg}$ bw/day when using the $\mathrm{Ka}$ $(0.27 / \mathrm{h})$ obtained by Qikprop software and to $3.3-14.6 \mathrm{mg} / \mathrm{kg}$ bw/day when using the $\mathrm{Ka}$ $(1.17 / \mathrm{h})$ obtained by the QSAR method of Hou et al. (2004). The PoDs for riddelliine obtained in rats when using the average $\mathrm{Ka}$ amount to $4.9-8.4 \mathrm{mg} / \mathrm{kg}$ bw/day (Chen et al. 2018). This outcome supports the outcome of the sensitivity analysis indicating that the $\mathrm{Ka}$ value has a large influence on the model predictions. This leads to the conclusions that further refinement of especially the $\mathrm{P}_{\text {app }}$ and resulting $\mathrm{Ka}$ value may improve the models and their predictions. However, given that the predicted $\mathrm{BMD}_{5}$ values for riddelliine in Chinese and Caucasian subjects amounting to 2.6 and $0.5 \mathrm{mg} / \mathrm{kg}$ bw/day, respectively are comparable to the literature reported in vivo data for human subjects amounting to $0.7-3 \mathrm{mg} / \mathrm{kg}$ bw/day (Culvenor 1983) when using the average Ka values, the use of the average Ka value for riddelliine seems a reasonable strategy. A recent study reported that the pyrrolizidine alkaloid monocrotaline (retronecine-type PA) is a high affinity substrate of the organic cation transporter 1 (OCT1), a transporter mainly expressed in liver and involved in uptake of chemicals from blood into the hepatocytes. This study suggested that active transport mechanisms may be involved in the cellular uptake of pyrrolizidine alkaloids into the liver tissue (Tu et al. 2013). In the present study, we used $\log \mathrm{K}_{\mathrm{ow}}$ to estimate the liver tissue:blood partition coefficients of lasiocarpine and riddelliine. In perfusion-limited PBK models, such as used in our study, an immediate partitioning between plasma and tissue is assumed, which is not dependent on chemical diffusion across cell membranes and/or active uptake processes. Therefore we do not expect to underestimate hepatic concentrations in our models. Given that the sensitivity analysis revealed that the $\mathrm{C}_{\max }$ of lasiocarpine and riddelliine in liver blood were not highly affected by the liver tissue:blood partition coefficients (see supplementary materials 6), estimation of this kinetic parameter for uptake of lasiocarpine 
and riddelliine into liver tissue based on partitioning between plasma and tissue is unlikely to affect the outcomes to a significant extent.

Given that the methods used in the present study and in the previous study (Chen et al. 2018) for predicting the in vivo liver toxicity of lasiocarpine and riddelliine in rat were similar, using species specific primary hepatocytes and species specific subcellular tissue fractions in similar in vitro incubations to define the species specific metabolic clearance, using a similar PBK model structure, the model predictions also provide insight in species differences in acute liver toxicity of lasiocarpine and riddelliine. This inter-species comparison, revealed that the predicted $\mathrm{BMDL}_{5}$ for liver toxicity of lasiocarpine and riddelliine in humans (average PoDs of Chinese and Caucasians) is up to 2.0-fold and 8.2-fold lower, respectively, than that for rat, indicating that humans are somewhat more sensitive towards acute liver toxicity of lasiocarpine and riddelliine than rats. It is of interest to note that the in vitro cytotoxicity of these two compounds in human Caucasian hepatocytes was lower than in rat hepatocytes, and that the predicted higher toxicity in vivo thus results from inter-species differences in the kinetics. The kinetics showed a 1.9- and 5.0-fold slower clearance of lasiocarpine and riddelliine in humans (Caucasian) compared to rats. According to literature, the metabolism of PAs in rodents is mainly catalyzed by CYP 2B and CYP 3A subfamilies, while in humans, metabolism is mainly catalysed by CYP 3A4 (Li et al. 2011; Miranda et al. 1991). This observation is of interest when considering rat toxicity data for human risk assessment. Extrapolating rodent toxicity data to the human situation usually includes the use of a default uncertainty factor of 10 to account for species differences, consisting of a factor of 4.0 for kinetic and a factor of 2.5 for dynamic differences (WHO 1999). In the present study, the interspecies differences in acute liver toxicity of lasiocarpine and riddelliine fall well within the default factor of 10. Such a compound specific uncertainty factor that could be smaller than the default value of 10 was also reported for aristolochic acid I for which in vitro-PBK model facilitated reverse dosimetry-based predicted PoDs differed about 1.8-fold between rat and human and about 1.9-fold between mouse and human (Abdullah et al. 2016). Also for the bioactivation of estragole the compound specific uncertainty factor for differences in kinetics between humans and rats was predicted to be 2-fold and thus smaller than the default factor of 4 for kinetic differences (Punt et al. 2009). Altogether, these results reveal that the in vitroPBK modelling-based reverse dosimetry may provide a way forward to define compound specific uncertainty factors, also called compound specific adjustment factors (CSAFs), in risk assessment.

Comparison of the predicted $\mathrm{BMDL}_{5}$ for lasiocarpine and riddelliine between Chinese and Caucasian subjects showed that for Chinese subjects, the predicted BMDL 5 for lasiocarpine was 2.0-fold higher and for riddelliine 5.0-fold higher than for Caucasians, suggesting that Chinese subjects might be less sensitive towards acute hepatotoxicity of lasiocarpine and riddelliine. This is mainly due to the differences in bioactivation of the parent compound, resulting in less bioactivation for both compounds at similar dose levels in average Chinese compared to average Caucasian subjects, and occurs in spite of less efficient clearance in Chinese as compared to Caucasians. In the present study, human hepatocytes used to study the in vitro cytotoxicity of lasiocarpine and riddelliine were derived from Caucasian subjects. 
Since human hepatocytes derived from Chinese subjects are not available, in vitro cytotoxicity for Chinese hepatocytes was defined by read-across from the Caucasian liver hepatocytes taking into account the difference in bioactivation between Chinese and Caucasian liver microsomes, as reflected by 7-GS-DHP formation in in vitro incubations. In the present study, we used GSH as a trapping agent to estimate the amount of reactive metabolites formed in liver microsomal incubations which is in line with the method described previously (Tamta et al. 2012). It has been reported that dehydro-PAs react with GSH forming three metabolites, 7-GS-DHP, 9-GS-DHP and 7,9-di-GSH-DHP, of which 7-GSDHP is the major metabolite formed in vivo and in vitro (Chen et al. 2016; Lame et al. 1990; Lin et al. 1998; Reed et al. 1992; Tamta et al. 2012), whereas 7,9-di-GS-DHP is only formed in vitro (Lin et al. 1998; Reed et al. 1992; Tamta et al. 2012). Other factors influencing the dynamics of liver toxicity caused by PAs, for example the relative potential for repair, cannot be taken into account in this way. Nevertheless the approach now taken provides an adequate first approach to study the potential ethnic differences in the toxicokinetic process of PAinduced liver toxicity.

The ethnic differences in metabolic clearance of lasiocarpine and riddelliine determined by substrate depletion using liver microsomess for both populations was 2- to 4-fold, with clearance by the average Caucasian being more efficient. The difference in efficiency for formation of bioactive metabolites for both compounds by the Chinese and Caucasian incubations amounted to 7- to 8-fold, the value for the average Caucasian being highest. It has been reported that besides CYP 450 enzymes, flavin-containing monooxygenases (FMO) and carboxylesterases are considered to be involved in detoxification pathways as shown in Figure 1 (Fu et al. 2004). FMO was found to be partly involved in the N-oxide formation and carboxylesterases are involved in hydrolysis of the ester groups of PAs leading to the formation of necine base and necic acid moieties, which is considered to be the major detoxification pathway ( $\mathrm{Fu}$ et al. 2004). Currently, two predominant carboxylesterases (CES) enzymes have been identified in human, i.e. CES 1 and CES 2 (Wang et al. 2018). A recent review reported that ethnic differences in carboxylesterases enzymes activity may be present due to single nucleotide polymorphisms (SNPs). For instance, CES 1 variants G1420E and D260fs, which are two important functional SNPs in Caucasian populations, showed reduced esterase activity in vitro, while these two variants were not found in the Asian populations (Cha et al. 2014; Wang et al. 2018). However, detailed studies of assessing ethnic differences in carboxylesterases activity are still lacking.

It is also of interest to note that in toxicological risk assessment, a default uncertainty factor of 10 is used to account for inter-individual variation, which can be divided into a factor 3.16 for kinetics and 3.16 for dynamic differences (WHO 1999). The ethnic human difference in predicted PoDs of lasiocarpine and riddelliine between Chinese and Caucasian subjects, were estimated to be 1.7-and 5.0-fold respectively, and are thus in line with the default value of 3.16 for inter-individual kinetic differences. However, various studies report much higher than 2 to 4-fold inter-individual variability in the expression of the major hepatic CYP enzyme (CYP 3A4) involved in PA metabolism, ranging up to 100-fold (Westlind-Johnsson et al. 2003), or even up to 400-fold when taking illness, inhibition, and induction-related 
interactions into account (Galetin et al. 2004; Wilkinson 2005). Another study reported that inter-individual variation in the expression of CYP $3 \mathrm{~A} 4$ varies 40- to 50-fold (IngelmanSundberg 2004). Therefore, the human inter-individual variation in kinetics is likely to be larger than the default value of 3.16. In a next step, to actually quantify inter-individual human variation in sensitivity to acute liver toxicity of lasiocarpine and riddelliine, the PBK model of the present study should be developed for individual subjects and combined with Monte Carlo modelling to predict hepatotoxicity within the human population. This Monte Carlo modelling should especially consider the variability in the PBK model parameters shown in the sensitivity analysis to influence the model predictions to the largest extent including the $\mathrm{Ka}$ and the kinetic parameters for metabolic clearance in the liver.

Given that the ultimate critical effect of lasiocarpine and riddelliine is not only acute hepatotoxicity but also genotoxicity and subsequently, carcinogenic transformation, it may also be of interest to extent the current PBK model to a physiologically based dynamic (PBD) model able to predict in vivo DNA binding as previously done for estragole (Paini et al. 2010). This would require definition of an in vitro concentration response curve for DNA adduct formation in the primary hepatocytes, which can subsequently be translated to an in vivo dose-response curve for DNA adduct formation. According to the study of Xia et al. (2013), DNA adduct formation is a common biological biomarker of PA-induced tumorigenicity in rats. Previous studies reported that the metabolism of riddelliine by human liver microsomes resulted in a similar metabolic pattern and DNA adduct profile to those formed in the rat liver, suggesting that the mode of action of PAs studied in experimental rodents is highly relevant to humans (Xia et al. 2003; Zhao et al. 2012). The current PBK model could also translate in vitro concentration-response curves for DNA adduct formation in human liver cells to in vivo dose-response curves for DNA adduct formation in the liver of human.

In conclusion, the present study shows that PBK modelling-based reverse dosimetry can identify the differences in inter-species and inter-ethnic human differences in liver toxicity of lasiocarpine and riddelliine. The species dependent variation in hepatotoxicity defined by the BMDL $_{5}$ (average PoDs of Caucasians and Chinese) is up to 2.0-fold for lasiocarpine and 8.2fold for riddelliine, with humans being more susceptible to lasiocarpine and riddelliineinduced liver toxicity than rat. The inter-ethnic human difference between average Chinese and average Caucasian subjects was estimated to be 2.0-fold for lasiocarpine and 5.0-fold for riddelliine, with the average Caucasian being more sensitive towards the acute liver toxicity of lasiocarpine and riddelliine, mainly due to more efficient reactive metabolite formation. Altogether, the present study shows proof-of-principle for a method to predict inter-species and inter-ethnic differences in in vivo liver toxicity for PAs by an alternative testing strategy integrating in vitro cytotoxicity assays with PBK modelling-based reverse dosimetry.

\section{Note}

The authors declare that they have no conflict of interest 


\section{References}

Abdullah R, Alhusainy W, Woutersen J, Rietjens IMCM, Punt A (2016) Predicting points of departure for risk assessment based on in vitro cytotoxicity data and physiologically based kinetic (PBK) modeling: The case of kidney toxicity induced by aristolochic acid I. Food Chem Toxicol 92:104-116

Adams IR, Hamlin KE, Jr., Jelinek CF, Phillips RF (1942) Structure of riddelliine, the alkaloid in Senecio riddellii. J Am Chem Soc 64:2760-2763

Bane A, Seboxa T, Mesfin G, Ali A, Tsegaye Y, M WT, S GS, Haile T (2012) An outbreak of venoocclusive liver disease in northern Ethiopia, clinical findings. Ethiop Med J 50 Suppl 2:9-16

Barter ZE, Tucker GT, Rowland-Yeo K (2013) Differences in Cytochrome P450-Mediated Pharmacokinetics Between Chinese and Caucasian Populations Predicted by Mechanistic Physiologically Based Pharmacokinetic Modelling. Clin Pharmacokinet 52:1085-1100

Bfr (2013) Pyrrolizidine alkaloids in herbal teas and teas. BfR opinion

Brown RP, Delp MD, Lindstedt SL, Rhomberg LR, Beliles RP (1997) Physiological parameter values for physiologically based pharmacokinetic models. Toxicol Ind Health 13:407-484

Cha Y-J, Jeong H-E, Shin J-G, Kim E-Y, Yu K-S, Cho J-Y, Hyun Yoon S, Lim K (2014) Genetic Polymorphisms of the Carboxylesterase 1 ( CES1 ) Gene in a Korean Population. Transl Clin Pharmacol 22:30-34

Chan PC, Haseman JK, Prejean JD, Nyska A (2003) Toxicity and carcinogenicity of riddelliine in rats and mice. Toxicol Lett 144:295-311

Chan SC, Liu CL, Lo CM, Lam BK, Lee EW, Wong Y, Fan ST (2006) Estimating liver weight of adults by body weight and gender. World J Gastroenterol 12:2217-2222

Chen L, Ning J, Louisse J, Wesseling S, Rietjens IMCM (2018) Use of physiologically based kinetic modelling-facilitated reverse dosimetry to convert in vitro cytotoxicity data to predicted in vivo liver toxicity of lasiocarpine and riddelliine in rat. Food Chem Toxicol 116(Pt B):216-226

Chen M, Li L, Zhong D, Shen S, Zheng J, Chen X (2016) 9-Glutathionyl-6,7-dihydro-1-hydroxymethyl5H-pyrrolizine Is the Major Pyrrolic Glutathione Conjugate of Retronecine-Type Pyrrolizidine Alkaloids in Liver Microsomes and in Rats. Chem Res Toxicol 29(2):180-189

Cubitt HE, Houston JB, Galetin A (2009) Relative importance of intestinal and hepatic glucuronidationimpact on the prediction of drug clearance. Pharm Res 26:1073-1083

Culvenor CC (1983) Estimated intakes of pyrrolizidine alkaloids by humans. A comparison with dose rates causing tumors in rats. J Toxicol Environ Health 11:625-635

DeJongh J, Verhaar HJM, Hermens JLM (1997) A quantitative property-property relationship (QPPR) approach to estimate in vitro tissue-blood partition coefficients of organic chemicals in rats and humans. Arch Toxicol 72:17-25

European Food Safety Authority (EFSA) (2011) Scientific opinion on pyrrolizidine alkaloids in food and feed. EFSA J 9: 2406 
European Food Safety Authority (EFSA) (2017) Risks for human health related to the presence of pyrrolizidine alkaloids in honey tea herbal infusions and food supplements. EFSA J 15:4908

Environmental Protection Agency (EPA) (2017) U.S. EPA Benchmark dose modeling guidance.

Evans MV, Andersen ME (2000) Sensitivity analysis of a physiological model for 2,3,7,8tetrachlorodibenzo-p-dioxin (TCDD): assessing the impact of specific model parameters on sequestration in liver and fat in the rat. Toxicol Sci 54:71-80

Fashe MM, Juvonen RO, Petsalo A, Rasanen J, Pasanen M (2015) Species-Specific Differences in the in Vitro Metabolism of Lasiocarpine. Chem Res Toxicol 28:2034-2044

Field RA, Stegelmeier BL, Colegate SM, Brown AW, Green BT (2015) An in vitro comparison of the cytotoxic potential of selected dehydropyrrolizidine alkaloids and some N-oxides. Toxicon 97:36-45

Fu PP, Xia Q, Lin G, Chou MW (2004) Pyrrolizidine Alkaloids - Genotoxicity, Metabolism Enzymes, Metabolic Activation, and Mechanisms. Drug Metab Rev 36:1-55

Galetin A, Brown C, Hallifax D, Ito K, Houston JB (2004) Utility of recombinant enzyme kinetics in prediction of human clearance: Impact of variability, CYP3A5, and CYP2C19 on CYP3A4 probe substrates. Drug Metab Dispos 32:1411-1420

Gerets HHJ, Tilmant K, Gerin B, Chanteux H, Depelchin BO, Dhalluin S, Atienzar FA (2012) Characterization of primary human hepatocytes, HepG2 cells, and HepaRG cells at the mRNA level and CYP activity in response to inducers and their predictivity for the detection of human hepatotoxins. Cell Biology and Toxicology 28:69-87

Hou TJ, Zhang W, Xia K, Qiao XB, Xu XJ (2004) ADME evaluation in drug discovery. 5. Correlation of Caco-2 permeation with simple molecular properties. J Chem Inf Comput Sci 44:1585-1600

International Agency for Research on Cancer (IARC) (2017) Agents Classified by the IARC Monographs. vol 1-118. International Agency for Research on Cancer, Lyon, France

Ingelman-Sundberg M (2004) Human drug metabolising cytochrome P450 enzymes: properties and polymorphisms. Naunyn Schmiedebergs Arch Pharmacol 369:89-104

Kanebratt KP, Andersson TB (2008) Evaluation of HepaRG cells as an in vitro model for human drug metabolism studies. Drug Metab Dispos 36:1444-1452

Kvist AJ, Kanebratt KP, Walentinsson A, Palmgren H, O'Hara M, Bjorkbom A, Andersson LC, Ahlqvist M, Andersson TB (2018) Critical differences in drug metabolic properties of human hepatic cellular models, including primary human hepatocytes, stem cell derived hepatocytes, and hepatoma cell lines. Biochem Pharmacol 155:124-140

Lambert CB, Spire C, Claude N, Guillouzo A (2009) Dose- and time-dependent effects of phenobarbital on gene expression profiling in human hepatoma HepaRG cells. Toxicol Appl Pharmacol 234:345-360

Lame MW, Morin D, Jones AD, Segall HJ, Wilson DW (1990) Isolation and identification of a pyrrolic glutathione conjugate metabolite of the pyrrolizidine alkaloid monocrotaline. Toxicol Lett 51:321-329

Li N, Xia QS, Ruan JQ, Fu PP, Lin G (2011) Hepatotoxicity and Tumorigenicity Induced by Metabolic Activation of Pyrrolizidine Alkaloids in Herbs. Curr Drug Metab 12:823-834 
Lin G, Cui YY, Hawes EM (1998) Microsomal formation of a pyrrolic alcohol glutathione conjugate of clivorine. Firm evidence for the formation of a pyrrolic metabolite of an otonecine-type pyrrolizidine alkaloid. Drug Metab Dispos 26:181-184

Lin KM, Lau JK, Smith R, Phillips P, Antal E, Poland RE (1988) Comparison of alprazolam plasma levels in normal Asian and Caucasian male volunteers. Psychopharmacology 96:365-369

Lubberstedt M, Muller-Vieira U, Mayer M, Biemel KM, Knospel F, Knobeloch D, Nussler AK, Gerlach JC, Zeilinger K (2011) HepaRG human hepatic cell line utility as a surrogate for primary human hepatocytes in drug metabolism assessment in vitro. J Pharmacol Toxicol 63:59-68

Medinsky MA, Leavens TL, Csanady GA, Gargas ML, Bond JA (1994) In vivo metabolism of butadiene by mice and rats: a comparison of physiological model predictions and experimental data. Carcinogenesis 15:1329-1340

Miranda CL, Reed RL, Guengerich FP, Buhler DR (1991) Role of cytochrome P450IIIA4 in the metabolism of the pyrrolizidine alkaloid senecionine in human liver. Carcinogenesis 12:515-519

Mohabbat O, Younos MS, Merzad AA, Srivastava RN, Sediq GG, Aram GN (1976) An outbreak of hepatic veno-occlusive disease in north-western Afghanistan. Lancet 2:269-271

National Health and Family Planning Commission (NHFPC) (2007) Reference individuals for use in radiation protection-Part2: Masses of main organs and tissues (In Chinese).

National Health and Family Planning Commission (NHFPC) (2014) Reference individiuals for use in radiation protection-Part3: main physiological parameters (In Chinese). vol GBZ/T 200.3.

Ning J, Louisse J, Spenkelink B, Wesseling S, Rietjens IMCM (2017) Study on inter-ethnic human differences in bioactivation and detoxification of estragole using physiologically based kinetic modeling. Arch Toxicol 91:3093-3108

National Toxicology Program (NTP) (1978) Bioassay of lasiocarpine for possible carcinogenicity. Natl Cancer Inst Carcinog Tech Rep Ser 39: 1-66

National Toxicology Program (NTP) (2003) Toxicology and carcinogenesis studies of riddelliine (CAS No 23246-96-0) in F344/N rats and B6C3F1 mice (gavage studies). Natl Toxicol Program Tech Rep Ser 508: $1-280$

Paini A, Punt A, Viton F, Scholz G, Delatour T, Marin-Kuan M, Schilter B, van Bladeren PJ, Rietjens IMCM (2010) A physiologically based biodynamic (PBBD) model for estragole DNA binding in rat liver based on in vitro kinetic data and estragole DNA adduct formation in primary hepatocytes. Toxicol Appl Pharmacol 245:57-66

Punt A, Paini A, Boersma MG, Freidig AP, Delatour T, Scholz G, Schilter B, van Bladeren PJ, Rietjens IMCM (2009) Use of physiologically based biokinetic (PBBK) modeling to study estragole bioactivation and detoxification in humans as compared with male rats. Toxicol Sci 110:255-269

Reed RL, Miranda CL, Kedzierski B, Henderson MC, Buhler DR (1992) Microsomal formation of a pyrrolic alcohol glutathione conjugate of the pyrrolizidine alkaloid senecionine. Xenobiotica 22:13211327 
Shi ZR, Yan LN, Li B, Wen TF (2009) Evaluation of standard liver volume formulae for Chinese adults. World J Gastroenterol 15:4062-4066

Slob W (2002) PROAST: Software for dose-response modeling and benchmark dose analysis. RIVM.

Smith LW, Culvenor CCJ (1981) Plant sources of hepatotoxic pyrrolizidine alkaloids. J Nat Prod 44:129152

Stegelmeier BL, Edgar JA, Colegate SM, Gardner DR, Schoch TK, Coulombe RA, Molyneux RJ (1999) Pyrrolizidine alkaloid plants, metabolism and toxicity. J Nat Toxins 8:95-116

Tamta H, Pawar RS, Wamer WG, Grundel E, Krynitsky AJ, Rader JI (2012) Comparison of metabolismmediated effects of pyrrolizidine alkaloids in a HepG2/C3A cell-S9 co-incubation system and quantification of their glutathione conjugates. Xenobiotica 42:1038-1048

Tandon HD, Tandon BN, Mattocks AR (1978) An epidemic of veno-occlusive disease of the liver in Afghanistan. Pathologic features. Am J Gastroenterol 70:607-613

Tu M, Sun S, Wang K, Peng X, Wang R, Li L, Zeng S, Zhou H, Jiang H (2013) Organic cation transporter 1 mediates the uptake of monocrotaline and plays an important role in its hepatotoxicity. Toxicology 311:225-230

van Liempd S, Morrison D, Sysmans L, Nelis P, Mortishire-Smith R (2011) Development and validation of a higher-throughput equilibrium dialysis assay for plasma protein binding. J Lab Autom 16:56-67

Wang D, Zou L, Jin Q, Hou J, Ge G, Yang L (2018) Human carboxylesterases: a comprehensive review. Acta Pharmaceutica Sinica B 8:699-712

Waters NJ, Jones R, Williams G, Sohal B (2008) Validation of a rapid equilibrium dialysis approach for the measurement of plasma protein binding. J Pharm Sci 97:4586-4595

Westlind-Johnsson A, Malmebo S, Johansson A, Otter C, Andersson TB, Johansson I, Edwards RJ, Boobis AR, Ingelman-Sundberg M (2003) Comparative analysis of CYP3A expression in human liver suggests only a minor role for CYP3A5 in drug metabolism. Drug Metab Dispos 31:755-761

World Health Organization (WHO) (1999) International Programme on Chemical Safety (IPCS). Principles for the assessment of risks to human health from exposure to chemicals.

Wiedenfeld H, Edgar J (2011) Toxicity of pyrrolizidine alkaloids to humans and ruminants. Phytochem Rev 10:137-151

Wilkinson GR (2005) Drug therapy - Drug metabolism and variability among patients in drug response. New Engl J Med 352:2211-2221

Xia Q, Chou MW, Kadlubar FF, Chan PC, Fu PP (2003) Human liver microsomal metabolism and DNA adduct formation of the tumorigenic pyrrolizidine alkaloid, riddelliine. Chem Res Toxicol 16:66-73

Xia Q, Zhao Y, Von Tungeln LS, Doerge DR, Lin G, Cai L, Fu PP (2013) Pyrrolizidine alkaloid-derived DNA adducts as a common biological biomarker of pyrrolizidine alkaloid-induced tumorigenicity. Chem Res Toxicol 26:1384-1396 
Yang JL, He MM, Niu W, Wrighton SA, Li L, Liu Y, Li C (2012) Metabolic capabilities of cytochrome P450 enzymes in Chinese liver microsomes compared with those in Caucasian liver microsomes. Brit $\mathrm{J}$ Clin Pharmaco 73:268-284

Yuan D, Lu T, Wei YG, Li B, Yan LN, Zeng Y, Wen TF, Zhao JC (2008) Estimation of standard liver volume for liver transplantation in the Chinese population. Transplant Proc 40:3536-3540

Zhao Y, Xia Q, Gamboa da Costa G, Yu H, Cai L, Fu PP (2012) Full structure assignments of pyrrolizidine alkaloid DNA adducts and mechanism of tumor initiation. Chem Res Toxicol 25:1985-1996

\section{Supplementary materials}

For convenience, supplementary materials 1, 2, 3 and 6 can be found in http://edepot.wur.nl/ $\underline{496944}$

\section{Supplementary materials 4}

Table S4 In vitro cytotoxicity data of lasiocarpine and riddelliine based on the experiments of the present study

\begin{tabular}{|c|c|c|c|c|c|}
\hline Compound & Cell model & $\begin{array}{l}\text { Compound } \\
\text { concentration } \\
(\mu \mathrm{M})\end{array}$ & $\begin{array}{c}\text { Mean of } \\
\text { cell } \\
\text { viability \% }\end{array}$ & $\begin{array}{c}\text { The effect } \\
\text { concentration } \\
\text { in human liver } \\
\text { blood }(\mu \mathrm{M})\end{array}$ & $\begin{array}{c}\text { Dose } \\
\text { reconstructed } \\
\text { using PBK } \\
\text { model } \\
(\mathrm{mg} / \mathrm{kg} \mathrm{bw})\end{array}$ \\
\hline \multirow{3}{*}{ Lasiocarpine } & HepaRG & $\begin{array}{c}0,4.7,9.4 \\
18.8,37.5,75 \\
150,300\end{array}$ & $\begin{array}{c}100,108, \\
108,89 \\
93,75,52, \\
40\end{array}$ & $\begin{array}{c}0,10.9,21.8 \\
43.6,87.2 \\
174.4,348.8 \\
697.7\end{array}$ & $\begin{array}{c}0,27.7,43.5, \\
63.2,86.4, \\
116.8,164.3, \\
248.4\end{array}$ \\
\hline & $\begin{array}{c}\text { Human } \\
\text { (Caucasian) } \\
\text { hepatocyte }\end{array}$ & $\begin{array}{c}0,4.7,9.4 \\
18.8,37.5,75 \\
150,300\end{array}$ & $\begin{array}{c}100,82, \\
62,52,33, \\
26,20,17\end{array}$ & $\begin{array}{c}0,10.9,21.8 \\
43.6,87.2 \\
174.4,348.8, \\
697.7\end{array}$ & $\begin{array}{c}0,27.7,43.5, \\
63.2,86.4, \\
116.8,164.3, \\
248.4\end{array}$ \\
\hline & $\begin{array}{l}\text { Corrected } \\
\text { human } \\
\text { (Chinese) } \\
\text { hepatocyte }\end{array}$ & $\begin{array}{c}0,34,70,104 \\
230,351,707 \\
1414\end{array}$ & $\begin{array}{c}100,82, \\
62,52,33, \\
26,20,17\end{array}$ & $\begin{array}{c}0,79.3,160.8 \\
242.5,535.9 \\
817.6,1645.2 \\
3290.5\end{array}$ & $\begin{array}{c}0,62.0,93.7, \\
120.4,202.2, \\
276.5,489.6, \\
904.1\end{array}$ \\
\hline
\end{tabular}


Table S4 (continued) In vitro cytotoxicity data of lasiocarpine and riddelliine based on the experiments of the present study

\begin{tabular}{|c|c|c|c|c|c|}
\hline Compound & Cell model & $\begin{array}{l}\text { Compound } \\
\text { concentration } \\
(\mu \mathrm{M})\end{array}$ & $\begin{array}{c}\text { Mean of } \\
\text { cell } \\
\text { viability \% }\end{array}$ & $\begin{array}{c}\text { The effect } \\
\text { concentration } \\
\text { in human liver } \\
\text { blood }(\mu \mathrm{M})\end{array}$ & $\begin{array}{c}\text { Dose } \\
\text { reconstructed } \\
\text { using PBK } \\
\text { model } \\
(\mathrm{mg} / \mathrm{kg} \mathrm{bw})\end{array}$ \\
\hline \multirow{3}{*}{ Riddelliine } & HepaRG & $\begin{array}{c}0,4.7,9.4 \\
18.8,37.5,75 \\
150,300\end{array}$ & $\begin{array}{c}100,90 \\
81,80,70 \\
67,49,37\end{array}$ & $\begin{array}{c}0,10.9,21.8, \\
43.6,87.2 \\
174.4,348.8 \\
697.7\end{array}$ & $\begin{array}{c}0,6.0,11.5 \\
22.9,43.9 \\
80.8,144.6 \\
253.7\end{array}$ \\
\hline & $\begin{array}{c}\text { Human } \\
\text { (Caucasian) } \\
\text { hepatocyte }\end{array}$ & $\begin{array}{c}0,4.7,9.4 \\
18.8,37.5,75 \\
150,300\end{array}$ & $\begin{array}{c}100,80 \\
71,63,54 \\
40,32,29\end{array}$ & $\begin{array}{c}0,10.9,21.8 \\
43.6,87.2 \\
174.4,348.8 \\
697.7\end{array}$ & $\begin{array}{c}0,6.0,11.5 \\
22.9,43.9 \\
80.8,144.6 \\
253.7\end{array}$ \\
\hline & $\begin{array}{l}\text { Corrected } \\
\text { Human } \\
\text { (Chinese) } \\
\text { hepatocyte }\end{array}$ & $\begin{array}{c}0,39.3,85.5 \\
192.8,307.4 \\
618.3,1236.7 \\
2236.1\end{array}$ & $\begin{array}{c}100,80, \\
71,63,54, \\
40,32,29\end{array}$ & $\begin{array}{c}0,91.4,198.7 \\
448.4,714.9 \\
1438.0 \\
2875.9,5200.2\end{array}$ & $\begin{array}{c}0,28.3,58.0, \\
122.4,187.3, \\
359.8,694.1, \\
1230.1\end{array}$ \\
\hline
\end{tabular}




\section{Supplementary materials 5}
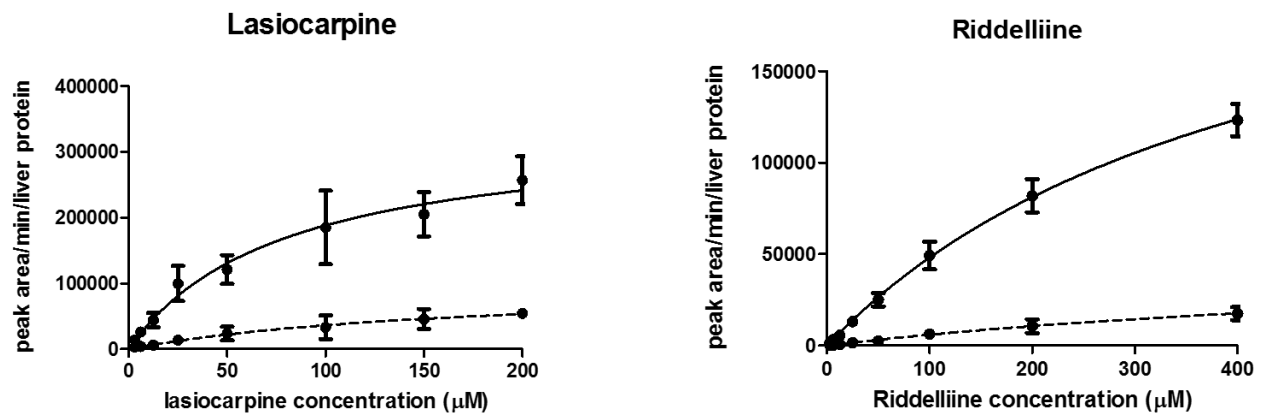

Fig. S5 Lasiocarpine and riddelliine concentration-dependent rate of formation of 7-GS-DHP by liver in incubations with Chinese (dotted line) and Caucasian (solid line) liver microsomes in the presence of the cofactor NADPH. Data points represent mean values \pm SD of three individual experiments.

Table S5 The ratio of the peak areas of 7-GS-DHP formed in incubations with Chinese and Caucasian liver microsomes at each substrate concentration.

\begin{tabular}{ccc}
\hline \multirow{2}{*}{$\begin{array}{c}\text { Substrate concentration } \\
(\mu \mathrm{M})\end{array}$} & \multicolumn{2}{c}{ Fold-difference $\pm \mathrm{SD}^{\mathrm{c}}$} \\
\cline { 2 - 3 } 3.1 & Lasiocarpine & Riddelliine \\
\hline 6.2 & $4.5 \pm 0.4$ & $5.1 \pm 0.6$ \\
12.5 & $6.4 \pm 0.6$ & $10.2 \pm 2.9$ \\
25 & $7.2 \pm 1.0$ & $8.4 \pm 0.9$ \\
50 & $7.3 \pm 1.5$ & $9.1 \pm 2.6$ \\
100 & $5.5 \pm 1.7$ & $10.3 \pm 0.8$ \\
$150^{\mathrm{a} / 200^{\mathrm{b}}}$ & $6.2 \pm 1.8$ & $8.2 \pm 0.8$ \\
$200^{\mathrm{a} / 400^{\mathrm{b}}}$ & $4.7 \pm 1.1$ & $8.2 \pm 1.1$ \\
\hline
\end{tabular}

a Lasiocarpine concentration

b Riddelliine concentration

c Calculated as peak areas of 7-GS-DHP in incubation with Caucasian liver microsomes divided by those in incubation with Chinese liver microsomes 

8
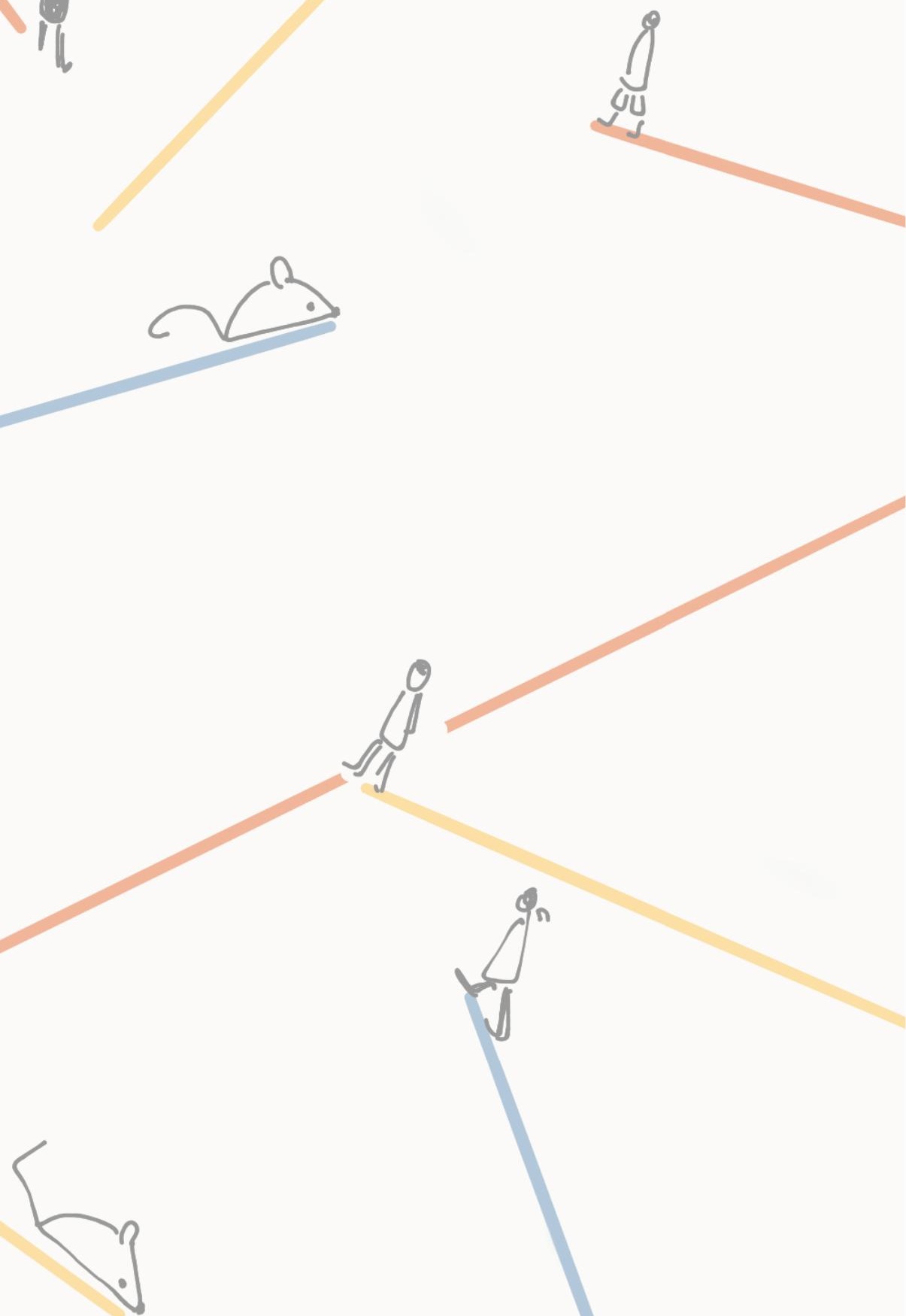


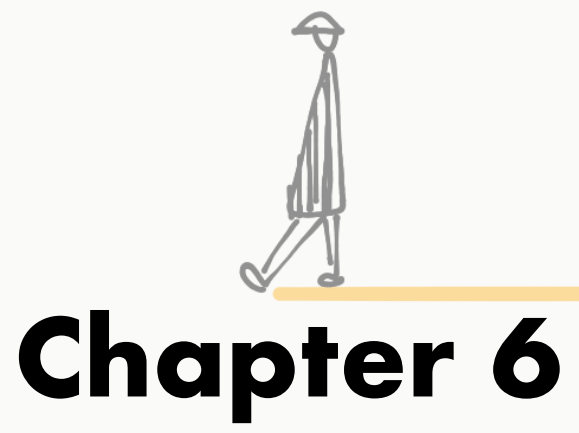

Integrating physiologically based kinetic (PBK) and Monte Carlo modelling to predict interindividual and inter-ethnic variation in Bioactivation and liver toxicity of lasiocarpine
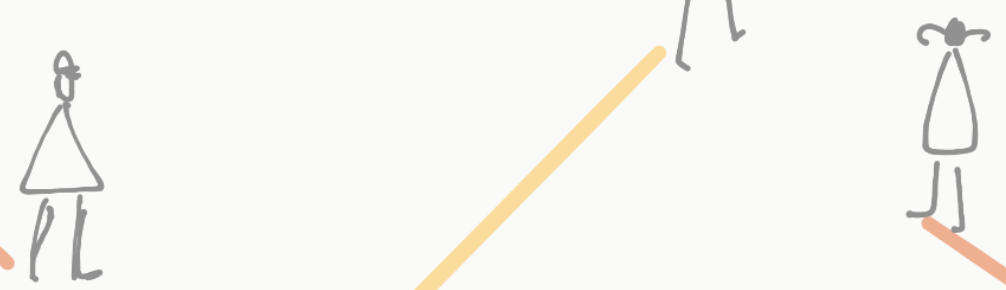

Jia Ning, Ivonne M.C.M. Rietjens, Marije Strikwold

Submitted 


\begin{abstract}
The aim of the present study was to predict the effect of inter-individual and inter-ethnic human kinetic variation on the sensitivity toward acute liver toxicity of lasiocarpine in the Chinese and the Caucasian population, and to derive chemical specific adjustment factors (CSAFs) by integrating variation in the vitro kinetic constants $\mathrm{V}_{\max }$ and $\mathrm{K}_{\mathrm{m}}$, physiologically based kinetic (PBK) modelling and Monte Carlo simulation. CSAFs were derived covering the $90^{\text {th }}$ and $99^{\text {th }}$ percentile of the population distribution of pyrrole glutathione adduct (7-GSDHP) formation, reflecting bioactivation. The results revealed that in the Chinese population, as compared to the Caucasian population, the predicted 7-GS-DHP formation at the geometric mean, the $90^{\text {th }}$ and the $99^{\text {th }}$ percentile were 2.1-, 3.3- and 4.3-fold lower respectively. The CSAFs obtained using the $99^{\text {th }}$ percentile values were 8.3, 17.0 and 19.5 in the Chinese, the Caucasian population and the two populations combined, respectively, while the CSAFs were generally 3.0-fold lower at the $90^{\text {th }}$ percentile. These results indicate that when considering the formation of 7-GS-DHP the Caucasian population may be more sensitive toward acute liver toxicity of lasiocarpine, and further point out that the default safety factor of 3.16 for inter-individual human kinetic differences may not be sufficiently protective. Altogether, the results obtained demonstrate that integrating PBK modelling with Monte Carlo simulations using human in vitro data is a powerful strategy to quantify inter-individual variations in kinetics, and can be used to refine the human risk assessment of pyrrolizidine alkaloids.
\end{abstract}




\section{Introduction}

Lasiocarpine is one of the 1,2-unsaturated pyrrolizidine alkaloids (PAs) which are secondary metabolites of certain flowering plants. Lasiocarpine is widely distributed in the Heliotropium and Symphytum genus (Smith and Culvenor 1981). Human exposure to lasiocarpine may result from consumption of contaminated staple foods, plant food supplements, herbal teas and herbal medicines (EFSA 2011; EFSA 2017). Exposure to lasiocarpine is known to cause acute liver toxicity in humans. The latest documented outbreak has been reported in Afghanistan in 2008, where 38 people suffered from massive ascites and four people died due to consumption of wheat flour contaminated with seeds of Heliotropium, containing lasiocarpine (EFSA 2011; Kakar et al. 2010). Next to acute liver toxicity, lasiocarpine is also classified as a Group 2B carcinogen due to its carcinogenicity induced by a genotoxic mode of action (IARC 2017; NTP 1978).

It is generally accepted that lasiocarpine exerts its hepatotoxicity and carcinogenicity upon metabolic activation (Fu et al. 2004; IPCS 1989; Prakash et al. 1999; Stegelmeier et al. 1999). Three major metabolic pathways of lasiocarpine can be distinguished, namely detoxification via (1) hydrolysis of lasiocarpine into a necine base or (2) N-oxidation to lasiocarpine Noxide, and bioactivation by (3) hydroxylation at the C-3 or C-8 position of the necine base, followed by dehydration into dehydrolasiocarpine (Figure 1). Dehydrolasiocarpine is highly reactive and is able to attack nucleophilic molecules such as DNA and proteins resulting in hepatotoxicity and carcinogenicity (Fu et al. 2004). A recent in vitro study using human liver microsomes provided convincing evidence that the metabolism of lasiocarpine is mainly catalyzed by the CYP 3A enzyme family, particularly CYP 3A4 (Fashe et al. 2015). Other studies also reported that PA metabolism is mainly catalyzed by the CYP 3A4 enzyme in human (Fu et al. 2004; Li et al. 2011; Miranda et al. 1991).

It is well known that the abundance and activity of CYP $3 \mathrm{~A} 4$ in the liver varies significantly in individuals and in different ethnic groups (Fu et al. 2004). Some studies reported that the variation in CYP 3A4 expression including mRNA and protein levels in the liver can be up to 100-fold and even 400-fold when taking illness, inhibition and induction-related interactions of drugs into account (Westlind-Johnsson et al. 2003; Wilkinson 2005). This large variation in CYP 3A activity may result in marked variations in the metabolic profile of PAs and thus different susceptibilities toward pyrrolizidine alkaloid intoxication (Fu et al. 2004). Therefore, human inter-individual and inter-ethnic (Chinese and Caucasian) variation upon PA metabolism may be higher than the default uncertainty factor of 3.16 for inter-individual human kinetic differences (IPCS 2005) that is used to derive health-based guidance values for the risk assessment of compounds with non-genotoxic critical effects. For risk assessment of compounds that are both genotoxic and carcinogenic, the default uncertainty factor of 3.16 for inter-individual human kinetic differences is part of the cut-off value of 10000 for evaluating the Margin of Exposure (MOE) (EFSA 2005; EFSA 2012; EFSA 2015).

Previously, we predicted the inter-ethnic differences in acute liver toxicity between the average Chinese and Caucasian subject to amount to 2-fold for lasiocarpine with the average 
Caucasian being more sensitive than the average Chinese. This was done by applying PBK modelling-based reverse dosimetry translating in vitro hepatic cytotoxicity data obtained in primary hepatocytes in vitro to in vivo acute liver toxicity of lasiocarpine and riddelliine for the average Chinese population and the average Caucasian population. Mixed gender pooled Chinese and Caucasian liver fractions were used to predict the kinetic constants for metabolism of these compounds in the liver which were subsequently included in the physiologically based kinetic (PBK) models for the average Chinese and Caucasians (Ning et al. 2019). However, this study did not characterize the inter-individual variations within the Chinese and the Caucasian population. Given the large variation among individuals in terms of CYP 3A4 variability, the aim of the present study was to study the effect of inter-individual and inter-ethnic (Chinese and Caucasian) kinetic variation on sensitivity towards bioactivation and acute liver toxicity of lasiocarpine using liver fractions from 25 male Chinese individuals and 25 male Caucasian individuals, and to derive chemical specific adjustment factors (CSAFs) by applying physiologically based kinetic (PBK) modelling together with Monte Carlo simulation.

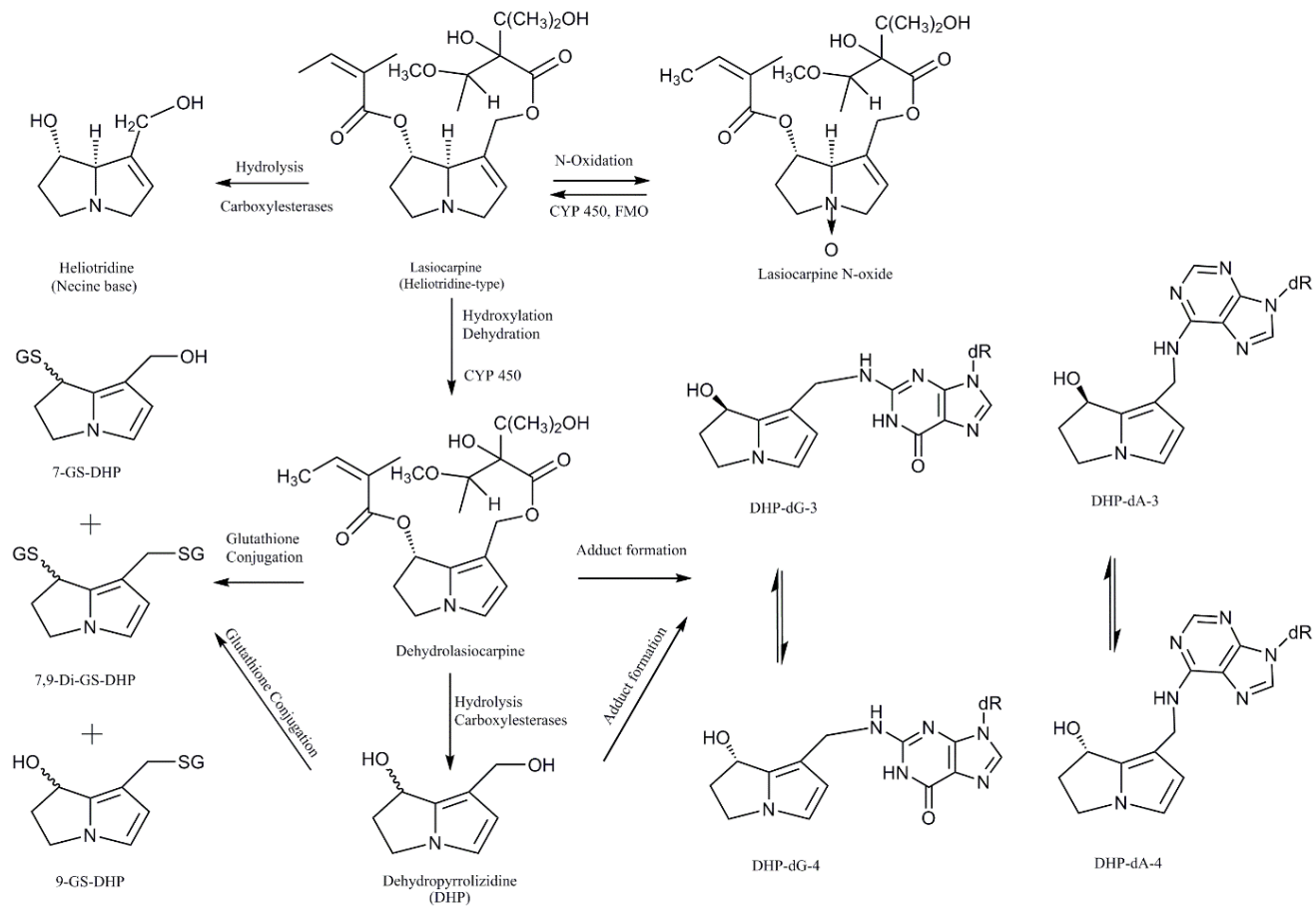

Fig. 1 Principal metabolism pathways of lasiocarpine in humans. FMO=flavin-containing monooxygenase, CYP 450=cytochromes P450 


\section{Materials and Methods}

\subsection{Compounds and biological materials}

Lasiocarpine (purity $>97 \%$ ) was purchased from Phytolab (Vestenbergsgreuth, Germany). Dehydromonocrotaline (purity $\geq 90 \%$ ) was purchased from Santa Cruz Biotechnology (Heidelberg, Germany). Potassium dihydrogen phosphate, trifluoroacetic acid (TFA) and formic acid were purchased from VWR International (Darmstadt, Germany). Phosphate buffered saline (PBS) was purchased from Gibco (Paisley, Scotland, UK). Phosphoric acid was obtained from Merck (Darmstadt, Germany). Reduced nicotinamide adenine dinucleotide phosphate (NADPH) was obtained from Roche Diagnostics (Mannheim, Germany). Acetonitrile (UPLC/MS grade) was obtained from Biosolve BV (Valkenswaard, The Netherlands). Glutathione (GSH), N,N-dimethylformamide (DMF) and dimethylsulfoxide (DMSO) were purchased from Sigma-Aldrich (Steinheim, Germany). Twenty-five individual male human liver microsomes of Caucasian origin were purchased from XenoTech (Lenexa, USA). Twenty-five individual male human liver microsomes of Chinese origin were purchased from PrimeTox (Wuhan, China). The characteristics of the human liver microsome donors are presented in Table $\mathrm{S} 1$ in the supplementary materials 1.

\subsection{General outline for PBK modelling with Monte Carlo simulation}

The outline of the present study consisted of the following steps: 1) the metabolism of lasiocarpine in the liver was studied using subcellular liver fractions of 25 male Chinese individuals and 25 male Caucasian individuals. From this the coefficient of variation (CV) values of lasiocarpine depletion and 7-GS-DHP formation were derived, 2) The interindividual variation in the Chinese and Caucasian population in the kinetic constants $\mathrm{V}_{\max }$ and $\mathrm{K}_{\mathrm{m}}$ of lasiocarpine depletion and 7-GS-DHP formation were included in the PBK model in connection with Monte Carlo simulations to predicted inter-ethnic and inter-individual variation of 7-GS-DHP formation by the liver, 3) CSAFs were derived from the results of the Monte Carlo simulations for the Caucasian population, the Chinese population and the two populations combined covering the $90^{\text {th }}$ and $99^{\text {th }}$ percentile of the population distribution of 7 GS-DHP formation in the liver compartment (IPCS 2005), 4) Finally, PBK modeling-based reverse dosimetry was used to convert in vitro liver toxicity data obtained in human hepatocytes to in vivo dose-response curves for acute liver toxicity for the average and the sensitive (90th and 99th percentile) individuals in the Chinese and Caucasian populations and the two populations combined.

\subsection{In vitro microsomal incubations of lasiocarpine depletion and 7-GS-DHP formation}

The in vitro incubations for lasiocarpine depletion and formation of 7-GS-DHP by 25 individual Chinese and 25 individual Caucasian liver microsomes were performed according to the method described by Ning et al. (2019). Briefly, individual male liver microsomes were incubated with lasiocarpine in the presence of NADPH either in the absence or presence of GSH. The incubation mixtures consisted of $0.1 \mathrm{M}$ potassium phosphate ( $\mathrm{pH} 7.4$ adjusted by phosphoric acid) containing (final concentrations) $0.04 \mathrm{mg} / \mathrm{ml}$ microsomal protein and $2 \mathrm{mM}$ NADPH. Under these incubation conditions conversion of lasiocarpine was linear in time and 
with the amount of microsomal protein (data not shown). In the final incubations, the substrate concentrations ranged from 3 to $200 \mu \mathrm{M}$ lasiocarpine (added from 100 times concentrated stock solutions in DMSO). The reactions were started after a 5 min preincubation at $37^{\circ} \mathrm{C}$ in a shaking water bath by adding NADPH. Incubations were performed for $30 \mathrm{~min}$ in a shaking water bath at $37^{\circ} \mathrm{C}$. The reactions were terminated by adding $20 \%$ $(\mathrm{v} / \mathrm{v})$ ice-cold acetonitrile and the samples were subsequently put on ice. Blank incubations were performed in the absence of the cofactor NADPH for each substrate concentration. Each incubation was performed in duplicate. All samples were centrifuged for $5 \mathrm{~min}$ at $16000 \mathrm{~g}$ and the supernatants were directly analyzed by UPLC.

The incubation conditions for 7-GS-DHP formation were similar to the conditions to measure lasiocarpine depletion outlined above, with the exception that $4 \mathrm{mM}$ GSH (final concentration) was added to the incubation mixtures before the $5 \mathrm{~min}$ pre-incubation. The level of GSH in the incubations was chosen to obtain the maximal scavenging of dehydrolasiocarpine (DHPA) and/or of dehydropyrrolizidine (DHP) forming 7-GS-DHP as the major detectable glutathione conjugate. Each incubation was performed in duplicate. All samples were centrifuged for $5 \mathrm{~min}$ at $16000 \mathrm{~g}$ and the supernatants were directly analyzed by LC-MS/MS.

\subsection{UPLC analysis of lasiocarpine depletion and LC-MS/MS analysis of 7-GS-DHP formation}

From each incubation, $3.5 \mu \mathrm{l}$ of supernatant from incubations for determination of depletion of lasiocarpine were injected into the UPLC (Acquity, Waters) using a BEH C18 (1.7 $\mu \mathrm{m}$ $2.1 \times 50 \mathrm{~mm}$ ) column with a guard column (Acquity, Waters) and a diode array detector (PAD) (Acquity, Waters). The flow rate was $0.6 \mathrm{ml} / \mathrm{min}$ and the mobile phase consisted of ultrapure water with $0.1 \%(\mathrm{v} / \mathrm{v})$ TFA and acetonitrile. For the analysis of lasiocarpine, a gradient was applied from 0 to $50 \%$ acetonitrile in ultrapure water in $2 \mathrm{~min}$, after which the percentage of acetonitrile was increased to $100 \%$ in $3 \mathrm{~min}$ and kept at this level for $2 \mathrm{~min}$, after which the starting conditions were reset. Under these conditions, lasiocarpine had a retention time of 1.9 min. Identification of lasiocarpine was achieved by comparison of the UV spectrum and retention time of this compound to those of the reference compound. Quantification of lasiocarpine was based on its peak area measured at a wavelength of $220 \mathrm{~nm}$, using a linear calibration curve $\left(\mathrm{R}^{2}=0.999\right)$ of the corresponding standard compound $(1.25-200 \mu \mathrm{M}$ lasiocarpine added from 100 times concentrated stock solutions in DMSO and further dissolved in 0.1 M potassium phosphate buffer ( $\mathrm{pH}$ 7.4)).

The glutathione conjugate of lasiocarpine, 7-GS-DHP was identified and quantified by LCMS/MS as described previously (Ning et al. 2019). Briefly, LC-MS/MS analysis was performed on a Shimadzu Nexera XR LC-20AD SR UPLC system coupled with a Shimadzu LCMS-8040 mass spectrometer. Aliquots of the incubation mixture of $1 \mu \mathrm{l}$ were loaded on a reverse phase $\mathrm{C} 18$ column (Phenomenex $1.7 \mu \mathrm{m} 2.1 \times 50 \mathrm{~mm}$ ). The flow rate was $0.3 \mathrm{ml} / \mathrm{min}$ and the mobile phase was made with ultrapure water with $0.1 \%(\mathrm{v} / \mathrm{v})$ formic acid and acetonitrile containing $0.1 \%(\mathrm{v} / \mathrm{v})$ formic acid. A linear gradient was applied from $0 \%$ to $5 \%$ acetonitrile over $8 \mathrm{~min}$ and further increased to $100 \%$ in $6 \mathrm{~min}$. This percentage was kept for 
$0.5 \mathrm{~min}$, and then reduced to the starting conditions in $0.1 \mathrm{~min}$. Under these conditions, 7-GSDHP eluted at $8.97 \mathrm{~min}$. For MS-MS analysis, a Shimadzu LCMS-8040 triple quadrupole with electrospray ionization (ESI) interface was used. The instrument was operated in positive ionization mode in the multiple reaction monitoring (MRM) mode with a spray voltage of $4.5 \mathrm{KV}$. The 7-GSH-DHP was monitored at the $[\mathrm{M}+\mathrm{H}]^{+}$of precursor to products of $443.2 \rightarrow 425.15(\mathrm{CE}=-7 \mathrm{kV}), 443.2 \rightarrow 118.1(\mathrm{CE}=-24 \mathrm{kV})$ and $443.2 \rightarrow 247.2(\mathrm{CE}=$ $-15 \mathrm{kV}$ ) $\mathrm{m} / \mathrm{z}$. The peak area of the total ion chromatogram (TIC) for the 7-GS-DHP formation was used for quantification (Ning et al. 2019).

Quantification of 7-GS-DHP was achieved by comparing the peak area of the 7-GS-DHP formed in the incubation samples to the calibration curve of 7-GS-DHP $\left(r^{2}=0.991\right)$ ranging from $0.078 \mu \mathrm{M}$ to $10 \mu \mathrm{M}$ at a wavelength of $220 \mathrm{~nm}$. 7-GS-DHP was synthesized based on the method described by Ma et al. (2015). Briefly, GSH (6.5 mg, $21 \mathrm{mM})$ in $70 \mu \mathrm{L}$ of PBS was added to dehydromonocrotaline $(10 \mathrm{mg}, 31 \mathrm{mM})$ in $1 \mathrm{ml}$ of DMF with molar ratio of dehydromonocrotaline : GSH of 1:0.67. The reaction mixture was stirred at ambient temperature for $2 \mathrm{~h}$ and quenched with $10 \mathrm{ml}$ of PBS. The reaction product was purified by preparative HPLC (Waters, Milford, MA) equipped with an Alltima C18 $5 \mu \mathrm{m}$ column (150 $\times$ $4.6 \mathrm{~mm})$ and a guard column $(7.5 \times 4.6 \mathrm{~mm})$ (Alltech, Bergen op Zoom, the Netherlands). Detection was performed between 200 and $350 \mathrm{~nm}$ using a Waters 2996 photodiode array detector (PAD) (Waters, Milford, MA). The flow rate was $1 \mathrm{ml} / \mathrm{min}$. The mobile phase used for purification consisted of (A) $0.01 \%$ formic acid in ultrapure water and (B) acetonitrile. A gradient was applied from $5 \% \mathrm{~B}$ with a linear increase to $15 \%$ in $40 \mathrm{~min}$, after which the gradient returned to the initial condition in $5 \mathrm{~min}$ and remained $5 \mathrm{~min}$ at this condition before the next injection. Figure S2 in the supplementary materials 2 shows the HPLC-PAD chromatogram of reaction products before purification. The peaks of 7-GS-DHP were collected and subsequently freeze dried. The purity of 7-GS-DHP was checked by HPLCPAD and was determined to be $82 \%$ by comparing the peak area of 7-GS-DHP and the peak area of the impurities after purification. The standard was dissolved in water and aliquots were stored in $-80^{\circ} \mathrm{C}$.

\subsection{Determination of kinetic constants of lasiocarpine depletion and 7-GS-DHP formation}

The depletion of lasiocarpine was quantified as the amount detected in the blank incubations performed in the absence of the cofactor NADPH minus the amount detected in the full corresponding incubations with NADPH. The amount of 7-GS-DHP formed in the full incubations in the presence of NADPH and GSH was corrected for the amounts detected in the blank incubations without NADPH for each substrate concentration. The data for the rate of conversion of lasiocarpine and 7-GS-DHP formation with increasing substrate concentration $[\mathrm{S}]$ were fitted to the standard Michaelis-Menten equation:

$\mathrm{v}=\mathrm{V}_{\max } /\left(1+\mathrm{K}_{\mathrm{m}} /[\mathrm{S}]\right)$

The apparent maximum velocity $\left(\mathrm{V}_{\max }\right)$ and the apparent Michaelis-Menten constant $\left(\mathrm{K}_{\mathrm{m}}\right)$ were determined by fitting the data to the Michaelis-Menten model using GraphPad 
(GraphPad Prism software version 5.04, San Diego California USA). The catalytic efficiency in vitro expressed in $\mu \mathrm{L} / \mathrm{min} / \mathrm{mg}$ liver microsomes was calculated as the $\mathrm{V}_{\max }$ in vitro divided by the $\mathrm{K}_{\mathrm{m}}$. In vitro derived $\mathrm{V}_{\max }$ values expressed in $\mathrm{nmol} / \mathrm{min} / \mathrm{mg}$ microsomal protein were scaled to in vivo $\mathrm{V}_{\max }$ values expressed in $\mu \mathrm{mol} / \mathrm{h} / \mathrm{g}$ liver using a microsomal protein yield of $35 \mathrm{mg} / \mathrm{g}$ human liver (Medinsky et al. 1994). The $\mathrm{K}_{\mathrm{m}}$ values in vitro were assumed to be equal to in vivo $K_{m}$ values.

\subsection{PBK modelling}

The PBK model previously developed for lasiocarpine in Chinese and Caucasians was used in the present study to evaluate the human inter-ethnic and inter-individual variation in lasiocarpine induced bioactivation that may result from differences in the kinetics of lasiocarpine metabolism in the liver. In our previous study (Ning et al. 2019), the fraction of blood flow to the small intestine for the Caucasian population was $18.1 \%$, obtained from Brown et al. (1997) which is the low of the portal vein including the sum of the blood flow to stomach, esophagus, small intestine, large intestine, spleen, and pancreas. In the present study, the value of the fraction of blood flow to the small intestine for the Caucasian population was further refined to $10 \%$ as reported from ICRP (2003). It is important to note that this refinement will only have a minor influence on the predicted $\mathrm{C}_{\max }$ of lasiocarpine in liver blood. In the present study, we further included the kinetic parameters of 7-GS-DHP formation in the PBK model code to determine the relative inter-ethnic and inter-individual differences in bioactivation of lasiocarpine. As an example the equation for the liver is described as follows:

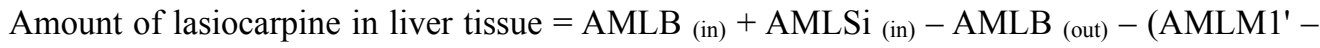
AMLM2') - AMLM2'

Where AMLB (in) is the total amount of lasiocarpine entering the liver from the blood ( $\mu \mathrm{mol} /$ h), AMLSi (in) is the amount of lasiocarpine entering the liver from the intestinal tissue ( $\mu \mathrm{mol}$ /h), AMLB (out) reflects the amount of lasiocarpine leaving the liver to the systemic blood circulation $(\mu \mathrm{mol} / \mathrm{h})$, AMLM1' is the total amount of lasiocarpine metabolized in the liver ( $\mu \mathrm{mol} / \mathrm{hr}$ ), AMLM2' is the amount of 7-GS-DHP formed in the liver ( $\mu \mathrm{mol} / \mathrm{hr}$ ). The model code and physiological parameters can be found in the supplementary materials 3 . With this equation, the change of lasiocarpine in the liver tissue by metabolism is solely based on the lasiocarpine depletion as measured in vitro, while next to that, 7-GS-DHP formation in the liver is modelled as an additional parameter. It is important to note that the amount of 7-GSDHP formation formally is already included in the AMLM1', which is the parameter describing clearance of lasiocarpine and this is why AMLM2', the formation of 7-GS-DHP is both added and subtracted. Adding it in this way allows quantification of the 7-GS-DHP formation without disturbing the mass balance.

\subsection{Sensitivity analysis}

A sensitivity analysis was performed to identify model parameters that influence the predicted 7-GS-DHP formation by the liver expressed in $\mu \mathrm{g} / \mathrm{kg}$ bw, 24 hours after a single oral dose of $8 \mathrm{ng} / \mathrm{kg}$ bw/day and $3 \mathrm{mg} / \mathrm{kg}$ bw/day representing respectively an average daily intake of PAs 
via consumption of herbal tea (BfR 2013) and a dose level known to cause liver toxicity in humans (Culvenor 1983). Normalized sensitivity coefficients (SC) were calculated using the following equation:

$$
\mathrm{SC}=\left(\mathrm{C}^{\prime}-\mathrm{C}\right) /\left(\mathrm{P}^{\prime}-\mathrm{P}\right) \times(\mathrm{P} / \mathrm{C})
$$

Where $\mathrm{C}$ is the initial value of the model output (amount of 7-GS-DHP formation by the liver); $\mathrm{C}^{\prime}$ is the modified model output resulting from a $5 \%$ increase of the parameter value; $\mathrm{P}$ is the initial parameter value; $\mathrm{P}^{\prime}$ is the modified parameter value (Evans and Andersen 2000).

\subsection{Individual PBK models and Monte Carlo simulation}

The kinetic constants shown to be of influence in the sensitivity analysis were determined from the incubation experiments with 25 individual Chinese male and 25 individual Caucasian male liver microsomes and were integrated into the PBK model resulting in 50 separate PBK models. Parameters that were not influential on the model outcome $(\mathrm{SC}<|0.5|)$ were kept as they were originally defined for the average Caucasian or average Chinese population. With the PBK models, the formation of 7-GS-DHP by the liver at an oral dose level of $8 \mathrm{ng} / \mathrm{kg}$ bw was predicted for each individual. Based on the incubations with 25 individual Chinese and 25 individual Caucasians, the mean and the $\mathrm{CV}$ of the $\mathrm{V}_{\max }$ and $\mathrm{K}_{\mathrm{m}}$ for lasiocarpine depletion and 7-GS-DHP formation were obtained.

Then, a Monte Carlo simulation was performed in connection with the PBK model to simulate the variation in the 7-GS-DHP formation by the liver that could occur in the Chinese, the Caucasian and the two populations combined at an oral dose of $8 \mathrm{ng} / \mathrm{kg}$ bw. For the Monte Carlo analysis, a total of 15000 simulations were performed, where in each simulation, the $\mathrm{V}_{\max }$ and $\mathrm{K}_{\mathrm{m}}$ of lasiocarpine depletion and of 7-GS-DHP formation were randomly taken from a log-normal distribution. The distributions were truncated at $\pm 3 \mathrm{SD}$ by excluding individuals with kinetic constants that were three times higher or lower than the geometric mean (GM) values from the Monte Carlo simulation (Punt et al. 2016; Strikwold et al. 2017). The kinetic constants for the lasiocarpine depletion and 7-GS-DHP formation in the liver were assumed to vary independently, but the maximum allowed ratio of the $V_{\max }$ of 7GS-DHP formation and the $\mathrm{V}_{\max }$ of lasiocarpine depletion was set to 1.0 to assure 7-GS-DHP formation cannot exceed the total lasiocarpine depletion. The mean, $\mu_{w}$ and standard deviation $\sigma_{w}$ describing the log-normal distribution of $\mathrm{V}_{\max }$ and $\mathrm{K}_{\mathrm{m}}$ values were derived using the following equation (Zhang et al. 2007):

$$
\mu_{w}=\ln \left(\mu_{x} / \sqrt{1+\mathrm{CV}_{x}^{2}}\right) \text { and } \sigma_{w}^{2}=\ln \left(1+\mathrm{CV}_{x}^{2}\right)
$$

Where $\mu_{x}$ is the mean of $V_{\max }$ or $\mathrm{K}_{\mathrm{m}}$ and $\mathrm{CV}_{x}$ is the coefficient of variation for each of the values. The mean and $\mathrm{CV}$ of the $\mathrm{V}_{\max }$ and $\mathrm{K}_{\mathrm{m}}$ for lasiocarpine depletion and 7-GS-DHP formation were obtained in the present study based on the incubations with 25 individual Chinese and 25 individual Caucasian human liver microsomes. The variation of 7-GS-DHP formation obtained from the Monte Carlo simulations for the Chinese, the Caucasian and the 
two populations combined were compared to each other and to the predicted 7-GS-DHP formation from the individual PBK models for the 25 Chinese individuals, 25 Caucasian individuals and 50 individuals, respectively at the same oral dose level.

In the Monte Carlo simulations, the kinetic constants $\mathrm{V}_{\max }$ and $\mathrm{K}_{\mathrm{m}}$ of lasiocarpine depletion and of 7-GS-DHP formation were randomly and independently taken from their respective log-normal distributions. However, a relatively high and statistically significant correlation (Pearson $\mathrm{r}$ of 0.83 ) between $\mathrm{V}_{\max }$ of lasiocarpine depletion and of 7-GS-DHP formation derived from the 50 human individuals was observed. There was also a strong correlation with Pearson $r$ of 0.88 between the catalytic efficiency of lasiocarpine depletion and of 7-GSDHP formation (see supplementary materials 4). This correlation indicates that when clearance is effective, 7-GS-DHP formation is effective as well, indicating that treating them as independent parameters in the Monte Carlo simulation could possibly result in unrealistic estimates of the inter-individual differences. With Berkeley Madonna version (8.0.1), covariation between parameters cannot be analyzed. Instead, to include the effect of covariation in our model predictions with our Monte Carlo simulations using Berkeley Madonna, we allowed the ratio of the $V_{\max }$ of 7-GS-DHP formation and the $V_{\max }$ of lasiocarpine depletion in each simulation to only vary between the lowest and the highest observed values for these $\mathrm{V}_{\max }$ ratios in the in vitro incubations from the 25 Chinese and 25 Caucasian individuals, which were 0.017 (derived from Chinese individual number 20) and 0.96 (derived from Caucasian individual 098H0150), respectively.

The model predictions with the Monte Carlo simulation were performed in the Berkeley Madonna software with version of 8.0.1 (Macey and Oster, UC Berkeley, CA, USA) applying Rosenbrock's algorithms for solving stiff systems. The output of the Monte Carlo simulations was statistically analyzed with GraphPad (GraphPad Prism software version 5.04, San Diego California USA) to obtain the different percentiles, i.e. GM, 90 th and $99^{\text {th }}$ percentile of the amount of 7-GS-DHP formed by the liver of the Chinese, the Caucasian and the two populations combined. In both the scenario of Monte Carlo simulation in which the $V_{\max }$ of 7GS-DHP formation and the $\mathrm{V}_{\max }$ of lasiocarpine depletion was set to 1.0, and the scenario to evaluate the effect of covariation, the CSAFs were obtained by dividing the $90^{\text {th }}$ or $99^{\text {th }}$ percentile of 7-GS-DHP formation by the GM (IPCS 2005).

\subsection{BMD analysis of predicted in vivo dose-response data obtained from PBK-based reverse dosimetry approach}

In our previous study (Ning et al. 2019), the in vitro concentration-response curves for cytotoxicity obtained in pooled ( 5 donors) human hepatocytes were translated to in vivo doseresponse curves for acute hepatotoxicity of lasiocarpine by PBK modelling-based reverse dosimetry. This quantitative in vitro to in vivo extrapolation (QIVIVE) was performed based on the $\mathrm{C}_{\max }$ (the maximum concentration in liver blood). For the QIVIVE, each free concentration of lasiocarpine in the cell culture medium of the cytotoxicity experiments, was set equal to the free $\mathrm{C}_{\max }$ of lasiocarpine in the liver blood in the PBK model to calculate the oral dose that results in this concentration (Ning et al. 2019). In the present study, a similar approach was applied to define the dose-response curve for the average population, while the 
dose-response curves of the sensitive individuals ( $90^{\text {th }}$ and $99^{\text {th }}$ percentile) in the Chinese, the Caucasian and the two populations combined, were derived by applying the obtained CSAF to the dose-response curve of the average populations.

\section{Results}

\subsection{Depletion of lasiocarpine by individual human liver microsomes}

Table 1 shows the kinetic constants $\mathrm{V}_{\max }$ and $\mathrm{K}_{\mathrm{m}}$, and the catalytic efficiency for depletion of lasiocarpine by 25 Chinese and 25 Caucasian individuals, derived from experiments with liver microsomal incubations with the cofactor NADPH at increasing concentrations of lasiocarpine. For the 25 Chinese individuals, the difference between the highest and the lowest catalytic efficiency amounted to 3.5 -fold with a CV of $35 \%$. For the 25 Caucasian individuals, the difference between the highest and the lowest catalytic efficiency amounted to 9.2-fold with a CV of $79 \%$. This relatively high $\mathrm{CV}$ is mainly caused by Caucasian individuals $098 \mathrm{H} 068$ and $098 \mathrm{H} 0397$ which have relatively high $\mathrm{V}_{\max }$ and low $\mathrm{K}_{\mathrm{m}}$ values resulting in catalytic efficiencies that are 3.0- and 3.6-fold higher respectively than the average catalytic efficiency. The average catalytic efficiencies of lasiocarpine depletion of the Chinese population and the Caucasian population were 1.7-fold lower and 1.4-fold higher, respectively than the average catalytic efficiency obtained when the data for the two populations were combined ( 25 Chinese +25 Caucasian individuals) (Table 2 ). The CVs for the catalytic efficiency of the Chinese population and the Caucasian populations were 2.5and 1.1-fold lower, respectively than the catalytic efficiency of $89 \%$ for the combined population.

For the inter-ethnic comparison, it can be noted that the average catalytic efficiency for depletion of lasiocarpine was 2.3-fold lower in the Chinese population than in the Caucasian population, which is mainly due to the a 2.3-fold lower average $\mathrm{V}_{\max }$ value obtained for the Chinese population. The average $\mathrm{K}_{\mathrm{m}}$ value in both populations is comparable. The $\mathrm{CV}$ of the catalytic efficiency for lasiocarpine depletion was 2.3-fold lower for the Chinese population than for the Caucasian population, indicating that the inter-individual variation is higher within the 25 Caucasian individuals than within the 25 Chinese individuals.

\subsection{Bioactivation of lasiocarpine to 7-GS-DHP by individual human liver microsomes}

Table 1 shows the kinetic constants $V_{\max }$ and $\mathrm{K}_{\mathrm{m}}$ and the catalytic efficiency for 7-GS-DHP formation obtained for the 25 Chinese and 25 Caucasian individuals. For the 25 Chinese individuals, the difference between the highest and the lowest catalytic efficiency amounted to 43.5 -fold with a $\mathrm{CV}$ of $77 \%$. This relatively high $\mathrm{CV}$ value is partly due to Chinese individuals 7 and 20 which both show an about 6.5-fold lower $\mathrm{V}_{\max }$ value, and a 1.1- and 2.2fold higher $\mathrm{K}_{\mathrm{m}}$ value, respectively, than the average kinetic constants for the 25 individuals, resulting in about 12-fold lower catalytic efficiency for these individuals compared to the average value. For 7-GS-DHP formation by the liver microsomes from the 25 Caucasian individuals, the difference between the highest and the lowest catalytic efficiency amounted to 792 -fold with a CV of $120 \%$. This large difference in catalytic efficiency for 7-GS-DHP formation within these 25 Caucasian individuals is partly due to the relatively low activity by 
Caucasian individual $098 \mathrm{H} 0495$ with a 25.4 -fold lower $\mathrm{V}_{\max }$ and a 5.5-fold higher $\mathrm{K}_{\mathrm{m}}$ than the average kinetic constants of the 25 Caucasian individuals, resulting in a 203 -fold lower catalytic efficiency than the average value. The average catalytic efficiencies of 7-GS-DHP formation of the Chinese population and the Caucasian population were 4.2-fold lower and 1.8-fold higher, respectively than the average catalytic efficiency obtained for the two populations combined (25 Chinese and 25 Caucasian individuals) (Table 2). The CVs of the catalytic efficiency of the Chinese population and the Caucasian populations were 2.2- and 1.4-fold lower, respectively than the catalytic efficiency of $167 \%$ for the combined human population.

For the 7-GS-DHP formation, the average catalytic efficiency obtained with the Chinese population is 7.3-fold lower than the average catalytic efficiency obtained with the Caucasian population which is mainly due to a 7.1-fold lower average $\mathrm{V}_{\max }$ value for 7-GS-DHP formation in the Chinese population compared to the average $\mathrm{V}_{\max }$ for the Caucasian population. The average $\mathrm{K}_{\mathrm{m}}$ value for 7-GS-DHP formation differed only 1.2-fold between the two populations. The CV of $77 \%$ and $120 \%$ for the catalytic efficiencies for 7-GS-DHP formation in the Chinese and Caucasian population respectively showed that the interindividual differences in kinetics for 7-GS-DHP formation are relatively high in both populations. 


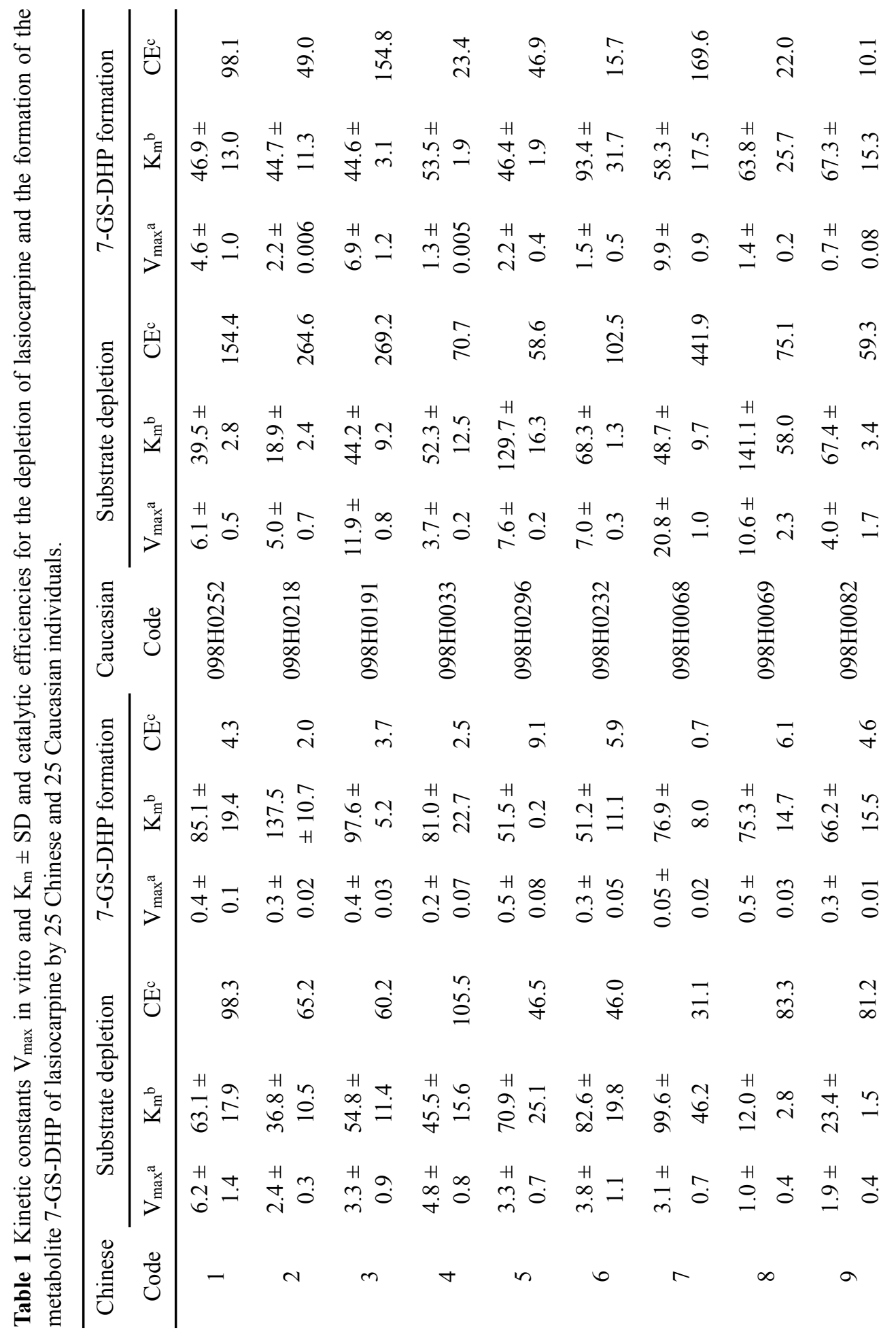




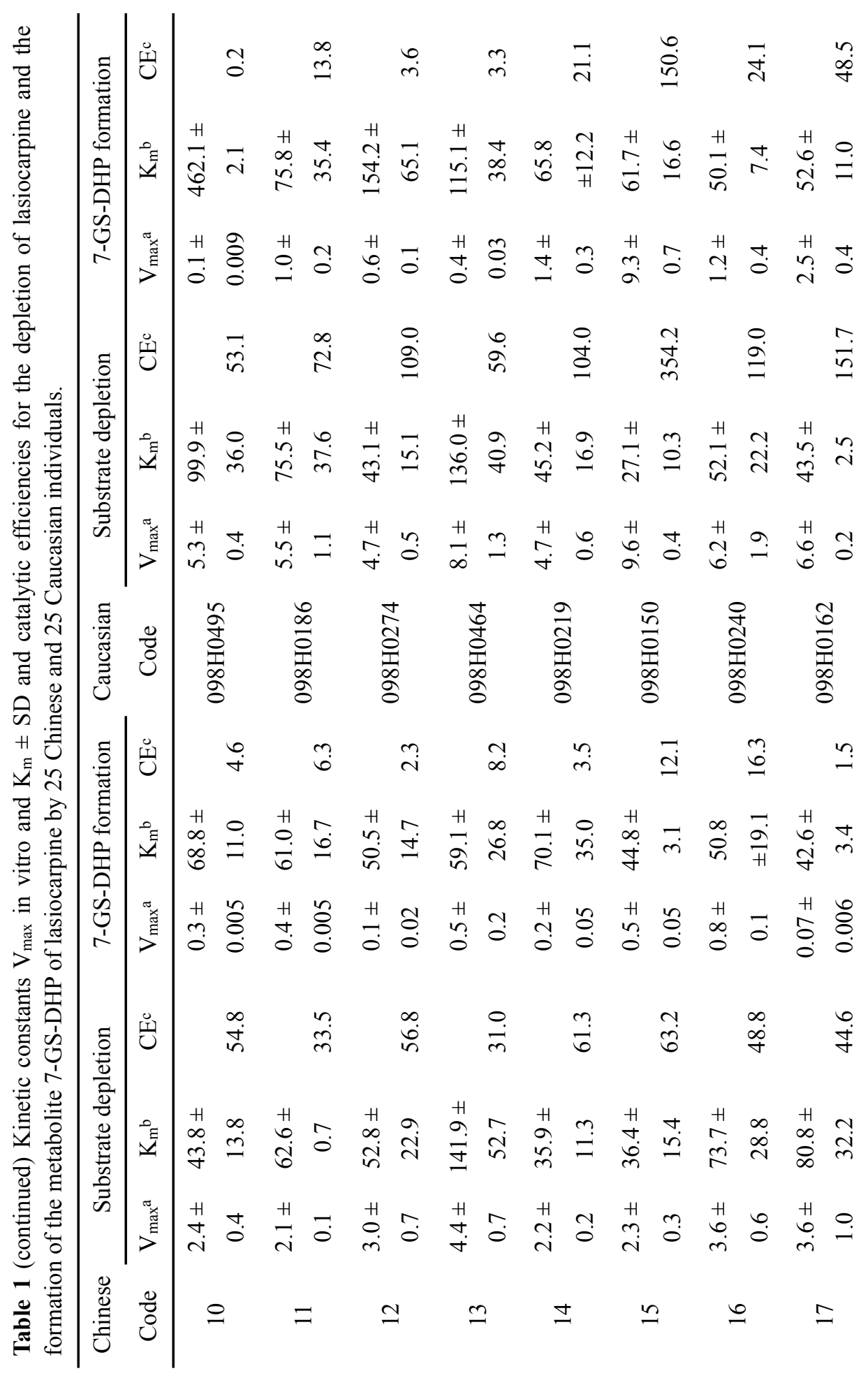




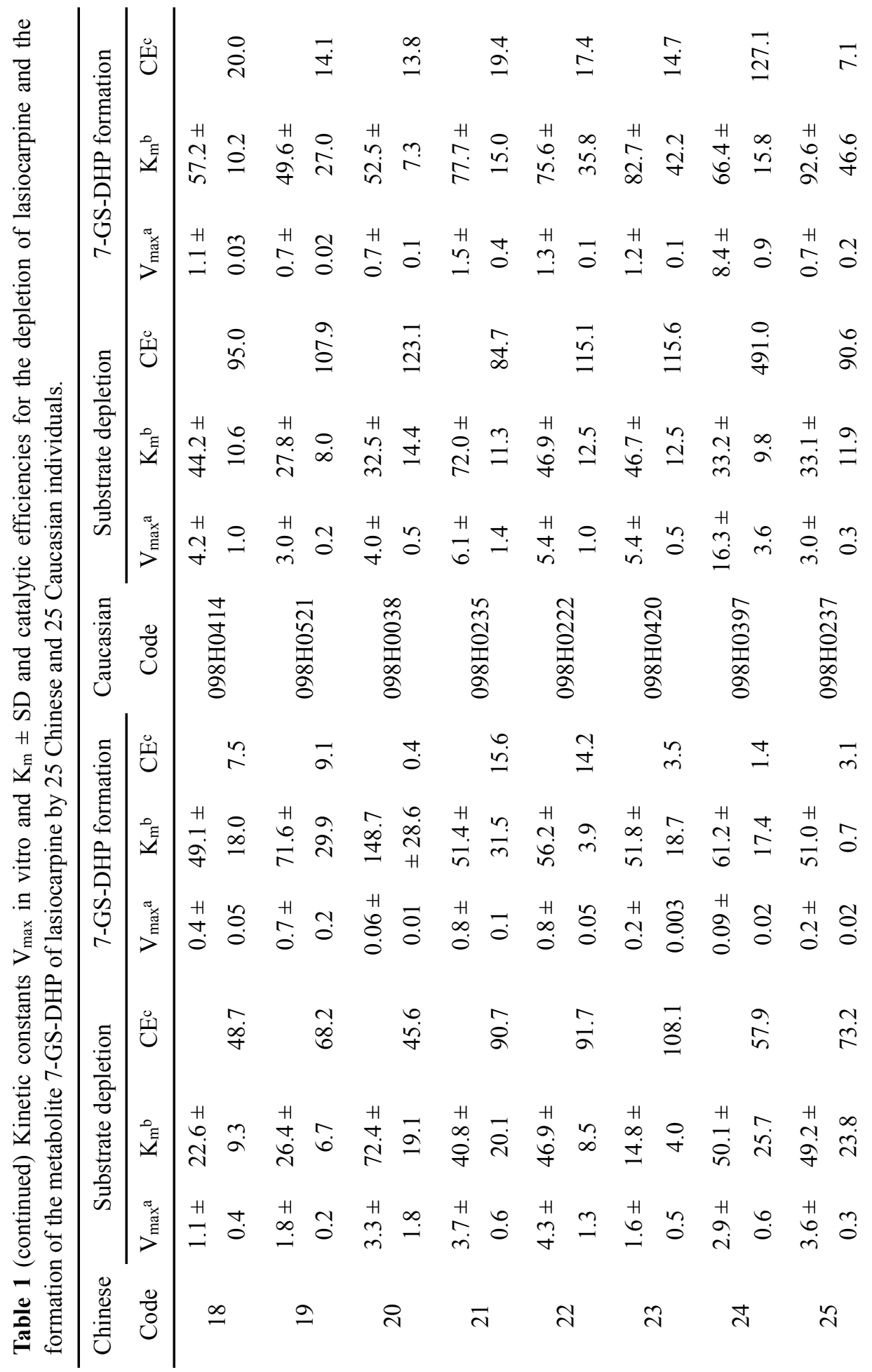




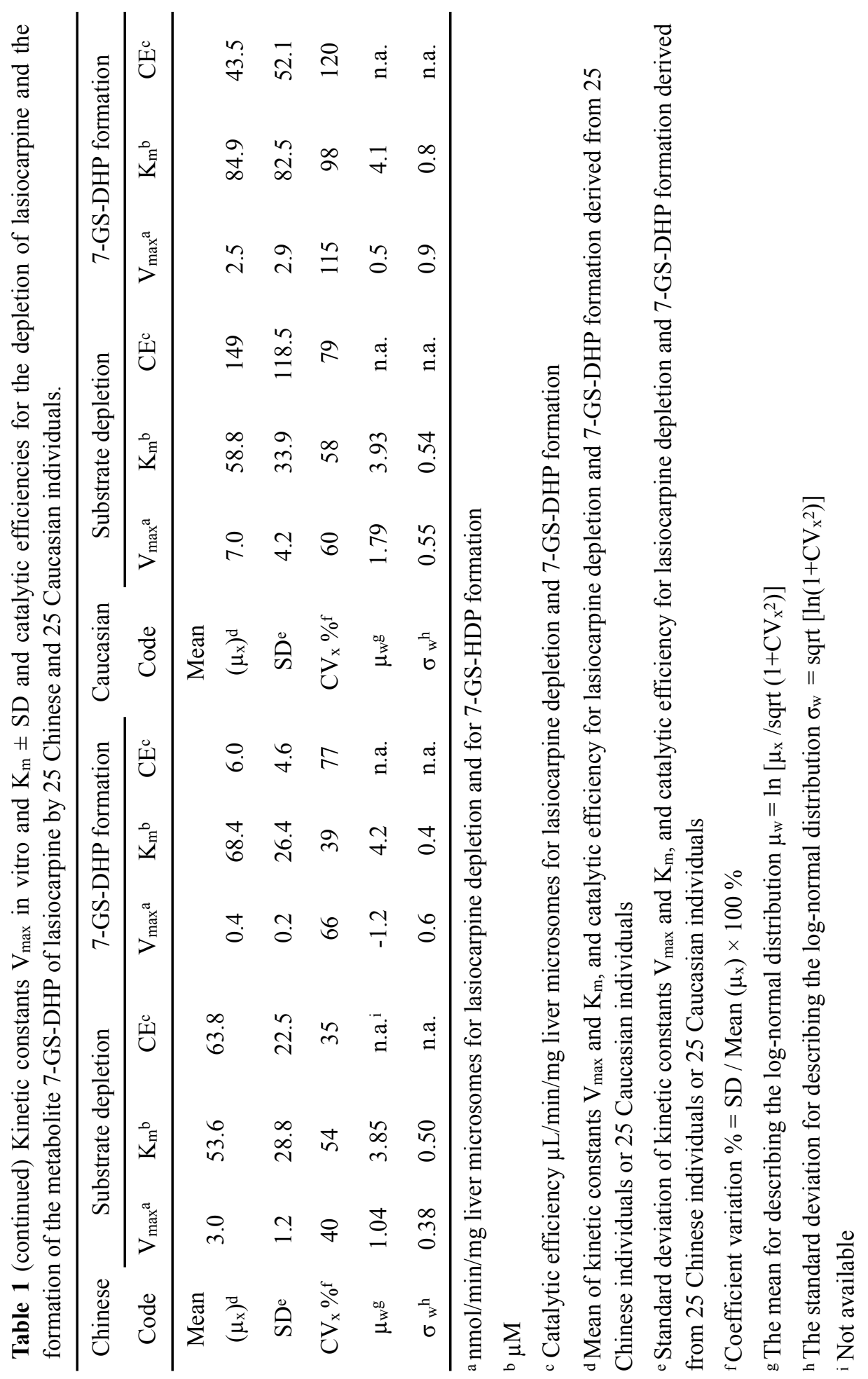




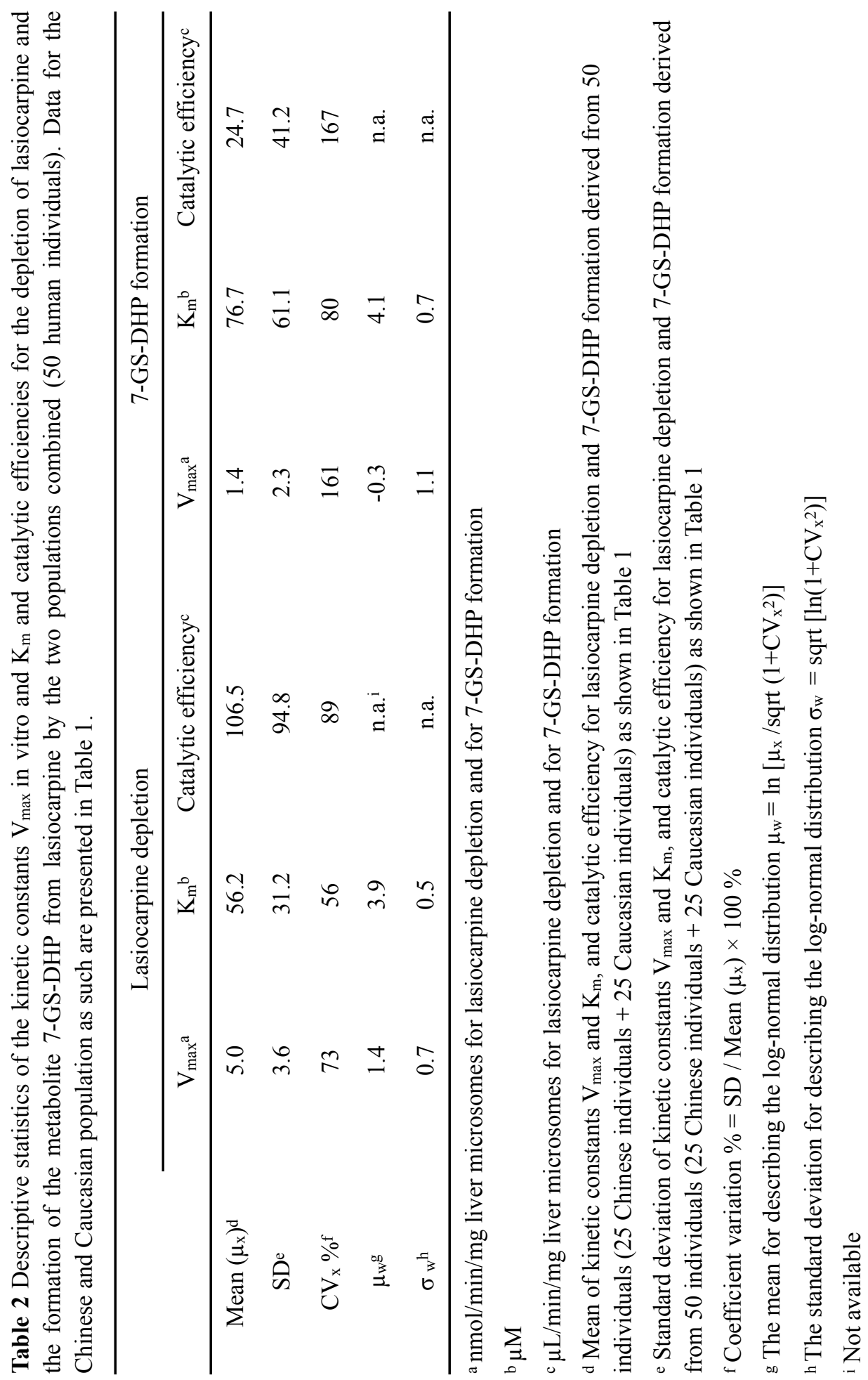




\subsection{Sensitivity analysis}

The sensitivity analysis showed that the kinetic constants $V_{\max }$ and $\mathrm{K}_{\mathrm{m}}$ for lasiocarpine depletion and 7-GS-DHP formation by the liver of the Caucasian population influenced the predicted formation of 7-GS-DHP by the liver to a large extent (Figure 2) both at a dose of 8 $\mathrm{ng} / \mathrm{kg}$ bw and $3 \mathrm{mg} / \mathrm{kg}$ bw. The results of the sensitivity analysis performed in the Chinese population were similar to the results obtained in the Caucasian population (data not shown). Given that 7-GS-DHP formation reflects the potential bioactivation of lasiocarpine, the Monte Carlo simulation and CSAFs analysis were based on 7-GS-DHP formation by the liver.

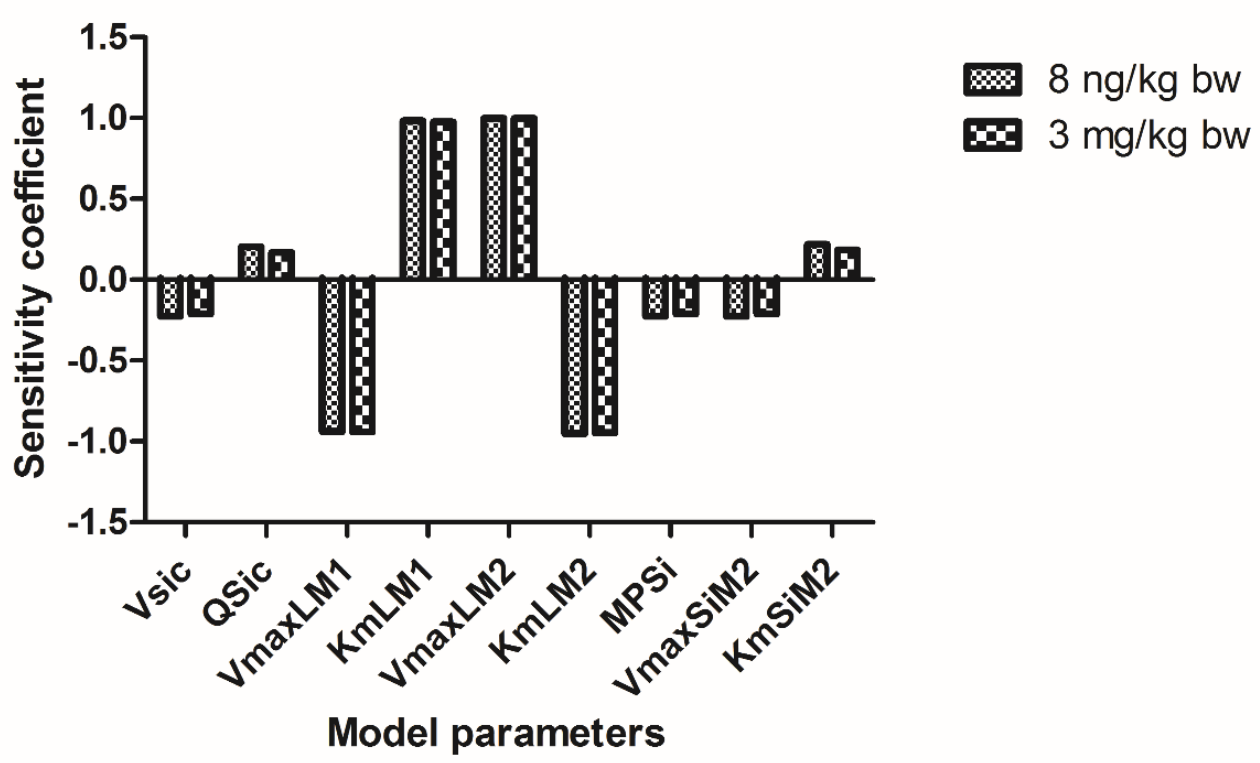

Fig. 2 Normalized sensitivity coefficients of PBK model parameters for the prediction of the formation of 7-GS-DHP by the liver expressed in $\mu \mathrm{g} / \mathrm{kg}$ bw, 24 hours after oral administration of $8 \mathrm{ng} / \mathrm{kg}$ bw and $3 \mathrm{mg} / \mathrm{kg}$ bw of lasiocarpine. VSic=fraction of small intestinal tissue, $\mathrm{QSic}=$ fraction of blood flow to small intestine, $\mathrm{MPSi}=$ microsomal protein yield in small intestine. The $\mathrm{V}_{\max } \mathrm{LM} 1$ and $\mathrm{V}_{\max } \mathrm{SiM} 2$ represent the maximum rate of lasiocarpine depletion in the liver and small intestine, respectively. The $\mathrm{K}_{\mathrm{m}} \mathrm{LM} 1$ and $\mathrm{K}_{\mathrm{m}} \mathrm{SiM} 2$ are the MichaelisMenten constant for the lasiocarpine depletion in the liver and small intestine, respectively. $\mathrm{V}_{\max } \mathrm{LM} 2$ and $\mathrm{K}_{\mathrm{m}} \mathrm{LM} 2$ represent the maximum rate of formation and the Michaelis-Menten constant for the 7-GS-DHP formation by the liver.

\subsection{Individual PBK models and predicted 7-GS-DHP formation}

Figure 3 shows the PBK model based predictions for the time-dependent liver blood concentration of lasiocarpine at an oral dose of $8 \mathrm{ng} / \mathrm{kg}$ bw (Figure 3A) and the dosedependent formation of 7-GS-DHP (Figure 3B) both for the average, and the most and least 
active of the 25 Chinese individuals, the 25 Caucasian individuals and the two populations combined (25 Chinese +25 Caucasians). The difference between the predicted $\mathrm{C}_{\max }$ of lasiocarpine in liver blood for the individual with the highest and the lowest efficiency in lasiocarpine depletion is 2.5-fold for the Chinese population, 7.4-fold for the Caucasian population and 12.7-fold for the two populations combined. Formation of 7-GS-DHP for each population was linear up to at least $80 \mathrm{mg} / \mathrm{kg}$ bw lasiocarpine. Figure 4 shows the box and whisker plots for the predicted formation of 7-GS-DHP by the liver at an oral dose of 8 $\mathrm{ng} / \mathrm{kg}$ bw for 25 Chinese individuals and 25 Caucasian individuals. Table 3 shows the GM and geometric CV of 7-GS-DHP formation predicted by individual PBK models in each population. For 7-GS-DHP formation, the difference between the highest and the lowest individual is 41-fold for the Chinese population, 200-fold for the Caucasian population and 200 -fold for the two populations combined. For the inter-ethnic comparison, the GM and geometric CV of the predicted 7-GS-DHP formation in 25 Chinese individuals is 2.2-fold lower and 1.1-fold higher, respectively than those values in 25 Caucasian individuals.
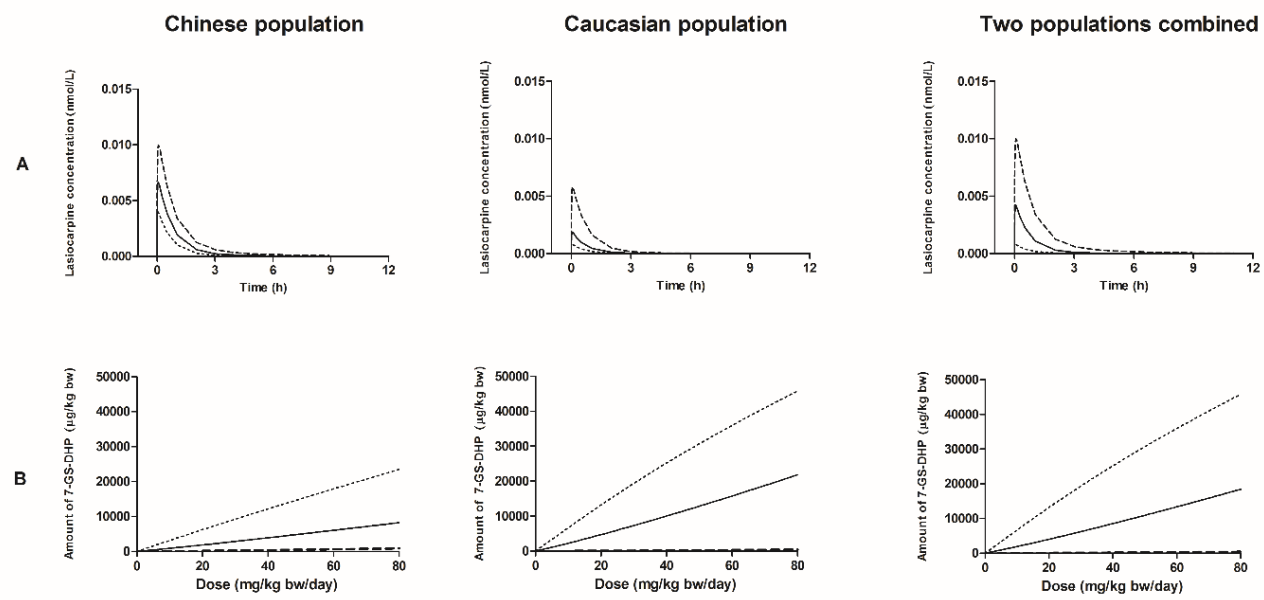

Fig. 3 PBK model predicted (A) time-dependent liver blood concentration of lasiocarpine at an oral lasiocarpine dose of $8 \mathrm{ng} / \mathrm{kg}$ bw and (B) dose-dependent formation of 7-GS-DHP by the liver, in the Chinese population, the Caucasian population and the two populations combined. The individuals with the highest (black dotted line), the lowest (black dashed line) and average (black solid line) efficiencies of lasiocarpine depletion and 7-GS-DHP formation are shown for each population.

\subsection{Monte Carlo simulations and CSAF}

Table 3 shows the GM and geometric CV of 7-GS-DHP formation derived by Monte Carlo simulation in each population. For the Chinese population, the GM and geometric CV values obtained by the Monte Carlo simulation were comparable to the GM and geometric CV obtained with the 25 Chinese individuals. The Monte Carlo simulation results for both the Caucasian population and the two populations combined revealed that the GM values of 7-GSDHP were comparable to the GM values obtained when analyzing the 25 Caucasian 
individuals and the 50 individuals, but that the geometric CV of the predicted 7-GS-DHP formation obtained from Monte Carlo simulation were about 3.0-fold higher than the values obtained from 25 Caucasian individuals and the 50 individuals. This indicates that the ethnic groups of 25 individuals each adequately represented the GM of 7-GS-DHP formation in a large population, but that the Monte Carlo simulation approach is more appropriate to identify sensitive individuals with respect to 7-GS-DHP formation within the population.

Table 3 Geometric mean, geometric mean $\mathrm{CV} \%$ and the fold difference between the minimum and maximum or the $1^{\text {st }}$ and $99^{\text {th }}$ percentile of the predicted formation of 7-GSDHP by the liver 24 hours after exposure to an oral lasiocarpine dose of $8 \mathrm{ng} / \mathrm{kg}$ bw by individual PBK models and in the population predicted by PBK modelling linked with Monte Carlo simulation with the maximum allowed ratio of the $\mathrm{V}_{\max }$ of 7-GS-DHP formation and the $\mathrm{V}_{\max }$ of lasiocarpine depletion set at 1.0 in the Chinese population, the Caucasian population and two populations combined.

\begin{tabular}{|c|c|c|c|c|c|c|}
\hline & \multicolumn{3}{|c|}{$\begin{array}{l}\text { 7-GS-DHP formation } \\
\text { individual PBK models }\end{array}$} & \multicolumn{3}{|c|}{$\begin{array}{l}\text { 7-GS-DHP formation } \\
\text { combination of PBK model with } \\
\text { Monte Carlo simulation }\end{array}$} \\
\hline & $\begin{array}{c}\text { Chinese } \\
\text { population }\end{array}$ & $\begin{array}{l}\text { Caucasian } \\
\text { population }\end{array}$ & $\begin{array}{l}\text { Two } \\
\text { populations } \\
\text { combined }\end{array}$ & $\begin{array}{l}\text { Chinese } \\
\text { population }\end{array}$ & $\begin{array}{l}\text { Caucasian } \\
\text { population }\end{array}$ & $\begin{array}{l}\text { Two } \\
\text { populations } \\
\text { combined }\end{array}$ \\
\hline $\begin{array}{c}\text { Geometric } \\
\text { mean } \\
(\mu \mathrm{g} / \mathrm{kg} \\
\mathrm{bw})\end{array}$ & 0.00054 & 0.0012 & 0.00079 & 0.00057 & 0.0012 & 0.00083 \\
\hline $\begin{array}{l}\text { Geometric } \\
\text { mean } \\
\mathrm{CV}(\%)\end{array}$ & 114 & 109 & 136 & 169 & 390 & 401 \\
\hline $\begin{array}{l}\text { Fold } \\
\text { difference }\end{array}$ & 41-fold ${ }^{a}$ & 200-fold ${ }^{\mathrm{a}}$ & $200-$ fold $^{\mathrm{a}}$ & 69-fold ${ }^{b}$ & 392-fold ${ }^{b}$ & 607-foldb \\
\hline \multicolumn{7}{|c|}{$\begin{array}{l}\text { a Fold differences of the minimum and maximum of the predicted formation of 7-GS-DHP by } \\
\text { individual PBK models }\end{array}$} \\
\hline $\begin{array}{l}\text { b Fold differe } \\
\text { DHP obtaine } \\
\text { the maximur } \\
\text { depletion set }\end{array}$ & $\begin{array}{l}\text { nces of the } 1 \mathrm{~s} \\
\text { from the co } \\
\text { allowed rati } \\
\text { at } 1.0 \text {. }\end{array}$ & $\begin{array}{l}\text { and } 99^{\text {th }} \text { perc } \\
\text { nbination of } \\
\text { of the } V_{\max }\end{array}$ & $\begin{array}{l}\text { PBK modelli } \\
\text { of 7-GS-DHF }\end{array}$ & $\begin{array}{l}\text { gopulation fo } \\
\text { formath Mont } \\
\text { fon an }\end{array}$ & $\begin{array}{l}\text { the formatio } \\
\text { Carlo simul } \\
d \text { the } V_{\max } \text { of }\end{array}$ & $\begin{array}{l}n \text { of } 7-G S- \\
\text { ations with } \\
\text { lasiocarpine }\end{array}$ \\
\hline
\end{tabular}




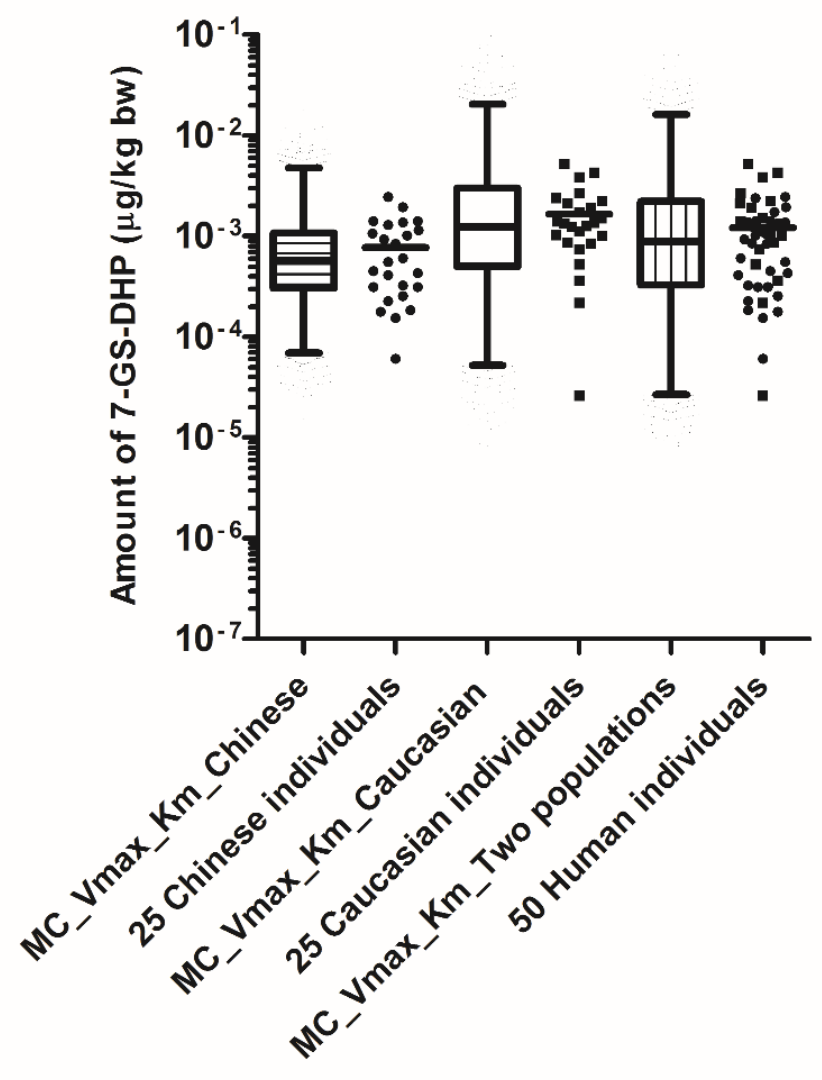

Fig. 4 Box and whisker plots representing the distribution of the predicted 7-GS-DHP formation by the liver, expressed in $\mu \mathrm{g} / \mathrm{kg}$ bw, 24 hours after exposure to an oral lasiocarpine dose of $8 \mathrm{ng} / \mathrm{kg}$ bw that represents an average daily intake of PAs via consumption of herbal tea (BfR 2013) for 14851 Chinese (box with horizontal lines), 13119 Caucasians and 13399 individuals from the two populations combined (box with vertical lines) obtained by Monte Carlo simulation including variation in $\mathrm{V}_{\max }$ and $\mathrm{K}_{\mathrm{m}}$ for lasiocarpine depletion and variation in $\mathrm{V}_{\max }$ and $\mathrm{K}_{\mathrm{m}}$ for 7-GS-DHP formation with the maximum allowed ratio of the $\mathrm{V}_{\max }$ of 7GS-DHP formation and the $\mathrm{V}_{\max }$ of lasiocarpine depletion set as 1.0. The whiskers represent the $1^{\text {st }}$ and $99^{\text {th }}$ percentile of the populations and the small dots represent those values that were higher or lower than the $1^{\text {st }}$ and $99^{\text {th }}$ percentile of the studied populations consisting of less than about $2 \%$ of the 14851 (Chinese), 13119 (Caucasian) and 13399 (two populations combined) Monte Carlo simulations. The figure also includes scatter plots representing the predicted amount of 7-GS-DHP formation by the liver 24 hours after exposure to an oral lasiocarpine dose of $8 \mathrm{ng} / \mathrm{kg}$ bw for the 25 Chinese individuals (circles), the 25 Caucasian individuals (squares) and the 50 human individuals (circles + squares). 
Figure 4 present the box and whisker plots for the predicted 7-GS-DHP formation by the liver obtained from the Monte Carlo simulations for the Chinese, the Caucasian and the two populations combined, applying a single oral dose of $8 \mathrm{ng} / \mathrm{kg}$ bw that represents an average daily intake of PAs via consumption of herbal tea (BfR 2013). Figure 5 presents the related frequency distribution for the predicted 7-GS-DHP formation by the liver for these modeled populations. The inter-ethnic comparison reveals that the GM of predicted 7-GS-DHP formation is 2.1-fold lower in the Chinese population than in the Caucasian population, while the differences in the predicted 7-GS-DHP formation between the populations amounted to 3.3- and 4.3-fold, respectively when the $90^{\text {th }}$ and $99^{\text {th }}$ percentile of populations were considered.

The CSAFs obtained by dividing the $90^{\text {th }}$ or $99^{\text {th }}$ percentile obtained with the maximum allowed ratio of the $\mathrm{V}_{\max }$ of 7-GS-DHP formation and the $\mathrm{V}_{\max }$ of lasiocarpine depletion set at 1.0, reflecting the worst-case scenario for the 7-GS-DHP formation (IPCS 2005), are presented in Table 4. The CSAF values for the 99 th percentile amounted to 8.3 for the Chinese population, 17.0 for the Caucasian population and 19.6 for the two populations combined, while these values were generally 3-fold lower in each population when the $90^{\text {th }}$ percentile was used. Applying Monte Carlo simulation with the observed minimum and maximum acceptable boundary to evaluate the effect of modelling covariation on the predicted CSAF value, indicated that for each population the CSAF values were 1.1 -fold $\left(90^{\text {th }}\right.$ percentile and 99th percentile) lower than the CSAF values derived when using the maximum allowed ratio of 1.0 as the worst-case scenario (see Table 4). 


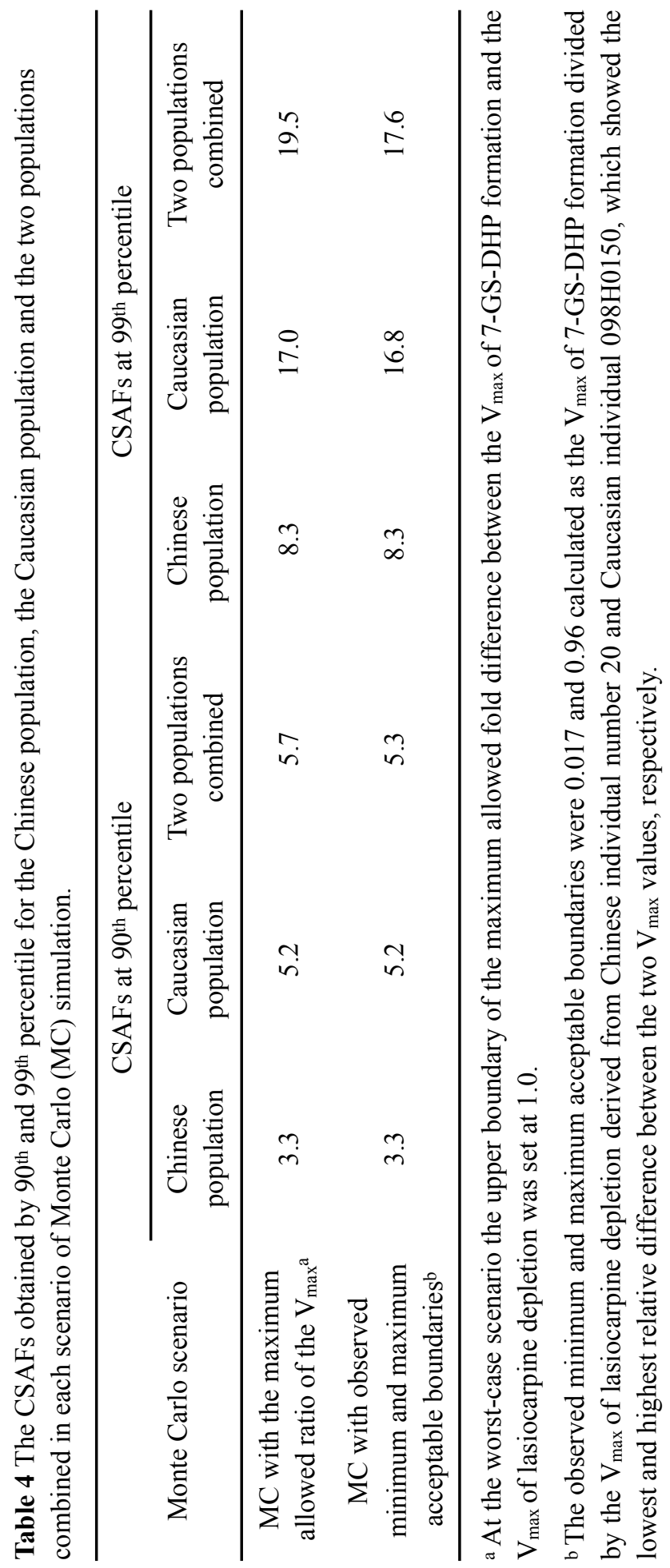


A

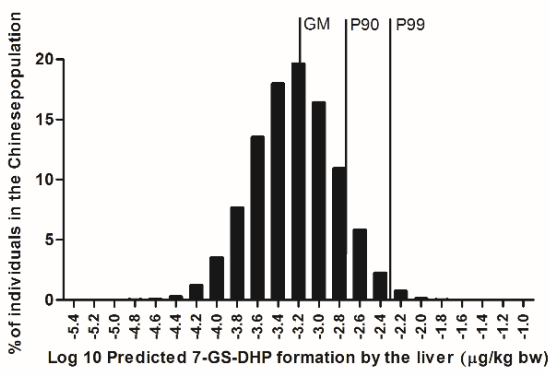

$\mathbf{B}$
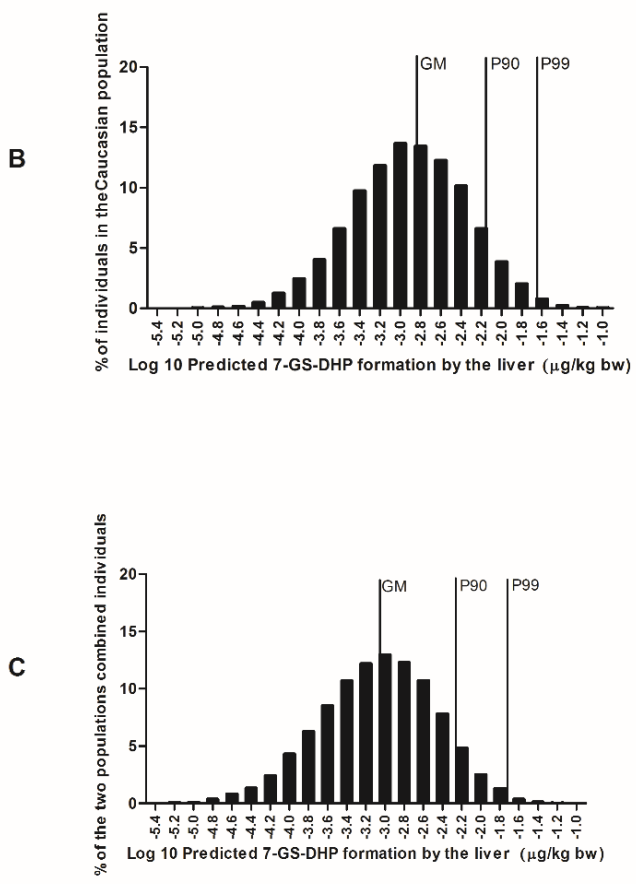

Fig. 5 Frequency distribution for the predicted total amount of 7-GS-DHP formed by the liver $24 \mathrm{~h}$ after a single oral lasiocarpine dose of $8 \mathrm{ng} / \mathrm{kg}$ bw exposure, for 14851 Chinese individuals (A), 13119 Caucasian individuals (B) and 13399 individuals in the two populations combined (C) obtained by Monte Carlo simulation including variation in the $V_{\max }$ and $\mathrm{K}_{\mathrm{m}}$ for lasiocarpine depletion and for 7-GS-DHP formation with the maximum allowed ratio of the $V_{\max }$ of 7-GS-DHP formation and the $V_{\max }$ of lasiocarpine depletion set at 1.0. The GM, P90 and P99 represent the geometric mean, the 90th and 99th percentile of the distribution. 


\subsection{Predicted dose-response curve for the most sensitive population}

Figure 6 shows the predicted in vivo dose-response curves for the hepatotoxicity of lasiocarpine for the average and the most sensitive ( $90^{\text {th }}$ and $99^{\text {th }}$ percentile of the population) individuals of the Chinese, the Caucasian and the two populations combined. The in vivo dose-response curves for the most sensitive individuals were obtained by dividing the doseresponse curves for the average population with the CSAF values for the $90^{\text {th }}$ and $99^{\text {th }}$ percentile of the population (using the CSAF values obtained with the maximum allowed ratio of the $\mathrm{V}_{\max }$ of 7-GS-DHP formation and the $\mathrm{V}_{\max }$ of lasiocarpine depletion set at 1.0). Table 5 shows the predicted PoD $\left(\mathrm{BMDL}_{5}-\mathrm{BMDU}_{5}\right)$ values for the $90^{\text {th }}$ percentile and $99^{\text {th }}$ percentile of each population derived from these dose-response curves. The BMDL 5 -BMDU 5 values for the $99^{\text {th }}$ percentile of the Chinese, the Caucasian and the combined population were $1.8-5.0,0.4-1.4,0.8-1.9 \mathrm{mg} / \mathrm{kg}$ bw, while the $\mathrm{BMDL}_{5}-\mathrm{BMDU}_{5}$ values for the $90^{\text {th }}$ percentile of each population were generally 3 -fold higher in each population. Applying the CSAF values obtained with the observed minimum and maximum acceptable boundaries to evaluate the effect of modelling covariation on the dose-response curves for the most sensitive individuals in each population, the PoDs were up to 1.2 -fold ( $90^{\text {th }}$ and $\left.99^{\text {th }}\right)$ higher than the PoDs derived when using the maximum allowed ratio of 1.0.

For the ethnic comparison, the predicted PoD was 3- to 4-fold higher for the $90^{\text {th }}$ and $99^{\text {th }}$ percentile of Chinese the population than for the $90^{\text {th }}$ and $99^{\text {th }}$ percentile of the Caucasian population in both scenarios of the Monte Carlo simulations, indicating that Caucasians may be more sensitive to the liver toxicity of lasiocarpine than the Chinese population when taking the most sensitive individuals into account. For the most sensitive individuals within the two populations combined, the PoD values were up to 2-fold higher than the PoDs of the most sensitive individuals in the Caucasian population. 

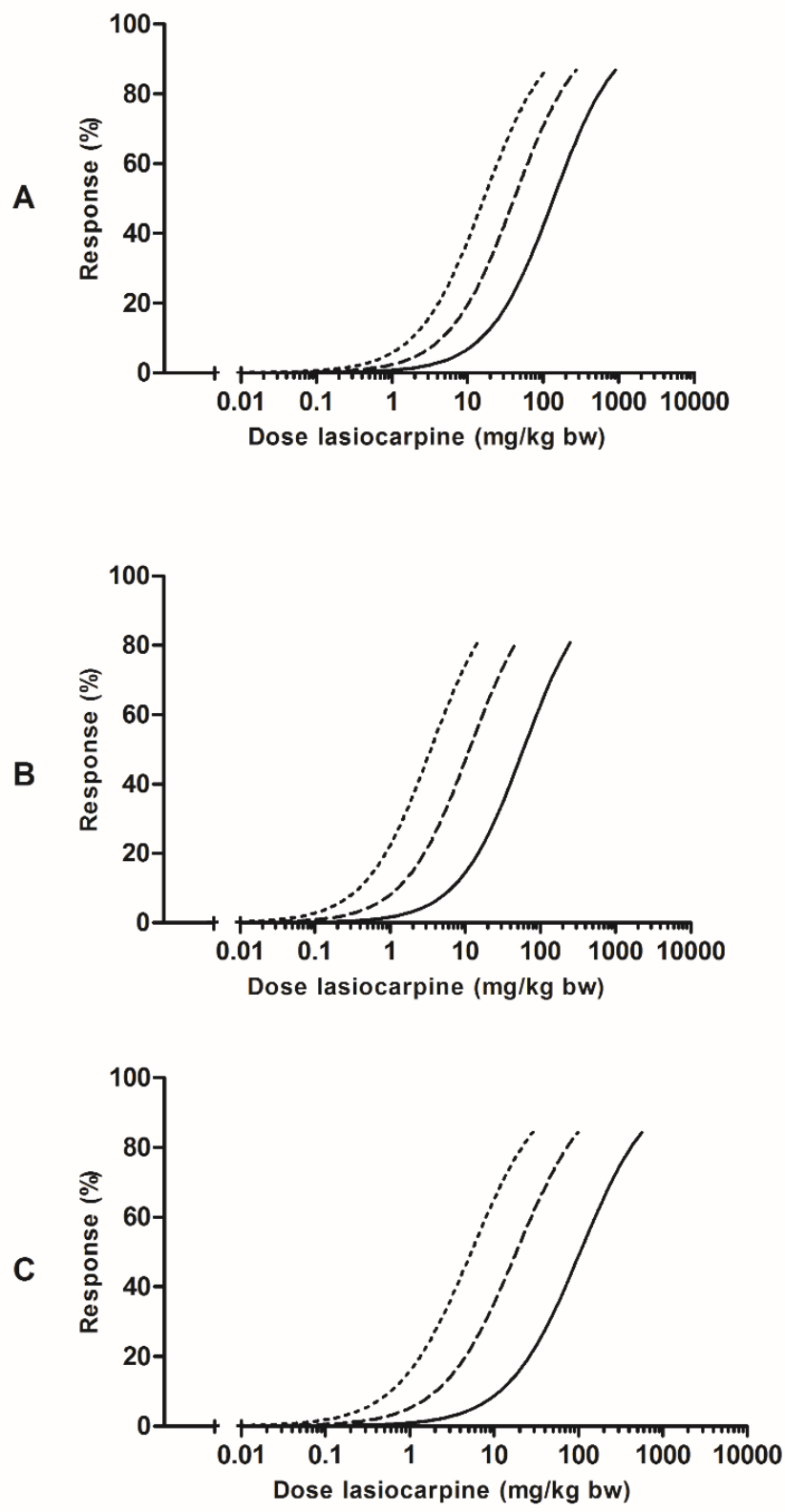

Fig. 6 Predicted dose-response curve for acute liver toxicity of lasiocarpine in the average (solid line), the 90th (dashed line) and 99th percentile (dotted line) of the Chinese (A), the Caucasian (B) and the two populations combined (C). 


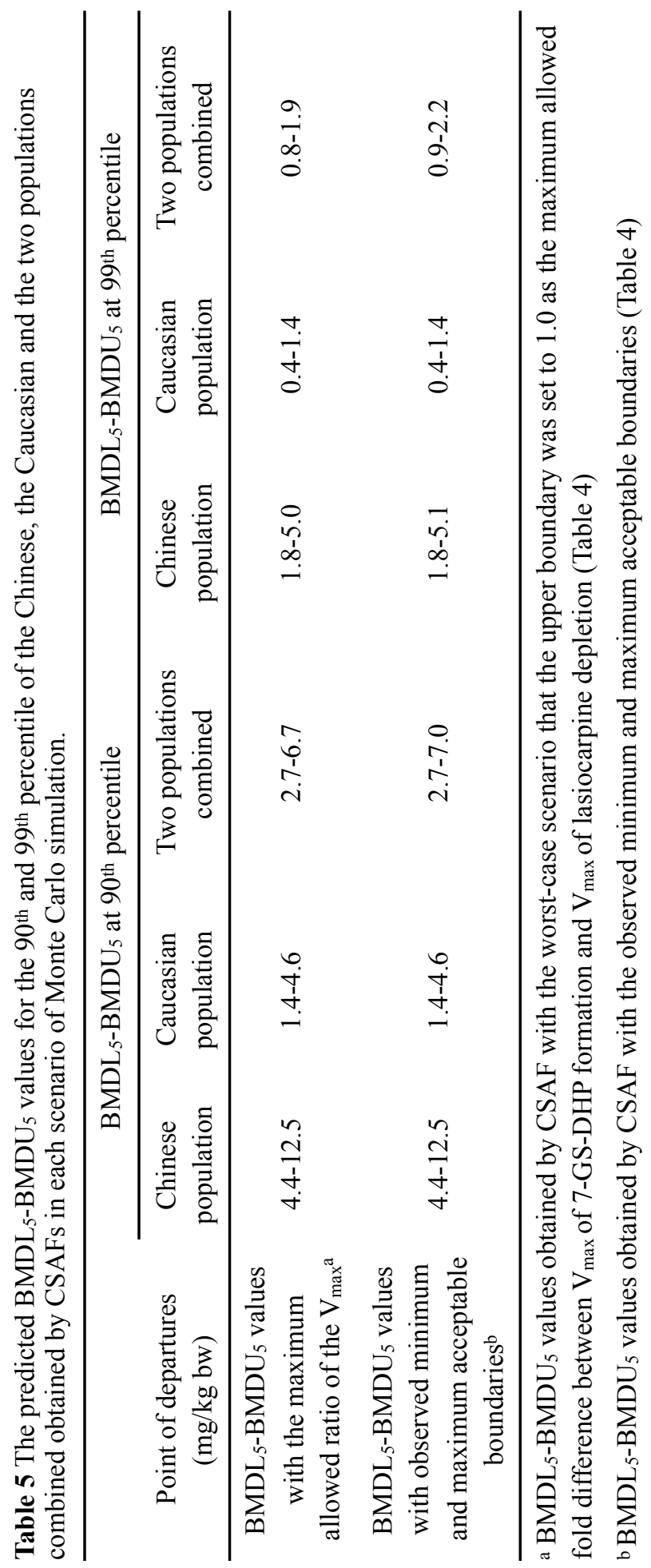




\section{Discussion}

The aim of the present study was to investigate the effect of the inter-individual and the interethnic human kinetic variation in the Chinese and the Caucasian populations for lasiocarpine induced acute liver toxicity and bioactivation, by integrating PBK modelling with Monte Carlo simulations using human in vitro data, and to ultimately derive CSAFs. The obtained CSAFs, with two scenarios of Monte Carlo simulations, were further used to derive the concentration-response curves for the most sensitive individuals in each population, i.e. the $90^{\text {th }}$ and $99^{\text {th }}$ percentile of the population.

In the present study, the sensitivity analysis revealed that the 7-GS-DHP formation was highly affected by kinetic constants $V_{\max }$ and $K_{m}$ of lasiocarpine depletion and the $V_{\max }$ and $K_{m}$ of 7GS-DHP formation and thus these parameters were included in the Monte Carlo simulation. Phase I metabolism of lasiocarpine, as shown in Figure 1, is mediated by flavin-containing monooxygenases, carboxylestereases and cytochrome P450 enzymes. The P450 isoenzyme CYP 3A4 is the primary metabolizing enzyme responsible for the metabolic activation of different types of PAs by human liver microsomes (Fashe et al. 2015; Fu et al. 2004; Li et al. 2011; Miranda et al. 1991). In the present study, the kinetic constants of depletion of lasiocarpine and 7-GS-DHP formation were derived from 25 male Chinese and 25 male Caucasian liver microsomes. It has been reported that male rodents are more sensitive to PA intoxication than female rodents. For humans, according to the review of $\mathrm{Fu}$ et al. (2004), there are no reports on a gender difference in the susceptibility of humans to pyrrolizidine alkaloid intoxication and recent reviews or studies regarding the gender difference in the sensitivity towards PAs are not available. However, many studies have shown that CYP 3A4 protein levels are about 2-fold higher in female livers than in male livers (Lamba et al. 2010; Nicolas et al. 2009; Schmidt et al. 2001; Wolbold et al. 2003; Yang et al. 2010; Zanger and Schwab 2013). In vivo, women had about $16 \%$ higher oral clearance and $20 \%$ higher systemic clearance rates than men for oral and intravenous administration of midazolam, a selective substrate of CYP 3A4 and CYP 3A5, but no significantly different area under the plasma concentration-time curve (AUC) of orally administered midazolam was observed between the sexes in a meta-analysis of ten studies toward midazolam disposition including 409 healthy volunteers (Hu and Zhao 2010). However, several studies evaluating the gender difference in clearance of CYP 3A4 substrates have reported conflicting results (Cotreau et al. 2005; Nicolas et al. 2009; Schwartz 2003; Soldin and Mattison 2009), and thus so far no consensus has been reached regarding differences in the metabolism of CYP 3A4 substrates in man and women (Manoranjenni et al. 2012). Comparing the catalytic efficiency values of lasiocarpine depletion and 7-GS-DHP formation obtained using mixed gender Chinese liver microsomes (15 female +25 male) in our previous study (Ning et al. 2019), the average catalytic efficiency values for depletion of lasiocarpine and 7-GS-DHP formation derived from 25 Chinese male individuals is 1.4-fold lower and 1.5-fold higher than values from mixed gender Chinese liver microsomes, respectively. For Caucasians, the catalytic efficiency for lasiocarpine depletion and 7-GS-DHP formation obtained from 25 Caucasian male individuals is 1.3-fold lower and 1.5-fold higher than those values derived from mixed gender Caucasian liver microsomes (16 female +17 male). Given that male individuals seem to be 
less efficient in lasiocarpine depletion but more active in bioactive metabolite formation, using the reported mean and CV of lasiocarpine depletion and 7-GS-DHP formation in the modelling approach applied in the present study will give insight in the most sensitive individuals for each population.

Besides the gender difference, other environmental factors such as age, smoking habits and alcohol consumption may affect CYP 3A4 activity. Rahmioglu et al. (2011) reported that about $20 \%$ of the inter-individual variations in induced CYP 3A4 activity were associated with Body Mass Index (BMI), alcohol use, and smoking habit, whereas age, diet and exercise levels were not associated with variations in CYP 3A4 activity. Hamilton et al. (2006) reported that in an analysis of thirty-two male Caucasians, $\mathrm{C}_{\max }$ and AUC of erlotinib in plasma, metabolized in the human liver primarily by CYP 3A4 but also by CYP 1A2 in smokers, were about 1.5- and 2.8-fold respectively lower in non-smokers. Thus, it seems that smoking may enhance the CYP 3A4 activity. Table S1 shows the information on history of alcohol consumption and smoking habit which was only available for the 25 Caucasian individuals. Given the large inter-individual variation in catalytic efficiency values of 7-GSDHP formation, and limited database regarding the history of alcohol consumption and smoking habit, conclusions on the association between PA metabolism and environmental factors have to await until further data become available in the future.

Previous studies have shown that CYP 3A4 plays an important role in PA metabolism, especially for the bioactivation pathway (Fashe et al. 2015; Li et al. 2011; Miranda et al. 1991). Inter-individual differences in CYP 3A4 abundance have been frequently reported. Rostami-Hodjegan and Tucker (2007) reported a CV of $119 \%$ of CYP 3A4 abundance in Caucasians by a meta-analysis and a CV of $87 \%$ for CYP $3 \mathrm{~A} 4$ abundance in Chinese (Inoue et al. 2006). Achour et al. (2014) reported that there were strong and significant correlations between the CYP 3A4 abundance in the liver and the CYP 3A probe testosterone metabolism mediated by CYP $3 \mathrm{~A} 4$ (spearman nonparametric correlation coefficient $\mathrm{r}_{\mathrm{s}}=0.79, \mathrm{P}<0.0001$ ) obtained by analysis of 23 individual liver microsomes from Caucasian subjects and one from an African American subject. Shu et al. (2001) also reported a good correlation (correlation coefficient of 0.79 ) between enzyme protein content and activity for CYP 3A4 by analysis of 42 Chinese liver microsomes (26 Chinese male individuals +16 Chinese female individuals). The CYP 3A4 protein level was determined by Western blot analysis and the activity was measured by omeprazole sulfoxidation, mediated by CYP 3A4. In this study, the CV of CYP 3A4 activity in 42 Chinese individuals was reported to be $53.4 \%$ and gender difference in CYP 3A4 activity was not observed. Thus, for the liver, it can be speculated that the CV \% of CYP 3A4 activity in vitro is likely to have a comparable value to the CV \% value of CYP $3 \mathrm{~A} 4$ abundance in the liver. In the present study, the CV of catalytic efficiency of 7-GS-DHP formation in Chinese and Caucasian population were $77 \%$ and $120 \%$, respectively, which are comparable to the CV of $87 \%$ in Chinese and $119 \%$ in Caucasians of CYP 3A4 abundance reported in the literature (Inoue et al. 2006; Rostami-Hodjegan and Tucker 2007). The study of Rahmioglu et al. (2011) reported a high degree (66 \%-88\%) of heritability in the CYP 3A4 inter-individual variation in twin studies as detected by repeated CYP 3A4-related 
drug administration, indicating that genetic polymorphism plays an important role in interindividual variations in CYP $3 \mathrm{~A} 4$ activity.

To date, 54 allelic variants of CYP 3A4 have been reported (https://www.pharmvar.org/), but only a few alleles are known to influence the CYP 3A4 expression or function. According to the literature (Wang et al. 2011), the CYP 3A4*22 mutation explains some of the genetic polymorphism showing a reduced activity in humans. For the inter-ethnic comparison, our previous study reported that the catalytic efficiencies of lasiocarpine depletion and of 7-GSDHP formation in average pooled Chinese microsomes were 2.3- and 7.5-fold lower, respectively than those values obtained in average pooled Caucasian microsomes (Ning et al. 2019). The predicted $C_{\max }$ of lasiocarpine in the liver blood was 2-fold higher in the average Chinese than in the average Caucasians at similar dose levels. The present study showed similar results for the average catalytic efficiencies of lasiocarpine depletion and 7-GS-DHP formation in 25 Chinese individuals which were 2.3- and 7.3-fold lower, respectively compared to those values obtained in 25 Caucasian individuals. A review of McGraw and Waller (2012) reported that the CYP 3A4*20 mutation has a relatively higher prevalence in the Chinese population (22\%) than in the Caucasian population $(6 \%)$. This allele has been shown to be devoid of any catalytic activity of CYP 3A4 in vitro (Westlind-Johnsson et al. 2006). The difference in CYP $3 \mathrm{~A} 4 * 20$ prevalence between the two populations may explain the observed lower catalytic efficiency of lasiocarpine depletion and 7-GS-DHP formation in the Chinese population to some extent. However, CYP 3A4 *20 cannot fully explain the predicted phenotype difference between these two ethnic groups indicating that other SNPs and or factors may contribute as well. The genetic polymorphisms in CYP 3A4 appeared to be more prevalent in Caucasians than in Asian (Li and Bluth 2011). This seems to be in line with our results in the present paper that the coefficient of variation for 7-GS-DHP formation, was higher in the 25 Caucasian individuals than in the 25 Chinese individuals. A major bottleneck for characterizing CYP 3A activity is the lack of a specific probe to separate the CYP 3A4 and CYP 3A5 activity, hampering the identification of the association between genotype and phenotype (McGraw and Waller 2012). Thus, the present study showed that ethnic differences in CYP 3A4 activity exist, but identification of all the underlying factors that result in this CYP 3A4 related ethnic differences in the metabolic phenotype, remains a topic for future research.

Comparing CSAF values obtained using the $90^{\text {th }}$ and the $99^{\text {th }}$ percentile for the formation of 7GS-DHP obtained from the Monte Carlo simulations, to the default uncertainty factor of 3.16 for inter-individual kinetic variation reveals that the default safety factor may not be adequately protective for lasiocarpine and/or PA toxicity in the studied populations. The IPCS document (IPCS 2005) stated that both inter-species and inter-individual variation in toxicokinetics would be ideally based on the free concentration of the reactive metabolite in the target tissue/organ. Based on this and the fact that the CSAFs derived from interindividual variations for the formation of the metabolite 7-GS-DHP reflecting bioactivation are higher than the CSAF values generated from the variations in $C_{\max }$ of the parent compound in liver blood (shown in the supplementary materials 5), the use of the CSAF of 5.7 and 19.5 derived from the formation of 7-GS-DHP for the $90^{\text {th }}$ percentile and $99^{\text {th }}$ 
percentile of the two populations combined, respectively were considered as the worst-case scenario for risk assessment.

For compounds that are both genotoxic and carcinogenic but also have thresholded adverse endpoints like acute liver toxicity, such as lasiocarpine, a Margin of Exposure (MOE) approach can be used to perform a risk assessment for both the acute liver toxicity and the carcinogenicity. In the MOE approach, the PoD like a BMDL 10 (the lower confidence limit of the benchmark dose resulting in 10\% extra effect above background values) is divided by the exposure level of the compound ( $\mathrm{mg} / \mathrm{kg}$ bw/day). In general, for a threshold endpoint such as acute liver toxicity, a MOE value of $>100$ is regarded to be of no or low concern for public health, while for evaluation of the carcinogenicity a MOE calculated using the BMDL 10 for induction of liver tumors should be at least 10000 (EFSA 2015). Both values include (1) a factor 10 for inter-species differences including both kinetic (a factor of 4) and dynamic (a factor of 2.5) differences between experimental animals and humans and (2) a factor 10 for inter-individual differences in kinetics (a factor of 3.16) and dynamics (a factor of 3.16) within the human population (EFSA 2012; EFSA 2015). The factor of 10000 additionally incudes (3) a factor 10 for inter-individual differences in cell control and DNA repair; and (4) a factor 10 for the fact that the $\mathrm{BMDL}_{10}$ or the reference point is not identical to a no observed adverse effect level (NOAEL) (EFSA 2005). Our previous studies showed that the inter-species differences in acute liver toxicity of lasiocarpine amount to about 2-fold taking toxicokinetic and toxicodynamic differences into account (Chen et al. 2018; Ning et al. 2019). Based on the inter-species differences in toxicokinetics and toxicodynamics, together with a CSAF of 19.5 defined for the inter-individual kinetic differences for the two populations combined and the default factor of 3.16 for inter-individual dynamics differences, the compound specific MOE cut-off value for non-genotoxic effects would be $123(2 * 3.16 * 19.5)$, which is 1.2-fold higher than the default threshold value of 100 used for inter- and intraspecies differences. Evaluating the endpoint of carcinogenicity, the default value of 10000 for the MOE for genotoxic carcinogens could be increased to $12324(19.5 * 2 * 3.16 * 10 * 10)$. When the CSAF obtained with the observed minimum and maximum acceptable boundaries, would be used, the compound specific MOE cut-off values for non-genotoxic effects and for genotoxic carcinogens would be 1.2-fold lower than these values derived when using the maximum allowed ratio of 1.0 .

In conclusion, the present study shows that linking Monte Carlo simulations with PBK modelling can be used to predict the effect of inter-individual and inter-ethnic human kinetic variation in lasiocarpine induced acute liver toxicity, and that based on this CSAF values can be derived. The CSAFs obtained using the $90^{\text {th }}$ and $99^{\text {th }}$ percentile for inter-individual variation in the Chinese, the Caucasian and the two populations combined were higher than default safety factor of 3.16 for inter-individual human differences in kinetics. The interethnic human differences between the Chinese and the Caucasian population was predicted to be 2.1-, 3.3- and 4.3-fold when comparing the GM, the 90th percentile and the 99th percentile of the populations, respectively, showing that the Caucasian population is more sensitive than the Chinese population. Overall, the present study shows that integrating PBK modelling with Monte Carlo simulations is a powerful strategy to quantify inter-individual 
variation in kinetics, which can be used to refine human risk assessment of PAs based on only in human vitro and in silico data.

\section{Note}

The authors declare that they have no conflict of interest

\section{Acknowledgements}

This work was funded by a grant from the China Scholarship Council (No. 201507720019 to NING JIA)

\section{References}

Achour B, Russell MR, Barber J, Rostami-Hodjegan A (2014) Simultaneous Quantification of the Abundance of Several Cytochrome P450 and Uridine 5 '-Diphospho-Glucuronosyltransferase Enzymes in Human Liver Microsomes Using Multiplexed Targeted Proteomics. Drug Metab Dispos 42:500-510

Bundesinstitut für Risikobewertung (BfR) (2013) Pyrrolizidine alkaloids in herbal teas and teas. BfR opinion

Brown RP, Delp MD, Lindstedt SL, Rhomberg LR, Beliles RP (1997) Physiological parameter values for physiologically based pharmacokinetic models. Toxicol Ind Health 13:407-484

Chen L, Ning J, Louisse J, Wesseling S, Rietjens IMCM (2018) Use of physiologically based kinetic modelling-facilitated reverse dosimetry to convert in vitro cytotoxicity data to predicted in vivo liver toxicity of lasiocarpine and riddelliine in rat. Food Chem Toxicol 116:216-226

Cotreau MM, von Moltke LL, Greenblatt DJ (2005) The influence of age and sex on the clearance of cytochrome P450 3A substrates. Clin Pharmacokinet 44:33-60

Culvenor CC (1983) Estimated intakes of pyrrolizidine alkaloids by humans. A comparison with dose rates causing tumors in rats. J Toxicol Environ Health 11:625-635

DeJongh J, Verhaar HJM, Hermens JLM (1997) A quantitative property-property relationship (QPPR) approach to estimate in vitro tissue-blood partition coefficients of organic chemicals in rats and humans. Arch Toxicol 72:17-25

European Food Safety Authority (EFSA) (2005) Opinion of the Scientific Committee on a request from EFSA related to A Harmonised Approach for Risk Assessment of Substances Which are both Genotoxic and Carcinogenic. EFSA J 3:282

European Food Safety Authority (EFSA) (2011) Scientific opinion on pyrrolizidine alkaloids in food and feed. EFSA J 9:2406

European Food Safety Authority (EFSA) (2012) Guidance on selected default values to be used by the EFSA Scientific Committee, Scientific Panels and Units in the absence of actual measured data. EFSA J $10: 2579$

European Food Safety Authority (EFSA) (2015) Scientific Opinion on acrylamide in food. EFSA J 13:4104 
European Food Safety Authority (EFSA) (2017) Risks for human health related to the presence of pyrrolizidine alkaloids in honey, tea, herbal infusions and food supplements. EFSA J 15:4908

Evans MV, Andersen ME (2000) Sensitivity analysis of a physiological model for 2,3,7,8tetrachlorodibenzo-p-dioxin (TCDD): assessing the impact of specific model parameters on sequestration in liver and fat in the rat. Toxicol Sci 54:71-80

Fashe MM, Juvonen RO, Petsalo A, Rasanen J, Pasanen M (2015) Species-Specific Differences in the in Vitro Metabolism of Lasiocarpine. Chem Res Toxicol 28:2034-2044

Fu PP, Xia Q, Lin G, Chou MW (2004) Pyrrolizidine Alkaloids-Genotoxicity, Metabolism Enzymes, Metabolic Activation, and Mechanisms. Drug Metab Rev 36:1-55

Hamilton M, Wolf JL, Rusk J, Beard SE, Clark GM, Witt K, Cagnoni PJ (2006) Effects of smoking on the pharmacokinetics of erlotinib. Clin Cancer Res 12:2166-2171

Hu ZY, Zhao YS (2010) Sex-Dependent Differences in Cytochrome P450 3A Activity as Assessed by Midazolam Disposition in Humans: A Meta-Analysis. Drug Metab Dispos 38:817-823

International Agency for Research on Cancer (IARC) (2017) Agents Classified by the IARC Monographs. vol 1-118. International Agency for Research on Cancer, Lyon, France

International Commission on Radiological Protection (ICRP) (2003) Basic Anatomical and Physiological Data for Use in Radiological Protection Reference Values. ICRP Publication 89. Ann ICRP 32:3-4

Inoue S, Howgate EM, Rowland-Yeo K, Shimada T, Yamazaki H, Tucker GT, Rostami-Hodjegan A (2006) Prediction of in vivo drug clearance from in vitro data. II: potential inter-ethnic differences. Xenobiotica 36:499-513

International Programme on Chemical Safety (IPCS) (1989) International Programme on Chemical Safety, Health and Safety Guide no. 26: Pyrrolizidine Alkaloids Health and Safety Guide. World Health Organization, Geneva, Switzerland

International Programme on Chemical Safety (IPCS) (2005) Chemical-specific adjustment factors for interspecies differences and human variability : guidance document for use of data in dose/concentrationresponse assessment. WHO

Kakar F, Akbarian Z, Leslie T, Mustafa ML, Watson J, van Egmond HP, Omar MF, Mofleh J (2010) An outbreak of hepatic veno-occlusive disease in Western afghanistan associated with exposure to wheat flour contaminated with pyrrolizidine alkaloids. J Toxicol 2010:313280

Lamba V, Panetta JC, Strom S, Schuetz EG (2010) Genetic predictors of interindividual variability in hepatic CYP3A4 expression. J Pharmacol Exp Ther 332:1088-1099

Li J, Bluth MH (2011) Pharmacogenomics of drug metabolizing enzymes and transporters: implications for cancer therapy. Pharmgenomics Pers Med 4:11-33

Li N, Xia QS, Ruan JQ, Fu PP, Lin G (2011) Hepatotoxicity and Tumorigenicity Induced by Metabolic Activation of Pyrrolizidine Alkaloids in Herbs. Curr Drug Metab 12:823-834 
Ma L, Zhao H, Xia Q, Cai L, Fu PP (2015) Synthesis and phototoxicity of isomeric 7,9-diglutathione pyrrole adducts: Formation of reactive oxygen species and induction of lipid peroxidation. J Food Drug Anal 23:577-586

Manoranjenni C, Donald M, Amin R-H (2012) Sex Differences in the Clearance of CYP3A4 Substrates: Exploring Possible Reasons for the Substrate Dependency and Lack of Consensus. Curr Drug Metab 13:778-786

McGraw J, Waller D (2012) Cytochrome P450 variations in different ethnic populations. Expert Opin Drug Metab Toxicol 8:371-382

Medinsky MA, Leavens TL, Csanady GA, Gargas ML, Bond JA (1994) In vivo metabolism of butadiene by mice and rats: a comparison of physiological model predictions and experimental data. Carcinogenesis 15:1329-1340

Miranda CL, Reed RL, Guengerich FP, Buhler DR (1991) Role of cytochrome P450IIIA4 in the metabolism of the pyrrolizidine alkaloid senecionine in human liver. Carcinogenesis 12:515-519

National Health and Family Planning Commission (NHFPC) (2007) Reference individuals for use in radiation protection-Part2: Masses of main organs and tissues. (in Chinese)

National Health and Family Planning Commission (NHFPC) (2014) Reference individiuals for use in radiation protection-Part3: main physiological parameters. vol GBZ/T 200.3 (in Chinese)

Nicolas JM, Espie P, Molimard M (2009) Gender and interindividual variability in pharmacokinetics. Drug Metab Rev 41:408-421

Ning J, Chen L, Strikwold M, Louisse J, Wesseling S, Rietjens IMCM (2019) Use of an in vitro-in silico testing strategy to predict inter-species and inter-ethnic human differences in liver toxicity of the pyrrolizidine alkaloids lasiocarpine and riddelliine. Arch Toxicol 93:801-818

National Toxicology Program (NTP) (1978) Bioassay of lasiocarpine for possible carcinogenicity. Natl Cancer Inst Carcinog Tech Rep Ser 39:1-66

Prakash AS, Pereira TN, Reilly PEB, Seawright AA (1999) Pyrrolizidine alkaloids in human diet. Mutat Res Genet Toxicol Environ Mutagen 443:53-67

Punt A, Paini A, Spenkelink A, Scholz G, Schilter B, van Bladeren PJ, Rietjens IMCM (2016) Evaluation of Interindividual Human Variation in Bioactivation and DNA Adduct Formation of Estragole in Liver Predicted by Physiologically Based Kinetic/Dynamic and Monte Carlo Modeling. Chem Res Toxicol 29:659-668

Rahmioglu N, Heaton J, Clement G, Gill R, Surdulescu G, Zlobecka K, Hodgkiss D, Ma Y, Hider RC, Smith NW, Ahmadi KR (2011) Genetic epidemiology of induced CYP3A4 activity. Pharmacogenet Genomics 21:642-651

Rostami-Hodjegan A, Tucker GT (2007) Simulation and prediction of in vivo drug metabolism in human populations from in vitro data. Nat Rev Drug Discov 6:140-148

Schmidt R, Baumann F, Hanschmann H, Geissler F, Preiss R (2001) Gender difference in ifosfamide metabolism by human liver microsomes. Eur J Drug Metab Pharmacokinet 26:193-200 
Schwartz JB (2003) The influence of sex on pharmacokinetics. Clin Pharmacokinet 42:107-121

Shu Y, Cheng ZN, Liu ZQ, Wang LS, Zhu B, Huang SL, Ou-Yang DS, Zhou HH (2001) Interindividual variations in levels and activities of cytochrome P-450 in liver microsomes of Chinese subjects. Acta Pharmacol Sin 22:283-288

Smith LW, Culvenor CC (1981) Plant sources of hepatotoxic pyrrolizidine alkaloids. J Nat Prod 44:129152

Soldin OP, Mattison DR (2009) Sex differences in pharmacokinetics and pharmacodynamics. Clin Pharmacokinet 48:143-157

Stegelmeier BL, Edgar JA, Colegate SM, Gardner DR, Schoch TK, Coulombe RA, Molyneux RJ (1999) Pyrrolizidine alkaloid plants, metabolism and toxicity. J Nat Toxins 8:95-116

Strikwold M, Spenkelink B, Woutersen RA, Rietjens IMCM, Punt A (2017) Development of a Combined In Vitro Physiologically Based Kinetic (PBK) and Monte Carlo Modelling Approach to Predict Interindividual Human Variation in Phenol-Induced Developmental Toxicity. Toxicol Sci $157: 365-376$

Wang D, Guo Y, Wrighton SA, Cooke GE, Sadee W (2011) Intronic polymorphism in CYP3A4 affects hepatic expression and response to statin drugs. Pharmacogenomics J 11:274-286

Westlind-Johnsson A, Hermann R, Huennemeyer A, Hauns B, Lahu G, Nassr N, Zech K, IngelmanSundberg M, von Richter O (2006) Identification and characterization of CYP3A4*20, a novel rare CYP3A4 allele without functional activity. Clin Pharmacol Ther 79:339-349

Westlind-Johnsson A, Malmebo S, Johansson A, Otter C, Andersson TB, Johansson I, Edwards RJ, Boobis AR, Ingelman-Sundberg M (2003) Comparative analysis of CYP3A expression in human liver suggests only a minor role for CYP3A5 in drug metabolism. Drug Metab Dispos 31:755-761

Wilkinson GR (2005) Drug metabolism and variability among patients in drug response. N Engl J Med 352:2211-2221

Wolbold R, Klein K, Burk O, Nussler AK, Neuhaus P, Eichelbaum M, Schwab M, Zanger UM (2003) Sex is a major determinant of CYP3A4 expression in human liver. Hepatology 38:978-988

Yang X, Zhang B, Molony C, Chudin E, Hao K, Zhu J, Gaedigk A, Suver C, Zhong H, Leeder JS, Guengerich FP, Strom SC, Schuetz E, Rushmore TH, Ulrich RG, Slatter JG, Schadt EE, Kasarskis A, Lum PY (2010) Systematic genetic and genomic analysis of cytochrome P450 enzyme activities in human liver. Genome Res 20:1020-1036

Zanger UM, Schwab M (2013) Cytochrome P450 enzymes in drug metabolism: regulation of gene expression, enzyme activities, and impact of genetic variation. Pharmacol Ther 138:103-141

Zhang X, Tsang AM, Okino MS, Power FW, Knaak JB, Harrison LS, Dary CC (2007) A physiologically based pharmacokinetic/pharmacodynamic model for carbofuran in Sprague-Dawley rats using the exposure-related dose estimating model. Toxicol Sci 100:345-359 


\section{Supplementary materials}

For convenience, supplementary materials 1-3 can be found in http://edepot.wur.nl/496945

\section{Supplementary materials 4}

A

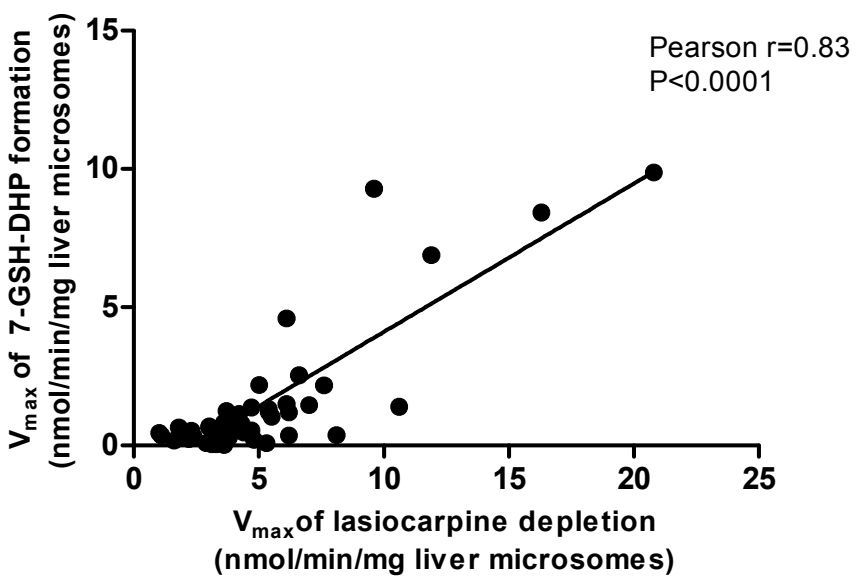

B

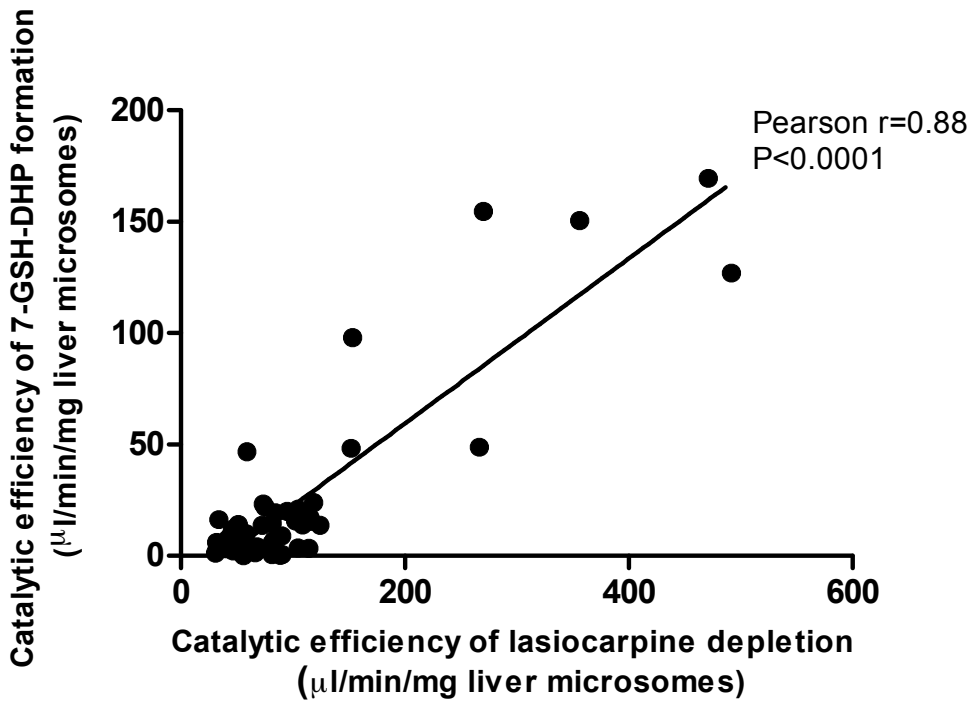

Fig. S4 Correlation plot for $\mathrm{V}_{\max }$ of lasiocarpine depletion and $\mathrm{V}_{\max }$ of 7-GS-DHP formation (A) and correlation plot for catalytic efficiency of lasiocarpine depletion and catalytic efficiency of 7-GS-DHP formation (B). The correlation analysis was analyzed by GraphPad (GraphPad Prism software version 5.04, San Diego California USA). 


\section{Supplementary materials 5}

A

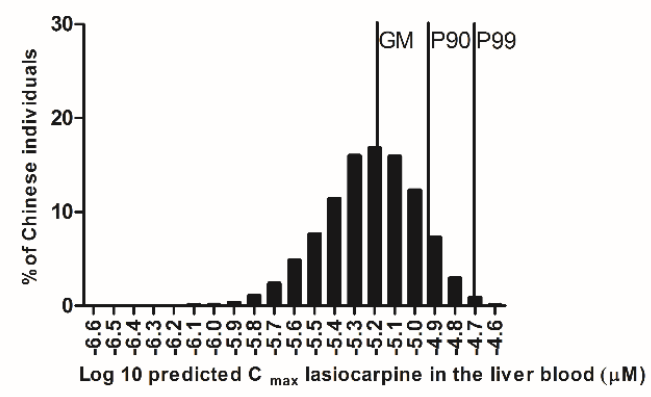

B
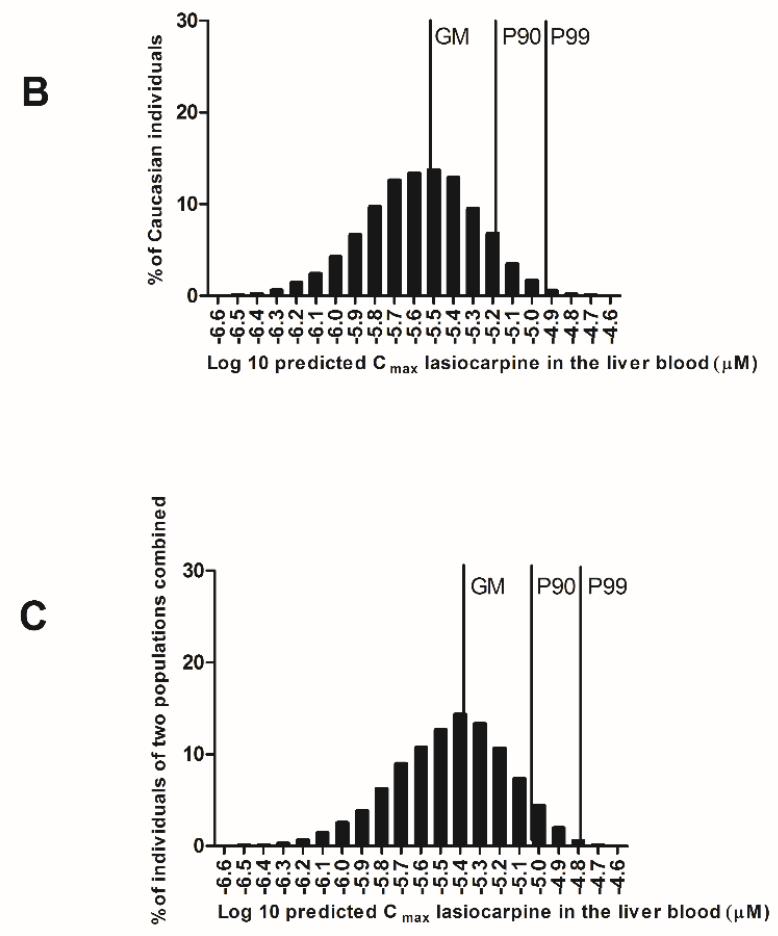

Fig. S5 Frequency distribution for the maximum predicted concentration of lasiocarpine in the liver blood for 14856 Chinese (A), 14823 Caucasians (B) and 14841 individuals of the two populations combined (C) at an oral dose of $8 \mathrm{ng} / \mathrm{kg}$ bw by Monte Carlo simulation including variation in the $\mathrm{V}_{\max }$ and $\mathrm{K}_{\mathrm{m}}$ of lasiocarpine depletion and $\mathrm{Ka}$ (oral absorption coefficient) in the PBK model of lasiocarpine. The GM, P90 and P99 represent the geometric mean, the $90^{\text {th }}$ and the $99^{\text {th }}$ percentile of the distribution. 
Table S5 The CSAFs for the inter-individual variation of $\mathrm{C}_{\max }$ of lasiocarpine in the liver blood for the $90^{\text {th }}$ and $99^{\text {th }}$ percentile in the Chinese, the Caucasian and the two populations combined.

\begin{tabular}{lccc}
\hline & $\begin{array}{c}\text { CSAF } \\
\text { Chinese } \\
\text { population }\end{array}$ & $\begin{array}{c}\text { CSAF } \\
\text { Caucasian } \\
\text { population }\end{array}$ & $\begin{array}{c}\text { CSAF } \\
\text { Two populations combined }\end{array}$ \\
\cline { 2 - 4 } $90^{\text {th }}$ percentile & 1.8 & 2.3 & 2.3 \\
$99^{\text {th }}$ percentile & 2.4 & 3.7 & 3.5 \\
\hline
\end{tabular}





$$
3
$$



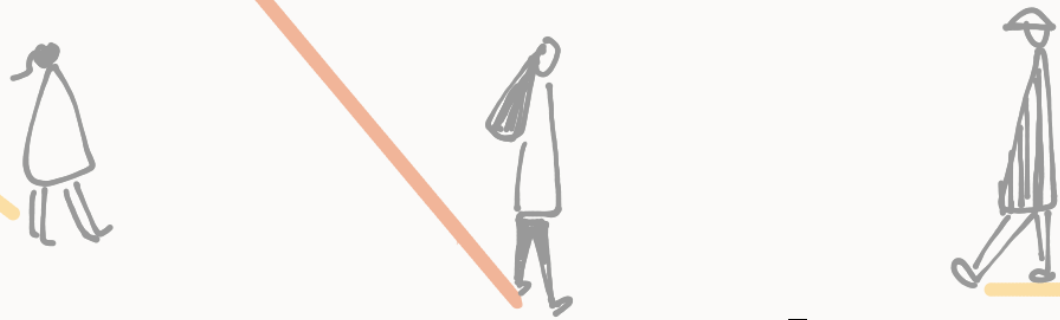

\section{Chapter 7}

General Discussion

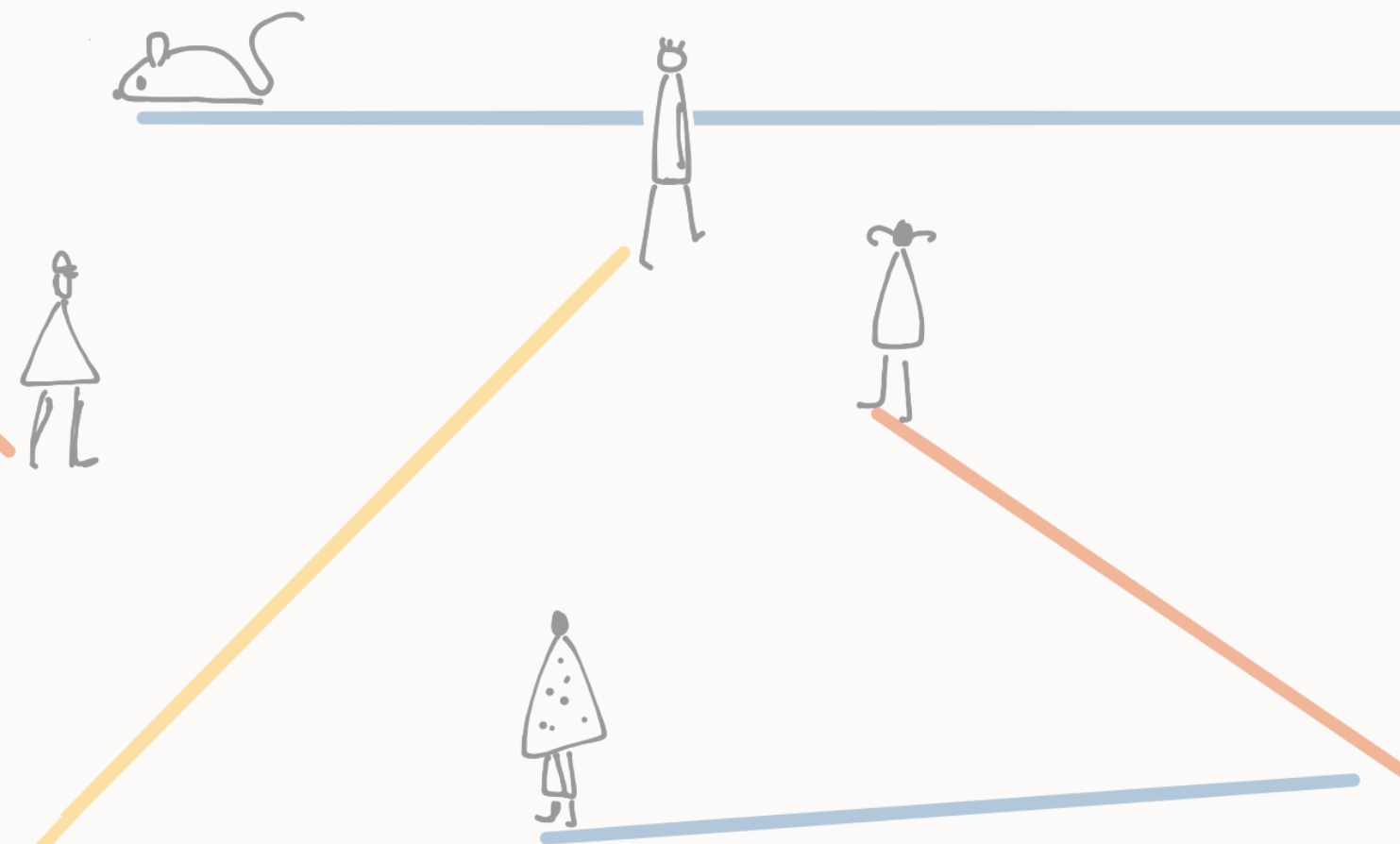


In China, a long history of using botanical preparations combined with the recent economic growth has led to active commercialization of the botanical market, without accompanying risk and safety assessments. As a result there is a need of performing risk assessment of botanical and botanical preparations. The aim of the present thesis was to perform the risk and safety assessment of two groups of natural toxins, namely alkenylbenzenes and pyrrolizidine alkaloids (PAs) for the Chinese population as compared to the overall Caucasian population using state-of-the-art methodologies including the Margin of Exposure (MOE) approach, physiologically based kinetic (PBK) modelling-based reverse dosimetry and the combination of PBK modelling and Monte Carlo simulation. In this chapter, the results of the thesis will be presented and discussed followed by an overview of future perspectives.

\subsection{Overview and discussion of the results}

Alkenylbenzenes and PAs are widely present in botanical preparations and are considered as genotoxic and carcinogenic compounds. Thus, the European Commission (EC) has prohibited the use of alkenylbenzenes such as estragole, methyleugenol and safrole as pure compound in foods based on Regulation No 1334/2008 of the European Parliament and of the Council (EC 2008). For PAs, different Member States in the EU have established specific limits for their occurrence in food including food supplements and/or in herbal medicines. For example, in Germany, the total intake of 1,2-unsaturated PAs and N-oxides of PAs must not exceed $0.1 \mu \mathrm{g}$ per day when the herbal medicines are orally consumed for a period of more than 6 months (Merz and Schrenk 2016). In the Netherlands, the total content of PAs in herbal preparations and herbal extracts must not exceed $1 \mu \mathrm{g} / \mathrm{kg}$ (Merz and Schrenk 2016). However, in China, such regulations and specific limitations for alkenylbenzenes and PAs are not in place. Despite of this, the Chinese health authorities have developed a list of dietary herbs (allowed as herbal medicines or food) and a list of herbs (allowed as health food products). Herbs potentially containing alkenylbenzenes or PAs can be found in these two lists without any restrictions regarding dose, frequency and time for consumption (CFDA 2013a; CFDA 2013b). For herbal medicines, the qualification and standard of those products are regulated based on the Chinese Pharmacopoeia, in which the purity, dosage, description, precaution and storage of each medicine are regulated (Liu and Salmon 2010), but the total content of alkenylbenzenes and PAs are not regulated. The estimated daily intake (EDIs) of PAs from some herbal medicines may exceed the maximum daily intake of $0.007 \mu \mathrm{g} / \mathrm{kg}$ bw/day set by the COT (2008), BfR (2011) and EFSA (2011) (Griffin et al. 2014). Thus, there is a need to perform a risk assessment for exposure to alkenylbenzenes and PAs, not only for the Caucasian population as generally done by regulatory bodies such as EFSA, but, given potential inter-ethnic variations in toxicokinetics and toxicodynamics, also for the Chinese population. This was done in the present thesis using the Margin of Exposure (MOE) approach, while also using PBK modelling-based reverse dosimetry and Monte Carlo simulation to quantify inter-species, inter-ethnic as well as inter-individual differences in sensitivity.

In chapter 2, a risk assessment of 10 plant food supplements (PFS), 23 traditional Chinese medicines (TCM) and 38 herbal teas containing alkenylbenzenes was performed using the 
MOE approach. The levels of alkenylbenzenes in 71 botanical preparations collected on the Chinese market were quantified and the combined EDI was determined using a dose addition approach. Based on the chemical analysis of 71 botanical preparations, estragole appeared to be the most frequently detected alkenylbenzene, being detected in 30 out of 71 samples, followed by methyleugenol, myristicin, elemicin, apiol and safrole. The combined EDI values obtained assuming equal potency of all alkenylbenzenes detected in the PFS, TCM and herbal teas were $0.3-14.3,0.05-539.4$ and $0.04-42.5 \mu \mathrm{g} / \mathrm{kg}$ bw/day, respectively. Calculating combined EDI values of alkenylbenzenes taking into account the toxic equivalency (TEQ) approach, the values for PFS, TCM and herbal teas were 0.3-7.7, 0.05-278.0 and 0.02-16.5 $\mu \mathrm{g}$ estragole equivalents/kg bw/day, respectively. Assuming daily life-long consumption, $60 \%$ of the PFS samples had MOE values for consumption of the individual alkenylbenzenes lower than 10000 and almost all PFS samples resulted in intakes that gave MOE values lower than 10000 when taking combined exposure of alkenylbenzenes into account, except for one PFS sample. For TCM samples, $43 \%$ of the samples had MOE values lower than 10000 when taking combined exposure into account. TCM number 6 had an individual MOE value even lower than 10, which means that the daily intake of the alkenylbenzene of interest, myristicin, would be within the range of dose levels predicted to cause liver tumors in experimental animals (Al-Malahmeh et al. 2017a; Van Den Berg et al. 2011). Assuming consumption of one cup of herbal tea per day, $50 \%$ of the tea samples would give rise to MOE values lower than 10000 based on combined exposure approaches. Given that life-long daily exposure may not represent a realistic exposure scenario, risk assessment for short-term consumption such as two weeks in a life time was also performed using Haber's rule to correct for shorter than life time exposure. Taking this short term exposure into account, the MOE values of most of the botanical preparations analysed were above 10000 , indicating a low priority for risk management. However, this does not hold for TCM 6, of which the MOE was still below 10000 based on assuming equal potency of the alkenylbenzenes in a combined risk assessment. Overall, the MOE values for PFS, TCM and herbal teas containing alkenylbenzenes indicate that there is a potential priority for risk management for several botanical preparations, especially when considering life-long daily exposure. The results also support the need for development of a method to take shorter than life time exposure into account since so far there is no officially accepted methodology to perform such a risk assessment, although the use of Haber's rule may provide a first tier approach for making this correction.

The objective of Chapter 3 was to further study the hazards and risks of exposure to alkenylbenzenes taking potential inter-ethnic differences in sensitivity between the Caucasian and Chinese population into account. This was done by developing physiologically based kinetic (PBK) models to predict the differences in bioactivation and detoxification of the food-borne genotoxic carcinogen estragole between Chinese and Caucasians. Estragole was selected as the model compound because estragole was the most frequently detected compound in the 71 botanical preparations collected from the Chinese market (Chapter 2). To define the PBK models for the Chinese and Caucasian populations, kinetic constants for the various metabolic reactions of estragole were derived from in vitro incubations with relevant 
tissue fractions of the two ethnic groups. The outcomes predicted by the PBK model for the Chinese population were compared to those predicted by the PBK model for Caucasians to evaluate the inter-ethnic differences in metabolic activation and detoxification of estragole and to demonstrate the potential of PBK modelling to study such inter-ethnic variability. The outcomes of the model predictions showed that the detoxification of the proximate carcinogenic metabolite 1'-hydroxyestragole to 1'-oxoestragole was similar in both ethnic groups. For the bioactivation pathway, at realistic daily intake of estragole, only $0.02 \%$ of the dose was converted to the ultimate carcinogenic metabolite, i.e. 1'-sulfooxyestragole, in the Chinese population, while this value amounted to $0.09 \%$ of the dose in Caucasian subjects. This 4.5-fold difference accompanied by similar rates of detoxification may indicate a lower risk of estragole for the Chinese population at similar levels of exposure. The study provides a proof-of-principle for how mode of action based PBK modelling can identify differences in ethnic sensitivity and provide a more refined risk assessment for a specific ethnic group for a compound of concern.

After studying the bioactivation, detoxification and inter-ethnic differences in potential hazards of alkenylbenzenes, also the hazards of a second groups of food-borne botanical ingredients of concern, the pyrrolizidine alkaloids were investigated. Especially the 1,2unsaturated pyrrolizidine alkaloids are converted to DNA reactive metabolites that cause the compounds to be genotoxic and carcinogenic. For the PAs, PBK modelling was applied to provide a means to study not only inter-ethnic, but also inter-species and inter-individual differences in sensitivity to PA toxicity. In addition, the PBK models were used to convert in vitro data on liver toxicity to predicted in vivo dose-response data in order to show the potential for quantitative in vitro to in vivo extrapolation (QIVIVE) and predict points of departure (PoD) for risk assessment. Given the large data gaps in the toxicity data base for PAs, development of alternative testing strategies could help to support read-across and fill the existing data gaps without the need for extensive animal testing. Chapter $\mathbf{4}$ demonstrated that combining in vitro cytotoxicity data obtained with primary rat hepatocytes with PBK modelling-based reverse dosimetry could adequately predict in vivo liver toxicity data of lasiocarpine and riddelliine for rats. Lasiocarpine and riddelliine were selected as the model compounds, because for these PAs in vivo data are available that enable evaluation of the predictions made. The PBK models for rats for lasiocarpine and riddelliine were developed based on the data derived from in silico methods, incubation experiments using subcellular tissue fractions and literature data to define the physiological parameters. The developed PBK model of riddelliine was evaluated by comparison of the predicted maximal blood concentration $\left(\mathrm{C}_{\max }\right)$ of riddelliine to the available in vivo kinetic data upon dosing $10 \mathrm{mg} / \mathrm{kg}$ bw of this PA to rats and mice, showing that the predicted $C_{\max }$ values are 2- and 9.5-fold higher than the reported $\mathrm{C}_{\max }$ for mouse and rat, respectively. Subsequently, the in vitro concentration-response curves for cytotoxicity of lasiocarpine and riddelliine obtained in primary rat hepatocytes were converted to in vivo dose-response curves for acute liver toxicity by PBK modelling-based reverse dosimetry, from which the PoDs, the benchmark dose lower and upper confidence limits for $5 \%$ effect (BMDL5-BMDU5) were derived using a BMD analysis. The predicted PoDs were compared to the available literature data on in vivo 
liver toxicity of lasiocarpine, showing that the predicted PoDs fall well within the range of PoDs obtained from the available oral single dose in vivo studies. In conclusion, this chapter showed that PBK modelling-based reverse dosimetry can translate in vitro concentrationresponse curves to in vivo dose-response curves to predict the acute liver toxicity of lasiocarpine and riddelliine in rats. The developed PBK models for lasiocarpine and riddelliine in rats were used as a starting point to construct PBK models for these two PAs in humans, and study inter-species and inter-ethnic differences in liver toxicity of these PAs (chapter 5).

After providing a proof-of-principle for extrapolation of in vitro toxicity data on PAs to the in vivo situation using PBK modelling-based reverse dosimetry in rats (chapter 4) and illustrating how PBK modelling can identify differences in ethnic sensitivity (chapter 3), chapter 5 investigated the inter-species and inter-ethnic human differences in acute liver toxicity of lasiocarpine and riddelliine using the same approach developed in chapter 4 . Thus, PBK models for lasiocarpine and riddelliine for the average Chinese and the average Caucasian were developed, based on the PBK model defined and validated in rats (chapter 4). Subsequently, these models were used to convert in vitro toxicity data obtained in pooled Caucasian primary hepatocytes to predicted in vivo dose-response data, from which the PoDs were derived and were compared to the PoDs for rats derived in chapter 4. Similarly, the PoDs obtained for the average Chinese and the average Caucasian were compared to each other to provide insights in inter-ethnic differences. The inter-species differences amounted to 2-fold for lasiocarpine and 8.2-fold for riddelliine with humans being more sensitive than rats. This is mainly due to the fact that the kinetics showed a slower clearance of lasiocarpine and riddelliine in humans than in rats. The inter-ethnic human differences amounted to 2-fold for lasiocarpine and 5-fold for riddelliine with the average Caucasian being more sensitive than the average Chinese. This could be ascribed to the fact that there was less bioactivation for both compounds at similar dose levels in average Chinese as compared to average Caucasian subjects. Altogether, this chapter showed a proof-of-principle for using PBK modelling as a method to predict inter-species and inter-ethnic differences in in vivo liver toxicity for PAs via an alternative testing strategy integrating in vitro cytotoxicity assays with PBK modellingbased reverse dosimetry.

After studying the ethnic human differences in acute liver toxicity of lasiocarpine between the average Chinese and the average Caucasian population using mixed gender pooled Chinese and Caucasian liver fractions applying PBK modelling-based reverse dosimetry, it was investigated to what extent alternative testing strategies could provide insights in the interindividual variations in kinetics and sensitivity to PAs, which may assist in defining chemical specific adjustment factors (CSAFs). Given the large variation among individuals in terms of CYP 3A4 activity, representing the major hepatic metabolic enzyme activity involved in conversion of lasiocarpine, the aim of chapter 6 was to study the effect of inter-individual and inter-ethnic (Chinese and Caucasian) kinetic variations on the sensitivity towards acute liver toxicity of lasiocarpine, and to derive CSAFs by applying PBK modelling and Monte Carlo simulation. To this purpose, the kinetic constants for lasiocarpine depletion and 7-GSDHP formation obtained from incubation experiments using liver fractions from 25 Chinese 
and 25 Caucasians were included in the previously developed PBK model (chapter 5) to develop individual PBK models and to predict 7-GS-DHP formation in 25 Chinese and 25 Caucasian individuals. Use of glutathione to scavenge the reactive dehydro-PAs forming the major pyrrole-GSH adduct, 7-GS-DHP, was applied to determine inter-individual differences in bioactivation and potential toxicity of lasiocarpine (IPCS 2005). For the inter-individual comparison, the predicted 7-GS-DHP formation in the liver varied 41-fold within the 25 Chinese individuals, 200-fold within the 25 Caucasian individuals and 200-fold within the data set as a whole ( 25 Chinese +25 Caucasians) when comparing the individuals with the highest and the lowest activity. For the inter-ethnic comparison, the geometric mean (GM) for the predicted 7-GS-DHP formation in incubations with liver samples of the 25 Chinese individuals appeared to be 2.2-fold lower than this value in incubations with liver samples from the 25 Caucasian individuals. Subsequently, a Monte Carlo simulation was performed to predict inter-individual and inter-ethnic variations in 7-GS-DHP formation in the liver and to generate frequency distributions of 7-GS-DHP formation in the Chinese, the Caucasian and the two populations combined. From these distributions CSAFs were obtained by dividing the $90^{\text {th }}$ or $99^{\text {th }}$ percentile by the GM of 7-GS-DHP formation in the liver. The results revealed an inter-ethnic variation of 2.1-, 3.3- and 4.3-fold when comparing the predicted 7-GS-DHP formation at the GM, 90th and 99th percentile of the Chinese population and the Caucasian population, respectively, at a dose of level of $8 \mathrm{ng} / \mathrm{kg}$ bw lasiocarpine, with the Caucasian population being more sensitive. The CSAFs obtained based on the GM and $90^{\text {th }}$ percentile individuals of the Chinese, Caucasian and the two populations combined were 3.3, 5.2 and 5.7, respectively. The CSAFs obtained based on the GM and the $99^{\text {th }}$ percentile were $8.3,17.0$ and 19.5 for inter-individual variations in the Chinese, the Caucasian population and the two populations combined, respectively. The CSAF values obtained indicate that the default safety factor of 3.16 for inter-individual human kinetic differences may not be sufficiently protective. Altogether, the results showed that integrating PBK modelling with Monte Carlo simulations based on only human in vitro and in silico data is a powerful strategy to quantify inter-individual variation in kinetics, which can be used to refine human risk assessment of PAs. 


\subsection{General discussion and future perspectives}

The present thesis demonstrates the potential of using the Margin of Exposure (MOE) approach, physiologically based kinetic (PBK) modelling-based reverse dosimetry and the combination of PBK modelling with Monte Carlo simulation to study the inter-species, interethnic and inter-individual differences in bioactivation, toxicity and detoxification of alkenylbenzenes and pyrrolizidine alkaloids, which are two groups of genotoxic and carcinogenic botanical ingredients present in the modern food chain. The results of the present thesis can be discussed in a wider perspective, providing some important considerations for future studies. The topics that are relevant to discuss include:

- The in vitro system selected for studying liver toxicity

- The dose metric used for conducting PBK modelling-based reverse dosimetry

- $\quad$ Use of PBK modelling to study inter-ethnic human differences

- Implications for risk assessment

- $\quad$ Future perspectives

\subsubsection{The in vitro system selected for studying liver toxicity}

Selection of an appropriate in vitro system to study liver toxicity is of importance when the in vitro data are used to perform quantitative in vitro to in vivo extrapolation (QIVIVE). For studying liver toxicity, maintaining metabolic activity is a crucial requirement for the cell model used since xenobiotic metabolic enzymes are highly expressed in the liver to metabolize drugs or chemicals, which may produce reactive metabolites in addition to the parent compounds. Ideally, a reliable in vitro hepatotoxicity model should resemble the phenotype observed in vivo and be able to conduct long-term studies and high-throughput applications (Lauschke et al. 2016). The current golden standard in vitro liver model consists of human primary hepatocytes (Bell et al. 2016; Gomez-Lechon et al. 2014). Human primary hepatocyte monolayers are able to maintain key hepatic-specific functions, such as carbohydrate metabolism, ureogenesis, plasma protein synthesis and secretion, lipid metabolism and transport, bile acid synthesis and uptake, and drug metabolism capacity (Gomez-Lechon et al. 2003; Gomez-Lechon et al. 2014). Human primary hepatocytes have been extensively used as a tool to assess the risk of xenobiotic bioactivation and hepatotoxicity in humans (Gomez-Lechon et al. 2014).

Liver toxicity studies can be performed using primary hepatocytes either in suspension or in monolayer culture. Use of primary hepatocytes in suspension is often preferable due to the ease in handling and relatively lower costs (Smith et al. 2012). However, it has been reported that the activity of biotransformation enzymes in primary hepatocytes in suspension may reduce dramatically within a few hours, whereas the enzyme activities in a hepatocyte monolayer can be maintained for 24 hours after plating the cells (Smith et al. 2012). Therefore, the use of primary human hepatocytes in suspension is preferable for determination of intrinsic metabolic clearance requiring only a limited incubation time, while 
the use of primary hepatocytes in cultured monolayers is more appropriate to study hepatotoxicity of compounds since hepatotoxicity is a process that occurs over several hours, and hepatocytes in suspension cannot maintain cell viability to develop toxicity (Soldatow et al. 2013). At present, cryopreserved hepatocytes provide an important source of commercially available cells. Although the hepatocyte viability and function can be reduced by cryopreservation, recent improvements in the freezing protocols have resulted in a more successful recovery and platability of the hepatocytes after thawing. Additionally, several studies have shown that the differences in CYP and conjugating enzyme activities, needed for metabolizing capability, between fresh and cryopreserved human hepatocytes from the same batch were limited (Donato et al. 2008; McGinnity et al. 2004). In chapter 4 and chapter 5 of this thesis, cryopreserved rat and human primary hepatocytes in monolayer culture were used to quantify the in vitro liver toxicity of lasiocarpine and riddelliine. When thawing and plating the cells, the thawing and plating mediums provided by the supplier were used for optimal cell attachment and monolayer formation. The cell viability was more than $90 \%$ after thawing. The concentration-response curve for the cytotoxicity of lasiocarpine obtained with cryopreserved primary rat hepatocytes in chapter 4 appeared to be comparable to in vitro concentration-response curves obtained with fresh isolated rat primary hepatocytes in culture previously reported by Hayes et al. (1984), indicating that the concentration-response curves obtained with cryopreserved primary rat and human hepatocytes can adequately be used to study liver toxicity of PAs.

One of the drawbacks of primary human hepatocytes is the donor-to-donor variability which may hamper the reproducibility. A recent study investigated the inter-donor variability of phase I (CYP) and phase II enzymes (UGT and SULT) enzymes in cryopreserved primary human hepatocytes obtained from 5 donors using three reference drugs related to druginduced liver injury (DILI), namely acetaminophen (APAP), diclofenac (DF) and tolcapone (TC), and the phase II substrate 7-hydroxycoumarin (7-HC). The results showed that the inter-donor variability in phase I and phase II metabolism of the test compounds was substantial. For example, relative CYP activity compared to total DF metabolism varied from $3.1 \%$ to $12.2 \%$ (4-fold). For SULT activity, the donor-activity expressed relative to total metabolism of 7-HC varied from $0.02 \%$ to $1.7 \%$ (85-fold) (den Braver-Sewradj et al. 2016). To better represent the average human population, in the present thesis pooled human primary hepatocytes made from 5 donors were used to study liver toxicity of PAs. It has been reported that in humans metabolism of PAs is mainly catalyzed by CYP 3A4 (Fashe et al. 2015; Li et al. 2011; Miranda et al. 1991). Gerets et al. (2012) reported that the variability in CYP 3A4 activity in three freshly isolated primary human hepatocytes quantified using midazolam as catalytic probe was up to 10 -fold. Thus, the use of pooled human hepatocytes made from 5 donors to study liver toxicity of PAs may represent the average human population only to some extent, because of the large variation among individuals in terms of CYP 3A4 activity. The effect of inter-individual variations in sensitivity towards liver toxicity of lasiocarpine was further investigated using liver fractions from 50 human individuals $(25$ Chinese +25 Caucasians) in chapter 6. A novel in vitro hepatotoxicity model, human induced-pluripotent stem cell (iPSC) derived hepatocytes, seems to be a promising option in the future to 
overcome the limitation of donor-to-donor variability. Human hepatocytes derived from iPSCs generated from multiple individuals with different polymorphic characteristics may provide a limitless supply of hepatocytes for high-throughput screening with minor batch variability to improve reproducibility (Gomez-Lechon et al. 2014).

Compounds that are capable of inducing liver damage are known as hepatotoxins. These toxins can directly interfere with cellular processes and affect the cell integrity or become toxic once they produce toxic metabolites by biotransformation. Thus, it is necessary to ascertain whether toxicity is associated with metabolism of a given compound. This should be assessed by comparing in vitro systems that are metabolically competent with cell models that may not contain the required biotransformation enzymes at a substantial or sufficient level. Thus when biotransformation is a prerequisite for toxicity this will become apparent when comparing the concentration-response curves obtained with 1) primary cultured hepatocytes, 2) non-hepatic cells and 3) hepatoma cell lines that lack P450 (Gomez-Lechon et al. 2003). As an example, Figure 1 shows the in vitro concentration-response curves for the cytotoxicity of lasiocarpine obtained with primary human hepatocytes, HepaRG and HepG2 cell lines upon 24 hours of exposure. HepaRG is a hepatoma cell line considered as a promising in vitro surrogate of primary human hepatocytes. One of the remarkable characteristics of HepaRG cells is that they can be differentiated into a mixed culture of hepatocyte-like and biliary-like cells (Aninat et al. 2006; Lambert et al. 2009). The differentiated cells display relatively higher levels of phase I and phase II enzymes than other hepatoma cell lines (Kanebratt and Andersson 2008). HepG2 is one of the most frequently used human cell models for hepatotoxicity evaluations. The results obtained in the present thesis and summarised in Figure 1 show that PAs are not toxic toward HepG2 cells up to a concentration of $300 \mu \mathrm{M}$, most likely because these cells apparently lack a sufficient amount of the required CYP activity, while the HepG2 cell line was reported to be the most predictive cell model after primary human hepatocytes for prediction of drug-induced liver injury DILI (SisonYoung et al. 2017). The $\mathrm{IC}_{50}$ value for lasiocarpine obtained with primary human hepatocytes was 3 -fold lower than the $\mathrm{IC}_{50}$ value derived with HepaRG cells and thus cytotoxicity data derived from primary hepatocytes were used to perform QIVIVE in chapter 4 and 5 . The fact that lasiocarpine is not cytotoxic towards HepG2 cells, corroborates that it is of importance to select in vitro systems that are metabolically competent instead of hepatoma cell lines that lack P450 such as HepG2 cells, especially when evaluating the toxicity of highly metabolized parent compounds or compounds that only become toxic once they produce toxic metabolites upon biotransformation. 


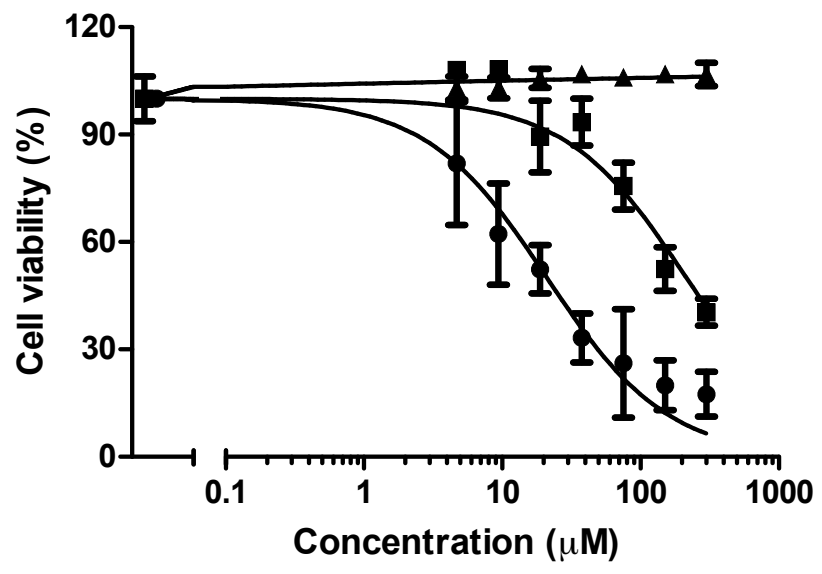

Fig. 1 In vitro concentration-response curves for the cytotoxicity of lasiocarpine obtained with primary human hepatocytes (circles), HepaRG cells (squares) and HepG2 (triangles) cells upon 24 hours exposure.

In chapter 4 and 5, the primary hepatocytes were exposed to PAs for 24 hours taking into consideration the major drawback of primary hepatocytes that the key hepatic-specific characteristics such as cell morphology, structure, polarity, bile canaliculi formation and more importantly, drug metabolizing capability undergo rapid changes or reduction once they are placed into culture (Godoy et al. 2013; Gomez-Lechon et al. 2008; Gomez-Lechon et al. 2014; Soldatow et al. 2013). Since HepaRG cells are able to retain metabolic activity for about several weeks (Klein et al. 2014), a single exposure of HepaRG cells to lasiocarpine with exposure duration of both 24 and 72 hours was performed (Figure 2) showing that the concentration-response curve of lasiocarpine obtained with primary human hepatocytes upon 24 hours exposure is highly comparable to the curve obtained with HepaRG cells upon 72 hours exposure. These results indicate that the use of HepaRG cells with exposure duration of 72 hours increases the toxicity of lasiocarpine as compared to 24 hour exposure, so that the cells may potentially reflect the cytotoxicity of lasiocarpine obtained with primary human hepatocytes. It is necessary to confirm this suggestion by testing more unsaturated PAs to corroborate that the use of HepaRG cells with extended exposure duration can be considered as an alternative for primary human hepatocytes when assessing the liver toxicity of PAs in the future. 


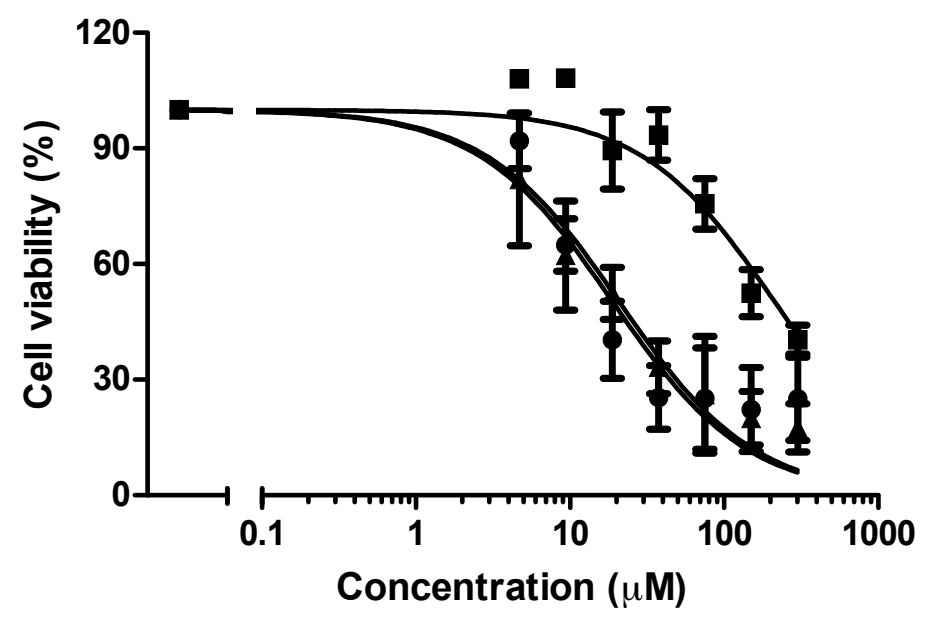

Fig. 2 In vitro concentration-response curves for the cytotoxicity of lasiocarpine obtained with primary human hepatocytes (triangles) upon 24 hours exposure, and with HepaRG cells upon 24 (squares) and 72 hours (circles) exposure.

Besides the acute liver toxicity test with short-term exposure, long-term exposure when studying hepatotoxicity is also of importance. Holmgren et al. (2014) reported that a significant shift of the cytotoxicity curves was observed upon 7-day and 14-day exposure compared with 2-day exposure of iPSCs derived hepatocytes to amiodarone, aflatoxin B1 and troglitazone, showing that cells are more sensitive upon long-term and repeated exposures. This is in line with in vivo observations. For PAs, a recent study reported a No Observed Adverse Effect Level (NOAEL) of lasiocarpine amounting to $0.6 \mathrm{mg} / \mathrm{kg}$ bw/day upon daily repeated oral exposure of rats for 28 days (Dalefield et al. 2016). This value is substantially lower than the reported Lowest Observed Adverse Effect Levels (LOAEL) of 80 and $120 \mathrm{mg}$ / $\mathrm{kg}$ bw obtained upon single oral exposure of lasiocarpine causing increased incidence of acute zonal hepatic necrosis and megalocytosis in rats (Nolan et al. 1966). Using an uncertainty factor of 3 to convert these LOAEL values for liver toxicity data to a NOAEL resulted in NOAEL values of 27 and $40 \mathrm{mg} / \mathrm{kg}$ bw/day. The in vitro PBK modelling based predicted $\mathrm{BMDL}_{5}$ BMDU $_{5}$ value for acute liver toxicity in rats reported in chapter 4 was 23-34 mg/kg bw/day, which is more in line with the PoDs obtained from the available oral single dose in vivo studies (Nolan et al. 1966). Therefore, it would be worthwhile and interesting to conduct a long-term and/or repeated exposure of the cell models to PAs in the future. To date, use of HepaRG cells maybe an option for studying such chronic or repeated exposure to PAs since these cells can be cultured for several weeks with constant metabolic enzyme activities (Klein et al. 2014). It has been shown that HepaRG 3D spheroids have greater CYP 3A4 activity, the key enzyme for PAs metabolism, than the HepaRG cultured in 2D monolayers (Mueller et al. 2014). Thus, the use of HepaRG 3D spheroids may provide an even more accurate assessment of human hepatotoxicity of PAs upon chronic exposure in the future. 
Altogether, primary hepatocytes in culture or hepatoma cell lines have been widely used for predictions of hepatotoxicity of compounds for decades. The limitations of these in vitro models have led to the development of novel in vitro systems such as 3D systems, bioartificial livers, complex co-culture system and iPSCs derived hepatocytes (GomezLechon et al. 2014; Gomez-Lechon et al. 2016; Soldatow et al. 2013). The need for in vitro liver systems capable of resembling the in vivo like function over long-term culture is essential to better mimic chronic exposures that may be most relevant for the toxicity of foodborne toxins like the alkenylbenzenes and PAs of the present thesis. Such novel systems will provide in vitro models enabling QIVIVE to predict chronic toxicity, thereby further reducing the need for animal use in toxicological studies.

\subsubsection{Dose metric for conducting PBK modelling-based reverse dosimetry approach}

In chapter 4 and 5 , the in vitro concentration-response curves for the cytotoxicity of lasiocarpine and riddelliine were translated to in vivo dose-response curves to predict the acute liver toxicity of these two compounds using PBK modelling-based reverse dosimetry. The primary hepatocytes and HepaRG cells were exposed to these two PAs in serum free medium. This was done because the serum in the culture medium may significantly reduce the effect concentration of a chemical in an in vitro system due to possible binding of the chemical to plasma proteins, and generally only the free form of the chemical is assumed to cause the adverse effect. Figure 3 compares the concentration-response curves for cytotoxicity of lasiocarpine in HepaRG cells exposed for 72 hours in medium with $10 \%$ serum and without serum, showing that protein binding can reduce the cytotoxicity of lasiocarpine to the cells, despite the fact that DelRaso et al. (2003) found that protein enhanced the uptake rate of cadmium to rat hepatocytes. Figure 3 indicates that serum is one of the important bioavailability reducing factors also reducing PA induced toxicity. Besides protein binding, other factors like evaporation and non-specific binding of compounds to the plastic of well plates may affect the effect concentration (Groothuis et al. 2015). Recent studies reported that sorption to plastic of relatively low to moderately lipophilic chemicals such as cisplatin with a $\log \mathrm{P}$ of -2.35 and adefovir dipivoxil with a $\log \mathrm{P}$ of 2.45 did not significantly reduce the free concentration (Crean et al. 2015; Wilmes et al. 2015). The $\log P$ of lasiocarpine and riddelliine were 0.48 and -0.28 , respectively, estimated by ChemBio 3D 2010 software (CambrigeSoft, USA), indicating that sorption to well plates of PAs would not likely substantially affect the freely available concentration in our test system. Solutions that may help to overcome these factors include the use of plate sealers to minimize evaporation and the use of cell suspension cultures in culture plates of non-binding materials to avoid the non-specific binding to the plastic (Groothuis et al. 2015). Groothuis et al. (2015) also proposed to use partition-controlled dosing systems in the in vitro assay, which can compensate for the loss of compounds due to evaporation. 


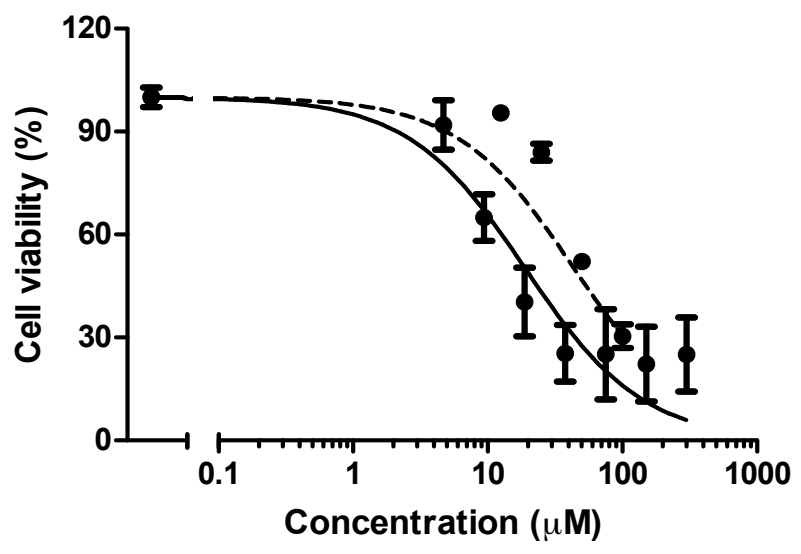

Fig. 3 In vitro concentration-response curves for the cytotoxicity of lasiocarpine in cells of the HepaRG cell line upon 72 hours exposure in the culture medium with $10 \%$ serum (dashed line) and without serum (solid line)

The importance of protein binding for the QIVIVE was further emphasized by Mielke et al. (2017) using ibuprofen as an example which is a highly protein-bound substance (fraction of unbound in blood $=0.1 \%$ ). Mielke et al. (2017) reported a partition coefficient between hepatic tissue and blood of 3.01 calculated by an algorithm method using tissue composition and physicochemical properties of the compound and the unbound fraction of the compound in blood (Schmitt 2008), and a partition coefficient of 11.1 obtained by using the ratio of the in vitro concentration in the hepatic cell lysate and in the supernatant. The author explained that this difference may result from the difference between protein binding in vivo (99.9\%) and in vitro (nearly zero), because the protein content in the blood and in the media for in vitro cultivation is different (Mielke et al. 2017). Thus, in chapter 4 and 5, before extrapolation of the in vitro concentration-response curve to the in vivo situation, a correction for the difference in the protein binding of lasiocarpine and riddelliine in the rat and human blood as compared to the in vitro medium was made. To this end protein binding in rat and human plasma was determined using rapid equilibrium dialysis. Protein binding in the culture medium was considered negligible because the in vitro toxicity was determined in serum free medium. The unbound fraction values for lasiocarpine in human and rat plasma were determined to be 0.43 and 0.64 , respectively. The relatively lower unbound fraction value observed in human plasma could be explained by the fact that the number of binding sites is about 3 times higher for human serum albumin than for rat serum albumin (Lin 1995).

Another important issue to be discussed is the selection of the dose metric to be used to relate the exposure scenario of a compound to its toxic effect. Dose metrics that are often used to correlate the in vitro and in vivo situation are the maximum concentration reached over the exposure period $\left(\mathrm{C}_{\max }\right)$ and the area under the concentration-time curve (AUC). The choice of the dose metric depends on the mode of action of the compound and the toxic endpoint of interest. When predicting toxicity for which a threshold exists and thus a concentration below 
which there will be no effect, $\mathrm{C}_{\max }$ may be the selected dose metric. AUC could be used when testing reactive chemicals causing cumulative damage, such as genotoxicity or carcinogenicity (Groothuis et al. 2015). In chapter 4 and 5, we quantified cytotoxicity using mitochondrial reduction of 3-(4,5-dimethylthiazol-2-yl)-2,5-diphenyltetrazolium bromide (MTT) as an endpoint and used $\mathrm{C}_{\max }$ as the dose metric to perform the reverse dosimetry to assess the acute liver toxicity of PAs. Despite the fact that cell viability assays such as the MTT assay typically reflect a late-stage indicator of toxicity, the predicted PoDs in chapter 4 based on $\mathrm{C}_{\max }$ as the dose metric appeared to fall well within the range of PoDs obtained from the available oral single dose in vivo studies. When using AUC as the dose metric, the predicted PoDs were out of the range of in vivo observed PoDs (Figure 4), indicating that extrapolation based on $\mathrm{C}_{\max }$ is more appropriate to predict acute liver toxicity, which may be supported by the fact that acute toxicity is generally a result of high exposure resulting from peak concentrations (Blaauboer et al. 2006). Abdullah and co-workers (2016) predicted acute kidney toxicity of aristolochic acids I using PBK modelling-based reverse dosimetry. They performed the in vitro cytotoxicity test of aristolochic acids I in the LLC-PK1 porcine kidney cells and MDCK canine cells and subsequently translated these in vitro data to an in vivo dose-response curve by using $\mathrm{C}_{\max }$. Abdullah and co-workers also reported that when the prediction was based on the AUC approach, the differences between in vivo observed PoDs and predicted PoDs were more than one order of magnitude (Abdullah et al. 2016), supporting the use of $\mathrm{C}_{\max }$ as a dose metric for acute toxicity. For use of AUC as the dose metric, a recent study reported that using the $\gamma \mathrm{H} 2 \mathrm{AX}$ assay in rat hepatocytes for prediction of the in vivo genotoxicity of lasiocarpine and riddelliine, use of the AUC as the dose metric to perform QIVIVE was able to predict the DNA adduct formation in rats (Chen et al. 2019).

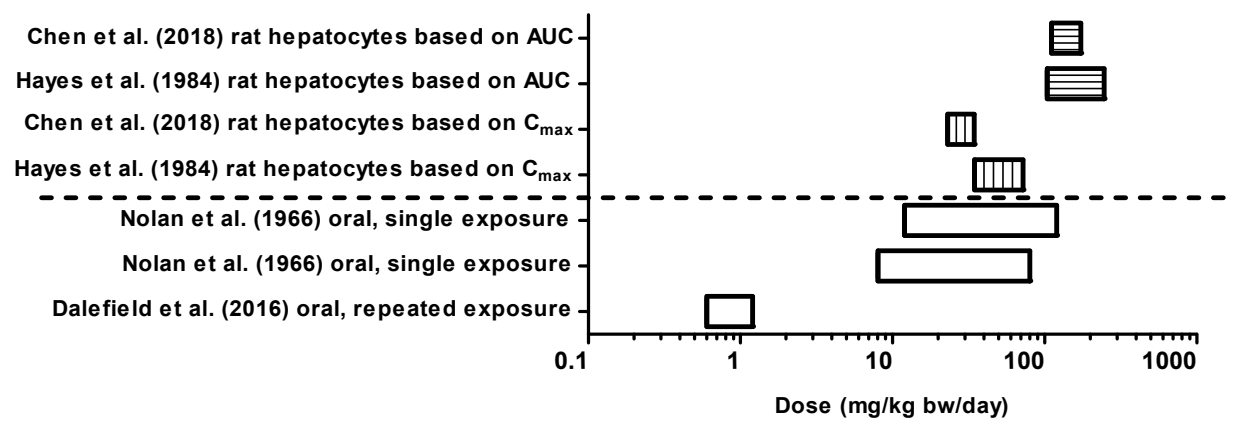

Fig. $4 \mathrm{BMDL}_{5}-\mathrm{BMDU}_{5}$ for acute liver toxicity of lasiocarpine in rat predicted by the in vitro PBK based-reverse dosimetry approach (above the dash line) based on $\mathrm{C}_{\max }$ (bars containing vertical lines) and AUC (bars containing horizontal lines) and observed in vivo PoDs for acute liver toxicity (below the dashed line) (adapted from chapter 4).

One of the limitations of cytotoxicity tests is that cell viability is a late-stage indicator of cell injury. It has been reported that the cytotoxicity tests are more likely to miss the toxicity of 
compounds that require chronic exposure or exert adverse but non-lethal effects (O'Brien et al. 2006). This is also the case for PAs since using cell viability data obtained by a single exposure to lasiocarpine resulting in the predicted BMDL5-BMDU 5 value of $23-34 \mathrm{mg} / \mathrm{kg} \mathrm{bw} /$ day, could not predict liver toxicity induced by daily repeated oral exposure to rats for 28 days as shown in Figure 4 and in chapter 4 . Thus, there is a need for evaluating not only viability, but also other hepatotoxic markers that can be used to detect early stage of liver toxicity upon long-term exposure. In general, metabolic parameters are more sensitive than cytotoxicity and can be used to detect the sub-lethal effect of hepatotoxins (Gomez-Lechon et al. 2010), although it remains to be established to what extent they represent effects related to adversity or rather to restoring cellular homeostasis. It has been reported that in micropatterned coculture models of primary hepatocytes, the sensitive parameters varied in the following order: albumin secretion $>$ urea secretion $>$ ATP levels $>$ GSH levels (Khetani et al. 2013). Using long-term repeated exposure of cells with constant metabolic enzyme activities, such as HepaRG cells, to low concentrations of PAs and detecting early markers of hepatotoxicity, could be an option to predict the liver toxicity of PAs upon daily low dose exposure, which could be validated against in vivo liver toxicity being measured by more sensitive and specific biomarkers of liver injury. For example, two new DILI markers microRNA-122 and glutamate dehydrogenase have shown promising results in animal models and clinical studies in patients with liver damage (Church and Watkins 2019; Hassan and Fontana 2019). Other limitations of using cell viability as the parameter to quantify the adverse effects are related to detecting specific cell injury and mechanisms of action. For these aspects, omics technologies allowing multi-parametric analysis in the same cell preparations may have the potential to identify new hepatotoxicity markers that will increase our understanding of the mechanism of hepatotoxicity.

To perform QIVIVE subsequently, the concentration in the serum free medium was assumed to be equal to the unbound concentration in the liver blood. Accordingly, using in vitro cytotoxicity data to estimate in vivo acute toxicity makes several assumptions, including 1) cytotoxicity in vitro is a good predictor of acute toxicity in vivo, 2) unbound cytotoxic concentrations in in vitro culture medium reflect the unbound blood concentrations, 3) blood concentrations reflect the concentrations to which the target tissue cells are exposed (KoppSchneider et al. 2013). The first issue has been addressed above. In theory, the most relevant dose metric to perform QIVIVE would be the concentration at the side of action within or on cells (at specific receptors) (Groothuis et al. 2015). Mielke et al. (2017) particularly stressed that measurements of intracellular concentrations in the target tissue are a must when applying QIVIVE to reach the same effect in vivo as seen in vitro. Mielke et al. (2017) reported that based on a validated human model, the in vivo concentration of ibuprofen in the liver was predicted to be 3-fold higher than in the blood and this was supported by postmortem data obtained in a human subject who committed suicide with an overdose of ibuprofen. In this subject, the ratio between the ibuprofen concentration in the liver and in the blood was 2.7 (Kunsman and Rohrig 1993). The unbound intracellular concentration is likely the most relevant concentration for predicting the adverse effect. However, quantification of such concentrations requires delicate work since extraction of tissues or cells and correction 
for intracellular protein binding in the cells remains difficult, since extraction procedures may shift the equilibria thus affecting the fraction unbound. This may be why Mielke et al. (2019) suggested the use of the total intracellular concentration, but this does not meet the concept that only the free unbound fraction is available for cellular interaction causing toxicity. Additionally, use of intracellular concentrations may hamper the step of model validation, because measuring tissue concentrations in vivo would require invasive approaches, making the approach less suitable for alternative testing strategies and especially challenging for the development and validation of human PBK models. Thus, it can be considered that use of unbound extracellular concentrations can serve as the first tier approach for QIVIVE to predict adverse effects, being fully adequate when the model can be validated (see Figure 5 in chapter 4) and the PoDs predicted for toxicity perfectly match the experimental in vivo data, as shown in the present thesis for the liver toxicity of lasiocarpine (see Figure 4). Using other approaches such as using intracellular concentrations may only become relevant when the first tier approach does not work out, although then determining intracellular unbound rather than total concentrations, both in the in vitro as well as the in vivo model, may become a challenge.

Altogether, a number of relevant choices with respect to dose metrics for QIVIVE have been discussed in this section. It appears that selection of the in vitro assay set up used for QIVIVE requires careful consideration of multiple parameters including the use of the free or unbound concentration, the in vitro model used, the exposure duration, the endpoint of interest measured in the in vitro assay, the dose metric to be used to relate in vitro and in vivo exposure (i.e. $\mathrm{C}_{\max }$ or AUC), and use of extracellular or intracellular concentrations. Adequate argumentation of choices made on all these aspects is important when performing QIVIVE.

\subsubsection{Use of PBK modelling to study inter-ethnic human kinetic differences}

Careful analysis of the literature indicates that the studies on alkenylbenzenes (chapter 3 ) and PAs (chapter 5 and 6) described in the present thesis are the first attempts to use PBK modelling to identify inter-ethnic kinetic differences in bioactivation and detoxification and thus sensitivity towards genotoxic and carcinogenic compounds. Currently, most developed PBK models for genotoxic and carcinogenic compounds in humans use parameters gathered for the Caucasian population (Abdullah et al. 2016; Al-Malahmeh et al. 2017a; Al-Subeihi et al. 2012; Alajlouni et al. 2016b; Martati et al. 2012; Punt et al. 2009; Van Den Berg et al. 2012). Thus, risk assessment of such compounds takes ethnic human differences into account via the uncertainty factor of 10 for human variability in kinetics (factor of 3.16) and in dynamics (factor of 3.16), while evidence to support that this approach is sufficiently protective is absent.

To date, several studies have developed physiologically based pharmacokinetic (PBPK) models particularly for the Chinese population by incorporating Chinese specific physiological and enzyme data to predict clearance of certain probe drugs, such as omeprazole (Barter et al. 2013; Feng et al. 2015), bitopertin (Feng et al. 2016), phenacetin (Barter et al. 2013), bupropion (Barter et al. 2013), tolbutamide (Barter et al. 2013), desipramine (Barter et al. 2013), alprazolam (Barter et al. 2013), and midazolam (Barter et al. 
2013; Wang et al. 2016). In these studies, compared to the Caucasian models, the main differences in the inputs for the Chinese models were average liver weight and relative abundance of CYP enzymes responsible for metabolism of these drugs. The intrinsic catalytic activity per unit amount of enzyme was generally assumed to be the same in Caucasian and Chinese (Barter et al. 2013; Feng et al. 2015; Wang et al. 2016). This approach was feasible because the selected model drugs were mainly metabolized by a single enzyme. However, the model compounds studied in the present thesis, alkenylbenzenes and PAs, are metabolized by a mix of CYPs and phase II enzymes. This hampers to use the relative CYP abundance to model the metabolic activity in the different ethnic groups, because the contribution of each CYP and phase II enzyme involved in metabolism of alkenylbenzenes and PAs are generally unknown.

Thus, in chapters 3, 5 and 6, incubations using Chinese and Caucasian liver fractions were performed to obtain kinetic data for the model compounds in these two ethnic groups. In chapter 3, the quality of Chinese and Caucasian liver samples was checked and compared to the study by Yang et al. (2012). In this study, 10 CYP activities were evaluated in Chinese and Caucasian liver microsomal samples each made from 30 donors. The differences in CYP activities between Chinese and Caucasian liver microsomes evaluated in chapter 3 were in line with results reported by Yang et al. (2012). Thanks to physiological data (in Chinese) provided by the National Health and Family Planning Commission of the People's Republic of China (NHFPC 2007; NHFPC 2014), all of the physiological parameters used in the PBK models could be defined specifically for the Chinese population. In this thesis, the main differences between Chinese and Caucasian PBK models appeared to relate to differences in physiological parameters and kinetic parameters. For the physiological parameters, liver weight was considered as one of the critical scaling factors for converting in vitro kinetic data to the in vivo situation (Pelkonen and Turpeinen 2007). Barter et al. (2013) noted that Chinese have significantly smaller livers than Caucasians based on extensive data from the literature (Chan et al. 2006; Shi et al. 2009; Yuan et al. 2008). Thus, a scaling factor of 0.85 was used to quantify the liver volume of the Chinese population (Barter et al. 2013). Li et al. (2014) summarized Chinese liver volume $(\mathrm{n}=9842)$ determined at autopsy from a series of Chinese deceased individuals (in Chinese) (Wang et al. 1995; Wang 1998; Li and Huang 2006), showing that the percentage of liver volume compared to the whole body was around $2.3 \%$ for the Chinese population aged from 20-60 years for both males and females. Thus, according to the data provided by NHFPC (NHFPC 2007; NHFPC 2014) and Li et al. (2014), a relative liver volume of $2.3 \%$ was used to represent the average Chinese population in the present thesis, which, taking also the difference in body weight into account, amounts to 0.76 times the relative liver volume of the Caucasian population. Thus, it can be assumed that our physiological parameters, especially the critical physiological parameter, liver volume, were adequately representing the average Chinese population. In chapter 5 , the unbound fractions of lasiocarpine and riddelliine in blood were corrected by rapid equilibrium dialysis (RED) using plasma from Caucasian subjects. According to the study of Zhou et al. (1990), there were no differences in serum albumin levels between Chinese and Caucasians. Thus, the unbound fraction of PAs in blood obtained with Caucasian subjects were used as default in 
the present study, which is in line with previously developed PBPK models for probe drugs for the Chinese population (Barter et al. 2013; Feng et al. 2015; Wang et al. 2016).

The major bottleneck of the developed PBK models for estragole (chapter 3), and for lasiocarpine and riddelliine (chapter 5) for the Chinese population is that the predicted kinetic data cannot be validated against in vivo data. Unlike previously developed PBPK models of probe drugs for the Chinese population (Barter et al. 2013; Feng et al. 2015; Wang et al. 2016) for which the predicted kinetic profiles could be evaluated against observed data in Chinese individuals, human in vivo kinetic profiles of the genotoxic and carcinogenic model compounds of the present studies were not available. In such cases validation can be made based on validation of the corresponding rat models. In chapter 3 and 5, the PBK models developed for estragole, lasiocarpine and riddelliine for the Chinese and Caucasian population were based on PBK models previously defined and validated in rats. Jones et al. (2006) validated this iterative simulation approach using 19 orally administrated compounds, showing that for these compounds for which rat PBK models presented good predictions, the accuracy of human PBK simulations was within 2-fold regarding the pharmacokinetic parameters such as AUC, $\mathrm{C}_{\max }$ and $\mathrm{T}_{\max }$ (Jones et al. 2009; Jones et al. 2006). In addition, in chapter 3 and chapter 5, the PBK models for the model compounds for the Chinese population were evaluated indirectly by comparing the predicted inter-ethnic differences in major metabolite formation or parent concentrations in the blood to reported ethnic differences in hepatic drug-metabolizing enzymes that mainly catalyze the formation of the major metabolites or cause the parent compound depletion. Recently, Paini et al. (2019) proposed several approaches that could be applied when there are no in vivo data to evaluate the model performance. Among those approaches, use of micro-scale systems, such as human-on-a-chip technology, which potentially could predict kinetics and whole body response to substances, could be used to validate the PBK model developed for the Chinese population in the future. Then, different cell types derived from the Chinese population would be required to develop such micro-scale systems, which is another obstacle as also shown by the studies in the present thesis. By far, most international biotechnology companies hardly provide the mammalian materials particularly made from specific ethnic groups other than the Caucasian population, which may be a reason why ethnic related-studies are relatively limited, especially the studies on ethnic differences in absorption, distribution and excretion. Furthermore, the level to which the micro-scale organ on a chip models mimics the actual in vivo kinetics also remains to be established. One of the critical challenges for the micro-scale systems is to design the correct scale of the organs on such chips to human physiological conditions. If the relative size of organs or the flow rates from model systems are not correctly scaled, it will have profound effects on the performance of body-on-a-chip systems and it will become difficult to use these models for validation of the PBK model predictions.

China is a multi-ethnic country with 56 ethnic minorities, in which the majority of the Chinese population is Han comprising $91.5 \%$ of the total Chinese population. The liver fraction of Chinese samples were a pool of 40 donors of Chinese Han. Despite the fact that Han Chinese have anatomical and physiological characteristics similar to those of other minorities, the differences in genetic polymorphisms of phase I and phase II enzymes 
between the majority and minority ethnic groups may contribute to the intra-national differences in metabolism of alkenylbenzenes and PAs within the Chinese population. Shu et al. (2001) reported the variations in hepatic abundance and activities of CYP 1A2, 2C9 and 3A4 in liver microsomes of Chinese subjects including 17 Han Chinese, 17 Zhuang Chinese and 8 Miao Chinese. The results showed no remarkable differences in the hepatic abundance of CYP 2C9 and CYP 3A4 among these Chinese ethnic groups, except for the fact that the activity of CYP 1A2 in Han Chinese was about 1.5-fold lower than that in the Zhuang and the Miao samples. The authors speculated this difference to be possibly due to the great variations of CYP 1A2 activity among individual samples. Besides differences quantified in in vitro studies, also in vivo pharmacokinetic differences between Han Chinese and non-Han Chinese have been reported such as for compounds like midazolam, losartan and tinidazole (Chang et al. 2009; Guo et al. 2011; Yang et al. 2012). The present thesis provides an initial insight of how PBK modelling can be used to identify differences between ethnic groups for bioactivation and detoxification of compounds that are both genotoxic and carcinogenic, and it would be of interest to perform similar studies using liver fractions from more Chinese ethnic groups to obtain more detailed insights for the Chinese population as a whole.

Besides the issue of composition of the liver fractions, another uncertainty lies in the number of liver fractions obtained from Chinese donors. By the end of 2017, the Chinese population reached 1390.08 million reported by the National Bureau of Statistics of China (NBS) (NBS 2018). In the present thesis, pooled liver microsomes and S9 made from 40 donors were applied to represent the average Chinese population when performing in vitro kinetic studies on estragole (in chapter 3) and on PAs (in chapter 5). In chapter 6, 25 Chinese individual microsomes were used to study the inter-individual kinetic differences for lasiocarpine (PA) within the Chinese population. The International Programme on Chemical Safety (IPCS) provided a guide to judge whether the number of subjects within the population is sufficient to define population variability allowing a reliable estimate of the central tendency (IPCS 2005). According to the IPCS, the standard error (standard deviation [SD] of the sample divided by the square root of the sample size) should be less than approximately $20 \%$ of the mean. Based on data obtained in chapter 6 , the standard error of predicted 7-GS-DHP formation in 25 Chinese individuals (i.e., standard deviation divided by the square root of the sample number: $\left.0.00062 / 25^{0.5}\right)$ is $16.0 \%$ of the mean $(0.00077)$, and therefore the sample number of 25 in chapter 6 is considered adequate. Thus, in chapter 5 , the use of Chinese liver microsomes made from a pool of 40 donors to represent the average Chinese population to study the PA metabolism can also be considered adequate. For estragole, a recent study reported that using a human PBK model for estragole, the mean of predicted formation of 1'sulfooxyestragole in 19 donors comprising 15 Caucasians, 2 Hispanic, 1 Asian and 1 AfricanAmerican was $0.06 \mathrm{nmol} / \mathrm{g}$ liver (Punt et al. 2016). With the standard deviation of 0.05 , the standard error is $19.1 \%$ of the mean, which is less than the threshold value of $20 \%$ provided by the IPCS, indicating that the number of Chinese subjects used to produce the pooled sample, amounting to 40 donors, would probably also be sufficient to study the in vitro kinetics of estragole in chapter 3. 
In chapter 3, there was 4.5-fold variation in formation of the ultimate carcinogenic metabolite of estragole accompanied by similar rates of detoxification for the Caucasian and Chinese population indicating a lower risk of estragole for the Chinese population at similar levels of exposure. In chapter 5, it was reported that the inter-ethnic human differences varied 2.0-fold for lasiocarpine and 5.0-fold for riddelliine with the Chinese being less sensitive to PA induced bioactivation and liver toxicity. Chapter 6 further supported the conclusion reported in chapter 5 that the geometric mean of predicted 7-GS-DHP formation, reflecting bioactivation of lasiocarpine, was 2.2-fold lower in the Chinese population compared to the Caucasian population. Based on the results of chapter 3 , chapter 5 and chapter 6 , compared to the Caucasian population, the Chinese population was generally less sensitive towards the natural toxins studied in the present thesis. This is because both alkenylbenzenes and PAs require biotransformation to exert their adverse effects, with the balance between bioactivation and detoxification determining the ultimate toxicity of the compounds. For both groups of natural toxins tested in the present thesis, reactive metabolite formation was predicted to be relatively lower in the Chinese population combined with comparable to relatively small differences in detoxification in both ethnic groups, resulting in the conclusion that there is a lower risk of alkenylbenzenes and PAs for the Chinese population compared to the Caucasian population at similar levels of exposure.

Both intrinsic and extrinsic factors can lead to these ethnic differences in kinetics. The intrinsic factors are those related to physiological properties and genetic variability, while the extrinsic factors are related to factors including diet, smoking habits, alcohol use, drug exposure, etc (ICH 1998). An important intrinsic factor relates to genetic polymorphisms in the expression and/or function of enzymes. Genetic polymorphisms in most of the phase I and phase II enzymes have been identified for both East Asians and Caucasians (Kim et al. 2004; $\mathrm{Li}$ and Bluth 2011; McGraw and Waller 2012). For the model compounds studied in the present thesis, several genetic polymorphisms in SULTs, important for bioactivation of alkenylbenzenes, and in CYP 3A4, essential for bioactivation of PAs, have been reported. The human SULTs involved in the sulfation reaction of 1'-hydroxyestragole have not been identified to date, but sulfation of the 1'-hydroxy metabolites of the related alkenylbenzene methyleugenol in the liver has been reported to be catalyzed mainly by SULT 1A1 and to a small extent by SULT 1E1 (Herrmann et al. 2012). The SULT 1A1 polymorphism has been reported to be associated with increasing risk of various cancer types (Xiao et al. 2014). The occurrence of SULT 1A1*2 (Arg213His) in the Asian population, amounting to $9.58 \%$, is less than in the Caucasian population with an occurrence of $35.2 \%$ ( $\mathrm{Li}$ and Bluth 2011; Xiao et al. 2014). Thus, it can be hypothesised that the SNP difference in SULT 1A1 between Asians and Caucasian may partly explain the predicted differences in 1'-sulfooxyestragole formation between the two ethnic groups. For CYP 3A4, 54 allelic variants have been identified (https://www.pharmvar.org/), but only a few of them are known to influence the CYP 3A4 expression or function. For example, only CYP $3 \mathrm{~A} 4 * 22$ showed a reduced activity in vivo (Wang et al. 2011). Other SNPs like CYP 3A4*2, CYP 3A4*8, CYP 3A4*11, CYP 3A4*13, CYP 3A4*17, CYP 3A4*18A and CYP 3A4*26 have been shown to alter CYP 3A4 function in vitro (Dai et al. 2001; Eiselt et al. 2001; Lee et al. 2005; Sata et al. 2000; Werk et 
al. 2014; Westlind-Johnsson et al. 2006). The genetic polymorphisms in CYP 3A4 appeared to be more prevalent in Caucasians than in Asians ( $\mathrm{Li}$ and Bluth 2011). This seems to be in line with our results in chapter 6 where the coefficient of variation for the predicted bioactivation, reflected by 7-GS-DHP formation, was higher in the 25 Caucasian individuals than in the 25 Chinese individuals. As discussed in chapter 6 , the occurrence of CYP $3 \mathrm{~A}^{*} 20$ in the Chinese population was higher $(22 \%)$ than in the Caucasian population $(6 \%)$ (McGraw and Waller 2012). This allele has been shown to be devoid of any catalytic activity of CYP 3A4 in vitro (Westlind-Johnsson et al. 2006). The difference in the occurrence of CYP $3 \mathrm{~A} 4 * 20$ between the Chinese and the Caucasian population may potentially explain a part of the predicted differences in sensitivity towards PA toxicity of the two ethnic groups. Besides intrinsic factors, an extrinsic factor resulting in ethnic differences important in China is the culture of using botanical preparations (Bajaj et al. 2019). Several preparations used in traditional Chinese medicine (TCM) have been reported to alter enzyme activities in preclinical models and/or in clinical drug-drug interaction studies (Qiu et al. 2016; Tang et al. 2016).

Altogether, for building ethnic-specific PBK models for compounds, metabolized by a mix of enzymes, it is necessary to study the inter-ethnic kinetic differences on a case-by-case basis. Besides the ethnic-specific physiological and metabolic parameters which have been included in the present thesis, other ethnic-specific related parameters like the PBK model parameters for absorption, distribution and excretion may need to be further refined when it would be essential to further improve the accuracy of the predictions made.

\subsubsection{Implication for risk assessment}

For refinement of the risk assessment of compounds that are both genotoxic and carcinogenic, the present thesis studied inter-species, inter-ethnic human and inter-individual human differences for alkenylbenzenes and PAs. This enabled definition of CSAFs for risk assessment. In the present thesis, the uncertainty factor of 10 for inter-species differences in kinetics (uncertainty factor of 4) and dynamics (uncertainty factor of 2.5) have been investigated in chapter 5 using PBK modelling-based reverse dosimetry to predict in vivo dose-response curves for acute liver toxicity of lasiocarpine and riddelliine. The inter-species differences in acute liver toxicity of lasiocarpine and riddelliine in rat and human appeared to fall well within the default factor of 10, indicating that the factor of 10 is sufficiently protective for inter-species differences for these PAs. Similar results were reported for other natural botanical toxins by Abdullah et al. (2016) and Punt et al. (2009), showing that the inter-species differences in kinetics of aristolochic acid I and estragole were smaller than the default factor of 4 for kinetic differences. The results reported in chapter 5 together with previous results (Abdullah et al. 2016; Punt et al. 2009) indicate that for botanical ingredients with genotoxic and carcinogenic characteristics that are of possible concern such as alkenylbenzenes, aristolochic acid and PAs, inter-species differences are likely covered by the default uncertainty factor of 10 , although it remains necessary to evaluate more compounds to determine if the default factor of 10 is sufficiently protective in all cases. This factor of 10 is part of the MOE of 10000 used in risk assessment of compounds that are both genotoxic and 
carcinogenic with a MOE > 10000 being of low priority for subsequent risk management actions (EFSA 2005). The threshold of 10000 is based on a factor 10 for inter-species differences (consisting of a default factor of 4.0 for toxicokinetics and of 2.5 for toxicodynamics) and a factor 10 for intra-species (consisting of a default factor of 3.16 for toxicokinetics and of 3.16 for toxicodynamics); (2) a factor 10 for inter-individual differences in cell control and DNA repair; and (3) a factor 10 for the fact that the BMDL 10 or the reference point is not identical to a no observed adverse effect level (NOAEL) (EFSA 2005).

For inter-ethnic human kinetic differences studied in chapter 3 for estragole and in chapter 5 for lasiocarpine and riddelliine, the inter-ethnic human variabilities in kinetics were about 2to 5-fold, which is generally in line with the default value of 3.16 for inter-individual kinetic differences. The Chinese population was generally less sensitive towards the natural toxins tested in the present thesis, indicating a relatively lower risk of alkenylbenzenes and PAs for the Chinese population at similar levels of exposure. In other words, because of differences in kinetics, intake levels in the Chinese population could be 2- to 5-fold higher before reaching the same risk as expected for the Caucasian population, assuming a similar relationship in both populations between the formation of the reactive metabolite and the adverse effect, i.e. in toxicodynamics. For alkenylbenzenes, chapter 2 reported the combined estimated daily intake (EDI) values assuming equal potency for the different alkenylbenzenes for consumption of PFS (10 samples), TCMs (23 samples) and one cup of herbal tea (38 samples) for the Chinese population. Comparison of these values to the EDI values of alkenylbenzenes resulting from consumption of PFS and herbal teas previously determined for the Caucasian population (Al-Malahmeh et al. 2017b; Alajlouni et al. 2016a; Alajlouni et al. 2017), reveals that the combined median EDI values of Chinese consumption of PFS and herbal teas determined in chapter 2 were 3-60 times lower than those reported in previous studies for Caucasians. The relatively lower EDI values obtained for consumption of PFS and herbal teas in chapter 2 probably result from the fact that the PFS and herbal tea samples consist of a mixture of botanicals, in which the percentage of the herbal ingredient containing alkenylbenzenes might be relatively low, resulting in lower concentrations of alkenylbenzenes in those samples compared to the samples analysed in previous studies for Caucasians (AlMalahmeh et al. 2017b; Alajlouni et al. 2016a; Alajlouni et al. 2017). Thus, although the number of analysed samples to define the EDI for the Chinese populations remains limited, the results of the present thesis suggest that for consumption of PFS and herbal teas containing alkenylbenzenes, compared to the Caucasian population, there is a relatively lower risk for the Chinese population not only because of the relatively lower level of bioactivation but also because of possibly lower levels of intake of alkenylbenzenes from these products. However, it is important to note that half of the herbal teas and PFS samples collected from the Chinese market resulted in EDI that gave rise to MOE values lower than 10000 , indicating a priority for risk management. For TCM, the comparison between the two ethnic groups could not be made because the EDI values for alkenylbenzenes resulting from use of TCM are not available for the Caucasian population. According to the literature, TCM are traditionally used as drugs to prevent and treat diseases and make up 30\%-50\% of the total medicinal consumption in China (Liu and Salmon 2010). Cases of liver injury associated with 
use of TCM have increasingly been reported in the last few years (Teschke 2014). In chapter 2 , it is reported that one TCM sample would result in levels of intake that gave rise to a MOE value lower than 10 upon daily life-long consumption, indicating that daily intake of the alkenylbenzene of interest, myristicin, would be within the range of dose levels causing liver tumors in experimental animals. Thus, it can be speculated that the risk resulting from consumption of TCM containing alkenylbenzenes may be higher for the Chinese that the Caucasian population based on the fact that within Chinese culture TCM has a long history of use providing drugs to prevent and treat diseases.

For PAs, levels present in and resulting EDI values for herbal teas and PFS for the Chinese population are not available, whereas the European Food Safety Authority (EFSA) reported that consumption of herbal teas and PFS for the Caucasian population may lead to exposure levels amounting to $890 \mathrm{ng} / \mathrm{kg}$ bw/day and $1800 \mu \mathrm{g} / \mathrm{kg}$ bw/day, respectively (EFSA 2017). This shows that the EDI of PFS has reached PA levels in the range of the lowest known dose associated with human acute adverse effects induced by PAs of approximately $2 \mathrm{mg} P A / \mathrm{kg}$ bw/day (EFSA 2017). Different authorities and Member States in the EU have set specific limits for PAs, while such limitations for PA content in food is not in place in China. The study of Griffin et al. (2014) analyzed 54 TCM samples and found that the estimated daily intake resulting from use of $40 \%$ of the positive samples (42 out of 54) would exceed the maximum daily intake for PAs of $0.007 \mu \mathrm{g} / \mathrm{kg}$ bw/day as set by the COT (2008), BfR (2011) and EFSA (2011). Ruan et al. (2015) reported that 15 hepatic sinusoidal obstruction syndrome (HSOS) patients were associated with consuming a PA-containing herb identified as Gynura segetum. In China, this herb is called Tusanqi and is a constituent of TCM and commonly used to treat traumatic injury and it is also one of the plant sources used as a folk medicinal herb by self-medication (Lin et al. 2011; Ruan et al. 2015). Thus, it is concluded that there is a high risk of PA exposure by herbal medicines for the Chinese population compared to the Caucasian population based on the fact that the limitation of PA content in foods is currently not in place in China and PA-containing herbs are commonly used in TCM and folk medicines for self-treatment. Overall, although the Chinese population was predicted to be less sensitive towards bioactivation and liver toxicity induced by alkenylbenzenes and PAs, this result should be integrated with expected levels of exposure resulting from consumption of botanical preparations, especially as a result of use of herbal medicines by the Chinese population as compared to the Caucasian population.

In chapter 6, the inter-individual kinetic differences underlying possible differences in liver toxicity of PAs were investigated using Monte Carlo simulation combined with PBK modelling, showing that for protecting of the $90^{\text {th }}$ percentile of the two populations combined (Chinese and Caucasians), the CSAF value for kinetic differences needs to be increased to 5.7, and for protecting the $99^{\text {th }}$ percentile of the human population, the CSAF needs to be further increased to 19.5. These results reveal that the kinetic differences among human individuals in bioactivation of lasiocarpine are higher than the default value of 3.16 for interindividual kinetic differences, and also higher than the 2-fold kinetic differences observed between the two average ethnic groups studied in chapter 5 when studying pooled liver samples of both ethnic groups. A similar result was previously reported for the inter- 
individual differences in kinetics of estragole (Punt et al. 2016), resulting in the conclusion that for protection of the $9^{\text {th }}$ percentile of the human Caucasian population, a larger CSAF for human variability in kinetics of 5.4 would be required (Punt et al. 2016). The CSAF values for estragole defined when taking inter-individual differences in kinetics into account are higher than the kinetic differences in bioactivation of this compound between the average Chinese and the average Caucasian population reported to amount to 4.5 -fold in chapter 3 . Overall, it can be concluded that the uncertainty factor of intra-species variability in kinetics for alkenylbenzenes and PAs could be refined by studying the effect of inter-individual variations. The CSAFs defined for inter-individual variations within an ethnic group are larger than those reflecting average inter-ethnic differences. This implies that the CSAF for inter-individual variation will also adequately cover the inter-ethnic differences.

Based on the results of inter-species, inter-ethnic and inter-individual variations observed in the present thesis for bioactivation and detoxification of alkenylbenzenes and PAs, the uncertainty factors to be used in the risk assessment of these two groups of compounds can be redefined. This would affect the MOE value of 10000 to be used by risk managers to evaluate the priority in risk management. For protecting the $99^{\text {th }}$ percentile of the human population as a whole when considering exposure to estragole, the CSAF of 5.4 defined for inter-individual kinetic differences in reactive metabolite formation (Punt et al. 2016), together with the default uncertainty factor of 3.16 for inter-individual dynamic differences, would give rise to a CSAF for intra-species differences of 17.1, which is 1.7 -fold higher than the default value of 10 for variation in kinetics (3.16) and dynamics (3.16) (IPCS 2005). Thus, it can be concluded that a MOE threshold value of 10000 would not be sufficiently protective for the human population. For PAs, the in vitro PBK modelling-based reverse dosimetry approach was used to predict the in vivo dose-response curves of acute liver toxicity of PAs. For the genotoxic and carcinogenic compounds that also have non-genotoxic endpoints, EFSA advised that a MOS (margin of safety) higher than 100 could be considered to be protective raising no concern for human health with respect to the non-genotoxic endpoints. The threshold of 100 is subdivided into a factor of 10 for inter-species and a factor of 10 for intra-species differences (EFSA 2012; EFSA 2015). Based on the inter-species differences upon acute liver toxicity of lasiocarpine amounting to 2-fold taking kinetics and dynamics into account, together with a CSAF of 19.5 defined for the inter-individual kinetic differences and the default factor of 3.16 for dynamics, the overall CSAF would be 123 , which is 1.2-fold higher than the default threshold value of 100 for the non-genotoxic endpoints. Besides the uncertainty factor defined for acute liver toxicity, the CSAF of 19.5 for inter-individual differences in reactive metabolite formation of lasiocarpine reported in chapter 6 combined with the default uncertainty factor for inter-individual differences in dynamics and the CSAF of 2 for inter-species differences, would also support an increase of the MOE default threshold of 10000 to $12324(19.5 * 3.16 * 2 * 10 * 10)$ for liver tumor formation induced by lasiocarpine.

Overall, based on the CSAFs defined for inter-species, inter-ethnic and inter-individual differences for the Chinese and the Caucasian population combined for alkenylbenzenes and PAs, the inter-species difference in kinetics and dynamics fall well within the default factor of 
10. The inter-individual human differences in kinetics within the Chinese population, the Caucasian population and the two populations combined were all shown to be higher than the average inter-ethnic kinetic differences and the CSAFs for inter-individual kinetic variations for both groups of compounds were higher than the default uncertainty factor of 3.16. These CSAFs would support an increase of the threshold value for the MOE of 10000 for evaluation of the carcinogenicity and the threshold value of 100 for evaluation of nongenotoxic endpoints of alkenylbenzenes and PAs.

\subsubsection{Future perspectives}

The present thesis revealed the proof-of-principle that the in vitro PBK modelling-based reverse dosimetry is able to predict in vivo acute liver toxicity of PAs in a quantitative way. To increase the impact and applicability of this approach, one may consider to provide proofsof-principle for other endpoints. For example, it would be interesting to study the sub-chronic adverse effect of PAs, resulting from long-term repeated exposure to low concentrations using cells with constant metabolic enzyme activities, such as HepaRG cells and detecting early markers of hepatotoxicity, in order to predict the liver toxicity of PAs upon daily low dose exposure. Besides sub-chronic liver toxicity, toxicity studies of other organs such as lung and kidney could also be studied. Subsequently, a complete toxicological risk assessment for PAs could be performed to see if the target organ is correctly predicted by the in vitro PBK modelling-based reverse dosimetry approach.

For further refinement of risk assessment, the present thesis studied the inter-individual variations in kinetics, whereas the effect of inter-individual dynamic differences remains to be elucidated in future studies. This could be done by extending our current PBK models to physiologically based dynamic (PBD) models, in which for example DNA adduct formation could be integrated. Xia et al. (2013) reported that 12 tumorigenic PAs and PA N-oxides undergo an identical bioactivation mechanism in rats by the formation of four major DNA adducts, with two predominant adducts, i.e. DHP-dG-3 and DHP-dG-4 and two minor adducts, i.e. DHP-dA-3 and DHP-dA-4. Additionally, previous studies reported a similar profile of DNA adducts formed in incubations with human liver microsomes and calf thymus DNA to those formed in similar incubations with rat liver microsomes and calf thymus DNA, suggesting that the mode of action of PAs studied in these rodents is highly relevant for humans (Xia et al. 2003; Zhao et al. 2012). Combining the current PBK model with an equation describing the concentration dependent DNA adduct formation of PAs in different human individual primary hepatocytes could provide a way forward to predict the in vivo dose-response curves for DNA adduct formation in the liver of humans.

A final point that can be considered in the future when using in vitro PBK modelling-based reverse dosimetry for risk assessment is the uncertainty factor to be used when a PoD for risk assessment is defined by a QIVIVE approach. This requires insight in the uncertainties inherent to the approach and the model applied. In the present thesis, a sensitivity analysis was performed to evaluate the impact of model parameters on the model output so that the effect of small changes in parameter values on the model outcome can be studied. When performing QIVIVE, the unbound extracellular concentration in vitro is considered to be a 
surrogate for the unbound concentration in the liver blood, which is considered the relevant concentration at the side of action. As discussed above, there are inherent uncertainties related to the choices made for this QIVIVE translation, although currently there is no means to evaluate or quantify the impact of the uncertainties of this extrapolation. Thus, an additional safety factor may need to be introduced. For the compounds studied in the present thesis, differences in predicted toxicity values of lasiocarpine and those values derived from in vivo toxicity studies were within 3.5-fold (chapter 4), indicating that an extra uncertainty factor of 3.5 can be used when a PoD is derived with the in vitro PBK approach to predict acute liver toxicity of PAs. Earlier studies have shown that the PoDs for developmental toxicity predicted with the in vitro PBK approach were within 0.2 to 10 -fold difference compared to those values derived from in vivo data (Louisse et al. 2015; Louisse et al. 2010; Strikwold et al. 2017; Strikwold et al. 2013). Thus, an uncertainty factor of 10 may be used when a PoD is derived with the in vitro PBK approach to predict developmental toxicity. To determine such an appropriate uncertainty factor when using in vitro PBK approach to perform QIVIVE, more examples and proofs-of principles for other chemicals and other endpoints are needed. When the in vitro PBK approach would be based on an adequate human in vitro model and a human PBK model describing the kinetics and dynamics directly for humans, it might be advocated to replace the default uncertainty factor 10 for inter-species differences by the uncertainty factor required when a PoD for risk assessment is defined by a QIVIVE approach.

\subsection{Conclusion}

In conclusion, the present thesis showed an alternative approach to define in vivo doseresponse curves that can define PoDs for risk assessment. The present thesis demonstrated that combining in vitro toxicity data with the kinetic compound characteristics integrated into a PBK model adequately predicted in vivo acute liver toxicity of PAs. The obtained results show the possibility to use the method to provide a PoD for risk assessment and also to define uncertainty factors for inter-species and intra-species differences. Additionally, this thesis provided the possibilities to define ethnic-specific PBK models that can be used to further refine risk assessment of both compounds that are genotoxic and carcinogenic, and compounds with non-genotoxic endpoints. Moreover, this thesis illustrated that there is a potential priority for risk management for several botanical preparations containing alkenylbenzenes or PAs for the Chinese population when considering daily life-long exposure. Altogether, this thesis provides a proof-of-principle to perform risk and safety assessment only based on in vitro and in silico data. 


\section{References}

Abdullah R, Alhusainy W, Woutersen J, Rietjens IMCM, Punt A (2016) Predicting points of departure for risk assessment based on in vitro cytotoxicity data and physiologically based kinetic (PBK) modeling: The case of kidney toxicity induced by aristolochic acid I. Food Chem Toxicol 92:104-116

Al-Malahmeh AJ, Al-Ajlouni A, Wesseling S, Soffers AEMF, Al-Subeihi A, Kiwamoto R, Vervoort J, Rietjens IMCM (2017) Physiologically based kinetic modeling of the bioactivation of myristicin. Arch Toxicol 91:713-734

Al-Subeihi AA, Spenkelink B, Punt A, Boersma MG, Van Bladeren PJ, Rietjens IMCM (2012) Physiologically based kinetic modeling of bioactivation and detoxification of the alkenylbenzene methyleugenol in human as compared with rat. Toxicol Appl Pharmacol 260:271-284

Alajlouni AM, Al-Malahmeh AJ, Isnaeni FN, Wesseling S, Vervoort J, Rietjens IMCM (2016a) Level of alkenylbenzenes in parsley and dill based teas and associated risk assessment using the Margin of Exposure approach. J Agr Food Chem 64:8640-8646

Alajlouni AM, Al-Malahmeh AJ, Wesseling S, Kalli M, Vervoort J, Rietjens IMCM (2017a) Risk assessment of combined exposure to alkenylbenzenes through consumption of plant food supplements containing parsley and dill. Food Addit Contam Part A:2201-2211

Al-Malahmeh AJ, Alajlouni AM, Ning J, Wesseling S, Vervoort J, Rietjens IMCM (2017b) Determination and risk assessment of naturally occurring genotoxic and carcinogenic alkenylbenzenes in nutmeg-based plant food supplements. J Appl Toxicol 37:1254-1264

Alajlouni AM, Al Malahmeh AJ, Kiwamoto R, Wesseling S, Soffers AEMF, Al-Subeihi AAA, Vervoort J, Rietjens IMCM (2016b) Mode of action based risk assessment of the botanical food-borne alkenylbenzene apiol from parsley using physiologically based kinetic (PBK) modelling and read-across from safrole. Food Chem Toxicol:138-150

Aninat C, Piton A, Glaise D, Le Charpentier T, Langouet S, Morel F, Guguen-Guillouzo C, Guillouzo A (2006) Expression of cytochromes P450, conjugating enzymes and nuclear receptors in human hepatoma HepaRG cells. Drug Metab Dispos 34:75-83

Bajaj G, Gupta M, Wang HH, Barrett JS, Tan M, Rupalla K, Bertz R, Sheng J (2019) Challenges and Opportunities With Oncology Drug Development in China. Clin Pharmacol Ther 105:363-375

Barter ZE, Tucker GT, Rowland-Yeo K (2013) Differences in Cytochrome P450-Mediated Pharmacokinetics Between Chinese and Caucasian Populations Predicted by Mechanistic Physiologically Based Pharmacokinetic Modelling. Clin Pharmacokinet 52:1085-1100

Bell CC, Hendriks DFG, Moro SML, Ellis E, Walsh J, Renblom A, Fredriksson Puigvert L, Dankers ACA, Jacobs F, Snoeys J, Sison-Young RL, Jenkins RE, Nordling A, Mkrtchian S, Park BK, Kitteringham NR, Goldring CEP, Lauschke VM, Ingelman-Sundberg M (2016) Characterization of primary human hepatocyte spheroids as a model system for drug-induced liver injury, liver function and disease. Sci Rep 6:25187

Blaauboer B, Hermens J, van Eijkeren J (2006) Estimating acute toxicity based on in vitro cytotoxicity: role of biokinetic modelling. ALTEX 23(suppl): 250-253 
Bundesinstitut für Risikobewertung (BfR) (2011) Analytik und Toxizität von Pyrrolizidinalkaloiden sowie eine Einschätzung desgesundheitlichen Risikos durch deren Vorkommen in Honig.

Chan SC, Liu CL, Lo CM, Lam BK, Lee EW, Wong Y, Fan ST (2006) Estimating liver weight of adults by body weight and gender. World J Gastroenterol 12:2217-2222

Chang XY, Guo T, Xia DY (2009) Pharmacokinetics of Tinidazole in Chinese subjects: comparison of Mongolian, Korean, Hui, Uighur and Han nationalities. J Pharm Pharm Sci 12:175-180

Chen L, Peijnenburg Ad, de Haan L, Rietjens IMCM (2019) Prediction of in vivo genotoxicity of lasiocarpine and riddelliine in rat liver using a combined in vitro-physiologically based kinetic modelling-facilitated reverse dosimetry approach (in press)

China Food and Drug Administration (CFDA) (2013a) List of dietary herbs (in Chinese)

China Food and Drug Administration (CFDA) (2013b) List of herbs allowed for health food products (in Chinese)

Church RJ, Watkins PB (2019) Serum biomarkers of drug-induced liver injury: Current status and future directions. J Dig Dis 20:2-10

COT (2008) COT Statement on Pyrrolizidine Alkaloids in Food.

Crean D, Bellwon P, Aschauer L, Limonciel A, Moenks K, Hewitt P, Schmidt T, Herrgen K, Dekant W, Lukas A, Bois F, Wilmes A, Jennings P, Leonard MO (2015) Development of an in vitro renal epithelial disease state model for xenobiotic toxicity testing. Toxicol In Vitro (1 Pt A):128-137

Dai D, Tang J, Rose R, Hodgson E, Bienstock RJ, Mohrenweiser HW, Goldstein JA (2001) Identification of variants of CYP3A4 and characterization of their abilities to metabolize testosterone and chlorpyrifos. J Pharmacol Exp Ther 299:825-831

Dalefield RR, Gosse MA, Mueller U (2016) A 28-day oral toxicity study of echimidine and lasiocarpine in Wistar rats. Regul Toxicol Pharmacol 81:146-154

DelRaso NJ, Foy BD, Gearhart JM, Frazier JM (2003) Cadmium uptake kinetics in rat hepatocytes: correction for albumin binding. Toxicol Sci 72:19-30

den Braver-Sewradj SP, den Braver MW, Vermeulen NP, Commandeur JN, Richert L, Vos JC (2016) Inter-donor variability of phase I/phase II metabolism of three reference drugs in cryopreserved primary human hepatocytes in suspension and monolayer. Toxicol In Vitro 33:71-79

Donato MT, Lahoz A, Montero S, Bonora A, Pareja E, Mir J, Castell JV, Gomez-Lechon MJ (2008) Functional assessment of the quality of human hepatocyte preparations for cell transplantation. Cell Transplant 17:1211-1219

European Commission (EC) (2008) No. 1334/2008 of the European Parliament and of the Council of 16 December 2008 on flavourings and certain food ingredients with flavouring properties for use in and on foods and amending Council Regulation (EEC) No. 1601/91, Regulations (EC) No. 2232/96 and (EC) No. 110/2008 and Directive 2000/13/EC. Official Journal of the European Communities 354:34-50 
European Food Safety Authority (EFSA) (2005) Opinion of the scientific committee on a request from EFSA related to a harmonised approach for risk assessment of substances which are both genotoxic and carcinogenic. EFSA J 282:1-31

European Food Safety Authority (EFSA) (2011) Scientific opinion on pyrrolizidine alkaloids in food and feed. EFSA J 9:2406

European Food Safety Authority (EFSA) (2012) Guidance on selected default values to be used by the EFSA Scientific Committee, Scientific Panels and Units in the absence of actual measured data. EFSA J $10: 2579$

European Food Safety Authority (EFSA) (2015) Scientific Opinion on acrylamide in food. EFSA J $13: 4104$

European Food Safety Authority (EFSA) (2017) Risks for human health related to the presence of pyrrolizidine alkaloids in honey, tea, herbal infusions and food supplements. EFSA J 15:4908

Eiselt R, Domanski TL, Zibat A, Mueller R, Presecan-Siedel E, Hustert E, Zanger UM, Brockmoller J, Klenk HP, Meyer UA, Khan KK, He YA, Halpert JR, Wojnowski L (2001) Identification and functional characterization of eight CYP3A4 protein variants. Pharmacogenetics 11:447-458

Fashe MM, Juvonen RO, Petsalo A, Rasanen J, Pasanen M (2015) Species-Specific Differences in the in Vitro Metabolism of Lasiocarpine. Chem Res Toxicol 28:2034-2044

Feng S, Cleary Y, Parrott N, Hu P, Weber C, Wang Y, Yin OQ, Shi J (2015) Evaluating a physiologically based pharmacokinetic model for prediction of omeprazole clearance and assessing ethnic sensitivity in CYP2C19 metabolic pathway. Eur J Clin Pharmacol 71:617-624

Feng S, Shi J, Parrott N, Hu P, Weber C, Martin-Facklam M, Saito T, Peck R (2016) Combining 'Bottom-Up' and 'Top-Down' Methods to Assess Ethnic Difference in Clearance: Bitopertin as an Example. Clin Pharmacokinet 55:823-832

Gerets HHJ, Tilmant K, Gerin B, Chanteux H, Depelchin BO, Dhalluin S, Atienzar FA (2012) Characterization of primary human hepatocytes, HepG2 cells, and HepaRG cells at the mRNA level and CYP activity in response to inducers and their predictivity for the detection of human hepatotoxins. Cell Biol Toxicol 28:69-87

Godoy P, Hewitt NJ, Albrecht U, Andersen ME, Ansari N, Bhattacharya S, Bode JG, Bolleyn J, Borner C, Bottger J, Braeuning A, Budinsky RA, Burkhardt B, Cameron NR, Camussi G, Cho CS, Choi YJ, Rowlands JC, Dahmen U, Damm G, Dirsch O, Donato MT, Dong J, Dooley S, Drasdo D, Eakins R, Ferreira KS, Fonsato V, Fraczek J, Gebhardt R, Gibson A, Glanemann M, Goldring CEP, GomezLechon MJ, Groothuis GMM, Gustavsson L, Guyot C, Hallifax D, Hammad S, Hayward A, Haussinger D, Hellerbrand C, Hewitt P, Hoehme S, Holzhutter HG, Houston JB, Hrach J, Ito K, Jaeschke H, Keitel V, Kelm JM, Park BK, Kordes C, Kullak-Ublick GA, LeCluyse EL, Lu P, Luebke-Wheeler J, Lutz A, Maltman DJ, Matz-Soja M, McMullen P, Merfort I, Messner S, Meyer C, Mwinyi J, Naisbitt DJ, Nussler AK, Olinga P, Pampaloni F, Pi JB, Pluta L, Przyborski SA, Ramachandran A, Rogiers V, Rowe C, Schelcher C, Schmich K, Schwarz M, Singh B, Stelzer EHK, Stieger B, Stober R, Sugiyama Y, Tetta C, Thasler WE, Vanhaecke T, Vinken M, Weiss TS, Widera A, Woods CG, Xu JJ, Yarborough KM, Hengstler JG (2013) Recent advances in 2D and 3D in vitro systems using primary hepatocytes, alternative hepatocyte sources and non-parenchymal liver cells and their use in investigating mechanisms of hepatotoxicity, cell signaling and ADME. Arch Toxicol 87:1315-1530 
Gomez-Lechon MJ, Castell JV, Donato MT (2008) An update on metabolism studies using human hepatocytes in primary culture. Expert Opin Drug Metab Toxicol 4:837-854

Gomez-Lechon MJ, Donato MT, Castell JV, Jover R (2003) Human hepatocytes as a tool for studying toxicity and drug metabolism. Curr Drug Metab 4:292-312

Gomez-Lechon MJ, Lahoz A, Gombau L, Castell JV, Donato MT (2010) In vitro evaluation of potential hepatotoxicity induced by drugs. Curr Pharm Des 16:1963-1977

Gomez-Lechon MJ, Tolosa L, Conde I, Donato MT (2014) Competency of different cell models to predict human hepatotoxic drugs. Expert Opin Drug Metab Toxicol 10:1553-1568

Gomez-Lechon MJ, Tolosa L, Donato MT (2016) Metabolic activation and drug-induced liver injury: in vitro approaches for the safety risk assessment of new drugs. J Appl Toxicol 36:752-768

Griffin C, Gosetto F, Danaher M, Sabatini S, Furey A (2014) Investigation of targeted pyrrolizidine alkaloids in traditional Chinese medicines and selected herbal teas sourced in Ireland using LC-ESI-MS/ MS. Food Addit Contam Part A Chem Anal Control Expo Risk Assess 31:940-961

Groothuis FA, Heringa MB, Nicol B, Hermens JLM, Blaauboer BJ, Kramer NI (2015) Dose metric considerations in in vitro assays to improve quantitative in vitro-in vivo dose extrapolations. Toxicology $332: 30-40$

Guo T, Mao GF, Xia DY, Su XY, Zhao LS (2011) Pharmacokinetics of midazolam tablet in different Chinese ethnic groups. J Clin Pharm Ther 36:406-411

Hassan A, Fontana RJ (2019) The diagnosis and management of idiosyncratic drug-induced liver injury. Liver Int 39:31-41

Hayes MA, Roberts E, Jago, MV., Safe SH, Farber E, Cameron RC (1984) Influences of various xenobiotic inducers on cytocidal toxicity of lasiocarpine and senecionine in primary cultures of rat hepatocytes. J Toxicol Environ Health 14:683-694

Herrmann K, Engst W, Appel KE, Monien BH, Glatt H (2012) Identification of human and murine sulfotransferases able to activate hydroxylated metabolites of methyleugenol to mutagens in Salmonella typhimurium and detection of associated DNA adducts using UPLC-MS/MS methods. Mutagenesis $27: 453-462$

Holmgren G, Sjögren A-K, Barragan I, Sabirsh A, Sartipy P, Synnergren J, Björquist P, IngelmanSundberg M, Andersson TB, Edsbagge J (2014) Long-Term Chronic Toxicity Testing Using Human Pluripotent Stem Cell-Derived Hepatocytes. Drug Metab Dispos 42:1401-1406

International Conference on Harmonisation (ICH) (1998) International Conference on Harmonisation: guidance on ethnic factors in the acceptability of foreign clinical data; availability--FDA. Notice. Fed Regist 63:31790-31796

International Programme on Chemical Safety (IPCS) (2005) Chemical-specific adjustment factors for interspecies differences and human variability : guidance document for use of data in dose/ concentration-response assessment. World Health Organization.

Jones HM, Gardner IB, Watson KJ (2009) Modelling and PBPK simulation in drug discovery. AAPS J $11: 155-166$ 
Jones HM, Parrott N, Jorga K, Lavé T (2006) A Novel Strategy for Physiologically Based Predictions of Human Pharmacokinetics. Clin Pharmacokinet 45:511-542

Kanebratt KP, Andersson TB (2008) Evaluation of HepaRG cells as an in vitro model for human drug metabolism studies. Drug Metab Dispos 36:1444-1452

Khetani SR, Kanchagar C, Ukairo O, Krzyzewski S, Moore A, Shi J, Aoyama S, Aleo M, Will Y (2013) Use of Micropatterned Cocultures to Detect Compounds That Cause Drug-Induced Liver Injury in Humans. Toxicol Sci 132:107-117

Kim K, Johnson JA, Derendorf H (2004) Differences in drug pharmacokinetics between east Asians and Caucasians and the role of genetic polymorphisms. J Clin Pharmacol 44:1083-1105

Klein S, Mueller D, Schevchenko V, Noor F (2014) Long-term maintenance of HepaRG cells in serumfree conditions and application in a repeated dose study. J Appl Toxicol 34:1078-1086

Kopp-Schneider A, Prieto P, Kinsner-Ovaskainen A, Stanzel S (2013) Design of a testing strategy using non-animal based test methods: Lessons learnt from the ACuteTox project. Toxicol In Vitro 27:1395-1401

Kunsman GW, Rohrig TP (1993) Tissue distribution of ibuprofen in a fatal overdose. Am J Forensic Med Pathol 14:48-50

Lambert CB, Spire C, Claude N, Guillouzo A (2009) Dose- and time-dependent effects of phenobarbital on gene expression profiling in human hepatoma HepaRG cells. Toxicol Appl Pharmacol 234:345-360

Lauschke VM, Hendriks DFG, Bell CC, Andersson TB, Ingelman-Sundberg M (2016) Novel 3D culture systems for studies of human liver function and assessments of the hepatotoxicity of drugs and drug candidates. Chem Res Toxicol 29: 1936-1955

Lee SJ, Bell DA, Coulter SJ, Ghanayem B, Goldstein JA (2005) Recombinant CYP3A4*17 is defective in metabolizing the hypertensive drug nifedipine, and the CYP3A4*17 allele may occur on the same chromosome as CYP3A5*3, representing a new putative defective CYP3A haplotype. J Pharmacol Exp Ther 313:302-309

Li GF, Yu G, Liu HX, Zheng QS (2014) Ethnic-specific in vitro-in vivo extrapolation and physiologically based pharmacokinetic approaches to predict cytochrome P450-mediated pharmacokinetics in the Chinese population: opportunities and challenges. Clin Pharmacokinet $53: 197-202$

Li J, Bluth MH (2011) Pharmacogenomics of drug metabolizing enzymes and transporters: implications for cancer therapy. Pharmgenomics Pers Med 4:11-33

Li N, Xia QS, Ruan JQ, Fu PP, Lin G (2011) Hepatotoxicity and Tumorigenicity Induced by Metabolic Activation of Pyrrolizidine Alkaloids in Herbs. Curr Drug Metab 12:823-834

Li ZS, Huang ZM (2006) Summary tables of the normal weight of internal organs of Chinese individuals across different age ranges. Guangxi Yi Ke Da Xue Xue Bao 23:155-156 (in Chinese)

Lin G, Wang JY, Li N, Li M, Gao H, Ji Y, Zhang F, Wang H, Zhou Y, Ye Y, Xu HX, Zheng J (2011) Hepatic sinusoidal obstruction syndrome associated with consumption of Gynura segetum. J Hepatol 54:666-673 
Lin JH (1995) Species similarities and differences in pharmacokinetics. Drug Metab Dispos 23:1008-1021

Liu FX, Salmon JW (2010) Herbal medicine regulation in China, Germany, and the United States. Integr Med 9:54-61

Louisse J, Bosgra S, Blaauboer BJ, Rietjens IMCM, Verwei M (2015) Prediction of in vivo developmental toxicity of all-trans-retinoic acid based on in vitro toxicity data and in silico physiologically based kinetic modeling. Arch Toxicol 89:1135-1148

Louisse J, de Jong E, van de Sandt JJ, Blaauboer BJ, Woutersen RA, Piersma AH, Rietjens IMCM, Verwei M (2010) The use of in vitro toxicity data and physiologically based kinetic modeling to predict dose-response curves for in vivo developmental toxicity of glycol ethers in rat and man. Toxicol Sci 118:470-484

Martati E, Boersma MG, Spenkelink A, Khadka DB, van Bladeren PJ, Rietjens IMCM, Punt A (2012) Physiologically based biokinetic (PBBK) modeling of safrole bioactivation and detoxification in humans as compared with rats. Toxicol Sci 128:301-316

McGinnity DF, Soars MG, Urbanowicz R, Riley RJ (2004) Evaluation of fresh and cryopreserved hepatocytes as in vitro drug metabolism tools for the prediction of metabolic clearance. Drug Metab Rev 36:106-106

McGraw J, Waller D (2012) Cytochrome P450 variations in different ethnic populations. Expert Opin Drug Metab Toxicol 8:371-382

Merz KH, Schrenk D (2016) Interim relative potency factors for the toxicological risk assessment of pyrrolizidine alkaloids in food and herbal medicines. Toxicol Lett 263:44-57

Mielke H, Partosch F, Gundert-Remy U (2019) Letter to the editor. Comment on 'Use of an in vitro-in silico testing strategy to predict inter-species and interethnic human diferences in liver toxicity of the pyrrolizidine alkaloids lasiocarpine and riddelliine' by Ning et al. Arch Toxicol

Mielke H, Di Consiglio E, Kreutz R, Partosch F, Testai E, Gundert-Remy U (2017) The importance of protein binding for the in vitro-in vivo extrapolation (IVIVE)-example of ibuprofen, a highly proteinbound substance. Arch Toxicol 91:1663-1670

Miranda CL, Reed RL, Guengerich FP, Buhler DR (1991) Role of cytochrome P450IIIA4 in the metabolism of the pyrrolizidine alkaloid senecionine in human liver. Carcinogenesis 12:515-519

Mueller D, Kramer L, Hoffmann E, Klein S, Noor F (2014) 3D organotypic HepaRG cultures as in vitro model for acute and repeated dose toxicity studies. Toxicol In Vitro 28:104-112

National Bureau of Statistics (NBS) (2018) Statistical Communiqué of the People's Republic of China on the 2017 National Economic and Social Development.

National Health and Family Planning Commission (NHFPC) (2007) Reference individuals for use in radiation protection-Part2: Masses of main organs and tissues. National Health and Family Planning Commission of the People's Republic of China (in Chinese) 
National Health and Family Planning Commission (NHFPC) (2014) Reference individiuals for use in radiation protection-Part3: main physiological parameters. vol GBZ/T 200.3. National Health and Family Planning Commission of the People's Republic of China (in Chinese)

Nolan JP, Scheig RL, Klatskin G (1966) Delayed hepatitis and cirrhosis in weanling rats following a single small dose of the senecio alkaloid, lasiocarpine. Am J Pathol 49:129-151

O'Brien PJ, Irwin W, Diaz D, Howard-Cofield E, Krejsa CM, Slaughter MR, Gao B, Kaludercic N, Angeline A, Bernardi P, Brain P, Hougham C (2006) High concordance of drug-induced human hepatotoxicity with in vitro cytotoxicity measured in a novel cell-based model using high content screening. Arch Toxicol 80:580-604

Paini A, Leonard JA, Joossens E, Bessems JGM, Desalegn A, Dorne JL, Gosling JP, Heringa MB, Klaric M, Kliment T, Kramer NI, Loizou G, Louisse J, Lumen A, Madden JC, Patterson EA, Proença S, Punt A, Setzer RW, Suciu N, Troutman J, Yoon M, Worth A, Tan YM (2019) Next generation physiologically based kinetic (NG-PBK) models in support of regulatory decision making. Comput Toxicol 9:61-72

Pelkonen O, Turpeinen M (2007) In vitro-in vivo extrapolation of hepatic clearance: biological tools, scaling factors, model assumptions and correct concentrations. Xenobiotica 37:1066-1089

Punt A, Paini A, Boersma MG, Freidig AP, Delatour T, Scholz G, Schilter B, Bladeren PJV, Rietjens IMCM (2009) Use of physiologically based biokinetic (PBBK) modeling to study estragole bioactivation and detoxification in humans as compared with male rats. Toxicol Sci 110:255-269

Punt A, Paini A, Spenkelink A, Scholz G, Schilter B, van Bladeren PJ, Rietjens IMCM (2016) Evaluation of Interindividual Human Variation in Bioactivation and DNA Adduct Formation of Estragole in Liver Predicted by Physiologically Based Kinetic/Dynamic and Monte Carlo Modeling. Chem Res Toxicol 29:659-668

Qiu F, Liu S, Miao P, Zeng J, Zhu L, Zhao T, Ye Y, Jiang J (2016) Effects of the Chinese herbal formula "Zuojin Pill" on the pharmacokinetics of dextromethorphan in healthy Chinese volunteers with CYP2D6*10 genotype. Eur J Clin Pharmacol 72:689-695

Ruan J, Gao H, Li N, Xue J, Chen J, Ke C, Ye Y, Fu PP-C, Zheng J, Wang J, Lin G (2015) Blood Pyrrole-Protein Adducts-A Biomarker of Pyrrolizidine Alkaloid-Induced Liver Injury in Humans. J Environ Sci Health C Environ Carcinog Ecotoxicol Rev, Part C 33:404-421

Sata F, Sapone A, Elizondo G, Stocker P, Miller VP, Zheng W, Raunio H, Crespi CL, Gonzalez FJ (2000) CYP3A4 allelic variants with amino acid substitutions in exons 7 and 12: evidence for an allelic variant with altered catalytic activity. Clin Pharmacol Ther 67:48-56

Schmitt W (2008) General approach for the calculation of tissue to plasma partition coefficients. Toxicol In Vitro 22:457-467

Shi ZR, Yan LN, Li B, Wen TF (2009) Evaluation of standard liver volume formulae for Chinese adults. World J Gastroenterol 15:4062-4066

Shu Y, Cheng ZN, Liu ZQ, Wang LS, Zhu B, Huang SL, Ou-Yang DS, Zhou HH (2001) Interindividual variations in levels and activities of cytochrome P-450 in liver microsomes of Chinese subjects. Acta Pharmacol Sin 22:283-288 
Sison-Young RL, Lauschke VM, Johann E, Alexandre E, Antherieu S, Aerts H, Gerets HHJ, Labbe G, Hoet D, Dorau M, Schofield CA, Lovatt CA, Holder JC, Stahl SH, Richert L, Kitteringham NR, Jones RP, Elmasry M, Weaver RJ, Hewitt PG, Ingelman-Sundberg M, Goldring CE, Park BK (2017) A multicenter assessment of single-cell models aligned to standard measures of cell health for prediction of acute hepatotoxicity. Arch Toxicol 91:1385-1400

Smith CM, Nolan CK, Edwards MA, Hatfield JB, Stewart TW, Ferguson SS, Lecluyse EL, Sahi J (2012) A comprehensive evaluation of metabolic activity and intrinsic clearance in suspensions and monolayer cultures of cryopreserved primary human hepatocytes. J Pharm Sci 101:3989-4002

Soldatow VY, Lecluyse EL, Griffith LG, Rusyn I (2013) In vitro models for liver toxicity testing. Toxicol Res (Camb) 2:23-39

Strikwold M, Spenkelink B, de Haan LHJ, Woutersen RA, Punt A, Rietjens IMCM (2017) Integrating in vitro data and physiologically based kinetic (PBK) modelling to assess the in vivo potential developmental toxicity of a series of phenols. Arch Toxicol 91:2119-2133

Strikwold M, Spenkelink B, Woutersen RA, Rietjens IMCM, Punt A (2013) Combining in vitro embryotoxicity data with physiologically based kinetic (PBK) modelling to define in vivo dose-response curves for developmental toxicity of phenol in rat and human. Arch Toxicol 87:1709-1723

Tang J, Ji H, Shi J, Wu L (2016) Ephedra water decoction and cough tablets containing ephedra and liquorice induce CYP1A2 but not CYP2E1 hepatic enzymes in rats. Xenobiotica 46:141-146

Teschke R (2014) Traditional Chinese Medicine Induced Liver Injury. J Clin Transl Hepatol 2:80-94

Van Den Berg SJ, Punt A, Soffers AE, Vervoort J, Ngeleja S, Spenkelink B, Rietjens IMCM (2012) Physiologically based kinetic models for the alkenylbenzene elemicin in rat and human and possible implications for risk assessment. Chem Res Toxicol 25:2352-2367

Van Den Berg SJ, Restani P, Boersma MG, Delmulle L, Rietjens IMCM (2011) Levels of genotoxic and carcinogenic compounds in plant food supplements and associated risk assessment. Food Sci Nutr 2:989-1010

Wang JX (1998) Organ mass reference values of Chinese. In: Wang JX, Chen RS, editors. Data of anatomical physiological and metabolic characteristics for Chinese reference man. Beijing: Atomic Energy Press 21-28 (in Chinese)

Wang D, Guo Y, Wrighton SA, Cooke GE, Sadee W (2011) Intronic polymorphism in CYP3A4 affects hepatic expression and response to statin drugs. Pharmacogenomics J 11:274-286

Wang HY, Chen X, Jiang J, Shi J, Hu P (2016) Evaluating a physiologically based pharmacokinetic model for predicting the pharmacokinetics of midazolam in Chinese after oral administration. Acta Pharmacol Sin 37:276-284

Wang JX, Li BX, Chen RS (1995) Reference values of main internal organs for Chinese. Zhonghua Fang She Yi Xue Yu Fang Hu Za Zhi 15:248-254 (in Chinese)

Werk AN, Lefeldt S, Bruckmueller H, Hemmrich-Stanisak G, Franke A, Roos M, Kuchle C, Steubl D, Schmaderer C, Brasen JH, Heemann U, Cascorbi I, Renders L (2014) Identification and characterization of a defective CYP3A4 genotype in a kidney transplant patient with severely diminished tacrolimus clearance. Clin Pharmacol Ther 95:416-422 
Westlind-Johnsson A, Hermann R, Huennemeyer A, Hauns B, Lahu G, Nassr N, Zech K, IngelmanSundberg M, von Richter O (2006) Identification and characterization of CYP3A4*20, a novel rare CYP3A4 allele without functional activity. Clin Pharmacol Ther 79:339-349

Wilmes A, Bielow C, Ranninger C, Bellwon P, Aschauer L, Limonciel A, Chassaigne H, Kristl T, Aiche S, Huber CG, Guillou C, Hewitt P, Leonard MO, Dekant W, Bois F, Jennings P (2015) Mechanism of cisplatin proximal tubule toxicity revealed by integrating transcriptomics, proteomics, metabolomics and biokinetics. Toxicol In Vitro (1 Pt A):117-127

Xia Q, Chou MW, Kadlubar FF, Chan PC, Fu PP (2003) Human liver microsomal metabolism and DNA adduct formation of the tumorigenic pyrrolizidine alkaloid, riddelliine. Chem Res Toxicol 16:66-73

Xia Q, Zhao Y, Von Tungeln LS, Doerge DR, Lin G, Cai L, Fu PP (2013) Pyrrolizidine alkaloid-derived DNA adducts as a common biological biomarker of pyrrolizidine alkaloid-induced tumorigenicity. Chem Res Toxicol 26:1384-1396

Xiao J, Zheng Y, Zhou Y, Zhang P, Wang J, Shen F, Fan L, Kolluri VK, Wang W, Yan X, Wang M (2014) Sulfotransferase SULT1A1 Arg213His polymorphism with cancer risk: a meta-analysis of 53 case-control studies. PLoS One 9:e106774

Yang JL, He MM, Niu W, Wrighton SA, Li L, Liu Y, Li C (2012) Metabolic capabilities of cytochrome P450 enzymes in Chinese liver microsomes compared with those in Caucasian liver microsomes. Brit J Clin Pharmaco 73:268-284

Yang L, Guo T, Xia DY, Zhao LS (2012) Pharmacokinetics of losartan and its active carboxylic acid metabolite E-3174 in five ethnic populations of China. J Clin Pharm Ther 37:226-231

Yuan D, Lu T, Wei YG, Li B, Yan LN, Zeng Y, Wen TF, Zhao JC (2008) Estimation of standard liver volume for liver transplantation in the Chinese population. Transplant Proc 40:3536-3540

Zhao Y, Xia Q, Gamboa da Costa G, Yu H, Cai L, Fu PP (2012) Full Structure Assignments of Pyrrolizidine Alkaloid DNA Adducts and Mechanism of Tumor Initiation. Chem Res Toxicol 25:1985-1996

Zhou HH, Adedoyin A, Wilkinson GR (1990) Differences in Plasma-Binding of Drugs between Caucasians and Chinese Subjects. Clin Pharmacol Ther 48:10-17 

Summary

Acknowledgements

About the author

List of publications

Overview of complèted training activities
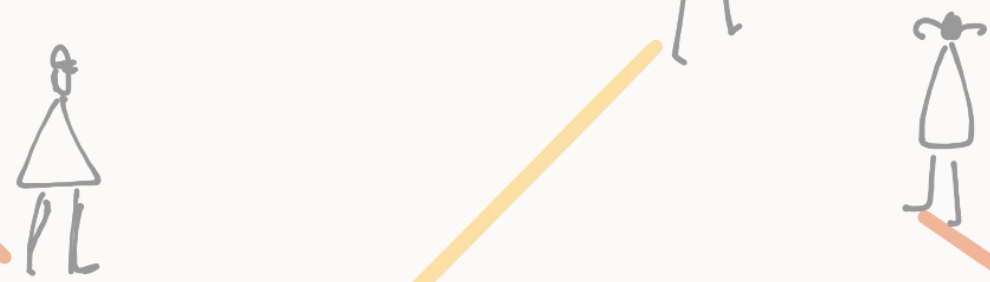


\section{Summary}

The aim of the present thesis was to perform the risk and safety assessment of two groups of natural food-borne toxins, namely alkenylbenzenes and pyrrolizidine alkaloids (PAs) for the Chinese population as compared to the overall Caucasian population using methods including the Margin of Exposure (MOE) approach, physiologically based kinetic (PBK) modelling-based reverse dosimetry and the combination of PBK modelling and Monte Carlo simulation.

Chapter 1 introduced the model compounds studied in the thesis, namely alkenylbenzenes and 1,2-unsaturated PAs, the aim of the thesis and the strategies applied to perform a mode of action based safety and risk assessment. The regulations and consumer behaviour towards botanical preparations in China and in several EU countries were also presented.

In chapter 2, a risk assessment of 10 plant food supplements (PFS), 23 traditional Chinese medicines (TCM) and 38 herbal teas containing alkenylbenzenes obtained at the Chinese market was performed using the Margin of Exposure (MOE) approach. Use of about half of the samples would result in estimated intakes upon daily life-time exposure that give rise to MOE values lower than 10 000, suggesting a potential priority for risk management. For shortterm exposure such as two weeks consumption, applying Haber's rule, only one TCM (TCM 6) still had a MOE value below 10 000. It is concluded that consumption of Chinese botanical preparations raise a concern because of exposure to alkenylbenzenes, especially when exposure is for longer periods of time.

Chapter 3 studied the differences in bioactivation and detoxification of the food-borne genotoxic carcinogen estragole between Chinese and Caucasians using a mode of action based PBK modelling approach. The outcomes of the model predictions showed that the detoxification of the proximate carcinogenic metabolite 1'-hydroxyestragole, mainly by conversion to 1'-oxoestragole, was similar in both ethnic groups. For the bioactivation pathway, at realistic daily intakes of estragole, in the Chinese population only $0.02 \%$ of the dose was converted to the ultimate carcinogenic metabolite 1'-sulfooxyestragole, while this value amounted to $0.09 \%$ of the dose in Caucasian subjects. This 4.5 -fold difference accompanied by similar rates of detoxification may indicate a lower risk of estragole for the Chinese population at similar levels of exposure.

In chapter 4, it was demonstrated that combining in vitro cytotoxicity data obtained with primary rat hepatocytes with PBK modelling-based reverse dosimetry could adequately predict in vivo acute liver toxicity of lasiocarpine and riddelliine for rats. The PBK models for rats for lasiocarpine and riddelliine were developed based on data derived from in silico approaches, incubation experiments using subcellular tissue fractions and literature to define the PBK model parameters. Concentration-response curves obtained from in vitro cytotoxicity assays in primary rat hepatocytes were converted to in vivo dose-response curves for acute liver toxicity by PBK-modelling based reverse dosimetry. From the in vivo dose-response curves thus obtained points of departure (PoDs) were derived that were compared to available literature data on in vivo liver toxicity of lasiocarpine. The predicted PoDs appeared to fall well within the range of PoDs obtained from the available oral single 
PoDs appeared to fall well within the range of PoDs obtained from the available oral single dose in vivo studies. In conclusion, this chapter showed that PBK modelling based-reverse dosimetry can translate in vitro concentration-response curves to in vivo dose-response curves to predict the acute liver toxicity of lasiocarpine and riddelliine in rats.

Chapter 5 investigated the inter-species and inter-ethnic human differences in acute liver toxicity of lasiocarpine and riddelliine using the approach developed in chapter 4 for rats. Thus, PBK models for lasiocarpine and riddelliine for the average Chinese and Caucasian were developed, based on the PBK models defined and validated for these PAs in rats (chapter 4). Subsequently, the models were used to convert in vitro toxicity data obtained in pooled Caucasian primary hepatocytes to predict in vivo dose-response curves for acute liver toxicity of lasiocarpine and riddelliine in humans, from which PoDs were derived that were compared to PoDs derived in a similar manner for rats in chapter 4 to obtain insight in interspecies differences. Similarly, the PoDs obtained for the average Chinese and Caucasian were compared to provide insights in inter-ethnic differences. The inter-species differences amounted to 2-fold for lasiocarpine and 8.2-fold for riddelliine with humans being more sensitive than rats. The inter-ethnic human differences varied 2-fold for lasiocarpine and 5fold for riddelliine with the average Caucasian being more sensitive than the average Chinese. Altogether, this chapter shows a proof-of-principle for a method to predict inter-species and inter-ethnic differences in in vivo liver toxicity for PAs by an alternative testing strategy integrating in vitro cytotoxicity assays with PBK modelling-based reverse dosimetry.

Chapter 6 investigated the effect of inter-individual and inter-ethnic (Chinese and Caucasian) kinetic differences in bioactivation of lasiocarpine, and definition of chemical specific adjustment factors (CSAFs) by applying PBK modelling and Monte Carlo simulation. The results revealed an inter-ethnic variation of 2.1-, 3.3- and 4.3-fold when comparing the predicted 7-GS-DHP formation at the GM, 90th and 99th percentile of the Chinese and Caucasian population, respectively, at a dose of level of $8 \mathrm{ng} / \mathrm{kg}$ bw lasiocarpine, indicating that the Caucasian population was more sensitive. The CSAFs obtained based on the GM and 90 th percentile individuals of the Chinese, Caucasian and the two populations combined were 3.3, 5.2 and 5.7, respectively. The CSAFs obtained based on the GM and the 99 ${ }^{\text {th }}$ percentile were 8.3, 17.0 and 19.5 for inter-individual variations in the Chinese, the Caucasian population and the two populations combined, respectively. The CSAF values obtained in this chapter indicate that the default safety factor of 3.16 for inter-individual human kinetic differences may not be sufficiently protective for any of the populations.

Chapter 7 presents the general discussion based on the results of the present thesis placing the results in a wider perspective while also presenting future perspectives. It is concluded that the present thesis showed an alternative to animal testing approach to define in vivo doseresponse curves for risk assessment, based on in silico and in vitro data. It was demonstrated that combining in vitro toxicity data with the kinetic processes integrated into a PBK model adequately predicts in vivo acute liver toxicity of PAs. The obtained results show the possibility to use the method to provide a PoD to perform risk assessment or to define CSAFs for inter-species and intra-species differences. Additionally, this thesis provided insight in the 
possibilities to build ethnic-specific PBK models that can be used to further refine the risk assessment of compounds that are genotoxic and carcinogenic and also have non-genotoxic endpoints. Moreover, this thesis illustrated that there is a potential priority for risk management for several botanical preparations containing alkenylbenzenes or PAs for the Chinese population when considering daily life-long exposure. Altogether, this thesis provided a proof-of-principle to perform a risk and safety assessment for food-borne toxins based on only in vitro and in silico data. 


\section{Acknowledgements}

First, I would like to express my sincere gratitude to my promotor Prof. Ivonne Rietjens for her support, supervision and knowledge. I have learned a lot from you, not only from the scientific perspective, but also from your attitude towards life, which motivated me a lot especially when I encountered some difficulties during my $\mathrm{PhD}$. You are very efficient, highly disciplined and a hard smart-working person. I really appreciated your quick response to my thesis and papers especially during the last stretch of my $\mathrm{PhD}$. Without your help and guidance, I do not think I would have been able to make everything happened (start from CSC application until finalizing my thesis).

I would like to thank as well to my co-promotor Marije Strikwold. Your guidance, critical comments, precision for science encouraged me to keep going on. Although sometimes I was a bit of shocked and stressful by your perfectionism, particularly during the last stretch when I even considered postponing my defence date, your courage and dedicated support encouraged me to carry on further. I am very grateful for the time you dedicated to work on my project, especially after working hours and even during your holidays. I still remember that we discussed how to revise my last experimental chapter during the last Easter holiday. I've really learned a lot from you, especially in computer modelling.

I want to express my gratitude to Jochem Louisse for being part of my supervisory committee and helping me during the first stage of this journey. It was a pleasure to work with you. I also want to thank Bert Spenkelink and Sebas Wesseling, without your dedicated technical support, I would not have been able to finalize my experiments on time. I appreciate the many interesting conversions we had and the personals interests we shared, you both made me feel relax when I was busy doing experiments in the lab. To Lidy, thank you for helping me out with the administrative things. Thank you Ixchel and Suparmi for being my paranymphs. I also would like to thank all the TOX colleagues for the nice working atmosphere: Annelies, Artem, Ashraf, Aziza, Ben, BiYao, BoHan, Chen, DanLei, Diego, Diana, Felicia, Georgia, Hans Bouwmeester, Hans van den Berg, Ignacio, Isaac, JingFang, JingJin, Karsten, Katja, Korphimol, Laura, Lenny, Letty, Lu, Nico, Marta, Menno, MengYing, MiaoYing, Mebra, QianRui, ShenSheng, Shuo, WeiJia. To all Msc students, SiJin, Riana, XiangNan, XinYue, YiFei I am deeply grateful for your interest and contributions to my project.

All the way from the Netherlands to China, I would like to thank my friends there for their support and encouragement throughout my PhD. To my parents and grandparents, a special acknowledgment for their love, support and understanding. Last but not least, I want to give many thanks to Xiao. Thank you for your support, encouragement and patience. I feel lucky that I met you in Wageningen. I am happy that we have similar hobbies and share similar values about life. I think the best from our relationship is that we can improve together and I am looking forward to the next stage of our life together. 


\begin{abstract}
About the author
Jia Ning was born on February 17th 1991 in Beijing, China. After completing her bachelor degree in HuNan University, China in 2013, she came to Wageningen University in the Netherlands for her master degree in food safety. During her master, she performed her master thesis at Division of Toxicology on the topic of risk assessment of PFS containing myristicin. She subsequently finished her internship in Dutch National Institute for Public Health and Environment (RIVM) on the topic of inflammasome activation by nanomaterials. From 2015, she started her PhD at the Division of Toxicology under supervision of Prof. Dr Ivonne M.C.M. Rietjens. During her $\mathrm{PhD}$ study, she followed a postgraduate education program in Toxicology, which enables her to register as an European Toxicologist.
\end{abstract}




\section{LIST OF PUBLICATIONS}

- $\quad$ Ning J, Cui XY, Kong XN, Tang YF, Wulandari R, Chen L, Wesseling S, Rietjens IMCM (2018) Risk assessment of genotoxic and carcinogenic alkenylbenzenes in botanical containing products present on the Chinese market. Food and Chemical Toxicology $115: 344-357$

- $\quad$ Ning J, Louisse J, Spenkelink B, Wesseling S, Rietjens IMCM (2017) Study on interethnic human differences in bioactivation and detoxification of estragole using physiologically based kinetic modeling. Archives of Toxicology 91:3093-3108

- $\quad$ Ning J, Chen L, Strikwold M, Louisse J, Wesseling S, Rietjens IMCM (2019) Use of an in vitro-in silico testing strategy to predict inter-species and inter-ethnic human differences in liver toxicity of the pyrrolizidine alkaloids lasiocarpine and riddelliine. Archives of Toxicology 93:801-818

- Ning J, Rietjens IMCM, Strikwold M (2019) Evaluation of the interindividual and interethnic variation in lasiocarpine induced liver toxicity by physiologically based kinetic (PBK) and Monte Carlo modelling (Submitted)

- Ning J, Chen L, Rietjens IMCM (2019) Role of toxicokinetics and alternative testing strategies in pyrrolizidine alkaloid toxicity and risk assessment. Food and Chemical Toxicology 131

- Chen L, Ning J, Louisse J, Wesseling S, Rietjens IMCM (2018) Use of physiologically based kinetic modelling-facilitated reverse dosimetry to convert in vitro cytotoxicity data to predicted in vivo liver toxicity of lasiocarpine and riddelliine in rat. Food and Chemical Toxicology 116:216-226

- $\quad$ Rietjens IMCM, Ning J, Chen L, Wesseling S, Strikwold M, Louisse J (2019) Selecting the dose metric in reverse dosimetry based QIVIVE. Archives of Toxicology. https:// doi.org/10.1007/s00204-019-02438-1

- Al-Malahmeh AJ, Alajlouni AM, Ning J, Wesseling S, Vervoort J, Rietjens IMCM (2017) Determination and risk assessment of naturally occurring genotoxic and carcinogenic alkenylbenzenes in nutmeg-based plant food supplements. Journal of Applied Toxicology 37:1254-1264 


\section{Overview of completed training activities}

\section{Discipline specific activities}

$\begin{array}{lll}\text { Molecular toxicology } & \text { PET } & 2016 \\ \text { Pathobiology } & \text { PET } & 2016 \\ \text { Epidemiology } & \text { PET } & 2016 \\ \text { Introduction Laboratory Animal science } & \text { PET } & 2016 \\ \text { Cell Toxicology } & \text { PET } & 2018 \\ \text { Organ toxicology } & \text { PET } & 2017 \\ \text { reproductive toxicology } & \text { PET } & 2016 \\ \text { Legal and regulatory toxicology } & \text { PET } & 2018\end{array}$

\section{Conferences}

Dutch Society of Toxicology (NVT) annual meeting, Doorn, the Netherlands, poster, 2017

Dutch Society of Toxicology (NVT) annual meeting, Hilversum, the Netherlands, poster, 2018

PA workshop, Kaiserslautern University, Germany, 2018

\section{General courses}

VLAG PhD week

Project and time management

Scientific Publishing

Brain Training

$\mathrm{PhD}$ peer consultation

Information Literacy including Endnote Introduction

Reviewing a scientific paper

Brain-friendly working \& writing

$\begin{array}{ll}\text { VLAG } & 2016 \\ \text { WGS } & 2017 \\ \text { WGS } & 2016 \\ \text { WGS } & 2016 \\ \text { WGS } & 2017 \\ \text { WGS } & 2017 \\ \text { WGS } & 2016 \\ \text { WGS } & 2018\end{array}$

\section{Optional}

$\begin{array}{ll}\text { WUR } & 2015 \\ \text { WUR } & 2016 \\ \text { WUR } & 2017 \\ \text { WUR } & 2018\end{array}$


The research described in this thesis was financially supported by the China Scholarship Council (No. 201507720019 to NING JIA)

Financial support from Wageningen University \& Research for printing the thesis is gratefully appreciated

Cover \& layout design: Jia Ning

Printed by: Ridderprint B.V. | Ridderkerk 